
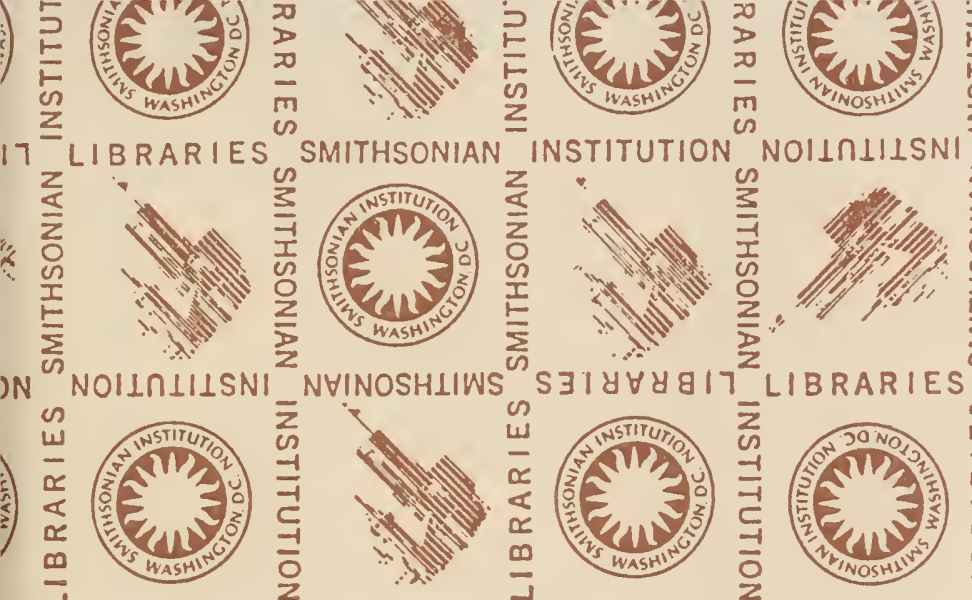

$\sum_{n}$
-1
$\overline{-1}$
$c$
$\bar{c}$
$\overline{0}$
$z$
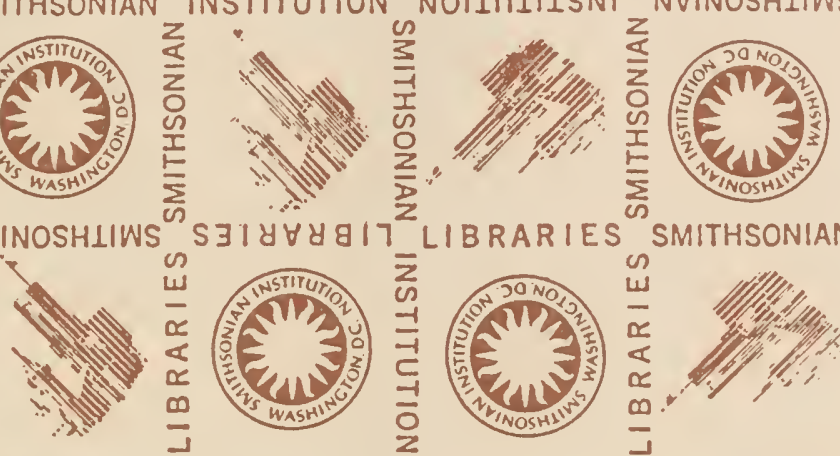

os

S $\exists I \forall \forall \forall$
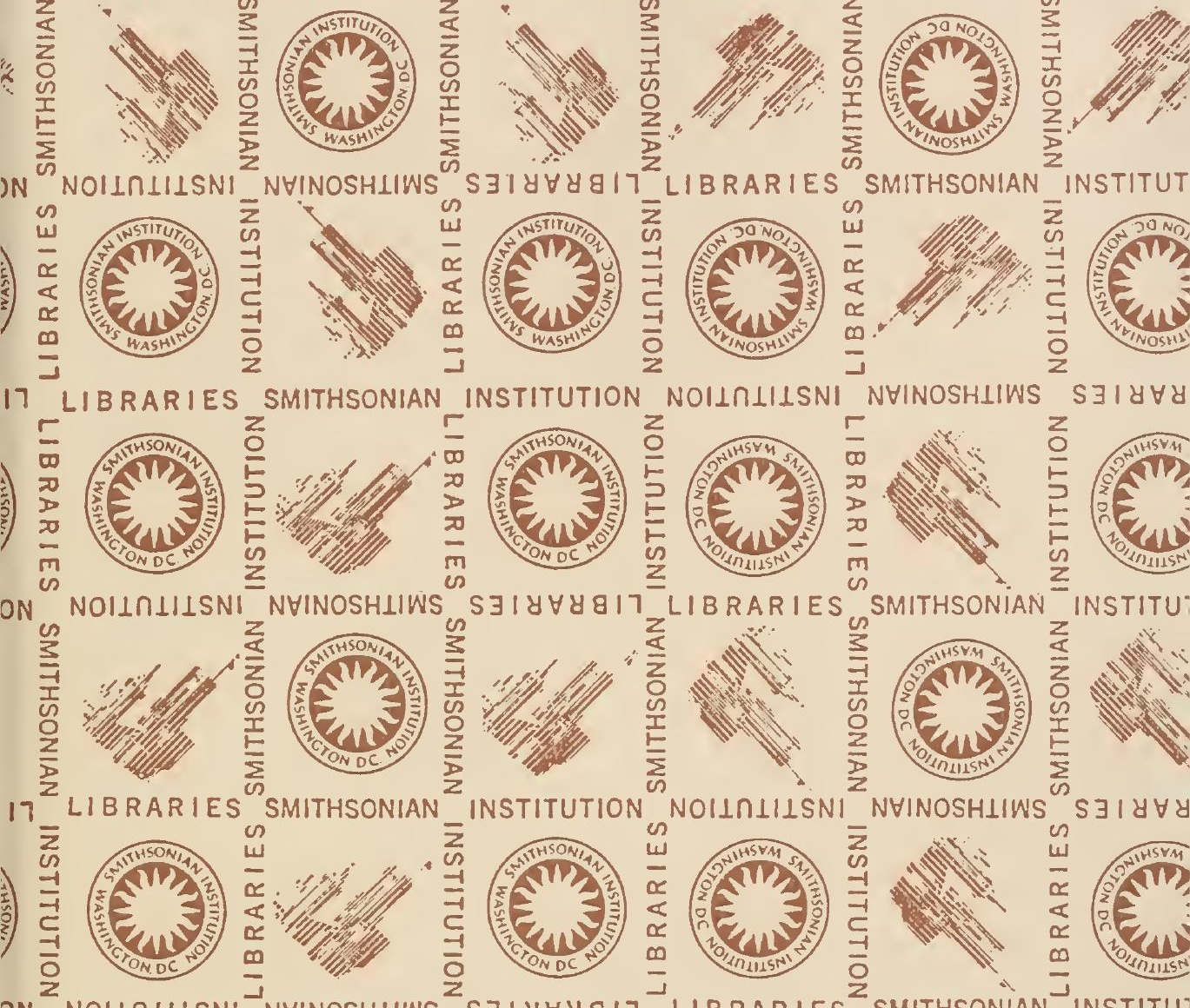

INSTITU
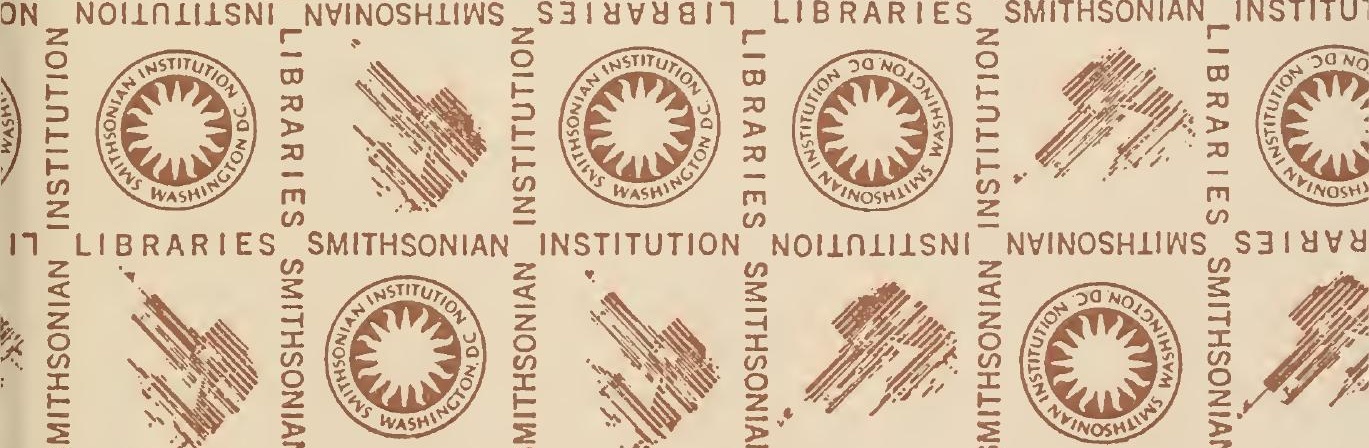

LIBRARIES SMITHSONIAN
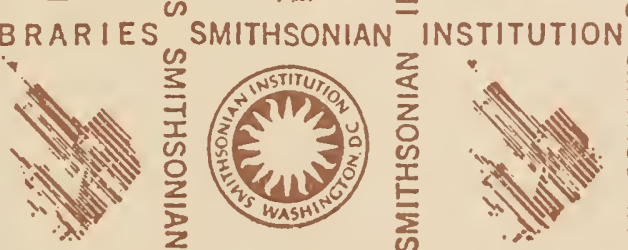

N NOIINIILSNI

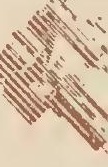

$S \exists I \forall \forall y$
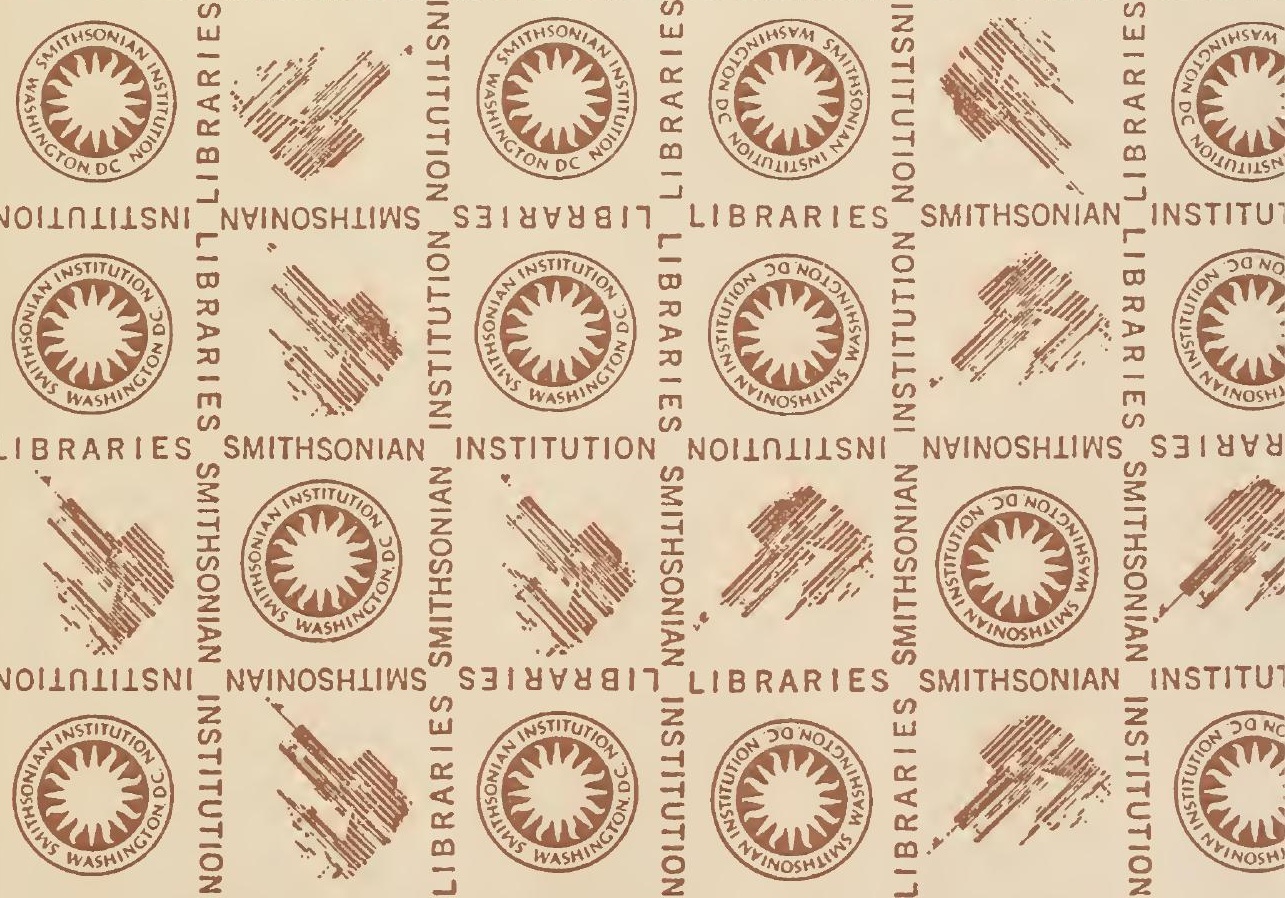

SMITHSONIAN

INSTITU

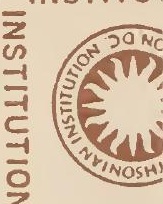

LIBRARIES SMITHSONIAN INSTITUTION
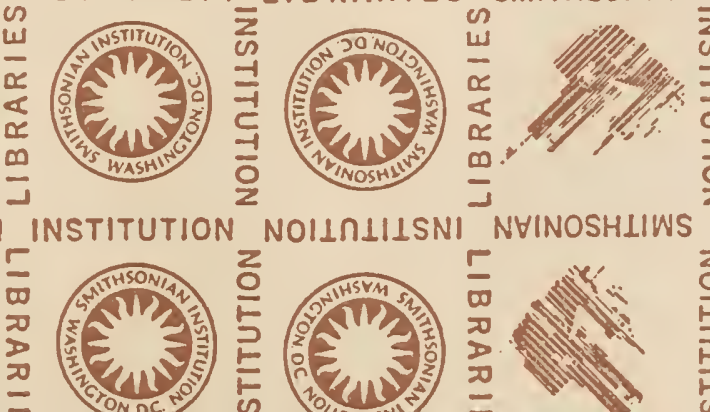

D

$S \exists I \forall \forall \forall$
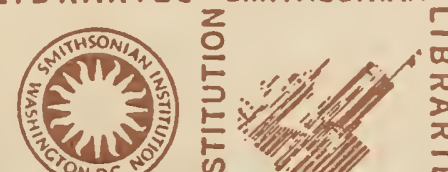



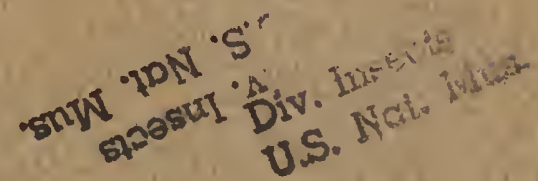

\title{
CHECK-LIST OF THE
}

INSECTS OF CONNECTICUT

\author{
By
}

WILTON EVERETT BRITTON, Ph.D.

State Entomologist and

Entomologist of the Agricultural Experiment Station, New Haven, Conn.

HARTFORD

Published by the State 1920 



\section{B ULLETINS}

OF THE

\section{State Geological and Natural History Survey of Connecticut}

I. First Biennial Report of the Commissioners of the State Geological and Natural History Survey, Igo3-I904.

2. A Preliminary Report on the Protozoa of the Fresh Waters of Connecticut: by Herbert William Conn. (Out of print. To be obtained only in Vol. I, containing Bulletins I-5. Price \$I.5O, postpaid.)

3. A Preliminary Report on the Hymeniales of Connecticut: by Edward Albert White.

4. The Clays and Clay Industries of Connecticut: by Gerald Francis Loughlin.

5. The Ustilagineæ, or Sniuts, of Connecticut: by George Perkins Clinton.

6. Manual of the Geology of Connecticut: by William North Rice and Herbert Ernest Gregory. (Out of print. To be obtained only in Vol. II, containing Bulletins 6-I2. Price \$2.45. postpaid.)

7. Preliminary Geological Map of Connecticut: by Herbert Ernest Gregory and Henry Hollister Robinson.

8. Bibliography of Connecticut Geology : by Herbert Ernest Gregory.

9. Second Biennial Report of the Commissioners of the State Geological and Natural History Survey, 1905-1906.

10. A Preliminary Report on the Algæ of the Fresh Waters of Connecticut: by Herbert William Conn and Lucia Washburn (Hazen) Webster.

II. The Bryophytes of Connecticut: by Alexander William Evans and George Elwood Nichols.

12. Third Biennial Report of the Commissioners of the State Geological and Natural History Survey, I907-I908.

I3. The Lithology of Connecticut: by Joseph Barrell and Gerald Francis Loughlin.

I4. Catalogue of the Flowering Plants and Ferns of Connecticut growing without cultivation : by a Committee of the Connecticut Botanical Society. 
I5. Second Report on the Hymeniales of Connecticut: by Edward Albert White.

I6. Guide to the Insects of Connecticut: prepared under the direction of Wilton Everett Britton. Part I. General Introduction: by Wilton Everett Britton. Part II. The Euplexoptera and Orthoptera of Connecticut: by Benjamin Hovey Walden.

17. Fourth Biennial Report of the Commissioners of the State Geological and Natural History Survey, I909-Io.

I8. Triassic Fishes of Connecticut: by Charles Rochester Eastman.

19. Echinoderms of Connecticut: by Wesley Roscoe Coe.

20. The Birds of Connecticut: by John Hall Sage and Louis Bennett Bishop, assisted by Walter Parks Bliss.

21. Fifth Biennial Report of the Commissioners of the State Geological and Natural History Survey, I9II-I9I2.

22. Guide to the Insects of Connecticut: prepared under the direction of Wilton Everett Britton. Part III. The Hymenoptera, or Wasp-like Insects, of Connecticut : by Henry Lorenz Viereck, with the collaboration of Alexander Dyer MacGillivray, Charles Thomas Brues, William Morton Wheeler, and Sievert Allen Rohwer.

23. Central Connecticut in the Geologic Past: by Joseph Barrell.

24. Triassic Life of the Connecticut Valley: by Richard Swann Lull.

25. Sixth Biennial Report of the Commissioners of the State Geological and Natural History Survey, I9I3-I9I4.

26. The Arthrostraca of Connecticut: by Beverly Waugh Kunkel.

27. Seventh Biennial Report of the Commissioners of the State Geological and Natural History Survey, I9I5-19I6.

28. Eighth Biennial Report of the Commissioners of the State Geological and Natural History Survey, I917-I918.

29. The Quaternary Geology of the New Haven Region, Connecticut: by Freeman Ward, Ph.D.

30. Drainage, Modification and Glaciation in the Danbury Region, Connecticut: by Ruth Sawyer Harvey, Ph.D.

3I. Check List of the Insects of Connecticut: by Wilton Everett Britton, Ph.D.

32. Ninth Biennial Report of the Commissioners of the State Geological and Natural History Survey, I9I9-1920. 
Bulletins I, 9, I2, I 7, 21, 25, 27, 28 and 32 are merely administrative reports containing no scientific matter. The other bulletins may be classified as follows:

Geology: Bullétins 4, 6, 7, 8, I3, 18, 23, 24, 29, 30.

Botany: Bulletins 3, 5, IO, I I, I 4, I5.

Zoölogy: Bulletins 2, 16, 19, 20, 22, 26, 31.

These bulletins are sold and otherwise distributed by the State Librarian. Postage, when bulletins are sent by mail, is as follows:

$\begin{array}{rrrrrr}\text { No. I } & \text { \$0.0I } & \text { No. I3 } & \text { \$0.08 } & \text { No. } 23 & \text { \$0.03 } \\ 3 & .08 & \text { I4 } & .08 & 24 & .10 \\ 4 & .06 & \text { I5 } & .06 & 25 & .02 \\ 5 & .03 & \text { I6 } & .07 & 26 & .06 \\ 7 & .06 & \text { I7 } & .02 & 27 & .02 \\ 8 & .05 & \text { I8 } & .07 & 28 & .02 \\ 9 & .02 & 19 & .08 & 29 & .03 \\ 10 & .08 & 20 & .14 & 30 & .03 \\ 11 & .07 & 21 & .02 & 31 & .06 \\ \text { I2 } & .02 & 22 & .08 & 32 & .02\end{array}$

The prices when the bulletins are sold are as follows, postpaid :

\begin{tabular}{|c|c|c|c|c|c|}
\hline I & $\$ 0.05$ & No. I3 & $\$ 0.40$ & No. 23 & \$o.I 5 \\
\hline 3 & .40 & I 4 & .75 & 24 & .65 \\
\hline 4 & .30 & I 5 & .35 & 25 & .05 \\
\hline 5 & .15 & I6 & .35 & 26 & .80 \\
\hline 7 & .60 & I7 & .05 & 27 & .05 \\
\hline 8 & .20 & I8 & .25 & 28 & .05 \\
\hline 9 & .05 & I9 &.+5 & 29 & .50 \\
\hline IO & .35 & 20 & .50 & 30 & .45 \\
\hline I I & .30 & $2 \mathrm{I}$ & .05 & $3^{I}$ & 1.50 \\
\hline 12 & .05 & 22 & 2.00 & 32 & .05 \\
\hline
\end{tabular}

A part of the edition of these Bulletins have been assembled in volumes substantially bound in cloth, plainly lettered, and sell for the following prices, postpaid:

Volume $\quad I$, containing Bulletins $I-5$

$\$ 1.50$

Volume II, containing Bulletins 6-I2

2.45

Volume III, containing Bulletins I3-I5

2.50

Volume IV, containing Bulletins I6-2I

2.15

Volume V, containing Bulletin 22

2.50

It is intended to follow a liberal policy in gratuitously distributing these publications to public libraries, colleges, and 
scientific institutions, and to scientific men, teachers, and others who require particular bulletins for their work, especially to those who are citizens of Connecticut.

Applications or inquiries should be addressed to

GEORGE S. GODARD,

State Librarian, Hartford, Conn.

In addition to the bulletins above named, published by the State survey, attention is called to three publications of the United States Geological Survey prepared in co-operation witl the Geological and Natural History Survey of Connecticut These are the following:

Bulletin 484. The Granites of Connecticut: by T. Nelson Dale and Herbert E. Gregory.

Water-Supply Paper 374. Ground Water in the Hartford, Stamford, Salisbury, Willimantic and Saybrook Areas, Connecticut: by Herbert E. Gregory and Arthur J. Ellis.

Water-Supply Paper 397. Ground Water in the Waterbury Area, Connecticut: by Arthur J. Ellis, under the direction of Herbert E. Gregory.

These papers may be obtained from the Director of the United States Geological Survey at Washington. 


\section{CATALOGUE SLIPS.}

Connecticut. State geological and natural history survey. Bulletin no. 31. Check-list of the insects of Connecticut. By Wilton Everett Britton. Hartford, 1920.

397 pp., $23^{\mathrm{cm}}$.

Britton, Wilton Everett.

Check-list of the insects of Connecticut. By Wilton Everett Britton. Hartford, 1920.

$397 \mathrm{pp} ., 23^{\mathrm{cm}}$

(Bulletin no. 31, Connecticut geological and natural history survey.) 



\section{CATALOGUE SLIPS.}

\section{Zoology.}

Britton, W. E. Check-list of the insects of Connecticut. Hartford, 1920.

397 pp., $23^{\mathrm{cm}}$.

(Bulletin 110. 31, Connecticut greological and natural history survey.)

\section{Insects.}

Britton, W. E. Check-list of the insects of Connecticut. Hartford, 1920.

397 pp., $2.3 \mathrm{em}$.

(Bulletin no. 31, Conmecticut geological and natural history survey.) 



\section{State of Conumertinut}

PUBLIC DOCUMENT No. 47

\section{State Geological and Natural History Survey}

HERBERT E. GREGORY, SUPERINTENDENT

\section{BULLETIN NO. 31}

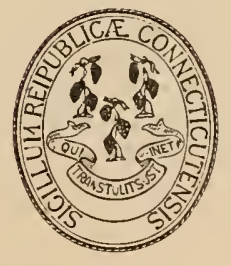

HARTFORD

Printed for the State Geological and Natural History Survey 1920 


\section{State Geological and Natural History Survey}

\section{COMMISSIONERS}

Marcus H. Holcomb, Governor of Connecticut Arthur Twining Hadley, President of Yale University William Arnold Shankin, President of Wesleyan University Remsen Brinckerioff Ogilby, President of Trinity College Charles Lewis Brach, President of Connecticut Agricultural College Benjamin Tinkiam Marshall, President of Connecticut College for Women SUPERINTENDENT

Herbert E. Gregory 


\section{CHECK-LIST OF THE INSECTS OF CONNECTICUT}

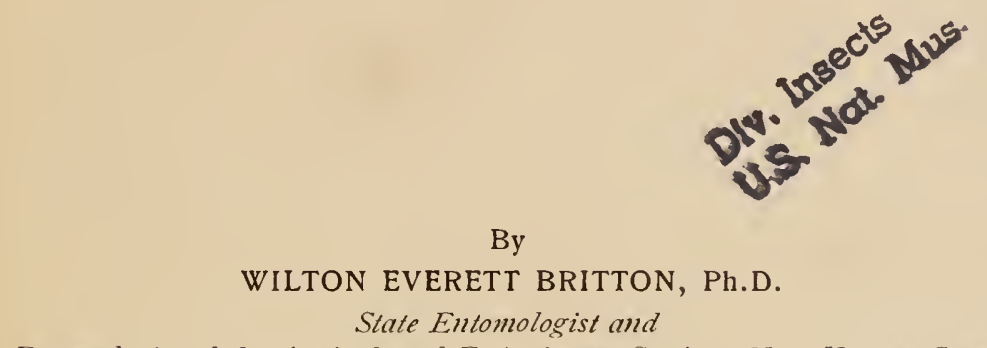

Entomologist of the Agricultural Experiment Station, New Haven, Conn.

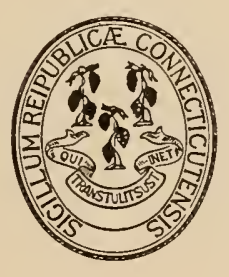

HARTFORD

Printed for the State Geological and Natural History Survey 1920 



\section{CONTENTS}

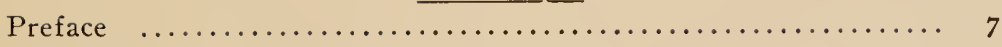

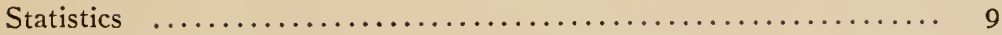

Key to initials of authorities for occurrence of species $\ldots \ldots \ldots \ldots$ ro

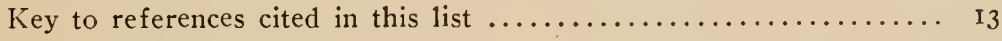

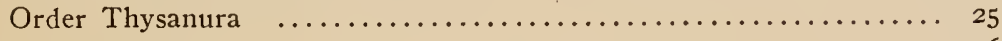

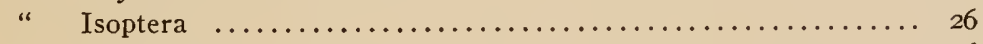

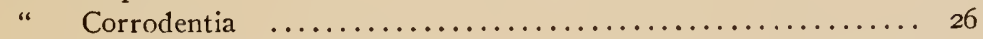

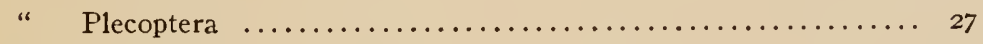

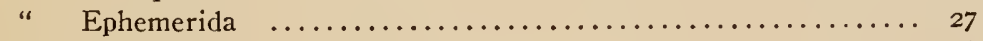

" Mallophaga $\ldots \ldots \ldots \ldots \ldots \ldots \ldots \ldots \ldots \ldots \ldots \ldots \ldots \ldots \ldots \ldots, 28$

" Neuroptera ............................... 29

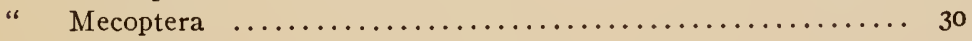

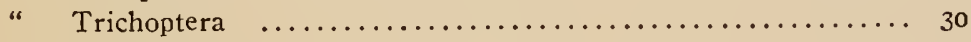

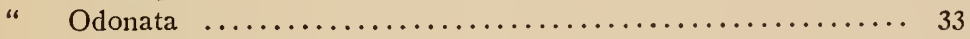

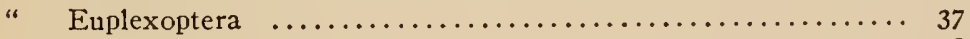

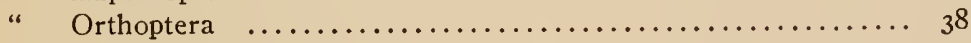

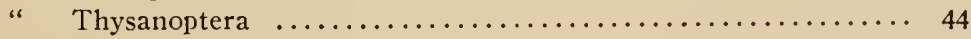

“ Hemiptera ............................... 45

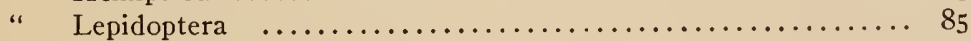

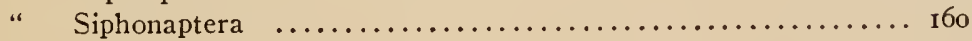

“ Diptera ..................................... 160

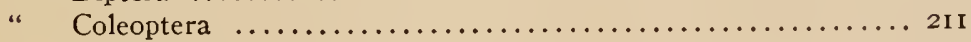

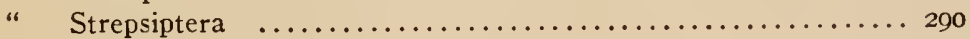

“ Hymenoptera .................................291

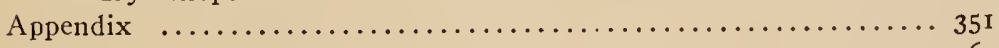

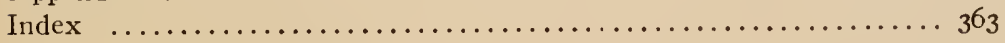



PREFACE.

The purpose of this list is to stimulate an interest in the collection and study of insects in the state, as has been done in New Jersey, and to serve as a check-catalogue of the species in the collections of the institutions and amateur collectors. A work entitled "Guide to the Insects of Connecticut," containing keys to orders, families, genera, and species, and including much information about life-histories, habits, distribution, etc., is already in progress; two papers, the Euplexoptera and Orthoptera, by B. H. Walden, and the Hymenoptera, by Henry L. Viereck, having already been published as Bulletins 16 and 22 of this Survey. It will be many years at least before the Guide can be finished so as to include all orders of insects, and the usefulness of a checklist for service during this time seemed to warrant its preparation and publication.

Only such species are included as are known to occur within the boundaries of the state, and are represented by specimens or reliable records. The list is based on the material in the collection of the Agricultural Experiment Station at New Haven, which has been accumulated during the past twenty years, and is now by far the most important collection of Connecticut insects in existence. Many records have been obtained from the collections of various institutions both in and outside of the state, and from many private collectors. No localities are mentioned in the list, but these will be given in the Guide. Where possible, one or more references are given for each species. These are not references to the original descriptions except in a few cases, but are usually references to the most accessible accounts, sometimes popular and sometimes scientific, containing descriptions, figures, or studies of life-histories and injuries that will aid one in recognizing the species. Only the well-known common names and 
synonyms are given, except where a different specific or generic name occurs in the work cited.

The arrangement of the orders, families, and genera is from the lowest to the highest, and in this respect is opposite to that of Dyar's List of Lepidoptera and Blatchley's Coleoptera of Indiana, but coincides with Aldrich's List of Diptera and Calvert's List of Odonata. These works have all been followed except where they have been shown to be in error or where new or additional genera and species were included. For convenient reference the species have been arranged alphabetically.

I wish to acknowledge here my obligations to the following collectors and specialists for furnishing records and identifying species listed in this work.

W. D. Kearfott, Montclair, N. J.; the late Professor John B. Smith, Agricultural Experiment Station, New Brunswick, N. J.; William Beutenmiiller and the late John A. Grossbeck, formerly of the American Museum of Natural History, New York, N. Y.; Charles W. Johnson, Boston Society of Natural History, Boston, Mass.; E. P. Van Duzee, Berkeley, Calif., formerly of Buffalo, N. Y.; George Dimmock, Springfield, Mass.; Professors Herbert Osborn and J. S. Hine, Ohio State University, Columbus, Ohio; Dr. E. P. Felt, State Entomologist, Albany, N. Y.; Doctors Philip L. Calvert and Henry Skinner, Philadelphia, Pa.; Professor J. G. Needham, Cornell University, Ithaca, N. Y.; Messrs. Charles W. Leng, John D. Sherman, Jr., Fred Wintersteiner, New York, N. Y.; J. R. de la Torre Bueno, White Plains, N. Y.; William T. Davis, New Brighton, Staten Island, N. Y.; Charles Schaeffer, Brooklyn, N. Y.; Professor W. E. Hinds, Auburn, Ala.; Kenyon F. Chamberlain, Cornwall, Conn.; Arthur I. Bourne, Amherst, Mass.; R. R. Parker, Bozeman, Mont.; J. F. Abbott, St. Louis, Mo.; Professor A. P. Morse, Wellesley College, Wellesley, Mass.; C. A. Frost, Framingham, Mass.; L. W. Swett, Boston, Mass.; Thomas L. Casey, Washington, D. C.; Professors J. W. Folsom and Alexander D. MacGillivray, University of Illinois, Urbana, I11.; Dr. Edith M. Patch, Agricultural Experiment Station, Orono, Me.; Professors William Morton 
Wheeler and Charles T. Brues, Bussey Institution, Forest Hills, Mass.; Charles R. Ely, Washington, D. C.; B. H. Walden, New Haven, Conn.; Dr. L. O. Howard, Dr. H. G. Dyar, the late Otto Heidemann, Henry L. Viereck, Nathan Banks, W. Dwight Pierce, S. A. Rolnwer, of Washington, D. C., and John J. Davis, Riverton, N. J., Harrison E. Smith, Arlington, Mass., and Alfred B. Champlain, Harrisburg, Pa., all now or formerly of the Bureau of Entomology; and finally to many collectors whose initials appear after the names of species in the list.

This list must necessarily be very incomplete, but it is believed to be accurate and reliable in regard to the species included. It is hoped that sometime a revised and enlarged edition may be issued, and the author requests that authentic records be sent him of any additional species taken within the state.

$$
\text { W. E. Britton. }
$$

Agricultural Experiment Station,

New Haven, Conn., April 6, 1920.

\section{STATISTICS.}

Statistics of the insects listed in this Bulletin are. as follows :-

\begin{tabular}{|c|c|c|c|}
\hline Order & $\begin{array}{c}\text { Number of } \\
\text { Families }\end{array}$ & $\begin{array}{c}\text { Number of } \\
\text { Genera }\end{array}$ & $\begin{array}{l}\text { Number of } \\
\text { Species and Varieties }\end{array}$ \\
\hline Thysanura & 5 & 10 & 16 \\
\hline Isoptera & I & I & $\mathbf{I}$ \\
\hline Corrodentia & I & 6 & 9 \\
\hline Plecoptera & I & 9 & II \\
\hline Ephemerida & I & 7 & I I \\
\hline Mallophaga & 2 & 4 & 7 \\
\hline Neuroptera & 5 & 10 & 16 \\
\hline Mecoptera & I & 2 & 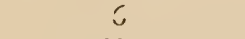 \\
\hline Trichoptera & 7 & I7 & 30 \\
\hline Odonata & 3 & 5 & IOI \\
\hline Euplexoptera & 1 & 2 & 2 \\
\hline Orthoptera & 6 & 50 & 106 \\
\hline Thysanoptera & 3 & 9 & 12 \\
\hline Hemiptera & 37 & 330 & 800 \\
\hline Lepidoptera & 46 & 609 & 1452 \\
\hline Siphonaptera & I & I & I \\
\hline Diptera & 53 & 455 & I I I I \\
\hline Coleoptera & 83 & $78 \mathrm{I}$ & 1825 \\
\hline Strepsiptera & 2 & 3 & 3 \\
\hline Hymenoptera & 74 & 635 & I $26 \mathrm{I}$ \\
\hline Total & 333 & 2946 & $678 I$ \\
\hline
\end{tabular}


KEY TO INITIALS OF AUTHORITIES FOR OCCURRENCE OF SPECIES.

A. B. C. - Alfred B. Champlain, Bureau of Economic Zoology, Harrisburg, Pa., formerly of Washington, D. C., Lyme and New Haven, Conn.

A. E. V. - Professor Addison E. Verrill, New Haven, Conn.

A. H. Me. - Rev. A. H. Manee, Southern Pines, N. C., formerly of New Milford, Conn.

A. H. Mn. - Dr. Anna H. Morgan, Mt. Holyoke College, South Hadley, Mass.

A. I. B. - Arthur I. Bourne, Amherst, Mass., formerly of New Haven, Conn.

Ald. Cat. - Aldrich's Catalogue of North American Diptera.

A. P. M. - Professor Albert P. Morse, Wellesley, Mass.

A. W. P. - A. W. Pearson, Norwich, Conn.

B. H. W. - B. H. Walden, New Haven, Conn.

C. C. - Rev. Charles Crozet, formerly of Hartford and Danielson, Conn., now of Rome, Italy.

C. D. J. - Professor C. D. Jarvis, formerly of Storrs, Conn.

C. H. Y. - Professor Clarence H. Young, New York City.

C. R. E. - Charles R. Ely, Wáshington, D. C. (Summers at East River, Conn.)

C. S. - Charles Schaeffer, Brooklyn, N. Y.

C. W. J. - Charles W. Johnson, Boston, Mass.

C. W. L. - Charles W. Leng, New York City.

D. J. C. - Donald J. Caffrey, Burean of Entomology, Washington, D. C., formerly of New Haven and Wallingford, Conn.

D. W. C. - D. W. Coquillett, Washington, D. C. Died I9I I. E. D. H. - Edward Doubleday Harris, New York City. Died I9I9.

E. F. - Ernst Frensch, Mystic, Conn.

E. L. D. - Edgar L. Dickerson, Newark, N. J.

E. N. - Edward Norton, Farmington, Conn. Died I894.

E. P. F. - Dr. E. P. Felt, State Entomologist, Albany, N. Y.

E. P. V. D. - Edward P. Van Duzee, San Francisco, Calif., formerly of Buffalo, N. Y. 
F. K. - Frederick Knab, Washington, D. C. Died 1918.

F. W. H. - F. IV. Holms, Norwich, Conn.

G. A. C. - G. A. Cromie, New Haven, Conn.

G. D. - George Dimmock, Springfield, Mass.

G. H. H. - George H. Hollister, Hartford, Conn.

G. M. - Gustav Mayr, Vienna, Austria. Died 1908.

H. B. - Henry Bird, Rye; N. Y.

H. B. K. - Harry B. Kirk, Harrisburg, Pa., formerly of New Haven, Conn.

H. F. B. - Homer F. Bassett, Waterbury, Conn. Died 1902.

H. H. A. - H. H. Abeling, Torrington, Conn.

H. J. G. - Henry J. Goodman, New Haven, Conn.

H. L. J. - Harry L. Johnson, South Meriden, Conn.

H. L. V. - Henry L. Viereck, Bureau of Biological Survey, Washington, D. C., formerly of New Haven, Conn.

H. M. R. - H. M. Russell, Bureau of Entomology, Washington, D. C., formerly of Bridgeport, Conn. Died I9I5.

H. W. F. - Professor H. W. Foote, New Haven, Conn.

H. W. W. - Rev. H. W. Winkley, formerly of Branford, Conn.

J. A. H. - James A. Hyslop, Bureau of Entomology, Washington, D. C.

J. K. L. - J. Kirby Lewis, New Haven, Conn.

J. R. T. B. - J. R, de la Torre Bueno, White Plains, N. Y.

J. S. H. - Professor J. S. Hine, Ohio State University, Columbus, Ohio.

J. T. K. - J. T. Klein, Torrington, Conn. Died 1904.

K. F. C. - Kenyon F. Chamberlain, Cornwall, Conn.

L. B. W. - Lewis B. Woodruff, New York City.

L. W. S. - L. W. Swett, Boston, Mass.

O. H. - Otto Heidemann, Bureau of Entomology, Washington, D. C. Died igi6.

P. L. B. - Philip L. Buttrick, New Haven, Conn.

R. H.- Robert Hochstein, Torrington, Conn. Died I920.

R. Hd. - Roland Hayward, Milton, Mass. Died Igo6.

S. I. S. - Professor Sidney I. Smith, New Haven, Conn.

S. N. D. - Stewart N. Dunning, Hartford, Conn.

S. W. W. - Professor S. W. Williston, Chicago, Ill., formerly of New Haven, Conn. Died i9r8. 
T. L. C. - Major Thomas L. Casey, Washington, D. C.

W. B. - William Beutenmüller, New York City.

W. C. W. - William C. Woods, Middletown, Conn.

W. E. B. - W. E. Britton, New Haven, Conn.

W. F. - William Fox, Philadelphia, Pa.

W. H. P.-William H. Patton, Hartford, Conn. Died, I9I8.

W. M. W. - Professor William M. Wheeler, Forest Hills, Mass.

W. S. B. - Professor W. S. Blatchley, Indianapolis, Ind. 
KEY TO REFERENCES CITED IN THIS LIST.

Act. Soc. Sci. Fenn. - Acta Societatis Scientiarum Fennicæ, Helsingfors, Finland.

Am. Ent. - American Entomologist, I868. St. Louis, Mo.

ii volumes; American Entomologist and Botanist, I870. 2nd ser. i volume, I88o. New York, N. Y. Am. Jour. Sci. - American Journal of Science and Arts. New Haven, Conn. I8I8-date.

Am. Mus. Jour. - Journal of the American Museum of Natural History. New York, N. Y.

Am. Nat. - The American Naturalist. Now published at New York, N. Y. Begun in 1867.

Annales - A series of papers on Diptera by Jacques Bigot in Annales de la Société Entomologique de France, Paris. I874-I892.

Ann. Ent. Soc. Am. - Annals of the Entomological Society of America. Columbus, Ohio. Igo8-date.

Ann. Lyc. Nat. Hist. N. Y. - Annals of the Lyceum of Natural History of New York, N. Y. 8 vols. I824-I867.

Ann. Mus. Nat. Hung.- Annals of the National Museum of Hungary.

Ann. N. Y. Acad. Sci. - Annals of the New York Academy of Science. New York, N. Y. Begun in 1877.

Ann. Soc. Ent. France.-Annales de la Société Entomologique de France. Paris. Begun in 1832.

Aphid. Minn. - Synopsis of the Aphidida of Minnesota, by O. W. Estlund, Bulletin 4, Geological and Natural History Survey of Minnesota. St. Paul, Minn. I887.

Berl. Ent. Zeit. or Zeitschr. - Berliner Entomologische Zeitschrift. Berlin, Germany. Begun in 1857 .

Bomb. Moths. - A Monograph of the Bombicine Moths of America North of Mexico, by A. S. Packard, Memoirs, National Academy of Sciences, Vol. vii, Part I. Notodontidx. Washington, D. C. I895.

Bost. Jour. Nat. Hist. - Boston Journal of Natural History. Boston, Mass. I834-I863.

British Aphid. - Monograph of the British Aphides, by G. B. Buckton. 4 vols. Ray Society. London, England. I875-I 883 . 
Brit. Ent. - British Entomology, by J. Curtis. London, England.

Bull. Am. Mus. Nat. Hist. - Bulletin of the American Museum of Natural History. New York, N. Y. Begun in $188 \mathrm{I}$.

Bull. Brook. Ent. Soc. - Bulletin of the Brooklyn (N. Y.) Entomological Society. 7 vols. I878-1885. Re-established in I9I2.

Bull. Buff. Soc. Nat. Sci. - Bulletin of the Buffalo Society of Natural Sciences. Buffalo, N. Y. Begun in 1874.

Bull. Geol. Geog. Surv. Terr. - Bulletin of the United States Geological and Geographical Survey of the Territories. Washington, D. C.

Bull. Ill. State Lab. Nat. Hist. - Bulletin of the Illinois State Laboratory of Natural History. Urbana, Ill.

Bull. Mosc. - Bulletin de la Société Impériale des Naturalistes de Moscow, Moscow, Russia.

Bull. Sci. Lab. Denison Univ. - Bulletin of the Science Laboratories, Denison University. Granville, Ohio.

Bull. Soc. Ent. Belg. - Bulletin de la Société Entomologique de Belgique. Brussels, Belgium. Begun in 1860.

Bull. Wis. Acad. Sci. - Bulletin Wisconsin Academy of Sciences, Milwaukee, Wis. Begun in I90I.

Bur. Ent. - Bureau of Entomology (formerly Division), United States Department of Agriculture, Washington, D. C. Bulletins, new series. Technical series, Circulars.

Butterfly Book. - The Butterfly Book, by W. J. Holland. Doubleday, Page \& Co., Garden City, N. Y.

Can. Bark Beetles. - Canadian Bark Beetles, by J. M. Swaine. Technical Bulletin, No. I4, Entomological Branch, Department of Agriculture, Dominion of Canada, Ottawa, Can. 1918.

Can. Ent. - Canadian Entọmologist. London, Ontario, Canada. Begun in 1869 .

Cat. Brit. Mus. - Catalogue of the British Museum, London, England.

Cat. Brit. Tin. - Catalogue of British Tineinæ.

Cat. Homop. N. Y. - Catalogue of New York Homoptera. 
Cat. Ins. N. Y. - Catalogue of New York Insects, by Asa Fitch. Fourth Annual Report of the Regents of the University of the State of New York, I85I. Reprinted in 9th Report of the N. Y. State Entomologist, I 892.

Cat. Lep. Eur. - Catalogue Méthodique des Lépidoptères d'Europe, by P. A. J. Duponchel. Paris, France. I $844-$ I 846 .

Cat. Noc. - Catalogue of Noctuidæ, by J. B. Smith. Bulletin 44. United States National Museum. Washington, D. C. I 893 .

Cent. - Diptera Americæ Septentrionalis Indigena, by $\mathrm{H}$. Loew. Originally published in to Centuries in Berliner Entonologische Zeitschrift, I86I-72. Berlin, Germany. (Usually cited as "Century.")

Cin. Quart. Jour. Sci. - Cincinnati Quarterly Journal of Science. Cincinnati, Ohio.

Coleop. Can. - Petite Faune Entomologique du Canada: Les Coleoptères, by L'Abbé L. Provancher, with two supplements. Quebec, Canada. I877 and 1879.

Coleop. Ind. - Coleoptera of Indiana, by W. S. Blatchley. Nature Publishing Company, Indianapolis, Ind.

Collem. Minn. - Collembola of Minnesota, by Joseph E. Guthrie, Zoological Series IV, Geological and Natural History Survey of Minnesota.

Compl. Writ. - Complete Writings on the Entomology of North America, by Thomas Say. 2 vols.

Comst. Butt. - How to Know the Butterflies, by J. H. and A. B. Comstock. D. Appleton \& Co., New York, N. Y.

Conn. Geol. Nat. Hist. Surv. - Connecticut Geological and Natural History Survey. Hartford, Conn. Begun in 1903.

Cornell Agr. Expt. Sta. - Cornell Agricultural Experiment Station. Ithaca, N. Y.

Diag. N. A. Phyc. - Diagnoses of North American Phycitidæe and Galleriidæ, by E. L. Ragonot. Paris, France. I 888 .

Dipt. Exot. (Suppl.) - Diptères Exotiques Nouveaux ou Peu Connus, by J. Macquart. 2 vols. 5 supplements. I838I855. Paris, France. 
Dipt. Saund. - Insecta Saundersiana, Diptera, by Francis Walker. 5 parts, London, England. I850-1856.

Dipt. Scand. - Diptera Scandinaviæ, by J. W. Zetterstedt. London, England. I842-I860.

Encycl. Meth. - Encyclopédie Méthodique, by A. G. Olivier. I789-179I et I825. Paris, France.

Ent. Am. - Entomologica Americana. Brooklyn Entomological Society, Brooklyn, N. Y. I885-I89o.

Ent. Cont. - Entomological Contributions, by J. A. Lintner. N. Y. State Museum of Natural History, Albany, N. Y.

Ent. Monthl. Mag. - The Entomologists' Monthly Magazine. London, England. Begun in I864.

Ent. News. - Entomological News. Philadelphia, Pa. Bégun in 1892.

Ent. Rec. - Entomologists' Record and Journal of Variation. London, England. Begun in I890.

Ent. Syst. Suppl. - Entomologia Systematica. Supplement, by J. C. Fabricius.

Enum. Hemip. - Enumeratio Hemipterorum, by C. Stål, Stockholm, Sweden, I870-1876.

Exot. Schmett. - Lepidopterorum Exoticorum Species Novæ aut Minus Cognitæ, by G. A. W. Herrich-Schæffer. Ratisbonæ. I850-I858.

Fauna Aust. - Fauna Austriaca (Diptera), by J. R. Schiner. 2 vols. Vienna, Austria. I862-I864.

Faun. Bor. Am. - Fauna Boreali-Americana, by William Kirby. 4 vols. London, England. I $829-1837$.

Faun. Germ. - Faunæ Insectorum Germanicæ Initia.

Gen. Ins. - Genera Insectorum. Fascicles I-I63. Edited by P. Wytsman. Brussels, Belgium. Begun in 1902.

Geol. Geog. Surv. - Bulletin of the United States Geological and Geographical Survey of the Territories. Washington, D. C.

Handb. Brit. Lep. - Handbook of British Lepidoptera, by E. Meyrick. London, England and New York, N. Y. I895.

Hayden's Geol. Surv. Mont. - Hayden's Geological Survey of Montana. Washington, D. C. 
Hemip. Col. - A Preliminary List of the Hemiptera of Colorado, by C. P. Gillette and C. F. Baker. Colorado Agricultural Experiment Station, Bull. 3i (Technical series No. I), Fort Collins, Col. I895.

Hemip. Gymn. Eur. - Hemiptera Gymnocerata Europæ, by O. M. Reuter. 5 vols. Helsingfors, Finland. I878I896.

Icon. Reg. An. Ins. - Iconographie du Règne Animal. Insectes. vii, by F. E. Guérin-Méneville. Paris, France. I829-I 844 .

I11. Exot. Ent. - Illustrations of Exotic Entomology, by Drury, London, England. I770-I775.

Ill. Lep. Het. Brit. Mus. - Illustrations of Typical Specimens of Lepidoptera Heterocera in the Collection of the British Museum, London, England. 1877-1893.

Ins. Aff. Park and Woodl. Trees. - Insects Affecting Park and Woodland Trees, by E. P. Felt, Memoir 8, N. Y. State Museum, Albany, N. Y.

Insect Book. - The Insect Book, by L. O. Howard, Doubleday, Page \& Co., Garden City, N. Y. Igor.

Ins. Galls Springfield. - Insect Galls of Springfield, Mass. and Vicinity, by Fannie A. Stebbins. Bulletin No. 2, Springfield Museum of Natural History, Springfield, Mass. I9I0.

Ins. Ill. - Reports of the State Entomologist on the Noxious and Beneficial Insects of the State of Illinois. Urbana, I11. Begun in I867.

Ins. Inj. Veg. - Insects Injurious to Vegetation, by $T$. W. Harris. (Flint Edition), Boston, Mass. I863.

Ins. Life. - Insect Life. 7 vols. U. S. Department of Agriculture, Washington, D. C. I888-I894.

Ins. N. Y. - Report on the Noxious and Beneficial Insects of New York, by Asa Fitch. I-I4. Albany, N. Y. I855I 870.

Insec. Insc. Menst. - Insecutor Inscitia Menstruus. Washington, D. C. Begun in I9I3.

Insects and Insecticides. - Insects and Insecticides, by C. M. Weed. Hanover, N. H. I89I. 
Isis. - Encyclopädische Zeitschrift, Herausgegeben von Oken, Leipzig, Germany. I8I 7-I848.

Jour. Acad. Nat. Sci. - Journal of the Academy of Natural Sciences, Philadelphia, $\mathrm{Pa}$.

Jour. Cin. Soc. Nat. Hist. - Journal Cincinnati (Ohio) Society of Natural History.

Jour. Econ. Ent. - Journal of Economic Entomology. Concord, N. H. Begun in I908.

Jour. Ent. Zool. - Journal of Entomology and Zoology, Pomona College. Begun in I909 as Pomona College Journal of Entomology, Claremont, Calif.

Jour. N. Y. Ent. Soc. - Journal of the New York Entomological Society. New York, N. Y. Begun in I893.

Kans. Acad. Sci. - Transactions of the Kansas Academy of Sciences. Topeka, Kans. Begun in 1874.

Kans. Univ. Quart. - Kansas University Quarterly. Lawrence, Kans.

La. Agr. Expt. Sta. - Louisiana Agricultural Experiment Station. Baton Rouge, La.

Lep. Brit. - Lepidoptera Brittanica, by A. H. Haworth, London, England. I803-I8I2.

Lep. Het. Brit. Mus. - Lepidoptera Heterocera of British Museum, London, England.

Lep. Ins. Ga. - The Natural History of the Rarer Lepidopterous Insects of Georgia, by J. E. Smith and J. Abbott. London, England. I797.

Life Hist. Am. Insects. - Life History of American Insects, by Clarence Moores Weed. The MacMillan Company, New York, N. Y. I90I.

Linn. Ent. - Linnæa Entomologica. Berlin, Germany. I846I 866.

List. - A List of the Specimens of Insects in the Collection of the British Museum, by Francis Walker. Diptera. 4 parts and 3 supplements. $1848-1855$. Lepidoptera Heterocera i-xxxv, I854-I866. London, England.

Mass. Agr. Expt. Sta. - Massachusetts Agricultural Experiment Station. Amherst, Mass.

Me. Agr. Expt. Sta. - Maine Agricultural Experiment Station. Orono, Maine. 
Mem. Coleop. - Memoirs on the Coleoptera, by Thomas L. Casey, New Era Printing Co., Lancaster, Pa. Begun in 1910 .

Mem. Ins. - Memoires pour Servir à L'Histoire des Insectes, by Charles DeGeer. 7 vols. Stockholm, Sweden. I $752-1758$.

Mich. Agr. Expt. Sta. - Michigan Agricultural Experiment Station. East Lansing, Mich.

Minn. Agr. Expt. Sta. - Minnesota Agricultural Experiment Station. St. Anthony Park, Minn.

Mon. Geom. - Monograph of Geometridx, by A. S. Packard, vol. x, Rept. U. S. Geological Survey of the Territories, Washington, D. C. 1876.

Mon. N. A. Dipt. - Monographs of the Diptera of North America, by H. Loew and Baron Osten Sacken. Vols. I-4. Smithsonian Miscellaneous Collections, Washington, D. C. $1862-1872$.

Mon. N. A. Ses. - Monograph of the Sesiidæ of America North of Mexico, by William Beutenmüller. Memoirs of the American Museum of Natural History, Vol. I. New York, N. Y.

Mon. Oestriden. - Monographie der Estriden, by Fr. Brauer. Vienna, Austria. I863.

Mosq. N. A. - Mosquitoes of North and Central America and the West Indies, by Howard, Dyar and Knab, Carnegie Institution, Washington, D. C. I912-I7.

Moth Book. - The Moth Book, by W. J. Holland. Doubleday, Page \& Co., Garden City, N. Y.

Myodaires. - Essai sur les Myodaires, by J. B. RobineauDesvoidy. Mémoires des Savants Étrangers de L'Académie des Sciences de Paris, Vol. iii, 390-4I3. Paris, France. 1827.

Nat. Can. - Le Naturaliste Canadien.

N. A. Cramb. - The Crambidx of North America, by Charles H. Fernald. Report, Massachusetts Agricultural College, Amherst, Mass. 1896.

N. A. Ent. - North American Entomologist. Buffalo, N. Y. I879-80. 
N. J. Agr. Expt. Sta. - New Jersey Agricultural Experiment Station, New Brunswick, N. J.

N. Y. Agr. Expt. Sta. - New York Agricultural Experiment Station, Geneva, N. Y.

N. Y. Agr. Rept. - Report of the New York Department of Agriculture.

N. Y. State Mus. Bull. - Bulletin of the New York State Museum, Albany, N. Y.

Ofv. Finsk. Vet. Soc. Förh. - Öfversigt af Finska VetenskapsSocietetens Förhandlingar-Helsingfors, Finland.

Ofv. Vet. Akad. Förh. - Ofversigt af Konglica Svenska Vetenskaps-Akademiens Förhandlingar. Stockholm, Sweden. Begun in 1844 .

Ohio Acad. Sci. - Ohio Academy of Sciences. Columbus, Ohio.

Ohio Nat. - Ohio Naturalist. Columbus, Ohio.

Ohio State Univ. Bull. - Bulletin of the Ohio State University. Columbus, Ohio.

Orthop. Ind. - Orthoptera of Indiana, by W. S. Blatchley, Indianapolis, Ind.

Pack. Guide. - A Guide to the Study of Insects, by A. S. Packard. 9th edition. Henry Holt \& Co., New York, N. Y. I889.

Papilio. - Papilio. New York Entomological Club. 4 vols. I 88I-I 884.

Proc. Acad. Nat. Sci. Phila. - Proceedings of the Academy of Natural Sciences, Philadelphia, Pa. Begun in I84I.

Proc. Am. Phil. Soc.- Proceedings of the American Philosophical Society. Philadelphia, Pa. Begun in 1860.

Proc. Bost. Soc. Nat. Hist. - Proceedings of the Boston Society of Natural History. Boston, Mass. Begun in I84I.

Proc. Cal. Acad. Sci. - Proceedings of the California Academy of Sciences. San Francisco, Calif. Begun in 1854 .

Proc. Dav. Acad. Sci. - Proceedings of the Davenport (Iowa) Academy of Natural Sciences. Davenport, Iowa.

Proc. Iowa Acad. Sci. - Proceedings of the Iowa Academy of Sciences. Des Moines, Ia. Begun in 1887. 
Proc. Phila. Ent. Soc. - Proceedings of the Entomological Society of Philadelphia, Pa. 6 vols. I86I-I867.

Proc. U. S. Nat. Mus. - Proceedings of the United States National Museum. Washington, D. C. Begun in 1878 .

Proc. Wash. Acad. Sci. - Proceedings of the Washington Academy of Science. Washington, D. C. Begun in I899.

Proc. Wash. Biol. Soc. - Proceedings of the Washington Biological Society. Washington, D. C.

Proc. Wash. Ent. Soc. - Proceedings of the Entomological Society of Washington, D. C. Begun in I884.

Proc. Zool. Soc. Lond. - Proceedings of the Zoological Society. London, England.

Prodrome. - Prodrome of a Monograph of the Tabanidæ of the United States, by C. R. Osten Sacken. Memoirs, Boston Society of Natural History, vol. II. Boston, Mass. $1875-78$.

Psyche.- Psyche. Cambridge (Mass.) Entomological Club. Begun in 1874 .

Pteroph. N. A. - The Pterophidæ of North America, by Charles H. Fernald. Massachusetts Agricultural Experiment Station, Special Bulletin. Revised edition, I 898 .

Rept. Conn. Agr. Expt. Sta. - Report of the Connecticut Agricultural Experiment Station. New Haven, Conn.

Rept. Geol. Ind. - Report of the Geologist of Indiana. Indianapolis, Ind.

Rept. Ill. State Ent. - Report of the State Entomologist of Illinois. Urbana, Ill. Begun in I867.

Rept. Ins. Mass. - Annual Report of the Injurious and Beneficial Insects of Massachusetts, by A. S. Packard, i-iii, Boston, Mass. I87I-I873.

Rept. Ins. Mo. - Annual Report of the State Entomologist of Missouri, by C. V. Riley, I-9. Jefferson City, Mo. I 869-I 877 .

Rept. N. Y. State Ent. - Report of the State Entomologist of New York. Albany, N. Y. Begun in 1882.

Rept. Peab. Acad. Sci. - Report of the Peabody Academy of Science. Salem, Mass. 
Rept. U. S. Dept. Agr. - Report of the United States Department of Agriculture. Washington, D. C.

Rept. U. S. Ent. Com. - Report of the United States Entomological Commission, U. S. Department of Agriculture. Washington, D. C.

Rev. \& Mag. Zool. - Revue et Magasin de Zoologie Pure et Appliquée. Paris, France. 1849-1879.

Revis. Tachinid. - Revision of the Tachinidæ, by D. W. Coquillett. Technical Series No. 7, Bureau of Entomology, U. S. Department of Agriculture. Washington, D. C.

Rhynchop. N. E. Am. - Rhynchophora or Weevils of North Eastern America, by W. S. Blatchley and C. W. Leng. Nature Publishing Co., Indianapolis, Ind. I9ı6.

Samml. Ausser. Schmett. - Sammlung Neuer oder Wenig Bekannter Ausser-Europäischer Schmetterlinge. Regensburg, Germany. I850-I858.

Samml. Exot. Schmett. - Sammlung Exotischer Schmetterlinge, by Jacob Hübner. Augsburg, Germany. I806I824.

Schmett. Eur. - Die Schmetterlinge von Europa, by F. Treitschke. Leipzig, Germany.

Smith. Misc. Coll. - Smithsonian Miscellaneous Collections. Smithsonian Institution, Washington, D. C. Begun I862.

Spec. Gén. - Species Général des Lepidoptères, by A. Gúenée. Paris, France. I852-1857.

Spec. Gyponæ. - Species Gyponæ Generis Homopterorum, by J. Spangberg. Stockholm, Sweden. I878.

Stand. Nat. Hist. - The Standard Natural History, edited by J. S. Kingsley. 6 vols. Boston, Mass. I884-1885.

Stett. Ent. Zeit. - Stettiner Entomologische Zeitung. Stettin, Germany. Begun in 1840 .

Storrs Agr. Expt. Sta. - Agricultural Experiment Station. Storrs, Conn.

Syn. N. A. Syrph. - Synopsis of the Syrphidre of North America, by S. W. Williston, U. S. National Museum, Bu1l. No. 31. WVashington, D. C. I886. 
Syn. Neurop. N. A. - Synopsis of the Neuroptera of North America, by H. A. Hagen, Smithsonian Miscellaneous Collections. W'ashington, D. C. I86r.

Syst. Antl. - Systema Antliatorum, by J. C. Fabricius. Brunsvigae. I805.

Syst. Beschr. - Systematische Beschreibung der Bekannten Europäischen Zweiflügeligen Insecten, by $\mathrm{F}$. W. Meigen. 7 vols. Aachen und Hamm, Germany. I 8 I $8-1838$.

Syst. Nat. - Systema Naturæ, by C. V. Linnæus. Stockholm, Sweden.

Syst. Rhyng. - Systema Rhyngotorum, by J. C. Fabricius. Brunsvigæe, 1803 .

Syst. Verz. Wien. - Systematisches Verzeichniss der Schmetterlinge der WVeiner Gegend, by I. Schiffermüller. Vienna, Austria. 1776.

Tab. Ohio. - Tabanidx of Ohio, by J. S. Hine. Ohio Academy of Science, Special Papers. Columbus, Ohio.

Tin. N. A. - The Tineina of North America, by Brackenbridge Clemens. John Van Voorst, London, England. 1872.

Trans. Am. Ent. Soc. - Transactions of the American Entomological Society. Academy of Natural Sciences, Philadelphia, Pa. Begun in 1868.

Trans. Conn. Acad. Sci. - Transactions of the Connecticut Academy of Arts and Sciences. New Haven, Conn.

Trans. Ent. Soc. Lond. - Transactions of the Entomological Society of London, England. Begun in 1836. N. S.

Trans. Linn. Soc. London. - Transactions of the Linnean Society of London, England. Begun in I79r.

Trans. Md. Acad. - Transactions of the Maryland Academy of Science.

Trans. N. Y. Agr. Soc. - Transactions of the New York Agricultural Society, Albany, N. Y.

Trans. St. Louis Acad. Sci. - Transactions of the St. Louis Academy of Science. St. Louis, No. Begun in 1856.

U. S. Dept. Agr. - United States Department of Agriculture. Washington, D. C.

U. S. Geol. Surv. - United States Geological Survey. WVashington, D. C. 
U. S. Nat. Mus. Bull. - Bulletins of the United States National Museum. Washington, D. C.

Verh. Zool-Bot. Ges. Wien. - Verhandlungen der ZoologischBotanischen Gesellschaft in Wien. Vienna, Austria. Begun in 1852 .

Verz. bek. Schmett. - Verzeichniss Bekannter Schmetterlinge, by Jacob Hübner. Augsburg, Germany. I8I6.

W. Va. Agr. Expt. Sta. - West Virginia Agricultural Experiment Station. Morgantown, W. Va.

West. Dipt. - Western Diptera, by C. R. Osten Sacken. U. S. Geological and Geographical Survey of the Territories, iii, 1877. Washington, D. C.

Wien. Ent. Mon. - Wiener Entomologische Monatsschrift. Vienna, Austria.

Yearbcok U. S. D. A. - Yearbook of the United States Department of Agriculture. Washington, D. C.

Zeitsch. f. Ges. Naturwiss. - Zeitschrift für die Gesammten Naturwissenschaften. Now Zeits. f. Naturwiss., pub. in Leipzig.

Zool. Bull. - Zoological Bulletin, Ginn \& Co., Boston, Mass. Zutr. Exot. Schmett. - Zuträge zur Sammlung Exotischer Schmetterlinge, by Jacob Hubner. Augsburg, Germany. I8I8-I825. 


\section{Order THYSANURA.}

Suborder CINURA.

Family MACHILIDÆ.

Machilis Latreille.

sp. probably variabilis Say. Pack. Guide, 9th Ed., pl. x, figs. 8 and 9 .

\section{Family LEPISMID Æ.}

Lepisma Linnæus.

quadriseriata Packard. Ctenolepisma. Bristle-tail. Silverfish. Fish moth. Pack. Guide, 9th Ed., 623.

saccharina Linnæus. Bristle-tail. Silverfisl., Bur. Ent., Bull. 4, new ser., 76 .

Thermobia Bergroth.

domestica Packard. Lepisma. Silverfish. Bristle-tail. Fish moth. Insect Book, 382 ; Bur. Ent., Bull. 4, new ser., 77.

Suborder COLLEMBOLA.

Family SMINTHURIDÆ.

Sminthurus Latreille.

hortensis Fitch. pruinosus. Garden flea. Rept. N. Y. State Ent., ii, 207.

\section{Family ENTOMOBRYID死.}

sp.

Entomobrya Rondani.

Seira Lubbock.

nigromaculata Lubbock. Collem. Minn., 36 .

Isotoma Bourlet.

albella Packard. Rept. Peab. Acad. Sci., v, 32.

sp. near palustris Müller. Collem. Minn., 70.

sp.

sp. 
Family PODURIDÆ.

Achorutes Templeton.

armatus Nicolet. Proc. U. S. Nat. Mus., 50, 491.

harveyi Folsom. Ibid., 50, 486.

nivicola Fitch. Rept. N. Y. State Ent., ii, 203.

Neanura MacGillivray.

muscorum Templeton. Proc. U. S. Nat. Mus., 50, 508.

Onychiurus Gervais.

fimetarius Linnæus. Proc. U. S. Nat. NIus., 53, 649.

\section{Order ISOPTERA.}

Family TERMITIDE.

Termes Linnæus.

flavipes Kollar. White ant. Bur. Ent., Bull. 4, new ser., 70.

\section{Order CORRODENTIA.}

Family PSOCIDÆ.

Troctes Burmeister.

divinatorius Müller. Atropos. Book louse. Bur. Ent., Bull. 4, new ser., 79.

pulsatorius Linnæus. Clothilla. Rept. N. Y. State. Ent., ii, 202.

Pterodela Kolbe.

pedicularis Linnæus. Psocus salicis. Hemerobius. Syn. Neurop. N. A., I3.

Cæcilius Curtis.

aurantiacus Hagen. Psocus. Syn. Neurop. N. A., I4.

Polypsocus Hagen.

corruptus Hagen. Psocus. Syn. Neurop. N. A., I3.

Psocus Latreille.

confraternus Banks. Trans. Am. Ent. Soc., xxxii, 2. ieidyi Aaron. Proc. Acad. Nat. Sci., Phila., I886, I5. sp.

Cerastipsocus Kolbe.

venosus Burmeister. Psocus. Syn. Neurop. N. A., Io. 


\section{Order PLECOPTERA.}

Family PERLID E.

Acroneuria Pictet.

abnormis Newman. Syn. Neurop. N. A. I7.

Perla Geoffrey.

postica Walker. Syn. Neurop. N. A., 23.

Perlesta Banks.

placida Hagen. Syn. Neurop. N. A., 28.

virginica Banks. Trans. Am. Ent. Soc., xxv, I99.

Isoperla Banks.

bilineata Say. Syn. Neurop. N. A., 3o.

Alloperla Banks.

imbecilla Say. Syn. Neurop. N. A., 3I.

lateralis Banks. Trans. Am. Ent. Soc., xxxvii, 337.

Tæniopteryx Pictet.

frigida Hagen. Syn. Neurop. N. A., 35.

Nemoura Latreille.

perfecta Walker. Syn. Neurop. N. A. 37.

Leuctra Stephens.

tenella Provancher. N. Y. State Mus., Bu1l. 47, $4 \mathrm{I} 6$.

Capnia Pictet.

necydaloides Pictet. Syn. Neurop. N. A., 32.

Order EPHEMERIDA.

Family EPHEMERID E.

Hexagenia Walsh.

bilineata Say. Batis. Compl. Writ., i, 203. (A.H.Mn.)

limbata Pictet. Batis. Palingenia bilineata Say. variabilis

Eaton. Syn. Neurop. N. A., 4I.

Ephemera Linnæus.

decora Walker. varia Eaton. Insect Book, pl. xxvi, 6.

Leptophlebia Westwood.

cupida Say. Batis ignava. Syn. Neurop. N. A., 47. 
Ephemerella Walsh.

cornuta Morgan. Ann. Ent. Soc. Am., iv, I14. (A.H.Mn.)

Callibætis Eaton.

ferrugineus Walsh. Trans. Am. Ent. Soc., xxvi, 250.

Siphlonurus Eaton.

aridus Say. Batis. Syn. Neurop. N. A., 46.

mirus Eaton. Can. Ent., xlvii, 248.

typicus Eaton. Ibid., xlvii, 253.

Heptagenia Walsh.

luridipennis Burmeister. Syn. Neurop. N. A., 49.

terminata Walsh. Pentagenia. Proc. Phila., Ent. Soc., ii, 203.

\section{Order MALLOPHAGA.}

Biting bird lice.

Family PHILOPTERIDÆ.

Goniocotes Burmeister.

abdominalis Piaget. gigas Taschenberg. Bur. Ent., Bull. 7 , 32; Storrs Agr. Expt. Sta., Bull. 86, 180.

hologaster Nitzsch. Bur. Ent., Bull. 7, 33; Bull. 5, new ser., I92.

Lipeurus Nitzsch.

heterographus Nitzscl. Chicken head louse. Bur. Ent., Bull. 5, new ser., 23r ; Storrs Agr. Expt. Sta., Bull. 86, 178 .

variabilis Nitzsch. Variable chicken louse. Bur. Ent., Bull. 5 , new ser., $2 \mathrm{O} 2$.

\section{Family LIOTHEIDÆ.}

\section{Menopon Nitzsch.}

biseriatum Piaget. Large body hen louse. Bur. Ent., Bull. 5, new ser., 212; Storrs Agr. Expt. Sta., Bu1l. 86, I74. pallidum Nitzsch. Small body hen louse. Common biting hen louse. Bur. Ent., Bull. 5, new ser., 210; Storrs Agr. Expt. Sta., Bull. 86, I74.

Nitzschia Denny. pulicaris Nitzsch. Jour. N. Y. Ent. Soc., x, 225. 


\section{Order NEUROPTERA.}

Family SIALID E.

Corydalis Latreille.

cornuta Linnzeus. Dobson. Hellgrammite. Insect Book, pl. xxiii, I5 and 16.

Chauliodes Latreille.

angusticollis Hagen. N. Y. State Mus., Bull. 68, 462.

pecticornis Linnaus. Ibid., Bull. 68, 46I : Insect Book, pl. xxxiii, I4.

rasticornis Rambur. N. Y. State Mus., Bull. 68, 460.

serricornis Say. Ibid., Bull. 68, 459.

Sialis Latreille.

infumata Newman. N. Y. State Mus., Bull. 68, 448: Insect Book, pl. xxiii, I8.

\section{Family HEMEROBIID E.}

\section{Hemerobius Linnæus.}

humuli Linnæus. Syn. Neurop. N. A., 205.

stigmaterus Fitcl.. Ibid., 202.

Sympherobius Banks.

amiculus Fitch. Hemerobius. Syn. Neurop. N. A., 200.

Micromus Rambur.

posticus Walker. Hemerobius. Syn. Neurop. N. A., 204.

Family CHRYSOPID无.

Chrysopa Leach.

harrisi Fitch. Trans. Am. Ent. Soc., xxix, I 55. nigricornis Burmeister. Insect Book, pl. xxvi, 2. oculata Say. Ibid., 223.

\section{Family MYRMELEONID王.}

Myrmeleon Linnæus.

immaculatus DeGeer. Insect Book, pl. xxv, 2.

Brachynemurus Hagen.

abdominalis Say. Insect Book, pl. xxvi, II. 


\section{Family ASCALAPHID Æ.}

Colobopterus Rambur.

excisus Hagen. Can. Ent., xix, I53. (H. J. G.)

\section{Order MECOPTERA.}

Family PANORPIDÆ.

\section{Panorpa Linnæus.}

americana Swederus. Bull. Sci. Lab., Denison Univ., xi, 250. confusa Westwood. Ibid., xi, $25 \mathrm{I}$. maculosa Hagen. Ibid., xi, 247. nebulosa Westwood. Ibid., xi, 247. rufescens Rambur. Insect Book, pl. xxiii, Io.

\section{Bittacus Latreille.}

stigmaterus Say. Bull. Sci. Lab., Denison Univ., xi, 260.

\section{Order TRICHOPTERA.}

Family PHRYGANEIDÆ.

\section{Phryganea Linnæus.}

cinerea Walker. Syn. Neurop. N. A., 252. interrupta Say. Compl. Writ., i, 98.

\section{Neuronia Leach.}

angustipennis Hagen. Verh. Zool-Bot. Ges. Wien., I873, 400. concatenata Walker. Phryganea irrorata. Syn. Neurop. N. A., 249.

postica Walker. Ibid., $25 \mathrm{I}$. stygipes Hagen. Verh. Zool-Bot. Ges. Wien., I873, 388.

\section{Family LIMNEPHILID瓜.}

\section{Limnephilus Leach.}

despectus Walker. Syn. Neurop. N. A., 259. indivisus Walker. Ibid., 260. submonilifer Walker. Ibid., 260. 
Platycentropus Ulmer.

maculipennis Kolenati. Hallesus hostis. Syn. Neurop. N. A., 266.

Pycnopsyche Banks. scabripennis Rambur. Hallesus. Syn. Neurop. N. A., 265.

Dicosmœcus McLachlan. argus Harris. Entomological Correspondence, 333.

Stenophylax Kolenati.

divergens Walker. Limnephilus. Syn. Neurop. N. A., 255.

Chilostigma McLachlan.

difficilis Walker. Limnephilus. Syn. Neurop. N. A., 268.

$$
\text { Family RHYACOPHILID Æ. }
$$

Chimarrhia Leach.

aterrima Hagen. Syn. Neurop. N. A., 297.

Family SERICOSTOMATIDÆ.

Helicopsyche Hagen.

borealis Hagen. Notidobia. Syn. Neurop. N. A., 27 I.

Family LEPTOCERIDÆ.

Leptocerus Leach.

sp.

Ecetina Banks.

avara Banks. Trans. Am. Ent. Soc. xxii, 316.

incerta Walker. Leptocerus. Syn. Neurop. N. A., 278.

sp. ?

Mystacides Latreille.

nigra Linnæus. Leptocerus. Syn. Neurop. N. A., 277.

sepulchralis Walk. Leptocerus. Ibid., 277.

Setodes Rambur.

grandis Banks. Proc. Wash. Ent. Soc. viii, 128.

Family HYDROPSYCHID无.

Macronema Pictet.

zebrata Hagen. Insect Book, pl. xxiii, 9. 


\section{Hydropsyche Pictet.}

alternans Walker. morosa. Syn. Neurop. N. A., 288.

analis Banks. Jour. N. Y. Ent. Soc., I903, 243.

scalaris Hagen. Syn. Neurop. N. A., 286.

sordida Hagen. Ibid., 290.

sp.

Family HYDROPTILID E.

Orthotrichia Eaton.

pictipes Banks. Trans. Am. Ent. Soc., xxxvii, 359. 


\section{Order ODONATA.}

Dragonflies.

Family AGRIONIDÆ.

Agrion Fabricius.

Caloptery.x Leach.

æquabile Say. Insect Book, pl. xlvi, I4. amatum Hagen. Psyche, 5, 244.

maculatum Beanvois. Insect Book, pl. xlvi, I2.

\section{Hetærina Hagen.}

americana Fabricius. Insect Book, pl. xlvi, 9, II.

\section{Lestes Leach.}

congener Hagen. Trans. Am. Ent. Soc., xx, 229.

disjunctus Selys. Ibid., xx, 23I.

eurinus Say. Insect Book, pl. xlvii, II.

forcipatus Rambur. Ibid., pl. xlvi, I, 5 .

inæqualis Walsh. Rept. Geol. Ind., 260.

rectangularis Say. Ibid., pl. xlvii, 9.

uncatus Kirby. Ibid., pl. xlviii, 3.

unguiculatus Hagen. Ibid., pl. xlvii, 15 .

vigilax Hagen. Ibid., pl. xlvii, 8.

Argia Rambur.*

mœsta Hagen var. putrida Hagen. Insect Book, pl. xlvii, IO, I3.

violacea Hagen. Trans. Am. Ent. Soc., xx, 233.

Nehalennia Selys.

irene Hagen. Insect Book, pl. xlvi, 3.

Amphiagrion Selys.

saucium Burmeister. Insect Book, pl. xlvi, 2.

Chromagrion Needham.

conditi:m Hagen. N. Y. State Mus., Bull. 68, pl. I3, I and 2.

Enallagma Charpentier.

aspersum Hagen. Trans. Am. Ent. Soc., xx, 237.

civile Hagen. Insect Book, pl. xlvii, I2, I4.

* See also appendix. 
carunculatum Morse. Psyche, 7, 208. cyathigerum Charpentier. annc.rum. Ent. News, xi, I54. divagans Selys. Trans. Am. Ent. Soc., xx, 238. durum Hagen. Insect Book, pl. xlviii, 13. ebrium Hagen. Rept. Geol. Ind., xxiv, 270. exsulans Hagen. Insect Book, pl. xlvi, 4, 8. geminatum Kellicott. Ent. News, vi, 239. signatum Hagen. Insect Book, pl. xlvii, I. traviatum Selys. Rept. Geol. Ind., I900, 27 I. vesperum Calvert. pollutum. Trans. Am. Ent. Soc., xlv, 38 I. Ischnura Charpentier.

posita Hagen. Insect Book, pl. xlvi, 7. verticalis Say. Ibid., pl. xlvii, 2, 3, 5 .

Anomalagrion Selys.

hastatum Say. Insect Book, pl. xlvi, 16, I8.

Family ESCHNIDÆ.

Hagenius Selys.

brevistylus Selys. Trans. Am. Ent. Soc., xx, $24 \mathrm{I}$.

\section{Ophiogomphus Selys.}

johannus Needham. Can. Ent., xxix, I82. rupinsulensis Walsh. Rept. Geol. Ind., xxiv, 298.

Lanthus Needham.

albistylus Hagen. Gomphus navius. Trans. Am. Ent. Soc., $\mathrm{xx}, 2+2$. (L. B. W.)

Gomphus Leach.

abbreviatus Hagen. Trans. Am. Ent. Soc., xx, 243. borealis Needham. N. Y. State Mus., Bull. 68, 265. brevis Hagen. N. Y. State Mus., Bull., 47, 449. exilis Selys. Trans. Am. Ent. Soc., xx, 243. sordidus Hagen. lividus Selys. Ent. News, xiv, 256. spicatus Hagen. Rept. Gcol. Ind., 292, 1899.

Dromogomphus Selys.

spinosus Selys. N. Y. State Mus., Bull. 47, 462.

Cordulegaster Leach.

diastotops Selys. latcralis. Proc. Bost. Soc. Nat. Hist., x, 2 II. 
erroneus Hagen. Trans. Am. Ent. Soc., xx, 246.

maculatus Selys. Insect Book, pl. xliv, 7.

obliquus Say. Rept. Geol. Ind., xxiv, 300.

\section{Anax Leach.}

junius Drury. Insect Book, pl, xl, I5.

\section{Eshna Fabricitıs.}

canadensis Walker. Can. Ent., x1, 384.

clepsydra Say. Ibid., xl, 383 .

constricta Say. Insect Book, pl. xli, 4. juncea Linnæus. Can. Ent., xl, 385 .

tuberculifera Walker. Can. Ent., xl, 385.

umbrosa Walker. Ibid., xl, 380 .

verticalis Hagen. Trans. Am. Ent. Soc., xx, 248.

\section{Epiæschna Selys.}

heros Fabricius. Insect Book, pl. xli, 7 .

Boyeria MacLachlan.

vinosa Say. Fonscolombia. Insect Book, pl. xliv, 4.

Basiæschna Selys.

janata Say. Insect Book, pl. xliv, I.

Gomphæschna Selys.

furcillata Say. Rept. Geol. Ind., xxiv, 302.

\section{Family LIBELLULIDÆ}

Didymops Rambur.

transversa Say. Insect Book, pl. xlv, 9.

Epicordulia Selys.

princeps Hagen. Insect Book, pl. xiv, 8.

Helocordulia Needham.

uhleri Selys. N. Y. State Mus., Bull. 47, 496.

Tetragoneuria Hagen.

cynosura Say. Trans. Am. Ent. Soc., xx, 252.

cynosura var. simulans Muttkowski. Bull. Wis. Acad. Sci., 95, I9II.

spinigera Selys. Rept. Geol. Ind., 3I I. 1899.

spinosa Hagen. N. Y. State Mus., Bull. 47, 495. 
Somatochlora Selys.*

elongata Scudder, var. minor Calvert. Ent. News, ix, 87. tenebrosa Say. Rept. Geol. Ind., 3I4. I899.

Cordulia Leach.

shurtleffi Scudder. N. Y. State Mus., Bull. 47, 506.

\section{Dorocordulia Needham.}

lepida Hagen. N. Y. State Mus., Bull. 47, 506.

libera Selys. Rept. Geol. Ind., 3I4. 1899.

Libellula Linnæus.

auripennis Burmeister. Insect Book, pl. xlv, 6.

cyanea Fabricius. Ibid., pl. xliv, 2, 3 .

exusta Say. Ibid., pl. xli, 5, 6.

incesta Hagen. Insect Book, pl. xliii, 3 .

luctuosa Burmeister. basalis. Ibid., pl, xliii, 2.

pulchella Drury. Ibid., pl. xl, 7.

quadrimaculata Linnæus. Ibid., pl. xl, 3.

semifasciata Burmeister. Ibid., pl, xlv, 5 .

vibrans Fabricius. Ibid., pl. xl, Ir.

Plathemis Hagen.

lydia Drury. trimaculata. Insect Book, pl. xl, I, 5 .

Erythrodiplax Brauer.

berenice Drury. Micrathyria. Insect Book, pl. xli, I, 2, 3 .

Nannothemis Brauer.

bella Uhler. Trans. Am. Ent. Soc., xx, 260.

Tramea Hagen.

carolina Linnæus. Insect Book, pl. xliii, 8.

Sympetrum Newman.

corruptum Hagen. Diplax. Insect Book, pl. xlii, 5.

costiferum Hagen. Ibid., pl. xliv., 8, 9.

rubicundulum Say. Diplax. Ibid., pl. xlii, 8, 9.

semicinctum Say. Diplax. Ibid., pl. xliii, 7.

vicinum Hagen. Diplax. Trans. Am. Ent. Soc., xx, 264.

Leucorhinia Brittinger.

frigida Hagen. Trans. Am. Ent. Soc., xvii, 23r. (L. B. W.) glacialis Hagen. N. Y. State Mus., Bull. 47, 5 I8. intacta Hagen. Insect Book, pl. xliv, 5, 6.

* See also appendix. 
Celethemis Hagen.

elisa Hagen. Insect Book, pl. xl, I4.

eponina Drury. Ibid., pl. xliii, 4.

monomelæna Williamson. Ohio Nat., Io. 1910.

Perithemis Hagen.

domitia Drury. tenera Say. Insect Book, pl. xl, 4, 6.

Erythemis Hagen.

simplicicollis Say. Mesothemis. Insect Book, pl. xli. 8, 9.

Pachydiplax Brauer.

longipennis Burmeister. Trans. Am. Ent. Soc., xx, 265.

Order EUPLEXOPTERA.

Earwigs.

Family FORFICULID Æ.

Anisolabis Fieber.

maritima Bonnel. Maritime earwig. Conn. Geol. Nat. Hist.

Surv., Bull. I6, 45 .

Labia Leach.

minor Linnæus. Conn. Geol. Nat. Hist. Surv., Bull. 16, 47. 


\section{Order ORTHOPTERA.}

Grasshoppers, Crickets, and Cockroaches.

Family BLATTIDÆ.

Parcoblatta Hebard.

pennsylvanica DeGeer. Pennsylvania cockroach. Conn. Geol. Nat. Hist. Surv., Bull. I6, 54.

uhleriana Saussure. Ibid., Bull. I6, 55 .

virginica Brunner, Ischnoptera borealis. Proc. Acad. Nat. Sci., Phila., 44. I910.

\section{Blattella Caudell.}

germanica Linnæus. Ectobia. Phyllodromia. Croton bug. German cockroach. Bur. Ent., Bull. 4, new ser., 92: Conn. Geol. Nat. Hist. Surv., Bull. 16, 56 .

\section{Blatta Linnæus.}

orientalis Linnæus. Oriental cockroach. Bur. Ent., Bull. 4, new ser., 91 : Conn. Geol. Nat. Hist. Surv., Bull. I6, 57. Periplaneta Burmeister.

americana Linnæus. American cockroach. Bur. Ent., Bull. 4, new ser., 84: Conn. Geol. Nat. Hist. Surv., Bull. I6, 58. australasiæ Fabricius. Australian cockroach. Bur. Ent., Bull.

4, new ser., 9I. Conn. Geol. Nat. Hist. Surv., Bu1l. I6, 59.

Nyctibora Burmeister.

noctivaga Rehn. mericana Saussure. In bananas. Conn. Geol.

Nat. Hist. Surv., Bull. I6, 53.

Pycnoscelus Scudder.

surinamensis Linnæus. Leucopluca. In greenhouse. Rept.

Conn. Agr. Expt. Sta., 307. I9I7.

Panchlora Burmeister.

cubensis Saussure. pocyi. viridis. Green cockroach. In bunches of bananas. Conn. Geol. Nat. Hist Surv, Bull. I6, 53.

Family PHASMIDÆ.

Diapheromera Gray.

femorata Say. Walking stick. Conn. Geol. Nat. Hist. Surv., Bull. 16,62 . 
Family MANTIDE.

Tenodera Burmeister.

sinensis Saussure. Paratenodera. Chinese praying mantis. Conn. Geol. Nat. Hist. Surv., Bull. I6, 60.

Manomera Rehn and Hebard.

blatchleyi Caudell. Proc. U. S. Nat. Mus., 44, 6r2. (A.P.M.)

Family ACRIDID E.

Nomotettix Morse.

cristatus Scudder. Crested grouse locust. Conn. Geol. Nat. Hist. Surv., Bull. I6, 66.

cristatus var. carinatus Scudder. Ibid., Bull. 16, 67.

Tettix Charpentier.

angustus Hancock. Trans. Am. Ent. Soc., xxiii, 238. (A.P.M.) granulatus Kirby. Conn. Geol. Nat. Hist. Surv., Bull. I6, 68. ornatus Say. Ornate grouse locust. Ibid., Bull. I6, 67.

Paratettix Bolivar.

cucullatus Burmeister. Hooded grouse locust. Conn. Geol. Nat. Hist. Surv., Bull. 16, 69.

Tettigidea Scudder.

parvipennis Harris. Conn. Geol. Nat. Hist. Surv., Bull. I6, 70. parvipennis var. pennata Morse. Ibid., Bull. I6, 70.

- Eritettix Bruner.

carinatus Scudder. Conn. Geol. Nat. Hist. Surv., Bull. I6, 75. Orphulella Giglio-Tos.

olivacea Morse. Conn. Geol. Nat. Hist. Surv., Bull. I6. 8I. pelidna Burmeister. Smaller spotted-winged locust. Ibid., Bull. 16,79 .

speciosa Scudder. Ibid., Bull. I6, 8o.

Dicromorpha Morse.

viridis Scudder. Short-winged green locust. Conn. Geol. Nat. Hist. Surv., Bull. $16,76$.

Chlœaltis Harris.

conspersa Harris. Sprinkled locust. Conn. Geol. Nat. Hist. Surv., Bull. I6, 83 .

\section{Stenobothrus Fischer.}

curtipennis Harris. Short-winged brown locust. Conn. Geol.

Nat. Hist. Surv., Bull. 16, 83 . 
Mecostethus Fieber.

lineatus Scudder. Conn. Geol. Nat. Hist. Surv., Bull. I6, 85. platypterus Scudder. Ibid., Bull. I6, 86. (A. P. M.)

\section{Arphia Stål.}

sulphurea Fabricius. Sulphur-winged locust. Conn. Geol. Nat. Hist. Surv., Bull. I6, 89.

xanthoptera Germar. Ibid., Bull. 16, 90.

Chortophaga Saussure.

viridifasciata DeGeer. Green-striped locust. Conn. Geol. Nat. Hist. Surv., Bull. 16, 9o.

\section{Encoptolophus Scudder.}

sordidus Burmeister. Clouded locust. Conn. Geol. Nat. Hist. Surv., Bull. $16,92$.

\section{Camnula Stål.}

pellucida Scudder. Clear-winged locust. Conn. Geol. Nat. Hist. Surv., Bull. 16, 92.

\section{Hippiscus Saussure.}

tuberculatus Beauvois. Coral-winged locust. Conr. Geol. Nat. Hist. Surv., Bull. I6, 94.

\section{Dissosteira Scudder.}

carolina Linnæus. Carolina locust. Conn. Geol. Nat. Hist. Surv., Bull. 16, 96.

Spharagemon Scudder.

bolli Scudder. Conn. Geol. Nat. Hist. Surv., Bull. 16, 99. collare Scudder, var. scudderi Morse. Ibid., Bull I6, 98. collare var. wyomingianum Thomas. Mottled sand locust. Ibid., Bull. I6, 98 . saxatile Morse. Ibid., Bull. I6, Ioo.

Scirtetica Saussure.

marmorata Harris. Conn. Geol. Nat. Hist. Surv., Bull. I6, Ior. Psinidia Stål.

fenestralis Serville. Long-horned locust. Conn. Geol. Nat.

Hist. Surv., Bull. 16, I03.

Trimerotropis Stål.

maritima Harris. Maritime locust. Conn. Geol. Nat. Hist. Surv., Bull. I6, I04.

Circotettix Scudder.

verruculatus Kirby. Conn. Geol. Nat. Hist. Surv., Bull. I6, 105. 
Pseudopomala Morse.

brachyptera Scudder. Conn. Geol. Nat. Hist. Surv., Bull. I6, 73 .

\section{Schistocerca Stål.}

alutacea Harris. Leather-colored locust. Conn. Geol. Nat. Hist. Surv., Bull. I6, I08.

americana Drury. American locust. Ibid., Bull. I6, Io8.

rubiginosa Harris. Rusty locust. Ibid., Bull. I6, Iog.

\section{Melanoplus Stål.}

atlanis Riley. Lesser locust. Conn. Geol. Nat. Hist. Surv., Bul1. I6, I 17.

bivittatus Say. Yellow-striped locust. Ibid., Bull. I6, I2I.

fasciatus Walker and Barnston. Ibid., Bull. I6, I I9.

femur-rubrum DeGeer. Red-legged locust. Ibid., Bull. I6, I I8. luridus Dodge. Ibid., Bull. I6, I20.

mancus Smith. Ibid., Bull. I6, II 5 .

minor Scudder. Ibid., Bull. I6, I I9.

punctulatus Uhler. Grizzly locust. Ibid., Bull. I6, I22.

scudderi Uhler. Scudder's short-winged locust. Ibid., Bull. I6, I I6.

\section{Paroxya Scudder.}

floridana Thomas. Conn. Geol. Nat. Hist. Surv., Bull. I6, I23

\section{Family LOCUSTID $Æ$.}

Scudderia Stål.

curvicauda DeGeer. Curve-tailed katydid. Conn. Geol. Nat. Hist. Surv., Bull. I6, 127.

furcata Bruner. Fork-tailed katydid. Ibid., Bull. I6, I28.

pistillata Bruner. Ibid., Bull. I6, I28.

septentrionalis Serville. Ibid., Bull. I6, I29.

texensis Saussure-Pictet. Ibid., Bull. I6, 126.

\section{Amblycorypha Stål.}

oblongifolia DeGeer. Oblong-winged katydid. Conn. Geol. Nat. Hist. Surv., Bull. I6, I30.

rotundifolia Scudder. Round-winged katydid. Ibid., Bull. I6, I30. 
Cyrtophyllus Burmeister.

perspicillatus Linnæus. concazus. True katydid. Conn. Geol. Nat. Hist. Surv., Bull. I6, I3I.

Conocephalus Thunberg.

ensiger Harris. Sword-bearer. Conn. Geol. Nat. Hist. Surv,, Bull. I6, I33.

exiliscanorus Davis. Ibid., Bull. I6, I34. robustus Scudder. Ibid., Bull. I6, I35. triops Linnæus. Ibid., Bull. I6, I35.

Orchelimum Serville.

concinnum Scudder. herbacenm. Conn. Sttate Geol. Nat. Hist. Surv., Bull. I6, I39.

gladiator Bruner. Can. Ent., xxiii, 7 I.

vulgare Harris. Common meadow grasshopper. Conn. Geol. Nat. Hist. Surv., Bull. I6, I39.

\section{Xiphidium Serville.}

brevipenne Scudder Short-winged meadow grasshopper.

Conn. Geol. Nat. Hist. Serv., Bull I6, I37.

ensiferum Scudder. Ibid., Bull. 16, 137. (W. S. B.)

fasciatum DeGeer. Slender meadow grasshopper. Ibid., Bull. I6, I37.

spartinæ Fox. Ent. News, xxiii, IrII. (A.P.M.)

\section{Atlanticus Scudder.}

dorsalis Burmeister. Conn. Geol. Nat. Hist. Surv., Bull. I6, I4I.

pachymerus Burmeister. Ibid., Bull. I6, I4I.

Ceutophilus Scudder.

brevipes Scudder. Conn. Geol. Nat. Hist. Surv., Bull. I6, I45. gracilipes Haldeman. Ibid., Bull. г6, I43.

grandis Scudder. Ibid., Bu1l. г6, I44.

latens Scudder. Ibid., Bull. I6, I46.

maculatus Harris. Ibid., Bull. 16, I45.

neglectus Scudder. Ibid., Bull. I6, I47.

pallidipes Walker. Ibid., Bull. І6, І 46.

terrestris Scudder. Ibid., Bull. 16, I44. 


\section{Family GRYLLID瓜.}

\section{Gryllotalpa Linnæus.}

borealis Burmeister. Northern mole cricket. Conn. Geol. Nat. Hist. Surv., Bull. 16, I49.

Tridactylus Olivier.

terminalis Uhler. Conn. Geol. Nat. Hist. Surv., Bull. 16, I50.

Nemobius Serville.

carolinus Scudder. Conn. Geol. Nat. Hist. Surv., Bull. I6, I52. fasciatus DeGeer. vittatus. Small black cricket. Striped ground cricket. Ibid., Bull. I6, I5I.

griseus Walker. Can. Ent., xxxiv, I82.

maculatus Blatchley. Orthop. Ind., 424.

palustris Blatchley. Marsh ground cricket. Conn. Geol. Nat.

Hist. Surv., Bull. I6, I52.

Gryllus Linnæus.

abbreviatus Serville. Common field cricket. Conn. Geol. Nat. Hist. Surv., Bull. I6, I 54.

domesticus Linnæus. House cricket. Conn. Geol. Nat. Hist. Surv., I6, I 54 .

pennsylvanicus Burmeister. Pennsylvania field cricket. Ibid., Bull. I6, I 54 .

\section{Ecanthus Serville.}

angustipennis Fitch. Narrow-winged tree cricket. Conn. Geol. Nat. Hist. Surv., Bull. 16, I 58.

exclamationis Davis. Ibid., Bull. I6, I 58 .

nigricornis Walker. fasciatus Fitch. Striped tree cricket. Ibid., Bull. I6, 159 .

niveus DeGeer. Snowy tree cricket. Ibid., Bull. I6, I57. pini Beutenmüller. Pine tree cricket. Ibid., Bull. I6, I60. (WV. B.)

quadripunctatus Beutenmüller. Four-spotted tree cricket. Ibid., Bull. I6, I 59.

Anaxipha Saussure.

exigua Say. Conn. Geol. Nat. Hist. Surv., Bull. I6, I62.

Xabea Walker.

bipunctatus DeGeer. Two-spotted tree cricket. Conn. Geol. Nat. Hist. Surv., Bull. I6, гбг. 


\section{Order THYSANOPTERA (PHYSOPODA).}

Family ÆOLOTHRIPID Æ.

\section{Æolothrips Haliday.}

fasciatus Linnæus. Coleothrips trifasciata. Proc, U. S. Nat. Mus., xxvi, I27.

$$
\text { Family THRIPIDÆ. }
$$

Euthrips Targioni-Tozzetti.

tritici Fitch. Thrips. Wheat thrips. Proc. U. S. Nat. Mus., - xxvi, 148.

\section{Anaphothrips Uzel.}

striatus Osborn. Grass thrips. Proc. U. S. Nat. Mus., xxvi, I6r.

Heliothrips Haliday.

femoralis Heeger. cestri. Proc. U. S. Nat. Mus., xxvi, I72. hæmorrhoidalis Bouché. Ibid., xxvi, 168.

\section{Parthenothrips Uzel.}

dracænæ Heeger. Proc. U. S. Nat. Mus., xxvi, I76.

Thrips Linnæus.

perplexus Beach. Proc. U. S. Nat. Mus., xxvi, I84. tabaci Lindeman. Onion thrips, Ibid., xxvi, I79: Rept. Conn. Agr. Expt. Sta., 1903, 266.

\section{Family PHLCEOTHRIPID旡.}

\section{Anthothrips Uzel.}

verbasci Osborn. Proc. U. S. Nat. Mus., xxvi, I89. sp.

\section{Liothrips Uzel.}

citricornis Hood. Can. Ent., xl, 306.

\section{Leptothrips Hood.}

aspersus Hinds. Cryptothrips. Proc. U. S. Nat. Mus., xxvi, 265. 


\section{Order HEMIPTERA.}

Bugs, Lice, Aphids, Scale $\cdot$ Insects, etc.

Suborder PARASITICA.

Family PEDICULIDÆ.

Pediculus Linnæus.

corporis DeGeer. vestimenti Leach. Body-louse of man. Bur.

Ent. Bull. 5, new series.

humanus Linnæus. capitis. Head-louse of man. Ibid., 166.

Phthirius Leach.

pubis Linnæus. inguinalis Leach. Crab-louse of man. Bur.

Ent., Bull. 5, new series, 165.

Hæmatopinus Leach.

eurysternus Nitzsch. Short-nosed ox-louse. Bur. Ent., Bull.

5. new series, 172.

Linognathus Enderlein.

piliferus Burmeister. Sucking dog-louse. Bur. Ent., Bull. 5, new series, 169.

vituli Linnæus. Long-nosed ox-louse. Ibid., I76.

Suborder HOMOPTERA.

Family FULGORID $\approx$.

Scolops Schaum.

angustatus Uhler. Bu1l. Geol. Geog. Surv., I, 350.

sulcipes Say. Compl. Writ., ii, 254.

Phylloscelis Germar.

atra Germar. Proc. Acad. Nat. Sci. Phila., I907, 472.

atra var. albovenosa Millichar. Ibid., 1907, 472.

Elidiptera Spinola.

Helicoptera Amyot and Serville.

variegata Van Duzee. Proc. Acad. Nat. Sci. Phila., I907, 479. sp. 
Catonia Uhler.

grisea Van Duzee. Proc. Acad. Nat. Sci. Phila., I907, 480, 482. impunctata Fitch. Myndus. Ibid., I907, 480, 482.

\section{Oliarus Stål.}

cinnamomeus Provancher. Proc. Acad. Nat. Sci. Phila., I907, $485,488$.

complectus Ball. Ibid., I907, 485, 488.

humilis Say. Ibid., I907, 485, 488.

Cixius Latreille.

basalis Van Duzee. Bull. Buff. Soc. Nat. Sci., v, I9I. colœpeum Fitch. Proc. Acad. Nat. Sci. Phila., I907, 489, 490. misellus Van Duzee. (erroneously stigmaticus) Can. Ent., xxxviii, 408.

pini Fitch. Ibid., xxxviii, 408.

\section{Ecleus Stål.}

borealis Van Duzee. Bull. Buff. Soc. Nat. Sci., x, 495.

campestris Ball. Can. Ent., xxxiv, I56.

decens Stål. Berl. Ent., Zeitschr., vi, 307.

\section{Myndus Stål.}

fulvus Osborn. Ohio Nat., iv, (2) 46.

\section{Bruchomorpha Newman.}

oculata Newman. Can. Ent., xxi, 7.

Acanalonia Spinola.

Amphiscepa Uhler.

bivittata Say. Flata. Insect Book, P1. xxviii, 20. Compl.

Writ., ii, 255 .

\section{Ormenis Stål.}

pruinosa Say. Flata. Insect Book, P1. xxvii, 28. Compl. Writ., ii, 373 .

septentrionalis Spinola. Insect Book, P1. xxvii, 24, 27 ; Ann.

Soc. Ent. France, Ser. i, viii, I839.

\section{Lamenia Stål.}

obscura Ball. Can. Ent. xxxiv, 262.

vulgaris Fitch. Cat. Ins. N. Y., 47, I85I.

Otiocerus Kirby.

amyoti Fitch. Ins. N. Y., iii, 394.

degeerii Kirby. Trans. Linn. Soc. London, xiii, I6. 
Stenocranus Fieber.

dorsalis Fitch, var. vittatus Stål. (lautus Van Duzee.) Bull. Buff. Soc. Nat. Sci., v, 23I.

\section{Kelisia Fieber.}

axialis Van Duzee. Bull. Buff. Soc. Nat. Sci., v, I9I.

\section{Prokelisia Osborn.}

marginata Van Duzee. Bull. Buff. Soc. Nat. Sci., v, 235.

Pissonotus Van Duzee.

ater Van Duzee. Bull. Buff. Soc.. Nat. Sci., v, I9o.

\section{Stobæra Stål.}

tricarinata Say. Delpha.t. Compl. Writ., ii, 255.

Liburniella Crawford.

ornata Stål. Berl. Ent. Zeitschr., vi, 3I5.

\section{Liburnia Stål.}

campestris Van Duzee. Bull. Buff. Soc. Nat. Sci., v, I9I. laminalis Van Duzee. Ibid., v, 25I.

lateralis $V$ an Duzee. Ibid., v, 253.

lutulenta Van Duzee. Ibid., v, I9I.

pellucida Fabricius. Ibid., v, I9I.

puella Van Duzee. Ibid., v, IgI.

\section{Family CICADELLID\&.*}

Jassoidea.

\section{Agallia Curtis.}

constricta Van Duzee. Can. Ent., xxvi, 90.

novella Sav. Proc. Acad. Nat. Sci. Phila., I831, 6, 309.

quadripunctata Provancher. Nat. Can., I872, 4, 376.

sanguinolenta Provancher. Ibid., I872, 4, 376.

\section{Idiocerus Lewis.}

lachrymalis Fitch. Ohio State Univ., Bu1l. 22, 507, 1905.

pallidus Fitch. Ibid., Bull, 22, 505, 1905.

fitchi Van Duzee. maculipennis. Proc. Dav. Acad. Sci., vii, $73,127$.

provancheri Van Duzee. Buff. Soc. Nat. Sci., Bull. 5, I94. scurra Germar. gemmisimulans Leonard and Crosby. Jour. Econ. Ent., 8, 542.

sp.

\footnotetext{
* See also appendix.
} 
Macropsis Lewis.

Pediopsis Burmeister.

trimaculata Fitch. insignis Van Duzee. Ent. Amer., v, I72. viridis Fitch. Rept. N. Y. State Ent., 9, 399.

virescens Gmelin, var. graminea Fabricius. Me. Agr. Expt. Sta., Bull. 239, 90.

Oncopsis Burmeister.

cognatus Van Duzee. Ent. Amer., vi, 225.

fitchi Van Duzee. fenestratus Fitch. Rept. N. Y. State Ent., 9, 400 .

minor Fitch. Ent. Amer., vi, 228.

sobrius Walker. Ibid., vi, 223.

variabilis Fitch. Ent. Amer., vi, 224.

sp.

\section{Oncometopia Stål.}

lateralis Fabricius, var. limbata Say. Compl. Writ., ii, 258.

Cicadella Latreille.

Tettigoniella Jacoby.

gothica Signoret, Tettigonia. Ohio State Univ. Bull., ser. 5, No. 2I, I9.

Kolla Distant.

bifida Say. Tettigonia. Bull. Ill. State Lab. Nat. Hist., iii, 27. tripunctata Fitch. Tettigonia. Ohio State Univ. Bull., Ser. 5, No. $21,26$.

Helochara Fitch.

communis Fitch. Ohio State Univ. Bull., Ser. 5, No. 21, 28. Graphocephala Van Duzee.

Diedrocephala Ball.

coccinea Fœrster. Tettigonia. Bull. Ill. State Lab. Nat. Hist., iii, 23.

coccinea var. teliformis Walker. Bull. Buff. Soc. Nat. Sci., ix, $2 I 4$.

Dræculacephala Ball.

angulifera Walker. Ohio State Univ. Bull., Ser. 5, No. 2I, 33,35 .

minor Walker. Bull. Buff. Soc. Nat. Sci., ix, 2 I4. 
mollipes Say. Tettigonia. Bull. Ill. State Lab. Nat. Hist., iii, 24. noveboracensis Fitch. Tettigonia. Ibid., iii, 24.

Penthimia Germar.

americana Fitch. Rept. N. Y. State Ent., ix, 397.

Gypona Germar.

bipunctulata Woodworth. Bull. I1l. State Lab. Nat. Hist., iii, 30 .

cinerea Uhler. Bull. Geol. Geog. Surv., 3, 460.

geminata Osborn. Rept. N. Y. State Ent., xx, 513.

melanota Spangberg. Spec. Gyponæ, I878, 23.

octolineata Say. striata. Aazilineata. Bull. Ill. State Lab.

Nat. Hist., iii, 30.

rugosa Spangberg. Rept. N. Y. State Ent., 20, 5 I3.

scarlatina Fitch. Ibid., ix, 397.

Stroggylocephalus Flor.

agrestris Fallen. Bull. Buff. Soc. Nat. Sci., v, 197.

Xerophlœa Germar.

major Baker. Psyche, viii, 285.

viridis Fabricius. Proc. Iowa Acad. Sci., iv, I79.

Acucephalus Germar.

albifrons Linnæus. Tettigonia mixta. Compl. Writ., ii, 258.

Parabolocratus Fieber.

viridis Uhler. Bull. Geol. Geog. Surv., 3, 462, I877.

Mesamia Ball.

vitellina Fitch. Paramesus. Rept. N. Y. State Ent., 9, 399.

Scaphoideus Uhler.

auronitens Provancher. Jour. Cin. Soc. Nat. Hist., xix, I94.

carinatus Osborn. Ohio Nat., xi, $25 \mathrm{I}$.

consors Uhler. Jour. Cin. Soc. Nat. Hist., xix, 196.

immistus Say. Ibid., xix, 204.

intricatus Uhler. Ibid., xix, 202.

lobatus Van Duzee. Ohio Nat., xi, 250.

luteolus Van Duzee. Jour. Cin. Soc. Nat. Hist., xix, 203.

melanotus Osborn. Ohio Nat., xi, 251.

opalinus Osborn. Rept. N. Y. State Ent., 20, 525.

productus Osborn. Jour. Cin. Soc. Nat. Hist., xix, 200.

unicolor Osborn. consors var. unicolor. Ibid., xix, 196.

sp. 
Platymetopius Burmeister.

acutus Say. Jassus. Compl. Writ., ii, 382.

frontalis Van Duzee. Can. Ent., xxii, II2.

frontalis var.

hyalinus Osborn. Ent. News, xi, 50I.

magdalensis Pròvancher. Tenn. State Bd. Ent., Bull. I7, 4 I.

Deltocephalus Burmeister.

apicatus Osborn. Me. Agr. Expt. Sta., Bull. 238, I20.

balli Van Duzee. nigrifrons. Bur. Ent., Bull., 198, 77 :

configuratus Uhler. Bull. Geol. Geog. Surv., iv, 511, I87I.

flavicosta Stål. flavocostatus. Can. Ent., xxiv, II6.

inimicus Say. Jassus. Compl. Writ., ii, 382; Me. Agr. Expt.

Sta., Bull. 238, I23.

littoralis Ball. Bull. Buff. Soc. Nat. Sci., ix, 22 I.

melsheimerii Fitch. Tenn. State Bd. Ent., Bull., I7, 48.

minimus Osborn and Ball. Ibid., I7, 47.

pascuellus Fallen. minki Fieber. Me. Agri. Expa. Sat., Bull., 238 , II 8 .

pictus Osborn. Tenn. State Bd. Ent., Bull. I7, 43.

sayi Fitch. Ibid., Bull. I7, 46.

simplex Van Duzee. Trans. Am. Ent. Soc., xix, 304.

sylvestris Osborn and Ball. Tenn. State Bd. Ent., Bull., I7, 49.

Driotura Osborn and Ball.

gammaroides Van Duzee. Me. Agr. Expt. Sta., Bull, 238, I32.

Euscelis Brullé.

Athysanus Burmeister.

anthracinus Van Duzee. Ohio Nat., ii, 24I.

curtisii Fitch. Ibid., ii, $25 \mathrm{I}$.

extrusus Van Duzee. Ibid., ii, 237.

relativus Gillette and Baker. obsoletus. Ibid., ii, 239.

striatulus Fallen. plutonius Uhler. Ibid., ii, 240.

striolus Fallen. Ibid., ii, 235.

uhleri Ball. Can. Ent., xliii, 200.

vaccini Van Duzee. Ohio Nat., ii, 242.

varus Ball. Ibid., ii, 239 .

Eutettix Van Duzee.

cinctus Osborn and Ball. Proc. Dav. Acad. Sci., xii, 64. johnsoni Van Duzee. Mesamia. Ibid., xii, $6 \dot{6}$. 
marmoratus Van Duzee. Ibid., xii, 35 .

seminudus Say. Athysanus. Ibid., xii, 42.

strobi Fitch. Ibid., xii, 44.

Phlepsius Fieber.

collitus Ball. Can Ent., xxxv, 227.

fulvidorsum Fitch. Trans. Am. Ent. Soc., xix, 74.

fuscipennis Van Duzee. Ibid., xix, 70.

incisus Van Duzee. Ibid., xix, 73.

irroratus Say. Ibid., xix, $7 \mathrm{I}$.

lobatus Osborn. Proc. Ia. Acad. Sci., v, 247.

majestus Osborn and Ball. Tenn. St. Bd. Ent., Bull. I7, 76.

nebulosus Van Duzee. Trans. Am. Ent. Soc., xix, 77.

solidaginis Walker. humidus. Ibid., xix, 76 .

Acinopterus Van Duzee.

acuminatus Van Duzee. Psyche, vi, 308.

Thamnottettix Zetterstedt.

brittoni Osborn. Proc. Dav. Acad. Sci., x, I66.

clitellarius Say. Jassus. Compl. Writ., ii, 384 .

eburatus Van Duzee. Can. Ent., xxi, ro.

kennicotti Uhler. Proc. Ent. Soc. Phila., ii, 16I.

melanogaster Provancher. Nat. Can., iv, 378.

nigrifrons Forbes. perpunctata Van Duzzee. Ins. I11., I4, 67.

Chlorotettix Van Duzee.

balli Osborn. Proc. Iowa Acad. Sci., v, 247.

galbanatus Van Duzee. Psyche, vi, 309.

lusorius Osborn and Ball. Proc. Iowa Acad. Sci., iv, 226.

tergatus Fitch. Psyche, vi, 309.

unicolor'Fitch. Ibid., vi, 308.

viridius Van Duzee. Ibid., vi, 309.

Jassus Fabricius.

olitorius Say. subbifasciatus. Jour. Acad. Nat. Sci. Phila., vi, 301,183 I.

\section{Cicadula Zetterstedt.}

sexnotata Fallen. Tenn. State Bd. Ent., Bull. I7, 94.

variata Fallen. Ibid., Bull. I7, 95. 
Balclutha Kirkaldy.

impicta Van Duzee. Gnathodus. Can. Ent., xxiv, I I3.

punctata Thunberg. Me. Agr. Expt. Sta., Bull., 238, I49.

Alebra Fiebẻr.

albostriella Fallen, var. fulveola Herrich-Schæffer. Proc. U. S. Nat. Mus., xx, $7 \mathrm{I} 3$.

Dicraneura Hardy.

mali Provancher. communis Gillette. Proc. U. S. Nat. Mus., $\mathrm{xx}, 7 \mathrm{I} 8$.

\section{Empoasca Walsh.}

flavescens Fabricius. Proc. U. S. Nat. Mus., xx, 745.

mali Le Baron. Apple leaf-hopper. Rept. Conn. Agr. Expt. Sta., I904, 2 I6.

smaragdula Fallen. Proc. U. S. Nat. Mus., xx, 725.

trifasciata Gillette. Ibid., $\mathrm{xx}, 726$.

unica Provancher. splendida Gillette. Ibid., xx, 73I.

Typhlocyba Germar.

Eupteryx Curtis.

flavoscuta Gillette. Proc. U. S. Nat. Mus., xx, 749.

melissæ Curtis. collina Flor. Bull. Buff. Soc. Nat. Sci., x, 5 II. nigra Osborn. Ohio State Univ., Bull. 22, 543.

Empoa Fitch.

Typhlocyba Sahlberg.

fabæ Harris. Ins. Inj. Veg., 230.

lethierryi Edwards. Me. Agr. Expt. Sta., Bull., 238, I 57.

querci Fitch. Proc. U. S. Nat. Mus., xx, 766.

querci Fitch, var. gilletti Van Duzee. bifasciata. Me. Agr. Expt. Sta., Bull., 238, I 57.

rosæ Linnæus. Rose leaf-hopper, Proc. U. S. Nat. Mus., xx, $77 \mathrm{x}$.

ulmi Linnæus. Proc. U. S. Nat. Mus., xx, 769.

Erythroneura Fitch.

comes Say. Grape leaf-hopper. Proc. U. S. Nat. Mus., xx, 759.

comes var. maculata Gillette. Ibid., $\mathrm{xx}, 764$.

obliqua Say. Ibid., $\mathrm{xx}, 756$.

vulnerata Fitch. Ibid., xx, 764 . 
Family MENBRACIDA.

Campylenchia Stål.

latipes Say. curvata Stål. Bull. Buff. Soc. Nat. Sci., ix, I II. Enchenopa Amyot and Serville.

binotata Say. Bull. Buff. Soc. Nat. Sci., ix, II2.

Ceresa Amyot and Serville.

albescens Van Duzee. Bull. Buff. Soc. Nat. Sci., ix, 35. (W.E.B.)

basalis Walker. turbida. Bull. Buff. Soc. Nat. Sci., ix, 33, 39. borealis Fairmaire. Ibid., ix, 34, 38 .

brevicornis Fitch. Ibid., ix, 34, 40.

brevis Walker. Ibid., ix, 34, 40.

bubalus Fabricius. Buffalo tree-hopper. Insect Book, Pl. xxvii, I8; Bull. Buff. Soc. Nat. Sci., ix, 33, 36.

constans Walker. Bull. Buff. Soc. Nat. Sci., ix, 37. (L.B.W.).

diceros Say. Ibid., ix, 33, 35 .

palmeri Van Duzee. Ibid., ix, 38. (L.B.IV.)

taurina Fitch. Ibid., ix, 34, 37.

\section{Stictocephala Stål.}

inermis Fabricius. Bull. Buff. Soc. Nat. Sci., ix, 44.

lutea Walker. Ibid., ix, 43, 49.

\section{Micrutalis Fowler.}

calva Say. Bull. Buff. Soc. Nat. Sci., ix, 53.

dorsalis Fitch. Ibid., ix, 53. (L.B.IV.)

\section{Carynota Fitch.}

mera Say. Bull. Buff. Soc. Nat. Sci., ix, 55, 56.

porphyrea Fairmaire. Ibid., ix, 56, 57 .

stupida Walker. muskokensis Goding. Ibid., ix, 56.

Thelia Amyot and Serville.

bimaculata Fabricius. Bull. Buff. Soc. Nat. Sci., ix, 57.

Glossonotus Butler.

acuminatus Fabricius. Bull. Buff. Soc. Nat. Sci., ix, 58, 59. cratægi Fitch. Ibid., ix, 58, 59.

godingi Van Duzee. Ibid., ix, 58, 59.

univittata Harris. Ibid., ix, 58 . 
[Bull.

Telamona Fitch.

ampelopsidis Harris. Bull. Buff. Soc. Nat. Sci., ix, 63, 68. barbata Van Duzee. Ibid., ix, 62, 65. (L.B.W.) coryli Fitch. Ibid., ix, 63, 68.

declivata Van.Duzee. Ibid., ix, 6r, 64.

decorata Ball. Proc. Wash. Biol. Soc., xvi, I79. (I.W.D.)

dubiosa Van Duzee. irrorata. Bull. Ill. St. Lab. Nat. Hist., iii, 418. (L.B.W.)

extrema Ball. Bull. Buff. Soc. Nat. Sci., ix, 64, 7r. (M.P.Z.) maculata Van Duzee. Ibid., ix, 63, 72 .

querci Fitch. Ibid., ix, 62, 67.

reclivata Fitch. Ibid., ix, 62, 67. (D.J.C.)

sinuata Fowler. Ibid., ix, 63, 69. (L.B.W.)

subfalcata Van Duzee. Ibid., $\mathrm{x}, 509$. (L.B.W.)

tristis Fitch. coryli. Ibid., ix, 63, 68. (H.L.V.)

unicolor. Fitch. Ibid., ix, 64, 7I.

\section{Smilia Germar.}

camelus Fabricius. Bull. Buff. Soc. Nat. Sci., ix, 74 . camelus var. viridis Goding. Bull. Ill. State Lab. Nat. Hist., iii, 426 .

sp.

\section{Cyrtolobus Goding.}

cinctus Van Duzee. Bull. Buff. Soc. Nat. Sci., ix, 8o, 86. (L.B.W.)

cinereus Emmons. Ibid., ix, 8r, $9 \mathrm{r}$.

discoidalis Emmons. Ibid., ix, 8o, go. (L.B.W.)

fenestratus Fitch. Ibid., ix, 79, 8r.

fuliginosus Emmons. Ibid., ix, 79, 82.

fuscipennis Van Duzee. Ibid., ix, 8r, 91. (L.B.W.)

inermis Emmons. Ibid., ix, 8r, 9o. (Q.S.L.)

intermedius Emmons. Ibid., ix, 8o, 9o. (L.B.W.)

maculipennis Emmons. Ibid., ix, 8I, 89. (L.B.W.)

muticus Fabricius. trilincatus Say. Xantholobus. Ibid., ix, 96.

ovatus Van Duzee. Ibid., ix, 96.

sculptus Fairmaire. Ibid., ix, 79, 85. (L.B.W.)

tuberosus Fairmaire. Ibid., ix, 79, 84. (L.B.W.)

vau Say. Ibid., ix, 8o, 87. 


\section{Atymna Stål.}

castaneæ Fitch. Bull. Buff. Soc. Nat. Sci., ix, 92, 93. inornata Say. Ibid., ix, 92, 93. (L.B.W.)

querci Fitch. Ibid., ix, 92, 93.

\section{Xantholobus Van Duzee.}

muticus Fabricius. trilineatus Say. Bull. Buff. Soc. Nat. Sci., ix, 96 .

Ophiderma Fairmaire.

flava Goding. Bu11. Buff. Soc. Nat. Sci., ix, 98, Ioo.

flavicephala Goding. Insect Life, v, 92. (M.P.Z.)

flaviguttula Goding. Bull. Ill. State Lab. Nat. Hist., iii, 438. flaviguttula var. definita Woodruff. Jour. N. Y. Ent. Soc., xxvii, 259. (L.B.W.)

grisea Woodruff. Ibid., xxvii, 254. (L.B.W.)

pubescens Emmons. Bull. Buff. Soc. Nat. Sci., ix, 99.

salamandra Fairmaire. Ibid., ix, 98, 99.

\section{Vanduzea Goding.}

arcuata Say. Bull. Buff. Soc. Nat. Sci., ix, IO2. (L.B.W.)

Entylia Germar.

bactriana Germar. carinata. Bull. Buff. Soc. Nat. Sci., ix, 105.

Publilia Stål.

concava Say. Bull. Buff. Soc. Nat. Sci, ix, Io6.

Family CERCOPID E.

Monecphora Amyot and Serville.

Tomaspis Stål.

bicincta Say var. ignipecta Fitch. Ins. N. Y., iii, 7I, in Rept.

Agr. Soc., 389 .

Aphrophora Germar.

parallela Say. Cercopis. Compl. Writ., i, 202.

quadrinotata Say. Compl. WV rit., ii, 38r.

saratogensis Fitch. Cat. Homop. N. Y., 53, 71 Io.

Lepyronia Amyot and Serville.

quadrangularis Say. Compl. Writ., ii, 256.

Philænus Stål.

lineatus Linnæus. Rept. N. Y. State Ent., iv, 240. 


\section{Philaronia Ball.}

bilineata Say. americanus Baker. Can. Ent., xxix, II2.

Clastoptera Germar.

obtusa Say var. obtusa Ball. Compl. Writ., ii, 256.

obtusa var. achatina Germar. Cat. Homop. N. Y., 53.

proteus Fitch. Cat. Ilomop. N. Y., 54, 722.

proteus var. saint-cyri Provancher. Nat. Can., iv, 35 I.

proteus var. pini Fitch. Cat. Homop. N. Y., 53.

\section{Family CICADID E.}

Tibicen Latreille.

auletes Germar. Large dog day harvest fly, Jour. N. Y. Ent. Soc., xxiii, 2.

canicularis Harris. Dog day harvest fly, Ent. News, xviii, I26. linnei Smith and Grossbeck. Ent. News, xviii, I27.

lyricen DeGeer. fuliula Osborn. Ibid,, xviii, r25.

lyricen var. englehardti Davis. Ibid., xxi, 457.

sayi Smith and Grossbeck. Ibid., xviii, I24.

\section{Tibicina Kollar.}

septendecim Linnæus. Seventeen year locust or periodical cicada. Bur. Ent., Bull. 7I ; Rept. Conn. Agr. Expt. Sta., I9I I, 296.

Family PSYLLIDÆ.

Livia Latreille.

maculipennis Fitch. Psyche xix, 6. marginata Patch. Ibid., xix, 8.

vernalis Fitch. Diraphia. Rept. Ins. N. Y., iv, 53: Psyche, xix, 7 .

Aphalara Forster.

veaziei Patch. Me. Agr. Expt. Sta., Bull. I87, I6.

Trioza Fœrster.

tripunctata Fitch. Rept. N. Y. State Ent., ix, 404.

Calophya Fr. Loew.

flavida Schwarz. Proc. Wash. Ent. Soc., vi, 243. nigripennis Riley. Ibid., vi, 244. 
Pachypsylla Riley.

celtidis-gemma Riley. Proc. Wash. Biol. Soc., ii, 74. celtidis-mamma Riley. Ibid., ii, 73.

celtidis-vesiculum Riley. Am. Mus. Jour., iv, 35.

venusta Osten Sacken. Proc. Wash. Biol. Soc., ii, 72.

Spanioneura Fœrster.

fonscolombii Fœrster. ${ }^{1}$ Verh. Zool-Bot. Ges. Wien., I879, pl. ix, 26.

Psylla Geoffroy.

annulata Fitch. U. S. Nat. Mus., Bull. 85, I52. (IV. C. W.) buxi Linnæus. Verh. Zool-Bot. Ges. Wien., I881, I69.

floccosa Patch. Can. Ent., xli, 301.

galeaformis Patch. Me. Agr. Expt. Sta., Bull. 187, I2. pyricola Fœrster. pyri. Pear psylla. Rept. Conn. Agr. Expt. Sta., 1903, 262.

striata Patch. Me. Agr. Expt. Sta., Bull. I87, I4.

Psyllopsis Fr. Loew.

fraxinicola Fœrster. U. S. Nat. Mus., Bull. 85, I32: Rept. N. Y. State Ent., xxvi, 39.

'This species on Buxus sempervirens has not been identified with certainty.

Family APHIDID E.

\section{Longistigma Wilson.}

caryæ Harris. Lachnus caryc, L. longistigma Monell, $L$. platanicola Riley. Can. Ent., 4I, 385 .

Anœcia Koch.

corni Fabricius. Aphis. Schizoneura panicola Thomas. U. S. Dept. Agr., Div. Ent., Bull. 22, 32.

Todolachnus Matsumura.

pinicola Kaltenbach. abictis. Fitch. 5th Rept. U. S. Ent. Com., 853.

strobi Fitch. Me. Agr. Expt. Sta. Bull. 202, 167.

Calaphis Walsh.

betulæcolens Fitch. Callipterus. Aphid. Minn., 43.

castaneæ Fitch. Ibid., 44.

Callipterinella van der Goot.

annulata Koch. Chaitophorus. Jour. Econ. Ent., I0, 427. 
Euceraphis Walker.

betulæ Koch. Callipterus. Jour. Econ. Ent., Io, 425.

mucida Fitch. Callipterus. Ibid., 425.

Monellia Oestlund.

caryæ Monell. Callipterus. U. S. Dept. Agr., Bull. ıoo, I9. castalis Fitch. Jour. Econ. Ent., IO, 424.

Myzocallus Passerini.

discolor Monell. Callipterus. Aphid. Minn., 4I. punctatellus Fitch. Aphis. Jour. Econ. Ent., Io, 420. ulmifolii Monell. Callipterus. Aphid. Minn., 42.

Therioaphis Walker.

tilliæ Linnæus. Aphis. Ann. Ent. Soc. Am., 2, 33.

Chaitophorus Koch.

nigræ Oestlund. cordata Williams. Jour. Econ. Ent., 429. viminalis Monell. stevensis Sanborn. Aphid. Minn., 40.

\section{Neothomasia Baker.}

bruneri Williams. Chaitophorus. Univ. Studies, Lincoln, Neb., II, No. 3, reprint 6.

populicola Thomas. Me. Agr. Expt. Sta., Bull. 2I3, 78 .

Periphyllus Van der Hoven.

americanus Baker. Jour. Econ. Ent., Io, 428.

lyropicta Kessler. Ibid., Io, 428.

Drepanaphis Del Guercio.

acerifoliæ Thomas. Siphonophora. Drepanosiphum. Kan. Univ. Sci., Bull., 3, 45.

Drepanosiphum Koch.

platanoides Schrank. Aphis. Jour. Econ. Ent., 2, 346.

Phyllaphis Koch.

fagi Linnæus. Beech aphid. Aphid. Minn., 32.

Melanoxantherium Schouteden.

media Baker. Jour. Econ. Ent., I0, 43I.

smithiæ Monell. Melanoxanthus: Jour. Econ. Ent., 2, 387.

Aphis Linnæus.

cardui Linnæus. pruni. prunifolic. Me. Agr. Expt. Sta., Bull. 233,263 . 
cerasifoliæ Fitch. Choke-cherry aphid. Ibid., 262.

davisi Patch. Jour. Econ. Ent., IO, 4IS. (N. N. for Aphis populifolice Fitch of Davis, I910.)

folsomi Davis. Ent. News, I9, I45.

gossypii Glover. malve Koch., cucurbiti Buckton. Melon

aphid. Rept. Conn. Agr. Expt. Sta., 1908, 813.

laburni Kaltenbach. Afid. Ital., I25.

maidi-radicis Forbes. Corn root aphid. Bur. Ent., Bull. 85,

97.

maidis Fitch. Bur. Ent., Tech. Ser., I2, I44.

nerii Boyer. Oleander aphid. Pom. Jour. Ent., iii, 530.

persicæ-niger Smith. Black peach aphid. Jour. Econ. Ent., i, 308 .

pomi DeGeer. Green apple aphid. Rept. Conn. Agr. Expt. Sta., I903, 259.

pseudobrassicæ Davis. Turnip aphid. Ibid., I9r6. Texas Agr. Expt. Sta., Bull. r8o; Rept. Conn. Agr. Expt. Sta., 1916, 98.

rumexicolens Patch. Jour. Econ. Ent., IO, 4I7.

rumicis Linnæus. Bean aphid. Aphid. Minn., 6r.

saliceti Kaltenbach. Jour. Econ. Ent., Io, 4I7.

sanborni Patch. Me. Agr. Expt. Sta., Bull. 225, 52.

spiræcola Patch. spircella Gillette. Me. Agr. Expt. Sta., 233, 270.

viburniphila Patch. Jour. Econ. Ent., Io, 4r6.

yuccæ Cowen. yucciola Williams. Yucca aphid. Hemip.

Col., 122.

Anuraphis Del Guercio.

malifoliæ Fitch. sorbi Kaltenbach. Rosy apple aphid. Rept. Conn. Agr. Expt. Sta., 1909, 343; Thirteenth Rept. Del. Agr. Expt. Station, 1900, I49.

tulipæ Boyer. Aphis gladioli Felt. Rept. N. Y. State Ent., 24, 19.

\section{Brevicoryne Das.}

brassicæ Linnæus. Cabbage aphid. Ins. Life, iii, 289; Cornell Univ. Agr. Expt. Sta., Bull. 300.

\section{Hyalopterus Koch.}

arundinis Fabricius. Aphis prini. Jour. Econ. Ent., 2, 354. 
Rhopalosiphum Koch.

nymphææ Linnæus. Aplis. butoni. aquaticus. prunorum.

Science, 42, I64. Me. Agr. Expt. Sta., Bull. 233, 263. prunifoliæ Fitch. (avence in part) Aphid. Minn., 65. serotinæ Oestlund. Ibid., 76.

Siphocoryne Passerini.

xylostei Schrank. Aphis. Afid. Ital., I42.

Amphorophora Buckton.

rhois Monell. Aphid. Minn., 75.

\section{Myzus Passerini.}

braggii Gillette. Capitophorus. Jour. Econ. Ent., 8, 375. cerasi Fabricius. Aphis. Cherry aphid. Aphid. Minn., 73; Me. Agr. Expt. Sta., Bull. 233, 258.

persicæ Sulzer. Green peach aphid. Ins. N. Y., iii, 359. ribis Linnaeus. Currant aphid. Aphid. Minn., 74. rosarum Kaitenbach. Me. Agr. Expt. Sta., Bull. 233, 269. violæ Pergande. Rhopalosiphum. Violet aphid. Can. Ent., xxxii, 29.

\section{Macrosiphum Passerini.}

albifrons Essig. Pom. Jour. Ent., 3, 543.

cratægi Monell. Siphonophora cratcgi. Me. Agr. Expt. Sta., Bull. 233, 255.

granarium Kirby. Jour. Econ. Biol., viii, $5^{8 .}$

illinoiensis Shimer. Science 4I, 834.

lilii Monell. Siphonophora. Rept. U. S. Entomologist, I879, 220.

liriodendri Monell. Ins. I1l., 8, I89; Jour. Econ. Ent., 4, 383. luteola Williams. Siphonophora. University Studies, Lincoln, Nebraska, II, No. 3, reprint 3I.

pisi Kaltenbach. U. S. Rept. Agr., Bull. 276.

rosæ Linnaus, Aphis rosc. Me. Agr. Expt. Sta., Bull. 233, 268. rudbeckiæ Fitch. Nectarophora rudbeckic. Proc. Ent. Soc. Phila., i, 298; Jour. Econ. Ent., 4, 383.

sanborni Gillette. Siphonophora chrysanthcmicolcns Williams. Can. Ent., x1, 65.

solanifolii Ashmead. Siphonophora. Can. Ent., xiv, 92 ; Me. Agr. Expt. Sta., Bull. 242. 
sonchi Linnæus. Aphis sonchi. Jour. Econ. Biol., viii, 64. tanaceti Linnæus. Aphis tanaceti. Ins. I11., viii, 68. Jour. Econ. Ent., 4, 382 .

Eriosoma Leach.

americana Riley. Schizoneura Hartig. Mé. Agr. Expt. Sta., Bull. 24I ; Bull. 256, 340 (Key). Jour. Agr. Research, 6, 359.

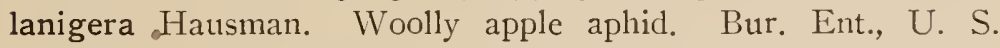
Dept. Agr., Rept. No. Ior ; Me. Agr. Expt. Sta., Bull. 256. lanuginosa Hartig. Me. Agr. Expt. Sta., Bull. 256, 340 (Key). rileyi Thomas. Me. Agr. Expt. Sta., Bull. 256, 340 (Key). ulmi Linneus. Jour. Agr. Research, 6, 359; Me. Agr. Expt. Sta., Bull. 256, 340 (Key).

Tetraneura Hartig.

graminis Monell. colophoidca Howard. ulmicola Gillette.

Asiphum Koch.

pseudobyrsa VValsh. Ent. News, 25, 269.

Prociphilus Koch.

approximatus Patch. Jour. Econ. Ent., IO, 4I8. corrugatans? Sirrene. Jour. Agr. Research, 5, III7.

imbricator Fitch. Woolly beech aphid.

tessellata Fitch. Schizoncura accrifolii. "Alder blight." Jour. Econ. Ent., ii, 35 .

Neoprociphilus Patch.

aceris Monell. Jour. Agr. Research, 5, i I I7.

Thecabius Koch.

populi-condupliformis Cowen. patchii Gillette. Hemip. Col., II 5 .

Pemphigus Hartig.

populicaulis Fitch. Me. Agr. Expt. Sta., Bull. 213, 77.

Hormaphis Osten Sacken.

hamamelidis Fitch. Aphid. Minn., 24; Bur. Ent., Tech. Series, No. 9, 9.

Hamamelistes Shimer.

spinosus Shimer. papyracer. Aphid. Minn., I9; Bur. Ent., Tech. Series, No. 9, 25. 
Chermes Linnæus.

abietis Kaltenbach. Spruce gall aphid. Rept. Conn. Agr. Expt. Sta., 1906, 302; Me. Agr. Expt. Sta., Bull. 173. cooleyi Gillette. Proc. Acad. Nat. Sciences Phila., 1907, reprint, 3-I4.

pinicorticis Fitch. Pine bark aphid. Ins. Aff. Park \& Wood. Trees, 192.

pinifoliæ Fitch. abieticolens. Me. Agr. Expt. Sta., Bull. I73. strobilobius Kaltenbach. Woolly larch aphid. Ins. Aff. Park \& Wood. Trees, I87.

Phylloxera Boyer.

caryæcaulis Fitch. Hickory gall aphid. Ins. Aff. Park and

Wood. Trees, 33I ; Proc. Davenport Acad. Sci., 9, 243.

castaneæ Haldeman. Proc. Davenport Acad. Sci., 9, 257.

foveola Pergande. Ibid., 9, 188.

rimosalis Pergande. Ibid., 9, 216.

vastatrix Planchon. Grape phylloxera. Insects and Insecticides, ist ed. Iog.

Family ALEYRODIDÆ.

Aleurochiton Tullgren.

forbesii Ashmead. Aleurodes aceris Forbes. Bur. Ent., Tech. Ser. $27,88$.

Aleyrodes Latreille.

asarumis Shimer. actece Britton. Ent. News, xvi, 65.

fernaldi Morrill. Psyche, x, 83 .

Trialeurodes Cockerell.

Asterochiton Quaintance and Baker.

Aleyrodes.

coryli Britton. Ent. News, xviii, 337.

morrilli Britton. Ibid., xviii, 340.

packardi Morrill. Can. Ent., xxxv, 25.

vaporariorum Westwood. Greenhouse white fly. Rept. Conn.

Agr. Expt. Sta., 1902, 148.

waldeni Britton. Ent. News, xviii, 339.

Tetraleurodes Cockerell.

mori Quaintance, var. maculata Morrill. Psyche, x, 8I. 
No. 3I.] insects OF CONNECTICUT: HeMIPTERA.

Family COCCID E.

Orthezia Bosc d'Antic.

insignis Douglas. Greenhouse orthezia. Rept. Conn. Agr. Expt. Sta., 1905, 235.

Asterolecanium Targioni-Tozzetti.

variolosum Ratzeburg. Pit-making oak scale. Rept. Conn. Agr. Expt. Sta., I905, 235.

Kermes Boitard.

kingii Cockerell. Ohio Acad. Sci., iv, part 2, 36.

pubescens Bogue. Ibid., iv, part 2, 37.

sassceri King. Jour. Ent. Zool., vi, 48.

waldeni King. Jour. Econ. Ent., vii, I50.

Gossyparia Signoret.

spuria Modeer. ulmi. Elm scale. Rept. Conn. Agr. Expt.

Sta., I905, 235.

Eriococcus Targioni-Tozzetti.

azaleæ Comstock. Cornell Agr. Expt. Sta., Bull. 372, 487.

Phenacoccus Cockerell.

acericola King. Woolly maple leaf scale. Rept. Conn. Agr. Expt. Sta., 1905, 226.

Pseudococcus Westwood.

adonidum Linnæus. Dactylopius longispinus. Long-tailed mealy bug. Jour. Econ. Ent., ii, 43I.

citri Risso. Dactylopius destructor. Common or short-tailed mealy bug. Rept. Conn. Agr. Expt. Sta., I905, 236.

Eriococcus Targioni-Tozzetti.

vitis Linnæus. innumerabilis. Cottony maple scale. Jour.

Econ. Ent., ii, 433.

\section{Coccus Linnæus.}

hesperidum Linnæus. Lecanium. Soft brown scale. In greenhouses. Jour. Econ. Ent., ii, 436.

Toumeyella Cockerell.

liriodendri Gmelin. Eulecanium tulipiferc. Tulip tree scale. Rept. Conn. Agr. Expt. Sta., I905, 239; Jour. Econ. Ent., ii, 447 . 
Lecanium Burmeister.

caryæ Fitch. Hickory Lecanium. Jour. Econ. Ent., ii, 442. corni Bouché. armeniacum, canadense, cerasifex. 'European fruit lecanium. Jour. Econ. Ent., ii, 443; Rept. Conn. Agr. Expt. Sta., I905, 237.

nigrofasciatum Pergande. Terrapin scale. Rept. Conn. Agr. Expt. Sta., 1905, 238.

Saissetia Deplanches.

hemisphærica Targioni-Tozzetti, filicum. Hemispherical scale. Rept. Conn. Agr. Expt. Sta., 1905, 239.

Physokermes Targioni-Tozzetti.

piceæ Schrank. Spruce scale. Can. Ent., 49, 3 I7.

Chionaspis Signoret.

americana Johnson. White elm scale. Rept. Conn. Agr. Expt. Sta., 1905, 239.

caryæ Cooley. Mass. Agr. Expt. Sta., Spec. Bu1l., I899, 40. corni Cooley. Ibid., Spec. Bull., I899, I 5.

euonymi Comstock. Euonymus scale. Rept. Conn. Agr. Expt. Sta., I905, 240.

furfura Fitch. harrisii. Scurfy scale. Ibid., I903, 227.

lintneri Comstock. Mass. Agr. Expt. Sta., Spec. Bull., 1899, 22.

pinifoliæ Fitch. Pine leaf scale. Rept. Conn. Agr. Expt. Sta., 1905, 240.

\section{Hemichionaspis Cockerell.}

aspidistræ Signoret. In greenhouses. Mass. Agr. Expt. Sta., Spec. Bull., I $899,45$.

\section{Diaspis Costa.}

boisduvalii Signoret. In greenhouses. Ohio Acad. Sci., 1904, 52.

carueli Targioni-Tozzetti. Juniper scale. Rept. U. S. Dept. Agr., I880, 3 Io.

echinocacti Bouché, var. cacti Comstock. In greenhouses. Cornell Agr. Expt. Sta., Butll. 372, $55^{2}$.

piricola Del Guercio. Epidiaspis. European pear scale. Ent. News, xi, 590. 
Aulacaspis Cockerell.

pentagona Targioni-Tozzetti. West Indian peach scale. Rept.

Conn. Agr. Expt. Sta., I9I3, 240.

rosæ Bouché. Diaspis. Rose scale. Ibid., I905, 241.

\section{Leucaspis Targioni-Tozzetti.}

japonica Cockerell. Psyche, viii, 53.

\section{Aspidiotus Bouché.}

abietis Schrank. Hemlock leaf scale. 5th Rept. U. S. Ent. Com., 878.

ancylus Putnam. Putnam's scale. Ohio Acad. Sci., I904, 57. cyanophylli Signoret. In greenhouses. Ibid., I904, 59.

forbesi Johnson. Cherry scale. Bull. Ill. State Lab. Nat.

Hist., iv, 380 .

hederæ Vallot. ncrii. White scale. Oleander scale. In greenhouses. Rept. Conn. Agr. Expt. Sta., 1905, 242. osborni Newell and Cockerell. Proc. Iowa Acad. Sci., v, 229. ostreæformis Curtis. European fruit scale. Bur. Ent., Bull. 20,76 .

perniciosus Comstock. San José scale. Conn. Agr. Expt. Sta., Bull. 165.

ulmi Johnson. Elm aspidiotus. Bull. Ill. State Lab. Nat. Hist., iv, 388 .

\section{Chrysomphalus Ashmead.}

aonidum Linnæus. ficus. Circular scale. Fig scale. In greenhouses. Rept. Conn. Agr. Expt. Sta., 1905, 243.

aurantii Maskell. Red orange scale. In greenhouses. Ohio Acad. Sci., 1904, 7I.

dictyospermi Morgan. Morgan's scale. In greenhouses.

Rept. Conn. Agr. Expt. Sta., 1905, 244. obscurus Comstock. Report U. S. Dept. Agr., I 80, 303.

\section{Lepidosaphes Shimer.}

beckii Newman. Mytilaspis citricola. Purple scale. In greenhouses. Ohio Acad. Sci., 1904, 73. newsteadi Sulc. Rept. Conn. Agr. Expt. Sta., I9I5, I40. ulmi Linnæus. Mytilaspis pomorum. Oyster-shell scale. Ibid. $1903,229$. 
Ischnaspis Douglas.

longirostris Signoret. filiformis. Thread scale. In greenhouses. Rept. Conn. Agr. Expt. Sta., 1905, 245.

Parlatoria Targioni-Tozzetti.

pergandii Comstock. Chaff scale. In greenhouses. Ohio Acad. Sci., 1904, 75.

\section{Suborder HETEROPTERA.}

Family CORIXIDÆ.

\section{Arctocorixa Wallengren.}

alternata Say. Compl. Writ., ii, $25 \mathrm{I}$. compressa Abbott. Wash. Univ. Studies, ii, 8I. compressa var. interrupta Say. Compl. Writ., ii, 250. kennicottii Uhler. Trans. Md. Acad., i, 393. lucida Abbott. Ent. News, xxvii, 341. nitida Fieber. Bull. Brook. Ent. Soc., viii, 88, 89. ornata Abbott. Ent. News, xxvii, 34I. scabra Abbott. Bull. Brook. Ent. Soc., viii, 88, 90. seriata Abbott. Ent. News, xxvii, 342.

\section{Corixa Geoffroy.}

verticalis Fieber. Bull. Brook. Ent. Soc., viii, 89, 90.

\section{Family BELOSTOMATIDÆ.}

\section{Benacus Stål.}

griseus Say. Insect Book, P1. xxvii, 4.

\section{Lethocerus Mayr.}

americanus Leidy. Belostoma. Giant water bug. Life Hist. Amer. Insects, 4.

obscurus Dufour. Belostoma. Ann. Soc. Ent. France, I863, $3^{8} 3$.

Belostoma Latreille.

flumineum Say. Compl. Writ., i, 364 . 
Family NEPID无.

Nepa Linnæus.

apiculata Uhler. Proc. Bos. Soc. Nat. Hist., xix, I40.

Ranatra Fabricius.

americana Montandon. quadridentata Uhler. Can. Ent., xxxvii, I87; Insect Book, P1. xxvii, fig. 2. (as fusca.).

\section{Family NAUCORIDÆ.}

\section{Pelocoris Stål.}

femoratus Beauvois. Naucoris poeyi. Stand. Nat. Hist., ii, 259.

\section{Family NOTONECTIDÆ.}

\section{Notonecta Linnæus.}

insulata Kirby. Jour. N. Y. Ent. Soc., xiii, 162. (J.R.T.B.) irrorata Uhler. Insect Book, Pl. xxvii, I2; Jour. N. Y. Ent. Soc., xiii, I59.

undulata Say. Jour. N. Y. Ent. Soc., xiii, I 52.

undulata var. latona Kirkaldy.

variabilis Fieber. Jour. N. Y. Ent. Soc., xiii, I55.

\section{Plea Leach.}

striola Fieber. Stand. Nat. Hist., ii, 253.

Family SALDID瓜:

\section{Pentacora Reuter.}

hirta Say. Salda. Compl. Writ., i, 359.

ligata Say. Salda. Ibid., i, 359.

Salda Fabricius.

coriacea Uhler. Bull. Geol. Geog. Surv. Terr., iii, (2) 436.

Saldula Van Duzee.

confluenta Say. Compl. Writ., i, 36r.

interstitialis Say. Salda. Compl. Writ., ii, 248.

pallipes Fabricius. Salda. Bull. Geol. Geog. Surv. Terr., iii, (2) 446.

reperta Uhler. Salda. Ibid., 446.

saltatoria Linnæus. Act. Soc. Sci. Fenn., xxi. (2) I5. 


\section{Family VELIIDÆ.}

\section{Microvelia Westwood.}

americana Uhler. Hemip. Col., 6r.

Family GERRIDÆ.

Gerris Fabricius.

buenoi Kirkaldy. Ent. News, xxii, 246. canaliculatus Say. Comp1. Writ., i, 363 . conformis Uhler. Trans. Am. Ent. Soc., xxxvii, 247. marginatus Say. Insect Book, P1. xxix, 38; Compl. Writ., i, 362.

remigis Say. Ibid., P1. xxvii, 6; Compl. Writ., i, 362. rufoscutellatus Latreille. Gen. Ins., iii, I34.

Metrobates Uhler.

hesperius Uhler. Proc. Bost. Soc. Nat. Hist., xiv, I08.

\section{Family MIRIDE (CAPSID E).}

Chlamydatus Curtis.

associatus Uhler. Hayden's Geol. Surv. Mont., I872, 4I9.

Campylomma Reuter.

verbasci Meyer. Hemip. Gymn. Eur., I (2), 53.

Apocremnus Fieber.

ancorifer Fieber. Psallus. Hemip. Gymn. Eur., I, IO4.

Plagiognathus Fieber.

annulatus Uhler. Hemip. Col., 5I.

blatchleyi Reuter. Ofv. Finska. Vet. Soc. Forh., liv., Afd., A, No. 7, 61.

confusus Reuter. Act. Soc. Sci. Fenn., xxxvi, (2) 80.

fraternus Uhler. Hemip. Col., 51.

obscurus Uhler. Hayden's Geol. Surv. Mont., I872, 4I8 (C.R.E.)

politus Uhler. Hemip. Col., 52 (C.R.E.)

Rhinocapsus Uhler.

vanduzeei Uhler. Trans. Md. Acad., I890, 82 (E.L.D.).

Microphylellus Reuter.

modestus Reuter. Finsk. Vet. Soc. Forh., Bd. liv., I912, 62. 
Reuteroscopus Kirkaldy.

ornatus Reuter. Episcopus. Ofv. Vet. Akad. Forh., 1875, 90. Lopus Hahn.

decolor Fallen chrysanthemi. Hemip. Gymn. Eur., II, 287.

Macrotylus Fieber.

amœnus Reuter. Nearktische Capsiden, Act. Soc. Sci. Fenn., xxvi, (2) 75 .

Halticus Hahn.

citri Ashmead. whleri Gerard. Garden flea hopper. Ent. Amer., iii, I55; Rept. Conn. Agr. Expt. Sta., 1904, 217. intermedius Uhler. Proc. U. S. Nat. Mus., xxvii, 360.

Strongylocoris Blanchard.

stygicus Say. Capsus. Stiphrosoma. Compl. Writ., i, 344.

Pseudoxenetus Reuter.

scutellatus Uhler. Xenetus. Trans. MId. Acad., I890, 80.

Sericophanes Reuter.

noctuans Knight. Ent. News, xxviii, 3; Proc. Iowa Acad.

Sci., V, 238.

Orectoderus Uhler.

obliquus Uhler. Bull. U. S. Geol. Sur. Terr., ii, 320.

Ceratocapsus Reuter.

fasciatus Uhler. Bull. U. S. Geol. Surv. Terr., viii, 42I (E.L.D.).

modestus Uhler. Melimna. Ent. Amer., iii, 69. (C.R.E.)

pumilus Uhler. Melinna. Ibid., iii, 69. (C.R.E.)

Lopidea Uhler.

cæsar Reuter. Nearktische Capsiden Act. Soc. Sci. Fenn., xxxvi, (2) 72.

heidemanni Knight. Ent. News, xxriii, 456. (H.L.J.)

instabilis Reuter. Ibid., xxxvi, (2) 72.

marginata Uhler. Proc. Cal. Acad. Sci., iv, 249.

media Say. Compl. Writ., i, 34I.

reuteri Knight. Ent. News, xxviii, 459. (B.H.W.)

robiniæ Uhler. Capsus. Proc. Phila. Ent. Soc., i, 24.

staphyleæ Knight var. sanguinea Knight. Ent. News, xxviii, 4 fir. (W.E.B.) 
Orthotylus Fieber.

catulus Van Duzee. Proc. Cal. Acad. Sci., (4th ser) VI, I916, I06.

chlorionis Say. Ibid., 1916, 98.

cruciatus Van Duzee. Ibid., 1916, II9.

flavosparsus Sahlberg. Ibid., I9I6, 97; Hemip. Gymn. Eur., I 883,360 .

uniformis Van Duzee. Proc. Calif. Acad. Sci., Ser. 4, vi, 99. Innacora Reuter.

malina Uhler. Bull. U: S. Geol. Surv. Terr., iii, 4 I9.

Hyaliodes Reuter.

vitripennis Say. Capsus. Compl. Writ., i, 345.

Dicyphus Fieber.

agilis Uhler. Bull. U. S. Geol. Surv., 1877, 425.

californicus Stål. Freg. Eugenies Resa, Hemip., I859, 259.

vestitus Uhler. Hemip. Col., 46. (E.L.D.)

Macrolophus Fieber.

separatus Uhler. Dicyphus. Proc. Zool. Soc. London, I893, 194. (C.R.E.)

Cylapus Say.

tenuicornis Say. Compl. Writ., i, 347.

Fulvius Stål.

brunneus Provancher. Lygus. Pamerocoris anthocoroides.

U. S. Geol. Surv., Bull. iii, I877, 425.

Monalocoris Dahlbom.

filicis Linnæus. Cimex. Syst. Nat., (I0) I758, 443.

Sixeonotus Reuter.

insignis Reuter. Ofv. Vet. Akad. Forh., 1875 (9) 78.

Cimatlan Distant.

catulum Uhler. Proc. Cal. Acad. Sci., (2) iv, I894, 257. venatorium Van Duzee. Bull. Buffalo Soc. Nat. Sci., X, I912, 479.

Camptobrochis Fieber.

grandis Uhler. Ent. Amer., ii, 230.

nebulosus Uhler. Hayden's Geol. Surv. Mont., I872, 4 I7. 
nitens Reuter. Nearktische Capsiclen, Act. Soc. Sci. Fenn., xxxvi, (2) I909, 56. (C.R.E.)

validus Renter var. cunealis Reuter. Nearktische Capsiden, Act. Soc. Sci. Fenn., xxxvi, 59.

\section{Deræocoris Kirschbaum.}

ruber Linnaus. Syst. Nat. Ed. X, I758, 446.

ruber var danicus Fabricius. Syst. Ent., iv, I\&I.

ruber var. segusinus Muller. Hemip. Gymı. Eur., V, 32.

\section{Garganus Stal.}

fusiformis Say. Capsus. Compl. IVrit., i, 344, I859.

Neurocolpus Renter.

nubilus Say. Capsus. Compl. ITrit., i, 34I, I859.

\section{Phytocoris Fallen.*}

eximius Renter. Nearktische Capsiden, Act. Soc. Sci. Fenn., xxvi, (2) 23, I909.

fenestratus Reuter. Ibid., xxvi, (2) 24, I909.

infuscatus Renter. Ibid., xxvi, (2) 20. (C.R.E.)

puella Reuter. Ibid., xxvi, (2) 20, (C.R.E.)

puella var. confluens Reuter. Ibid., xxvi, (2) 20. (C.R.E.) tibialis Reuter. Ibid., xxvi, (2) 20.

Paracalocoris Distant.

scrupeus Say. Capsus. Compl. Writ., i, 342, I859; Ann. Ent. Soc. Am., IX, 368.

Stenotus Jakolevsky.

hinotatus Fabricius. Oncognathus. Ins. Life, v, 90. (O.H.)

Adelphocoris Renter.

rapidus (Say) Reuter. Calocoris. Compl. Writ., i, I859, 339.

Dichrooscytus Ficber.

suspectus Reuter. Act. Soc. Sci. Fenn., xxxvi, (2) 37, Igog.

Pœciloscytus Fieber.

basalis Reuter. scriccus. Bull. U. S. Geol. Surv., iii, 422, IS75.

venaticus Uhler, var.

* See also appendix. 


\section{Pœcilocapsus Reuter.}

lineatus Fabricius. Capsus 4-vittatus. Four-lined leaf bug. N. Y. (Cornell) Agr. Expt. Sta. Bull., 58; Compt. Writ., i, 339 .

\section{Horcias Distant.}

dislocatus Say. Het. New Harmony, 2I, I832; Bull. Buffalo Soc. Sci., X, 483 .

dislocatus var. affinis Reıter. Nearktische Capsiden, Act. Soc. Sci Fenn., 4I.

dislocatus var. goniphorus Say. Compl. Writ., i, 34I.

dislocatus var. limbatellus Walker. Nearktische Capsiden, Act. Soc. Sci. Fenn., xxxvi (2), 4I.

dislocatus var. nigrita Reuter. Ibid., 4I.

dislocatus var. scutellatus Van Duzce. Bull. Buff. Soc. Nat. Sci., $x, 484$.

\section{Capsus Fabricius.}

ater Linnæus. Hemip. Gymn. Eur., v, r896, 15. ater var. semiflavus Linmæus. Syst. Nat., Ed. 12, i, 725.

\section{Coccobaphes Uhler.}

sanguinareus Uhler. Proc. Bos. Soc. Nat. Hist., xix, 40I, I878. Tropidosteptes Uhler.

cardinalis Uhler. Proc. Bos. Soc. Nat. Hist., xix, 404, 1878.

Neoborus Distant.

amœnus Reuter var. Neoborus sateus. Proc. Cal. Acad. Sci. (2) iv.

geminus Say. Capsus. Compl. Writ., i, 344.

palmeri Reuter. Bemerk. u. Nearktische Capsiden, I909, 49.

Xenoborus Reuter.

pettiti Renter. Nearlitische Capsiden, Act. Soc. Sci. Fenn., 5o Lygidea Renter.

mendax Reuter. N. Y. (Cornell) Agr. Expt. Sta., Bull. 291.

\section{Lygus Hahn.}

belfragei Renter. Ofv. Vet. Acad. Forlı, I875, 7 I. caryæ Knight. N. Y. (Cornc1l) Agr. Expt. Sta., Bull. 39r, 6I5. (B.H.W.)

caryæ var. subfuscus Knight. Ibid., 616. 
campestris Linneus. Orthops scutcllatus. Bull. U. S. Geol. Sur. Terr., iii, 420, I877; Hemip. Gymn. Eur., v, 79. communis Knight. Can. Ent. xlviii, $3+6$.

fasciatus Reuter. Ofv. Vet. Akad. Forh., 1875, 72. (L.B.IV. hirticulus Van Duzee. tenellus. N. Y. (Cornell) Agr. Expt. Sta., Bull. 391, 633.

inconspicuus Knight. Ibid., 612. (B.H.W.)

invitus Say. Capsus. dark var. Compt. Writ., i, I859, 345. johnsoni Knigltt. N. Y. (Cornell) Agr. Expt. Sta., Bull. 39I, 629. (C.W.J.)

olivaceus Reuter. Ofv. Finska Vet. Soc. Forh., lxix, I907, 7. omnivagus Knight. N. Y. (Cornell) Agr. Expt. Sta., Bull. 39I, 627.

nyssæ Knight. Bull. Brook. Ent. Soc., xiii, 43.

pabulinus Linnæus. Hemip. Gym11. Eur., v, II4.

pabulinus var. signifer Reuter. Nearktische Capsiden, Act. Soc. Sci. Fenn., xxxvi, I909, 42.

pratensis Linnæus. Tarnished plant bug. Rept. Conn. Agr. Expt. Sta., I904, 218.

pratensis var. oblineatus Say. N. Y. (Cornell) Agr. Expt. Sta., Bull., 39I, 564.

rubicundus (Uhler) Reuter. Hemip. Gymn. Eur., v, 72.

tiliæ Knight. N. Y. (Cornell) Agr. Expt. Sta., Bull. 39г, 6rз. (H.L.V.)

vanduzeei Knight var. rubroclarus Knight. Ibid., Bull. 39 I. 567 .

viburni Knight. Ibid., 609. (B.H.W.)

vitticollis Reuter. monachus. Bur. Ent., Bull. I3, old ser., 63.

\section{Platytylellus Reuter.}

confraternus Uhler. Resthenia. Hayden's Surv. Terr. Rept. for I87I, 4II.

insignis Say. Capsus. Compl. Writ., i, I859, 342.

insitivus Say. Capsus. Ibid., i, I859, 340.

Trigonotylus Fieber.

brevipes Jakolevsky. Act. Soc. Sci. Fen11., xxxri, (2) I909, 6. (C.R.E.)

ruficornis Fourcroy. Ent. Paris, 1785, 209; Fallen, Hem.

Suec., I829, I33. 
tarsalis Reuter. Callimiris. Act. Soc. Sci. Fenn., xxxvi, (2) I909, 6.

uhleri Reuter. Callimiris. Ofv. Vet. Akad. Forh., I875, 60.

Stenodema Laporte.

trispinosum Reuter. Brachytropis calcaratus. Nearktische Capsiden, Act. Soc. Fenn., xxxvi (2) I909, 4.

vicinum Provancher. Miris instabilis. affinis. Rept. Geol. Geog. Surv., v, 1875,836 .

Miris Fabricius.

dolobratus Linnæus. Hayden's Geol. Surv. Mont., I872, 409. Mesomiris Reuter.

curtulus Reuter. Nearktische Capsiden, Act. Soc. Sci. Fenn., xxxvi, 5 .

Collaria Provancher.

meilleurii Provancher. Nabidea coracina. Nat. Can., iv, I 872,79 .

oculata Reuter. Trachelomiris. Ofv. Vet. Akad. Forh., 1875（9) 6I.

\section{Family ANTHOCORIDE.}

Xylocoris Dufour.

Piezostcthus Fieber.

cursitans Fallen. Ofv. Vet. Akad. Forh., I87I, 4II.

sordidus Reuter. Ibid., 560. (J.R.T.B.)

Triphleps Fieber.

insidiosa Say. Compl. Writ., i, 357.

\section{Family CIMICID E.}

Cimex Linnæus.

lectularius Linnæus. Bed bug. Bur. Ent., Bull. 4, new series, 32 .

\section{Eciacus Stål.}

vicarius Horvath. Barn swallow bug. Insect Book, 288; Bur.

Ent., Bull. 5, new series, г6r. 
Family NABID E.

Pagasa Stål.

fusca Stein. Berl. Ent. Zeit., i, 90.

Nabis Latreille.

Reduviolus Kirby.

annulatus Reuter. Ofv. Vet. Akad. Forh., xxix, $\$ 6$.

ferus Linnæus. Coriscus. Syst. Nat., Ed. 10, 449.

inscriptus? Kirby. Coriscus. Can. Ent., x, 2 I 3.

roseipennis Reuter. Coriscus. Ofv. Vet. Akad. Forh., I872, 87. (H.W.W.)

rufusculus Reuter. Coriscus. Ibid., 92.

subcoleoptratus Kirby. Coriscus. Insect Book, Pl. xxix, zo.

Family MESOVELIIDE.

Mesovelia Mulsant and Rey.

mulsanti White. bisignata. Stand. Nat. Hist., ii, 273 (H.L.V.)

Family HEBRID Æ.

Hebrus Curtis.

concinnus Uhler. Noogeus. Proc. Zool. Soc., Lond., I894, 221.

Family REDUVIID Æ.

Ploiaricola Reuter.

tuberculata Banks. Ploiariodes. Psyche, xvi, 46.

Emesa Fabricius.

brevipennis Say. (longipes DeGeer, preoccupied.) Compl. Writ., i, 106.

\section{Barce Stål.}

annulipes Stål. Entmm. Henip., iii, I27.

Pygolampis Germar.

pectoralis Say. Reducitus. Compl. Writ., I, 306.

Reduvius Fabricius.

personatus Linnæus. Bur. Ent., Bull. 22, 24. (D.J.C.) 
Melanolestes Stål.

abdominalis Herrich-Schæffer. Insect Book, P1. xxxi, 38. picipes Herrich-Schæffer. Ibid., P1. xxxi, 39.

\section{Apiomerus Hahn.}

crassipes Fabricius. Insect Book, P1. xxix, 20.

Zelus Fabricius.

audax Banks. Ent. News, xxi, 325.

exsanguis Stål. luridus. Insect Book, P1. xxix, 22 ; Stett. Ent. Zeit., I862, 452 .

Pselliopus Bergroth.

Milyas Stål.

cinctus Fabricius. Insect Book, P1. xxxi, 43.

Fitchia Stål.

aptera Stål. Ofv. Vt. Akad. Forh., I859, 37 I

Acholla Stål.

multispinosa DeGeer. Menn. Ins., iii, 23.

Sinea Amyot and Serville.

diadema Fabricius. Insect Book, P1. xxxi, 44; Jour. N. Y. Ent. Soc. ix, 3 .

spinipes Herrich-Schæffer. Enum. Hemip., ii, 7I. (L.B.W.)

\section{Family PHYMATID在.}

Phymata Latreille.

erosa Linnæus. Insect Book, 297.

erosa var. fasciata Gray. Griffith's Animal Kingdom, xv, 242. (E.L.D.)

erosa var. wolfii Stål, pennsylvanica Handlirsch. Insect Book, Pl. xxxi, 36 .

vicina Handlirsch. Bull. Buff. Soc. Nat. Sci. ix, 174.

Family PIESMID $\mathrm{E}$.

Piesma Lepeletier and Serville.

cinera Say. Tingis. Compl. Writ., i, 349. 


\section{Family TINGID无.}

\section{Corythucha Stål.}

arcuata Say. Tingis. Insect Book, 299; Compl. Writ., i, 350. ciliata Say. Tingis. Compl. Writ., I, $3+9$.

cratægi Osborn and Drake. Bull. Ohio St. Univ., xx, 229.

juglandis Fitch. Tingis. Insects N. Y., iii, 466.

marmorata Uhler. Tingis. Proc. Bos. Soc. Nat. Hist., xix, 415.

pallipes Parshley. Trans. Am. Ent. Soc., xliv, 82. (H.M.P.) pergandei Heidemann. Proc. Wash. Ent. Soc., viii, Io.

\section{Leptobyrsa Stål.}

rhododendri Horvath. explanata Heidemann. Proc. Wash.

Ent. Soc., x, 105.

\section{Gargaphia Stål.}

angulata Heidemann. Can. Ent., xli, 301.

tiliæ Walsh. Proc. Ent. Soc. Phila., iii, 408. (L.B.W.)

\section{Leptostyla Stål.}

clitoriæ Heidemann. heidemanni Osborn and Drake. Proc.

Wash. Ent. Soc., xiii, I37.

\section{Physatocheila Fieber.}

plexa Say. Compl. Writ., i, 349. (E. L. D.)

variegata Parshley. Psyche, xxiii, I66.

\section{Leptoypha Stål.}

mutica Say. Compl. Writ., i, 3+9.

\section{Melanorhopala Stål.}

clavata Stå1. Tingis. Enum. Hennip., iii, I30.

Hesperotingis Parshley.

antennata Parshley. Psyche, xxiv, 2I.

$$
\text { Family LYGÆID E. }
$$

Oncopeltus Stål.

fasciatus Dallas. Enum. Hemip., iv, IO3. (G.H.H.)

\section{Lygæus Fabricius.}

kalmii Stål. Enum. Hemip., iv, 10\%.

reclivatus Say. Compl. Writ., i, 329. 
Ortholomus Stå1.

longiceps Stål. Enum. Hemip., iv, I20.

Nysius Dallas.

californicus Stål. Enum. Hemip., iv, I20.

ericæ Schilling. angustatus. Insect Book, 3I I ; Hayden's Geol. Surv. Mont., 406.

Ischnorrhynchus Fieber.

geminatus Say. Lygaus. Compl. Writ., I, 330.

Cymus Halın.

angustatus Stål. Enum. Hemip., iv, 126.

luridus Stål. Ibid., iv, I26.

Ischnodemus Fieber.

falicus Say. Compl. Writ., i, 33I.

Blissus Burmeister.

hirtus Montandon. Ann. Soc. Ent. Belg., xxxvii, 405.

leucopterus Say. Chinch bug. Insect Book, 3I I Bur. Ent., Bi1l. 69.

Geocoris Fallen.

bullatus Say. Compl. Writ., i, 336 .

bullatus var. discopterus Stål. Enum. Hemip., iv, I36.

uliginosus Say var. limbatus Stål. Ibid., iv, I36.

uliginosus var. speculator Montandon. Buli. Soc. Sci. Buc., xvi, 227.

Isthmocoris McAtee.

piceus Say. Compl. Writ., i, 336.

Phlegyas Stål.

abbreviatus Uhler. Pcliopelta. Phygadicus. Bull. Geol.

Geog. Surv. Terr., ii, 3 I 3.

CEdancala Amyot and Serville.

dorsalis Say. Compl. Writ., i, 335 .

Crophius Stål.

disconotus Say. Compl. Writ., i, 330.

Myodocha Latreille.

serripes Olivier. Insect Book, Pl, xxix, 23.

Heræus Stål.

plebejus Stål. Enum. Hemip., iv, 147 . 


\section{Ligyrocoris Stål.}

diffusus Uhler. Proc. Bos. Soc. Nat. Hist., xiv, Ior.

Perigenes Distant.

constrictus Say. falla.r Heidemann. Compl. Writ., i, 332.

costalis Van Duzee. Can. Ent., xli, 373.

Orthæa Dallas.

basalis Dallas. List. Hemip., ii, 575 .

Ptochiomera Say.

clavigera Uhler. Hemip. Col., 24.

Cnemodus Herrich-Schæffer.

mavortius Say. Compl. Writ., i. 337.

Pseudocnemodus Barber.

canadensis Provancher. bruneri Barber. Jour. N. Y. Ent. Soc., xix, 26.

\section{Ozophora Uhler.}

picturata Uhler. Proc. Bost. Soc. Nat. Hist., xiv, 102.

Rhyparochromus Curtis.

plenus Distant. Biol. Centr. Amer., Heterop., i, 2 I6.

Sphragisticus Stål.

nebulosus Fallen. Traperonotus. List. Hemip., ii, 564.

Aphanus Distant.

plenus Distant. Biol. Centr. Amer., Heterop., i, 409.

Emblethis Fieber.

vicarius Horvath. Ann. Mus. Nat. Hung., vi, 563.

Eremocoris Fieber.

ferus Say. Pamera. Compl. Writ., i, 333.

Xestocoris Van Duzee.

nitens Van Duzee. Ent. News, xvii, 390.

\section{Cryphula Stål.}

parallelogramma Stål. Enum. Hemip., iv, I65.

Scolopostethus Fieber.

atlanticus Horvath. Rev. d'Entom., xii, 239.

thomsoni Reuter. Ann. Soc. Ent. Fr., Ser. 5, ii, 562. 


$$
\text { Family NEIDID E. }
$$

Neides Latreille.

muticus Say. Compl. Writ., i, 328.

\section{Jalysus Stål.}

spinosus Say. Berytus. Compl. Writ., i, 28.

\section{Family ARADID蚱.}

Aradus Fabricius.

abbas Bergroth. Bull. Soc. Ent. Belg., I889. clxxx. falleni Stål. Enum. Hemip., iii, ı 36. (M.P.Z.) quadrilineatus Say. Compl. Writ., ii, 249. robustus Uhler. Proc. Bos. Soc. Nat. Hist., xiv, I04. similis Say. Compl. Writ., i, 35I.

\section{Aneurus Curtis.}

fiskei Heidemann. Proc. Wash. Ent. Soc., vi, I64. (E.L.D.) inconstans Uhler. Proc. Bost. Soc. Nat. Hist., xiv, I05.

\section{Family COREID E.}

\section{Merocoris Pert \\ Corynocoris Mayr.}

typhæus Fabricius var. distinctus Dallas. Ent. S, st., Suppl. 537 ; List. Henip., ii, 419. (L.B.W.)

\section{Acanthocephala Laporte.}

terminalis Dallas. Metapodius. Insect Book, P1. xxxi, 19; Bull. Geol. Geog. Surv. Terr., ii, (5) 298.

\section{Euthochtha Mayr.}

galeator Fabricius. Acanthocerus. Insect Book, P1. xxxi, 31 ; Syst Rhyng., I9I.

Anasa Anyot and Serville.

armigera Say. Compl. Writ., ii, 244. repetita IJeidemann. Proc. Wash. Ent. Soc., vii, II. tristis DeGeer. Squash 1ung. Rept. Conn. Agr. Expt. Sta., 1908, 811; 1919, 44 . 
Family ALYDIDA.

Protenor Stål.

belfragei Haglund. Stett. Ent. Zeit., I868, I62.

Megalotomus Fieber.

quinquespinosus Say. Lygaus. Insect Book, Pl. xxxi, 25; Compl. Writ., ii, 247.

Alydus Fabricius.

eurinus Say. Lygaus. Insect Book, P1. xxxi, 24; Compl. Writ., ii, 247.

pilosulus Herrich-Schæffer. Ibid., P1. xxix, ii; Proc. U. S. Nat. Nus., xvi, 5 o.

\section{Family CORIZID E.}

Harmostes Burmeister.

reflexulus Say. Insect Book, P1. xxix, It; Compl. Writ., i, 323.

Corizus Fallen.

lateralis Say. Ann. Ent. Soc. Am., i, I 40.

bohemani Signoret. nigristernum. Ibid., i, I43.

Family PENTATOMIDA.

Podops Laporte.

cinctipes Say. Trans. Am. Ent. Soc., xxx, 22.

Brochymena Amyot and Serville.

carolinensis IVestwood, anmulata Fabricius. Trans. Am. Ent. Soc., $\mathrm{xxx}, 27$.

arborea Say. Ibid., xxx, 27.

quadripustulata Fabricius. Ibid., xxx, 28.

Peribalus Mulsant and Rey.

limbolarius Stål. Holcostethus. Trans. Am. Ent. Soc, xxx, 32.

Trichopepla Stål.

semivittata Say. Insect Book, P1. xxx, 6; Trans. Am. Ent. Soc., $\mathrm{xxx}, 34$.

6 
Rhytidolomia Stål.

saucia Say. Lioderma. Trans. Am. Ent. Soc., xxx, 36.

senilis Say. Lioderma. Ibid., xxx, 37.

Chlorochroa Stål.

uhleri Stål. persimilis Horvath. Pcntatoma juniperina. Lioderma. Trans. Am. Ent. Soc., xxx, 39.

Mormidea Amyot and Serville.

lugens Fabricius. Insect Book, P1. xxxi, 3; Stett. Ent. Zeit. xxiii, I03.

Solubea Bergroth.

pugnax Fabricius. Ebalus. Enum. Hemip., ii, 22.

Euschistus Dallas.

euschistoides Vollenhoven. fissilis Uhler. Trans. Am. Ent. Soc., $\mathrm{xxx}, 44$.

ictericus Linnæus. Ibid., xxx, 47.

tristigmus Say. Ibid., xxx, 47.

variolarius Beaurois. Spined tobacco bug. Rept. Conn. Agr.

Expt. Sta., 1906, 274.

Cœnus Dallas.

delius Say. Insect Book, P1. xxx, I I C Compl. Writ., i, 320.

Hymenarcys Amyot and Serville.

nervosa Say. Pentatoma. Compl. Writ., i, 32 I.

Neottiglossa Kirby.

undata Say. Trans. Am. Ent. Soc., xxx, 50.

Cosmopepla Stål.

bimaculata Thomas. carnifer Fabricius. Insect Book, P]. xxxi, 6.

\section{Menecles Stil.}

insertus Say. Pentatoma. Compl. Writ., i, 3 I7.

Thyanta Stål.

calceata Say. Pentatoma. Compl. Writ., i, 320.

custator Fabricius. Insect Book, Pl. xxx, fig. I7.

Murgantia Stål.

histrionica Hahn. Harlequin cabbage bug. Bur. Ent., Circ. IO.3. (H.L.J.) 
Acrosternum Fieber.

hilare Say. Insect Book, P1. xxxi, Io; Trans. Am. Ent. Soc., xxx, 58 .

pennsylvanicum DeGeer. Ibid., xxx, 57 .

Banasa Stål.

calva Say. Insect Book, I'l. xxx, 30: Trans. Am. Ent. Soc., $\mathrm{xxx}, 59$.

dimidiata Say. Ibid., xxx, 59.

Dendrocoris Bergroth.

humeralis ['hler. Liotropis. linll. Geol. Geog. Surv., xxx, foo.

Meadorus 11 ulsant and Rey.

lateralis Say. Elusmucha. Trans. Am. Ent. Soc., xxx, 73.

Elasmostethus Fieber.

cruciatus Say. borealis Westwoor. Compl, Writ., i, 3II. (L.B.II.)

Stiretrus Laporte.

anchorago Fabricius. Spec, Ins., ii, 3+I.

Perillus Stål.

circumcinctus Stål. Trans. Am. Ent. Soc., xxx, 67. exaptus Say. Ibid., xxx, 65.

\section{Mineus Stål.}

strigipes Herrich-Schæeffer. Trans. Am. Ent. Soc., xxx, 67.

Apateticus Dallas.

bracteatus Fitch. Podisus. Trans, Am. Ent, Soc., xxx, 70. cyn:cus Say. Podisus. Ibid., xxx, 68.

Podisus Herrich-Schreffer.

maculiventris Say. spinosus. Spined soldier bug. Rept.

Conn. Agr. Expt. Sta., Igo8, Pl. lv, d.

modestus Dallas. Trans. Am. Ent. Soc., xxx, 7 I.

placidus Uhler. Ibid., xxx, 7 I.

serieventris Uhler. Ibid., xxx, 7 . 
Family CYDNIDE.

Thyreocoris Schrank.

ater Amyot and Serville. Corimelcna micolor Beauvois. Trans. Am. Ent., Soc., xxx, 4.

lateralis Fabricius. gilletti. Corimelana. Ibid., xxx, 7.

nitiduloides Wolff. Corimelana. Ibid., xxx, 5.

pulicarius Germar. Corimelcna. Ibid., xxx, 9.

Amnestus Dallas.

pallidus Zimmer. Can. Ent., xlii, I66.

pusillus Uhler. subferrugineus. Trans. Am. Ent. Soc., xxx, 25. (H.L.V.)

spinifrons Say. Ibid., $\mathrm{xxx}, 25$.

Sehirus Amyot and Serville.

cinctus Beauvois. Canthophorus. Bull. Geol. Geog. Surv., Terr. iii, 397.

Family SCUTELLERID瓜.

Homæmus Dallas.

æneifrons Say. Trans. Am. Ent. Soc., xxx, I3.

Eurygaster Laporte.

alternata Say. Trans. Am. Ent. Soc., xxx, I8. 


\section{Order LEPIDOPTERA.}

Butterflies and Moths.

Suborder HETEROCERA.

Moths.

Family MICROPTERYGIDE.

Eriocephala Curtis.

griseocapitella Walsingham. Ent. Rec., x, I6I.

Family HËPIALID $Æ$.

Sthenopis Packard.

argenteomaculatus Harris. Silver-spotted ghost moth. Moth Book, 443 .

quadriguttatus Grote. Proc. Phila. Ent. Soc., iii, 73; Moth

Book, 443.

Family TINEID无.

Pseudanaphora Walsingham.

arcanella Clemens. Proc. Acad. Nat. Sci. Phila., I859, 262.

Acrolophus Poey.

plumifrontellus Clemens. Proc. Acad. Nat. Sci. Phila., 1859, 26r; Moth Book, 443.

Pronuba Riley.

yuccasella Riley. Rept. Ins. Mo., v, I50; Ins. Life, iv, 360;

Noth Book, 44I.

Pitys Chambers.

fasciella Chambers. Can. Ent., v, iii. (C.R.E.)

miscecristatella Chambers. Ibid., v, iii. (C.R.E.)

Hybroma Clemens.

servulella Clemens. Proc. Phila. Ent. Soc., i, I37. (C.R.E.)

Cyane Chambers.

visaliella Chambers. Can. Ent., v, Ir3. (C.R.E.) 
Amadrya Clemens.

effrenatella Clemens. Proc. Acad. Nat. Sci. Phila., 1859, 260. confusella Dietz. Trans. Anl. Ent. Soc., xxxi, 8. (C.R.E.)

\section{Homosetia Clemens.}

costisignella Clemens. Trans. Am. Ent. Soc., xxxi, 83 . (C.R.E.)

Tinea Linnaeus.

acapnopennella Clemens. Trans. An. Ent. Soc., xxxi, 63. arcella Fabricius. Ibid., xxxi, 62.

biflavimaculella Clemens. Ibid., x, I 7o. (C.R.E.)

carnariella Clemens. Proc. Acad. Nat. Sci. Phila., I859, 256. (C.R.E.)

dorsistrigella Clemens. subjunctella. Ibid., I859,.257.

fuscipunctella Haworth. frigidella. Trans. Am. Ent. Soc., x, IтI. (C.R.E.)

granella Linnaeus. variatclla. Ibid., x, I7I.

marginistrigella Chambers. Can. Ent., v, 88. (C.R.E.)

obscurostrigella Chambers. Ibid., vi, 232. (C.R.E.)

pellionella Linnaens. Fur moth. Trans. Am. Ent. Soc., xxxi, 51: Moth Book, 433. (C.R.E.)

rileyi Dietz. Trans. An. Ent. Soc., xxxi, 59.

Monopsis Itïbner.

ferruginella Hübner. crocicapitella. Trans. Am. Ent. Soc., x, I, I70; xxxi, 33.

Tineola Herrich-Schaffer.

bisselliella Itummel. Clothes moth. Bur. Ent., Bull. 4, new ser., 66: Moth Book, 432.

Xylestia Clemens.

pruniramiella Clemens. Proc. Acad. Nat. Sci. Phila., I859, 257. (C.R.E.)

Scardia Treitschke.

approximatella Dietz. Trans. An. Ent. Soc., xxxi, 27.

Acrolepia Curtis.

incertella Chambers, dorsimaculclla. Can. Ent., v, I74. (C.R.E.) 
Argyresthia Hübner.

andereggiella Duponchel. oreasella. Tin. ‥ A., 93.

annettella linsck. Proc. L. S. Nat. Mus., xxxii, 12.

austerella Zeller. undulatella (hanbers. Ibid., xxxii, 22.

freyella Walsingham. Ibid., xxii, II. (C.R.E.)

gœdartella Linnæeus. Ibid., xxxii, 12. (C.R.E.)

Swammerdamia Hübner.

castanæ Pusck. Proc. Wash. Ent. Soc., xvi, I 4 8. (C.R.E.)

Tischeria Zeller.

citrinipennella Clemens. Tin. Х. A., 39. (C.R.E.)

malifoliella Clemens. Apple leaf-miner. Can. Ent., iii, 2o8;

Storrs Agr. Expt. Sta., Iinll. 45.

solidaginifoliella Clemens. Tin. N, A., S I. (C.R.E.)

Lyonetia Hübner.

s1. (scveral species probably synonymous.)

Philonome Chambers.

clemensella Chambers. Can. Ent., vi, 97. (C.R.E.)

Proleucoptera Busck.

albella Chambers. Can. Ent., iii, 23. (C.R.E.)

Marmara Clemens.

elotella Pusck. Jourr. Econ. Ent., 10, 488 .

salictella Clemens. I'roc. P'hila. Ent. Soc., ii, 7. (C.R.E.)

Leucanthiza Clemens.

amphicarpeæfoliella Clemens. Tin. N. A., 85. (C.R.E.)

Ornix Treitschke.

cratægifoliella Clemens. Can. Ent., $v, 48$ : Trans. Am. Ent.

Soc., xxxiii, 292. (C.R.E.)

kalmiella Dietz. Trans. Am. Ent. Soc., xxxiii, 29r. (C.R.E.) oblitereila Dietz. Ibid., xxxiii, 297. (C.R.E.)

præciosella Dietz. Ibid., xxiii, 291. (C.R.E.)

prunivorella Chambers. Ibid., xxxiii, 294. (C.R.E.)

quadripunctella Clemens. Tin. N. A., 15\%. (C.R.E.)

Gracillaria Haworth.

alchimiella Scopoli. superbifrontella. Trans. Am. Ent. Soc., $x, 191$. 
belfrageella Chambers. Can. Ent., vii, 92. (C.R.E.)

bimaculatella Ely. Insec. Insc. Menst., iii, 53. (C.R.E.)

blandella Clemens. Tin. N. A., 257. (C.R.E.)

burgessiella Zeller. Insec. Insc. Menst., iii, 5I. (C.R.E.)

cornusella Ely. Ibid., iii, 53. (C.R.E.)

coroniella Clemens. Tin. N. A., 243, (C.R.E.)

elongella Linnæus. Trans. Am. Ent. Soc., x, I93. (C.R.E.)

fasciella Chambers. Ibid., x, 20 r. (C.R.E.)

flavella Ely. Insec. Insc. Menst., iii, 56. (C.R.E.)

flavimaculella Ely. Ibid., iii, 57. (C.R.E.)

fraxinella Ely. Ibid., iii, 58. (C.R.E.)

fulgidella Clemens. Tin. N. A., 92. (C.R.E.)

glutinella Ely. Insec. Insc. Menst., iii, 55. (C.R.E.)

juglandiella Chambers. Can. Ent., vii, 9. (C.R.E.)

lespedezæfoliella Clemens. robiniella. Tin. N. A., I4t, 207. (C.R.E.)

minimella Ely. Insec. Insc. Menst., iii, 58. (C.R.E.)

ostryæella Chambers. Ent. News, xxiii, I67. (C.R.E.)

packardella Chambers. Can. Ent., iv, 27. (C.R.E.)

pennsylvaniella Engel. Ent. News, xviii, 276. (C.R.E.)

purpuriella Chambers. Can. Ent., ix, I26. (C.R.E.)

quercinigrella Ely. Insec. Insc. Menst., iii, 6o. (C.R.E.)

rhoifoliella Chambers, Can. Ent., viii, 3I. (C.R.E.)

sassafrasella Chambers. Ibid., viii, 33. (C.R.E.)

serotinella Ely. Coriscium. Ent. News, xix, 57. (C.R.E.)

stigmatella Fabricius. Can. Ent. xi, 74. (C.R.E.)

strigifinitella Clemens, Tin. N. A., 92. (C.R.E.)

venustella Clemens. eupatoriclla. Ibid., 92. (C.R.E.)

violacella Clemens. desmodifoliella. Ibid., 93, 268. (C.R.E.)

Lithocolletes Hïbner.

aceriella Clemens. Tin. N. A., 65. (C.R.E.)

argentifimbriella Clemens. Trans. Am. Ent. Soc., xxxiv, 28I.

(C.R.E.)

basistrigella Clemens. Ibid., xxxiv, 308. (C.R.E.)

bethuniella Chambers. Can. Ent., iii, Io9. (C.R.E.)

betulivora Walsingham. Ins. Life, iii, 326. (C.R.E.)

caryæalbella Cliambers. Can. Ent., iii, 5S. (C.R.E.)

caryæfoliella Clentens. Tin. N. A., 65. (C.R.E.) 
No. 3I.] INSECtS OF CONNEC'TICUT: LeplinolterA.

cincinnatiella Chambers. Trans. Am. Ent. Soc., xxxiv, 329. (C.R.E.)

corylisella Chambers. Ibid., xxxiv, 344. (C.R.E.)

cratægella Clemens. Tin. N. A., 76. (C.R.E.)

desmodiella Clemens. Ibid., 68. (C.R.E.)

fitchiella Clemens. Ibid., I39. (C.R.E.)

guttifinitella Clemens. Ibid., 65. (C.R.E.)

hamadryella Clemens. White oak blotch leaf miner. Can. Ent., iii, 55.

lentella Braun. Trans. Am. Ent. Soc., xxxiv, 326. (C.R.E.)

lucidicostella Clemens. Ibid., xxxiv, 28I. (C.R.E.)

nemoris Walsingham. Ins. Life, ii, I 6. (C.R.E.)

ostryarella Chambers. Can. Ent., iii, III. (C.R.E.)

picturatella Braun. Ent. News, xxvii, 8+. (C.R.E.)

saccharella Braun. Trans. Am. Ent. Soc., xxxiv, 327. (C.R.E.)

salicifoliella Clemens. Tin. N. A., I69. (C.R.E.)

ulmella Chambers. Can. Ent., iii, I+8. (C.R.E.)

Bucculatrix Zeller.

canadensisella Chambers, Birch leaf skeletonizer. Rept.

Conn. Agr. Expt. Sta., I909-IO, joI.

magnella Chambers. Can. Ent., vii, 54. (C.R.E.)

nivella Chambers. Ibid., vii, 5+. (C.R.E.)

pomifoliella Clemens. Apple Bucculatrix. Ribbed cocoonmaker. Cornell Agr. Expt. Sta., Bull. 214: Moth Book, 43 I.

sp.

Opostega Zeller.

bosqueella Cliambers. Bull. Geol. Geog. Surv. Terr., iv, I06. (C.R.E.)

magnella Chambers. Can. Ent., vii, 54. (C.R.E.)

niveella Chambers. Ibid., vii, 54. (C.R.E.)

quadristrigella Chambers. Cin. Quart. Jour. Sci., ii, Io6. (C.R.E.)

\section{Trifurcula Zeller.}

obrutella Zeller. Verh. Zool.-Bot. Ges. Wien., xxiii, $3^{16 .}$ (C.R.E.) 
Nepticula Von Heyden.

amelanchierella Clemens. Tin. N. A., 174. (C.R.E.)

virginiella Clemens. Ibid., I72. (C.R.E.)

sp.

Family ELACHISTID E.

Walshia Clemens.

amorphella Clemens. Tin. N. A., 2.t. (C.R.E.)

Mompha Ilübner.

cephalonthiella Chambers. Can. Ent., iii, 22I. (C.R.E.)

eloisella Clemens. Tin. N. A., I3I.

Stagmatophora Herrich-Schaffer.

ceanothiella Cosens. Can. Ent., xl, io7. sexnotella Chambers. Jour. N. Y. Ent. Soc., x, 97. sp.

Psacaphora Herrich-Scheffer.

passerella Busck. Proc. Wash. Ent. Soc., xi, 95. (C.R.E.) sp.

Theisoa Chambers.

constrictella Zeller. bifasciella. Can. Ent., vi, 76. (C.R.E.)

Stilbosis Clemens.

tesquella Clemens. Tin. N. A., I 29. (C.R.E.)

Antispila Hübner.

cornifoliella Clemens. Tin. N. A., Io3. (C.R.E.)

Scythris II ïbner.

albapennella Chambers. Can. Ent. vii, II.

eboracensis Zeller. Linn. Ent., x, 205.

Lymnæcia Stainton.

phragmitiella Stainton. Cat. Brit. Tin., Suppl., 4, 1?51.

Elachista Treitschke.

albapalpella Chambers. Jour. Cin. Soc. Nat. Hist., viii, 240.

(C.R.E.)

curvilineella Chambers. Can, Ent., ir, I72. (C.R.E.) sp. 
Erineda Pusck.

elyella Busck. I'roc. Wash. Ent. Soc., xi, 94. (C.R.E.)

Cosmopteryx Ijübner.

gemmiferella Clemens. Proc. Acad. Nat. Sci. Phila., I860, Io.

Batrachetra Stainton.

trichella Busck. ('an. Ent., xl, Ior) (C.R.E.)

\section{Coleophora Ilïbner.}

cratipennella Clemens. Proc. Hhila. Ent. Soc., iii, 506.

(C.R.E.)

fletcherella Fernald. Can. Ent., ir, I 22.

laricella Hübner. Ins. Aff. l'ark and lloudl. Trees, i, iтo.

limosipennella Duponchel. Ibid., i, if 5 .

spissicornis Haworth. cormscipennella. Tin. N. A., 88. (C.R.E.)

sp.

$$
\text { Family RIASTOB.ASID.E. }
$$

Holcocera Clemens.

chalcofrontella Clemens. T'roc. Phila. Ent. Soc., ii, I22.

(C.R.E.)

elyella Dietz. Trans. An. Ent. Soc., xxxvi, 49. (C.R.E.)

melanostriatella Dietz. Ibid., xxxri. 66. (C.R.E.)

Valentina Malsingham.

modestella Clemens. glandulella. Trans. Am. Ent. Soc., xxxvi, 33. (C.R.E.)

\section{Pseudopigritia Dictz.}

equitella Dietz. Trans. Am. Ent. Soc., xxrii, I I2.

Pigritia Clemens.

laticapitella Clemens. Trans. An. Ent. Soc., xxтii, Io6. ornatella Dietz. Ibid., xxvii, I07. (C.R.E.)

sp.

$$
\text { Family GEOPIORIDA. }
$$

Borkhausenia Hiibner.

ascriptella Busck. Can. Ent., xl, II)t.

shalleriella Chambers. Geophort. Cin. Ouart. Jour. Sci., ii. I 14. 
Decantha Busck.

borkhauseni Zeller. Verh. Zool-Bot. Ges. Wien., xxiii, 290.

(C.R.E.)

Ecophora Latreille.

newmanella Clemens. Dasycera. Tin. N. A., 252.

Gerdana Busck.

caritella Busck. Pros. U. S. Nat. Mus., xxxv, I93. (C.R.E.)

Epicallima Dyar.

argenticinctella Clemens. Proc. Acad. Nat. Sci. Phila., I860, I67.

Euclemensia Grote.

bassettella Clemens. Proc. Phila. Ent. Soc., ii, 423: Rept. Conn. Agr. Expt. Sta., I916, I43.

Semioscopsis Hübner.

allenella Walsinghain. 'Trans. Am. Ent. Soc., x, I74. (C.R.E.)

Depressaria Haworth.

atrodorsella Clemens. Proc. Acad. Nat. Sci. Phila., ii, I24. betulella Busck. Proc. U. S. Nat. Mus., xxiv, 746. (C.R.E.) fernaldella WValsingham. Ins. Life, i, 256.

gracilis Walsingham. Ibid., i, 257. (C.R.E.)

heracliana DeGeer. ontariella. Can. Ent., ii, 3, 19. (C.R.E.) pulvipennella Clemens. Proc. Phila. Ent. Soc., ii, 42 I.

robiniella Packard. Pack. Guide, 349.

sp. near pallidella Busck.

Cryptolechia Zeller.

tentoriferella Clemens. Machima. Tin. N. A., I 4 S. (C.R.E.)

Psilocorsis Clemens.

quercicella Clemens. Tin. N. A. I49. (C.R.E.)

Agnopteryx Hübner.

flavicomella Engel. Ent. News, xviii, 276. (C.R.E.)

lecontella Clemens. Tin. N. A., I37.

fernaldella Walsingham. a'alsinghamiclla Busck. Ins. Life, i, 256. 
Family XYLORICTIDE.

\section{Brachyloma Clemens.}

querciella Busck. Proc. W ash. Ent. Soc., x, Ir2. (C.R.E.)

Stenoma Zeller.

leucillana Zeller; Ins. Life, ii, I53.

schlægeri Zeller. Ibid., ii, I52.

\section{Family GELECIIID E.}

\section{Gelechia IIübner.}

argentipunctella Ely. Proc. WTash. Ent. Soc., xii, 7o. (C.R.E.) conclusella IValker. crescontifasciclla. Can. Ent., vi, 237. (C.R.E.)

disco-ocellella Chambers. Ibid., iv, I94. (C.R.E.)

fluvialella Busck. Ibid., x1, I94. (C.R.E.)

hibiscella Busck. Proc. U. S. Nat. Mus., xxv, 869. (C.R.E.) maculimarginella Chambers. Can. Ent., vi, 24I. (C.R.E.) mediofuscella Clemens. Proc. Phila. Ent. Soc., ii, I I, I20.

nigrimaculella Busck. Proc. U. S. Nat. Mius., xxv, 880. (C.R.E.)

pseudoacaciella Chambers. Can. Ent., iv, I07.

pseudofondella Busck. Proc. WVash. Ent. Soc., ix, 87. (C.R.E.) rileyella Chambers. Can. Ent., iv, ıo6. (C.R.E.)

trialbamaculella Chambers. Cin. Quart. Jour. Sci., ii, 250. (C.R.E.)

vernella Murtfeldt. formosclla. Can. Ent., xiii, 243. (C.R.E.)

\section{Anacampsis Curtis.}

innocuella Zeller. Proc. U. S. Nat. Mus., xxv, 845. (C.R.F.) rhoifructella Clemens. Can. Ent., iii. 68. (C.R.E.) tristrigella WValsingham. Trans. An. Ent. Soc., x, r8ı.

Dichomeris Hübner.

herculella Busck. Proc. Wash. Ent. Soc., xi, 89. (C.R.E.) marginellus Fabricius. Juniper webworm. Rept. N. Y. State Ent., 26, 35: Rept. Conn. Agr. Expt. Sta., I9I5, I37.

\section{Aproærema Durrant.}

nigratomella Clemens. apicilinclla. Proc. Phila. Ent. Soc., ii, I I, I20. (C.R.E.) 


\section{Anarsia Zeller.}

lineatella Zeller. Peach twig borer. Rept. Conn. Agr. Expt. Sta., I918, 306.

Ypsolophus Fabricius.

bipunctellus IValsingham. Trans. Am. Ent. Soc., x, I86. (C.R.E.)

ligulellus Hïbner. reedella. Can. Ent. iv, 222.

ventrellus Fitch. Trans. N. Y. Agr. Soc., xiii, 254. (C.R.E.)

Anorthosia Clemens.

punctipennella Clemens. Can. Ent., iv, 225. (C.R.E.)

\section{Glyphidocera Walsingham.}

speratella Busck. Proc. WVash. Ent. Soc., ix, 88. (C.R.E.,

Trichotaphe Clemens.

alacella Clemens. Proc. I'hila. Ent. Soc., i, I32. (C.R.E.) flavocostella Clemens. Ibid., i, I 3 I. inversella Zeller. 'Can. Ent., x, 54. (C.R.E.) juncidella Clemens. dubitclla. Ibid., iv, 92. (C.R.E.) serrativittella Zeller. Ibid., ix, 24. (C.R.E.) setosella Clemens. costolutella. Ibid., iv, 209. (C.R.E.) trimaculella Chambers. Ibid., vi, 238. (C.R.E.) trinotella Busck. Ibid., xxxviii, I22, (C.R.E.)

Polyhymno Chambers.

luteostrigella Chambers. Can. Ent., vi, 247. (C.R.E.)

Gnorimoschema Busck.

artemisiella Kearfott. Jour. N. Y. Ent. Soc., xi, ı6o. (C.R.E.) banksiella Busck. Proc. U. S. Nat. Mus., xxv, 832 . (C.R.E.) gallæasteriella Kellicott. Can. Ent., x, 203. (C.R.E.) gallæsolidaginis Riley. Rept. Ins. Mo., i, I73. saphirinella Chambers. Cin. Quart. Jour. Sci., ii, 250. (C.R.E.)

Trypanisma Clemens.

prudens Clemens. Tin. N. A., I25. (C.R.E.)

Epithectis Meyrick.

attributella $1 \mathrm{~V}$ alker. difficilisclla. Can. Ent., iv, 66. 
Recurvaria Haworth.

apicitripunctella Clemens. Trans. An. Ent. Soc., x, is2. (C.R.E.)

obliquestrigella Chambers. Can. Ent., iv, 65. (C.R.E.)

Aristotelia Hïbner.

absconditella Wtalker. Trans. Ant. Ent. Soc., x, ISI. (C.R.E.) fungivorella Clemens. Proc. I'hila. Ent. Soc., iii, 507. (C.R.E.) minimella Chambers. Can. Ent., vi, 243. (C.R.E.)

pudibundella Zeller. intermedicll. Verh. Zool-Bot. Ges. Wien., xxiii, 273. (C.R.E.)

quinquepunctella Busck. Proc. L. S. Nat. Mus., xxv, Soł. (C.R.E.)

roseosuffusella Clemens. Froc. Phila. Ent. Soc., ii, I21.

rubidella Clemens, Ibid., ii, I2I. (C.R.E.)

Telphusa Chambers.

glandiferella Zeller. sclla. Can. Ent., vi, 238. (C.R.E.)

latifasciella Chambers. Cin. Quart. Jour. Sci., ii, 25 I.

palliderosacella Chambers. Bull. Geol. Geog. Surv. Terr., iv. go.

\section{Sitotroga Heinemann.}

cerealella Olivier. Angoumois grain moth. N. J. Agr. Expt. Sta., Bull. I 47 .

Paltodora Meyrick.

anteliella Busck. Proc. U. S. Nat. Mus., xxv, 778. (C.R.E.) cilialineella Chambers. Can. Ent., vi., 2+2. (C.R.E.) similiella Chambers. Ibid., iv, 193. (C.R.E.)

\section{Ptyocerata Ely.}

busckella Ely. Proc. Mrash. Ent. Soc., xii, 69. (C.R.E.)

Metzneria Zeller.

lappella Linnaeus. Syst. Nat., i758, 537.

$$
\begin{aligned}
& \text { Family YPONOMELTIDE. } \\
& \text { Yponomeuta Latreille. }
\end{aligned}
$$

multipunctella Clemens. Tin. N. A., 95. (C.R.E.)

Martyringa Busck.

latipennis Walsingham. Trans. Am. Ent. Soc., x, 190. (C.R.E.) 
Harpipteryx Hübner.

xylostella Linnæus. Contr. Nat. Hist. Lepidop, iv, 246, pl. xxviii, fig. I2.

Plutella Schrank.

maculipennis Curtis. crucifcrarum. dubiosella. Brit. Ent., I832, pl. 420 : Can. Ent., xxi, 27. (C.R.E.)

Choreutis Hübner.

inflatella Clemens. Proc. Phila. Ent. Soc., ii, 5.

Family TORTRICID E.

Evetria Hübner.

comstockiana Fernald. Can. Ent., xi, 157.

Polychrosis Ragonot.

vernoniana Kearfott. Trans. Am. Ent. Soc., xxxiii, 7. (C.R.E.)

viteana Clemens. Grape berry moth. Cornell Agr. Expt. Sta., Bull. 223 .

sp.

Bactra Stephens.

lanceolana Hübner. Schmett. Eur. Tort., f. 8o. i8oo. (C.R.E.)

Exartema Clemens.

concinnanum Clemens. Proc. Phila. Ent. Soc., v, I34. (C.R.E.)

fasciatanum Clemens. Ibid., v, I34.

inornatanum Clemens. Proc. Phila. Ent. Soc., v, I34.

olivaceanum Fernald. Trans. An. Ent. Soc., x, 7r.

permundanum Clemens. Proc. Phila. Ent. Soc., v, I34. punctanum Walsingham. I1l. Lep. Het. Brit. Mus., iv, 87. (C.R.E.)

sericoranum Walsingham. Ibid., iv, $3^{6}$.

versicoloranum Clemens. Proc. Acad. Nat. Sci. Phila., I860, 357. (C.R.E.)

zellerianum Fernald. nitidanum. Verh. Zool-Bot. Ges. Wein., xxv, 27o. (C.R.E.) 
Olethreutes Hübner.*

abietana Fernald. Can. Ent., x1, 340.

agilana Clemens. Proc. Acad. Nat. Sci. Phila., ı860. 359. (C.R.E.)

bipartitana Clemens. Ibid., is6o. 346.

campestrana Zeller. Terh. Zool-Bot. Ges. MTien., xxv. 282.

corruscana Clemens. Proc. I'hila. Acad. Sci., I860. 346.

cyanena Murtfeldt. Am. Ent., III. It.

dimidiana Sodoffsky. Bull. Mosc., I830. 73. (C.R.E.)

griseoalbana Malsingham. Ill. Lep. Het., Brit. Mus., iv. 38 .

(C.R.E.)

hartmanniana linnaeus. Fiann. Stec. 345. (C.R.E.)

hartmanniana var. albeolana Zeller. Terh. Zool-Bot. Ges.

IVien., xxv. 262.

hebesana Malker. I11. Lep. IIet., Prit Nus., iv. 3I.

impudens Wralsingham. Trans. Ent. Soc. Lond., I884." I35 (C.R.E.)

instrutana Clemens. Proc. Phila. Ent. Soc., v. I35. (C.R.E.) interruptolineana Fernald. Pcnthina. Trans An. Ent. Soc, x. 7o. (C.R.E.)

nimbatana Clemens. Proc. Acad. Nat. Sci. Plila., I860. 346.

ochromediana Keariott. Trans. Anı. Ent. Soc, xxxiii. I . (C.R.E.)

separatana Kearfott. Ibid. xxxiii. I3. (C.R.E.)

sp.

Pseudogalleria Ragonot.

inimicella Zeller. Verh. Zool-Bot. Ges. Wien., xxii. 559. (C.R.E.)

Eucosma Hiijner.

abbreviatana Walsingham. 111. Lep. Ilet., Rrit. Mus., iv. 54. agricolana Malsingham. Ibid. iv. 42. (C.R.IE.)

albiguttana Zeller. Verh. Zool-Bot. Ges. Wien., xxv. 3I3. (C.R.E.)

boxcana Kearfott. Trans. An. Ent. Soc., xxiii. 87. (C.R.E.) cataclystiana Wralker. Padisca ochreana. Proc. I'hila. Ent. Soc., III. 520 .

confana Kearfott. Proc. U. S. Nat. Mus., xxviii. 355. (C.R.E.) culminana Nralsingham. I1l. Lep. Het., iv. $3^{8}$.

* See also appendix. 
dorsisignatana Clemens. Proc. Acad. Nat. Sci. Phila., I860. 353: Noth Book, 4Is.

fulminana Walsingham. 111. Lep. Het., iv. 5o. (C.R.E.) gracilana Kearfott. Proc. U. S. Nat. Mus., xxviii. 352. (C.R.E.)

haracana Kearfott. Proteoptery.r. Trans. Am. Ent. Soc., xxxiii. 46. (C.R.E.)

juncticiliana Walsingham. Ill. Lep. Het., iv. 75. (C.R.E.) minutana Kearfott. Proc. U. S. Nat. Mus., xxviii. 356.

otiosana Clemens. Proc. Acad. Nat. Sci. Phila., I850. 354. (C.R.E.)

pergandeana Fernald. Can. Ent. xxxvii. 399. (C.R.E.)

perplexana Fernald. Epiblema. Jour. N. Y. Ent. Soc., ix. 5 I. (C.R.E.)

scudderiana Clemens. Saligneana. Proc. Phila. Ent. Soc., v. I4I. (C.R.E.)

solicitana Walker. Cat. Brit. Mus., xxviii. 387. (C.R.E.)

strenuana Walker. Ill. Lep. Het., iv. 52.

tomonana Keariott. Can. Ent., xxxix. 78. (C.R.E.)

transmissana Walker. Ill. Lep. Het., iv. 52.

zomonana Kearfott. Can. Ent., xxxix. 8o. (C.R.E.)

\section{Cydia Hübner.}

cinereodorsana Kearfott. Trans. Am. Ent. Soc., xxxiii. 39. (C.R.E.)

raracana Kearfott. Ibid., xxxiii. 44. (C.R.E.)

Thiodia Hübner.

essexana Kearfott. Trans. Am. Ent. Soc., xxxiii. 39. (C.R.E.) formosana Clemens. Proc. Acad. Nat. Sci. Phila., I860. 360. imbridana Fernald. Can. Ent., xxxvii. 400. (C.R.E.) ochrotermenana Kearfott. Ibid., xxxix. 57. (C.R.E.) olivaceana Riley. Trans. St. Louis Acad. Sci., iv. 320. (C.R.E.) signatana Clemens. Ins. Aff. Park and Woodl. trees, i. I6S. striatana Clemens. Proc. Acad. Nat. Sci. Phila., I860. 349. Episimus Walsingham.

argutanus Clemens. Proc. Acad. Nat. Sci. Phila., I860. 358. (C.R.E.)

Proteopteryx Walsingham.

bolliana Slingerland. Jour. N. Y. Ent. Soc., xvi, I73. cressoniana Clemens. Proc. Phila. Ent. Soc., III. 5 I4. 
Epinotia Hübner.

crispana Clemens. Proc. Phila. Ent. Soc., v. 137.

fasciolana Clenens. Ibid., I I I. 5 I I. (C.R.E.)

illicifoliana Kearfott. Bull. Am. Mus. Nat. Hist., xxiii. I 58 . (C.R.E.)

saliciana Clemens. Proc. Phila. Ent. Soc., I I I. 5I5. (C.R.E.)

Tmetocera Lederer.

ocellana Schiffermüller. Bud moth. Rept. Conn. Agr. Expt. Sta., Ig09-10. 353.

Eudemis Hübner.

vacciniana Packard. Black-headed cranberry worm. N. J. Agr. Expt. Sta., Spec. Bull. K. (C.R.E.)

Cenopis Zeller.

diluticostana Walsingham. quercana. Trans. Am. Ent. Soc., x, 69.

reticulatana Clemens. Ibid., ii, 272. (C.R.E.)

testulana Zeller. Verh. Zool-Bot. Ges. Wien., xxr, 241. (C.R.E.)

\section{Cœlostathma Clemens.}

discopunctana Clemens. Amphisa. Proc. Acad. Nat. Sci. Phila., I860, 355 .

Sparganothis Hübner.

inconditana WValsingham. I11. Lep. Het. Brit. Mus., iv, I6. (C.R.E.)

irrorea Robinson. Trans. Am. Ent. Soc., ii, 274.

xanthoides IValker. breviomatana. Proc. Phila. Ent. Soc., v, I 40.

\section{Archips Hübner.}

argyrospila WValker. Cat. Brit. Mus., xxviii, 373: Moth Book, 422.

cerasivorana Fitch. Trans. Am. Ent. Soc., ii, 275: Moth Book, 422.

clemensiana Fernald. Can. Ent., xi, I55.

fervidana Clemens. paludana. Trans. Am. Ent. Soc., ii, 275. (C.R.E.)

infumatana Zeller. Verh. Zool-Bot. Ges. Wien., xxr, 2 I6. (C.R.E.)

parallela Robinson. Trans. Am. Ent., Soc., ii, 267.

purpurana Clemens. Proc. Phila. Ent. Soc., v, iz6: Moth Book, 422 
rileyana Grote. Trans. Am. Ent. Soc., ii, I2I. (F.W.H.) rosaceana Harris. Oblique banded leaf-roller. Jour. Econ.

Ent. ii, 39I : Moth Book, 422.

rosana Linnæus. Rept. Conn. Agr. Expt. Sta., I9I3, 223.

semiferana Walker. Tortri. flaccidana Robinson. Trans. Am.

Ent. Soc., ii, 277.

virescana Clemens. Proc. Phila. Ent. Soc., v, I fo.

Ancylis Hübner.

angulifasciana Zeller. Verh. Zool-Bot. Ges. Wien., xxv, 256. (C.R.E.)

burgessiana Zeller. Ibid., xxv, 252.

comptana Frölich. Strawberry leaf-roller. N. J. Agr. Expt. Sta., Bull. I49: Moth Book, 4I9.

diminutana Kearfott. Proc. U. S. Nat. Mus., xxviii, 36r. (C.R.E.)

divisana Walker. Cat. Brit. MIus., xxviii, 385. (C.R.E.)

dubiana Clemens. Proc. Phila. Ent. Soc., iii, 5 I2.

laciniana Zeller. Verh. Zool-Bot. Ges. Wien., xxv, 253.

muricana Walsingham. 1ll. Lep. Het. iv, 74.

platanana Clemens. Proc. Acad. Nat. Sci. Phila., I860, 349. (C.R.E.)

pulchellana Clemens. Proc. Phila. Ent. Soc., iii, 5II. (C.R.E.) subrequana Zeller. Verh. Zool-Bot. Ges. Wien., xxv, 254. (C.R.E.)

Enarmonia Hübner.

americana Walsingham. Ill. Lep. Het. Brit. Mus., iv, 67. caryana Fitch. N. Y. Agr. Rept., xvi, 459. (C.R.E.) interstinctana Clemens. Proc. Acad. Nat. Sci. Phila., IS60, 35I. prunivora Walsh. Scmasia. Lesser apple worm. Rept. Ill. State Ent., i, 2d ed., I08.

\section{Laspeyresia IIübner.}

gallæsaliciana Riley. Enarmonia. Trans. St. Louis Acad. Sci., iv, 320. (C.R.E.)

molesta Busck. Oriental peach moth. Rept. Conn. Agr. Expt. Sta., I9I8, 298.

\section{Gymnandrosoma Dyar.}

punctidiscanum Dyar. Proc. Wash. Ent. Soc, vi, 6o. 
Ecdytolopha Zeller.

insiticiana Zeller. Verh. Zool-Bot. Ges. Wien., xxv, 266.

Carpocapsa Treitschke.

pomonella Linnæus. Cydia. Codling-moth. Apple worm. Cornell Agr. Expt. Sta., Bull. I42; Moth Book, 4 I9.

\section{Phthinolophus Dyar.}

indentanus Dyar. Proc. Wasl. Ent. Soc., v, 307. (C.R.E.)

Acleris Hübner.

boscana Fabricius. Ent. Syst. Suppl., iii, 2, 269. (C.R.E.) hastiana Linnaus. Trans. Am. Ent. Soc., ii; 2So. (C.R.E.) logiana Schiffermïller var. viburnana Clemens. Ibid., ji, 28 I. subnivana Walker. deflcctana. Ibid., ii, 283. (C.R.E.) trisignana Robinson. Ibid,, ii, $2 \mathrm{~S}_{2}$. (C.R.E.)

\section{Peronea Curtis.}

commandrana Fernald. Can. Ent., xxiv, I24. (C.R.E.)

ferrugana Schiffermüller. gallicolana. Proc. Phila. Ent. Soc., iii, 5I6. (C.R.E.)

heindelana Fernald. Am. Nat., xxxix, I70, 859, 873. (C.R.E.) pulverosana Valker. I1l. Lep. Het. Brit. Mus., iv, 3. (C.R.E.) trisignana Robinson. Trans. Am. Ent. Soc., ii, 282. (C.R.E.) sp.

Epagoge Hübner.

sulfureana Clemens. Trans. Am. Ent. Soc., ii, 273. virescana Clemens. Proc. I'hila. Ent. Soc., v, I fo.

\section{Platynota Clemens.}

flavedana Clemens. Trans. Am. Ent. Soc., ii, 278: Moth Book, 422 .

\section{Pandemis Hïbner.}

lamprosana Robinson. Trans. An. Ent. Soc., ii, 264. (C.R.E.) limitata Robinson. Ibid., ii, 264.

\section{Tortrix Linnaeus.}

albicomana Clemens. Proc. Plila. Ent. Soc., v, I37: Moth Tiook, 423.

fumiferana Clemens. Ibid., v, I39. 
nervosana Kearfott. Trans. Am. Ent. Soc., xxxiii, 7 I. (C.R.E.)

pallorana Robinson. Ibid., ii, 266.

peritana Clemens. Ibid., ii, 277. (C.R.E.)

quercifoliana Fitch. N. Y. Agr. Rept., I858, 826.

\section{Eulia Hübner.}

alisellana Robinson. Trans. Am. Ent. Soc., ii, 267.

politana Haworth. Rept. U. S. Dept. Agr., I880, 264. (C.R.E.) quadrifasciana Fernald. Trans. Am. Ent. Soc., x, 67.

velutinana Walker. trifcrana. incertana. lutosana. Trans. Am. Ent. Soc., ii, 279.

\section{Amorbia Clemens.}

humerosana Clemens. Trans. Am. Ent. Soc., ii, 275: Moth Book, 423.

\section{Phalonia Hübner.}

angulatana Robinson. Trans. Am. Ent. Soc., ii, 286. (C.R.E.) argentilimitana Robinson. Ibid., ii, 287. (C.R.E.)

atmosana Busck. Jour. N. Y. Ent. Soc., xv, 22. (C.R.E.) aurorana Kearfott. Trans. Am. Ent. Soc., xxxiii, 83. (C.R.E.) biscana Kearfott. Ibid., xxxiii, 75. (C.R.E.) bunteana Robinson. Ibid., ii, 288 . (C.R.E.) dorsimaculana Robinson. Ibid., ii, 285. hospes Walsingham. Trans. Ent. Soc. Lond., I884, I3I. interruptofasciana Robinson. Trans. Am. Ent. Soc., ii, 287. (C.R.E.)

maiana Kearfott. Ibid., xxxiii, 82. (C.R.E.) marloffiana Busck. Jour. N. Y. Ent. Soc., xv, 26. (C.R.E.) nonlavana Kearfott. Trans. Am. Ent. Soc., xxxiii, 85. (C.R.E.) œnotherana Riley. Trans. St. Louis Acad. Sci., iv, 316. rana Busck. Jour. N. Y. Ent. Soc., xv, 22. (C.R.E.) temerana Busck. Ibid., xv, 28. (C.R.E.) winniana Kearfott. Can. Ent., xxxvii, ỊO. (C.R.E.)

Commophila Hübner.

contrastana Kearfott. Can. Ent., xxxix, I6o.

Pharmacis II übner.

sartana Hübner. Zutr. Exot. Schmett., ii, III. (C.R.E.) 
Hysterosia Stephens.

baracana Busck. Jour. N. Y. Ent. Soc., xv, 33. (C.R.E.) terminana Busck. Ibid., xv, 33. (C.R.E.)

\section{Family PTEROPHORIDE.}

Oxyptilus Zeller.

ningoris Walsingham. Pteroph. N. A., 20. (C.R.E.)

periscelidactylus Fitch. Grape plume moth. Ibid., I7.

tenuidactylus Fitch. Ibid., 20.

Platyptilia Hübner.

cosmodactyla Hübner. Pteroph. N. A., 25.

marginidactyla Fitch. Ibid., 34.

\section{Pterophorus Geoffroy.}

cretidactylus Fitch. Pteropli. N. A., 52.

elliottii Fernald. Can. Ent., xxr, 95.

homodactylus WValker. Pteroph. N. A., 4I.

inquinatus Zeller. Ibid., 56. (C.R.E.)

paleaceus Zeller. Ibid., 45 .

sp.

Family PYRALID.E.

Glaphria IIübner.

fulminalis Lederer. Wien. Ent. Mon., vii, 487.

glaphyralis Guenée. albolineata. Trans. An. Ent. Soc., i, 28. invisalis Guenée. Spec. Gén., viii, 36r. (C.R.E.)

sequistrialis Hübner. Zutr. Exot. Schmett., ii, 29. (C.R.E.)

Lipocosma Lederer.

fuliginosalis Fernald. Ent. Am., iv, 37.

\section{Desmia $W^{T}$ estwood.}

funeralis Hübner. Schmett. Eur., f. 103, 1796: Moth Book, 392.

funeralis var. subdivisalis Grote. Can. Ent., iii, I26.

\section{Diastictis Hübner.}

argyralis Hübner. Zutr. exot. Schmett., i, 2 I.

argyralis var. ventralis Grote and Robinson. Trans. Am. Ent.

Soc., i, 2I. (C.R.E.) 
Blepharomastix Lederer.

ranalis Guenée. Botys gracilis. Trans. Am. Ent. Soc., i, 25. (C.R.E.)

\section{Pantographa Lederer.}

limata Grote and Robinson. Can. Ent., xvi, 26: Moth Book, 393 .

\section{Diaphania Hübner.}

hyalinata Linnæus. Glyphodes. Melon caterpillar. Rept. N. Y. State Ent., xi, I34: Moth Book, 394. nitidalis Stoll. Glyphodes. Pickle worm. Rept. N. Y. State. Ent., xi, 126: Moth Book, 394. (A.H.Me.)

\section{Evergestis Hübner.}

simulatilis Grote. brunneogrisea. Ent. Am., ii, I7I. (C.R.E.) straminalis Hübner. Handb. Brit. Lep., 424.

\section{Crocidophora Lederer.}

tuberculalis Lederer. Wien. Ent. Mon., vii, 476. (C.R.E.)

\section{Nomophila Hübner.}

noctuella Denis and Schiffermüller. Can. Ent., xxv, I29.

\section{Loxostege Hübner.}

dasconalis Walker. Cat. Brit. Mus., xviii, 273.

helvialis Walker. Ibid., xix, 98I. (F.W.H.)

obliteralis Walker. Bur. Ent., Bull. 27, new ser., 102. similalis Guenée. Spec. Gén., viii, 405.

Diasemia Hübner.

pisusalis Walker.

Tholeria Hübner.

reversalis Guenée. Spec. Gén., viii, 409. (F.W.H.)

Perispasta Zeller.

cæculalis Zeller. immixtalis. ' Can. Ent., xiii, 232.

Phlyctænia Hübner.

acutella Walker. Cat. Brit. Mus., xxxv, I753.

ferrugalis Hübner. rubigalis. Greenhouse leaf-tyer. Rept. Conn. Agr. Expt. Sta., Igog-10, 360 .

terrealis Treitschke. Schm. Eur., vii, i 10. tertialis Guenée. plectilis. Trans. Am. Ent. Suc., i, 27: Moth Book, 397. 
Cindaphia Lederer.

bicoloralis Guenée. Spec. Génı., viii, 205: Noth Book, 397.

\section{Pyrausta Schrank.}

ainsliei Heinrich. Rept. Conn. Agr. Expt. Sta., IgIO, I73.

acrionalis Walker. Cat. Brit. Mus, xix, 925.

æglealis Wralker. Ibid., xviii, 565 .

chalybealis Fernald. (MIS. name.) (C.R.E.)

fissalis Grote. Bull. Geol. Geog. Surv. Terr., vi, 273. (C.R.E.)

fumalis Gtenée. Spec. Gén., viii, 358: Noth Book, 397.

funebris Ström. octomaculata. Moth Book, 398. (F.W.H.)

futilalis Lederer. ercctalis. Can. Ent., viii, 99.

illibalis Hübner. Zutr. Exot. Schmett., i, I().

insequalis Guenée. Spec. Gén., viii, 447: Moth Book, 398.

niveicilialis Grote. Bull. Buff. Soc., Nat. Sci., ii, 232: Noth Book, 398 (F.IV.H.)

orphisalis Tralker. adipaloides. Trans. Am. Ent. Soc., i, 26. (C.R.E.)

oxydalis Guenée. Spec. Gén., viii, 328.

penitalis Grote. Can. Ent., viii, 98. (C.R.E.)

pertextalis Lederer. Wien. Ent. Mon., vii, 466: Moth Book, 397.

phœnicealis Hübner. Zutr. Exot. Schmett., i, 22.

rubricalis Hübner. similalis. Schmett. Eur., 22, pl. i6, f. 106. (F.IV.H.)

signatalis Walker. rinulentu Grote and Robinson. Trans. Am. Ent. Soc., i, I6.

theseusalis Walker. Cat. Brit. Mrus., xriii, 562.

thestealis Walker. Ibid., xviii, 733 .

sp. (Undescribed.)

\section{Eustixia Hübner.}

pupula Hübner. Noth Book, 398.

\section{Nymphula Schrank.}

allionealis Walker. Cat. Brit. Mus., xvii, 453.

badiusalis Walker. Ibid., xix, 955.

icciusalis Walker. Ibid., xix, $97 \mathrm{I}$.

maculalis Clemens. Proc. Acarl. Nat. Sci. Phila.. I860, 218. obscuralis Grote. Papilio, i, I8: Moth Book, 399. 
Geshna Dyar.

primordialis Dyar. Jour. N. Y. Ent. Soc., xiv, 97.

Elophila Hübner.

fulicalis Clemens. Proc. Acad. Nat. Sci. Phila., I860, 217.

Diathrausta Lederer.

reconditalis Walker. Cat. Brit. Mus., xix, 943.

Scoparia Haworth.

basalis Walker. Cat. Brit. Mus., xxxiv, I 497.

centuriella Denis and Schiffermüller. ninguidalis. Trans. Am. Ent. Soc., xiii, I47. (C.H.Y.)

rectilinea Zeller. Verh. Zool-Bot. Ges. Wien., xxiv, 5.

Aglossa Latreille.

cuprealis Hübner. Verz. bek. Schmett., I $826,348$.

Hypsopygia Hübner.

costalis Fabricius. Clover hay worm. Rept. Conn. Agr. Expt.

Sta., 1900, 315: Moth Book, 399.

Pyralis Linnæus.

cuprina Zeller. Verh. Zool-Bot. Ges. Wien., xxii, 497. (C.R.E.)

farinalis Linnæus. Meal snout moth. Bur. Ent., Bull. 4, new ser., II9: Moth Book, 400.

Herculia Walker.

cohortalis Grote. Can. Ent., x, 233.

intermedialis Walker. Trans. Ent. Soc. Lond., i, in8.

olinalis Guenée. Spec. Gén., viii, i 8: Moth Book, 401.

Omphalocera Lederer.

dentosa Grote. Bull. Geol. Geog. Surv. Terr., vi, 272.

Tosale Walker.

oviplagis Walker. anthacioides. Trans. Am. Ent. Soc., i, I5. (C.R.E.)

Arta Grote.

statalis Grote. Bull. Buff. Soc., Nat. Sci., ii, 230.

Condylolomia Grote.

participialis Grote. Bull. Buff. Soc., Nat. Sci., i, I77. (C.R.E.) 
Galasa IValker.

rubidana WValker. Cat. Brit. Mus., xxxv, ISo2.

Schœnobius Duponchel.

clemensellus Robinson. Ent. Ann., iv, i 38 . (C.H.Y.)

forficellus Thumberg. longirostrellus. Ibid., iv, I39.

tripunctellus Robinson. Ibid., iv, I36.

unipunctellus Robinson. Ibid., iv, I 36.

Crambus Fabricius.

agitatellus Clemens. N. A. Cramb., 37.

albellus Clemens. Ibid., 38 .

alboclavellus Zeller. Ibid., 36: Moth Book, 402.

bidens Zeller. N. A. Cramb., 32. (C.R.E.)

caliginosellus Clemens. Ibiul., 6r.

decorellus Zincken. Ibid., 52.

elegans Clemens. Ibid., 45.

girardellus Clemens. Ibid., 28.

hortuellus Hübner. Ibid., to.

laqueatellus Clemens. Ibid., 35: Moth Book, 402.

leachellus Zincken. N. A. Cramb., 29.

luteolellus Clemens. Ibid., 64. (C.R.E.)

mutabilis Clemens. Ibid., 57.

præfectellus Zincken. Ibid., 3I.

ruricolellus Zeller. Ibid., 49.

teterrellus Zincken. Ibid., 5I. (C.R.E.)

trisectus TValker. Ibid., 59: Moth Book, 403.

turbatellus Malker. N. A. Cramb., +4: Moth Book, 402.

vulgivagellus Clemens. N. A. Cramb., 47.

zeellus Fernald. Ibid., 62. (C.R.E.)

\section{Haimbachia Dyar.}

placidellus Haimbach. Ent. News, xriii, 44.

\section{Argyria Hübner.}

auratella Clemens. N. A. Cramb., 72.

multilineatella Hulst. Ent. An1., iii, I3t. (C.R.E.)

nivalis Drury. Ibid., iii, $7 \mathrm{I}$.

Platytes Guenée.

vobisne Dyar. Insec. Insc. Menst., viii, I62. 
Diatræa Guilding.

alleni Fernald. Ent. An., iv, I20. (C.R.E.)

differentialis Fernald. Ibid., iv, I2O. (C.R.E.)

Chilo Zincken.

densellus Zeller. N. A. Cramb., 79.

plejadellus Zincken. Ibid., 7S. (C.H.Y.)

Dicymolomia Zeller.

julianalis Walker. Cat. Brit. Mus., xvii, 438 . (C.R.E.)

Galleria Fabricius.

mellonella Linnaus. Wax moth. Bee moth. Bur. Ent., Bull. 75, I9: Moth Book, 406.

Paralipsa Butler.

fulminalis Zeller. Verh. Zool-Bot. Ges. Wien., xxii, 560. (C.R.E.)

furellus Zeller. Ibid., xxxiii, 2I2. (C.R.E.)

Achroia Haworth.

grisella Fabricius. Cat. Lep. Eur., ii, No. 3 .

Epipaschia Clemens.

superatalis Clemens. Ent. Am., v, 5I.

Oneida Hulst.

lunulalis Hulst. Ent. Am., iii, I3O. (C.R.E.)

Benta Walker.

asperatella Clemens. Proc. Acad. Nat. Sci., Phila., I860, 207. (C.R.E.)

\section{Lanthape Clemens.}

platanella Clemens. Proc. Acad. Nat. Sci. Phila., I860, 207. (C.R.E.)

$$
\text { Wanda Hulst. }
$$

baptisiella Fernald. Ent. Am., iii, r28.

Tetralopha Zeller.

asperatella Clemens, form nephelotella Hulst. clemensalis

Dyar. Ent. Am., v, 7o.

militella Zeller. Ibid., v, 7x.

robustella Zeller. diluculclla. Ibid., v, 7 I. 
Myelois Hübner.

obnupsella Hulst. Trans. Am. Ent. Soc., xvii, iı8.

Acrobasis Zeller.*

angusella Grote. clicllo Dyar. Proc. Wasli. Ent. Soc., x, +3 . (C.R.E.)

aurorella Ely. Ibid., xii, 67.

betulella Hulst. Trans. Am. Ent. Soc., xrii, I25. (C.R.E.)

caryalbella Ely. Insec. Insc. Menst., i, 52. (C.R.E.)

caryæ Grote. Walnut bud moth. Rept. Conn. Agr. Expt.

Sta., I912, 253.

caryivorella Ragonot. Proc. Wash. Ent. Soc., x, 42.

comptoniella Hulst. Ibid., $\mathrm{x}, 42$.

coryliella Dyar. Ibid., $\mathrm{x}, 47$.

dyarella Ely. Ibid., xii, 67. (C.R.E.)

feltella Dyar. Ibid., xi, 2I 4. (C.R.E.)

hebescella Hulst. Ibid., x, +4. (C.R.E.)

irrubriella Ely. Ibid., x, I6I. (C.R.E.)

latifasciella Dyar. Ibid., $\mathrm{x}, 45$.

malipennella Dyar. Ibid., $\mathrm{x}, 47$. (C.R.E.)

nebulella Riley. Mincola. Rept. Ins. Mo., iv, 4I. (C.R.E.)

normella Dyar. Proc. IVash. Ent. Soc., x, 46. (C.R.E.)

ostryella Ely. Insec. Insc. Menst., i, 54. (C.R.E.)

palliolella Ragonot. Trans. Am. Ent. Soc., xvii, I2I. (C.R.E.) rubrifasciella Packard. Ann. Lyc. Nat. Iist., N. Y., x, 267. secundella Ely. Insec. Insc. Menst., i, 55. (C.R.E.)

stigmella Dyar. Proc. W Vash. Ent. Soc., x, 43. (C.R.E.)

sylviella Ely. Ibid., $\mathrm{x}, 48 . \quad$ (C.R.E.)

sp.?

\section{Mineola Hulst.}

indigenella Zeller. Apple leaf crumpler. Isis, 65I: Moth Book, 409.

juglandis LeBaron. Ins. I11., ii, I23. (C.R.E.)

vaccinii Riley. Cranberry fruit worm. Can. Ent., xvi, 237. (C.R.E.)

\section{Dioryctria Zeller.}

abietella Denis and Schiffermüller. Syst. Verz. Wien., x, IzS. reniculella Grote. N. A. Ent., i, 67. (C.R.E.)

* See also appendix. 


\section{Pin:pestis Grote.}

zimmermani Grote. Pine tip moth. Rept. Conn. Agr. Expt. sta., I916, I22.

Tacoma Hulst.

nyssæcolella Dyar. Proc. Wash. Ent. Soc., vi, II2. (C.R.E.)

\section{Glyptocera Ragonot.}

consobrinella Zeller. Verh. Zool-Bot. Ges. Wien., 528. (C.R.E.)

\section{Ortholepis Ragonot.}

jugosella Ragonot. Diag. N. A. Phyc., 6. (C.R.E.)

\section{Ambesa Grote.}

busckella Dyar. Proc. Wash. Ent. Soc., vi, Io8. (C.R.E.)

\section{Nephopteryx IIübner.}

fasciolalis HuIst. Trans. Am. Ent. Soc., xiii, I62. (C.R.E.) gilvibasella Hulst. Ibid., xvii, I45. (C.R.E.)

ovalis Packard. latifasciclla. N. A. Ent., i, II. (C.R.E.) scobiella Grote. Ibid., i, 5 I.

\section{Meroptera Grote.}

unicolorella Hulst. Ent. Am., iii, I36.

Salebria Zeller.

contatella Grote. N. A. Ent., i, 50.

\section{Elasmopalpus Blanchard}

lignosellus Zeller. Isis, I848, 883 .

\section{Zophodia Hübner.}

grossulariæ Riley. Gooseberry fruit worm. Trans. Am. Ent. Soc., xvii, 173 .

Immyrla Dyar.

nigrovittella Dyar. Jour. N. Y. Ent. Soc., xiv, I08.

Euzophera Zeller.

ochrifrontella Zeller. Trans. Am. Ent. Soc., xvii, I77.

semifuneralis Walker. Ibid., xvii, I75.

Vitula Ragonot.

edmandsii Packard. Trans. Am. Ent. Soc., xvii, I78. (C.R.E.)

Canarsia Hulst.

ulmiarrosorella Clemens. Trans. Am. Ent. Soc., xvii, ISo.

Hulstea Ragonot.

undulatella Clemens. Trans. Am. Ent. Soc., xvii, I87. 


\section{Homœosoma Curtis.}

mucidellum Ragonot. Trans. An. Ent. Soc., xrii, 193. (C.R.E.) reliquella Dyar. Proc. Wash. Ent. Soc., vi, iा2. uncanale IIulst. Ibid., xvii, I92. (C.R.E.)

\section{Ephestia Guenée.}

kuehniella Zeller. Mediterranean flour moth. Special Rept. Ninn. State Ent., I904: Noth Book, 4I2.

Ephestiodes Ragonot.

infimella Ragonot. Trans. Am. Ent. Soc., xvii, 194. (C.R.E.)

Varneria Dyar.

postremella Dyar. Proc. Wash. Ent. Soc., vi, II5. (C.R.E.)

\section{Eurythmia Ragonot.}

angulella Ely. Froc. Wrash. Ent. Soc., xii, 202. (C.R.E.)

diffusella Ely. Ibid., xii, 202. (C.R.E.)

furnella Ely. Ibid., xii, 202. (C.R.E.)

parvulella Ely. Ibid., xii, 202. (C.R.E.)

Moodna Hulst.

ostrinella Clemens. Proc. Acad. Nat. Sci. Phila., I860, 206.

Plodia Guenée.

interpunctella Hübner. Indian meal moth. Rept. Conn. Agr. Expt. Sta., 1905, 252: Moth Book, 4I5.

Atacosa Hulst.

glareosella Zeller. Trans. Am. Ent. Soc., xvii, 2 I I.

Cayuga Hulst.

gemmatella Hulst. Ent. Am., iii, i3t. (C.R.E.)

Peoria Ragonot.

approximeila Walker. Cat. Brit. Mus., xxxv, I722.

Bandera Ragonot.

binotella Zeller. Trans. Am. Ent. Soc., xvii, 202. 
Family SESIIDA.

Sesia Fabricius.

bolteri Hy. Edwards. Synanthedon. Papilio, iii, I55.

scitula Harris. Synanthedon. Mon. N. A. Ses., 298: Moth Book, 387 .

tipuliformis Clerck. Synanthedon. Mon. N. A. Ses., 285:

Moth Book, 385 .

Bembecia Hübner.

marginata Harris. Mon. N. A. Ses. 260: Moth Book, 38,3.

marginata var. albicoma Hulst. Mon. N. A. Ses., 262.

\section{Synanthedon Hübner.}

exitiosa Say. Samninoidea. Peach borer. Rept. Conn. Agr. Expt. Sta., Igog-IO, 359: Noth Book, 384.

pictipes Grote and Robinson. AEgeria. Sesia. Mon. N. A. Ses., 291 .

\section{Memythrus Newman.}

asilipennis Boisduval. Mon. N. A. Ses., 252.

tricinctus Harris. Ibid., 247.

Podosesia Moeschler.

syringæ Harris. Lilac borer. Mon. 'N. A. Ses., 24t: Moth Book, 382.

\section{Alcothoë Hy.-Edwards.}

caudata Ilarris. Mon. N. A. Ses., 240: Moth Book, 382. (F.W.H.)

\section{Melittia Hübner.}

satyriniformis Hübner. Squash borer. Rept. Conn. Agr. Expt. Sta., I907-08, 806: Moth Book, 380.

\section{Family COSSIDA. \\ Prionoxystus Grote.}

macmurtrei Guérin-Méneville. Cossus. querciperda. Ins. N. Y., Fourth Rept., Rept. N. I. State Agr. Soc., I857, 790. robiniæ Peck. Carpenter worm. Ins. Aff. Park and IVoodland Trees, 79: Moth Book, 378.

\section{Zeuzera Latreille.}

pyrina Linnaus. Leopard moth. Rept. Conn. Agr. Expt. Sta., I9I I, 317: Motli Book, 376. 
Family THYRIDE.

Thyris Laspeyres.

lugubris Boisduval. Spec. Gén. Pl. I 4. f. I I, I852: i, 490, I874. maculata Harris. Am. Jour. Sci., xxxvi, 313: Moth Book, 374.

\section{Family PIROMORPHIDA.}

Harrisina Packard.

americana Guérin-Méneville. Icon. Reg. An. Ins., 500: Moth Book, 372.

Pyromorpha Herricl-Schæffer.

dimidiata Herrich-Schæffer. Moth Book, 37I.

Family MEGALOPYGIDE.

Lagoa Harris.

crispata Packard. Crinkled flannel moth. Proc. Phila. Ent. Soc., iii, 335: Noth Book, 369.

Family COCHLIDIIDE.

Tortricidia Packard.

flexuosa Grote. Jour. N. IT. Ent. Soc., vi, 94: Moth Book, 368.

Packardia Grote and Robinson.

elegans Packard. nigripunctata. Proc. Phila. Ent. Soc., iii, 342: Noth Book, 367. (C.R.E.)

elegans var. fusca Packard. Proc. Phila. Ent. Soc., iii, 343. (C.R.E.)

testacea Packard. Ibid., iii, 348: Moth Book, 368. (C.R.E.)

Lithacodes Packard.

fasciola Herrich-Schaffer. Jour. N. Y. Ent. Soc., v, I : Moth Book, 367 .

fiskiana Dyar. Ent. News, xi, 333 .

Cochlidion Hïbner.

biguttata Packard. Jour. N. Y. Ent. Soc., v, r67: Moth Book, 367 .

rectilinea Grote and Robinson var. latomia Harvey. Can. Ent. ix, 75: Moth Book, 367. (C.H.Y.) 
Prolimacodes Schaus.

scapha Harris. Jour. N. Y. Ent. Soc., iv, I72: Moth Book, 367.

Phobetron Hübner.

pithecium Smith and Abbot. Hag-moth. Jour. N. Y. Ent. Soc., iv, I78: Moth Book, 366.

Sisyrosea Grote.

textula Herrich-Schæffer. inornata. Jour. N. Y. Ent. Soc., iv, I85: Moth Book, 366 . (C.R.E.)

Adoneta Clemens.

spinuloides Herrich-Schæffer. Jour. N. Y. Ent. Soc., v, 5: Moth Book, 365 .

\section{Euclea Hübner.}

chloris Herrich-Schæffer. viridis. Jour. N. Y. Ent. Soc., v, 6i : Moth Book, 365.

delphinii Boisduval var. interjecta Dyar. Ent. News, ii, 6r. delphinii var. querceti Herrich-Schæffer. Samml. Ausser. Schmett., f. I74.

delphinii var. viridiclava Walker. Cat. Brit. Mus., v, I I54: Moth Book, 365. (C.H.Y.)

indeterminata Boisduval. z'crnata. Jour. N. Y. Ent. Soc., v, Io: Moth Book, 365. (C.H.Y.)

Sibine Herrich-Schaffer.

stimulea Clemens. Empretia. Saddle-back caterpillar. Jour. N. Y. Ent. Soc., iv, I : Moth Book, 364.

\section{Family PSYCHID $\mathrm{E}$.}

Solenobia Zeller.

walshella Clemens. Proc. Phila. Ent. Soc., i, r32.

Eurycyttarus Hampson.

confederata Grote and Robinson. Trans. Am. Ent. Soc., ii, I9I : Moth Book, 363 .

Thyridopteryx Stephens.

ephemeræformis Haworth. Bag-worm. Bur. Ent., Circ. 97: Moth Book, 36r. 


\section{Family LACOSOMIDÆ.}

\section{Lacosoma Grote.}

chiridota Grote. Proc. Phila. Ent. Soc., iii, 78: Noth Book, 359.

Cicinnus Blanchard.

melsheimeri Harris. Ins. Inj. Ves., Flint Ed., 4I5: Moth Book; 359. (C.H.Y.)

\section{Family EPIPLEMIDA:}

\section{Calledapteryx Grote.}

dryopterata Grote. Trans. Am. Ent. Soc., ii, I20: Moth Book, 356. (C.R.E.)

\section{Family GEOMETRIDA.}

\section{Brephos Ochsenheimer.}

infans Moschler. U. S. Nat. Mus., Bull., No. 44, 396: Noth Book, 355 .

Abbotana Hulst.

clemataria Smith and Abbot. Mon. Geom., 561: Moth Book, 353. (F.IV.H.)

\section{Sabulodes Guenée.}

arcasaria IValker. depontanata. Mon. Geom., 483: Moth Book, 353.

lorata Grote. Mon. Geom., 547: Moth Book, 353. sulphurata Packard. Mon. Geom., 48.: Moth Book, 353. transversata Drury. Mon. Geom., 559: Noth Book, 353.

Tetracis Gulenée.

crocallata Gienée. Mon. Geom., 548: Noth Book, 353.

Caberodes Guenée.

confusaria Hübner. Mon. Geom., 534: Moth Book, 352.

confusaria var. metrocamparia Gillenée. Spec. Gén., ix, I37. (F.IV.H.)

majoraria Guenée. Non. Geom., 536: Noth Pook, 352. 
Syssaura Hübner.

infensata Guenée. Spec. Gén., ix, 67.

infensata var. biclaria Walker. varus. Mon. Geom., 538:

Moth Book, 352.

Azelina Guenée.

ancetaria Hübner. hubnerata. Mon. Geom., 520: Moth Book, 352.

Priocycla Guenée.

armataria Herrich-Schæffer. Mon. Geom., 510: Moth Book, $35 \mathrm{I}$.

Metanema Guenée.

determinata Walker. Cat. Brit. Mus., xxxv, I55I : Moth Book, $35 \mathrm{I}$.

inatomaria Guenée. Mon. Geom., 543.

Eutrapela Hübner.

alciphearia Walker. Cat. Brit. Mus., xx, I84. (H.W.F.)

kentaria Grote. Trans. Am. Ent. Soc., i, 359. (C.R.E.)

Euchlæna Hübner.

effectaria Walker. Mon. Geom., 5I4.

johnsonaria Fitch. bilincaria. Ibid., 5 II.

marginata Minot. Ibid., 505: Proc. Bost. Soc. Nat. Hist., xi, 169.

obtusaria Hübner. Mon. Geom., 516: Moth Book, 350.

serrata Drury. serrataria. Mon. Geom., 517: Moth Book, 350. vinulentaria Grote and Robinson. Mon. Geom., 506. (C.R.E.)

Gonodontis Hübner.

duaria Guenée. Mon. Geom., 502: Moth Book, 350.

hypochraria Herrich-Schæffer. Mon. Geom., 504: Moth Book, 350 .

obfirmaria Hübner. Mon. Geom., 499: Moth Book, 350. (F.W.H.)

warneri Harvey. apiciaria. Mon. Geom., 502.

Ania Stephens.

limbata Haworth. filamentaria. Mon. Geom., 47I: Moth Book, 349.

Hyperitis Guenée.

amicaria Herrich-Schxffer. nyssaria. Mon. Geom., 46r: Moth Book, 349. 
Plagodis II übner.

keutzingaria Packard. Mon. Geom., 468: Moth Book, 349.

serinaria Herrich-Schæffer. Mon. Geom., 469: Moth Book, 349.

\section{Xanthotype Warren.}

crocataria Fabricius. Mon. Geom., 474: Moth Book, 349. Ennomos Treitschke.

magnarius Guenée. alniaria. Mon. Geom., 529: Moth Book, 348.

subsignarius Hübner. Eugonia. Snow-white linden moth. Elm span-worm. Mon. Geom., 528: Moth Book, 348.

Eugonobapta Warren.

nivosaria Guenée. nivosata. Mon. Geom., 338: Moth Book, 348.

Metrocampa Latreille.

prægrandaria Guenée. perlaria. Mon. Geom., 49I: Moth Book, 348.

\section{Therina Hübner.}

athasiaria Walker. seminudaria. Mon. Geom., 495: Moth Book, 348. (F.W.H.)

fervidaria Hübner. Mon. Geom., 493: Moth Book, $3+8$.

Anagoga Hïbner.

pulveraria Linnæus. Syst. Nat., 521 .

Cingilia Walker.

catenaria Drury. Chain-dotted Geometer. Mon. Geom., 217: Moth Book, 347.

Erranis Hübner.

tiliaria Harris. Hybernia. Lime tree moth. Mon. Geom. 409:

Noth Book, 347.

\section{Phigalia Duponchel.}

titea Cramer. Mon. Geom., 442: Noth Book, 347.

Nacophora Hulst.

quernaria Smith and Abbot. Mon. Geom., 4rr. (H.W.F.)

Lycia Hübner.

cognataria Guenée. Non. Geom., 413: Moth Book, 345.

ursaria Walker. Mon. Geom., $4 \mathrm{I} 4$. 
Epimecis Hübner.

virginaria Cramer. hortaria. Mon. Geom1., 443: Moth Book, 344. (F.W.H.)

Ectropis Hübner.

crepuscularia Denis and Schiffermüller. Mon. Geom., 428: Moth Book, 344 .

Ethaloptera Hulst.

intextata Walker. anticaria. Mon. Geom., 423. (C.R.E.)

Melanophia Hulst.

canadaria Guenée. Tephrosia. Mon. Geom., 425: Moth Book, 344.

Cleora Curtis.

larvaria Guenée. Non, Geom., 437.

pampinaria Guenée. Ibid., 432: Moth Book, 344.

takenaria Pearsall. Can. Ent., xli, I19.

Selidosema Hübner.

humarium Guenée. Mon. Geom., 435.

umbrosarium Hübner. Ibid., 439. (H.W.F.)

Tornos Morrison.

scolopacinarius Guenée. Mon. Geom., 565. (C.R.E.)

Alcis Curtis.

multilineata Packard. Mon. Geom., 287.

Paraphia Guenée.

subatomaria Wood. Mon. Geom., 417.

Nepytia Hulst.

semiclusaria Walker. Cat. Brit. Mus., xxiv, 1506.

Catopyrrha Hülner.

coloraria Fabricius. Mon. Geom1, 206: Moth Book, 342.

coloraria var. dissimilaria Hübner. Mon. Geom., 208: Moth

Book, 342.

Apæcasia Hulst.

defluata Walker. Mon. Geom., 245: Moth Book, 342. detersata Guenée. Mon. Geom., 243.

Homoclodes Hulst.

fritillaria Guenée. Mon. Geom., 489.

Cymatophora Hübner.

ribearia Fitch. Mon. Geom., 248: Moth Book, 340. 
Macaria Curtis.

aquiferaria Walker. Mon. Geom., 295.

bisignata Walker. Ibid., 290.

minorata Packard. Ibid., 29I. (C.R.E.)

Philobia Duponchel.

enotata Guenée. Mon. Geom., 288: Motl Book, 339. (F.W.H.)

Sciagraphia Hulst.

continuata Nalker. orillata. Mon. Geom., 265. (C.R.E.)

granitata Guenée. Ibid., 285.

heliothidata Gnenée. occllinata. Ibid., 283: Moth Book, 339.

Deilinea Hübner.

erythremaria Guenée. Mon. Geom., 306.

liberaria IValker. integraria. lintneraria. Ibid., 209. (C.R.E.) variolaria Guenée. intentata. Ibid., 306: Moth Book, 33S.

Gueneria Packard.

basiaria Nalker. basiata. Mon. Geom., 308.

Psysostegania Warren.

pustularia Guenée. Mon. Geom., 3I0: Moth Book, 338.

Heliomata Grote.

cycladata Grote. Proc. Phila. Ent. Soc., vi, 30: Moth Book, 338. (F.IV.H.)

Anaplodes Packard.

iridaria Guenée. Mon. Geom., 394. (II.W.F.)

remotaria $\mathrm{N}^{\mathrm{a}}$ alker. Cat. Brit. Mus., xxii, 530.

Aplodes Guenée.

mimosaria Guenée. Mlon. Geom., $386,388$.

rubrifrontaria Packard. Ibid., 386.

Synchlora Guenée.

ærata Fabricius. glancaria. Mon. Geom., 392.

denticulata Walker. cxcuraria. Ibid., $3{ }^{S I}$.

Eucrostis II ïl)ner.

incertata Walker. N'cmoria gratata. Non. Geom., 372. (G.D.)

Nemoria Hïbner.

subcroceata Walker. Mon. Geom., 372. (F.IV.II.) 
Chlorochlamys Hulst.

chloroleucaria Guenée. Mon. Geom., 370: Moth Book, 336.

Eois Hübner.

demissaria Hübner var. russata Hulst. Ent. Am., ii, I87.

inductata Guenée. Mon. Geom., 340: Moth Book, 335.

ossularia Hübner. ossulata. Mon. Geom. 329: Moth Book, 335 .

Leptomeris Hübner.

quinquelinearia Packard. Mon. Geom., 348: Moth Book, 333. (C.H.Y.)

Cinglis Guenée.

purata Guenée. Mon. Geom., 353.

similaria Walker. quadrilincata. Ibid., 345 .

Synelis Hulst.

ennucleata Guenée. alabastaria. Mon. Geom., 347: Moth Book, 333 .

ennucleata var. adornata Prout. Can. Ent., xxxix, 4I2. ennucleata var. relevata Swett. Ibid., xxxix, I4I.

Cosymbia Hübner.

lumenaria Hübner. Mon. Geom., 365: Moth Book, 333.

Pleuroprucha Mœschler.

insulsaria Guenée. Deptalia. Mon. Geom., 335.

Erastria Hübner.

amaturaria Walker. Mon. Geom., 3I7: Moth Book, 333.

Hæmatopsis Hübner.

grataria Fabricius. Mon. Geom., 219: Moth Book, 332.

Petrophora Hübner.

ferrugata Clerck. Xanthorhö̈ ferrugaria. Mon. Geom., I46. (C.R.E.)

Gypsochroa Hübner.

designata Hufnagel. Mon. Geom., I44: Moth Book, 332.

Hydriomena IIübner.

latirupta Walker. Mon. Geom., I69: Moth Book, 33I. multiferata Walker. Mon. Geom., 8I. 
Mesoleuca Hübner.

implicata Guenée. multilineata. MIon. Geom., 7I. (F.M.H.) intermediata Guenée. Mon. Geom., I57: Moth Book, 330. lacustrata Guenée. Mon. Geom., I 58: Moth Book, 330. ruficilliata Guenée. Mon. Geom., I53: Moth Book, 330.

Orthonama Hübrier.

Percnoptilota Hulst.

obstipata Fabricius. fluciata Hübner. Mon. Geom., 78: Moth Book, 330 .

Rheumaptera Hübner.

hastata Linnæus. Mon. Geom., I64: Moth Book, 329.

Eustroma Hübner.

diversilineata Hïbner. Mon. Geom., I26: Moth Book, 329. diversilineata var. gracilineata Guenée. Spec. Gén., x, 476.

Hydria Hübner.

undulata Linnæus. Calocalpe. Mon. Geom., i72: Moth Book, 329.

\section{Euchœca Hübner.}

albovittata Guenée. Mon. Geom., IS9: Moth Book, 32 S.

\section{Venusia Curtis.}

comptaria W'alker. Cat. Brit. Mus., xxi, 406; Mtoth Book, $32 \mathrm{~S}$. Eucymatoge Hübner.

intestinata Guenée. Mon. Geom., I7o: Moth Book, $32 \mathrm{~S}$.

Eupithecia Curtis.

conformata Pearsall. Tephroclystis. Ent. Nerrs. xix, I2S. interruptofasciata Packard. Rept. Peab. Acad. Sci., v, 59. miserulata Grote. Proc. Phila. Ent. Soc., ii, 32. nebulosa Hulst. Trans. Am. Ent. Soc., xxiii, 266. packardata Taylor. absinthiata. Mon. Geom., 49. (L.IV.S.) Heterophleps I- Ierrich-Schaffer. triguttaria Ierrich-Schxffer. Mon. Geom., I93: Notlı Book, 327.

Eudule Hübner.

mendica Nalker. Beggar. Cat. Brit. Mus., ii, 576: Moth Book, 327. 
Alsophila Hübner.

pometaria Ilarris. Fall canker worm. Rept. Conn. Agr. Expt.

Sta., Igo8, $78_{3}$ : Moth Book, 326.

Paleacrita Riley.

vernata Peck. Spring canker worm. Rept. Conn. Agr. Expt.

Sta., Igo8, 782: Moth Book, 324.

Nyctobia IIulst.

limitata Walker. Mon. Geom., 457: Moth Book, 324.

Dyspteris Iïbner.

abortivaria Herrich-Schæffer. Mon. Geom., 368: Moth Book, 323 .

\section{Family PLATYPTERYGIDA.}

Falcaria Haworth.

bilineata Packard. Proc. Phila. Ent. Soc., iii, 376: Moth Book, $32 \mathrm{I}$.

\section{Drepana Schrank.}

arcuata WValker. Cat. Brit. Mus., v, I I6+: Moth Book, 32 I. Oreta Walker.

irrorata Packard. Moth Book, $32 \mathrm{I}$.

rosea Walker. Ibid., $32 \mathrm{I}$.

Eudeilinea Packard.

herminiata Guenéc. Spec. Gén., x, 58.

\section{Family LASIOCAMPIDA.}

\section{Epicnaptera Rambur.}

americana Harris. Gastropacha. Moth Book, 3I4.

Malacosoma Hübner.

americana Fabricius. Clisiocampa. Tent-caterpillar. Conn. Agr. Expt. Sta., Rept. I902, I42; I9I3, 226; Bull. I77 : Moth Book, 3i2.

disstria IIübner. Clisiocampa sylvatica. Forest tent-caterpillar. N. Y. Agr. Expt. Sta., Bull. I59: Moth Book, 3 I3. Tolype Hiibner.

velleda Stoll. Moth Book, 3i2. 
Family LIPARID E.

Euproctis Hübner.

chrysorrhœa Linnaeus. Brown-tail moth. Conn. Agr. Expt. Sta., Rept., I907, $3^{1} 3$; Bull. I82; Moth Book, 309.

\section{Porthetria Hübner.}

dispar Linneus. Ocneria. Gipsy moth. Conn. Agr. Expt. Sta., Rept., I905, 246; Bull. I86: Moth Book, 308.

Olene Hübner.

achatina Smith and Abbot. Lep. Ins. Ga., ii, 77 : Moth Book, 308.

cinnamonea Grote. Proc. Phila. Ent. Soc., vi, 6.

atomaria Wralker. obliquata. Contr. Nat. Hist. Lepidop., ii, 67 : Proc. Phila. Ent. Soc., vi, 4.

atomaria rar. parallela Grote and Robinson. Contr. Nat. Hist. Lepidop, ii, 69.

leucophæa Smith and Abbot. Lep. Ins. Ga., ii, 78: Moth Book, 308.

plagiata Walker. pinicola Dyar. Cat. Brit. Nus., iv, 109.

\section{Hemerocampa Dyar.}

definita Packard. Orgyia. Proc. Phila. Ent. Soc., iii, 332 : Moth Book, 3o8. (C.R.E.)

leucostigma Smith and Abbot. Orgyia. White-marked tussock moth. Rept. Conn. Agr. Expt. Sta., I905, 230: Moth Book, 306.

\section{Notolophus Germar.}

antiqua Linnaus. Rusty tussock moth. Moth Book, 306.

\section{Family THYATIRIDE.}

Pseudothyatira Grote.

cymatophoroides Guenée. Spec. Gén., v, I3: Moth Book, 304. expultrix Grote. Proc. Phila. Ent. Soc., ii, 5S: Moth Book, 304.

\section{Habrosyne Hübner.}

scripta Gosse. Thyatira. Cat. Noc., 27: Moth Book, 303. 


\section{Family NOTODONTIDÆ.}

Ellida Grote.

caniplaga Walker. Bomb. Moths, 169: Moth Book, 300.

Gluphisia Boisduval.

septentrionalis Walker. Bomb. Moths, 90: Moth Book, 30o.

Fentonia Butler.

marthesia Cramer. Bomb. Moths, 257: Moth Book, 300.

Harpyia Ochsenheimer.

borealis Boisduval. Bomb. Moths, 264: Moth Book, 299. cinerea Walker. Bomb. Moths, 273: Moth Book, 299.

Cerura Schrank.

scitiscripta Walker var. multiscripta Riley. Bomb. Moths, 276: Moth Book, 299.

Hyparpax Hübner.

aurora Smith and Abbot. Bomb. Moths, 284: Moth Book, 299.

Schizura Doubleday.

badia Packard. Bomb. Moths, 208: Moth Book, 299.

concinna Smith and Abbot. Red-htmped caterpillar. Bomb. Moths, 2I2: Moth Book, 298.

ipomœæ Doubleday. Bomb. Moths, 194: Moth Book, 298. ipomœæ var. cinereofrons Packard. Proc. Phila. Ent. Soc., iii, 366 .

leptinoides Grote. Bomb. Moths, I99: Moth Book, 299.

semirufescens Walker. cximia. Bomb. Moths, 2ro: Cat. Brit. Mus., xxxii, 450 .

unicornis Smith and Abbot. Bomb. Noths, 203; Noth Book, 298.

Ianassa Walker.

lignicolor Walker. Bomb. Moths, I89: Moth Book, 298.

Misogada Walker.

unicolor Packard. Hetcrocampa. Bomb. Moths, 254: Moth Book, 297. 


\section{Heterocampa Doubleday.}

bilineata Packard. Bomb. Moths, 2I8: Moth Book, 297.

biundata Walker. Bomb. Moths, 235: Moth Book, 297.

guttivitta Walker. Saddled prominent. Bomb. Moths, 230:

Me. Agr. Expt. Sta., Bull. i6r.

manteo Doubleday. Bomb. Moths, 224: Moth Book, 297. (C.R.E.)

obliqua Packard var. trouvelottii Packard. Proc. Phila. Ent. Soc., iii, 369.

umbrata Walker. Bomb. Noths, 249. (A.B.C.)

\section{Symmerista Hübner.}

albifrons Smith and Abbot. Bomb. Moths, 179: Moth Book, 296.

Nerice Walker.

bidentata Walker. Bomb. Moths, I7I: Moth Book, 296.

Nadata Walker.

gibbosa Smith and Abbot. Bomb. Noths, I 42 : Moth Book, 296.

Lophodonta Packard.

angulosa Smith and Abbot. Bomb. Moths, I 47 : Moth Book, 295.

ferruginea Packard. Bomb. Moths, 150: Moth Book, 295. (F.W.H.)

Hyperæschra Butler.

georgica Herrich-Schæffer. Drymonia. Bomb. Moths, I53:

Noth Book, pl. xl, 7 .

stragula Grote. Notodonta. Bomb. Noths, 165: Noth Book, pl. xl, I.

Pheosia Hübner.

rimosa Packard. dimidiata. Bomb. Moths, I58: Moth Book, 295 .

Datana Walker.

angusii Grote and Robinson. Bomb. Moths, ino: Moth Book, 293.

contracta Wralker. Bomb. Moths, I22.

integerrima Grote and Robinson. Bomb. Moths, i20: Moth Book, 294. 
major Grote and Robinson. Bomb. Moths, I I3.

ministra Drury. Yellow-necked caterpillar. Bomb. Moths, I06: Moth Book, 293.

perspicua Grote and Robinson. Bomb. Moths, II7: Moth Book, 294.

Melalopha Hïbner.

albosigma Fitch. Bomb. Moths, I 38 : Moth Book, 293.

albosigma var. specifica Dyar. Can. Ent., xxiv, I8o.

apicalis Walker. vau. Bomb. Moths, I25: Moth Book, 293. inclusa Hübner. Ichthyura americana. Bomb. Moths, I3I : Moth Book, 293.

inclusa var. inversa Packard. palla. Proc. Phila. Ent. Soc., iii, 352. (H.W.F.)

strigosa Grote. Bomb. Moths, I30. (H.W.F.)

\section{Apatelodes Packard.}

angelica Grote. Bomb. Moths, IO3: Moth Book, 293.

torrefacta Smith and Abbot. Bomb. Moths, Ioo: Moth Book, 292.

\section{Family NOCTUID $\mathrm{E}$.}

Hypena Schrank.

humuli Harris. U. S. Nat. Mus. Bull. No. 48, I I4: Moth Book, 287.

\section{Plathypena Grote.}

scabra Fabricius. Green clover worm. Rept. Conn. Agr. Expt. Sta., I9o8-9, 828: I919, I65: Moth Book, 287.

Bomolocha Hübner.

abalinealis Walker. U. S. Nat. Mus. Bull., No. 48, Ioo: Moth Book, 286.

atomaria Smith. Trans. Am. Ent. Soc., xxix, 216. (L.B.W.) baltimoralis Guenée. U. S. Nat. Mus. Bull., No. 48, 96: Moth Book, 286.

bijugalis Walker. U. S. Nat. Mus. Bull., No. 48, 97 : Moth Book, 286.

madefactalis Guenée. profecta. U. S. Nat Mus. Bull., No. 48: I02: Moth Book, 286.

manalis Walker. U. S. Nat. Mus. Bull., No. 48, 95: Moth Book, 286. 
scutellaris Grote. U. S. Nat. Mus. Bull., No. 48, 98: Moth Book, 286.

toreuta Grote. U. S. Nat. Mus. Bull., No. I05; Moth Book, 286.

\section{Lomanaltes Grote.}

eductalis Malker. U. S. Nat. Mus. Bull., No. 48, rog: Moth Book, 285 .

Salia Hübner.

interpuncta Grote. U. S. Nat. Mus. Bull., No. 48, 90: Trans. Am. Ent. Soc., iv, 93.

Capis Grote.

curvata Grote. Can. Ent., xiv, 20: Moth Book, 285.

Palthis Hübner.

angulalis Hïbner. U. S. Nat. Miıs. Bull., No. 48, 86: Moth Book, 285.

asopialis Guenée. U. S. Nat. Mus. Bull., No. 48, 87: Moth Book, $28_{5}$.

\section{Dircetis Grote.}

vitrea Grote. Bull. Geol. Geog. Surv. Terr., iv, I86.

Gaberasa Walker.

ambigualis Walker. U. S. Nat. Mus., Bull. No. 48, 8I : Moth Book, 284.

\section{Heterogramma Guenée.}

pyramusalis Walker. U. S. Nat. Nus. Bull., No. 48, 79: Moth Book, 284.

\section{Menopsimus Dyar.}

caducus Dyar. Jour. N. Y. Ent. Soc., xv, IIo.

\section{Tetanolita Grote.}

floridana Smith. U. S. Nat. Mus. Bull., No. $48,63$.

Bleptina Guenée.

caradrinalis Guenée. U. S: N’at. Mus. Bull., No. 48, 58: Moth Book, $28_{3}$.

\section{Renia Guenée.}

clitosalis Walker. U. S. Nat. Mus. Bull., No. 48, 72. discoloralis Guenée. Ibid., No. 48, 69: Moth Book, 283. factiosalis Walker. U. S. Nat. Mus. Bull., No. 48, 73. flavipunctalis Geyer. Ibid., No. 48, 74. 
Chytolita Grote.

morbidalis Guenée. U. S. Nat. Mus. Bull., No. 48, 56: Moth Book, 282.

\section{- Philometra Grote.}

eumelusalis Walker. U. S. Nat. Mus. Bull., No. 48, 53.

hanhami Smith. Can. Ent., xxxi, 207. (C.R.E.)

Hormisa Walker.

absorptalis Walker. U. S. Nat. Mrıs. Bull., No. 48, 46: Moth Book, 282.

litophora Grote. U. S. Nat. Mus. Bull., No. 48, 48.

Zanclognatha Lederer.

cruralis Guenée. U. S. Nat. Mus. Bull., No. 48, 40.

lavigata Grote. Ibid., No. 48, 36: Moth Book, $28 \mathrm{~s}$.

lævigata var. reversata Dyar. U. S. Nat. Mlus. Bull. No. 52, 24I. (C.R.E.)

lituralis Hübner. U. S. Nat. Mus. Bull., No. 48, 32 : Moth Book, $28 \mathrm{I}$.

marcidilinea Grote. U. S. Nat. Mus. Bull., No. 48, 43: Trans. Am. Ent. Soc., iv, 97.

ochreipennis Grote. U. S. Nat. Mus. Bull., No. 48, 44: Moth Book, $28 \mathrm{I}$.

pedipilalis Guenée. U. S. Nat. Mus. Bull., No. 48, 39.

Epizeuxis Hübner.

æmula Hïbner. U. S. Nat. Mus. Bull., No. 48, 26: Moth Book, $2 \mathrm{SI}_{\mathrm{I}}$.

americalis Guenée. U. S. Nat. Mus. Bull., No. 48, 23: Moth Book, 28I.

denticulalis Harvey. U. S. Nat. Mus. Bu1l., No. 48, I9: Moth Book, 28r.

diminuendis Barnes and McDumnough. Camptylochila. Contr. Nat. Hist. Lepidop., iv, 126.

humerata Smith. Ann. N. Y. Acad. Sci., xviii, t23. (C.R.E.) julia Barnes and McDunnough. Camptylochila. Contr. Nat. Hist. Lepidop., iv, I 26.

lubricalis Geyer. U. S. Nat. Mus. Bull., No. 48, I7: Moth Book, 28I.

majoralis Smith. U. S. Nat. Mus. Bull., No. 48, 25. (C.R.E.) merricki Smith. Can. Ent.. xxxvii, 260. rotundalis Walker. U. S. Nat. Mus. Bull., No. 48, 20. scobialis Grote. Ibid., No. 48, 21 : Moth Book, $28 \mathrm{r}$. 
Erebus Latreille.

odora Linnæus. Moth Book, 279.

Phæocyma Hübner.

æruginosa Guenée. Ypsia. Proc. U. S. Nat. Mus., xxxv, 233. cingulifera Walker Homoptera. woodii. Ibid., xxxv, 26I. (H.W.F.)

halata Smith. Proc. U. S. Nat. Mus., xxxv, 252. (A.B.C.) horrida Hübner. Zalc. Ibid., xxxv, 270: Moth Book, 277. lunata Drury. Homoptera. Edusa. Proc. U. S. Nat. Mus., xxxv, 225: Noth Book, 278.

minerea Guenée. Homoptera. Proc. U. S. Nat. Mus., xxxv, 238. (H.W.F.)

undularis Drury. Ipsia. Ibid., xxxv, 23I: Moth Book, 278. unilineata Grote. Homoptera. Proc. U. S. Nat. Mus., xxxv, 245: Moth Book, 278.

\section{Strenoloma Grote.}

lunilinea Grote. Bull. Buff. Soc. Nat. Sci., i, I27: Moth Book, $276 . \quad$ (C.R.E.)

Celiptera Guenée.

frustulum Guenée. Noth Book, 275.

Remigia Guenée.

repanda Fabricius. Noth Book, 274. (C.H.Y.)

Agnomonia Hübner.

analis Drury. Moth Book, 275. (C.R.E.)

Parallelia Hübner.

bistriaris Hübner. Moth Book, 273.

Panapoda Guenée.

rufimargo Hübner. Noth Book, 273.

rufimargo var. carneicosta Guenée. Moth Book, 273.

Phoberia Hübner.

atomaris Hübner. Moth Book, 273.

Euparthenos Grote.

nubilis Hübner. Moth Book, 272.

Allotria Hübner.

elonympha Hübner. Moth Book, 272. 


\section{Catocala Schrank.}

amatrix Hübner. Noth Book, 263.

amatrix var. nurus Walker. Ibid., 263. (C.R.E.)

amica IHibner. Ibid., 269.

amica var. androphila Guenée. Trans. Am. Ent. Soc., iv, I8. (C.R.E.)

amica var. lineeila Grote. Ibid., iv, I8: Moth Book, 269.

amica var. nerissa Hy. Edwards. Bull. Brook. Ent. Soc., iii, 6r : Moth Book, 269. (C.H.I.)

antinympha Hübner. Trans. Am. Ent. Soc., iv, I3: Moth Pook, 267.

badia Grote and Robinson. Proc. Phila. Ent. Soc., vi, 22: Moth Book, 267.

blandula Intst. polygama. Trans. Am. Ent. Soc., iv, I5. briseis Edwards. Proc. Phila. Ent. Soc., ii, 508: Moth Book, 264 .

cara Guenée. Trans. Am. Ent. Soc., iv, 7: Moth Book, 263. cara var. carissima Hulst. Bull. Brook. Ent. Soc., ii, 97. (C.R.E.)

cerogama Guenée. Trans. Am. Ent. Soc, iv, 9: Moth Book, 266.

coccinata Grote. Trans. Am. Ent. Soc., iv, 6: Moth Book, 265. concumbens Walker. Trans. Am. Ent. Soc., iv, 7 : Moth Book, 263.

dejecta Strecker. Bull. Brook. Ent. Soc., ii, 97: Moth Book, $26 \mathrm{I}$.

epione Drury. Trans. Am. Ent. Soc., iv, 2: Moth Book, 260. gracilis Edwards. Proc. Phila. Ent. Soc., ii, 5I I : Moth Book, 260.

grynea Cramer. Trans. Am. Ent. Soc., iv, i6: Moth Book, 269. habilis Grote. Trans. Enı. Ent. Soc., iv, II : Moth Pook, 268. ilia Cramer. Trans. An. Ent. Soc., iv, 8: Moth Book, 265. ilia, var. uxor Guenée. Moth Book, 265.

innubens Guenée. Spec. Gén., vii, 98: Moth Book, 265. (F.M.H.)

innubens var. flavidalis Grote. Trans. An. Ent. Soc., v, 95. (F.IV.II.)

marmorata Elwards. Proc. Phila, Ent. Soc., ii, 508: Moth Book, 263. (C.R.E.) 
micronympha Guenée var. fratercula Grote and Robinson. Proc. Phila. Ent. Soc., vi, 24: Moth Book, 269. micronympha var. gisela Neyer. Bull. Brook. Ent. Soc., ii, 96: Noth Book, 269.

neogama Smith and Abbot. Trans. Am. Ent. Soc., iv, 9: Moth Book, 266.

paleogama Guenée. Trans. Am. Ent. Soc., iv, io: Moth Book, 266.

paleogama var. annida Fager. Can. Ent., xiv, r 20.

parta Guenée. Trans. Am. Ent. Soc., iv, 6: Moth Book, 264. piatrix Grote. Trans. Am. Ent. Soc., iv, Io: Moth Book, 266. præclara Grote and Robinson. Proc. Phila. Ent. Soc., vi, 25: Noth Book, 269.

relicta WTalker. Trans. Am. Ent. Soc., iv, 4: Moth Book, 262. (C.R.E.)

relicta var. bianca Hy. Edwards. Bull. Brook. Ent. Soc., iii, 54: Moth Book, 262.

relicta var. clara Bentennïller. Bull. Am. NIus, Nat. Hist., xix, 506.

relicta var. phrynia Hy. Edwards. Bull. Brook. Ent. Soc., iii, 54. (C.R.E.)

residua Grote. Proc. Bost. Soc. Nat. Hist., xvi, 242.

retecta Grote. Trans. Am. Ent. Soc., iv, 4: Moth Book, 262. robinsonii Grote. Trans. Anl. Ent. Soc., iv, 20: Moth Book, 262.

sancta Hulst. commbialis. amasia. Trans. An. Ent. Soc. iv, I6: Noth Book, 268.

subnata Grote. Trans. Am. Ent. Soc.. iv, 9: Moth Book, 266. (C.R.E.)

tristis Edwards. Proc. Phila. Ent. Soc., ii, 51 I : Moth Book, 262.

ultronia Hübner. Moth Book, 265.

ultronia var. adriana Hy. Edwards. Bull. Brook. Ent. Soc., iii, $5 \%$.

unijuga VValker. Trans. Am. Ent. Soc., iv, 5: Moth Book, 264. vidua Snith and Abbot. viduata. Trans. Am. Ent. Soc., iv, 3: Moth Book, 26 I.

Syneda Guenée.

graphica Hübner. Moth Book, 259. 
Meliopotis Hübner.

limbolaris Geyer. Moth Book, 258.

Euclidea Ochsenheimer.

cuspidea Hübner. Moth Book, 258.

Drasteria Hïbner.

crassiuscula Haworth. Lep. Brit., 259: Moth Book, 257. (C.H.Y.)

erechtea Cramer. Moth Book, 257.

Homopyralis Grote.

contracta Walker. tactus. Moth Book, 256.

discalis Grote. Can. Ent., viii, 206.

Melanomma Grote.

auricinctaria Grote. Trans. Am. Ent. Soc., v, ri7.

Hyamia Walker.

perditalis Walker. Spargoloma umbrifascia. Moth Book, 254.

Pangrapta Hübner.

decoralis Hübner. Moth Book, 254.

Phalænostola Grote.

larentioides Grote. Trans. Am. Ent. Soc., iv, 302: Moth Book, 254.

Cydosia Westwood.

aurivitta Grote and Robinson. Trans. Am. Ent. Soc., ii, I86:

Moth Book, 253.

Euherrichia Grote.

mollissima Guenée. Moth Book, 253.

Metathorasa Moore.

monetifera Guenée. Spec. Gén., vi, 295: Moth Book, 252. (F.W.H.)

Tarache Hübner.

candẹfacta Hübner. Acontia. Trans. Am. Ent. Soc., xxvii, 82. delecta Walker. Ibid., xxvii, 60: Moth Book, 25 I.

erastrioides Guenée. Trans. Am. Ent. Soc., xxvii, 7I: Moth Book, $25 \mathrm{I}$.

Characoma Walker.

nilotica Rogenhofer. Nycteola proteclla Dyar. Jour. N. Y. Ent. Soc., vi, 40. 
Sarrothripus Curtis.

revayana Scopoli. Nycteola rezidana. Ann. Hist. Nat., v. i I6.

Chamyris Guenée.

cerintha Treitschke. Noth Book, 250.

Thalpochares Lederer.

fractilinea Smith. Ann. N. Y. Acad. Sci., xviii, I25.

Prothymia Hübner.

semipurpurea Walker. Moth Book, $2+8$.

Lithacodia Hübner.

bellicula Hübner. Moth Book, 248.

Galgula Guenée.

hepara Guenée. Moth Book. 247.

hepara var. partita Guenée. Noth Book, 247.

Eustrotia Hübner.

æria Grote. Papilio, i, II.

albidula Guenée. Moth Book, 247.

apicosa Haworth. Ibid., $2+7$.

carneola Guenée. Ibid., 247.

includens Walker. Cat. Brit. Mus., xii, 8I 3 .

muscoscula Guenée. Moth Book, 247.

nigellus Strecker. immuna. Ann. N. Y. Acad. Sci., xviii, I24. puncticosta Smith. Ibid., xviii, I22.

synochitis Grote and Robinson. Trans. Am. Ent. Soc., i, 357 :

Moth Book, $2+7$.

Phiprosopus Grote.

callitrichoides Grote. Trans. Am. Ent. Soc,, iv, 90: Moth Book, 245.

Doryodes Guenée.

bistriaris Geyer. Noth Book, 245.

Rivula Guenée.

propinqualis Guenée. Moth Book, 245.

Amolita Grote.

fessa Grote. Bull. Buff. Soc. Nat. Sci., ii, I 58: Moth Book, 244.

Eucalyptera Morrison.

humeralis Snith. Trans. Am. Ent. Soc., xxix, 220. (C.R.E.) 
Scoleocampa Guenée.

likurna Geyer. Noth Book, 24.

Alabama Grote.

argillacea Hübner. Cotton-worm moth. Moth Book, 243.

Marasmalus Grote.

inficita Walker. Moth Book, 242.

ventilator Grote. Trans. Am. Ent. Soc., iv, 89. (A.B.C.)

Pæctes Hübner.

abrostoloides Guenée. Noth Book, 24I.

oculatrix Guenée. Moth Book, 24I.

Ogdoconta Butler.

cinereola Guenée. Moth Book, 24I.

Autographa Hübner.

basigera Walker. Plusia. Cat. Brit. Mus., xxxiii, 836: Moth Book, 240. (C.H.Y.)

biloba Stephens. Plusia. Moth Book, 238.

brassicæ Riley. Plusia. Cabbage looper. Cabbage Plusia. Ibid., 238.

falcigera Kirby var. simplex Guenée. Ibid., 240. precationis Guenée. Ibid., 238.

\section{Eosphoropteryx.}

thyatiroides Guenée. Moth Book, 237. (A.H.Me.)

Plusia Hübner.

ærea Hübner. Moth Book, 237.

balluca Geyer. Ibid., 237. (H.H.A.)

\section{Panchrysia Hübner.}

purpurigera Walker. Noth Book, 236.

Calpe Treitschke.

canadensis Bethune. Proc. Phila. Ent. Soc., iv, 213: Moth Book, 236.

\section{Euthisanotia Hübner.}

grata Fabricius. Eudryas. Pearl wood-nymph. Jour. N. Y. Ent. Soc., ii, 25: Moth Book, 232.

unio Hübner. Eudryas. Beantiful wood-nymph. Jour. N. Y.

Ent. Soc., ii, 24: Moth Book, 232. 
Psychomorpha Harris.

epimenis Drury. Jour. N. Y. Ent. Soc., ii, I9: Noth Book, 232.

\section{Schinia Hübner.}

arcifera Guenée. Spec. Gén., vi, ISf: Moth Book, 228.

brevis Grote. Proc. Phila. Ent. Soc., iii, 530: Noth Book, 228. marginata Haworth. Lep. Brit., 374, 1810: Moth Book, 228. nundina Drury. I11. Exot. Ent., i, 36: Moth Book, 227.

Eupanychis Grote.

spinosæ Guenée. hirtclla. Spec. Gén., vi, I82: Moth Book, 226.

\section{Rhodophora Guenée.}

florida Guenée. Alaria. Spec. Gén., vi, I7ı : Moth Book, 224. Derrima II alker.

stellata Walker. Cat. Brit. Mus., xxii, 77o: Moth Book, 224. Heliothis Ochsenheimer.

obsoleta Fabricius. armiger. Cotton boll-worm. Corn earworm. Moth Book, 222.

Chloridea IVestwood.

virescens Fabricius. rhexic. Tobacco bud-worm. Rept. Conn. Agr. Expt. Sta., I909, 367: Moth Book, 222.

\section{Atethmia Hübner.}

rectifascia Grote. Proc. Bost. Soc. Nat. Hist., xvi, 242. (H.W.F.)

Ipimorpha Hïbner.

pleonectusa Grote. Bu11. Buff. Soc. Nat. Sci., i, I9I : Moth Book, 220.

Calymn:a Hübner.

orina Guenée. Moth Book, 2 I9.

\section{Epiglæa Grote.}

decliva Grote. Moth Book, 2Jo. (J.T.K.)

Glæa IIübner.

inulta Grote. Rept. Peab. Acad. Sci., vi, 30: Moth Book, 2 IS. (E.F.)

sericea Morrison. Proc. Bost. Soc. Nat. Hist., xvii, I5I ; Moth Book, 2is. (E.F.) 
Scopelosoma Curtis.

ceromatica Grote. Bull. Buff. Soc. Nat. Sci., ii, 70: Moth Book, 2 I 8.

devia Grote. Proc. Acad. Nat. Sci., Phila., I874, 209.

morrisoni Grote. Bull. Buff. Soc. Nat. Sci., ii, 7o.

sidus Guenée. Spec. Gén., v, 386.

tristigmata Grote. Can. Ent., ix, I 56.

walkeri Grote. Proc. Phila. Ent. Soc., ii, 439: Moth Book, 2 I8.

Orthosia Ochsenheimer.

bicolorago Guenée var. ferruginoides Guenée. Spec. Gén., v, 389.

helva Grote. Bull. Buff. Soc. Nat. Sci., ii, 3ro: Moth Book, 217.

inops Grote. Bull. Geol. Geog. Surv. Terr., vi, 270.

lutosa Andrews. Can. Ent., ix, 99.

ralla Grote and Robinson. Trans. Am. Ent. Soc., i, 346.

rubrescens Walker.

Cosmia Ochsenheimer.

paleacea Esper. Schmett. Eur., pl. I22, f. 504: Moth Book, 2I7. (C.R.E.)

Fagitana Walker.

littera Guenée. Moth Book, 217.

Tapinostola Lederer.

variana Morrison. Moth Book, 2I6.

Scoliopteryx Germar.

libatrix Linnaus. Moth Book, 2 I5.

Eucirrœdia Grote.

pampina Guenée. Moth Book, 215.

Conservula Grote.

anodonta Guenée. Moth Book, 2 I5.

Trigonophora Hïbner.

periculosa Guenée. Spec. Gén., vi, 65.

periculosa var. v-brunneum Grote. Moth Book, 2 I 5.

Brotolomia Lederer.

iris Guenée. Moth Book, 2 I 5. 
Jodia Hübner.

rufago Hübner. Moth Book, 2I5.

Pyrrhia Hübner.

umbra Hufnagel. Moth Book, 2 I4.

\section{Papaipema Smith.}

astuta Bird. Can. Ent., xxxix, 272. (H.B.)

baptisiæ Bird. Hydracia. Ibid., xxxiv, Iog.

cataphracta Grote. Hydrocia. Trans. Am. Ent. Soc., xxvi, 4I: Noth Book, 2 I4.

cerussata Grote. Trans. Am. Ent. Soc., xxvi, 38. (H.B.)

duovata Bird. Can. Ent., xxxiv, I I5. (H.B.)

duplicata Bird. Ibid., xl, 25. (H.B.)

frigida Smith. Trans. Am. Ent. Soc., xxvi, 39. (H.B.)

impecuniosa Grote. Ibid., xxvi, 42. (H.B.)

inquæsita Grote and Robinson. Ibid., i, 344: Moth Book, 213.

marginidens Guenée. birdii. Spec. Gén., v, I23.

maritima Bird. Can. Ent., xli, 59. (H.B.)

nitela Guenée. Trans. Am. Ent. Soc., xxvi, 34.

nitela var, nebris Guenée. Ibid., xxvi, 34: Moth Book, 2I3.

pterisii Bird. Can. Ent. xxxix, 3ro. (H.B.)

purpurifascia Grote and Robinson. Trans. Am. Ent. Soc., xxvi, 33. (H.B.)

rigida Grote. Can. Ent., ix, 87. (C.R.E.)

rutila Guenée. Trans. Am. Ent. Soc., xxvi, 43.

sciata Bird. Can. Ent., x1, 28. (H.B.)

Gortyna Ochsenheimer.

nictitans Borkhausen. Moth Book, 2 I 2.

nictitans var. americana Speyer. atlantica. Trans. Am. Ent.

Soc., xxvi, I8.

velata Walker. scra. Ibid,, xxvi, I6: Moth Book, 2 I2.

Achatodes Guenée.

zeæ Harris. Moth Book, 21 2.

Nonagria Ochsenheimer.

oblonga Grote. subcarnca. Can. Ent., xv, I75.

Sphida Grote.

obliqua Walker. Moth Book, 2II. 


\section{Cucullia Schrank.}

asteroides Guenée. Noth Book, 208.

convexipennis Grote and Robinson. Trans. Am. Ent. Soc., ii, 20I: Moth Book, 208.

speyeri Lintner. Twenty-sixth Rept. N. Y. State Mus., I872, I68.

Calocampa Stephens.

cineritia Grote. Proc. Acad. Nat. Sci. Phila., I874, 2 Io. curvimacula Morrison. Bull. Buff. Soc. Nat. Sci., ii, I9I: Moth Book, 208.

nupera Lintner. Bull. Buff. Soc. Nat. Sci., ii, is8: Moth Book, 208.

\section{Xylina Ochsenheimer.}

antennata NValker. cincrea. Cat. Brit. Mus., xiv, I733: Moth Pook, 206.

bethunei Grote and Robinson. Trans. Am. Ent. Soc., i, 354. disposita Morrison. Bull. Buff. Soc. Nat. Sci., ii, i I6: Moth Book, 206. fagina Morrison. Bull. Buff. Soc. Nat. Sci., ii, I I5.

ferrealis Grote. Rept. Peab. Acad. Sci., vi, 32. grotei Riley. Papilio, ii, IO2. pexata Grote. Rept. Peab. Acad. Sci., vi, 35: Moth Book, 207. thaxteri Grote. Bull. Puff. Soc. Nat. Sci., ii, I96: Moth Book, 207 .

unimoda Lintner. Ent. Cont., ix, 96: Moth Book, 207.

Tricholita Grote.

signata WValker. Moth Book, 205.

\section{Graphiphora Hübner.}

alia Guenée. Noth Book, 204.

furfurata Grote. Proc. Acad. Nat. Sci., Phila., I874, 20 I.

oviducta Gıenée. Spec. Gén., v, 357: Moth Book, 204. (C.R.E.)

revicta Morrison. Proc. Bost. Soc. Nat. Hist., xviii, $24 \mathrm{I}$. subterminata Smith. Proc. U. S. Nat. Mus., x, 476.

Crocigrapha Grote.

normani Grote. Can. Ent., vi, I 5: Moth Book, 20.4. 
Orthodes Guenée.

crenulata Butler. Moth Pook, 203.

cynica Guenée. Spec. Gén., v, 375: Moth Book, 203.

vecors Guenée. Spec. Gén., v. 376: Moth Book, 203.

Heliophila Hübner.

Lencania Ochsenheimer.

albilinea Hübner. Moth Book, 2OI.

commoides Guenée. Ibid., 203.

luteopallens Smith. Proc. U. S. Nat. Mus., xxv, I8o.

multilinea Walker. Cat. Brit. Mus., ix, 97: Moth Book, 202. phragmitidicola Guenée. Spec. Gén., v, 89.

pseudargyria Gutenée. Noth Book, 20 I.

unipuncta Haworth. Army worm. Rept. Conn. Agr. Expt. Sta., I014, I57: Moth Jiook, 200.

Nephelodes Guenée.

minians Guenée var. violans Guenée. Spec. Gén., r, Izo.

\section{Morrisonia Grote.}

confusa Tübner. Proc. U. S. Nat. Mus., xv, 85: Moth Book, 197.

sectilis Guenée. cricta. Proc. U. S. Nat. Mus., xv, 83 : Moth Book, 196.

\section{Mamestra Ochsenheimer.}

adjuncta Boiscluval. Proc. U. S. Nat. Mus., xvi, 237: Moth Book, I94.

assimilis Morrison. Proc. U. S. Nat. Mus., xvi, 234. (C.R.E.) congermana Morrison. Ibid., xvi, 229: Moth Book, I93.

cristifera Nalker. lubcus. Proc. U. S. Nat. Mus., xvi, 233. cuneata Grote. Ibid., xvi, $26 \mathrm{r}$.

detracta Walker. Ibid., xiv, 2 I I: Moth Book, I92.

distincta Hïbner. Proc. U. S. Nat. Mus., xiv, 2 I2.

goodellii Grote. Can. Ent., vii, 223.

grandis Boisdural. Proc. U. S. Nat. Nus., xvi, 224: Noth Book, I93.

latex Guenée. Spec. Gén., vi, 78 .

legitima Grote. Proc. Phila. Ent. Soc., iii, 82.

lilacina Harvey. Moth Book, I94. (H.W.F.) 
lorea Guenée. Proc. U. S. Nat. Mus., xvi, 262: Moth Book, 195.

meditata Grote. Proc. U. S. Nat. Mus., xiv, 210: Moth Book, 192.

picta Harris. Proc. U. S. Nat. Mus., xvi, 23I: Moth Book, 193.

purpurissata Grote. Proc. Phila. Ent. Soc., iii, 82: Moth Book, I92. (C.R.E.)

renigera Stephens. Proc. U. S. Nat. Mus., xvi, 250: Moth Book, I95.

rosea Harvey. Proc. U. S. Nat. Mus., xvi, 229: Moth Book, I93.

subjuncta Grote and Robinson. Proc. U. S. Nat. Mus., xvi, 223: Moth Book, I93.

trifolii Rottemburg. Proc. U. S. Nat. Mus., xvi, 226: Moth Book, 193.

vicina Grote. Moth Book, 195. (H.W.F.)

\section{Anytus Grote.}

privatus Walker. sculptus. Moth Book, I9I.

\section{Paragrotis Pratt.}

bostoniensis Grote. Proc. Acad. Nat. Sci., Phila., I874, 203. detersa Walker. pitychrous. Cat. Brit. Mus., ix, 212: Moth Book, i88. (E.F.)

insulsa Walker. Euxoa. Cameades. Moth Book, 189.

messoria Harris. Euxoa. Cameades. U. S. Nat. Mus. Bull., 38, I69: Moth Book, I88.

obeliscoides Guenée. Euxoa. Carneades. U. S. Nat. Mus. Bull., 38, I99: Moth Book, I90. (C.R.E.)

perpolita Morrison. Eunoa. Carneades. U. S. Nat. Mus. Bull., 38, I5I: Moth Book, is8.

redimicula Morrison. Euxoa. Cameades. U. S. Nat. Mus. Bull, 38, 202: Moth Book, I9o.

tessellata Harris. Euxoa. Cameades. U. S. Nat. Mus. Bull., 38, 192: Moth Book, I89.

velleripennis Grote. Euxoa. Camcades. U. S. Nat. Mus. Bu1l., 38, I53: Moth Book, I 88. 
Porosagrotis Smith.

vetusta Walker. murcmula. U. S. Nat. MIus. Bull., 38, 2I2: Moth Book, i87. (E.F.)

\section{Feltia Walker.}

annexa Treitschke. U. S. Nat. Mus. Bull., 38, 122: Moth Book, 187 .

gladiaria Morrison. U. S. Nat. Mus. Bull., 38, II7: Moth Book, i86.

jaculifera Guenée. U. S. Nat. Mus. Bull., 38, II2.

jaculifera var. herilis Grote. Ibid., 38, I 3 : Moth Book, I86. subgothica Haworth. U. S. Nat. Mus. Bull., 38, III: Moth Book, I86.

venerabilis Walker. U. S. Nat. Mus. Bull., 38, I 7 : Moth Book, I86.

volubilis Harvey. U. S. Nat. Mus. Bull., 38, I21 : Moth Book, I86.

Noctua Linnæus.

bicarnea Guenée. U. S. Nat. Mus. Bull., 38, 8I : Moth Book, 183 .

clandestina Harris. U. S. Nat. Nus. Bull., 38, 94: Moth Book, I84.

c-nigrum Linnæus. U. S. Nat. Mus. Bu11., 38, 82: Moth Book, I 83.

cynica Snith. Jour. N. Y. Ent. Soc., vi, IOI. (C.R.E.)

haruspica Grote. Bull. Buff. Soc. Nat. Sci., ii, 212: Moth Book, I84. (C.H.Y.)

lubricans Guenée. U. S. Nat. Mus. Bull., 38, 96: Moth Book, I 85 .

normaniana Grote. U. S. Nat. Mus. Bull., 38, 79: Moth Book, 183 .

phyllophora Grote. U. S. Nat. Mus. Bull., 38, 85 .

plecta Linnæus. Ibid., 38, 89: Moth Book, I84.

smithii Snellen. baja. Jour. N. Y. Ent. Soc., vi, 99.

Peridroma Hübner.

incivis Guenée. U. S. Nat. Mus. Bull., 38, 72: Moth Book, I83. (F.W.H.)

margaritosa Haworth var. saucia Hübner. Variegated cut worm. U. S. Nat. Mus. Bull., 38, 70: Moth Book, I82. 
Agrotis Ochsenheimer.

badinodis Grote. Can. Ent., vi, I3: Moth Book, ISI. (E.F.) geniculata Grote and Robinson. U. S. Nat. Mus. Bull., 38, 64: Noth Book, I82.

ypsilon Rottemburg. Black cut worm. Rept. Conn. Agr. Expt. Sta., I906, 265: Moth Book, I82.

\section{Epipsilia Hübner.}

Pachnobia Guenée.

fishi Grote. Bull. Geol. Surv. Terr., iv, 175.

manifesta Morrison. U. S. Nat. Mus. Bull., 38, 55 .

monochromatea Morrison. Ent. News, xхx, 6г.

salicarum Nalker. U. S. Nat. Nius. Bull., 38, 56: Moth Book, I 80 .

\section{Semiophora Stephens.}

elimata Guenée. U. S. Nat. Mus. Bu11., 38, 51 : Moth Book, ISo. (F.W.H.)

opacifrons Grote. U. S. Nat. Mus. Bull., 38, 97: Noth Book, ISo. (C.R.E.)

\section{Eueretagrotis Snith.}

sigmoides Guenée. L. S. Nat. Mus. Bull., 38, 48: Moth Book, 179 .

Adelphagrotis Smith.

prasina Fabricius. herbacca. U. S. Nat. Mus. Bull., 38, 4I : Ioth Book, I79.

Rhynchagrotis Smith.

alternata Grote. U. S. Nat. Mus. Bull., 38, 23; Moth Book, 179.

anchocelioides Guenée var. brunneipennis Grote. Can. Ent., vii, IS7: Moth Book, i 78.

placida Grote. U. S. Nat. Mus. Bull., 38, 2 I : Moth Book, i 78.

\section{Psaphidia Walker.}

grotei Morrison. Bull. Buff. Soc. Nat. Sci., i, 274: Moth Book, 177 .

resumens $\mathrm{W}^{\top}$ alker. Cat. Brit. Mus., xxxii, 448: Moth Book, 177. 


\section{Eutolype Grote.}

bombyciformis Smith. Proc. L. S. Nat. Mus., xv, 59: Moth Book, I77.

rolandi Grote. vernalis. Proc. U. S. Nat. MIus., xv, 6o.

Copipanolis Grote.

cubilis Grote. P'roc. L. S. Nat. Mus,, xv, 63: Moth Book, I77.

Adita Grote.

chionanthi Smith and Abbot. Lep. Ins. Ga., ii, I95: Moth Book, I\%7. (C.R.E.)

\section{Lepipolys Guenée.}

perscripta Gnenée. Spec. Gen., vi, I7ł.

\section{Magusa V'alker.}

dissidens Felder. divaricata. Proc. Wash. Ent. Soc., iv, 453: Moth Book, i75. (C.R.E.)

Laphygma Guenée.

frugiperda Snith and Abbot. Fall army worm. Rept. Conn. Agr. Expt. Sta., 1912, 284: Moth Book, I74.

\section{Prodenia Guenée.}

ornithogalli Guenée. lineatclla. Spec. Gén., v, I63: Moth Book, i 74 . ornithogalli var. eudiopta Gnenée. Spec. Gén., v, I64. (E.F.)

Heliotropha Lederer.

reniformis Grote. Can. Ent., vi, If: Moth Book, 173.

reniformis var. atra Grote. Proc. Acad. Nat. Sci., Phila., I874, 200: Noth Book, I 73 .

\section{Pyrophila Hübner.}

pyramidoides Gitenée. Spec. Gén., vi, 4I3: Moth Book, I73.

Actinotia Hïbner.

ramosula Guenée. Spec. Gén., vi, I It: Moth Book, I72.

Dipterygia Stephens.

scabriuscula Linnæus. Syst. Nat., 5i6: Noth Book. I72.

\section{Euplexia Stephens.}

lucipara Linnæens. Syst. Nat., 5IS: Moth Book, I72.

Feralia Grote.

jocosa Guenée. Spec. Gén., v, 37: Moth Book, I7I. 
Hyppa Duponchel.

xylinoides Guenée. Spec. Gén., vi, ıo6: Moth Book, r7ı.

Hadena Schrank.

apamiformis Guenée. Proc. U. S. Nat. Mus., xiii, $4 \mathrm{I} 6$. (C.R.E.)

arctica Boisduval. Ibid., xiii, 430: Moth Book, I69.

bridghami Grote and Robinson. Proc. Phila. Ent. Soc., vi, I7: Moth Book, I66.

burgessi Morrison. Proc. U. S. Nat. Mus., xiii, 4I2: Moth Book, I68.

cariosa Guenée. Proc. U. S. Nat. Mus., xiii, 438.

cogitata Smith. Ibid., xiii, 42I. (E.F.)

devastatrix Brace. Ibid., xiii, 426: Moth Book, I69.

diversicolor Morrison. Proc. Bost. Soc. Nat. Hist., xvii, I32. dubitans Walker. sputatrix. Proc. U. S. Nat. Mus., xiii, 422: Moth Book, I69.

finitima Guenée. Proc. U. S. Nat. Mus., xiii, 4I9. (C.H.Y.) fractilinen Grote var. vulgivaga Morrison. Can. Ent., vi, I5. impulsa Guenée. Ibid., xiii, 425. (H.W.F.)

lignicolor Guenée. Ibid., xiii, 442: Moth Book, I69.

mactata Guenée. Spec. Gén., v, 207: Moth Book, I67. (C.R.E.)

miseloides Guenée. Noth Book, I67.

misera Grote. Bu1l. Geol. Geog. Surv. Terr., vi, 582.

modica Guenée. Spec. Gén., v, 207: Moth Book, I67.

nigrior Smith. Proc. U. S. Nat. Mus., xiii, 437: Moth Book, I69. (C.R.E.)

passer Guenée. Proc. U. S. Nat. Mus., xiii, 4I I.

remissa Hübner. Ibid., xiii, 4I.4. (C.R.E.)

semicana Walker. Cat. Brit. Mus., xxxii, 675.

semicana var. fractilinea Grote. Can. Ent., vi, I5: Moth Book, I 68.

turbulenta Hübner. Moth Book, I67.

verbascoides Guenée. Proc. U. S. Nat. Mus., xiii, 437: Moth Book, I69.

viridimusca Smith. Can. Ent., xxxi, 264.

vultuosa Grote. Proc. U. S. Nat. Mus., xiii, 4I6: Moth Book, I68 (C.H.Y.) 
Hillia Grote.

algens Grote. Can. Ent., x, 236 .

discinigra Walker. Cat. Brit. Mus., ix, 27: Moth Book, I66. (C.R.E.)

\section{Oligia Hübner.}

chalcedonica Hübner. Ent. Am., v, r 48 .

festivoides Guenée. Moth Book, I65: Ent. Am., v, I47.

nucicolora Guenée. Spec. Gén., v, 24.

\section{Perigea Guenée.}

sutor Guenée. Spec. Gén., r, 23I.

vecors Guenée. lura. Ibid., v, 230: Moth Book, I65.

xanthoides Guenée. Spec. Gén., v, 227: Moth Book, 165.

Caradrina Ochsenheimer.

miranda Grote (?). Bull. Buff. Soc. Nat. Sci., i, I I.

Anorthodes Smith.

prima Smith. Trans. Am. Ent. Soc., xviii, I 5: Moth Book, I64. (C.R.E.)

Balsa Walker.

malana Fitch. Nolophana obliquifera. Ins. N. Y., ii, 244. tristigella Walker. zelleri. Cat. Brit. Mus., xxxv, I734.

Platysenta Grote.

videns Guenée. Spec. Gén., v, 78: Moth Book, r63.

Catabena Walker.

lineolata Walker. Cat. Brit. Mus., xxxii, 63I: Moth Book, I63.

Baileya Grote.

dormitans Guenée. Spec. Gén., v, I 5 . doubledayi Guenée. Ibid., v, I5: Moth Book, I62. opthalmica Guenée. Spec. Gén., v, I5: Moth Book, i62.

Chytonix Grote.

palliatricula Guenée. Spec. Gén., v, 26: Moth Book, I6r.

Polygrammata Hübner.

hebraicum Hübner. Zutr. Exot. Schmett., f. 25: Moth Book, I6o.

Dipthera Hïbner.

fallax Herrich-Schæeffer. Exot. Schmett., f. 2I I: Moth Book, I60. 


\section{Microcœlia Guenée.}

diptero:des Guenée. Proc. Phila., Ent. Soc., iii, 78: Moth Book, i6o.

dipteroides var. obliterata Grote. Proc. Phila. Ent. Soc., iii, 79.

Arsilonche Lederer.

albovenosa Goeze. Proc. U. S. Nat. Mus., xxi, I75: Moth Book, I59.

Apatela Hübner. Acronycta Ochsenheimer.

americana Harris. Proc. U. S. Nat. Mus., xxi, 44: Moth Book, I 53 .

clarescens Guenée. Proc. U. S. Nat. Mus., xxi, r40.

dactylina Grote. Ibid., xxi, 5I: Moth Book, 153.

distans Grote. Can. Ent., xi, 58. (C.R.E.)

furcifera Guenée. Proc. U. S. Nat. Muss, xxi, 85: Moth Book, I 55 .

hæsitata Grote. Proc. U. S. Nat. Mus., xxi, I 40.

hamamelis Guenée. Ibid., xxi, I4I.

hasta Guenée. Ibid., xxi, 87.

hastulifera Smith and Abbot. Ibid., xxi, 47.

impleta Walker. lutcicoma. Moth Book, I57.

impressa Walker. Proc. U. S. Nat. Mus., xxi, 159: Moth Book. 57.

innotata Guenée. Proc. U. S. Nat. Mus., xxi, 73: Moth Book, I55. (C.R.E.)

interrupta Guenée. occidcntalis. Proc. U. S. Nat. Mus., xxi, 79: Moth Book, 155.

lobeliæ Guenée. Proc. U. S. Nat. Muss, xxi, 82: Moth Book, 155 .

noctivaga Girote. Proc. U. S. Nat. Mu1s., xxi, 156: Moth Book, 157.

oblinita Smith and Abbot. Proc. U. S. Nat. Muss, xxi, I69:

Noth Book, I 57.

ovata Grote. Proc. U. S. Nat. Mus., xxi, r35. (C.R.E.)

pruni Harris. Ibid., xxi, I23.

retardata Walker. Ibid., xxi, 145.

sperata Grote. Ibid., xxi, 154 .

vinnula Grote. Ibid., xxi, 93: Noth Book, I 56.

xyliniformis Gnenée. longa. Proc. U. S. Nat. Mus., xxi, 166. 
Charadra Walker.

deridens Guenée. Proc. U. S. Nat. Mus., xxi, 25: Moth Book, I 52 .

Demas Stepliens.

flavicornis Smith. Bull. Brook. Ent. Soc., vii, 3.

propinquilinea Grote. Charadra. Trans. Am. Ent. Soc., iv, 293.

\section{Family AGARISTIDAE.}

Alypia Hübner.

octomaculata Fabricius. Eight-spotted forester. Bull. Am. Mus. Nat. Iist., x, 357: Moth Book, I44.

\section{Family ARCTIIDE.}

\section{Halisidota Hübner.}

caryæ Harris. Hickory tussock moth. Rept. Conn. Agr. Expt. Sta., I907, 332: Moth Book, I38.

tessellaris Smith and Abbot. Bull. Am. Mus. Hist., x, 370: Noth Book, 137.

\section{Euchætias Lyman.}

egle Drury. Milk-weed moth. Pull. Am. Mus. Nat. Hist. x, 368: Noth Book, i35.

\section{Ammalo $1 \mathrm{~T}$ alker.}

tenera IIübner. collaris. Bull. Am. Mus. Nat. Hist., x, 368.

Apantesis Walker.

anna Grote var. persephone Grote. Proc. Ent. Soc., I'hila., ii, 433 .

arge Drury. Arctia. Bull. Am. Mus. Nat. Hist., x, 379: Moth Book, I 30.

figurata Drury. Moth Book, I32. (H.M.F.) intermedia Stretch. Bull. Am. Mus. Nat. Tist., x, 379: Moth Book, I29. (C.I1.Y.)

michabo Grote. Arctia. Bu1l. Am. Mis. Nat. Hist, x, 380 : Moth Book, I3o. (A.H.Me.) nais Drury. Arctia. Bull. Am. Mus. Nat. Hist., x, $3 \mathrm{SI}_{\mathrm{I}}$ parthenice Kirby. Arctia. Ibid., x, 379: Noth Book, I29. 
phyllira Drury. Arctia. Bull. Am. Mus. Nat. Hist., x, 380:

Moth Book, 132.

virgo Linnæus. Arctia. Bull. Am. Mus. Nat. Hist., x, 377:

Noth Book, I29.

virguncula Kirby. Arctia. Bull. Am. Mus. Nat. Hist., x, 378:

Noth Book, I3I.

vittata Fabricius. Arctia. Hist., $\mathrm{x}, 3^{S_{\mathrm{I}}}$ : Bull. Am. Mus. Nat.

Moth Book, I32. (A.H.Me.)

\section{Diacrisia Hübner.}

latipennis Stretch. Spilosoma. Bull. Am. Mus. Nat. Hist., x, 375: Noth Book, I28.

virginica Fabricius. Virgin tiger moth. Bull. Am. Mus. Nat.

Hist., $\mathrm{x}, 374$ : Moth Book, I27.

Phragmatobia Stephens.

fuliginosa Linnæus. rubricosa. Bull. Am. Mus. Nat. Hist., x, 373: Moth Book, I26.

\section{Isia Walker.}

isabella Smith and Abbot. Pyrrharctia. Isabella tiger moth. Bull. Am. Muss. Nat. Hist., x, 373: Moth Book, I25.

\section{Hyphantria Harris.}

cunea Drury. Fall web worm. Bull. Am. Mus. Nat. Hist., x, 376: Noth Book, I23.

textor Harris. Rept. Ins. Mass., 255: Moth Book, I24.

\section{Estigmene Hübner.}

acræa Drury. Lencarctia. Salt march caterpillar. Bull. Am.

Muts. Nat. Hist., x, 372: Moth Book, I23.

congrua Walker. antigone. Cat. Brit. Mus., iii, 669: Moth Book, I23. (C.H.Y.)

\section{Ecpantheria Hübner.}

deflorata Fabricius. scribona. ocularia. Bull. Am. Mus. Nat.

Hist., $\mathrm{x}, 372$ : Moth Book, I2O.

Haploa Hübner.

clymene Brown. Callimorpha. Bull. Am. Mus. Nat. Hist., x, 366: Moth Book, IIs.

lecontei Boisduval var. militaris Harris. Bull. Am. Mus. Nat.

Hist., $x, 366$ : Moth Book, II8. 
Utetheisa Hübner.

bella Linnæus. Bull. Am. Mus. Nat. Hist., x, 367: Moth Book, iा 7 .

\section{Eubaphe IIübner.}

aurantiaca Hübner var. ferruginosa Walker. Homomelina. Bull. Am. Mus. Nat. Hist., $x, 365$. immaculata Reakirt. Proc. Phila. Ent. Soc., ii, 372. immaculata var. trimaculosa Reakirt. Proc. Ent. Soc., Phila., ii, 372 .

Rœselia Hübner.

minuscula Zeller var. phylla Dyar. Jour. N. Y. Ent. Soc., vi, 43 .

Nigetia Walker.

formosalis Walker. mclanopa. Cat. Brit. Mus., xxxiv, I506: Moth Book, 358. (C.R.E.)

Celama Walker.

clethræ Dyar. Nola. Can. Ent., xxxi, 62. ovilla Grote. Nola. Ibid., vii, 221 : Moth Book, 357.

pustulata Walker. nigrofasciata. Cat. Brit. Mus., xxxiii, 795: Moth Book, 357. (C.R.E.) triquetrana Fitch. Jour. N. Y. Ent. Soc., vi, 42.

\section{Family LITHOSIIDE.}

Illice Walker.

subjecta Walker. Cisthenc. Bull. Am. Mus. Nat. Hist., x, 363: Moth Book, Io9.

Clemensia Packard.

albata Packard. Bull. Am. Mus. Nat. Hist., x, 36r : Moth Book, IOS.

\section{Hypoprepia Hübner.}

fucosa Hübner. Bull. Am. Mus. Nat. Hist., x, 362: Moth Book, 106.

miniata Kirby. Bu1l. Am. Mus. Nat. Hist., x, 362: Moth Book, 106.

Crambidia Packard.

pallida Packard. Proc. Phila. Ent. Soc., iii, 99. 


\section{Family SYNTOMIDÆ.}

Lycomorpha Harris.

pholus Drury. Bull. Am. Mus. Nat. Hist., x, 355: Moth Book, IOI.

\section{Scepsis Walker.}

fulvicollis Hübner. Bu1l. Am. Mus. Nat. Hist., x, 353: Moth Book, ior.

Ctenucha Kirby. virginica Charpentier. Moth Book, IO2.

\section{Family CERATOCAMPIDA.}

Basilona Boisduval.

imperialis Drury. Eacles. Imperial moth. Bull. Am. Mus. - Nat. Hist., x, 442: Moth Book, 97.

Citheronia Hübner.

regalis Fabricius. Regal moth. Bull. Am. Mus. Nat. Hist., x, 4.I : Moth Book, 97.

Anisota Hübner.

rubicunda Fabricius. Dryocampa. Green-striped maple worm. Bull. Am. Mus. Nat. Hist., x, 440: Moth Book, 95.

senatoria Snith and Abbot. Orange-striped oak worm. Senator moth. Bull. Am. Mus. Nat. Hist., x, 439.

stigma Fabricius. Ibid., x, 439: Moth Book, 94.

virginiensis Drury. Bull. Am. Nus. Nat. Hist., x, 440: Noth Book, 95.

\section{Family SATURNIIDÆ. \\ Hemileuca Walker.}

maia Drury. Bull. Am. Mus. Nat. IIist., x, 438: Moth Book, 9I. (A.W.P.)

Automeris IIübner.

io Fabricius. Hyperchiria. Io moth. Bull. Am. Mus. Nat. Hist., x, 438: Noth Book, 89 .

\section{Telea Irübner.}

polyphemus Cramer. American silk-worm moth. Bull. An. Mus. Nat. Hist., x, 436: Moth Book, 87. 


\section{Tropæa Hübner.}

luna Linneus. Actias. Luna moth. Bu1l. Am. Mus. Nat. Hist., $x,+37$ : Moth Book, 87 .

Callosamia Packard.

angulifera Walker. Tulip tree silk moth. Bull. Am. Mus. Nat. Hist., x, 436: Noth Book, 86 .

promethea Drury. P'romethea moth. Bu1l. Am. Mus. Nat. Hist., $x, 435$ : Moth Book, $S_{4}$.

\section{Samia Hübner.}

cecropia Limieus. Attacus. Cecropia moth. Bull. Am. Mus. Nat. Hist., x, 435: Noth Book, 83.

\section{Philosamia Grote.}

cynthia Drury. Cynthia moth. Bull. Am. Mus. Nat. Hist., x, 434: Moth Book, 82.

$$
\text { Family SPHINGIDE. }
$$

Cressonia Grote and Robinson.

juglandis Smith and Abbot. Bull. Am. Mus. Nat. Hist., vii, 317: Moth Book, 57 .

\section{Paonias Hübner.}

astylus Drury. Calasymbolus. Bull. Am. Mus. Nat. Iist., vii, 3I6: Moth Pook, 56. (C.R.E.)

excæcatus Snith and Abbot. Liull. Am. Mus. Nat. Hist., vii, 3It: Moth Book, 56 .

myops Smith and Abbot. Pull. Am. Mus. Nat. Hist., vii, 3r5:

Moth Book, 56 .

\section{Smerinthus Latreille.}

jamaicensis Drury. geminatus. Bull. Am. Mus. Nat. Hist., vii, 313: Moth Book, 55.

\section{Marumba Moore.}

modesta Harris. Triptogon. Pachysphinx. Bull. Am. Mus. Nat. Hist., vii, 312: Moth Book, 57. (H.H.A.), (A.B.C.), (F.I'.H.)

\section{Lapara IValker.}

bombycoides Walker. Ellema harrisii. Bull. Am. Mus. Nat. Hist., vii, 3IO: Moth Book, 53. (H.H.A.)

coniferarum Snith and Abbot. Noth Book, 53. 
Ceratomia Harris.

amyntor Geyer. quadricornis. Bull. Am. Mus. Nat. Hist., vii, 307: Moth Book, 47.

undulosa Walker. Bull. Am. Mus. Nat. Hist., vii, 308: Moth Book, 48. (A.H.Me.)

Chlænogramma Smith.

jasminearum Boisduval. Bull. Am. Mus. Nat. Hist., 306: Moth Book, 46.

Dolba Walker.

hylæus Drury. Bull. Am. Mus. Nat. Hist., vii, 309: Moth Book, 46.

\section{Sphinx Linnæus.}

chersis Hübner. Hyloicus. Bull. Am. Mus. Nat. Hist., vii, 303: Noth Book, 50.

convolvuli Linnæus var. cingulata Fabricits. Herse. Bull. Am. Mus. Nat. Hist., vii, 298: Moth Book, 43. (H.H.A.) drupiferarum Smith and Abbot. Bull. Am. Mus Nat. Hist., vii, 30o: Moth Book, 52.

eremitis Hübner. Bu1l. Am. Mus. Nat. Hist., vii, 304: Moth Book, 49. (A.H.Me.) (G.D.)

gordius Stoll. Bull. Am. Mus. Nat. Hist., vii, 302: Moth Book, 5I.

kalmiæ Smith and Abbot. Bull. Am. Mus. Nat. Hist., vii, zor : Moth Book, 5I.

luscitiosa Clemens. Bull. Am. Mus. Nat. Hist., vii, 302: Moth Book, 52.

\section{Phlegethontius Hübner.}

quinquemaculatus Haworth. Protoparce celeus Hübner. Northern tobacco or tomato worm. Rept. Conn. Agr. Expt. Sta., 1906, 269: Moth Book, 45.

sexta Johanssen. Protoparce carolina Linneus. Southern tobacco or tomato worm. Rept. Conn. Agr. Expt. Sta., 1906, 269: Moth Book, 45.

Ampelophaga Bremer and Grey.

chœrilus Cramer. Darapsa. Pholus. Bull. Mus. Nat. Hist., vii, 29I: Moth Book, 68.

myron Cramer. Bull. Am. Mus. Nat. Hist., vii, 292: Moth Book, 68. 
versicolor Harris. Bull. Am. Mus. Nat. Hist., vii, 293: Moth Book, 69.

Pholus Hübner.

achemon Drury. Bull. Am. Mus. Nat. Hist., vii, 289: Moth Book, 66. (H.M.R.)

pandorus Hübner. Bu1l. Am. Mus. Nat. Hist., vii, 288: Moth Book, 65. (A.H.Me.)

Thereta Hübner.

tersa Linnæus. Chorocampa. Xylophanes. Bull. Am. Mus. Nat. Hist., vii, 287: Mioth Book, 75. (R.H.) (H.M.R.)

Deilephila Ochsenheimer.

lineata Fabricius. Celerio. White-lined sphinx. Bull. Am. Mus. Nat. Hist., vii, 285: Noth Book, 76.

Deidamia Clemens.

inscriptum Harris. Bull. Am. Mus. Nat. Hist., vii, 284: Moth Book, 7 r.

Sphecodina Blanchard.

abbotii Swainson. Thyreus. Abbot sphinx. Bull. Am. Nus. Nat. Hist., vii, 283: Moth Book, 70.

Amphion Hübner.

nessus Cramer. Bu1l. Am. Mus. Nat. Hist, vii, 282: Moth Book, 72.

Hemaris Dalman.

diffinis Boisduval. Hamorrhagia. Bull. Am. Mus. Nat. Hist., vii, 278 : Noth Book, 63 .

gracilis Grote and Robinson. Bu1l. Am. Mus. Nat. Hist., vii, 278: Moth Book, 63.

thysbe Fabricius. Bull. Am. Mus. Nat. Hist., vii, 277: Moth Book, 62 .

thysbe var. ruficaudis Kirby. Faun. Bor. Am., iv, 303.

\section{Suborder RHOPALOCERA.}

Butterflies.

Family HESPERIIDE.

Thanaos Boisduval.

icelus Lintner. Dreamy dusky-wing. Comst. Butt., 298. juvenalis Fabricius. Juvenal's dusky-wing. Ibid., 300. martialis Scudder. Martial's dusky-wing. Ibid., 299. persius Scudder. Persius' dusky-wing. Ibid., 299. 
Pholisora Scudder.

catullus Fabricius. Sooty-wing. Comst. Butt., 297.

Thorybes Scudder.

bathyllus Smith and Abbot. Eudamus. Southern cloudy-wing. Comst. Butt., 295.

pylades Scudder. Northern cloudy-wing. Ibid., 296.

Achlarus Scudder.

lycidas Smith and Abbot. Eudamus. Hoary-edge. Comst. Butt., 295.

Epargyreus Hübner.

tityrus Fabricius. Endamus. Silver-spotted skipper. Comst. Butt., 293.

\section{Eudamus Swainson.}

proteus Linnæus. Long-tailed skipper. Comst. Butt., 292. (S.I.S.)

\section{Phycanassa Scudder.}

vitellius Fabricius, delaware. logan. Vitellius skipper. Comst. Butt., 288. (C.H.Y.) (G.D.)

\section{Limochroes Scudder.}

bimacula Grote and Robinson. Pamphila. Ann. N. Y. Lyc. Nat. Hist., viii, 433 .

manataaqua Scudder. Cross-line skipper. Comst. Butt., 286. (F.IV.II.) pontiac Edwards. Black-dash. Ibid., 285.

Euphyes Scudder.

verna Edwards. Pamplila. Little glass-wing. Comst. Butt., $2 \mathrm{~S}_{3}$.

vestris Boisduval var. metacomet Harris. Ibid., $28_{3}$.

\section{Polites Scudder.}

peckius Kirby. Pamphila. Yellow spot. Comst. Butt., 282. Thymelicus Hübner.

cernes Boisduval and LeConte. Tawny-edged skipper. Comst. Butt., $28 r$. mystic Scudder. Long dash. Ibid., $28 \mathrm{I}$. otho Smith and Abbot. Otho skipper. Ibid., 279. 
Erynnis Sclirank.

metea Scudder. Cobweb skipper. Const. Butt., 277.

sassicus Harris. Pamphila. Indian skipper. Ibid., 276.

Atrytone Scudder.

hobomok Harris. Pamphila. Mormon. Comst. Butt., 275.

hobomok var. pocahontas Scudder. Ibid., 275.

zabulon Boisduval and Le Conte. Zabulon skipper. Ibid., 274.

Poanes Scudder.

massasoit Scudder. Pamplita. Mulberry wing. Comst. Butt., 273 .

Ancyloxypha Felder.

numitor Fabricius. Least skipper. Comst. Butt., 272.

Amblyscirtes Scudder.

vialis Edwards. Roadside skipper. Comst. Butt., 270. (F.W.H.)

Family LYCENIDE.

Everes Hübner.

comyntas Godart. Tailed blue. Comst. Butt., 204.

Cyaniris Dalman.

ladon Cramer. var. lucia Kirby. Spring azure. Comst. Butt., 249.

ladon var. marginata Edwards. Ibid., 249.

ladon var. neglecta Edwards. Ibid., 250.

Heodes Dalman.

hypophlæas Boisduval. Chrysophanus. Anterican copper.

Comst. Butt., $24 \mathrm{I}$.

hypophlæas var. fasciata Strecker.

Chrysophanus Hübner.

thoë Boisduval. Bronze copper. Comst. Butt., 239.

Feniseca Grote.

tarquinius Fabricius. Wanderer. Comst. Butt., 237.

Strymon Hübner.

titus Fabricius. Thecla. Coral hair-streak. Comst. Butt., 234. 
Incisalia Minot.

irus Godart. Hoary elfin. Comst. Butt., 233. (J.T.K.)

niphon Hübner. Banded elfin. Ibid., 234. (R.H.)

Mitoura Scudder.

damon Cramer. smilacis. Comst. Butt., 229.

Thecla Fabricius.

acadica Edwards. Acadian hair-streak. Comst. Butt., 224. (R.H.)

calanus Hübner. Banded hair-streak. Ibid., 226.

edwardsii Saunders. Edwards' hair-streak. Butterfly Book, 243. (J.T.K.)

liparops Boisduval and Le Conte. strigosa. Striped hair-streak.

Comst. Butt., 228. (R.H.)

\section{Uranotes Scudder.}

melinus Hübner. Gray hair-streak. Comst. Butt., 223.

\section{Family LIBYTHEID仺.}

Hypatus Hübner.

bachmani Kirtland. Libythea. Snout butterfly. Comst. Butt., 210.

\section{Family LYMNADID $\mathrm{E}$.}

Anosia Hübner.

plexippus Linnæus. Danais archippus. Monarch. Milkweed butterfly. Comst. Butt., 204.

\section{Family AGAPETID E.}

Cissa Doubleday.

eurytus Fabricius. Neonymplar. Little wood-satyr. Comst. Butt., 197.

\section{Satyrodes Scudder.}

canthus Linnæus. Neonympha. Eyed brown. Comst. Butt., I9I.

Enodia Hïbner.

portlandia Fabricius. Debis. Pearly eye. Comst. Butt., I89. (H.H.A.) 
Cercyonis Speyer.

alope Fabricius. satyrus. Blue-eyed grayling. Comst. Butt., IS4.

alope var, nephele Kirby. Dull-eyed grayling. Ibid., 185.

Family NYMPHALIDE.

Basilarchia Scudder.

archippus Cramer. Limenitis dissippus. Viceroy. Comst. Butt., I70.

arthemis Drury. Banded purple. Ibid., I67. (H.H.A.)

arthemis var. proserpina Edwards. Hybrid purple. Ibid., I7o. (J.T.K.)

astyanax Fabricius. ursula. Red-spotted purple. Ibid., I66.

Junonia Hübner.

cœnia IIübner. Buckeye. Comst. Butt., 160.

Vanessa Fabricius.

atalanta Linnæus. Pyrameis. Red admiral. Comst. Butt, I54.

cardui Linnæus. Thistle butterfly. Cosmopolite. Ibid., I58. huntera Fabricius. Painted beauty. Ibid., 156.

Aglais Dalman.

milberti Godart. Vanessa. American tortoise-shell. Comst. Butt., I5I.

\section{Euvanessa Scudder.}

antiopa Linnæus. Vanessa. Mourning cloak. Comst. Butt., I48.

Eugonia Hübner.

j-album Boisduval and Le Conte. Grapta. Compton tortoise.

Comst. Butt., I 46 .

Polygonia Hübner.

comma Harris. Grapta harrisii. Hop-merchant. Comst. Butt., Ifo.

faunus Edwards. Grapta. Green comma. Ibid., I38.

interrogationis Fabricius. Grapta. Violet-tip. Ibid., I34.

interrogationis var. umbrosa Lintner. Ibid., 135 .

progne Cramer. Grapta. Gray comma. Ibid., I43. 
Phyciodes Hübner.

tharos Drury. Pearl crescent. Comst. Butt., I29.

tharos var. morpheus Fabricius. Ibid., I29.

Charidryas Scudder.

nycteis Doubleday and Hewitson. Silver crescent. Comst. Butt., I27. (R.H.)

Cinclidia Hübner.

harrisii Scudder. Harris' butterfly. Comst. Butt., I26. (H.H.A.)

Euphydryas Scudder.

phaeton Drury. Mclitca. Baltimore. Comst. Butt., I25.

Brenthis Hübner.

bellona Fabricius. Argynnis. Meadow fritillary. Comst. Butt., II9.

myrina Cramer. Argynnis. Silver-bordered fritillary. Ibid., 122.

Argynnis Fabricius.

aphrodite Fabricius. Silver-spot fritillary. Comst. Butt., II7. atlantis Edwards. Mountain silver spot. Ibid., II8. (F.W.H.) cybele Fabricius. Great spangled fritillary. Ibid., II4.

Speyeria Scudder.

idalia Drury. Argynnis. Regal fritillary. Comst. Butt., II2.

Euptoieta Doubleday.

claudia Cramer. Variegated fritillary. Comst. Butt., Iog.

Family PIERID蚱.

Eurema Hübner.

euterpe Méné. Terias lisa. Little sulphur. Comst. Butt., 98. euterpe var. alba Strecker. Cat. Macrolep., 85.

Eurymus Swainson.

philodice Godart. Colias. Clouded sulphur. Comst. Butt., 92. Zerene Hübner.

cæsonia Stoll. Dog's head butterfly. Comst. Butt., 90. 
Callidryas Boisduval and LeConte.

eubule Linnaus. Catopsilia. Cloudless sulphur. Comst. Butt., SS.

Synchloë IIübner.

genutia Fabricius. Anthocaris. Falcate orange-tip. Comst. Butt., S2.

\section{Pontia Fabricius. \\ Picris Schrank.}

napi Linnæus var. oleracea Harris. Ins. Inj. Veg., 27o.

napi var. virginiensis Edwards. Comst. Butt., 76. (H.L.J.) protodice Boisduval and Le Conte. Picris. Checkered white.

Southern cabbage butterfly. Ibid., 73.

rapæ Linnæus. Imported cabbage butterfly. Ibid., 78.

rapæ var. immaculata Skinner and Aaron. Can. Ent. xxi, I 28.

Family PAPILIONIDA.

Laertias Hübner.

philenor Linnæus. Papilio. Blue swallow-tail. Comst. Butt., 66.

\section{Papilio Linnæus.}

glaucus Linneus var, turnus Linnæeus. Tiger swallow-tail. Comst. Butt., 51.

polyxenes Fabricius. asterias. Black swallow-tail. Celery butterfly. Ibid., 62.

thoas Linnæus. cresphontes. Giant swallow-tail. Orange dog. Ibid., 56 .

troilus Linnæus. Green-clouded swallow-tail. Ibid., 59.

\section{Iphidicles Hübner.}

ajax Linnzeus. Papilio. Zebra swallow-tail. Comst. Butt., 49. (F.IV.H.) 


\section{Order SIPHONAPTERA.}

Fleas.

Family PULICID疢.

Ctenocephalus Kolenati.

canis Curtis. Pulex serraticeps. Common cat and dog flea. Bur. Ent., Bull. 5, New ser., I50.

\section{Order DIPTERA.}

Flies, Mosquitoes, etc.

\section{Family TIPULID E.}

Geranomyia Haliday.

canadensis Westwood. Mon. N. A. Dipt., iv, So.

distincta Doane. Jour. N. Y. Ent. Soc., viii, I86.

rostrata Say. Compl. Writ., ii, 47. (C.W.J.)

\section{Rhipidia Meigen.}

domestica Osten Sacken. Mon. N. A. Dipt., iv, 84. (C.W.J.) fidelis Osten Sacken. Ibid., iv, 83 . maculata Meigen. Ibid., iv, 82 .

Discobola Osten Sacken.

argus Say. Compl. Writ., i, 243.

\section{Dicranomyia Stephens.}

badia Walker. Mon. N. A. Dipt., iv, 72. (Ald. Cat.)

hæretica Osten Sacken. Ibid., iv, 70.

immodesta Osten Sacken. Ibid., iv, 62.

liberta Osten Sacken. Ibid., iv, 69.

longipennis Schummel. Ibid., iv, 6r.

simulans Walker. List., i, 45. (C.W.J.)

\section{Limnobia Meigen.}

immatura Osten Sacken. Ibid., iv, 89. (H.L.J.)

indigena Osten Sacken. Mon. N. A. Dipt., iv, 94. (C.W.J.) triocellata Osten Sacken. Ibid., iv, 92. (C.W.J.) 


\section{Toxorhina Loew.}

muliebris Osten Sacken. Mon. N. A. Dipt., iv, II5.

Rhamphidia Meigen.

flavipes Macquart. Dipt. Exot. (Suppl.), v, I7. (C.W.J.)

Elephantomyia Osten Sacken.

westwoodi Osten Sacken. Mon. N. A. Dipt., iv, Iog. (C.W.J.)

Atarba Osten Sacken.

picticornis Osten Sacken. Mon. N. A. Dipt., iv, I28.

Teucholabis Osten Sacken.

complexa Osten Sacken. Mon. N. A. Dipt., iv, Iz2.

Antocha Osten Sacken.

saxicola Osten Sacken. opalizans. Mon. N. A. Dipt., iv, I26. (C.W.J.)

Ormosia Rondani.

luteola Dietz. Rhypholophus. Trans. Am. Ent. Soc., xlii, I38. (C.R.E.)

monticola Osten Sacken. Rhypholophus. Mon. N. A. Dipt., iv, I45.

pygmæa Alexander. pilosa Dietz. Rhypholophus. Trans. Am. Ent. Soc., xlii, I38. (C.R.E.)

Erioptera Meigen.

armata Osten Sacken. Mon. N. A. Dipt., iv, I6o. (C.W.J.) armillaris Osten Sacken. Ibid., iv, I58.

caloptera Say. Compl. Writ., ii, 44. chlorophylla Osten Sacken. Mon. N. A. Dipt., iv, I57. (C.W.J.)

dulcis Osten Sacken. West. Dipt. I98. (C.W.J.) needhami Alexander. Can. Ent., 1, 383 . parva Osten Sacken. Mon. N. A. Dipt., iv, I62. septemtrionalis Osten Sacken. Ibid., iv, I62. straminea Osten Sacken. Ibid., iv, I 57. venusta Osten Sacken. Ibid., iv, I58. (C.W.J.) vespertina Osten Sacken. Ibid., iv, I57.

Molophilus Curtis.

hirtipennis Osten Sacken. Mon. N. A. Dipt., iv, I63. 
Gonomyia Osten Sacken.

blanda Osten Sacken. Mon. N. A. Dipt., iv, I82. cognotella Osten Sacken. Ibid., iv, ISI. subcinerea Osten Sacken. Ibid., iv, I8I. (C.W.J.) sulphurella Osten Sacken. Ibid., iv, ıSo. (C.W.J.)

Symplecta Migigen.

Hclobia St. Fargeau.

hybrida Meigen. Syst. Beschr., vi, 282. (H.L.J.)

Trichocera Meigen.

bimacula Walker. MIon. N. A. Dipt., iv, 236.

brumalis Fitch. Rept. N. I. State Ent., ii, 243.

Epiphragma Osten Sacken.

fascipennis Say. Limnobia. Compl. Writ., ii, 45. (H.L.J.)

\section{Limnophila Macquart.}

adusta Osten Sacken. Mon. N. A. Dipt., iv, 2I5. (C.IV.J.) contempta Osten Sacken. Ibid., iv, 2 I 8.

fuscovaria Osten Sacken. Ibid., iv, 225. (C.IV.J.)

lenta Osten Sacken. Ibid., iv, 23I.

macrocera Say. Limnobia. Compl. Writ., ii, 46.

marchandi Alexander. Jour. N. Y. Ent. Soc., xxiv, I I8.

montana Osten Sacken. Non. N. A. Dipt., iv, 227. (H.L.J.)

recondita Osten Sacken. Mon. N. A. Dipt., iv, 2 I 2.

rufibasis Osten Sacken. Ibid., iv, 225. (C.IV.J.)

ultima Osten Sacken. Ibid., iv, 222. (H.L.J.)

Pseudolimnophila Alexander.

luteipennis Osten Sacken. Limmoplita. Mon. N. A. Dipt., iv, 217.

noveboracensis Alexander. Limmoplita. Psyche, xviii, 196.

Eulimnophila Alexander.

tenuipes Say. Compl. Nrit., ii, 46.

Hexatoma Latreille.

Anisomera Meigen.

megacera Osten Sacken. Anisomcra. Mon. N. A. Dipt., iv. 243 .

Eriocera Macquart.

longicornis Walker. Mon. N. A. Dipt., iv. 253. (C.MV.J.) 
Penthoptera Schiner.

albitarsis Osten Sacken. Mon. N. A. Dipt., iv, 257. (Ald. Cat.)

Tricyphona Zetterstedt.

inconstans Osten Sacken. Amalopis. Mon. N. A. Dipt., iv, 266.

(C.IV.J.)

Pedicia Latreille.

albivitta Walker. Insect Book, pl. xvi, I2.

Liogma Osten Sacken.

nodicornis Osten Sacken. Mon. N. A. Dipt., iv, 30I. (C.W.J.)

Bittacomorpha Westwood.

clavipes Fabricius. Insect Book, pl. xv, S.

Ptychoptera Meigen.

rufocincta Osten Sacken. Mon. N. A. Dipt., iv, 3I3.

Oropeza Needlianl.

dorsalis Johnson. I'roc. Bost. Soc. Nat. Hist., xxxiv, I I9. sayi Johnson. anmlata. Ibid., xxxiv, IIS. (C.W.J.)

subalbipes Johnson. Ibid., xxxiv, I2I.

Tanyptera Latreille.

Xiphura Brullé

frontalis Osten Sacken. Proc. Phila. Ent. Soc., iii, 48.

fumipennis Osten Sacken. Ibid., iii, 47.

topazina Osten Sacken. Ibid., iii, 47.

Ctenophora Meigen.

apicata Osten Sacken. Proc. I'hila. Ent. Soc., iii, 46.

nubecula Osten Sacken. Ibid., iii, 45. (C.W.J.)

Nephrotoma Meigen.

Pachyrhina Macquart.

eucera Loew. Cent., iv, 39.

ferruginea Fabricius. Insect Book, p1. xv, I7.

incurva Loew. Cent., iv. 32 .

lugens Loew. Ibid., v. 26.

macrocera Say. Compl. Nrit., ii, $35^{\circ}$.

sodalis Loew. Cent., v, 29.

tenuis Loew. Ibid., iv, 4I.

virescens Loew. Ibid., v, 25.

xanthostigma Loew. Ibid., v, 3 I. 
Tipula Linnæus.

abdominalis Say. Insect Book, pl. xvi, 4.

angustipennis Loew. Cent iv, I9. (H.L.J.)

annulicornis Say. Compl. Writ., ii, 350.

bella Loew. Ibid., iv, 29.

caloptera Loew. Ibid., iv, 30. (C.IV.J.)

cunctans Say. Ibid., ii, 48.

dejecta Walker. Dipt. Saund., 442. (C.W.J.)

eluta Loevv. Cent., iv, 27.

fuliginosa Say. speciosa Loew. Compl. Writ., ii, 44.

hebes Loew. Cent., iv, i8. (Ald. Cat.)

hermannia Alexander. fasciata Loew. Cent., iv, 6; N. Y.

(Cornell) Agr. Expt. Sta., Memoir 25, 946. (C.W.J.)

latipennis Loew. Cent., v, 20. (C.W.J.)

longiventris Loew. Ibid., iv, 5. (C.W.J.)

oropezoides Johnson. Proc. Bost. Soc. Nat. Hist., xxxiv, I3I. pallida Loew. Ibid., iv, I6. (C.W.J.)

sayi Alexander. costalis Say. Jour. Acad. Nat. Sci., iii, 23. serta Loew. Cent., iv, I4.

strepens Loew. Ibid., iv, 28. (C.W.J.)

submaculata Loew. Ibid., iv, 23.

tephrocephala Loew. Ibid., v, 23. (C.W.J.)

tricolor Fabricius. Dipt. Exot., i, 22.

trivittata Say. Compl. Writ., ii, 50.

ultima Alexander. flavicans Fabricius. Dipt. Exot. (Suppl.), i, 25 ; N. Y. (Cornell) Agr. Expt. Sta., Memoir 25, 944. umbrosa Loew. inermis. Cent., iv, $3 \mathrm{I}$. valida Loew. Cent., iv, 2I. (C.W.J)

\section{Family DIXIDE}

Dixa Meigen.

clavulus Williston. modesta. N. Y. State Mus. Bull., 68, 429. (C.W.J.)

fusca Loew. Ibid., 68, 432. (C.W.J.)

notata Loew. Ibid., 68, 432. 
Family PSYCHODIDE.

\section{Psychoda Latreille.}

alternata Say. Can. Ent., xxvi, 330. cinerea Banks. Ibid., xxvi, 33I.

marginalis Banks. Ibid., xxvi, 333.

minuta Banks. Ibid., xxvi, 33I.

\section{Family CHIRONOMIDÆ.}

Ceratopogon Meigen.

fusculus Coquillett. Proc. U. S. Nat. Mus., xxiii, 605.

piceus Winnertz. Linn. Ent., vi, $2 \mathrm{I}$.

\section{Palpomyia Meigen.}

rufus Loewv. Cent., i, I2. (C.W.J.)

tibialis Meigen. Fauna Aust., ii, 586.

trivialis Loew. Cent., i, 4. (C.IV.J.)

\section{Chironomus Meigen.}

cristatus Fabricius. N. Y. State Mus. Bull., 86, 242.

dispar Meigen. Ibid., 86, 2 г3. (C.W.J.)

dorsalis Meigen. Syst. Beschr., i, 25.

dux Johanssen. N. Y. State Mius. Bull. 86, 23I. (C.W.J.)

festivus Say. Compl. Writ., ii, 4I. (C.W.J.)

modestus Say. Ibid., ii, 4I: N. Y. State Mus. Bull., 86, 227.

nigricans Johanssen. N. Y. State Mus. Bull., 86, 219.

pulchripennis Coquillett. Ibid., 86, 204. (C.W.J.)

tæniapennis Coquillett. Ibid., 86, 203.

Orthocladius Van der Wulp.

sp.

Camptocladius Van der Wulp.

byssinus Schrank. Fauna Aust., ii, 6r2.

Cricotopus Van der Wulp.

sylvestris Fabricius. Fauna Aust., ii, 6ri.

Eurycnemus Van der Wulp.

scitulus Coquillett. Proc. U. S. Nat. Mus., xxiii, 6o8. (C.IV.J.) 
Tanypus Meigen.

carnea Fabricius. Syst. Ant1., 4I, i805. (H.L.J.)

dyari Coquillett. Ent. News, xiii, 85 .

johnsoni Coquillett. Proc. U. S. Nat. Mus., xxiii, 609.

monilis Linnaus. anmulatus. Compl. Writ., ii, 43.

Psilotanypus Kieffer.

thoracicus Loew. Tanypus. Cent., vii, 3 .

Chasmatonotus Loew.

bimaculatus Osten Sacken. West. Dipt., I9r.

\section{Family CULICIDE.}

Wyeomyia Theobald.

smithii Coquillett. Ä̈des smithii. Mosq. N. A., iii, 94.

Culex Linnæus.

dyari Coquillett. brittoni. Mosq. N. A., iii, 457.

pipiens Linneus. House or rain barrel mosquito. Rept. Conn.

Agr. Sta., I904, 266: Mosq. N. A., iii, 360.

salinarius Coquillett. Mosq. N. A., iii, 373.

territans Walker. Ibid., iii, 293.

\section{Mansonia Blanchard.}

perturbans Walker. Culcx. Taniorhynchus. Mosq. N. A., iii, 505 .

Aëdes Meigen.

atropalpus Coquillett. Mosq. N. A., iv, 63 S.

aurifer Coquillett. Ibid., iv, 766.

canadensis Theobold. Ibid., iv, 6.7.

cantator Coquillett. Brown salt marsh mosquito. Ibid., iv, 700.

fitchii Felt and Young, or abfitchii Felt., Ibid., iv, 682, 688.* fuscus Osten Sacken. Ibid., iv, 729.

hirsuteron Theobald. pretans. Ibid., iv, 743.

onondagensis Felt. currei. Ibid., iv, 629.

* Several females formerly identified as Culex cantans have been taken in Connecticut. As it is said to be impossible to separate fitchii and abfitchii from captured females, the species cannot be definitely placed here. 
sollicitans WValker. Banded salt marsh mosquito. Ibid., 658 .

Rept. Conn. Agr. Expt. Sta., I904, 268.

sylvestris Theobald. Mosq. N. A., iv, 694.

tæniorhynchus IViedeman. Ibid., iv, 667.

triseriatus Say. Ibid., iv, 762 .

trivittatus Coquillett. Ibid., iv, 773 .

Psorophora Robineau-Desvoidy.

ciliata Fabricius. Gallinipper. Rept. Conn. Agr. Expt. Sta., Ig04, 27I: Mosq. N. A., iv, 530.

Anopheles Meigen.

punctipennis Say. Rept. Conn. Agr. Expt. Sta,, I904, 27I :

Mosq. N. A., iv, Ioog.

quadrimaculatus Say. maculipcmis. Nalarial mosquito. Rept.

Conn. Agr. Expt. Sta., I904, 27 I : Mosq. N. A., I028.

Corethra Meigen.

cinctipes Coquillett. Can. Ent., xxxv, Igo.

Sayomyia Coquillett.

americana Johanssen. Corcthra plumicornis var, americana.

N. Y. State Mus. Pul1., 68, 395.

Family MYCETOPHILIDA.

Mycomya Rondani.

Sciophila Meigen.

littoralis Say. Compl. Writ., i, $2+5$.

Neoemphẹria Osten Sacken.

balioptera Leow. Cent., ix, I3.

\section{Leia Meigen.}

bivittata Say. Neoglaphyroptera. Compl. Writ., ii, 35 I.

opima Loew. Cent., ix, 29.

Rhymosia Winnertz.

filipes Loew. Cent., ix, $3^{6 .}$

Exechia Winnertz.

absoluta Johanssen. Me. Agr. Expt. Sta. Bull., 200, 72. 
Mycetophila Meigen.

contigua Walker. List., i, 46.

discoidea Say. Compl. Writ., ii, 35 I.

obscura Walker. List, i, Ior.

scalaris Loew. Dynatosoma. Cent., ix, 48.

Mycetobia Meigen.

divergens Walker. Dipt. Saund., 418.

Macrocera Meigen.

clara Loew. Cent., ix, 6. (C.W.J.)

formosa Loew. Ibid., ix, 8. (C.W.J.)

hirsuta Loew. Ibid., ix, 5. (C.W.J.)

inconcinna Loew. Ibid., ix, 7. (C.W.J.)

Family SCIARIDÆ.

Sciara Meigen.

actuosa Johannsen. Me. Agr. Expt. Sta., Bu1l. 200, I34.

inconstans Fitch. Ibid., Bull. 200, I39.

jucunda Johannsen. Ibid., Bull. 200, I3I.

mellea Johannsen. Ibid., Bull. 200, r29.

ochrolabis Loew. Ibid., Bull. 200, I25.

pauciseta Felt. Ibid., Bu1l. 200, I 30.

prolifica Felt. Ibid., Bull. 200, I28.

sciophila Loew. Ibid., Bull. 200, г26.

\section{Family CECIDOMYIDÆ (ITONIDIDÆ).*}

\section{Monardia Kieffer.}

modesta Felt. Psyche, xx, I42.

\section{Miastor Meinert.}

americanus Felt. Rept. N. Y. State Ent., No. 26, 82.

Rhabdophaga IVestwood.

salicifoliz Felt. Rept. N. Y. State Ent., No. 29, 106.

strobiloides Walsh. Cecidomyia. Pine cone willow gall. Proc.

Phila. Ent. Soc., iii, 58o; Rept. N. Y. State Ent., No. 29, I 12 .

* For additional species in this family see appendix. 


\section{Dasyneura Rondani.}

communis Felt. Jour. Econ. Ent., iv, 478; Rept. N. Y. State Ent., No. 29, I8I.

gleditschiæ Osten Sacken. Rept. Conn. Agr. Expt. Sta., I904, 21 2 ; Rept. N. Y. State Ent., No. 29, I63.

serrulatæ Osten Sacken. Rept. N. Y. State Ent., No. 29, I86.

Cystiphora Kieffer.

viburnifolia Felt. Jour. Econ. Ent., iv, 480; Rept. N. Y. State Ent. No. 29, 201.

Phytophaga Rondani.

destructor Say. Cecidomyia. Mayetiola. Hessian fly. Bur. Ent., Circ. 7o.

rigidæ Osten Sacken. Cecidomyia. Willow club gall. Rept. N. Y. State Ent., No. 23, $37 \mathrm{r}$.

Rhopalomyia Rubșamen.

anthophili Osten Sacken. Ins. Galls Springfld., 50; Rept. N. Y. State Ent., No. 25, 364. 365. (G. D.)

asterifloræ Felt. Rept. N. Y. State Ent., No. 23, 366.

capitata Felt. Ibid., No. 23. 364.

hirtipes Osten Sacken. Ins. Galls. Springfld., 52. (G.D.)

lateriflori Felt. Rept. N. Y. State Ent. No. 23, 364, 365 .

pedicellata Felt. Ibid., 30, 262.

\section{Sackenomyia Felt.}

viburnifolia Felt. Rept. N. I. State Ent., No. 30, 280.

Cincticornia Felt.

connecta Felt. Rept. N. Y. State Ent., No. 23, 38I.

globosa Felt. Jour. Econ. Ent., ii, 29r.

majalis Osten Sacken. Ins. Galls. Springfld., I7. (G.D.)

pustulata Felt. Jour. Econ. Ent., ii, $29 \mathrm{I}$.

\section{Asphondylia Loew.}

conspicua Osten Sacken. Ins. Galls Springfld., 54.

monacha Osten Sacken. Rept. N. Y. State Ent., No. 23, 376.

Neolasioptera Felt.

perfoliata Felt. Ins. Galls, Springfld., 49. (G.D.)

ramuscula Beutenmüller. Rept. N. Y. State Ent., No. 23, 333.

sambuci Felt. Ins, Galls, Springfld., 48. (G.D.) 
Lasioptera Meigen.

corni Felt. Rept. N. Y. State Ent., No. 23, 324. impatientifolia Felt. Ins. Galls Springfld., 43; Rept. N. Y. State Ent., No. 23, 324. (G.D.) querciperda Felt. Rept. N. Y. State Ent., No. 23, 324. vitis Osten Sacken. Ibid., No. 4, I83; No. 23, 324.

\section{Asteromyia Felt.}

carbonifera Felt. Baldratia. Rept. N. Y. State Ent., No. 23, 328. divaricata Felt. Baldratia. Ibid., No. 23, 330.

læviana Felt. Baldratia. Ibid., No. 23, 330.

nigrina Felt. Jour. Econ. Ent., iv, 48 I.

reducta Felt. Ibid., iv, 48r.

rosea Felt. Baldratia. Rept. N. Y. State Ent., No. 23, 328.

rubra Felt. Baldratia. Ibid., No. 23, 329.

sylvestris Felt. Can. Ent., xlvii, 228.

vesiculosa Felt. Jour. Econ. Ent., ii, 286.

Camptoneuromyia Felt.

rubifolia Felt. Rept. N. Y. State Ent., No. 23, 334.

Contarinia Rondani.

canadensis Fielt. State Mus. Bull., 200, i 78.

pyrivora Riley. Pear midge. Rept. Conn. Agr. Expt. Sta., I 9 I $3,239$.

tritici Kirby. Wheat midge. Jour. Econ. Ent., 5, 289.

violicola Coquillett. Violet Midge. Bur. Ent., N. S., Bull. 27, 47. virginiana Felt. Ins. Galls Springfld., 4O. (G.D.)

Thecodiplosis Kieffer.

liriodendri Osten Sacken. Cecidomyia. Mon. N. A. Dipt., i, 202. Youngomyia Felt.

umbellicola Osten Sacken. Trans. Am. Ent. Soc., iii, 52.

Clinodiplosis Kieffer.

florida Felt. Rept. N. Y. State Ent., No. 23, 4I I.

\section{Caryomyia Felt.}

carya Osten Sacken. Hormomyia. Rept. N. Y. State Ent., No. 23,388 .

consobrina Felt. Hormomyia. Ibid., No. 23, 387. persicoides Bentenmüller. Cocidomyia. Mon. N. A. Dipt., i, 193. 
sanguinolenta Osten Sacken. Cccidomyia. Ibid., i, I92.

thompsoni Felt. Hormomyia. Rept. N. Y. State Ent., No. $23,3^{8 S .}$

Hormomyia Loew.

modesta Felt. Psyche, xx, I45.

\section{Itonida Neigen.}

foliora Russell and Hooker. Cccidomyia crubescens. Ent.

News, xix, $3+9$.

\section{Retinodiplosis Kieffer.}

resinicola Osten Sacken. Trans. Am. Ent. Soc., iii, 345.

\section{Cecidomyia Meigen.}

deserta Patton. Can, Ent., xix, 247. (Ald. Cat.)

eupatorifloræ Bentenmüller. Ins. Galls Springfld., 49 (G.D.)

impatientis Osten Sacken. Ibid., 43. (G.D.)

ocellaris Osten Sacken. Sciara. Ocellate leaf gall. Rept.

U. S. Dept. Agr., ISS I-2, 202.

pudibunda Osten Sacken, Ins. (Galls Springlld., I5. (G.D.) squamulicola Stebbins. Ibid., i6.

verrucicola Osten Sacken. Can. Ent., vii, 201.

Family BIBIONIDA.

Bibio Geoffroy.

albipennis Say. Insect Book, pl. xr, IS.

femoratus Weidemann. Dipt. Exot. (Suppl.), i, 87.

longipes Loew. Cent., V, I2.

pallipes Say. Compl. Irrit., ii, 68.

xanthopus Tiedemann. Dipt. Exot. (Supp1.), i, 88.

Dilophus Meigen.

dimidiatus Loew. Cent., viii, 3. (C.IV.J.)

Family SCATOPSID E。

Rhegmoclema Enderlein.

atrata Say. Seatopse. Wash. Igr. Expt. Sta., Bull. I3o, I2.

Reichertella Enderlein.

femoralis Macquart. Scatopse pulicaria Loew. Wash. Agr. Expt. Sta., Bull. I $30,9$.

* See also appendix. 
Family SIMULIID无.

Simulium Latreille.

bracteatum Coquillett. Bur. Ent. Bull., ro, New ser., 69. pecuarum Riley. invenustum. Bur. Ent., Bull. Io, New ser., 68.

Family RYPHIDÆ.

Ryphus Latreille.

alternatus Say. Compl. Writ., ii, 5 r.

fenestralis Scopoli. Fauna Aust., ii, 495.

punctatus Fabricius. marginatus. Compl. Writ., ii, 50.

Family STRATIOMYID玉.

Allognosta Osten Sacken.

fuscitarsis Say. Beris. Compl. Writ., ii, 52.

obscuriventris Loew. Cent., iv, 45.

Ptecticus Loew.

sackenii Williston. Can. Ent., xvii, I24. (C.W.J.)

trivittatus Say. Sargus. Compl. Writ., ii, 355.

Macrosargus Bigot.

Sargus Fabricius.

cuprarius Linnæus. Fauna Aust., i, 21 ; Psyche, xxii, 29.

decorus Say. Insect Book, pl. xvi, II.

elegans Loew. Cent., vii, Io.

viridis Say. Compl. Writ., ii, 77 .

Stratiomyia Geoffroy.

badius Walker. Trans. Am. Ent. Soc., xxii, 243. (A.B.C.)

discalis Loew. Ibid., xxii, 240.

meigenii Wiedemann. Ibid., xxii, 238.

normula Loew. Ibid., xii, 255. (Ald. Cat.)

quaternaria Loew. Ibid., xxii, 24I. (Ald. Cat.)

Odontomyia Meigen.

cincta Olivier. Trans. Am. Ent. Soc., xxii, 253.

flavicornis Olivier. Ibid., xxii, 269.

hydroleonoides Jolnnson. Ibid., xxii, 26r. (C.W.J.)

interrupta Olivier. Ibid., xxii, 265. 
microstoma Loew. Ibid., xxii, 264.

pubescens Day. Ibid., xxii, 264.

virgo Wiedemann. Ibid., xxii, 262.

Euparyphus Gerstaecker.

bellus Loew. Cent., vii, I8. (C.W.J.)

Nemotelus Geoffroy.

carbonarius Loew. Psyche, $\mathrm{x}, \mathrm{I} 77$.

unicolor Loew. Ibid., x, I 76. (C.IV.J.)

Oxycera Meigen.

maculata Olivier. Encycl. Meth., viii, 6oo. (C.W.J.)

Family TABANIDÆ.

Pangonia Latreille.

rasa Loew. Tab. Ohio, 45.

Chrysops Meigen.

callidus Osten Sacken. Tab. Ohio, 35.

carbonarius Walker. Ohio Nat., v, 2I9.

celer Osten Sacken. Tab. Ohio, 36.

cuclux Whitney. Can. Ent., xi, 35.

cursim Whitney. Ibid., xi, 36 .

delicatulus Osten Sacken. Prodrome, i, 380.

dimmocki Hine. Ohio Nat., vi, 393.

fallax Osten Sacken. Tab. Ohio, 36.

flavidus Wiedemann. Ibid., 37.

frigidus Osten Sacken. Prodrome, i, 384.

hilaris Osten Sacken. Insect Book, pl. xvi, I7.

indus Osten Sacken. Tab. Ohio, 33, 34.

lugens Wiedemann var. morosus Osten Sacken. Prodrome, i, 377. (J.S.H.)

mœchus Osten Sacken. Tab. Ohio, 39.

montanus Osten Sacken. Ibid., 40.

niger Nacquart. Insect Book, pl. xv, 36 .

obsoletus Wiedemann. Tab. Ohio, 42.

plangens Wiedemann. fuliginosus. Dipt. Exot. Suppl., i, I9.

pudicus Osten Sacken. Prodrome, i, 38r.

sackeni Hine. Tab. Ohio, 42.

univittatus Macquart. Ibid., 4 .

vittatus Wiedemann. Insect Book, pl. xv, 37 . 
Tabanus Linnæus.

abdominalis Fabricius. Prodrome, ii, 434. acteon Osten Sacken. Ibid., ii, 443. (Ald. Cat.) astutus Osten Sacken. Ibid., ii, 47I. (Ald. Cat.) atratus Fabricius. Black horse fly. Insect Book; pl. xv, 42. bicolor Wiedemann. Ibid., pl. xvi, I3. cinctus Fabricius. Prodrome, ii 464 . coffeatus Macquart. Ibid., ii, 44I. (A.B.C.) costalis Wiedemann. Insect Book, pl. xv, 38 . epistates Osten Sacken. Tab. Ohio, 5O. (A.B.C.) giganteus DeGeer. Prodrome, ii, 464. (C.IV.J.) hinei Johnson. Psyche, x1, 15. lasiophthalmus Macquart. Insect Book, pl. xv, 4r. lineola Fabricius. Ibid., pl. xv, 35. longus Osten Sacken. Tab. Ohio, 52. microcephalus Osten Sacken. Prodrome, ii, 470. nigrescens Beauvois. Ibid., ii, 453 . nigrovittatus Macquart. Ibid., ii, 449. nivosus Osten Sacken. Tab. Ohio, 52. ohioensis Hine. Ibid., 53 . orion Osten Sacken. Prodrome, ii, 442. pumilis Nacquart. Tab. Ohio, 53. (A.B.C.) recedens Walker. catcuatus. Prodrome, ii, 433. sparus Whitney. Can. Ent., xi, 38 . (Ald. Cat.) stygius Say. Tab. Ohio, 54. (Ald. Cat.) superjumentarius Whitney. Can. Ent., xi, 37. trimaculatus Beanvois. Tab. Ohio, 55. (A.B.C.) trispilus Wiedemann. Insect Book, pl. xvi, 5. vivax Osten Sacken. Tab. Ohio, 56.

Merycomyia Hine.

whitneyi Johnson. geminata Hine. Psyche, xi, I5; Ohio Nat., xii, 5 I 5 .

Family $\cdot$ LEPTID E.

Cœnomyia Latreille.

ferruginea Scopoli. cinereibarbis Bigot. Can. Ent., xvii, r22. Xylophagus Meigen.

lugens Loew. Cent., iii; 8 . persequus Walker. Dipt. Sannd., i. (A.B.C.) 


\section{Solva Valker.}

Xylomyia Rondani.

pallipes Loew. Cent. iii, I22. (IV.E.B.)

Dialysis $\mathrm{W}$ alker.

elongata Say. Compl. Writ., ii, 58. (C.IV.J.)

Rhagio Fabricits.

Leptis Fabricius.

mystacea Macquart. Insect Book, pl. xvi, I5.

punctipennis Say. Compl. Writ., ii, 55.

scapularis Loew. Cent., i, 22. (S.IV.IV.)

vertebrata Say. Compl. Writ., i, 27.

Chrysophila Macquart.

basilaris Say. Trans. Am. Ent. Soc., v, 285.

fasciata Say. Compl. Nrit., i, 28.

ornata Say. Insect Rook, pl. xr, 2.

propinqua Valker. List, i, 215.

proxima Walker. Ibid., i, 2 I 4 .

quadrata Say. Leptis. Compl. Writ., ii, 55. (IV.E.B.)

thoracica Fabricius. Insect Book, pl. xvi, I8.

\section{Atherix Meigen.}

variegata Wralker. List, i, 2IS. (H.L.J.)

Symphoromyia Frauenfield.

hirta Johnson. Ent. News, viii, I20.

Glutops Burgess.

singularis Burgess. Proc. Bost. Soc. Nat. Hist., I878, 320. (H.L.J.)

\section{Fanily CYRTID E.}

Oncodes Latreille.

costatus Loew. Cent., ix, 67. (C.MY.J.)

pallidipennis Loew. Ibid., ri, 32.

\section{Acrocera Meigen.}

nigrina Westwood. Trans. Ent. Soc. Lond., r, 98. (C.IV.J.) 


\section{Family BOMBYLIID无.}

\section{Spogostylum Macquart.}

anale Say. Compl. Writ., ii, 6o. œdipus Fabricius. Anthra.t irrorata. Ibid., ii, 6I. (S.W.W.) simson Fabricius. Insect Book, pl. xvii, II.

\section{Exoprosopa Macquart.}

fasciata Macquart. Insect Book, pl. xviii, I8. fascipennis Say. Ibid., pl. xvii, 5.

\section{Villa Lioy.}

Anthra.r Scopoli.

alternata Say. Trans. Am. Ent. Soc., xiv, I66. fulvohirta Wiedemann. Ibid., xiv, I74. lateralis Say. Ibid., xiv, I66; xix, I69. lepidota Osten Sacken. Trans. Am. Ent. Soc., xix, I76. (C.W.J.)

morio Linnæus. Trans. Am. Ent. Soc., xxi, 98. (C.W.J.) sinuosa Wiedemann. Insect Book, pl. xvii, 7. webberi Johnson. Psyche, xxvi, II.

Bombylius Linnæus.

fraudulentus Johnson. Psyche, xiv, 99. fulvibasis Macquart. atriceps Loew. Cent. iv, 49. major Linnæus. Insect Book, pl. xvii, 6. pulchellus Loew. Cent., iv, 47. (S.W.W.) pygmæus Fabricius. Insect Book, pl. xviii, I9. varius Fabricius. Ibid., pl. xviii, 22. (A.B.C.)

\section{Sparnopolius Loew.}

fulvus Wiedemann. Insect Book, pl. xviii, 26.

Systropus Wiedemann.

macer Loew. Cent., iv, 56.

Geron Meigen.

senilis Fabricius. Cent., ix, I908.

\section{Family THEREVIDÆ.}

Tabuda Walker.

fulvipes Walker. Proc. Phila. Ent. Soc., i, 217. 
Psilocephala Zetterstedt.

aldrichi Coquillet. Can. Ent., xxv, 227. hæmorrhoidalis Macquart. Insect Book, pl. xviii, 27. melanoprocta Loew. Cent., viii, I5. (H.L.J.) pictipennis Wiedemann. Dipt. Exot. Suppl., II3. (H.L.J.)

Thereva Latreille.

albiceps Loew. Cent., ix, 69. (C.IV.J.) candidata Loew. Can. Ent., xxv, 197. frontalis Say. Compl. Writ., i, 252. (C.W.J.)

Family SCENOPINIDE.

Scenopinus Latreille.

fenestralis Linnæus. pallipcs. Compl. Writ., ii, 86. (C.W.J.) glabrifrons Meigen. Fauna Aust., i, I60.

Family MYDAIDA.

Mydas Fabricius. clavatus Drury. filatus. Dipt. Exot. Suppl., i I6.

\section{Family ASILID E.}

\section{Leptogaster Meigen.}

badius Loew. Trans. Am. Ent. Soc., xxxv, I6o.

flavillaceus Loew. Cent., ii, I2.

flavipes Loew. Trans. Am. Ent. Soc., xxxv, I63.

loewi Banks. Psyche, xxi, I33. (C.W.J.)

testacea Loew. Cent., ii, Io. (C.W.J.)

Ceraturgus Wiedemann.

cruciatus Say. Insect Book, pl. xvi, 29.

\section{Dioctria Meigen.}

albius Walker. Insect Book, pl. xvii, I2. baumhaueri Meigen. Syst. Beschr, ii, 245. brevis Banks. Psyche, xxiv, II7.

\section{Cyrtopogon Loew.}

falto Walker. chry'sopogon Loew. Insect Book, pl. xix, 28. marginalis Loew. Trans. Am. Ent. Soc., xxxv, 267. 
Lasiopogon Loew.

Danlopogon Loew.

opaculus Loew. Trans. Am. Ent. Soc., xxxy, 299. (C.W.J.) terricola Johnson. Ent. News, xi, 326.

tetragrammus Loew. Trans. Am. Ent. Soc., xxxy, 301. (C.IV.J.)

Holcocephala Jrennicke.

abdominalis Say. Trans. Am. Ent. Soc., xxxv, 309.

Holopogon Loew.

guttula Wiedemann. Trans. Am. Ent. Soc., xxxv, 3 I3.

Stichopogon Loew.

trifasciatus Say. Trans. Am. Ent. Soc., xxxv, 335.

Deromyia Philippi.

discolor Loew. Trans. Am. Ent. Soc., xxxv, $35^{8}$.

umbrina Loew. Ibid., xxxv, 367.

winthemi Wiedemann. Ibid., xxxv, $37 \mathrm{I}$.

Taracticus Loew.

octopunctatus Say. Trans. Am. Ent. Soc., xxxv, 373.

Atomosia Macquart.

puella Wiedemann. Psyche, x, II4.

\section{Lampria Macquart.}

bicolor Wiedemann. Insect Book, pl. xvi, 27.

Dasyllis Loew.

champlainii Walton. Ent. News, xxi, 243. (A.B.C.)

flavicollis Say. Laphria. Compl. Writ., i, 255.

grossa Fabricius. Laphria tergissa. Ibid., ii, 67.

posticata Say. Laphria. Compl. IVrit., i, 255.

thoracica Fabricius. Insect Book, pl. xviii, II.

\section{Laphria Meigen.}

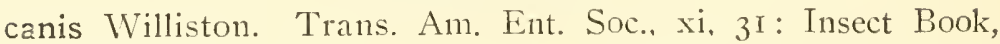
pl. xvii, Io.

gilva Linnaus, bilineata. Trans. Am. Ent. Soc., xi, 30. (D.J.C.)

Ommatius Wiedemann.

marginellus Fabricius. tibialis. Insect Book, pl. xvi, 25. 
Proctacanthus Macquart.

brevipennis Wiedemann. Trans. Am. Ent. Soc., xii, 73. philadelphicus Macquart. Insect Book, pl. xvii, 24.

\section{Erax Scopoli.}

æstuans Linnæus. bastardii Nacquart. Insect Book, pl. xvii, 14.

albibarbis Macquart. cinerascens. furax. Trans. Am. Ent. Soc., xii, 67.

rufibarbis Macquart. Dipt. Exot. Suppl., I, 2, I I6; Psyche, xvi, 33. (C.IV.J.)

Promachus Loew.

bastardii Macquart. Trans. Am. Ent. Soc., xii, 63.

fitchii Osten Sacken. Ibid., xiii, 61. (H.L.J.)

Asilus Linnaus.

auricomus Hine. Ann. Ent. Soc. Am., ii, I48.

erythocnemius Hine. Ibid., ii, I63. (J.S.H.)

flavofemorata Hine. flavipes. Ibid., ii, 153.

notatus Wiedemann. Ibid., ii, 157.

novæ-scotiæ Macquart. Ibid., ii, I57.

orphne Walker. distinctus. Ibid., ii, I54.

paropus Walket. Ibid., ii, I6r. (J.S.H.)

sadyates Walker. Ibid., ii, 156.

sericeus Say. Insect Book, pl. xrii, 20.

snowii Hine. anmulatus. Ann. Ent. Soc. Am., ii, I6o.

\section{Family DOLICHOPODIDE.}

\section{Psilopus Meigen.}

caudatus Miedemann. candatulus. Mon. N. A. Dipt., ii, 27 I. filipes Loew. Ibid., ii, 286. (S.W.W.)

pallens IViedemann. Ibid., ii, 275. (C.W.J.)

patibulatus Say. Compl. Writ., ii, 76.

scaber Loew. Mon. N. A. Dipt., ii, 250. (C.M.J.)

scobinator Loew. Ibid., ii, 268. (C.M.I.)

sipho Say. Compl. Writ., ii, 75.

tener Loew. Cent., ii, 7 I.

unifasciatum Say. Compl. Writ., ii, 75. (C.IV.J.) 
Diaphorus Meigen.

leucostoma Loew. Mon. N. A. Dipt., ii, ı66. (C.W.J.)

mundus Loew. Ibid., ii, I6I. (C.W.J.)

sodalis Loew. Ibid., ii, I63. (C.W.J.)

spectabilis Loew. Ibid., ii, I62. (C.W.J.)

Asyndetus Loew.

johnsoni Van Duzee. Psyche, xxiii, 93. (C.W.J.)

Chrysotus Meigen.

affinis Loew. Mon. N. A. Dipt., ii, I78. (C.W.J.)

auratus Loew. Ibid., ii, 183 .

discolor Loew. Ibid., ii, I82.

obliquus Loew. Ibid., ii, I76.

Argyra Macquart.

albicans Loew. Mon. N. A. Dipt., ii, I25. (C.W.J.)

aldrichi Johnson. Psyche, $\mathrm{x}, \mathrm{I} 8$.

calcitrans Loew. Mon. N. A. Dipt., ii, I30.

Leucostola Macquart.

cingulata Loew. Mon. N. A. Dipt., ii, I52. (C.W.J.)

Porphyrops Meigen.

longipes Loew. Mon. N. A. Dipt., ii, I40. (C.W.J.)

melampus Loew. Ibid., ii, I4I. (C.W.J.)

nigricoxa Loew. Ibid., ii, I45. (C.W.J.)

\section{Syntormon Loew.}

cinereiventris Loew. Mon. N. A. Dipt., ii, I37. (S.W.W.)

Sympycnus Loew.

lineatus Loew. Mon. N. A. Dipt., ii, I89.

Neurigona Rondani.

carbonifer Loew. Cent., $x, 84$. (C.W.J.)

rubella Loew. Mon. N. A. Dipt., ii, 227. (C.W.J.)

tarsalis Van Duzee. Ann. Ent. Soc. Am., vi, 5 I.

Hydrophorus Fallen.

intentus Aldrich. Psyche, xviii, $5 \mathrm{I}$.

parvus Loew. Mon. N. A. Dipt., ii, 216. (C.IV.J.)

pirata Loew. Ibid., ii, 2 I4. 
Rhaplium Meigen.

lugubre Loew. Pelastoneura. Mon. N. A. Dipt., ii, 105. (C.W.J.)

Dolichopus Latreille.

albicoxa Aldrich. Kans. Univ. Quart., ii, Io. (Ald. Cat.)

brevimanus Loew. Mon. N. A. Dipt., ii, 39. (C.W.J.)

calcaratus Aldrich. Kans. Univ. Quart., ii, 8. (C.W.J.)

comatus Loew. Mon. N. A. Dipt., ii, 69.

cuprinus Wiedemann. Ibid., ii, 55 .

detersus Loew. Kans. Univ. Quart., ii, 8. (C.W.J.)

eudactylus Loew. Mon. N. A. Dipt., ii, 48. (C.W.J.)

incisuralis Loew. Ibid., ii, 74. (C.W.J.)

laticornis Loew. Ibid., ii, 29. (Ald. Cat.)

latipes Loew. Ibid., ii, I7. (Ald. Cat.)

longipennis Loew. Ibid., ii, 57. (C.W.J.)

marginatus Aldrich. Kans. Univ. Quart., ii, I7.

melanocerus Loew. Mon. N. A. Dipt., ii, 33 o.

palæstricus Loew. Ibid., ii, 328. (C.W.J.)

præustus Loew. Ibid., ii, 68. (C.W.J.)

quadrilamellatus Loew. Ibid., ii, 33I. (C.W.J.)

ramifer Loew. Ibid., ii, 52. (C.W.J.)

setifer Loew. Ibid., ii, 30. (C.WV.J.)

variabilis Loew. Ibid., ii, 50.

vittatus Loew. Ibid., ii, 55 .

Gymnopternus Loew.

barbatulus Loew. Mon. N. A. Dipt., ii, 82. (S.W.11.)

crassicauda Loew. Ibid., ii, 95.

flavus Loew. Ibid., ii, So.

lævigatus Loew. Ibid., ii, 87 .

opacus Loew. Ibid., ii, 93.

ventralis Loew. Ibid., ii, 97.

sp.

Pelastoneurus Loew.

lætus Loew. Mon. N. A. Dipt., ii, Io6.

lamellatus Loew. Ibid., ii, 338 .

vagans Loew. Ibid., ii, I07. (C.W.J.) 
Family EMPIDID E.

Drapetis Meigen.

gilvipes Loew. Trans. Am. Ent. Soc., xxviii, 2r2. (C.W.J.) spectabilis Melander. Ibid., xxviii, $2 \mathrm{I} 2$.

Platypalpus Macquart.

æqualis Loew. Trans. Am. Ent. Soc., xxviii, 222.

Tachydromia Meigen.

pusilla Loew. Trans. Am. Ent. Soc., xxviii, 229.

\section{Hemerodromia Meigen.}

defecta Loew. Trans. Am. Ent. Soc., xxviii, 235. (C. W. J.) empiformis Say. Ibid., xxviii, 236.

\section{Litanomyia Melander.}

elongata Melander. Trans. Am. Ent. Soc., xxviii, 232.

Clinocera Meigen.

lineata Loew. Cent., ii, 250. (C.W.J.)

sp.

Syneches Walker.

rufus Loew. Trans. Am. Ent. Soc., xxviii, 253.

simplex Walker. Ibid., xxviii, 254.

Syndyas Loew.

polita Loew. Trans. Am. Ent. Soc., xxviii, 254.

Hybos Meigen.

electus Melander. Trans. Am. Ent. Soc., xxviii, 247. (C.W.J.)

slossonæ Coquillett. Ibid., xxviii, 247.

triplex Walker. Ibid., xxviii, 248.

\section{Leptopeza Macquart.}

compta Coquillett. Trans. Am. Ent. Soc., xxviii, 258. (C.W.J.)

\section{Empis Linneus.}

distans Loew. Trans. An. Ent. Soc., xxviii, 295.

otiosa Coquillett. Ibid., xxviii, 302. (A.B.C.)

pœciloptera Loew. Ibid., xxviii, 298. (C.W.J.)

sordida Loew. Cent., i, 29. (H.L.J.)

spectabilis Loew. Trans. Am. Ent. Soc, xxviii, 3I I.

tridentata Coquillett. Ibid., xxviii, 301. 
Hilara Meigen.

leucoptera Loew. Trans. Am. Ent. Soc., xxviii, 266.

mutabilis Loew. Ibid., xxviii, 265. (C.IV.J.)

trivittata Loew. Ibid., xxviii, 265. (C.IV.J.)

umbrosa loew. Ibid., xxviii, 264.

velutina Loew. Ibid., xxviii, 264. (C.W.J.)

Rhamphomyia Meigen.

angustipennis Loew. Cent., i, 55.

aperta Loew. Ibid., ii, 27. (H.L.J.)

brevis Loew. Proc. L. S. Nat. Mus., xviii, 4I4.

fumosa Loew. Cent., i, 39.

gilvipes Loew. Ibid., i, 48 . (C.W.J.)

irregularis Loew. Ibid., $\mathrm{v}, 60$.

longicauda Loew. Proc. U. S. Nat. Mus., xviii, 4 II.

manza Coquillett. Ibid., xviii, +15-427.

mutabilis Loew. Ibid., xviii, 4I I.

nana Loew. Ibid., xviii, 4I4.

pulla Loew. Ibid., xriii, 4II-4I2. (Ald. Cat.)

pusio Loew. Ibid., xviii, $4 \mathrm{I} 4$.

scolopacea Say. Compl. Writ., ii, 38. (C.W.J.)

umbilicata Loew. Cent., i, 65. (C.W.J.)

\section{Family LONCHOPTERIDE.}

Lonchoptera Meigen.

lutea Panzer. Fann. Germ., cviii, 20.

riparia Neigen. Syst. Besch1r., ir, IoS.

Family PHORIDA.

Paraspinophora Malloch.

multiseriata Aldrich. Trans. Am. Ent. Soc., xxix, 345 (C.MV.J.)

Dohrniphora Dahlbom.

concinna Meigen. nitidifrons Brues. Trans. Am. Ent. Soc., xix, 347.

Chætoneurophora. Malloch.

spinipes Coquillett. Can. Ent., xxvii, 105. 


\section{Aphiochæta Brues.}

approximata Malloch. Proc. U. S. Nat. Mus., 43, 483.

epeiræ Brues. Trans. Am. Ent. Soc., xxix, 358.

nigriceps Loew. Cent., vii, 99. (G.A.C.)

rufipes Meigen. Trans. Am. Ent. Sac., xxix, 368: Rept. Conn. Agr. Expt. Sta., I909-I0, 693.

\section{Phora Latreille.}

Trineura Meigen.

aterrima Fabricius. Trans. Am. Ent. Soc., xxix, 378. (C.W.J.)

\section{Conocera Meigen.}

atra Meigen. Trans. Am. Ent. Soc., xxix, 380.

\section{Family PIPUNCULIDA.}

Chalarus Walker.

spurius Fallen. Trans. Am. Ent. Soc., xxxvi, 274.

Pipunculus Latreille.

atlanticus Hough. Trans. Am. Ent. Soc., xxxvi, 294. (C.IV.J.)

cingulatus Loew. Ibid., xxxvi, 299.

minor Cresson. Ibid., xxxvi, 293.

nitidiventris Loew. Proc. Bost. Soc. Nat Hist., xxix, 82.

pallipes Johnson. Ent. News, xiv, I07. (C.W.J.)

similis Hough. Trans. Am. Ent. Soc., xxxvi, 315. (C.W.J.)

subvirescens Loew. Proc. Bost. Soc. Nat. Hist., xxix, 84 .

\section{Family SYRPHIDE.}

\section{Microdon Meigen.}

cothurnatus Bigot. Syn. N. A. Syrph., 8. (C.W.J.)

fuscipennis Macquart. Ibid., 4. (C.W.J.)

globosus Fabricius. Ibid., 4.

megalogaster Snow. Kans. Univ. Quart., i, 34. (C.W.J.)

ruficrus Williston. Syn. N. A. Syrph. 7. (Ald. Cat.)

tristis Loew. Ibid., 6. (A.B.C.)

Chrysotoxum Meigen.

derivatum Walker. Syn. N. A. Syrph., i6. (C.W.J.)

laterale Loew. Ibid., I4.

pubescens Loew. Ibid., I5. (A.B.C.) 
Chrysogaster Loew.

nigripes Loew. Syn. N. A. Syrph., 33.

nitida Wiedemann. Ibid., 35 .

pictipennis Loew. Ibid., 37.

pulchella Williston. Ibid., 35.

Pipiza Falleri.

albipilosa Williston. Syn. N. A. Syrph., 2s

calcarata Loew. Ibid., 24. (A.B.C.)

femoralis Loew. Ibid., 26. (C.W.J.)

modesta Loew. Ibid., 24.

pulchella Williston. Syn. N. A. Syrph., 29. (Ald. Cat.)

radicum Walsh and Riley. pistica. Ibid., 29. (Ald. Cat.)

Paragus Latreille.

bicolor Fabricius. Syn. N. A. Syrph., i8.

tibialis Fallen. Ibid., I9.

Chilosia Meigen.

cyanescens Loew. Syn. N. A. Syrph., 42. (Ald. Cat.)

pallipes Loew. Ibid., 4I.

tristis Loew. Ibid., 293.

Myiolepta Newman.

nigra Loew. Cent. x, 52. (C.W.J.)

Baccha Fabricius.

cognata Loew. Syn. N. A. Syrph., iz2.

fascipennis Wiedemann. aurinota Harris. Ibid., I20.

Ocyptamus Macquart.

fuscipennis Say. Baccha. Syn. N. A. Syrph., irg. (Ald Cat.)

Pyrophæna Schiner.

granditarsus Fœrster. ocymi. Syn. N. A. Syrph. 55. (C.W.J.)

Platychirus St. Fargeau and Serville.

hyperboreus Stæeger. Syn. N. A. Syrph., 57.

peltatus Meigen. Ibid., 58. (C.IV.J.)

quadratus Say. Ibid., 57.

Melanostoma Schiner.

mellinum Linnæus. Syn. N. A. Syrph., 49.

obscurum Say. Ibid., 48. 
Didea Macquart.

fasciata Macquart. Syn. N. A. Syrph., 89. (A.B.C.)

Syrphus Fabricius.

amalopis Osten Sacken. Syn. N. A. Syrph., 69. (S.W.W.) americanus IViedmann. Ibid., $\mathrm{S}_{2}$.

arcuatus Fallen. Ibid., 68.

grossulariæ Meigen. lesucurii. Syn. N. A. Syrph., 80.

latifasciatus Macquart. abbreviatus Zetterstedt. Ibid., 8I.

perplexus Osburn. Jour. N. Y. Ent. Soc., xviii, 155.

ribesii Linneus. Syn. N. A. Syrph., 77.

torvus Osten Sacken. Ibid., 79.

xanthostomus Villiston. Ibid., 86

Allograpta Osten Sacken.

obliqua Say. Syn. N. A. Syrph., 96.

\section{Xanthogramma Schiner.}

æqualis Loew. Syn. N. A. Syrph., 95. (A.B.C.)

emarginata Say. Ibid., 93. (A.B.C.)

felix Osten Sacken. Ibid., 9г. (S.W.IV.)

flavipes Loew. Ibid., 94.

Mesogramma Loew.

geminata Say. Mesograpta. Syn. N. A. Syrph., 102.

marginata Say. Mesograpta. Ibid., Ioo.

polita Say. Mesograpta. Ibid., 98.

\section{Sphærophoria St. Fargeau and Serville.}

cylindriça Say. Syn. N. A. Syrph., I05.

Sphegina Meigen.

campanulata Robertson. Can. Ent., xxxiii, 284. (C.W.J.)

keeniana Williston. Syn. N. A. Syrph., Ir3. (C.W.J.)

lobata Loew. Ibid., I I5. (C.IV.J.)

rufiventris Loew. Ibid., I I 4. (H.W.W.)

Neoascia Williston.

distincta Williston. Syn. N. A. Syrph., I 2. (C.IV.J.)

globosa Walker. Ibid., I I .

Rhingia Scopoli.

nasica Say. Syn. N. A. Syrph., 130. 
No. 3r.] INSECTS OF CONNECTICUT: DIPTERA.

Brachyopa Meigen.

media Williston. Syn. N. A. Syrph., I32.

Volucella Geoffroy.

evecta Walker. Syn. N. A. Syrph., I36. (A.B.C.)

vesiculosa Fabricius. Ibid., I4I. (Ald. Cat.)

Sericomyia Meigen.

chrysotoxoides Macquart. Syn. $\mathrm{X}$. A. Syrph., I57.

militaris Walker. Ibid., I55. (A.B.C.)

Eristalis Latreille.

æneus Scopoli. Syn. N. A. Syrph., I6r.

arbustorum Linnæus. Jon1. N. Y. Ent. Soc., xxiii, I39.

bastardii Nacquart. Syn. N. A. Syrph., 168.

brousi Williston. Ibid., 165.

compactus Walker. Ibid., I69. (S.W.W.)

dimidiatus Weidemann. Ibid., I62.

flavipes IValker. Ibid., I68.

saxorum IViedemann. Ibid., I63.

tenax Linnæus. Ibid., I60.

transversus IVicdemann. Ibid., I70.

Tropidia Meigen.

quadrata Say. Syn. N. A. Syrph., 207.

Helophilus Meigen.

chrysostomus Wiedemann. Syn. N. A. Syrplı, Igo.

conostoma Williston. Ibid., I93.

distinctus Williston. Ibid., I92.

hamatus Loew. Ibid., I95.

integer Loew. Ibid., I95.

lætus Loew. Ibid., I89. (Ald. Cat.)

latifrons Loew. Ibid., I88. (S.W.W.)

similis Macquart. Ibid., I89.

Mallota Meigen.

cimbiciformis Fallen. Syn. N. A. Syrph., 202.

posticata Fabricius. Ibid., 2OI. (A.B.C.)

Triodonta Williston.

curvipes WViedemann. Syn. N. A., Syrph., 206. 


\section{Pterallastes Loew.}

thoracicus Loew. Cent., iv, 8o. (A.B.C.)

Teuchocnemis Osten Sacken.

lituratus Loew. Syn. N. A. Syrph., 200. (S.W.W.)

Syritta St. Fargeau and Serville.

pipiens Linnæus. Syn. N. A. Syrph., 240.

Xylota Meigen.*

angustipennis Loew. Syn. N. A. Syrph., 231.

bicolor Loew. Ibid., 229. (A.B.C.)

chalybea Wiedemann. Ibid., 233.

ejuncida Say. Ibid., 229.

fraudulosa Loew. Ibid., 230. (A.B.C.)

obscura Loew. Ibid., 233. (C.W.J.)

Ferdinandea Rondani.

dives Osten Sacken. Chrysochlamys. West. Dipt., 340; Syn. N. A. Syrph., 24I. (C.W.J.)

Brachypalpus Macquart.

frontosus Loew. Syn. N. A. Syrph., 221. (A.B.C.)

\section{Criorhina Meigen.}

analis Macquart. Syn. N. A. Syrph., 2r4. (A.B.C.)

badia Walker. intersistens Walker. Ibid., $2 \mathrm{I} 2$.

decora Macquart. Somula. Ibid., 216. (A.B.C.)

umbratilis Williston. Cynorhina. Ibid., 212.

Milesia Latreille.

virginiensis Drury. ornata. Syn. N. A. Syrph., 255.

Spilomyia Meigen.

fusca Loew. Syn. N. A. Syrph., 246.

hamifera Loew. Ibid., 247. (A.B.C.)

longicornis Loew. Ibid., 245. (A.B.C.)

quadrifasciata Say. Ibid., 248.

\section{Sphecomyia.}

vittata Wiedemann. Syn. N. A. Syrph., 257. (C.W.J.)

Temnostoma St. Fargeau and Serville.

alternans Loew. Syn. N. A. Syrph., 252.

bombylans Fabricius. Ibid., 250. (Ald. Cat.)

* See also appendix. 
Ceriodes Rondani.

Ceria Fabricius.

abbreviata Loew. Syn. N. A. Syrph., 26r. (S.W.W.)

Eumerus Meigen.

strigatus Fallen. Lunate onion fly. Rept. N. Y. State Ent., 27, II 9 .

\section{Family CONOPID Æ.}

Conops Linnæus.

brachyrhynchus Macquart. Trans. Conn. Acad. Sci., iv, 34I. sylvosus Williston. Ibid., iv, 329.

xanthopareus Williston. Insect Book, pl, xxi, 37.

Physocephala Schiner.

sagittaria Say. Trans. Conn. Acad. Sci., vi, 39I. (C.W.J.), tibialis Say. Insect Book, pl. xv, I.

\section{Zodion Latreille.}

fulvifrons Say. Insect Book, pl. xxi, 35 .

\section{Stylogaster Macquart.}

biannulata Say. Myopa. Compl. IVrit., ii, 72. (S.W.W.) neglecta IVilliston. Trans. Con11. Acad. Sci., vi, 9I. (S.W.W.)

Dalmannia Robineat1-Desvoidy. nigriceps Loew. Trans. Conn. Acad. Sci., vi, 94. (S.W.W.)

Oncomyia Robineau-Desvoidy.

abbreviata Loew. Trans. Conn. Acad. Sci., vi, 97. baroni Williston. Ibid., vi, 97. (C.W.J.) loraria.Loew. Ibid., vi, 98 . modesta Williston var. melanopoda Williston. Ibid., vi, 96, 393.

\section{Myopa Fabricius.}

clausa Loew. Trans. Conn. Acad. Sci., vi, 385.

vesiculosa Say. Compl. Writ., ii, 72.

vicaria Walker. List, iii, 679. (H.L.J.) 
Fanily ESTRIDÆ.

Gastrophilus Leach. equi Clark. Horse bot fly. Bur. Ent., Bull. 5, 76.

Estrus Linnæus.

ovis Linnæus. Sheep bot fly. Bur. Ent., Bull. 5, 102.

Hypoderma Clark.

bovis DeGeer. Ox warble or bot $\mathrm{fl}_{2}$. . Bur. Ent., Bull. 5, 95. lineata DeVilliers. Ent. News, iv, 299.

Cuterebra Clark.

buccata Fabricius. Mon. Estriden, 247. (C.W.J.)

cuniculi Clark. Bur. Ent., Bull. 5, Io8.

\section{Family TACHINIDÆ.}

Gymnoclytia Brater and Bergenstamm.

immaculata Macquart. Cistogaster. Revis. Tachinid., 42. occidua Walker. Cistogaster. Smith. Misc. Coll., li, 127.

Gymnosoma Meigen.

fulginosa Desvoidy. Insect Book, pl. xxii, 2.

Phorantha Rondani.

occidentis Walker. Revis. Tachinid., 44.

Alophora Desvoidy.

ænoventris Williston. Revis. Tachinid., 45, 47.

fumosa Coquillett. Ibid., 46. (C.W.J.)

Trichopoda Latreille.

pennipes Fabricius. Insect Book, pl. xv, 25.

plumipes Fabricius. Revis. Tachinid., 48. (A.B.C.)

Myiophasia Brauer and Bergenstamm.

ænea Wiedemann. Revis. Tachinid., 50. (A.B.C.)

Cryptomeigenia Brauer and Bergenstamm.

theutis Walker. Revis. Tachinid., 52.

Dichætoneura Johnson.

leucoptera Johnson. Psyche, xiv, 9.

Polychætoneura Walton.

elyii Walton. Proc. Wash. Ent. Soc., vi, 9I. 
No. 3I.] INSECTS OF CONNECTICUT: DIPTERA.

Actia Desvoidy.

pilipennis Fallen. Revis. Tachinid., 59.

Chætophleps Coquillett.

setosa Coquillett. Revis. Tachinid., 59. (C.IV.J.)

Celatoria Coquillett.

spinosa Coquillett. Revis. Tachinid., 6o. (C.IV.J.)

Hypostena Meigen.

Tachinophyto Tower.

barbata Coquillett. Revis. Tachinid., 62. (C.IV.J.)

dunningii Coquillett. Ibid., 60.

flaveola Coquillett. Ibid., 6r .

floridensis Townsend. Ibid., 62.

variabilis Coquillett. Ibid., 62.

Macquartia Desvoidy.

pristis Walker. Revis. Tachinid., 64.

Polidea Macquart.

areos Walker. Revis. Tachinid., 64.

Leskia Desvoidy.

analis Say. Revis. Tachinid., 67. (A.B.C.)

thecata Coquillett. Ibid., 67. (A.B.C.)

Leskiomima Brauer and Bergenstamm.

tenera Viedemann. Revis. Tachinid., 67.

Leucostoma Meigen.

senilis Townsend. Revis. Tachinid., 69. (C.IV.J.)

Hyalomyodes Townsend.

triangulifera Loew. Revis. Tachinid., 7o.

Estrophasia Brauer and Bergenstamm.

signifera Van der Wulp. Biol. Dipt., ii, I67. (H.L.J.)

Xanthomelanodes Townsend.

arcuata Say. Xanthomclana. Revis. Tachinid., 75. (H.L.V.)

Hemyda Desroidy.

aurata Desvoidy. Revis. Tachinid., 73.

Eutrixa Coquillett.

exile Coquillett. Reris. Tachinid., 72. (C.M.J.) 
Epigrymyia Townsend.

polita Townsend. Trans. Am. Ent. Soc., xviii, 376. (C.W.J.)

Siphona Meigen.

brevirostris Coquillett. Revis. Tachinid., 76. (C.W.J.)

geniculata DeGeer. Ibid., 75 .

Cyrtophlœba Rondani.

horrida Coquillett. Revis. Tachinid., 78.

Paraplagia Brauer and Bergenstamm.

spinosula Bigot. Revis. Tachinid., 77. (W.E.B.)

Senotainia Macquart.

trilineata Van der Wulp. Revis. Tachinid., 8I.

Belvosia Desvoidy.

bifasciata Fabricius. Smith Misc. Coll., li, I04. (C.W.J.)

Goniomima Townsend.

unifasciata Desvoidy. Revis. Tachinid., 84. (A.B.C.)

Melanophrys Williston.

insolita Walker. Revis. Tachinid., 85. (C.IV.J.)

Ocyptera Latreille.

carolinæ Desvoidy. Insect Book., pl. xv, 29.

dosiades Walker. Revis. Tachinid., 86.

Linnæmyia Desvoidy.

hæmorrhoidalis Fallen. picta Meigen. Revis. Tachinid., 87.

Bonnetia Desvoidy.

compta Fallen. Revis. Tachinid., 87.

Ernestia Desvoidy.

Panzeria.

radicum Fabricius. Insect Book, pl. xv, 28.

ruficauda Brater. Hystricia. aldrichii Townsend. Smith. Misc. Coll., li, ı09. (H.E.S.)

Macromeigenia Brater and Bergenstamm. chrysoprocta Wiedemann. Revis. Tachinid., S9. (A.B.C.)

Gymnochæta Desvoidy.

alcedo Loew. Cent., viii, 6r.

fuficornis Williston. Trans. Am. Ent. Soc., xiii, 302. (C.W.J.)

Exoristoides Coquillett.

slossonæ Coquilett. Revis. Tachinid., 90. (H.E.S.) 


\section{Exorista Meigen.}

boarmiæ Coquillett. Revis. Tachinid., 95. (H.E.S.) confinis Fallen. Ibid., 97.

eudryæ Townsend. Ibid., ıoo. (C.IV.J.)

futilis Osten Sacken. Ibid., 98. (S.IV.W.)

petiolata Coquillett. Ibid., 98.

cheloniæ Rondani. Ibid., 92. (H.E.S.)

vulgaris Fallen. Ibid., 93. (H.E.S.)

pyste Walker. Ibid., 93.

Compsilura Bouché.

concinnata Meigen. U. S. Dept. Agr., Bull. 204, fig. 2.

Phorocera Desvoidy.

claripennis Macquart. Insect Book, pl. xxii, 6.

erecta Coquillett. Proc. U. S. Nat. Mus., xxv, II2.

Neopales Coquillett.

einaris Smith. Proc. Wash. Ent. Soc., xiv, II9.

slossonæ Townsend. Smith. Misc. Coll., li, IoS. (H.E.S.)

leucaniæ Coquillett. Revis. Tachinid., I04. (C.W.J.)

tortricis Coquillett. Ibid., I03. (A.B.C.)

\section{Doryphorophaga Townsend.}

doryphoræ Riley. Revis. Tachinid., I04.

Frontina Meigen.

aberrans Townsend. Ent. News., xxvii, 2I7.

aletiæ Riley. Can. Ent., xi, I62.

ancilla Walker. Revis. Tachinid., Io6.

frenchii Williston. Ibid., I07.

Sturmia Desvoidy.

albifrons Walker. Revis. Tachinid., Iog.

nidicola Townsend. Zygobothria. Bur. Ent., Bull. 9I, 289.

nigrita Townsend. Trans. Am. Ent. Soc., xviii, $35^{8}$.

phyciodis Coquillett. Revis. Tachinid., I09. (C.IV.J.)

Masicera Nacquart.

myoidea Desvoidy. Revis. Tachinid., II4.

rutila Meigen. Ibid., I I4. (H.L.V.)

Acemyia Desvoidy.

dentata Coquillett. Revis. Tachinid., i I6. (D.IV.C.) 
Tachina Meigen.*

mella Walker. Revis. Tachinid., II $\mathbf{I Q}$

robusta Townsend. Ibid., II9.

rustica Fallen. Ibid., II9.

Neofischeria Townsend.

flava Townsend. Taxonomy, 75. (C.W.J.)

Blepharipeza Macquart.

adusta Loew. Insect Book, pl. xxii, I8, 22. (A.B.C.)

leucophrys Wiedemann. Revis. Tachinid., I24.

Winthemia Desvoidy.

quadripustulata Fabricius. Insect Book, pl. xxii, 4.

Paradidyma Brauer and Bergenstamm.

singularis Townsend. Revis. Tachinid., ז26.

Metachæta Coquillett.

helymus Walker. Revis. Tachinid., I26.

Phorichæta Rondani.

sequax Williston. Revis. Tachinid,, I26. (B.H.W.)

Metopia Meigen.

lateralis Macquart. Revis. Tachinid., I27. (C.IV.J.)

leucocephala Rossi. Can. Ent., xliii, 3I4; xliv, I7O.

Araba Desvoidy.

tergata Coquillett. Jour. N. Y. Ent. Soc., iii, io3. (G.D.)

Opsidia Coquillett.

gonoides Coquillett. Revis. Tachinid., I28. (J.A.H.)

Hilarella Rondani.

fulvicornis Coquillett. Revis. Tachinid., I28. (H.L.V.) polita Townsend. Ibid., I29.

Brachycoma Rondani.

apicalis Coquillett. Revis. Tachinid., I3I. (D.W.C.)

Gonia Meigen.

capitata DeGeer. Insect Book, pl. xv, 26, $3 \mathrm{I}$.

distincta H. E. Smith. Psyche, xxii, 99.

senilis Williston. Can. Ent., xix, Io.

Spallanzania I Jesvoidy.

bucephala Meigen. hebes Rondani. Revis. Tachinid., I35. (C.W.J.)

hesperidarum Williston. Ibid., I35.

\footnotetext{
* See also appendix.
} 
Gædiopsis Braner and Bergenstamm.

ocellaris Coquillett. Proc. U. S. Nat. Mus., xxv, II8. (A.B.C.)

Microphthalma Macquart.

disjuncta Wiedemann. Revis. Tachinid., I38.

Tricophora Macquart.

ruficauda Tan der Wulp. Revis. Tachinid., I39.

Cuphocera Macquart.

fucata Van der IVulp. Revis. Tachinid., I40.

prompta Zetterstedt. Ibid., I 4 I.

Peleteria Desvoidy.

robusta Wiedemann. Revis. Tachinid., IfI.

prompta Zetterstedt. Ibid., I fI.

Archytas Jænnicke.

analis Fabricius. Insect Book, pl. xxii, Io.

aterrima Desvoidy. Ibid., pl. xv, 33 .

hystrix Fabricius. Revis. Tachinid., I42.

lateralis Macquart. Insect Book, pl. xxii, I4.

Echinomyia Dumeril.

algens Wiedemann. Insect Book, pl. xv, 27.

decisa Walker. Revis. Tachinid., I 43 . (S.W.W.)

florum Walker. Ibid., I 44. (H.L.J.)

Epalpus Rondani.

signifera Walker. Insect Book., p1. xxii, I2.

Bombyliomyia Brater and Bergenstamm.

abrupta Wiedemann. Insect Book, pl. xv, 22.

Jurinia Desvoidy.

adusta Van der Wulp. metallica. Insect Book, pl. xv, 27;

pl. xxii, $16,27$.

Family DEXIID E.

Myiocera Desvoidy.

simplex Bigot? Annales, I888, 266.

Melanophora Meigen.

roralis Linneus. Syst. Beschr., iv, 284. 
Ptilodexia Brauer and Bergenstamm.

harpasa Walker. tibialis. Myiocera. Myodaires, 306.

Thelaira Desvoidy.

leucozona Panzer. Insect Book., pl. xxii, 24.

Theresia Desvoidy.

tandrec Desvoidy. Myodaires, 326.

\section{Family SARCOPHAGIDE.}

Sarcophaga Meigen.

bullata Parker. Can. Ent., xlviii, 359.

cimbicis Townsend. Ibid., xxiv, I 26.

communis Parker. Proc. Bos. Soc. Nat. Hist., 35, 55.

fernaldi Parker. Ibid., 35, 72.

hæmorrhoidalis Fallen. Sarcoph. N. A., I89.

helicis Townsend. Ibid., I58.

latisetosa Parker. Proc. Bos. Soc. Nat. Hist., 35, 63.

latisterna Parker. Ibid., 35, 67. (C.W.J.)

peniculata Parker. Ibid., 35, 58.

quadrisetosa Coquillett. Helicobia. Ent. News, xii, I7.

scoparia Pandellé. Sarcoph. N. A., 2 I4.

scoparia var. nearctica Parker. Can. Ent., xlviii, 422.

sinuata Meigen. Sarcoph. N. A., 67.

tuberosa Pandelé var. sarracenioides Aldrich. Ibid., 227.

Sarcofahrtia Parker.

atlantica Parker. Ent. News. xxxiii, 203.

ravinia Parker. Psyche, xxiii, I3I.

Family MUSCID正.

Pollenia Desvoidy.

rudis Fabricius. Cluster fly. Insect Book, pl. xxii, 28.

Chrysomyia Desvoidy.

macellaria Fabricius. Screw worm fly. La. Agr. Expt. Sta., Bu11. 2, Sec. ser.

Cynomyia 1)esvoidy.

cadaverina Desvoidy. Insect Book, pl. xv, 2 I. 
Calliphora Desvoidy.

erythrocephala Meigen. Common blow fly. Insect Book, pl. xv, I6.

vomitoria Linnæus. Blue bottle fly. Minn. Agr. Expt. Sta., Bull. 48, I88.

\section{Protocalliphora Hough.}

azurea Fallen. Zool. Bull., ii, 289. (C:W.J.)

\section{Lucilia Desvoidy.}

cæsar Linnæus. Insect Book, p1. xv, 20.

sericata Meigen. Mutsca. Syst. Beschr., v, 53.

sylvarum Meigen. Ibid., v, 53.

Phormia Desvoidy.

regina Meigen. Musca. Syst. Beschr., v, 58 .

terræ-novæ Desvoidy. Zool. Bull., ii, 289.

Orthellia Desvoidy.

Pseudopyrellia Girschner.

cornicina Fabricius. Syst. Beschr., v, 57.

Pyrellia Desvoidy.

serena Meigen. cyanicolor Zetterstedt. Dipt. Scand., iv, I323.

Morellia Desvoidy.

micans Macquart. Insect Book., pl. xv, II.

Musca Linnæus.

domestica Linnæus. House fly. Insect Book, pl. xv, 6 .

Graphomyia Desvoidy.

maculata Scopoli. Syst. Beschr., v, 78 .

Stomoxys Geoffroy.

calcitrans Linnæus. Biting house fly. Proc. Bost. Soc. Nat.

Hist., xvi, г 36.

Hæmatobia St. Fargeau and Serville.

irritans Linnaus. scrrata. comicola. Horn fly. Rept. N. Y.

State Ent., No. 5, 220.

Myiospila Rondani.

meditabunda Fabricius. Syst. Beschr., v, 79.

Muscina Desvoidy.

assimilis Fallen. Syst. Beschr., v, 76. (H.L.V.)

stabulans Fallen. Stable fly. Insect Book, pl. xxii, 33. 


\section{Family ANTHOMYIDE.}

Hydrotæa Desvoidy.

armipes Fallen. Fauna Aust., i, 6г6. (H.L.V.)

Ophyra Desvoidy.

leucostoma Wiedemann. Syst. Beschr., v, I6o.

Fannia Desvoidy.

Homalomyia Bouché.

canalicularis Linnæus, Fauna Aust., i, 654. (C.W.J.). scalaris Fabricius. Syst. Beschr., v, I4I.

Hyetodesia Rondani.

deleta Stein. Berl. Ent. Zeitsch., xlii, I78. (C.W.J.)

lucorum Fallen. Syst. Beschr., v, 85. (H.L.V.)

Spilogaster Macquart.

uniseta Stein. Berl. Ent. Zeitsch., vlii, I92. (H.L.J.)

urbana Meigen. Ibid., xlii, 196.

\section{Limnophora Desvoidy.}

diaphana Wiedemann. Syst. Beschr., v, I89.

narona Walker. Cyrtoncurina. Berl. Ent. Zeitsch., xvii, 203.

Anthomyia Meigen.

albicincta Fallen. Syst. Beschr., v, г6 .

pluvialis Linnæus. Ibid., v, I63. (H.L.J.)

pratincola Panzer. Ibid., v, I63.

radicum Linnæus. Rept. N. Y. State Ent., No. I, I9I.

Hylemyia Desvoidy.

alcathoe Walker. List., iv, 937. (C.W.J.)

johnsoni Stein. Berl. Ent. Zeitschr., xvii, 215, 285. (C.W.J.)

Phorbia Desvoidy.

brassicæ Bouché. Cabbage maggot. Cornell Agr. Expt. Sta.,

Bu1l. 78 ; Rept. Conn. Agr. Expt. Sta., 19I4, I42.

ceparum Meigen. Onion maggot. Rept. Conn. Agr. Expt.

Sta., I9I I, 286.

cepetorum Meade. Ent. Month1. Mag., I882, 2i8. (C.IV.J.) cinerella Fallen. Proc. Wash. Acad. Sci., ii, 583.

fusciceps Zetterstedt. cilicrura. Seed-corn maggot. Bur.

Ent., Bull. 43, 68. 
latipennis Zetterstedt. Dipt. Scand., iv, I509. (C.W.J.) rubivora Coquillett. Raspberry cane maggot. Rept. Conn.

Agr. Expt. Sta., I902, I67.

Pegomyia Desvoidy.

calyptrata Zetterstedt. Berl. Ent. Zeitschr., xlii, 237.

hyoscyami Panzer. ricina Lintner. Beet or spinach leaf-

miner. Ins. Life, vii, 379.

vittiger Zetterstedt. Ins. Lapp., 1838, 164, 697.

winthemi Meigen. latitarsis Zetterstedt. Fauna Aust., i, 635 . (C.IV.J.)

Dialyta Meigen.

flavitibia Johannsen. Trans. An. Ent. Soc., 1xii, 394.

Caricea Desvoidy.

albicornis Meigen. Fauna Aust., i, 664. (C.W.J.)

Cœnosia Meigen.

ausoba Walker. Berl. Ent. Zeitschr., xlii, 260.

calopyga Loew. Cent., x, 7I. (C.IV.J.)

flavipes Stein. Berl. Ent. Zeitschr., xlii, 268.

lata Walker. Dipt. Saund., 368.

nivea Loew. Cent., $x, 70$.

tibialis Stein. Berl. Ent. Zeitschr., xlii, 275. (C.W.J.)

Dexiopsis Pokorny.

lacteipennis Zetterstedt. Dipt. Scand., iv, I722.

Schœnomyza Halliday.

chrysostoma Loew. Cent., ix, 86.

dorsalis Loew. Cent., $\mathrm{x}, 73$.

Lispa Latreille.

albitarsis Stein. Berl. Ent. Zeitschr., xlii, 277. (C.W.J.)

uliginosa Fallen. Fauna Aust., i, 66r.

Fucellia Desvoidy.

marina Macquart. fucorum. Proc. Wash. Acad. Sci., ii, 453.

\section{Family SCATOPHAGID尼.}

\section{Cordylura Fallen.}

adusta Loew. Cent., iii, 4I. confusa Loew. Ibid., iii, 43. ( C.W.J.) gilvipes Loew. Ibid., iii, 49. (C.W.J.) munda Loew. Ibid., iii, 9I. 
pleuritica Loew. Ibid., iii, 42. (A.B.C.)

setosa Loew. Ibid., iii, 44.

slossonæ Coquillett. Jour. N. Y. Ent. Soc., vi, r64. (H.L.J.)

variabilis Loew. Zeitsch. f. Ges Naturwiss, I876, 326.

Mosina Desvoidy.

variapes Walker. List. Dipt., 4, Iо-6. (H.L.J.)

Scatophaga Meigen.

furcata Say. Compl. Writ., ii, 85 .

merdaria Fabricius. Dipt. Scand., v, I970. (C.W.J.)

stercoraria Linnæus. Insect Book, pl. xv, I9.

Family HETERONEURIDÆ.

Clusia Halliday.

lateralis Walker. spectabilis. Cent., iv. 92.

\section{Family HELOMYZID瓜.}

Helomyza Fallen.

quinquepunctata Say. Compl. Writ., ii, 86. (C.W.J.)

Scoliocentra Loew.

helvola Loew. Cent., ii, 8o.

Ecothea Haliday.

fenestralis Fallen. Syst. Beschr., vi, 56 .

Tephrochlamys Loew.

rufiventris Meigen. Syst. Beschr., vi, $5^{8}$.

Family BORBORIDÆ.

Leptocera Olivier.

Limosina Macquart.

limosa Meigen. Syst. Pieschr., vi, 207.

Borborus Meigen.

equinus Fallen. Proc. IVash. Acad. Sci., ii, 594.

Sphærocera Latreille.

subsultans Fabricius. Proc. Wash. Acad. Sci, ii, 596. 
Family DRYOMYZIDÆ.

Neuroctena Rondani.

anilis Fallen. Dryomyza pallida Day. Can. Ent., xiii, 89. (S.IV.W.)

Family SCIONIYZIDE.*

Sciomyza Fallen.

nana Fallen. Mon. N. A. Dipt., i, ro4. pubera Loew. Ibid., i, Io6. (H.L.V.)

Pœcilomyia Melander.

decora Loew. Sapromyza. Cent., v, 96.

Hedroneura Hendel.

rufa Panzer. lineata Day. Can. Ent., xiii, 88.

Tetanocera Dumeril.

arcuata Loew. Mlon. N. A. Dipt., i, ir5. (C.W.J.)

clara Loew. Insect Book, pl. xxii, 36. (A.B.C.)

combinata Loew. Can. Ent., xiii, 85 .

pallida Loew. Mon. N. A. Dipt., i, II 3 .

plebeia Loew. Ibid., i, I20.

plumosa Loew. Insect Book, pl. xv, I4.

saratogensis Fitch. Ins. N. Y., 68.

setosa Coquillett. Proc. U. S. Nat. Muss, xxiii, 6r5. (C.W.J.)

sparsa Loew. Mon. N. A. Dipt., i, i I7. (S.W.W.)

umbrarum Linneus. Can. Ent., xiii, 85.

valida Loew. Mon. N. A. Dipt., i, i Io. (A.B.C.)

Sepedon Latreille.

armipes Loew. Mon. N. A. Dipt., I, I26. (C.W.J.)

fuscipennis Loew. Insect Book, pl. xxii, 4I.

pusillus Loew. Mon. N. A. Dipt., i, I27.

Family SAPROMYZIDE.

Lonchæa Fallen.

polita Say. Comp1. Writ., ii, 37I. (A.B.C.)

rufitarsus Macquart. Dipt. Exot. (Suppl.), iv, 300.

Lauxania Latreille.

cylindriformis Fabricins. Proc. Wash. Acad. Sci., ii, 400.

gracilipes Loew. Cent., i, 84. (C.IV.J.)

obscura Loew. Ibid., i, $8_{4}$.

* See also appendix. 
Minettia Desvoidy.

annulata Melander. Psyche, xx, 73. (C.W.J.)

lupulina Fabricius. Proc. Wash. Acad. Sci., ii, 460.

quadrilineata Loew. Cent., i, 78 .

valida Walker. macula Loew. Ibid., x, 82. (C.W.J.)

Sapromyza Fallen.

compedita Loew. Cent., i, 76 .

disjuncta Johnson. Psyche, xxi, 22. (C.W.J.)

fraterna Loew. Cent., i, 77. (C.IV.J.)

houghii Coquillett. Can. Ent., xxx, 277.

notata Fallen. Psyche, xx, 68. (C.W.J.)

philadelphica Macquart. Dipt. Exot. (Suppl.), ii, 3, I9I.

Camptoprosopella Hendel.

vulgaris Fitch. Ins. N. Y., i, 300.

\section{Family ORTALIDE.}

\section{Pyrgota Wiedemann.}

chagnoni Johnson. Can. Ent., xxxii, 246. (A.B.C.)

undata Wiedemann. Insect Book, pl. xv, 4.

valida Harris. Ibid., pl. xv, 9.

\section{Amphicnephes Loew.}

pulla Wiedemann. pertusus. Mon. N. A. Dipt., iii, 84.

Rivellia Desvoidy.

conjuncta Loew. Mon. N. A. Dipt., iii, 88.

flavimanus Loew. Ibid., iii, 92.

pallida Loew. Ibid., iii, 95.

quadrifasciata Macquart. Ibid., iii, 90.

viridulans Desvoidy. Ibid., iii, 88.

Myrmecothea Hendel.

Myrmecomyia Desvoidy.

myrmecoides Loew. Mon. N. A. Dipt., iii, ıoo. (A.B.C.)

Tritoxa Loew.

flexa Wiedemann. Mon. N. A. Dipt., iii, IO2.

incurva Loew. Insect Book, pl. xxii, 40.

Camptoneura Macquart.

picta Fabricius. Insect Book, pl. xv, Io. 
Idana Loew.

marginata Say. Compl. IVrit., ii, 368. (A.B.C.)

Tephronota Loew.

narytia Walker. ruficeps. humilis. Mon. N. A. Dipt., iii, I23. (C.W.J.)

Melieria Desvoidy.

similis Loew. Mon. N. A. Dipt., iii, I27. (C.IV.J.)

Tetanops Fallen.

luridipennis Loev. Mon. N. A. Dipt., iii, I I9. (C.IV.J.)

Callopistria Loew.

annulipes Macquart. Mon. N. A. Dipt., iii, I 4I. (C.W.J.)

Pseudotephritis Jolnson.

corticalis Loew. Mon. N. A. Dipt., iii, I36. (C.IV.J.)

Chrysomyza Fallen.

demandata Fabricius. Fanna Aust., ii, 85 .

Euxesta Loew.

notata Wiedemann. Mon. $\mathrm{N}$. A. Dipt., iii, I56.

\section{Chætopsis Loew.}

ænea WViedemann. Compl. Nrit., ii, 368.

apicalis Johnson. Ent. News, xi, 326.

fulvifrons Macquart. Dipt. Exot. (Suppl.), v, I25. (C.IV.J.)

Seoptera Kirby.

vibrans Linnæus. Mon. N. A. Dipt., ii, I53.

Stenomyia Loew.

tenuis Loew. Mon. N. A. Dipt., iii, I73.

Eumetopia Macquart.

rufipes Macquart. Mon. N. A. Dipt., iii, I75.

Family TRYPETID王.

Straussia Desvoidy.

longipennis Wiedemann. Mon. N. A. Dipt., i, 65.

Stenopa Loew.

vulnerata Loew. Mon. N. A. Dipt., iii, 232. (Psyche.)

Acidia Desvoidy.

fratria Loew. Mon. N. A. Dipt., i, 67. (C.W.J.)

suavis Loew. Ibid., iii, 235. (C.IV.J.) 
Spilographa Loew.

electa Say. Compl. Writ., ii, 396. (Ald. Cat.)

atra Loew. Cent., ii, 74.

\section{CEdaspis Loew.}

polita Loew. Mon. N. A. Dipt., i, 77. (Ald. Cat.)

Rhagoletis Loew.

cingulata Loew. Trypeta. Cherry maggot. Cornell Agr. Expt. Sta., Bull. I72.

pomonella Walsh. Apple maggot. Railroad worm. Rept. Me. Agr. Expt. Sta., I889, I90; Rept. Conn. Agr. Expt. Sta., I909-IO, 593 .

\section{Eutreta Loew.}

sparsa Wiedemann. Compl. Writ., ii, 370.

\section{Eurosta Loew.}

comma Wiedemann. Mon. N. A. Dipt., i, 93.

conspurcata Doane. Jour. N. Y. Ent. Soc., vii, I86.

elsa Dæcke. Ent. News, xxi, 342.

latifrons Loew. Mon. N. A. Dipt., i, 89.

reticulata Snow. Kans. Univ. Quart., ii, I7o.

solidaginis Fitch. Non. N. A. Dipt., i, 82.

Aciura Desvoidy.

limata Coquillett. Jour. N. Y. Ent. Soc., vii, 263. (C.W.J.)

\section{Icterica Loew.}

seriata Loew. Mon. N. A. Dipt., i, 84.

Tephritis Latreille.

platyptera Loew. Mon. N. A. Dipt., iii, 306.

Neaspilota Osten Sacken.

albidipennis Loew. Cent., i, 73. (C.W.J.)

\section{Euaresta Loew.}

bella Loew. Mon. N. A. Dipt., ii, 88.

festiva Loew. Ibid., i, 86.

pura Loew. Ibid., iii, 320. (II.L.J.)

Family MICROPEZID Æ.

Tæniaptera Macquart.

antennipes Say. Compl. Writ., ii, 83 .

divaricata Cresson. Ent. News., xxv, 459. (C.W.J.) 
Calobata Meigen.

alesia Walker. List. iv, I048. (C.W.J.) univitta Walker. Ibid., iv, IO+9. (C.IV.J.)

\section{Family SEPSID E.}

Sepsis Fallen.

neocynipsea Melander. Wash. Agr. Expt. Sta., Bull. I43, 28. signifera Melander. curritibia Melander. Ibid., Bull. I43, 28. violacea Meigen. Ibid., Bull. I+3, 20.

\section{Nemopoda Desvoidy.}

cylindrica Fabricius. Syst. Beschr., v, 290.

Meroplius Rondani.

stercoraria Desvoidy. Nemopoda minuta. Myodaires, 745 .

Themira Desvoidy.

flavicoxa Melander. Wash. Agr. Expt. Sta., Bull. I 43,46 .

Piophila Fallen.

casei Linnæus. Cheese skipper. Rept. N. Y. State Ent., xii, 229.

Family PSILID无.

Loxocera Meigen.

cylindrica Say. Compl. Writ., ii, 84. (H.L.V.)

pectoralis Loew. Cent., viii, 6. (A.B.C.)

pleuritica Loew. Ibid., viii, 65. (C.IV.J.)

pleuritica var, obsoleta Johnson. Psyche, xxvii, I6.

Chyliza Fallen.

apicalis Loew. Cent., viii, 72. (C.W.J.)

notata Loew. annulipes Macquart. Ibid., ix, 99. (A.B.C.)

Psila Meigen.

bivittata Loew. Cent., viii, 67. (Ald. Cat.)

collaris Loew. Ibid., viii, 68. (Ald. Cat.)

Family DIOPSID E.

Sphyracephala Say.

brevicornis Say. Compl. Writ., i, ı 6. (C.W.J.) 
Family EPHYDRIDA.

Dichæta Meigen.

caudata Fallen. Mon. N. A. Dịpt., i, I33.

Notiphila Fallen.

bella Loew. Mon. N. A. Dipt., i, I35. (C.W.J.)

bispinosa Cresson. Trans. Am. Ent. Soc., xliii, 58.

scalaris Loew. Mon. N. A. Dipt., i, I34.

olivacea Cresson. Trans. Am. Ent. Soc., xliii, 52.

Gastrops Williston.

nebulosa Coquillett. Can. Ent. xxxii, 34.

Typopsilopa Cresson.

atra Loew. Mon. N. A. Dipt., i, I42.

Hydrellia Desvoidy.

formosa Loew. Cent., i, 9ł.

Psilopa Fallen.

atrimanus Loew. Zeitsch. f. Ges. Naturwiss., I878, 197.

flavida Coquillett. Can. Ent., xxxii, 33. (C.W.J.)

Ilythea Haliday.

spilota Curtis. Brit. Ent., 4I3. (C.W.J.)

Ochthera Latreille.

mantis DeGeer. Ent. News., vii, I23. (W.M.IV.)

Parydra Stenhammar.

bituberculata Loew. Mon. N. A. Dipt., i, I65.

breviceps Loew. Ibid., i, I67.

imitans Loew. Zeitsch. f. Ges., Naturwiss., I878, 201.

Mosillus Latreille.

tibialis Cresson. Ent. News, xxvii, I49.

Ephydra Fallen.

atrovirens Loew. Mon. N. A. Dipt., i, I69.

subopaca Loew. Cent., v, 99.

Cirrula Cresson.

gigantea Cresson. Ent. News, xxvi, 7I. (C.IV.J.)

Scatella Desvoidy.

lugens Loew. Mon. N. A. Dipt., i, I7I.

stagnalis Fallen. Proc. Wash. Acad. Sci., ii, 462.

Dimecænia Cresson.

spinosa Loew. Cent. v, Ioo. 
Family CHLOROPIDE.

\section{Meromyza Meigen.}

americana Fitch. Greater wheat stem maggot. Bur. Ent., Bull.

42. New ser., 43.

flavipalpis Malloch. Can. Ent., xlvi, iı7. (C.W.J.)

Chlorops Meigen.

abdominalis Coquillett. Proc. Acad. Nat. Sci. Phila., I895, 318. (C.IV.J.)

crocota Loew. Cent., iii, 80. (C.IV.J.)

melanocera Loew. Ibid., iii, 9 I.

rufescens Coquillett. Can. Ent., xlii, 45. (C.W.J.)

Chloropisca Loew.

glabra Meigen. assimilis Nacquart. Jour. N. I. Ent. Soc., vi, 47 .

grata Loew. Cent., iii, 92. (C.IV.J.)

variceps Loew. Ibid., iii, 86. (H.L.V.)

Parectecephala Becker.

eucera Loew. Cent., iii, 85 .

Cetema Hendel.

procera Loew. Cent., x, 92. (C.W.J.)

Anthracophaga Loew.

sanguinolenta Loew. Cent., iii, S4.

Diplotaxa Loew.

versicolor Loew. Cent., iii, 97.

Hippelates Loew.

flavipes Loew. Cent., vii, 95.

nobilis Loew. Ibid., iii, 97.

pusio Loew. Ibid., $\mathrm{x}, 87$.

Crassiseta Von Roser.

Elachiptera Macquart.

costata Loew. Cent., iii, 62.

\section{Melanochæeta Bezzi.}

eunota Loew. Ibid., x, S9. (C.W.J.)

longula Loew. Ibid., iii, 84 .

Siphonella Macquart.

cinerea Loew. Cent., iii, SI. (C.IV.J.)

lævigata Fallen. Fauna Aust., ii, 229. (C.W.J.) 
Botanobia Livy.

Oscinis Latreille.

coxendix Fitch. Ins. N. Y., 30I.

soror Macquart. American frit-fly. Bur. Ent., Bull. 42, New ser., 57.

\section{Family DROSOPHILID E.}

Phortica Schiner.

vittata Coquillett. Proc. U. S. Nat. Mus., xxiii, 6 I8. (C.W.J.)

\section{Drosophila Fallen.}

adusta Loew. Scaptomy a. Native cabbage leaf-miner. Bur. Ent., New ser., Bull. 33, 76.

amœna Loew. Pretty pomace fly. Rept. U. S. Dept. Agr., I88I-2, 201.

ampelophila Loew. melanogaster Meigen. Vine-loving pomace fly. Ibid., I88I-2, 198.

busckii Coquillett. Ent. News, xii, I8. (C.W.J.)

flaveola Meigen. Scaptomyza. Imported turnip leaf-miner.

Bur. Ent., New ser., Bull. 33, 75.

funebris Fabricius. Proc. U. S. Nat. Mus., xxii, 264.

graminum Fallen. Scaptomy a. Imported cabbage leaf-miner.

Bur. Ent., New ser., Bull. 33, 76 .

ordanaria Coquillett. Proc. Ent. Soc. Wash., vi, I9o. (C.W.J.) quinaria Loew. Cent., vi, 9o.

\section{Family GEONYZIDE.}

Diastata Meigen.

nebulosa Fallen. Syst. Beschr., vi, 98.

\section{Family AGROMYZID Æ.}

Phytomyza Fallen.

aquilegiæ Hardy. Columbine leaf-miner. Rept. Conn. Agr. Expt. Sta., I894, 145.

diminuta Walker. Trans. Ent. Soc. Lond., New ser., iv, 232. 
Napomyza Haliday.

chrysanthemi Kowarz. Phytomyza. Chrysanthemum leaf-miner. Bur. Ent., Bull. IO, New ser., 79.

Cerodontha Rondani.

dorsalis Loew. Odontocera. Ceratomyza. Corn leaf-miner. Rept. Conn. Agr. Expt. Sta., I894, I43.

\section{Agromyza Fallen.}

æneiventris Fallen. Bur. Ent., Bull. Iо, New ser., 78 .

angulata Loew. Ann. Ent. Soc., Am., vi, 304.

longispinosa Nalloch. Ibid., vi, 276. (C.W.J.)

melampyga Loew. Ibid., vi, 282. (C.W.J.)

parvicornis Loew. Ibid., vi, 3I2.

platyptera Thomson. coronata Loew. Ibid., vi, 293.

posticata Meigen. terminalis Coquillett. Ibid., vi, 308.

pusilla Meigen. dimidiata Walker. trifolii Burgess. Ibid., vi, 278 .

simplex Loew. N. Y. Agr. Expt. Sta., Bull. I89; Ann. Ent. Soc. Am., vi, 3 5.

Mallochiella Melander.

halteralis Coquillett. Desmometopa. Proc. U. S. Nat. Mus., xxii, 267.

Desmometopa Loew.

latipes Meigen. Jour. N. Y. Ent. Soc., xxi, 24 I.

m-nigrum Zetterstedt. Ibid., xxi, 2fI.

Rhynchomilichia Hendel.

indecora Loew. Milichia. Proc. U. S. Nat. Mus., xxii, 263.

Odinia Desvoidy.

maculata Meigen. Jour. N. Y. Ent. Soc., xxi, 248. (A.B.C.) picta Loew. Cent., i, 99; Jour. N. Y. Ent. Soc., xxi, 248. (C.W.J.)

\section{Leucopis Meigen.}

nigricornis Egger. Jour. N. Y. Ent. Soc., xxi, 232. (C.IV.J.)

Ochthiphila Fallen.

elegans Panzer. Jour. N. Y. Ent. Soc., xxi, 233. (C.W.J.) polystigma Meigen. Ibid., xxi, 233. 
Family HIPPOBOSCIDE.

Olfersia Wiedemann.

americana Leach. Hippobosca bubonis. Pack. Guide, 417.

Melophagus Latreille.

ovinus Linnæus. Sheep tick. Bur. Ent., Bull. 5, New ser., I38. 


\section{Order COLEOPTERA.}

Beetles.

\section{Family CICINDELIDA.}

\section{Cicindela Linnæus.}

duodecimguttata Dejean. Coleop. Ind., 34. (E.D.H.)

formosa Say var. generosa Dejean. Ibid., 33.

hirticollis Say. Ibid., 35. (G.D.)

punctulata Fabricius. Ibid., 35.

puritana Horn. Trans. An. Ent. Soc, xxvii, I64.

purpurea Olivier. Coleop. Ind., 33.

purpurea var. limbalis Klug. Ibid., 33 .

repanda Dejean. Ibid., 34.

sexguttata Fabricius. Ibid., 32.

sexguttata var. harrisii Leng. Trans. An. Ent. Soc., xxviii, I 28. (E.D.H.)

tranquebarica Herbst. c'ulgaris. Coleop. Ind., 33.

\section{Family CARABIDA.}

\section{Cychrus Fabricius.}

elevatus Fabricius. Coleop. Ind., 43.

lecontei Dejean. Ibid., 43 .

viduus Dejean. Ibid., 42.

Carabus Linneus.

limbatus Say. Coleop. Ind., 45.

nemoralis Mulsant. Jour. N. Y. Ent. Soc., ii, I39.

serratus Say. Coleop. Ind., 45.

sylvosus Say. Ibid., 45.

vinctus IVeber. Ibid., 45 .

Calosoma IVeber.

calidum Fabricius. Coleop. Ind., 47.

externum Say. Ibid., $4^{6}$. 
frigidum Fabricius. Ibid., 47.

scrutator Fabricius. Caterpillar hunter. Ibid., 47.

sycophanta Linnæus. Bur. Ent., Bull. IOI.

willcoxi LeConte. Coleop. Ind., 47.

\section{Elaphrus Fabricius.}

cicatricosus LeConte. Coleop. Ind., 49. (C.S.)

fuliginosus Say. Ibid., 49. (A.B.C.)

ruscarius Say. Ibid., 49. (A.B.C.)

Notiophilus Dumeril.

æneus Herbst. Coleop. Ind., $5 \mathrm{r}$.

novemstriatus LeConte. Ibid., 52.

semistriatus Say. Ibid., $5 \mathrm{r}$.

Nebria Latreille.

pallipes Say. Coleop. Ind., 53.

Scarites Fabricius.

subterraneus Fabricius. Coleop. Ind., 56.

Dyschirius Bonelli.

globulosus Say, Coleop. Ind., 57. (A.B.C.) (C.C.)

hæmorrhoidalis Dejean. Ibid., 58. (C.C.)

hispidus LeConte. Ibid., 58. (A.B.C.)

sphæricollis Say. Ibid., 58.

Clivina Latreille.

americana Dejean. Coleop. Ind., 6r.

bipustulata Fabricius. Ibid., 6r.

impressifrons LeConte. Ibid., 60.

rubicunda LeConte. Ibid., 60.

Schizogenius Putzeys.

ferrugineus Putzeys. Coleop. Ind., 62. (CC..)

lineolatus Say. Ibid., 62. (C.C.)

Panageus Latreille.

fasciatus Say. Coleop. Ind., 66. (G.D.) (C.C.)

Bembidion Latreille.

americanum Dejean. Coleop. Ind., 72. (C.C.)

assimile Gyllenhal. Ibid., 78. (A.B.C.)

contractum Say. Trans. Am. Ent. Soc., xxiv, Io9.

coxendix Say. Coleop. Ind., 7o. (C.C.)

fugax LeConte. Ibid., 73. 
guexi Chaudoir. Trans. Am. Ent. Soc., xxiv, 7I. (R.Hd.)

graciliforme Hayward. Coleop. Ind., 76.

honestum Say. Ibid., 72.

inæquale Say. Ibid., 69. (C.C.)

nigrum Say. Ibid., 73.

oberthuri Hayward. Ibid., 74.

picipes Kirby. Ibid., 73. (K.F.C.)

punctatostriatum Say. Ibid., 7o. (C.C.)

quadrimaculatum Linnæus. Ibid., 78.

scopulinum Kirby. Trans. Am. Ent. Soc., xxiv, 82. (R.Hd.)

semistriatum Haldeman. Coleop. Ind., 79.

ustulatum Linneus, rupestre. Trans. Am. Ent. Soc., xxiv. 8o. (C.C.) (K.F.C.)

variegatum Say. Coleop. Ind., 77.

versicolor LeConte. Ibid., 76. (C.C.)

sp.

Tachys Schaum.

corruscus LeConte. Coleop. Ind., 86. (A.B.C.)

flavicauda Say. Ibid., 82.

incurvus Say. Ibid., 82.

lævus Say. Ibid., 85. (C.C.)

nanus Gyllenhal. Ibid., 82.

proximus Say. Compl. Writ., 502. (K.F.C.)

scitulus LeConte. Coleop. Ind., 85. (C.C.)

xanthopus Dejean. Ibid., 83 .

Patrobus Dejean.

longicornis Say. Coleop. Ind., 87.

rugicollis Randall. Jour. N. Y. Ent. Soc,, iii, 75.

Trechus Clairville.

chalybeus Dejean. Coleop. Ind., 87. (A.B.C.)

Myas Dejean.

coracinus Say. Coleop. Ind., 89. (G.D.)

cyanescens Dejean. Ibid., 89 .

Pterostichus Bonelli.

adoxus Say. Coleop. Ind., 9r.

apalachius Horn. Ibid., 9I.

caudicalis Say. Ibid.; 96.

convexicollis Say. Compl. Writ., ii, 475. (C.C.) 
coracinus Newman. Coleop. Ind., 93.

corusculus LeConte. Proc. Acad. Nat. Sci. Phila., I873, 314. corvinus Dejean. Coleop. Ind., 97.

erythropus Dejean. Ibid., 99.

honestus Say. Ibid., 9I.

lachrymosus Newman. Ibid., 93.

luctuosus Dejean. Ibid., 96.

lucublandus Say. Ibid., 95.

lustrans LeConte. Am. Lyc. Nat. Hist., N. Y. v, I8I. (G.D.)

mancus LeConte. Jour. Acad. Nat. Sci., Phila., ii, 234. (C.C.)

mutus Say. Coleop. Ind., 98.

patruelis Dejean. Ibid., 99.

pennsylvanicus LeConte. Ibid., 98. (K.F.C.)

rostratus Newman. Ibid., 9I. (K.F.C.)

sayi Brullé. Ibid., 95.

scrutator LeConte. Ibid., 97.

stygicus Say. Ibid., 93.

vitreus Dejean. Jour. N. Y. Ent. Soc., iii, IS8.

Amara Bonelli.

angustata Say. Coleop. Ind., I05.

apricaria Paykull. Trans. Am. Ent. Soc., xxxiv, 4I.

avida Say. Coleop. Ind., IO3.

basillaris Say. Ibid., гоб. (C.C.)

chalcea Dejean. Ibid., Iog.

crassispina I.eConte. Ibid., I07.

cupreolata Putzeys. Ibid., Io6.

devincta Casey. Mem. Coleop., viii, 307. (T.L.C.)

exarata Dejean. Coleop. Ind., Io4.

fallax LeConte. Ibid., ro6. (C.C.)

latior Kirby. Ibid., Io6.

impuncticollis Say. Ibid., Iо6.

musculus Say. Ibid., Iog.

obesa Say. Ibid., Ios.

pennsylvanica IIayward. Ibid., Io3.

rubrica Haldeman. Ibid., Iog.

Diplochila Brullé.

laticollis LeConte. Coleop. Ind., I I 3 . 
Dicælus Bonelli.

elongatus Bonelli. Coleop. Ind., i I6.

dilatatus Say. Ibid., i I6. (K.F.C).

politus Dejean. Ibid., I I6.

purpuratus Bonelli. I bid., I I 5 .

teter Bonelli. Ibid., I I6.

Badister Clairville.

micans LeConte. Coleop. Ind., I I9.

pulchellus LeConte. Ibid., i I 8.

Calathus Bonelli.

gregarius Say. Coleop. Ind., I20.

impunctatus Say. Ibid., I I I.

opaculus LeConte. Ibid., I 20.

Platynus Bonelli.

æruginosus Dejean. Coleop. Ind., I33.

affinis Kirby. Ibid., I 30.

anchomenoides Randall. Bost. Jour. Nat. Hist., ii, 2. (C.C.)

atratus LeConte. Coleop. Ind., I3o. (C.C.)

bogemanni Gyllenhal. Ibid., I33.

boopis Case: Mem. Coleop., ix, I37.

carbo LeConte. Coleop. Ind., i 28.

cincticollis Say. Ibid., 125.

cren:striatus I.eConte. Ibid., I34.

cupripennis Say. Ibid., I30.

decens Say. Ibid., 124. (C.C.)

decorus Say. Ibid., I 26.

excavatus Dejean. Ibid., I3I. (A.B.C.)

extensicollis Say. Ibid., 126.

ferreus Haldeman. Ibid., I 3 I.

frater LeConte. Proc. Acall. Nat. Sci. Phila., vii, 35-59. (C.C.)

lutulentus LeConte. Ibid., I3.5.

melanarius Dejean. Ibid., IzO.

nutans Say. Ibid., I3I.

octopunctatus Fabricius. Ibid., I32.

picipennis Kirby. Ibid., I35.

placidus Say. Ibid., I32.

punctiformis Say. Compl. Writ., ii, 48I.

reflexus LeConte. Coleop. Ind., I 25.

rubripes Zimmerman. Ibid., I 34 . (A.P.C.) 
ruficornis LeConte. Ibid., I35.

sinuatus Dejean. Ibid., I24.

tenuis LeConte. Ibid., I29.

Olisthopus Dejean.

micans LeConte. Ann. Lyc. Nat. Hist. N. Y., iv, 230. (A.B.C.) (H.B.K.)

parmatus Say. Coleop. Ind., I36.

Casnonia Latreille.

pennsylvanica Linnæus. Coelop. Ind., I39.

Galerita Fabricits.

bicolor Drury. Coleop. Ind. I40.

janus Fabricius. Ibid., 140.

Tetragonoderus Dejean.

fasciatus Haldeman. Coleop. Ind., I42.

Lebia Latreille.

analis DeGeer. Coleop. Ind., I47.

atriventris Say. Ibid., I44.

furcata LeConte. Ibid., I48. (G.D.)

fuscata Dejean. Ibid., I47.

grandis Hentz. Ibid., I44.

ornata Say. Ibid., 146.

pectita Horn. Trans. Am. Ent. Soc., xii, I33.

pulchella Dejean. Coleop. Ind., I45.

pumila Dejean. Ibid., I 46.

scapularis Dejean. Ibid., I48.

viridipennis Dejean. Ibid., I46.

viridis Say. Ibid., I 46.

vittata Fabricius. Ibid., I48.

Dromius Bonelli.

piceus Dejean. Coleop. Ind., I49.

Apristus Chandoir.

subsulcatus Dejean. Coleop. Incl., I 50.

Metabletus Schmidt.

americanus Dejean. Coleop. Ind., I5 г.

Axinopalpus LeConte.

biplagiatus Dejean. Coleop. Ind., I5I (A.B.C.)

Pinacodera Schaum.

limbata Dejean. Coleop. Ind. I52.

platicollis Say. Ibid., I53. 
Cymindis Latreille.

americana Dejean. Coleop. Ind., I 53.

neglecta Haldeman. Trans. Am. Ent. Soc., x, I 55.

pilosa Say. Coleop. Ind., I53.

Apenes LeConte.

lucidula Dejean. Coleop. Ind., I54.

sinuata Say. Ibid., I 54 .

Helluomorpha Laporte.

bicolor Harris. Coleop. Ind., I 55.

Brachynus Weber.

alternans Dejean. Coleop. Ind., I59. (C.C.)

ballistarius LeConte. Ibid., I6o.

conformis Dejean. Ann. Lyc. Nat. Hist. N. Y., iv, 207.

cordicollis Dejean. Coleop. Ind., I60.

cyanipennis Say. Ibid., I6r. (C.C.)

fumans Fabricius. Ibid., I6r.

perplexus Dejean. Ibid., I6I.

tormentarius LeConte. Ibid., I59.

Chlænius Bonelli.

æstivus Say., Coleop. Ind., I65.

impunctifrons Say. Ibid., I68.

laticollis Say. Ibid., I66.

leucoscelis Chevrolat. Ibid., I65. (C.C.)

nemoralis Say. Ibid., I69.

pennsylvanicus Say. Ibid., I68.

prasinus Dejean. Ibid., 165 .

purpuricollis Randall. Ibid., I68. (C.C.)

sericeus Fœrster. Ibid., I66.

solitarius Say. Ibid., I65.

tomentosus Say. Ibid., I67.

tricolor Dejean. Ibid., I69.

Anomoglossus Chaudoir.

emarginatus Say. Coleop. Ind., I69.

pusillus Say. Ibid., I jo.

Brachylobus Chandoir.

lithophilus Say. Coleop. Ind., I7o. (C.C.)

Lachnocrepis LeConte.

parallelus Say. Coleop. Ind., I7I. (H.L.J.) 
Oödes Bonelli.

americanus Dejean. Coleop. Ind., ı72. (A.B.C.)

Geopinus LeConte.

incrassatus Dejean. Coleop. Ind., I74.

Agonoderus Dejean.

lineola Fabricius. Coleop. Ind., I77.

pallipes Fabricius. Ibid., I77.

partiarius Say. Ibid., I77. (A.B.C.)

pauperculus Dejean. Ibid., I77. (C.C.)

testaceus Dejean. Ibid., I77.

Gynandropus Dejean.

hylacis Say. Coleop. Ind. I79.

Harpalus Latreille.

autumnalis Say. Coleop. Ind., 185 .

caliginosus Fabricius. Ibid., $1 \mathrm{~S}_{\mathrm{I}}$.

compar LeConte. Ibid., IS2.

convivus LeConte. Ibid., ISI. (C.C.)

dichrous Dejean. Ibid., I $8_{4}$.

erraticus Say. Ibid., I $\mathrm{S}_{\mathrm{I}}$.

erythropus Dejean. Ibid., ${ }_{1} S_{3}$.

faunus Say. Ibid., $1 S_{I}$.

herbivagus Say. Ibid., $18_{5}$.

laticeps LeConte. Ibid., I $S_{4}$.

lewisii LeConte. Proc. Acad. Nat. Sci., Phila., I865, I03.

longior Kirby. longicollis. Coleop. Ind., I83.

megacephalus LeConte. Ann. Lyc. Nat. Hist. N. Y., iv, 397.

nitidulus Chaudoir. Coleop. Ind., I $8_{5}$. (C.C.)

pennsylvanicus Dejean. Ibid., I 82 .

pleuriticus Kirby. Ibid., I $8_{+}$.

spadiceus Dejean. Can. Ent., ii, 6г .

vagans LeConte. Coleop. Ind., IS2.

viduus LeConte. Ibid., ı86. (C.C.)

viridiæneus Beanvois. Ibid., I $S_{1}$.

vulpeculus Say. Ibid., IS4.

Selenophorus Dejean.

gagatinus Dejean. Coleop. Ind., IS7.

opalinus LeConte. Ibid., IS7.

pedicularis Dejean. Ibid., I 87 . 
Stenolophus Dejean.

conjunctus Say. Coleop. Ind., Isg.

fuliginosus Dejean. Ibid., I 88.

humilus Hamilton. Can. Ent., xxy, 306. (C.C.)

ochropezus Say. Coleop. Ind., is8.

plebius Dejean. Ibid., I 88 .

Acupalpus Latreille.

carus LeConte. Coleop. Ind., igo. (A.B.C.)

hydropicus LeConte. Ibid., Igo. (A.B.C.)

Bradycellus Erichson.

rupestris Say. Coleop. Ind., I92.

Tachycellus Morawitz.

badiipennis Haldeman. Coleop. Ind,, I93. (A.B.C.)

kirbyi IIorn. Ibid., 193. (A.B.C.)

nigrinus Dejean. Ibid., 192. (A.B.C.)

Anisodactylis Dejean.

baltimorensis Say. Coleop. Ind., 197.

carbonarius Say. Ibid., 195.

discoideus Dejean. Ibid., I97.

harrisii LeConte. Ibid., I96.

interpunctatus Kirby. Ibid., 196.

interstitialis Say. Ibid., 200.

lugubris Dejean. Ibid., I99.

melanopus HaIdeman. Ibid., 197.

nigerrimus Dejean. Ibid., I96.

nigrita Dejean. Ibid., I97. (C.C.)

nitid:pennis LeConte. Ibid., I99.

rusticus Say. Ibid., 194.

sericeus Ilarris. Ibid., 200.

terminatus Say. Ibid., I99.

verticalis LeConte. Ibid., IgS.

\section{Family OMOPHRONIDÆ.}

Omophron Latreille.

americanum Dejean. Coleop. Ind., to.

labiatum Fabricius. Ibid., 40. (K.F.C.)

tessellatum Say. Ibid., 41 . 


\section{Family HALIPLIDÆ.}

\section{Haliplus Latreille.}

connexus Matheson.' Jour. N. Y. Ent. Soc., xx, I64. (K.F.C.) cribrarius LeConte. Ibid., xx, I7O. (K.F.C.)

fasciatus Aubé. Coleop. Ind., 202. (C.C.)

leopardus Roberts. Jour. N. Y. Ent. Soc., xxi, 98. (K.F.C.) ruficollis DeGeer. Coleop. Ind., 203. (C.C.)

triopsis Say. punctatus. Ibid., 202.

\section{Peltodytes Regimbart.}

Cncmidotus Illiger.

duodecimpunctatus Say. I2-punctatus. Coleop. Ind., 204. edentulus LeConte. Ibid., 204. (K.F.C.) muticus LeConte. Ibid., 204. (K.F.C.) shermani Roberts. Jour. N. Y. Ent. Soc., xxi, I 6. (K.F.C.)

\section{Family DYTISCIDÆ.}

Hydrocanthus Say.

iricolor Say. Coleop. Ind., 208. (C.C.)

\section{Laccophilus Leach.}

maculosus Germar. Coleop. Ind., 209.

undatus Aubé. Ibid., 2IO. (F.K.)

\section{Desmopachria Babington.}

convexa Aubé. Coleop. Ind., 2 I2.

\section{Bidessus Sharp.}

affinis Say. Coleop. Ind., 213.

granarius Aubé. Hydroporns. Trans. Am. Ent. Soc., iv, 390.

Celina Aubé.

angustata Aubé. Coleop. Ind., 2 I4.

Colambus Thomson.

dispar LeConte. dissimilis. Coleop. Ind., 215. (G.D.) inæqualis Fabricius. puntatus Say. Ibid., 215.

\section{Deronectes Sharp.*}

catascopium Say. Coleop. Ind., 2I6. (G.D.)

\section{Hydroporus Clairville.*}

americanus Aubé. Coleop. Ind., 220. (C.C.)

dimidiatus LeConte. semirufus. Ibid., 2 I9.

* See also appendix. 
niger Say. modestus. Ibid., 220.

pulcher LeConte. Ibid., 2 I8.

signatus Mannerheim. Bull. Moscow, I853, I6 I.

solitarius Sharp. On Dytiscidre, 443.

stagnalis Gemminger and Harold. Coleop. Ind., 2 I 8.

tenebrosus LeConte. Ibid., 2 I 7.

undulatus Say. Ibid., 2I9. (C.C.)

vitiosus Sharp. blanchardi. On Dytiscidæe, 443.

Ilybius Erichson.

biguttulus Germar. Coleop. Ind., 222.

confusus Aubé. Ibid., 222. (A.B.C.)

subæneus Erichson. Ibid., 222. (K.F.C.)

Coptotomus Say.

interrogatus Fabricius. Coleop. Ind., 223.

Copelatus Erichson.

glyphicus Say. Coleop. Ind., 223.

Matus Aubé.

bicarinatus Say. Coleop. Ind., 223.

Agabetes Crotch.

acuductus Harris. Coleop. Ind., 224. (C.C.)

Agabus Leach.

æruginosus Aubé. Species Gén., vi, 298. (C.C.)

disintegratus Crotch. Coleop. Ind., 227.

erythropterus Say. Ibid., 226. (A.B.C.)

gagates Aubé. Ibid., 226.

obtusatus Say. Ibid., 226. (A.B.C.)

punctatus Melsheimer. Ibid., 226.

reticulatus Aubé. Ibid., 225. (K.F.C.)

semipunctatus Kirby. Ibid., 227.

seriatus Say. parallelus. Ibid., 226.

stagninus Say. Ibid., 226.

Rhantus Eschscholtz.

binotatus Harris. Coleop. Ind., 228.

bistriatus Bergstrasser. Ibid., 228. (C.C.) 
Colymbetes Clairville.

sculptilis Harris. Coleop. Ind., 229 .

Dytiscus Linnæus.

fasciventris Say. Coleop. Ind., 23I.

harrisii Kirby. Ibid., 23 I.

hybridus Aubé. Ibid., 23I. (C.C.)

verticalis Say. Ibid., 23I.

Hydaticus Leach.

piceus LeConte. Coleop. Ind., 232.

stagnalis Fabricius. Ibid., 232.

Acilius Leach.

fraternus Harris. Coleop. Ind., 234.

mediatus Say. Ibid., 2.33 .

semisulcatus Aubé. Ibid., 233.

Thermonectes Eschscholtz.

ornaticollis Aubé. Coleop. Ind., 234. (C.C.)

Graphoderes Eschischoltz.

fasciatocollis Harris. cinereus. Coleop. Ind., 235.

liberus Say. Ibid., 235. (C.C.)

\section{Family GYRINIDÆ.}

Gyrinus Linnæus.

borealis Aubé. Coleop. Ind., 240.

limbatus Say. Ibid., 239.

lugens LeConte. Ibid., 240.

ventralis Kirby. Ibid., 239.

sp.

Dineutes MacLeay.

assimilis Aubé. Coleop. Ind., 242.

discolor Aubé. Ibid., 24I.

emarginatus Say. Ibid., 24I. (H.L.J.)

hornii Roberts. Ibid., 242.

nigrior Roberts. Trans. Am. Ent. Soc., xxii, 284.

vittatus Germar. Coleop. Ind., 24I. 
Family HYDROPHILIDA.

Hydræna Kingelan.

pennsylvanica Kiesenwetter. Coleop. Ind., 254. (C.C.)

Helophorus Fabricius.

lineatus Say. Coleop. Ind., 25 I.

\section{Hydrochus Leach.}

scabratus Mirlsant. Coleop. Ind., $25 \mathrm{I}$.

subcupreus Randall. Ibid., 252. (C. C.)

Berosus Leach.

infuscatus LeConte. Can. Ent., xxvii, I85. (C.C.)

peregrinus Herbst. Coleop. Ind., 258.

striatus Say. Ibid., 259.

\section{Hydrous Dahl.}

ovalis Ziegler. Coleop. Ind., 255.

triangularis Say. Ibid., 255.

Hydrophilus DeGeer.

obtusatus Say. Coleop. Ind., 257.

Tropisternus Solier.

glaber Herbst. Coleop. Ind., 256. (K.F.C.)

mixtus LeConte. Hydrophilus. Ibid., 256.

nimbatus Say. Ibid., 256.

\section{Hydrobius Leach.*}

fuscipes Linneus. Coleop. Ind., 262.

globosus Say. Ibid., 263.

\section{Paracymus Thomson.}

Creniphilus Motschulsky.

despectus LeConte. Coleop. Ind., 264.

digestus LeConte. Trans. Am. Ent. Soc., xvii. 274.

infuscatus Motschulsky. Coleop. Ind., 264.

subcupreus Say. Ibid., 264.

\section{Enochrus Thomson.}

Phillydrus Solier.

cinctus Say. Coleop. Ind., $26 \mathrm{r}$.

hamiltoni Horn. Ibid., 260.

ochraceus Melsheimer. Ibid., 260.

perplexus LeConte. Ibid., $26 \mathrm{I}$.

reflexipennis Zimmerman. angustulus Casey. Trans. Am. Ent.

Soc., xvii., 250.

* See also appendix. 
Laccobius Erichson.

agilis Randall. Coleop. Ind., 259.

\section{Cymbiodyta Bedel.}

fimbriata Melsheimer. Trans. Am. Ent. Soc., xvii., 258: Coleop. Ind., 262.

lacustris LeConte. Coleop. Ind., 262.

Chætarthria Stephens.

atra LeConte. Proc. Acad. Nat. Sci. Phila., vi, 24.

Cercyon Leach.

analis Paykull. Coleop. Ind., 269.

depressus Stephens. Trans. Am. Ent., Soc., vii, 293. (C.C.) hæmorrhoidalis Fabricius. Coleop. Ind., 267.

indistinctus Horn. Ibid., 267.

lateralis Marsham. Trans. Am. Ent. Soc., xvii, 297.

littoralis Gyllenhal. Ibid., 29 I.

navicularis Zimmerman. Coleop. Ind., 269. (C.C.)

ocellatus Say. Ibid., 269. (C.C.)

prætextatus Say. Ibid., 268.

pygmæus Illiger. Ibid., 268.

unpunctatus Linnæus. Ibid., 268.

Sphæridium Fabricius.

bipustulatus Fabricius. Jour. N. Y. Ent. Soc., xx, 69. scarabæoides Linnæus. Coleop. Ind., 265.

Cryptopleurum Mulsant.

minutum Fabricius. Coleop. Ind., 270.

Family SILPHIDA.

Necrophorus Fabricius.

americanus Olivier. Coleop. Ind., 274.

marginatus Fabricius. Ibid., 275.

orbicollis Say. Ibid., 275.

pustulatus Herschel. Ibid., 275.

sayi Laporte. Ibid., 274.

tomentosus IVeber. Ibid,, 274.

vespilloides Herbst. Trans. Am. Ent. Soc., viii, 234. 
Silpha Linnæus.

americana Linnæus. Coleop. Ind., 277.

inæqualis Fabricius. Ibid., 276.

noveboracensis Fœrster. Ibid., 277.

surinamensis Herbst. Ibid., 276 .

Choleva Latreille.

clavicornis LeConte. Coleop. Ind., 280.

terminans LeConte. Ibid., 28o. (C.C.)

\section{Prionochæta Horn.}

opaca Say. Coleop. Ind., 280.

Ptomophagus Illiger.

consobrinus LeConte. Coleop. Ind., 28I. (C.C.)

parasitus LeConte. Ibid., 282.

pusio LeConte. Ibid., 282. (C.C.)

Colon Herbst.

bidentatum Sah1berg. Trans. Am. Ent. Soc., viii, 269. (C.C.)

Anisotoma Illiger.

alternata Melsheimer. Trans. Am. Ent. Soc., viii, 285.

Leiodes Latreille.

discolor Melsheimer. Coleop. Ind., 287. (C.C.)

globosa LeConte. Ibid., 287. '(C.C.)

Agathidium Illiger.

exiguum Melsheimer. Coleop. Ind., 290.

oniscoides Beauvois. Ibid., 290.

Family SCYDMIENIDE.

Euconnus Thomson.

bicolor LeConte. Ann. N. Y. Acad. Sci., ix, 370.

salinator LeConte. Coleop. Ind., 295. (C.C.)

Connophron Casey.

fossiger LeConte. Coleop. Ind., 298. (C.C.)

hirtellus LeConte. Ann. N. Y. Acad. Sci., ix, 429.

lacunosum Casey. Ibid., ix, 459. (C.C.)

oreophilum Casey. Ibid., ix, 405.

sp.

Scydmænus Latreille.

perforatus Schaum. Coleop. Ind., 30I. (C.C.) 


\section{Family CORYLOPHIDA.}

Sericoderus Stephens.

favidus LeConte. Coleop. Ind., 504.

Molamba Casey.

fasciata Say. Sacimm. Coleop. Ind., 505 . (C.C.)

lunata LeConte. Sacium. Ibid., 505. (C.C.)

Rhypobius LeConte.

marinus LeConte. Jour. N. Y. Ent. Soc., viii, 65. (C.W.L.)

\section{Family STAPHYLINIDA.}

Thoracophorus Motschulsky.

Glyptoma Erichson.

costale Erichson. Coleop. Ind., 484.

\section{Triga Fauvel.}

picipennis LeConte. Coleop. Ind., 484 .

Olisthærus Heer.

nitidus LeConte. Trans. Am. Ent. Soc., v, 50.

Anthobium Leach.

convexum Fauvel. Coleop. Ind., 48 I.

Olophrum Erichson.

obtectum Erichson. Coleop. Ind., 479.

Oxytelus Gravenhorst.

insignitus Gravenhorst. Coleop. Ind., 468.

sculptus Gravenhorst. Ibid., 468.

Homalium Gravenhorst.

rivulare Paykull. Coleop. Neerland., i, 359.

Platystethus Mannerheim.

americanus Erichson. Coleop. Ind., 467.

Bledius Leach.

pallipennis Say. mandibularis. Coleop. Ind., 464.

Stenus Latreille.

colonus Erichson. Coleop. Ind., 408.

ingratus Casey. Ibid., 406.

morio Gravenhorst. subgriscus Casey. Ibid., 4 II.

stygicus Say. Coleop. Ind., 400 . 
Areus Casey.

annularis Erichson. Stenus. Coleop. Ind., 4 I3.

arculus Erichson. Stenlls. Ibid., $4 \mathrm{I} 4$.

cribratum LeConte. Smith Misc. Coll., I 865, 46.

flavicornis Erichson. Stemus. Coleop. Ind., 4I3.

Hesperobium Casey.

pallipes Gravenhorst. Haiticome. Can. Ent., xvii, I8o.

Lathrotaxis Casey.

longiusculum Gravenhorst. Lathrobium. Coleop. Ind., 427.

Gasterolobium Casey.

bicolor Gravenhorst. Cryptobimm. Coleop. Ind., 418.

Pæderus Gravenhorst.

littorarius Gravenhorst. Coleop. Ind., 437.

Lathrobium Gravenhorst.

simile LeConte. Coleop. Ind., 424.

simplex LeConte. Ibid., 423.

Trachysectus Casey.

confluentus Say. Lithocharis. Coleop. Ind., 43 I.

Astenus Stephens.

Sunius Erichson.

discopunctatus Say. longiusculus. Coleop. Ind., 439.

Gyrohypnus Mannerhein.

Xantholinus Serville.

emmesus Gravenhorst. Coleop. Ind., 396.

hamatus Say. obscurus. Ibid., 396.

obsidianus Melsheimer. Ibid., 395.

pusillus Sachse. Ibid., 398.

sanguinipennis LeConte. Trans. Am. Ent. Soc, viii, I74.

Nudobius Thonson.

cephalus Say. Tantholinus. Coleop. Ind., 395.

Neobisnius Ganglbatrer.

pæderoides LeConte. Coleop. Ind., 393.

Philonthus Curtis.

blandus Gravenhorst. Coleop. Ind., 387.

brunneus Gravenhorst. Ibid., 386.

cyanipennis Fabricius. Ibid., 387 . 
debilis Gravenhorst. Ibid., 383 .

fusiformis Melsheimer. Ibid., 385 .

lomatus Erichson. Ibid., 386.

longicornis Stephens. Ibid., 383 .

microphthalmus Horn. Ibid., 388 .

politus Linnæus. aneus Rossi. Trans. Am. Ent. Soc., xi, I8r. schwarzi Horn. Ibid., 385 .

sp.

Ocypus Kirby.

ater Gravenhorst. Coleop. Ind., 379.

Staphylinus Linnæus.

badipes LeConte. Coleop. Ind., 376 .

cinnamopterus Gravenhorst. Ibid., 377.

fossator Gravenhorst. Ibid., 377.

maculosus Gravenhorst. Ibid., 377.

mysticus Erichson. Ibid., 377.

prælongus Mannerheim. Trans. Am. Ent. Soc., vii, 197. (A.B.C.)

violaceus Gravenhorst. Coleop. Ind., 378.

vulpinus Nordmann. Ibid., 376 .

Ortholestes Ganglbaner.

Listotrophus Perty.

capitatus Bland. Proc. Phila. Ent. Soc., iii, 65. (K.F.C.)

cingulatus Gravenhorst. Coleop. Ind., 374.

Creophilus Mannerheim.

villosus Gravenhorst. Coleop. Ind., 374.

Acylophorus Nordmann.

pronus Erichson. Coleop. Ind., 368.

Quedius Stephens.

vernix LeConte. Coleop. Ind., 373.

Oxyporus Fabricius.

lateralis Gravenhorst. Coleop. Ind., 46r. major Gravenhorst. Ibid., 459. (A.B.C.) (H.B.K.)

vittatus Gravenhorst. Ibid., 460.

Habrocerus Erichson.

schwarzii Horn. Coleop. Ind., 457. 
Tachinus Gravenhorst.

fimbriatus Gravenhorst. Coleop. Ind., 446.

fumipennis Say. Trans. Am. Ent. Soc., vi, Ioo.

limbatus Nelsheimer. Coleop. Ind., 446.

Tachyporus Gravenhorst.

chrysomelinus Linnæus. Coleop. Ind., 448.

jocosus Say. Ibid., 448.

maculipennis LeConte. Ibid., 447.

Erchomus Motschulsky.

ventriculus Say. Coleop. Ind., 449.

Conosoma Kraatz.

basale Erichson. Coleop. Ind., 45I.

crassum Gravenhorst. Ibid., 450.

pubescens Paykull. Ibid., 45r.

Boletobius Stephens.

cincticollis Say. Coleop. Ind., 453.

cinctus Gravenhorst. Ibid., 454.

intrusus Horn. Ibid., 454.

niger Gravenhorst. Ibid. 452.

trinotatus Erichson. Ibid., 454.

Bryoporus Kraatz.

rufescens LeConte. Coleop. Ind., 457.

Hoplandria Kraatz.

brittoni Casey. Mem. Coleop., ii, 161.

Meronea Sharp.

venustula Erichson. Coleop. Ind., 349.

Atheta Thomson.

irvingi Casey. Mem. Coleop., i, 5.

vierecki Casey. Ibid., ii, IO3. (H.L.V.)

Datomicra Casey.

mina Casey. Mem. Coleop., ii, I 45. (H.L.V.)

Sableta Casey.

brittoni Casey. Mem. Coleop., ii, r45. (IV.E.B.)

Strigota Casey.

recta Casey. Mem. Coleop., ii, I65. (H.L.V.) 
Falagria Mannerheim.

dissecta Erichson. Coleop. Ind., 347.

Gyrophæna Mannerheim.

dissimilis Erichson. Coleop. Ind., 34I.

lobata Casey. Ibid., 34I.

Xenodusa Wasmann.

cava LeConte. Lomechusa. Coleop. Ind., 359.

cava' var. major Wasmann. Deutsche Ent. Zeit., I899, 4 Io. (C.W.L.)

Aleochara Gravenhorst.

bimaculata Gravenhorst. Coleop. Ind., 365 .

lata Gravenhorst. Ibid., 364.

marion Gravenhorst. Ent. News., xix, 64.

Homœusa Kraatz.

crinitula Casey. Jour. N. Y. Ent. Soc., xviii, 54.

Haploglossa Kraatz.

sp.

Family PSELAPHIDÆ.

Batrisodes Reitter.

fossicauda Casey. Ann. N. Y. Acad. Sci., ix, 575. (C.C.)

globosus LeConte. Coleop. Ind., 326.

harringtoni Casey. Ann. N. Y. Acad. Sci., ix, 578. (C.C.)

nigricans LeConte. Coleop. Ind., 328. (C.C.)

Nisaxis Casey.

tomentosa Aubé. Coleop. Ind., 3I7.

Brachygluta Thomson.

Bryaris Aubé

abdominalis Aubé. Coleop. Ind., 318. (A.B.C.)

terbrata Casey. perforata. Ibid., 3I8. (C.C.)

Reichenbachia Leach.

puncticollis LeConte. Coleop. Ind., 322. (C.C.)

rubicunda Aubé. Ibid., 321.

Decarthron Brendel.

abnorme LeConte. Coleop. Ind., 317. (C.C.)

longulum LeConte. Ibid., 3i6. (C.C.)

stigmosum Brendel. Proc. Phila. Ent. Soc., vi, 35. 
Pselaphus Herbst.

bellax Casey. Coleop. Ind., 3I4. (A.B.C.)

$$
\text { Tyrus Aubé. }
$$

humeralis Aubé. Coleop. Ind., 3 I 3 .

\section{Pilopius Casey.}

Ctcnistes Reichenbach.

lacustris Casey. piccus LeConte. Coleop. Ind., 3II. (C.C.)

Ceophyllus LeConte.

monilis LeConte. Coleop. Ind., 309. (C.W.L.)

Family CLAVIGERIDE.

Adranes LeConte.

lecontei Brendel. Coleop. Ind., 307. (C.C.)

Fanily PTILIDE.

Trichopteryx Kirby.

haldemani LeConte. Coleop. Ind., 489. (C.W.L.)

Family SCAPHIDIID E.

Scaphidium Olivier.

quadriguttatum Say. Coleop. Ind., 492. (C.C.)

quadriguttatum var. piceum Melsheimer. Ibid., 492.

Bæocera Erichson.

sp.

Scaphisoma Leach.

convexa Say. Coleop. Ind., 496. (C.C.)

suturalis LeConte. Ibid., 496. (C.C.)

Family HISTERIDE.

Hololepta Paykull.

fossularis Say. Coleop. Ind., 601. 
Hister Linnaens.

abbreviatus Fabricius. Coleop. Ind., 607.

americanus Paykull. Ibid., 609.

arcuatus Say. Compl. Writ., ii, 262. (C.C.)

bimaculatus Linnæus. Coleop. Ind., 609.

biplagiatus LeConte. Ibid., 603.

cognatus LeConte. Ibid., 604.

depurator Say. Ibid., 607.

egregius Casey. Mem. Coleop., vii, 288.

fœdatus LeConte. Coleop. Ind., 606.

furtivus LeConte. Ibid., 608.

immunis Erichson. Ibid., 605.

interruptus Beauvois. Ibid., 605.

lecontei Latreille. Ibid., 612.

merdarius Hoffman. Ibid., 6I2.

parallelus Say. Ibid., 6I2.

perplexus LeConte. Ibid., 609. (C.C.)

sedecimstriatus Say. Ibid., 609.

stygicus LeConte. Ibid., 606.

Phelister Marseıl.

subrotundus Say. Hister. Coleop. Ind., 6I I.

Gnathoncus Duval.

communis Marseul. rotundatus. Coleop. Ind., 6I9.

Psiloscelis Marseul.

harrisii LeConte. planipes. Mem. Coleop., vii, 204. (C.C.) perpunctatus LeConte. Trans. Am. Ent. Soc., viii, I9o.

Platysoma Leach.

carolinus Paylull. Coleop. Ind., 6г2.

coartatus LeConte. Ibid., 6I3. (A.B.C.) (H.B.K.)

Hetærius Erichson.

blanchardi LeConte. Proc. Am. Phil. Soc., xvii, 6o9. (C.C.) brunneipennis Randall. Coleop. Ind., 6 5 .

Dendrophilus Leach.

punctulatus Say. Coleop. Ind., 615.

Paromalus Erichson.

bistriatus Erichson. Coleop. Ind., 616. 
Saprinus Erichson.

assimilis Paykull. Coleop. Ind., 620.

conformis LeConte. Ibid., 620.

dimidiatipennis LeConte. Ibid., 608.

fraternus Say. Ibid., 623. (K.F.C.)

neglectus Marseul. Trans. An. Phil. Soc., xiii, 335. (C.C.)

oregonensis LeConte var. distinguendis Marseul. Mon. Hister., $44 \mathrm{I}$.

patruelis LeConte. Coleop. Ind., 624.

pennsylvanicus Paykull. Coleop. Ind., 620.

posthumus Marseul. Ibid., 6 I9.

Plegaderus Erichson.

transversus Say. Coleop. Ind., 625. (C.C.)

Acritus LeConte.

politus LeConte. Coleop. Ind., 627. (C.C.)

Family LYCID $\mathrm{E}$.

Lycostomus Motschulsky.

lateralis Melsheimer. Coleop. Ind., Sio.

Calopteron Guérin-Ménéville.

reticulatum Fabricius. Coleop. Ind., Si I. terminale Say. Ibid., 8Io.

Cæniella Cockerell.

Cania Newman.

dimidiata Fabricius. Coleop. Ind., Si I.

Celetes Newman.

basalis LeConte. Coleop. Ind., Si I.

Lopheros LeConte.

fraternus Randall. Coleop. Ind., EI I.

Eros Newman.

aurora Herbst. Coleop. Ind., 8I 2.

trilineatus Melsheimer. Ibid., 8iz.

Plateros Bourgeois.

canaliculatus Say. Coleop, Ind., 8I 5.

modestus Say. Ibid., Si 4 . 
Family LAMPYRIDÆ.

Lucidota Laporte.

atra Fabricius. Coleop. Ind., 8 I 7 .

corrusca Linnzeus. Ellychnia. Ibid., 8I8.

decipiens Harris. Pyropyga. Ibid., 8I9.

fenestralis Melsheimer. Pyropyga. Ibid., 819.

nigricans Say. Ibid., 8I9.

Lecontea Olivier.

augulata Say. Pyractonema. Coleop. Ind., 820.

lucifera Melsheimer. Pyractoncma. Coleop. Ind., 821.

Photinus Laporte.*

scintillans Say. Coleop. Ind., 823. (H.L.J.)

Photuris LeConte.

pennsylvanica DeGeer. Common firefly. Coleop. Ind., 823.

Family TELEPHORIDE.

Omethes LeConte.

marginatus LeConte. Coleop. Ind., 825. (C.C.)

Chauliognathus Hentz.

pennsylvanicus DeGeer. Soldier beetle. Coleop. Ind., 827.

Podabrus Fischer.

basillaris Say. Coleop. Ind., 830.

frater LeConte. Ibid., 830 .

modestus Say. Ibid., 830.

modestus var. flavicollis LeConte. Ibid., 83I.

protensus LeConte. Ibid., 83I. (C.C.)

rugulosus LeConte. Ibid., 830 .

tricostatus Say. Ibid., 829.

Telephorus Schæffer.

bilineatus Say. Coleop. Ind., 835 .

carolinus Fabricius. Ibid., 833 .

collaris LeConte. Proc. Acad. Nat. Sci., Phila., v. 540.

dentiger LeConte. Coleop. Ind., 833 .

excavatus LeConte. Ibid., 833 .

fraxini Say. Compl. Writ., ii, II8.

* See also appendix. 
lineola Fabricius. Coleop. Ind., 834 .

nigritulus? LeConte. Trans. Am. Ent. Soc., ix, 52.

rectus Walsh. Coleop. Ind., $8_{34}$.

rotundicollis Say. Ibid., 835 .

scitulus Say. Ibid., 834 .

tuberculatus LeConte. Ibid., 835 .

Polemius LeConte.

laticornis Say. Coleop. Ind., 836 .

Ditemnus LeConte.

bidentatus Say. Coleop. Ind., 837 .

Trypherus LeConte.

latipennis Germar. Coleop. Ind., $8_{3} 8$.

Malthodes Kiesenwetter.

concavus LeConte. Coleop. Ind., 839 .

Family PHENGODIDA.

Phengodes Illiger.

sp.

Family MELYRIDE (MALACHIDE).

Collops Erichson.

nigriceps Say. cximius. Trans. Am. Ent. Soc., iii, 8 o.

quadrimaculatus Fabricius. Coleop. Ind., 8+I.

tricolor Say. Ibid., S4I.

vittatus Say. Trans. Am. Ent. Soc., iii, SI.

Malachius Fabricius.

æneus Linnarus. Trans. Am. Ent. Soc., iv, II3.

Anthocomus Erichson.

erichsoni LeConte. Coleop. Ind., 842. (C.C.)

Pseudebæus Horn.

oblitus LeConte. Coleop. Ind., S42.

Attalus Erichson.

morulus LeConte. Coleop. Ind., $8+4$.

otiosus Say. Ibid., 845. (C.C.)

pallifrons Motschulsky. Ibid., S44. (C.C.)

scincetus Say. Ibid., 846.

terminalis Erichson. Ibid., 844. 


$$
\text { Family CLERIDAE. }
$$

Monophylla Spinola.

terminatus Say. Elasmocerus. Coleop. Ind., 849. (E.P.F.)

\section{Cymatodera Gray.}

bicolor Say. Coleop. Ind., 850. (D.J.C.)

inornata Say. Ibid., 850 .

Thanasimus Latreille.

dubius Say. Coleop. Ind., 854 .

Enoclerus Gahan.

analis LeConte. Coleop. Ind., 853. (F.K.)

nigrifrons Say. Ibid., 853.

quadriguttatus Olivier. nigripes. Ibid., 853 .

rosmarus Say. Ibid., 854 .

Phlœopterus Wolcott.

thoracicus Olivier. Enoclerus. Coleop. Ind., 854.

Trichodes Herbst.

nuttalli Kirby. Coleop. Ind., 852.

Hydnocera Newman.

humeralis Say. Coletop. Ind., 856 .

humeralis var. difficilis LeConte. Ibid., 856 .

pallipennis Say. Ibid., 856 .

tabida LeConte. Ibid., 857 . (A.B.C.)

unifasciata Say. Ibid., 855 .

verticalis Say. Ibid., 857 .

Zenodosus Wolcott.

sanguineus Say. Coleop. Ind., 855 .

Phyllobænus Spinola.

dislocatus Say. Coleop. Ind., 858. (A.B.C.)

Neichnea Wolcott and Chapin.

laticornis Say. Ichnea. Ellipotoma. Coleop. Ind., 859.

Chariessa Perty.

pilosa Forster. Coleop. Ind., 859 .

Corinthiscus Fairmaire.

Pelonium Spinola.

leucophæum Klug. Cregya z'estusta Spinola. Coleop. Ind., 860.

Necrobia Latreille.

ruficollis Fabricius. Coleop. Ind., 86r.

rufipes DeGeer. Ibid., 86r.

violacea Linnaeus. Ibid., 862. 
Family LYMEXYLID E.

Hylecœtus Latreille.

lugubris Say. Coleop. Ind., 894. (A.B.C.)

Melittomma Murray.

sericeum Harris. Lymerylon. Chestnut timber beetle. Coleop, Ind., 895 .

Family CEPHALOIDE.

Cephaloön Newman.

lepturides Newman. Ann. N. Y. Acad. Sci., ix, 650.

Family CEDENERID.E.

Nacerdes Schmidt.

melanura Linnæus. Coleop. Ind., I 306.

Asclera Schmidt.

ruficollis Say. Coleop. Ind., I 307.

Family MORDELLIDAE.

Anaspis Geoffroy.

flavipennis Haldeman. Coleop. Ind., I 3 IO.

rufa Say. Ibid., I3IO.

Tomoxia Costa.

bidentata Say. Coleop. Ind., I3I I.

Mordella Linnreus.

marginata Melsheimer. Coleop. Ind., I3 I3.

melæna Germar. Ibid., I3 I 2.

oculata Say. Ibid., I3I4.

scutellaris Fabricius. Ibid., I3I3.

triloba Say. Ibid., I3I4. (F.K.)

Glipodes LeConte.

helva LeConte. Proc. Acad. Nat. Sci., Phila., xiv, 48.

Mordellistena Costa.

aspersa Melsheimer. Coleop. Ind., I3I9.

comata LeConte. Ibid., I 3 I9.

comata var. cervicalis LeConte. Ibid., I3I9.

grammica LeConte var. varians LeConte. Ibid., I32I. (F.K.)

pubescens Fabricius. Ibid., I335. 
Family RHIPIPIIORID E.

Rhipiphorus Bosc d'Antic.

fasciatus Say. Myoditcs. walshii. Coleop. Ind., I367.

Macrosiagon. Hentz.

flavipennis LeConte. Rhipiphorus. Coleop. Ind., I366. limbatum Fabricius. Ibid., I367.

Family MELOIDE (or CANTHARIDA.)

Epicauta Redtenbacher.

cinerea Forster. Coleop. Ind, I362.

cinerea var. marginata Fabricius. Ibid., 1362.

pennsylvanica DeGeer. Ibid., I362.

vittata Fabricius. Ibid., I 36 I.

Macrobasis LeConte.

unicolor Kirby. Coleop. Ind., I 359.

Pomphopæa LeConte.

ænea Say. Coleop. Ind., I 364.

sayi LeConte. Ibid., I 364.

unguicularis LeConte. Ibid., I 354.

Meloe Linnatus.

angusticollis Say. Coleop. Ind., I353.

Zonitis Fabricius.

bilineata Say. Coleop. Ind., I356.

Tricrania LeConte.

sanguinipennis Say. Coleop. Ind., I354.

Family PYTHIDA.

Pytho Latreille.

niger Kirby. Trans. Am. Ent. Soc., xv, 46.

planus Herbst. americanus Kirby. Ins. Aff. Park and Woodl.

Trees, 368: Trans. Am. Ent. Soc., xv, 46.

Rhinosimus Latreille.

viridiæneus Randa11. Coleop. Ind., I304. 
Family PYROCHROIDA.

Pyrochroa Geoffroy.

femoralis LeConte. Coleop. Ind., I 348.

flabellata Fabricius. Ibid., 1348 .

Schizotus Newman.

cervicalis Newman. Coleop. Ind., I 349.

Dendroides Latreille.

bicolor Newman. canadensis. Coleop. Ind., I349. (G.D.)

concolor Newman. Ibid., I349.

Fanily PEDILID E.

Pedilus Fischer.

Corphyra Say.

collaris Say. Coleop. Ind., I33I.

elegans Hentz. Ibid., I330.

lugubris Say. Ibid., I33I.

Macratria Newman.

confusa LeConte. Coleop. Ind., I332.

Family ANTHICIDA:

Notoxus Geoffroy.

anchora Hentz. Coleop. Ind., I335.

bifasciatus LeConte. Ibid., I 335.

mondon Fabricius. Ibid., I336.

Malporus Casey.

formicarius Laferté. Coleop. Ind., I338. (A.B.C.) (H.B.K.)

Thicanus Casey.

rejectus LeConte. Ann. N. Y. Acad. Sci., viii, 67I. (A.B.C.)

(H.B.K.)

Lappus Casey.

sturni Laferté. clegans. Ann. N. Y. Acad. Sci., viii, 657. (G.D.)

Hemantus Casey.

floralis Linneus. Ann. N. Y. Acad. Sci., viii, 683 .

Anthicus Paykull.

cervinus Laferté. Coleop. Ind., I34 I.

Sapintus Casey.

pubescens LeConte. Ann. N. Y. Acad. Sci., viii, 733. 
Family ELATERIDÆ.

Tharops Castner.

ruficornis Say. Coleop. Ind., 703.

Adelocera Latreille.

discoidea Weber. Coleop. Ind., 7 5 .

marmorata Fabricius. Ibid., 714 .

obtecta Say. Ibid., 7I5.

Alaus Eschscholtz.

oculatus Linnæus. Eyed elater or click-beetle. Coleop. Ind., 7 I7. Monocrepidius Eschscholtz.

auritus Herbst. Coleop. Ind., 728.

bella Say. Ibid., 728.

vespertinus Fabricius. Ibid., 728.

Campylus Fischer.

denticornis Kirby. Coleop. Ind., 759.

Limonius Eschscholtz.

basillaris Say. Coleop. Ind., 758. (C.C.)

confusus LeConte. Ibid., $75^{8}$.

ectypus Say. agonus. Ibid., 756 .

griseus Beauvois. Ibid., 757 .

nimbatus Say. Ann. Lyc. Nat. Hist. N. Y., i, 255. (C.C.)

plebejus Say. Coleop. Ind., 758.

quercinus Say. Ibid., $75^{8}$.

stigma Herbst. Kafer, x, 286. (A.B.C.)

Leptoschema Horn.

discalceatum Say. Coleop. Ind., 761.

Athous Eschscholtz.

acanthus Say. Coleop. Ind., 760. (C.C.)

brightwelli Kirby. Ibid., 759 .

cucullatus Say. Ibid., 870.

Ludius Eschscholtz.

Corymbites Latreille.

æripennis Kirby. Trans. Anı. Phil. Soc., x, 439.

æthiops Herbst. Coleop. Ind., 766.

cylindriformis Herbst. Ibid., 765.

hieroglyphicus Say. Ibid., 766.

inflatus Say. Ibid., 767 . 
nigricornis Panzer. metallicus. Faun. Germ., 1xi, 5. pyrrhos Herbst. Coleop. Ind., 767 .

splendens Ziegler. Ibid., 768.

sulcicollis Say. Ibid., 768. (C.C.)

tarsalis Melsheimer. Ibid., 765.

tessellatus Linnæus. Syst. Nat., (Ed. Io) 406.

triundulatus Randall. Trans. Am. Phil. Soc., x, 477.

Hemicrepidius Germar.

bilobatus Say. Coleop. Ind., 770.

Asaples Kirby.

decoloratus Say. Ibid., 770.

memnonius Herbst. Ibid., 770.

Cryptohypnus Eschscholtz

abbreviatus Say. Coleop. Ind., 722.

Mielanactes LeConte.

morio Fabricius. Coleop. Ind., 772. (G.D.)

piceus DeGeer. Ibid., $77 \mathrm{I}$.

Estodes LeConte.

tenuicollis Randall. Trans. Am. Phil. Soc., x, 424.

Parallelosthetus Schwarz.

attenuatus Say. Ludius. Coleop. Ind., 736.

Trichopherus Mulsant and Godart.

abruptus Say. Ludius. Coleop. Ind., 737.

Agriotes Eschscholtz.

oblongicollis Melsheimer. Coleop. Ind., 74I.

Oxygonus LeConte.

obesus Say. Coleop. Ind., 769 .

Dolopius Eschscholtz.

lateralis Eschscholtz. Scricosomus. Coleop. Ind., 742.

Sericus Eschscholtz.

silaceus Say. Sericosomus. Coleop. Ind., 763. (C.C.)

viridanus Say. Sericosomms. Compl. writ., ii, 603.

Glyphonyx Candeze.

recticollis Say. Coleop. Ind., $7+3$.

testaceus Melsheimer. Ibid., $7+3$.

Eolus Eschscholtz.

dorsalis Say. Drasterius elegans. Coleop. Ind., 735. 
Elater Linnæus.

areolatus Say. obliquus. Coleop. Ind., 734.

collaris Say. Ibid., 734 .

linteus Say. Ibid., 732.

manipularis Candeze. Ibid., 732.

militaris Harris. Trans. Am. Ent. Soc., xii, ro. (A.B.C.)

mixtus Herbst. Trans. Am. Phil. Soc., x, 469.

mixtus var. fusculus LeConte. Ibid., 468.

nigricans Germar. Ibid., 468.

nigricollis Herbst. Coleop. Ind., 732.

nigrinus Paykull. Trans. Am. Ent. Soc., xii, Io. (F.K.)

pullus Germar. Ibid., xii, Io.

rubricollis Herbst. Coleop. Ind., 733.

rubricus Say. Ibid., 733.

sanguinipennis Say. Ibid., 734.

semicinctus Randall. Trans. Am. Phil. Soc., x., 465. (A.B.C.)

vitiosus LeConte. Ibid., 465 .

Megapenthes Kiesenwetter.

limbalis Herbst. Coleop. Ind., 736.

Melanotus Eschscholtz.*

americanus Herbst. Coleop. Ind., 747.

communis Gyllenhal. Ibid., 750.

cribulosus LeConte. Trans. Am. Phil. Soc., ser. 2, x, 479.

decumanus Erichson. Coleop. Ind., 749.

dubius LeConte. Trans. Am. Phil. Soc., Ser. 2, x, 479.

exuberans LeConte. Ibid., Ser. 2, x, 479.

fissilis Say. Coleop. Ind., $75^{\circ}$.

insipiens Say. Compl. Writ., ii, 622.

parumpunctatus Melsheimer. Coleop. Ind., 754

pertinax Say. Ibid., 755 .

sagittarius LeConte. Ibid., $75 \mathrm{r}$.

tenax Say. Ibid., 755 .

trapezoideus LeConte. Ibid., 752.

verberans LeConte. Ibid., 753 .

Cardiophorus Eschscholtz.

cardisce Say. Coleop. Ind., 720.

convexus Say. Ibid., 72r.

* See also appendix. 
gagates Erichson. Ibid., $72 \mathrm{I}$. robustus LeConte. Ibid., $72 \mathrm{I}$.

sp.

curiatus Say. Coleop. Ind., 721.

Horistonotus Candeze.

simplex Say. Trans. Am. Ent. Soc., xii, $3^{8 .}$

Family MELASIDE.

(EUCNEMID正.)

Melasis Olivier.

pecticornis Melsheimer. Coleop. Ind., 703.

Isorhipis Lacordaire. Tharops.

ruficornis Say. Coleop. Ind., 7o3.

\section{Deltometopus Bonvouloir.}

amœnicornis Say. Coleop. Ind., 704.

Entomophthalmus Bonvouloir.

rufiolus LeConte. Coleop. Ind., 709.

Family THROSCIDA or TRIXAGID无.

Throscus Latreille.

carinicollis Schaffer. Trans. Am. Ent. Soc., xliii, 22. chevrolati Bonvouloir. Coleop. Ind., 776.

Family BUPRESTID E.

Acmæodera Eschscholtz.

tubulus Fabricius. culta. Coleop. Ind., 794.

Chalcophora Solier.

fortis LeConte. Trans. Am. Phil. Soc., ii, I9I.

liberta Germar. Coleop. Ind., 7So.

virginiensis Drury. Ibid., 7So.

Dicerca Eschscholtz.

americana Herbst. Kafer, ix, 107.

caudata LeConte. Coleop. Ind., 7 SI.

divaricata Say. Ibid., 78I.

lurida Fabricius. Ibid., 782.

obscura Fabricius. Ibid., 782 .

prolongata LeConte. Trans. Am. Phil. Soc., xii, I94.

pugionata Germar. Coleop. Ind., $78 \mathrm{I}$. 
punctulata Schonherr. Syn. Ins., 3, I23.

tenebrosa Kirby. Coleop. Can., 348.

Melanophila Eschscholtz.

acuminata DeGeer. appendiculata. longipes. Coleop. Ind., 786.

fulvoguttata Harris. Entomological Correspondence, $35^{8}$.

Anthaxia Eschscholtz.

quercata Fabricius. cyanella. Coleop. Ind., 787. (F.K.)

viridifrons Laporte. Ibid., 787 .

Chrysobothris Eschscholtz.

azurea LeConte. Coleop. Ind., 79I.

dentipes Germar. Ibid., 78S. (K.F.C.)

femorata Fabricius. Flat-headed apple borer. Ibid., 789.

femorata var. quadriimpressa. Laporte and Gory. iv-impressa. Mon. Bupr., 2, 48.

floricola Gory. Coleop. Ind., 790.

harrisii Hentz. Jour. Acad. Nat. Sci. Phila., v, 373.

lesueuri Laporte and Gory. misella. Mon. Bupr., 2, 49.

pusilla Laporte and Gory. Coleop. Ind., 79r.

scabripennis Laporte and Gory. Mon. Bupr., 2, 53. (K.F.C.)

sexsignata Say. Coleop. Ind., 791.

Actenodes Lacordaire.

acornis Say. Coleop. Ind., 792.

Eupristocerus Deyrolle.

cogitans Weber. Coleop. Ind., 796.

Agrilus Stephens.

acutipennis Mannerheim. Coleop. Ind., 800.

arcuatus Say. Ibid., 799.

arcuatus var. obliquus LeConte. Trans. Am. Phil. Soc., ii, 243 .

auricomus Frost. Can. Ent., xliv., 250. (A.B.C.)

bilineatus Veber. Coleop. Ind., 800.

cephalicus LeConte. Can., Ent., xliv., 249.

champlaini Frost. Ibid., xliv., 245.

defectus LeConte. Trans. An1. Phil. Soc., ii, 23 .

egenus Gory. Coleop. Ind., 804.

horni Kerremans. blanchardi. Ibid., 8ог.

juglandis Knull. Ent. News, xxxi, 7.

lateralis Say. Coleop. Ind., 798.

masculinus Horn. Ibid., 799. 
No. 3i.] insects of CONNeCticut : COLEOpterA.

obsoletoguttata Gory. Ibid., 802.

otiosus Say. Ibid., 798.

politus Say. Ibid., 8or.

ruficollis Fabricius. Ibid., 798.

vittaticollis Randall. Ibid., Soo. (A.H.Me.)

Pachyscelus Solier.

ovatus Say. larigatus. Coleop. Ind., 807. purpureus Say. Ibid., 8o7.

Brachys Solier.

ærosa Melsheimer. Coleop. Ind, So6.

æruginosa Gory. Ibid., So6.

ovata Weber. Ibid., So6.

Taphrocerus Solier.

gracilis Say. Coleop. Ind., 805.

Mastogenius Solier.

subcyaneus LeConte. Coleop. Ind., 795.

Family PSEPIENIDE.

Psephenus Haldeman.

lecontei LeConte. Coleop. Ind., 677.

Family DRIOPIDA.

Dryops Olivier.

fastigiatus Say. Coleop. Ind., 679.

lithophilus Germar. Ibid., 678 .

productus LeConte. Trans. Am. Ent. Soc., iii, 32.

Family. ELMIDA.

Macronychus Mïller.

glabratus Say. Coleop. Ind., 682.

Stenelmis Dufour.

crenatus Say. Coleop. Ind., 68I.

Elmis Latreille.

elegans LeConte. Trans. Ant. Ent. Soc., iii, $3^{6 .}$

foveatus LeConte. Ibid., v, 53.

latiusculus LeConte. Ibid., iii, 37.

quadrinotatus Say. Coleop. Ind., 679.

sp.

sp. 
Family HETEROCERIDÆ.

Heterocerus Fabricius.

tristis Mannerheim. Coleop. Ind., 685 .

ventralis Melsheimer. Ibid., 684. (C.C.)

\section{Family GEORYSSID E}

Georyssus Latreille.

pusillus LeConte. Coleop. Ind., 675 .

\section{Family DASCILLID Æ.}

Eurypogon Motschulsky.

niger Melsheimer. Coleop. Ind., 689.

Ptilodactyla Latreille.

serricollis Say. Coleop. Ind., 69I.

Eucinetus Germar.

terminalis LeConte. Coleop. Ind., 692.

Ectopria LeConte.

nervosa Melsheimer. Coleop. Ind., 692.

\section{Family HELODIDÆ (or CYPHONID无.)}

Helodes Latreille.

pulchella Guérin-Ménéville. Coleop. Ind., 694. (C.C.) thoracia Guérin-Ménéville, Ibid., 694.

Scirtes Illiger.

orbiculatus Fabricius. Coleop. Ind., 694. tibialis Guérin-Ménéville. Ibid., 694. (F.K.)

Cyphon Paykull.

collaris Guérin-Ménéville. Coleop. Ind., 696. (C.C.)

obscurus Guérin-Ménéville. Ibid., 695.

ruficollis Say. Ibid., 695.

variabilis Thunberg. Ibid., 696 .

\section{Family BYTURIDÆ.}

Byturus Latreille.

unicolor Say. Raspberry fruit worm. Raspberry byturus Coleop. Ind.. $5^{89}$. 
Family DERMESTID Æ.

Dermestes Linnæus.

caninus Germar. Coleop. Ind., 590.

lardarius Linnæus. Larder beetle. Ham beetle. Coleop. Ind., 59I: Bur. Ent., Bull. + new ser. I07.

talpinus Mannerheim. Coleop. Ind., 59I. (G.D.) (K.F.C.)

vulpinus Fabricius. Ibid., 59I.

Attagenus Latreille.

pellio Linneus. Coleop. Ca11., 305. (C.C.)

piceus Olivier. Black carpet beetle. Coleop. Ind., 592: Bur. Ent., Bull. 4 new ser. 6r.

Trogoderma Latreille.

ornatum Say. Coleop. Ind., 593. (C.C.)

tarsale Melsheimer. Ibid., 593.

Cryptorophalum Guérin-Ménéville.

triste LeConte. Coleop. Ind., 594.

Anthrenus Geoffroy.

scrophulariæ Linnæus. Carpet Beetle. "Buffalo bug."

Coleop. Ind., 595: Bur. Ent., Bull. + new ser. 59.

scrophulariæ var. lepidus LeConte. Jour. N. Y. Ent. Soc., viii, I 60.

verbasci Lin11eus. Coleop. Ind., 597.

Family BYRRHID Æ.

Cytilus Erichson.

alternatus Say. sericeus. Coleop. Ind., 673.

Syncalypta Stephens.

spinosa Rossi. Mem. Coleop., iii, I7. (H. E. Smith.)

Percinolus Mulsant.

undatus Melsheimer. murinus. Coleop. Ind., 673.

Byrrhus Linnæus.

americanus LeConte. Coleop. Ind., 673.

Family OSTOMID Æ.

(TROGOSITID E.)

Nemosoma Latreille.

parallelum Melsheimer. Proc. Acad. Nat. Sci. Phila., ii, Io8. 
Tenebroides Piller and Metterpacker.

americana Kirby. Coleop. Ind., 665. (K.F.C.)

bimaculata Melsheimer. Ibid., 665. (C.S.)

corticalis Melsheimer. Ibid., 664.

mauritanica Linnæus. Ibid., 663.

Thymalus Duftschmid.

fulgidus Erichson. Coleop. Can., 733.

Family NOSODENDRONID $\approx$.

Nosodendron Latreille.

unicolor Say. Coleop. Ind., 672.

Family NITIDULID瓜.

Brachypterus Erichson.

urticæ Fabricius. Coleop. Ind., 630.

Cercus Latreille.

abdominalis Erichson. Coleop. Ind., 630.

Carpophilus Stephens.

brachypterus Say. Coleop. Ind., 633 .

dimidiatus Fabricius. Ibid., 632 .

hemipterus Linnæus. Ibid., $\sigma_{32}$.

niger Say. Ibid., 632.

Colopterus Erichson.

Colastus.

maculatus Erichson. Trans. Am. Ent. Soc., vii, 282.

semitectus Say. Coleop. Ind., 634.

trunctatus Randall. Ibid., 634 .

unicolor Say. Ibid., 634.

Conotelus Erichson.

obscurus Erichson. Coleop. Tnd., 635 .

Epuræa Erichson.

avara Randall. Coleop. Ind., 638 .

helvola Erichson. Ibid., 637.

labilis Erichson. Ibid., 640. 
Nitidula Fabricits.

rufipes Linnæus. Coleop. Ind., 64I.

ziczac Say. Ibid., 6+1.

Stelidota Erichson.

geminata Say. Coleop. Ind., 64I.

octomaculata Say. Ibid., 6+i. (M.P.Z.)

Phenolia Erichson.

grossa Fabricius. Coleop. Ind., 642 .

Osmosita Erichson.

colon Linnæus. Coleop. Ind., $6+3$.

Lobiopa Erichson.

undulata Say. Soronia. Coleop. Ind., 643.

Oxycnemis Erichson.

histrina LeConte. Psilopyga. Coleop. Ind., 645. (A.B.C.)

(H.B.K.)

Meligethes Stephen.

æneus Fabricius. brassica Scopoli. Coleop. Ind., 6łt.

Pallodes Erichson.

silaceus Erichson. pallidus. Coleop. Ind., 646.

Cychramus Kugelan.

adustus Erichson. Coleop. Ind., 647. (C.C.)

Cryptarcha Shuckard.

ampla Erichson. Coleop. Ind., 648 .

Glischrochilus Reitter.

$$
\text { Ips. }
$$

confluentus Say. Coleop. Ind., 650.

quadriguttatus Fabricius. fasciatus. Ibid., 649.

sanguinolentus Olivier. Ibid., 649 .

\section{Family RHIZOPHAGIDA.}

Rhizophagus Herbst.

approximatus LeConte? Trans. Am. Ent. Soc., vii, 330.

kipunctatus Say. Coleop. Ind., 651. (C.C.)

minutus Mannerheim. Ibid., 65 I. (C.C.) 
Family MONOTOMIDE.

Europs Wollaston.

pallipennis LeConte. Coleop. Ind., 669. (C.S.)

Bactridium LeConte.

hudsoni Casey. Mem. Coleop., vii, 99.

Family CUCUJID Æ.

Silvanus Latreille.

bidentatus Fabricius. Coleop. Ind., 562.

imbellis LeConte. Ibid., $5^{6} 3$.

planatus Germar. Trans. Amt. Ent. Soc., xi 72. (F.K.)

Oryzæphilus Ganglbauer.

surinamensis Linnæus. Saw-toothed grain weevil. Coleop. Ind., 562 ; Bur. Ent., Bull iv. new ser. I2 I.

Catogenus IVestwood.

rufus Fabricius. Coleop. Ind., 564.

Cucujus Fabricius.

clavipes Fabricius. Coleop. Ind., 565 .

Læmophlæus Laporte.

adustus LeConte. Coleop. Ind., 567. (C.C.)

biguttatus Say. Ibid., 567.

convexulus LeConte. Ibid., 566 .

fasciatus Melsheimer. Ibid., 567 .

pusillus Schonherr. Trans. Am. Ent. Soc., xi. 93.

testaceus Fabricius. Coleop. Ind., 567. (C.C.)

Lathropus Erichson.

vernalis LeConte. Coleop. Ind., 568. (C.C.)

Dendrophagus Schonherr.

glaber LeConte. Coleop. Ind., 566.

Brontes Fabricius.

debilis LeConte. Coleop. Ind., 568. (C.C.)

dubius Fabricius. Ibid., 568. (G.D.)

Telephanus Erichson.

velox Haldeman. Coleop. Ind., 567. 
Family EROTYLID E.

Languria Latreille.

gracilis Newman. Coleop. Ind,, 543.

lecontei Crotch. Ibid., 543.

mozardi Latreille. Clover stem borer. Ibid., $5+2$. tædata LeConte. Jour. N. Y. Ent. Soc., xii. I99.

Acropteroxys Gorham.

lecontei Crotch. Coleop. Ind., 543.

\section{Megalodacne Crotch.}

fasciata Fabricius. Coleop. Ind., 545.

heros Say. Ibid., 545.

Mycotretus Lacordaire."

sanguinipennis Say. Coleop. Ind., 546. (K.F.C.)

Tritoma Fabricius.

angulata Say. Coleop. Ind., 548 .

biguttata Say. Ibid., 548 .

flavicollis LeConte. Ibid., 549.

humeralis Fabricius. Ibid., 547.

thoracica Say. Ibid., 549.

unicolor Say. Ibid., 548 .

Family DERODON'TIDAE.

Laricobius Rosenhauer.

erichsoni Rosenhauer. rubidus. New Spec. N. A. Coleop., 99.

- Family CRYPTOPHAGID $\mathrm{E}$.

Telmatophilus Heer.

americanus LeConte. Coleop. Ind., 573.

Loberus LeConte.

impressus LeConte. Coleop. Ind., 573. (C.C.)

Tomarus LeConte.

pulchellus LeConte. Coleop. Ind., 574.

Antherophagus Latreille.

ochraceous Melsheimer. Coleop. Ind., 574.

* See also appendix. 
Cryptophagus Herbst.

cellaris Scopoli. Coleop. Can., 295. (C.C.)

croceus Zimmerman. Coleop. Ind., 576 .

nodangulus Zimmerman. Ibid., 577.

Cænoscelis Thomson.

ferruginea Sahlberg. Coleop. Ind., 579.

Atomaria Stephens.

curtula Casey. Jour. N. Y. Ent. Soc., viii, I22.

ephippiata Zimmerman. Coleop. Ind., 58 I.

ovalis Casey. Ibid., $5^{81}$.

pusilla Paykull. Ibid., 580. (C.W.L.)

\section{Family MYYETOPHAGID E.}

Mycetophagus Hellwig.

bipustulatus Melsheimer. Coleop. Ind., 584. (C.C.)

flexuosus Say. Ibid., 58.

melsheimeri LeConte. Ibid., $5^{8} 4$.

punctatus Say. Ibid., 584 .

Litargus Erichson.

didesmus Say. Coleop. Ind., 586.

tetraspilotus LeConte. Ibid., 586. (A.B.C.)

Typhæa Stephens.

fumata Linnæus. Coleop. Ind., 587.

$$
\text { Family COLYDIIDÆ. }
$$

Synchita Hellwig.

fuliginosa Melsheimer. Coleop. Ind., $55 \mathrm{I}$.

Bitoma Herbst.

quadriguttata Say. Coleop. Ind., $55^{2}$.

Colydium Fabricius.

lineola Say. Coleop. Ind., 555. (C.S.)

Cerylon Latreille.

castaneum Say. Coleop. Ind., 557.

sticticum Casey. Ann. N. Y. Acad. Sci., 1896, 336.

Philothermus Aubé.

glabriculus LeConte. Coleop. Ind., 557. (C.C.) 
Family LATHRIDIID E.

Lathridius Herbst.

liratus LeConte. Coleop. Ind., 653 .

Enicmus Thomson.

minutus Linnæus. Lathridius. Coleop. Inc., 655.

Cartodere Thomson.

filiformis Gyllenhal. Coleop. Ind., 656.

ruficollis Marshan. publicarius. Trans. Am. Ent. Soc., xxvi, I37. (G.D.)

Corticaria Marsham.

ferruginea Marsham. delcta. Coleop. Ind., $6_{5} 8$.

serrata Payku1l. Ibid., 658 .

Melanophthalma Motschulsky

Corticaria.

cavicollis Mannerheim. Coleop. Ind., 66r.

distinguenda Comolli. Ibid., 660.

picta LeConte. Ibid., 659. (C.C.)

pumila LeConte. Ibid.., 660. (C.C.)

\section{Family MYCETEIDE.}

Mycetæa Stephens.

hirta Marsham. Trans. Ann. Ent. Soc., iv, 362. (G.D.)

Family ENDOAICHIDE.

Endomychus Panzer.

biguttatus Say. Coleop. Ind., 537.

Lycoperdina Latreille.

ferruginea LeConte. Coleop. Ind., $53^{8}$.

Aphorista Gorham.

vittata Fabricius. Coleop. Ind., $53^{8}$.

$$
\text { Mycetina Mulsant. }
$$

perpulchra Newman. Coleop. Ind., 539. (C.C.)

Stenotarsus Perty.

hispidus Herbst. Coleop. Ind., 539. (C.C.)

testacea Ziegler. Mycetina. Trans. Am. Ent. Soc., iv, 360. (G.D.) 
Family PHALACRIDE.

Acylomus Sharp.

ergoti Casey. Coleop. Ind., 500.

Olibrus Erichson.

consimilis Marsham. Eustilbus apicalis. Coleop. Ind., 500. (C.C.)

pallipes Say. Ibid., 499.

semistriatus LeConte. Ibid., 499.

Phalacrus Paykull.

penicillatus Say. Compl. Writ., ii, 230.

politus Melsheimer. Coleop. Ind., 498.

pumilio LeConte. Ibid., 499.

Family COCCINELLIDÆ.

Hyperaspis Redtenbacher.

fimbriolata Nelsheimer. Coleop. Ind., 522.

signata Olivier var. binotata Say. Ibid., 523: Conn. Agr. Expt. Sta., Bull. r8r, 8.

signata var. proba Say. Coleop. Ind., 523.

undulata Say. Ibid., 522.

Brachyacantha Chevrolat.

ursina Fabricius. Coleop. Ind., 520: Conn. Agr. Expt. Sta., Bull. r8r, 9.

Microweisia Cockerell.

misella LeConte. Pentilia. Smilia. Conn. Agr. Expt. Sta., Rept. 1902, 128, Bull. 181, 8: Coleop. Ind., 524.

Scymnus Kugelan.

americanus Mulsant. Coleop. Ind., 528 .

brullei Mulsant. Trans. Am. Ent. Soc., xxii, ror.

collaris Melsheimer. Coleop. Ind., 530.

fraternus LeConte. Ibid., 529.

puncticollis LeConte. Ibid., 530 .

tenebrosus Mulsant. Ibid., 53I.

Delphastus Casey.

pusillus LeConte. Coleop. Ind., 519: Conn. Agr. Expt. Sta., Bu1l. I8I, 9. 
Coccidula Kugelan.

lepida LeConte. Coleop. Ind., 533: Conn. Agr. Expt. Sta., Bull. I8I, 6.

\section{Fsyllobora Chevrolat.}

viginti-maculata Say. 20-maculata. Twenty-spotted lady beetle. Coleop. Ind., 517: Conn. Agr. Expt. Sta., Bull. I8I, I2.

Anisosticta Duponchel.

seriata Melsheimer. Namia. Jour. N. Y. Ent. Soc., vii, 75: Conn. Agr. Expt. Sta., Bull. i8 i, 22.

strigata Thunberg. Coleop. Ind., 5io: Conn. Agr. Expt. Sta., Bull. ISI, 23.

\section{Ceratomegilla Crotch.}

fuscilabris Mulsant, Megilla. Spotted lady beetle. Erroneously maculata DeGeer, a tropical species. Coleop. Ind., 5IO: Conn. Agr. Expt. Sta., Bull. i8I, 2 I.

\section{Hippodamia Mulsant.}

convergens Guérin-Ménéville. Coleop. Ind., 5I2: Conn. Agr. Expt. Sta., Bull. isi, is.

glacialis Fabricitus. Coleop. Ind., 512: Conn. Agr. Expt. Sta., Bull. I8I, 20.

parenthesis Say. Coleop. Ind., 5II: Conn. Agr. Expt. Sta., - Bull. I8I, 20.

tredecempunctata Linnæus. I3-punctata. Thirteen-spotted lady beetle. Coleop. Ind., 5I I : Conn. Agr. Expt. Sta., Bu1l. ISI, 20.

\section{Coccinella Linnæus.}

monticola Mulsant. Jour. N. Y. Ent. Soc., vii, 89.

novemnotata Herbst. 9-notata. Nine-spotted lady beetle. Coelop. Ind., 514: Conn. Agr. Expt. Sta., Bull. I8 I, I5. perplexa Melsheimer. trifasciata. Jour. N. Y. Ent. Soc., vii, 89: Conn. Agr. Expt. Sta., i8 i, i6.

transversoguttata Fabricius. 5-notata. Five-spotted lady beetle. Jour. N. Y. Ent. Soc, vii, 89: Conn. Agr. Expt Sta., Bull. г8 г, I6.

\section{Cycloneda Crotch.}

munda Say. Red lady beetle. Erroneously Coccinclla sansuinca, a tropical species. Coleop. Ind., 515: Conn. Agr. Expt. Sta., Bu1l. ISI, I 7 . 
Adalia Mulsant.

bipunctata Linnæus. Two-spotted lady beetle. Coleop. Ind., 515: Conn. Agr. Expt. Sta., Bull. I8I, I7.

frigida Schneider. Coleop. Ind., 5 I6.

frigida var. opthalmica Mu1sant. Ibid., 5I6. (K.F.C.)

humeralis Say. bipunctata (or frigida) var. humeralis. Jour. N. Y. Ent. Soc., vii, 85 : Conn. Agr. Expt. Sta., Bull. I81, I8.

Anisocalvia Crotch.

duodecimmaculata Gebler. I2-maculata. Jour. N. Y. Ent. Soc., vii, 97.

Anatis Mulsant.

quindecimpunctata Olivier. I5-punctata. Fifteen-spotted lady beetle. Coleop. Ind., 516: Conn. Agr. Expt. Sta., Rept. 1909-10, 705. Bu1l. I8г, I3.

quindecimpunctata var. mali Say. Coleop. Ind., 5 I6. (K.F.C.)

Neomysia Casey.

pullata Say. Mysia. Jour. N. Y. Ent. Soc., vii, 99.

\section{Chilocorus Leach.}

bivulnerus Mulsant. Twice-stabbed lady beetle. Conn. Agr. Expt. Sta., Rept. 1902, I27: Bull. I65, I5: Bull. I81, I0: Coleop. Ind., 5 r8.

Harmonia Authors not Mulsant.

picta Randall. Clcis. Jour. N. Y. Ent. Soc., xi, 205 : Conn. Agr. Expt. Sta., Bull. ISI, I4.

similis Randall. Clcis. Anisocalzia. Jour. N. Y. Ent. Soc., xi, 206.

Epilachna Chevrolat.

borealis Fabricius. Squash lady beetle. Contn. Agr. Expt. Sta., Rept. 1908, 810: Bull. I81, I I : Coleop. Ind., 532.

\section{Family ALLECULIDA.}

\section{(CISTELIDA.)}

Lobopoda Solier (or Allecila Fabricius). atra Say. Ann. N. Y. Acad. Sci., vi, 82. (T.L.C.)

\section{Hymenorus Mulsant.}

niger Melsheimer. nigrinus. Coleop. Ind., I274. pilosus Melsheimer. Ibid., I274: Amm. N. Y. Acarl. Sci., vi, 93. 
Pseudocistela Crotch.

brevis Say. Coleop. Ind., 1276.

Isomira Mulsant.

quadriseriata Couper. Coleop. Ind., I277.

sericea Say. Cistela. Ibid., x 277.

Mycetochara Berthold.

binotata Say. My'cetochares. Coleop. Ind., I28I.

Chromatica LeConte.

amœna Say. Coleop. Ind., I 276 . (A.B.C.) (H.B.K.)

Capnochroa LeConte.

fuliginosa Melsheimer. Coleop. Ind., I 282.

Androchirus LeConte.

erythropus Kirby. Coleop. Ind., I $28_{3}$.

\section{Family TENEBRIONID更}

Hopatrinus Latreille.

minimus Beauvois. notus. Coleop. Ind., I252.

Blapstinus Latreille.

interruptus Say. Coleop. Ind., I253.

metallicus Fabricius. Ibid., I 253.

mœstus Melsheimer. Ibid., 1252.

Phaleria Latreille.

testacea Say. Long's Exp., ii, 280.

Bolitotherus Candeze.

cornutus Panzer. bifurcus. Coleop. Ind., I267.

Bolitophagus Illiger.

corticola Say. Coleop. Ind., I267.

Diaperis Geoffroy.

maculata Olivier. Coleop. Ind., I26r.

Hoplocephala Laporte.

bicornis Olivier. Arrhenoplita. Coleop. Ind., г 26г.

Platydema Laporte.

ellipticum Fabricius. Coleop. Ind., I 263. (C.C.)

erythrocera Laporte. Ibid., I263.

excavatum Say. Ibid., I262.

ruficorne Sturm. Ibid., I263.

subcostatum Laporte. Ibid., I264.

* See also appendix.

I7 
Tribolium MacLeay.

confusum Duval. Confused flour beetle. Coleop. Ind., I255:

Bur. Ent., new ser., Bull, iv, II3.

Diœdus LeConte.

punctatus LeConte. Coleop. Ind., I256.

Palorus Mulsant.

depressus Fabricius, melinus. Illus. Besttabl. Kaf. Deutsch. 74I.

Uloma Leporte.

impressa Melsheimer. Coleop. Ind., I257.

Alphitobius Stephens.

diaperinus Panzer. Coleop. Ind., I257. (C.C.)

piceus Olivier. Illus. Besttabl. Kaf. Deutsch., 748.

Hypophlœus Fabricius.

parallelus Melsheimer. Coleop. Ind., I266. (G.D.)

tenuis LeConte. Proc. Am. Phil. Soc., xvii, 422.

\section{Scotobates Horn.}

calcaratus Fabricits. Coleop. Ind., I248.

Xylopinus LeConte.

ænescens LeConte. Coleop. Ind., I249.

saperdioides Olivier. Ibid., I249. (G.D.)

Hoplandrus LeConte.

fulvipes Herbst. femoratus. Coleop. Ind., I 248

Iphthimus Truqui.

opacus LeConte. Coleop. Ind., I247. (G.D.)

Alobates Motschulsky.

pennsylvanica DeGeer. Nyctobates. Coleop. Ind., I247.

Upis Fabricius.

ceramboides Linnæus. Ent. Syst., ii, 5I5. (G.D.)

Tenebrio Linneus.

molitor Linnæus. Yellow meal worm. Bur. Ent, new ser.

Bull. iv, II6: Coleop Ind., I250.

obscurus Fabricius. Dark meal worm. Bur. Ent., new ser. Bull.

iv, II 7 : Coleop. Ind., I 250.

picipes Herbst. tencbroides. Coleop. Ind., I250.

Helops Fabricius.

æreus Germar. Coleop. Ind., I268.

Tarpela Bates.

micans Fabricius. Coleop. Ind., I 268. 
Meracantha Kirby.

contracta Beauvois. Coleop. Ind., I 269.

Strongylium Kirby.

tenuicolle Say. Coleop. Ind., I270.

Family LAGRIID玉.

Arthromacra Kirby.

ænea Say. Coleop. Ind., I284.

Statira Latreille.

gagatina Melsheimer. Coleop. Ind., I 285.

Family MELANDRYIDE.*

Penthe Newman.

obliquata Fabricius. Coleop. Ind., I290.

pimelia Fabricius. Ibid., r290. (G.D.)

Synchroa Newman.

punctata Newman. Coleop. Ind., I290.

Eustrophinus Seidletz."

bicolor Say. Eustrophus. Trans. Am. Ent. Soc., xv. 34; Coleop. Ind., I292.

Holostrophus Horn.

bifasciatus Say. Coleop. Ind., r293. (G.D.)

Orchesia Latreille.

castanea Melsheimer. Coleop. Ind., 1294.

gracilis Melsheimer. Ibid., I294. (F.K.)

Melandrya Fabricitus.

striata Say. Coleop. Ind., r296.

Emmesa Newman.

connectens Newman. Ent. Nag., 5, 376.

labiata Say. Coleop. Ind., I296.

Dircæa Fabricius.

quadrimaculata Say. liturata. Coleop. Ind., I298.

Hypulus Paykull.

concolor LeConte. Coleop. Ind., I298. (C.C.)

* See also appendix. 
Symphora LeConte.

rugosa Haldeman. Coleop. Ind., I299. (C.C.)

Canifa LeConte.

pallipes Melsheimer. Coleop. Ind., Izor.

Mycterus Clairville.

scaber Haldeman. Trans. Am. Ent. Soc., vii. 337.

\section{Family PTINIDÆ.}

Ptinus Linnæus.

fur Linnæus. Coleop. Ind., 865. (K.F.C.)

villiger Reitter. Ibid., 865 .

\section{Family ANOBIIDE.}

Xestobium Motschulsky.

rufovillosum DeGeer. tessellatum. Coleop. Ind., 868.

Ernobius Thomson.

mollis Linnæus. Coleop. Ind., 868.

Sitodrepa Thomson.

panicea Linnæus. Drug-store beetle. Coleop. Ind., 870.

Trichodesma LeConte.

gibbosa Say. Coleop. Ind., 87I.

klagesi Fall. Ibid., 87I. (A.B.C.)

Hadrobregmus Thomson.

carinatus Say. Coleop. Ind., 872.

Trypopitys Redtenbacher.

sericeus Say. Coleop. Ind., 872.

Xyletinus Latreille.

peltatus Harris.' Coleop. Ind., 874 .

Lasioderma Stephens.

testaceum Duftschmid. serricorne. Cigarette beetle. Coleop. Ind., 875: Rept. Conn. Agr. Expt. Sta., I906. 278.

Dorcatoma Herbst.

setulosum LeConte. Coleop. Ind., 883. (C.C.)

Cænocara Thomson.

oculata Say. Coleop. Ind., 884. 


\section{Family BOSTRICHID蚱.}

\section{Endecatomus Mellié.}

reticulatus Herbst. Coleop. Ind., 887.

rugosus Randall. Ibid., 887.

$$
\text { Xylobiops Casey. }
$$

basilare Say. Sino.rylon. Coleop. Ind., 888.

\section{Lichenophanes Lesne.}

armiger LeConte. Bostrichus. Coleop. Ind., 888.

\section{Bostrichus Geoffroy.}

angustus Casey. Jour. N. Y. Ent. Soc., vi, 72.

bicornis Weber. Coleop. Ind., 889. (C.C.)

\section{Dinoderus Stephens.}

rugosus Oliver. porcatus LeConte. Coleop. Ind., 890. punctatus Say. Ibid., 890.

\section{Family LYCTID Æ.}

\section{Lyctus Fabricius.}

opaculus LeConte. Coleop. Ind., 892.

linearis Goeze. striatus. unipunctatus. Powder post beetle.

Coleop. Ind., 89r : Bur. Ent., Circ. 55.

\section{Famîly SPHINDIDÆ.}

\section{Sphindus Chevrolat.}

americanus LeConte. Coleop. Ind., 902. (C.C.)

$$
\text { Family CISID Æ. }
$$

\section{Orthocis Casey.}

punctulatus Mellié. Coleop. Ind., 899.

\section{Octotemnus Mellié.}

denudatus Casey. Jour. N. Y. Ent. Soc., vi, 91.

Ceracis Mellié.

sallei Mellié. Coleop. Ind., 900.

\section{Cis Latreille.}

dubius Mellié. Ann. Soc. Ent. Fr., 2d. vi, 272.

fuscipes Mellié. Coleop. Ind., 898.

sp. 
Xestocis Casey.

levettei Casey. Coleop. Ind., 899.

Ennearthron Mellié.

thoracicorne Ziegler. Coleop. Ind., 900.

sp.

Family SCARAB ÆID Æ.

Canthon Hoffman.

lævis Drury. Coleop. Ind., 9I4.

nigricornis Say. Ibid., 9I4. (G.D.)

Chœridium Lepeletier.

histeroides Weber. Coleop. Ind., 9I 5.

Copris Geoffroy.

minutus Drury. Coleop. Ind., 916.

tullius Olivier. anaglypticus. Ibid., gr6.

Pinotus Erichson.

carolina Linnæus. Copris. Coleop. Ind., 916.

Phanæus MacLeay.

carnifex Linnæus. Coleop. Ind., 9r7.

Onthophagus Latreille.

hecate Panzer. Coleop. Ind., 9I8.

janus Panzer. Ibid., 919.

janus var. orpheus Panzer. Ibid., 9I9. (A.H.Me.)

janus var. striatulus Beatvois. Ibid., 919.

pennsylvanicus Harold.' Ibid., 920.

Aphodius Illiger.*

congregatus Mann. Trans. Am. Ent. Soc., xiv. I2. (C.C.)

distinctus Müller. inquinatus Herbst. Coleop. Ind., 933.

femoralis Say. Ibid., 934. (K.F.C.)

fimetarius Linnæus. Ibid., 930.

fossor Linnæus. Trans. Am. Ent. Soc., xiv. 4.

granarius Linnæus. Coleop. Ind., 93I.

terminalis Say? Ibid., 933.

vittatus Say.. Ibid., 93I.

Atænius Harold.

abditus Haldeman. Coleop. Ind., 926.

cognatus LeConte. Ibid., 926.

gracilis Melsheimer. Ibid., 925.

* See also appendix. 
Eucanthus IVestwood.

lazerus Olivier. Bolboccras. Coleop. Ind., 937.

Bolbocerasoma Schaffer.

farctum Fabricius. Bolboceras. Coleop. Ind., 937.

Odontæus Megerle.

cornigerus Melsheimer. Coleop. Ind., $93^{8 .}$ (C.C.)

filicornis Say. Ibid., 93S. (C.C.)

Geotrupes Fabricius.

balyi Jekel. Trans. Am. Ent. Soc., viii. I45.

blackburnii Fabricius. Coleop. Ind., 939.

egeriei Germar. Trans. Am. Ent. Soc., viii. I45.

hornii Blanchard. Psyche v. Io7.

semiopacus Jekel. Coleop. Ind., 939.

splendidus Fabricius. Ibid., 939.

Amphicoma Latreille.

vulpina Hentz. Trans. Am. Ent. Soc., x. I20.

Trox Fabricius.

æqualis Say. Coleop. Ind., 946.

capillaris Say. Ibid., 945.

erinaceus LeConte. Ibid., 944. (C.C.)

foveicollis Harris. Trans. Am. Ent. Soc., v, Io.

insularis Chevrolat. Coleop. Ind., 945.

scaber Linnzens. Ibid., 946.

sordidus LeConte. Ibid., 945.

terrestris Say. Ibid., 946.

unistriatus Beaurois. Ibid., 945.

Serica Mac Leay.

cucullata Dawson. Jour. N. Y. Ent. Soc., xxvii, 34. sericea Illiger. Coleop. Ind., $95^{8}$.

tristis LeConte. Ibid., $95^{8}$.

trociformis Burmeister. Ibid., 958.

vespertina Gyllenhal. Ibid., 956.

\section{Diplotaxis Kirby.}

atlantis Fall. Trans. Am. Ent. Soc., xxxv. 44.

liberta Germar. Coleop. Ind., 954.

sordida Say. Ibid., 955.

tristis Kirby. Ibid., 954. (F.K.) 
Phyllophaga Harris.

Lachnosterna Hope.

May or June Beetles.

anxia LeConte. dubia. insperata. Coleop. Ind., 970. (J.K.L.)

crenulata Frohlich. Ibid., 979.

drakei Kirby. grandis. Ibid., 970.

fœrsteri Burmeister. nova. Ibid., 972.

fraterna Harris. Ibid., 972.

fusca Frohlich. Ibid., 968.

gracilis Buirmeister. Ibid., 964.

hirsuta Knoch. Ibid., 974. (A.B.C.)

hirticula Knoch. Ibid., 976.

ilicis Knoch. ciliata. Ibid., 978.

marginalis LeConte. Ibid., 970. (A.B.C.)

micans Knoch. Ibid., 967. (C. W. F. Wagner.)

tristis Fabricius. Ibid., 979.

Polyphylla Harris.

variolosa Hentz. Trans. Am. Ent. Soc., ix, 75. (G.D.) (J.K.L.)

Dichelonyx Harris.

albicollis Burmeister. Coleop. Ind., 953.

diluta Fall. Ibid., 952.

elongata Fabricius. Ibid., 952.

testacea Kirby. Ibid., 951. (K.F.C.)

Hoplia Illiger.

equina LeConte. Trans. Am. Ent. Soc., viii. 193.

limbata LeConte. modesta. Coleop. Ind., 950.

trifasciata Say. Ibid., 949.

Macrodactylus Latreille.

subspinosus Fabricius. Rose chafer. Rose beetle. Bur. Ent., Circ. I I : Rept. Conn. Agr. Expt. Sta., I9r6, i I r.

Anomala Samouelle.

binotata Gyllenhal. Coleop. Ind., 982: Rept. Conn Agr. Expt.

Sta., 1902, I 76.

Hemispilota Casey.

lucicola Fabricius. Anomala. Coleop. Ind., 985. 
Pelidnota MacLeay.

punctata Linnæus. Spotted grape-vine beetle. Coleop. Ind., 986.

Cotalpa Burmeister.

lanigera Linnæus. Goldsmith beetle. Coleop. Ind., 987.

Dyscinetus Harold.

trachypygus Burmeister. Coleop. Ind., 990.

Ligyrus Burmeister.

gibbosus DeGeer. Coleop. Ind., 991.

Ligyrodes Casey.

relictus Say. Coleop. Ind., 992.

Aphonus LeConte.

castaneus Melsheimer. Mem. Coleop., vi, 220.

tridentatus Say. Coleop. Ind., 992. (G.D.)

Xyloryctes Hope.

satyrus Fabricius. Coleop. Ind., 992.

Cotinis Burmeister.

nitida Linnæus. Allorhina. Coleop. Ind,, 996.

Euphoria Burmeister.

fulgida Fabricius. Coleop. Ind., 998.

inda Linnæus. Bumble flower beetle. Ibid., 998.

\section{Stephanucha Burmeister.}

areata Fabricius. Coleop. Ind., 997.

Cremastocheilus Knoch.

canaliculatus Kirby. Coleop. Ind., IOoO.

castaneæ Knoch. Ibid., I000.

harrisii Kirby. Ibid., Iooo. (C.C.)

variolosus Kirby. Ibid., 999.

Osmoderma Lepeletier.

eremicola Knoch. Coleop. Ind., IOOI.

scabra Beauvois. Ibid., I002.

Gnorimella Casey.

maculosus Knoch. Gnorimus. Coleop. Ind., I002.

Trichionotus Casey.

affinis Gory. Coleop. Ind., 1004.

piger Fabricius. Ibid., I0O3.

Valgus Scriba.

squamiger Beaurois. Coleop. Ind., 1005. 
Family LUCANIDÆ.

Lucanus Linnæus.

capreolus Linnæus. dama. Stag beetle. Coleop. Ind., 905.

Dorcus MacLeay.

parallelus Say. Coleop. Ind., 906.

Platycerus Geoffroy.

quercus Weber. Coleop. Ind., 907.

Ceruchus MacLeay.

piceus WVeber. Coleop. Ind., 907. (G.D.)

Family PASSALID Æ.

Passalus Fabricius.

cornutus Fabricius. Coleop. Ind., 908.

Family CERAMBYCIDÆ.

Parandra Latreille.

brunnea Fabricius. Coleop. Ind., I007.

Derobrachus Serville.

brunneum Fœrster. Orthosona. Lesser Prionus. Coleop. Ind., IOII.

Prionus Geoffroy.

laticollis Drury. Broad-horned Prionus. Coleop. Ind., IoI I. pocularis Dalman. Ibid., Ioi2. (A.H.Me.)

Distenia Serville.

undata Olivier. Coleop. Ind., IO43.

Smodicum Haldeman.

cucujiforme Say. Coleop. Ind., ioi6.

Asemum Eschscholtz.

mœstum Haldeman. Coleop. Ind., IOI4.

Tetropium Kirby.

cinnamopterum Kirby. Colcop. Ind., IOI5.

Criocephalus Mutsant.

argestis Kirby. Colcop. Ind., IOI 5.

obsoletus Randall. Ibid., Iог 5.

Eme Nervman.

rigida Say. Bull. Brook. Ent. Soc., vii, ir6. 
Tylonotus Haldeman.

bimaculatus Haldeman. Coleop. Ind., I026.

Stromatium Serville.

pubescens Haldeman. Coleop. Ind, 1022.

Chion Newman.

cinctus Drury. Coleop. Ind., I022. (A.H.Me.)

Romaleum White.

atomarium Drury. Coleop. Ind., I023.

rufulum Haldeman. Ibid., ro23.

Hypermallus Lacordaire.

villosum Fabricius. parallchtm. Twig pruner. Coleop. Ind., 1025.

Elaphidion Serville.

mucronatum Say. Coleop. Ind., I024.

Pseudibion Casey.

unicolor Randall. Coleop. Ind., Ioz6.

\section{Heterachthes Newman.}

ebenus Newman. Mem. Coleop., iii, 307.

quadrimaculatus Haldeman. Coleop. Ind, I027.

Obrium Serville.

rufulum Gahan. rubrum. Coleop. Ind., I028.

Rhagium Fabricius.

lineatum Olivier. Coleop. Ind., Iot8.

\section{Centrodera LeConte.}

decolorata Harris. Colcop. Ind., IO48. (K.F.C.) picta Haldeman. Ibid., Iot8.

\section{Stenocorus Fabricius.}

trivittatus Say. To.rotus. Coleop. Ind., r047.

Hapalosalia Casey.

vibex Newman. Leptura. Coleop. Incl., I059. (F.K.)

Acmæops LeConte.

directa Newman. Coleop. Ind., I050.

thoracica IJaldeman. Ent. Am., vi, I04.

\section{Gaurotes LeConte.}

cyanipennis Say. Coleop. Ind., I050.

Anthophilax LeConte.

malachiticus Haldeman. Ent. Amer., vi, 98. (M.P.Z.) 
Judolia Mulsant.

cordifera Olivier. Ent. Am., vi, I88.

Brachyleptura Casey.

rubrica Say. Coleop. Ind., I057.

Parallelina Casey.

exigua Newman. Coleop. Ind., I056.

Strongalepta Casey.

lineola Say. Coleop. Ind., I055.

pubera Say. Ibid., I059.

vittata Germar. Ibid., I059.

vittata var. Ibid., I059.

Xestoleptura Casey.

octonotata Say. Coleop. Ind., I058.

Strophiona Casey.

nitens Fœrster. zebra. Coleop. Ind., I056.

Leptura Linnæus.

biforis Newman. Mem. Coleop., iv, 27I. (E.L.D.)

canadensis Olivier. Coleop. Ind., I057. (K.F.C.)

emarginata Fabricius. Ibid., I055. (A.B.C.)

hæmatites Newman. Entomologist, 73. (C.W.L.)

mutabilis Newman. Coleop. Can., 623.

vagans Olivier. Coleop. Ind., I057. (K.F.C.)

Typocerus LeConte.

acuticauda Casey. Mem. Coleop., iv, 274. (H.L.J.)

velutinus Olivier. Coleop. Ind., Io53.

Ophistomis Thomson.

Strangalia.

acuminata Olivier. Coleop. Ind., I05 I.

famelica Newman. Ibid., I05I.

luteicornis Fabricius. Ibid., I052.

Encyclops Fabricius.

cæruleus Say. Coleop. Ind., I045. (G.D.)

Desmocerus Serville.

palliatus Fœrster. Coleop. Ind., IO44.

Molorchus Fabricins.

bimaculatus Say. Coleop. Ind., io28. 
Merium Kirby.

proteus Kirby. Coleop. Ind., Ior9. (C.C.)

\section{Anacomis Casey.}

ligneus Fabricius. Hylotrupes. Coleop. Ind., Ioi9.

\section{Callidium Fabricius.}

antennatum Newman. Coleop. Ind., IoIg.

frigidum Casey. Mem. Coleop., viii, 289.

janthimum LeConte. Coleop. Ind., Iorg.

Phymatodes Mulsant.

æreum Newman. Coleop. Ind., IOI9.

amœnus Say. Ibid., IoI8.

dimidiatus Kirby. Ibid., IOI8.

variabilis Fabricius. Ibid., IOIS.

varius Fabricius. Ibid., IоI8.

Cyllene Newman.

caryæ Gahan. pictus. Hickory borer. Coleop. Ind., I033.

robiniæ Fœrster. Locust borer. Ibid., I034: Bur. Ent., Cir., 83.

Arhopalus Serville.

fulminans Fabricius. Coleop. Ind., I035.

Glycobius LeConte.

speciosus Say. Plagionotus. Maple borer. Rept. Conn. Agr. Expt. Sta., 1900. 337: 1907. 336: Coleop. Ind., I034.

Calloides LeConte.

nobilis Harris. Coleop. Ind., ro35.

Xylotrechus Chevrolat.

aceris Fisher. Proc. Wash. Ent. Soc., xviii, 2I5.

colonus Fabricius. Coleop. Ind., IO37.

quadrimaculatus Haldeman. Ibid., IO36. (A.B.C.)

saggitatus Germar. Ibid., I037. (A.B.C.)

undulatus Say. Ibid., I037.

undulatus var, fuscus Kirby. Ibid., I037.

Neoclytus Thomson.

acuminatus Fabricius. crythrocephalus. Coleop. Ind., I038. capræa Say. Ibid., I039.

muricatulus Kirby. Ibid., IO38.

Anthoboscus Chevrolat.

ruricola Olivier. Clytanthus. Coleop. Ind., IO39.

Clytus Laichartig.

marginicollis Laporte. Coleop. Ind., I040. 
Cyrtophorus LeConte.

verrucosus Olivier. Coleop. Ind., Iofo.

Tillomorpha Blanchard.

geminata Haldeman. Coleop. Ind., Io4o. (A.B.C.)

Euderces LeConte.

picipes Fabricius. Coleop. Ind., Io+r.

Stenosphenus Haldeman.

notatus Olivier. Coleop. Ind., I032.

Purpuricenus Serville.

axillaris IJaldeman. Coleop. Ind., Io3I. (A.B.C.)

humeralis Fabricius. Ibid., I030.

Batyleoma Casey.

suturalis Say. Batyle. Coleop. Ind., I0zr.

\section{Cyrtinus LeConte.}

pygmæus Haldeman. Coleop. Ind., roб2. (A.B.C.)

Psenocerus LeConte.

supernotatus Say. Coleop. Ind., I062.

Monohammus Serville.

notatus Drury. confusor. Trans. Am. Ent. Soc., xxiii, Iog.

marmorator Kirby. Ibid., xxiii, I09.

scutellatus Say. Coleop. Ind., I065.

titillator Fabricius. Ibid., I064.

Dorcaschema LeConte.

nigrum Say. Coleop. Ind., Io66. (D.J.C.)

Hetœmis Haldeman.

cinerea Olivier. Coleop. Ind., Io66.

Cacoplia LeConte.

pullata Haldeman. Trans. Am. Ent. Soc., xxiii, I I .

Goes LeConte.

pulchra Haldeman. Coleop. Ind., I067.

pulverulenta Haldeman. Ibid., I067.

tigrina DeGeer. Coleop. Ind., Iо67.

Fgomorphus Haldeman.

decipiens Haldeman. Acanthoderes. Coleop. Ind., Iozo.

Leptostylus LeConte.*

collaris Haldeman. Coleop. Ind., IO7I.

guttata Say. sexguttata. Ibid., Io72.

* See also appendix. 
Astylopsis Casey.

macula Say. Coleop. Ind., I072.

\section{Leiopus Serville.}

alpha Say. Coleop. Ind., I074.

punctatus Haldeman. Ibid., Io74. (C.C.)

variegatus Haldeman. Ibid., Io74.

\section{Lepturges Bates.}

querci Fitch. Coleop. Ind., Iо77.

facetus Say. Ibid., Iо77.

signatus LeConte. Ibid., 1076.

symmetricus Haldeman. Ibid., го76.

Hyperplatys Haldeman.

aspersus Say. Coleop. Ind., Ioz8.

maculatus Haldeman. Ibid., IozS.

\section{Urographis Horn.}

fasciatus DeGeer. Graphisurus. Coleop. Ind., Io79.

Dectes LeConte.

spinosus Say. Coleop. Ind., I075.

Hoplosia Mulsant.

nubila LeConte. Trans. Am. Ent. Soc., xxiii, I34.

Pogonocherus Latreille.

mixtus Haldeman. Coleop. Ind., Io8r.

Encyrus LeConte.

dasycerus Say. Coleop. Ind., Io8ı.

Eupogonius LeConte.

vestitus Say. Coleop. Ind., io82. (A.B.C.) (H.B.K.)

Oncideres Serville.

cingulata Say. Hickory twig-girdler. Fifth Rept.. U. S. Ent. Com., 288.

\section{Saperda Fabricius.}

candida Fabricius. Round-lieaded apple borer. Rept. Conn. Agr. Expt. Sta., I907. 333: N. Y. State Mus., Bull. 74. 23. calcarata Say. Poplar borer. Coleop. Ind., Io86. N. Y. State Mus. Bull. 74. 39 .

concolor LeConte. var. unicolor Joutel. Willow Borer. N. Y.

State Mus. Bull. 74. 74: Coleop. Ind., Io86. 
cretata Newman. Spotted apple tree borer. Coleop. Ind., I088. N. Y. State Mus., Bull. 74. 50. (C.C.)

discoidea Fabricius. Hickory borer. Coleop. Ind., Io89. N. Y. State Mus., Bull. 74. 52.

lateralis Fabricius. Red-edged Saperda. Coleop. Ind., I088: N. Y. State Mus., Bull. 74, 59.

mœsta LeConte. Coleop. Ind., I085. N. Y. State Mus., Bull. 74. 7I. (C.C.)

obliqua Say. Alder borer. N. Y. State Mus., Bull. 74, 18. puncticollis Say. Coleop. Ind., I085. (A.B.C.)

tridentata Olivier. Elm borer. Coleop. Ind., I087. N. Y. State Mus., Bull. 74, 44.

vestita Say. Linden borer. Coleop. Ind., Io89: N. Y. State Mus. Bull. 74. 54 .

Oberea Mulsant.*

bimaculata Olivier var. basalis. LeConte. Coleop. Ind., Iog2. ocellata Haldeman. Ibid., rog2.

ruficollis Fabricius. Trans. Am. Ent. Soc., vii. 47.

schaumii LeConte. Coleop. Ind., I09I. (F.K.)

tripunctata Swederus. Ibid., Iog2.

tripunctata var. mandarina Fabricius. Trans. Am. Ent. Soc., vii, 47 .

Tetraopes Serville.

canteriator Drapiez. Coleop. Ind., I094. tetraophthalmus Forster. Ibid., I094.

Family CHRYSOMELID尼.

Donacia Fabricius.

cincticornis Newman. Coleop. Ind., r IOz.

dives LeConte. Proc. Acad. Nat. Sci. Phila., v, 3 I4.

emarginata Kirby. Coleop. Ind., I Io6.

flavipes Kirby. Ibid., I Io6.

hirticollis Kirby. Trans. Am. Ent. Soc., xviii, I64.

metallica Ahrens. Coleop. Ind., I ro6.

palmata Olivier. Ibid., I IO2.

piscatrix Lacordaire. Ibid., IIO3. (K.F.C.)

rufa Say. Ibid., IIO7.

subtilis Kunze. Ibid., I IO3.

torosa LeContc. Ibid., I Ioz.

* See also appendix. 
tuberculata Lacordaire. Trans. Am. Ent. Soc., xviii, I7I. harrisi LeConte. Ibid., I72.

sp.

Orsodacna Latreille.

atra Ahrens. var. hepatica Say. Coleop. Ind., I Io8. atra Ahrens var. vittata Say. Ibid., I IoS.

\section{Zeugophora Kunze.}

consanguinea Crotch. Coleop. Ind., I Iog. scutellaris Suffrian. Ibid., I Io9.

Syneta Lacordaire.

ferruginea Germar. Coleop. Ind., i I Io.

\section{Lema Fabricius.}

brunnicollis Lacordaire. Coleop. Ind., i I I. (A.B.C.)

conjuncta Lacordaire. Proc. Phila. Acad. Nat. Sci., IS73, 25. trilineata Olivier. Old-fashioned potato beetle. Coleop. Ind., I I I I.

Crioceris Geoffroy.

asparagi Linnæus. Common asparagus beetle. Conn. Agr. Expt. Sta., I902, I72: Bur. Ent., Circ., I02.

duodecimpunctata Linnæus. Twelve-spotted asparagus beetle.

Bur. Ent., Circ. I02. 9.

Antipus DeGeer.

laticlavia Fœrster. Anomá. Coleop. Ind., Iri3.

Coscinoptera Lacordaire.

dominicana Fabricius. Coleop. Ind., I I 3 .

Babia Chevrolat.

quadriguttata Olivier. Coleop. Ind., I I I4.

Saxinis Lacordaire.

omogera LeConte. Coleop. Ind., I I I4. (G.D.)

Chlamys Knoch.

gibbosa Fabricius. plicata. Coleop. Ind., I I 5.

Exema Lacordaire.

conspersa Mannerheim. Proc. U. S. Nat. Mus., xx, 480.

Bassareus Haldeman.

formosus Melsheimer. Coleop. Ind., I I I9.

formosus var. sulphuripennis Melsheimer. Ibid., III9.

literatus Fabricius. Ibid., I I2O. 
literatus var. lativittus Germar. Ibid., I I20. mammifer Newman. Ibid., I I 9. (G. D.)

sellatus Suffrian. Ibid., I I 9.

\section{Cryptocephalus Geoffroy.}

insertus Haldeman. Coleop. Ind., I I 23.

mutabilis Melsheimer. Ibid., I I23.

notatus Fabricius var. quadrimaculatus Say. Ibid., II22.

ornatus Fabricins. Trans. Am. Ent. Soc., xviii, 20I. (H.L.J.)

quadruplex Newman. Coleop. Ind., I I22. (A.B.C.)

trivittatus Olivier. Ibid., I I 23.

venustus Fabricius. Ibid., I 123.

venustus var. cinctipennis Randall. Ibid., I I23.

venustus var. hamatus Melsheimer. Ibid., I I23. (A.B.C.)

venustus var. ornatus Fabricius. Ibid., II23. (H.L.J.)

Pachybrachys Chevrolat.

atomarius Melsheimer. infaustus. Coleop. Ind., I I 29.

carbonarius Haldeman. Ibid., I I27.

confederatus Fall. Trans. Am. Ent. Soc., xli, 387.

femoratus Olivier. Coleop. Ind., I I3I.

hepaticus Melsheimer. Ibid., I I 30.

luridus Fabricius. Ibid., I I 30.

m-nigrum Melsheimer. intricatus. Trans. Am. Ent. Soc., viii, 207.

othonus Say. Coleop. Ind., i 128.

pubescens Olivier. morosus. Trans. Am. Ent. Soc., viii, 206.

subfasciatus LeConte. Coleop. Ind., i I 30.

tridens Melsheimer. Ibid., I I29.

trinotatus Melsheimer. Ibid., I 128.

Monachus Chevrolat.

saponatus Fabricius. Coleop. Ind., I I33.

Diachus LeConte.

auratus LeConte. Coleop. Ind., I1 33.

pallidicornis Suffrian. Ibid., I I 33 .

Triachus LeConte.

atomus Suffrian. Coleop. Ind., I I 34.

cerinus LeConte. Trans. An. Ent. Soc., viii, I97. (G.D.)

Chrysodina Baly.

globosa Say. Trans. Am. Ent. Soc., xix, 233. 
Nodonota Leférre.

puncticollis Say. Coleop. Ind., I 149 : 'l'rans. An. Ent. Soc., xix, 232.

Colaspis Fabricius.

brunnea Fabricius. Brown Colaspis. Coleop. Ind., I I47.

Graphops LeConte.

pubescens Melsheimer. Coleop. Ind., I I 44.

Xanthonia Baly.

decemnotata Say. Coleop. Ind., I I42.

villosula Melsheimer. Ibid., II 42.

Fidia Baly.

viticida Walsh. Grape vine root worm. Coleop. Ind., I I 43 :

N. Y. State Mus., Bull. 72.

Metachroma LeConte.

pallida Say. Coleop. Ind., I I 46.

Adoxus Kirby.

obscurus Linnæus. Coleop. Ind., I I 36.

Tymnes Chapuis.

tricolor Fabricius. Coleop. Ind., I I 4 I.

Glyptoscelis LeConte.

barbata Say. Coleop. Ind., I I37.

pubescens Fabricius. Ibid., I I37.

Paria LeConte.

Typophorus.

canellus Fabricius var. atterrimus Olivier. Coleop. Ind., I I39.

canellus var. gilvipes Horn. Trans. Anl. Ent. Soc., xix, 208.

canellus var. notatus Say. Compl. IVrit., ii, 2 I3.

canellus var. pumilis LeConte. Coleop. Ind.. I I 39.

canellus var. quadriguttatus Horn. Coleop. Ind., I I 40.

canellus var. quadrinotatus Say. Ibid., I I39.

canellus var. thoracicus Melsheimer. Ibid., I I39.

Chrysochus Chevrolat.

auratus Fabricius. Coleop. Ind., I I 4 I.

Prasocuris Latreille.

phellandrii Linnens. Coleop. Ind., I I 5 I.

vittata Olivier. Coleop. Ind., I I5I. (K.F.C.) 


\section{Labioderma Chevrolat.}

clivicollis Kirby. Coleop. Ind., I I52: Jour. N. Y. Ent. Soc., iv, 195 .

\section{Leptinotarsa Stål.}

decemlineata Say. Doryphora. Io-lineata. Colorado potato beetle. Potato bug. Coleop. Ind., I I 53: Conn. Agr. Expt. Sta., Bull. 208, го6.

\section{Zygogramma Clievrolat.}

suturalis Fabricins. Coleop. Ind., I I 54: Jour. N. Y. Ent. Soc, iv, 197 .

Calligrapha Erichson.

elegans Olivier. Chrysomela. Coleop. Ind., i 56 : Jour. N. Y. Ent. Soc., iv, I98.

lunata Fabricius. hybrida. Jour. N. Y. Ent. Soc., iv, I9S. multipunctata Say. var. bigsbyana Kirby. Chrysomela. Coleop. Ind., I I 58: Jour. N. Y. Ent. Soc., iv, I99. philadelphica Linnæeus. Coleop. Ind., i i 58. philadelphica var. spireæ Say. Jour. N. Y. Ent. Soc, xiv, I99. rhoda Knab. Proc. Wash. Ent. Soc., xi, 83. (G.D.)

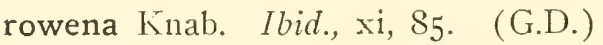
scalaris LeConte. Chrysomela. Coleop. Ind., I I57: Jour. N. Y. Ent. Soc., iv, r99. similis Rogers. Coleop. Ind., I I 56.

Phædon Latreille.

armoraciæ Linnæus cochlearic Gyllenhal. Plagiodera. Can. Ent., xxviii, 202.

Gastroidea Hope.

cyanea Melsheimer. Coleop. Ind., I I59. polygoni Linnæus. Ibid., I I 59.

Lina Megerle.

lapponica Linnæus. Melasoma. interrupta. Coleop. Ind., I I6o. scripta Fabricius. Cottonwood leaf beetle. Ibid., i I6o. N.

Y. Agr. Expt. Sta., Bull. I 43. tremulæ Fabricius. Coleop. Ind., I I6o.

Phyllodecta Kirby.

vulgatissima Linnæeus. Can. Ent., xxviii, 203.

Cerotoma Chevrolat.

trifurcata Forster. Coleop. Ind., I I63: Trans. Am. Ent. Soc., xx, I29: Rept. Conn. Agr. Expt. Sta., I918, 327. 
Trirhabda LeConte.

canadensis Kirby. Coleop. Ind., I I65.

virgata LeConte. Ibid., I 166.

Agelasa Motschulsky.

halensis Linnæus. Coleop. Ind., I I64.

Galerucella Crotch.

americana Fabricius. Coleop. Ind., I I67.

cavicollis LeConte. Ibid., I I69.

decora Say. Ibid., I 7 o.

integra LeConte. Ibid., I I67.

luteola Mïller. Conn. Agr. Expt. Sta., Bull. 55 and Rept., Igo8. notata Fabricius. Coleop. Ind., i i 68.

nymphææ Linneus. Ibid., I I69: Bur. Ent. Soc., Bull. 54, 58.

Monoxia LeConte.

puncticollis Say. maritima. Trans. Am. Ent. Soc., xx, 83 .

Diabrotica Chevrolat.*

atripennis Say var. cristata Harris. Trans. Am. Ent. Soc., $\mathrm{xx}, 95$.

atripennis var. fossata LeConte. Ibid., xx, 95.

duodecimpunctata Olivier. I2-punctata. Coleop. Ind., II72: Rept. Conn. Agr. Expt. Sta., I908, Sog.

vittata Fabricius. Striped cucumber beetle. Coleop. Ind., I I73:

Rept. Conn. Agr. Expt. Sta., I9o8, So7: 1917, 262.

Phyllobrotica Redtenbacher.

discoidea Fabricius. Coleop. Ind., i I 75.

limbata Fabricius. Ibid., I I75. Trans. Am. Ent. Soc., xx, I00.

Luperodes Motschulsky.

meraca Say. Coleop. Ind., i п 76.

Blepharida Rogers.

rhois Fœrster. Coleop. Ind., I 208.

Edionychis Latreille.

fimbriata Forster. Coleop. Ind., I I 83 .

limbalis Melsheimer var. subvittata Horn. Trans. An. Ent. Soc., xvi, I93.

miniata Fabricius. Coleop. Ind., I 183.

quercata Fabricius. Ibid., I $8_{5}$. (G.D.)

sexmaculata Illiger. Ibid., I I 84.

thoracica Fabricius. Ibid., II 82.

vians Illiger. Ibid., I I82.

* See also appendix. 


\section{Disonycha Chevrolat.}

caroliniana Fabricius. Coleop. Ind., I I 88.

collata Fabricius. Ibid., I IgI. (G.D.)

funerea Randall. Trans. Am. Ent. Soc., xvi, 208.

pennsylvanica Illiger. Coleop. Ind., I I 87 .

quinquevittata Say. Ibid., I I 88.

triangularis Say. Ibid., Igo.

xanthomelæna Dalman. collaris. Ibid., I Igo. (G.D.)

\section{Luperaltica Crotch.}

fuscula LeConte. Coleop. Ind., I 203.

\section{Altica Geoffroy:}

bimarginata Say. Alder flea beetle. Coleop. Ind., I20 I. corni Vroods. Me. Agr. Expt. Sta., Bull., 273, I56.

chalybea Illiger. Coleop. Ind., I20I.

fuscoænea Melsheimer. Ibid., I 202. (G.D.)

ignita Illiger. Ibid., I20I.

marevagans Horn. Trans. Am. Ent. Soc., xvi, 226.

rosæ Woods. Me. Agr. Expt. Sta., Bull. 273, I74.

rufa Illiger. Coleop. Ind., I 200.

torquata LeConte. Me. Agr. Expt. Sta., Bull. 273, 194. ulmi Woods. Ibid., Bull. 273, I82.

Crepidodera Chevrolat.

atriventris Melsheimer. Coleop. Ind., I2I4. (G.D.)

helxines Linnaus. Ibid., I 2 I 4 .

modeeri Linnæus. Ibid., I 2 I 4.

\section{Epitrix Foudras.}

cucumeris Harris. Cucumber flea beetle. Coleop. Ind., I2I7. parvula Fabricius. Ibid., I 2 I8. (G.D.)

Orthaltica Crotch.

copalina Fabricius. Coleop. Ind., I2 I5.

Mantura Stephens.

floridana Crotch. Coleop. Ind., I219. (G.D.)

Chætocnema Stephens.

confinis Crotch. Coleop. Ind., I212.

denticulata Illiger. Ibid., I 2 Iо.

minuta Melsheimer. Ibid., I2 IO.

pulicaria Melsheimer. Ibid., I2 I2. 


\section{Systena Clark.}

frontalis Fabricits. Coleup. Ind., r220.

hudsonias Forster. Ibid., 1 220 .

marginalis Illiger. Ibid., I221.

tæniata Say. Ibid., I 221.

tæniata var. blanda Melsheimer. Ibid., I22I.

Longitarsus Latreille.

insolens Horn. Trans. Am. Ent. Soc., xvi, 286.

melanurus Melsheiner. Coleop. Int., I Ioz.

Phyllotreta Foudras.

armoriaceæ Koch. Culeop. Ind., i ig8.

bipestulata Fabricitus. Ibid.. I Iog.

chalybeipennis Crotch. Trans. Am. Ent. Soc., xri, 300.

sinuata Stephens. Coleop. Ind., 1197.

vitteta Fabricius. Ibid., I I97.

Dibolia Chevrolat.

borealis Chevrolat. area. Coleop. Ind., I222.

Fsylliodes Latreille.

pinctulata Melsheimer. Coleop. Ind., 1222.

Stenispa Baly.

metallica Fabricius. Colcop. Incl., 1228.

Anoplitis Chapuis.

nervosa Panzer. Chalepus. Odontotu. Coleop. Ind., 1228.

Chalepus Thumberg.

bicolor Olivier. Odontota. Coleop. In1., i 226.

dorsalis Thunberg. Ibid., 1227.

hornii Smith. Odontota. Ibid., 1227.

notzta Olivier. Odontota. Trans. Am. Ent. Soc., x, 296.

scapularis O'ivier. Odontota. Coleop. Ind., I 226.

Baliosus Weise.

rubra Weber. Chalcpus. Odontota. Coleop. Ind., I227.

\section{Uroplata Baly.}

porcata Melsheimer. Trans. Am. Ent. Soc., x, 294.

Microrhopala Chevrolat.

vittata Fabricius. Coleop. Ind., 1224.

zercne Newman. Ibid., I 225. 
Chelymorpha Chevrolat.

cassidea Fabricius. argus. Coleop. Ind., I233.

Chirida Chapuis.

guttata Olivier. Coptocycla. signifera. Coleop. Ind., I232.

Deloyala Chevrolat.

clavata Fabricius. Coptocycla. Coleop. Ind., I232: Rept. Conn. Agr. Expt. Sta., I918, I Io.

Metriona Weise.

bicolor Fabricius. Coptocycla. aurichalcea. Coleop. Ind., I23I. purpurata Boheman. Coptocycla. Ibid., I232. (K.F.C.)

\section{Family MYLABRIDE.}

(BRUCHIDE.)

\section{Spermophagus Schonherr.}

hoffmannseggi Gyllenhal. robinic Fabricius. Coleop. Ind., I235.

Bruchus Linnæus.

calvus Horn. Coleop. Ind., I239.

nigrinus Horn. Trans. Am. Ent. Soc., iv, 327.

obtectus Say. Bean weevil. Coleop. Ind., I240.

pisorum Linnæus. Pea weevil. Ibid., I236.

quadrimaculatus Fabricius. Trans. Am. Ent. Soc., iv, 3 I8.

Zabrotes Horn.

subnitens Horn. Trans. Am. Ent. Soc., xii, I58.

\section{Family BRENTIDA.}

Eupsalis LeConte.

minuta Drury. Oak timber worm. Ins. Aff. Park and Woodl. Trees, 26I : Rhyn. N. E. Am., 20.

Family BELIDE.

Ithycerus Schonherr.

noveboracensis Fœrster. New York weevil. Rept. Conn. Agr. Expt. Sta., 1908, 845: Rhyn. N. E. Am., 92. 


$$
\text { Family PLATISTOMIDA. }
$$

$$
\text { (ANTIRIBIDE.) }
$$

Eurymycter LeConte.

fasciatus Olivier. Rhyn. N. E. Am., 30.

$$
\text { Euparius Schonherr. }
$$

marmoreus Olivier. Cratoparis lunatus. Rhyn. N. E. Am., 37; Ins. Aff. Park and Woodl. Trees, 499.

Brachytarsus Schonherr.

sticticus Boheman. rariegatus. Rhy. N. E. Am., 39.

tomentosus Say. Ibid., 40.

Anthribulus LeConte.

rotundatus LeConte. Rhỵn. N. E. Am., 4I.

\section{Family CURCULIONIDE.}

Auletes Schonherr.

albovestita Blatchley. Rhyn. N. E. An., 54.

ater LeConte. Ibid., 53 .

Eugnamptus Schonherr.

angustatus Gyllenhal. Rhyn. N. E. Am., $5^{6}$.

collaris Fabricitus var. nigripes Melsheimer. Ibid., 56 .

Rhynchites Herbst.

æratus Say. Rhyn. N. E. Am., 59.

bicolor Fabricius. Rose weeril. Ibid., 58.

hirtus Fabricius. Ibid., 59.

Attelabus Linnæus.

analis Illiger. Rhyn. N. E. Am., 62.

bipustulatus Fabricius. Ibid., 63. (A.B.C.) (H.B.K.)

nigripes LeConte. Ibid., 62.

rhois Boheman. Ibid., 64 .

Pterocolus Schonherr.

ovatus Fabricius. Rhyn. N. E. Am., 65. 
Apion Herlost.

coxale Fall. Rhyn. N. E. Am., 77.

griseum Smith. Ibid., So.

herculanum Smith. Ibid., S9. (A.B.C.) (H.B.K.)

nigrum Herbst. Ibid., 82.

patruele Sinith. Ibid., 78.

rostrum Say. Ibid., SI.

Hormorus Horn.

undulatus Uhler. Rept. Conn. Agr. Expt. Sta., I905, 259: Rhy 11. N. E. Am., Ioo.

Panscopus Schonherr.

erinaceus Say. Barynotus. Rhyn. N. E. Am., IO4.

Phylexis Schonherr.

rigidus Say. Barynotus. Rhyn. N. E. Ann., Iо7.

Otiorhynchus Germar.

ovatus Linnzeus. Strawberry crown girdler. Rept. Comn. Agr. Expt. Sta., I909, 370: Rhyn. N. E. Am., i I .

sulcatus Fabricius. Rept. Conn. Agr. Expt. Sta., I913, 230: Rhyn. N. E. Anr., I I.

Pandeletejus Schonherr.

hilaris Herbst. Rhyn. N. E. Am., I20.

\section{Aphrastus Schonherr.}

tæniatus Gyllenhal. Rhyn. N. E. Am., I27.

Polydrusus Germar.

impressifrons Gyllenhal. Rhyn. N. E. Am., Izo: N. Y. (Genera) Agr. Expt. Sta., Tech. Bull. $5^{6}$.

Scythropus Schomherr.

elegans Couper. Rhyn. N. E. Am., I3I.

Barypeithes Duval.

pellucidus Boheman. Rhyn. N. E. Amı., I33.

Sitona Schonherr.

flavescens Marsham. Rhỹn. N. E. Am., I 42. hispidula Germar. Ibid., I4I.

\section{Hypera Germar.}

punctatus Fabricits. Clover leaf beetle. Ins. Ill., xxv, $8_{3}$ : Rhyn. N. E. Am.. I 46. 
Phytonomus Schonherr.

meles Fahricius. Rhyn. N. E. Am., I nigrirostris Fabricius. Ibid., I 50.

sp.

\section{Listronotus Jekel.}

inæqualipennis Boheman. Rhyn. N. E. Am., I 56.

latiusculus Boheman. Ibid., I61.

tuberosus LeConte. Ibid., I5t.

Hyperodes Jekel.

humilis Gyllenhal. Rhyn. ․ E. Am., г;6.

solutus Boheman. Ibid., I66.

sp.

\section{Pissodes Germar.}

affinis Randall. Rhyn. N. E. Am., 183.

approximatus Ilopkins. Ibid., I $\mathrm{S}_{\mathrm{I}}$.

strobi Peck. White Pine Weevil. Ibid., ISo. Rept. Conn. Agr.

Expt. Sta., 1919, 144.

\section{Hylobius Germar.}

pales Herbst. Proc. Am. Plhil. Soc., xv, Iło: Ryn. N. E. Am. I 86.

confusus Kirby. Rhyn. N. E. Am., is7.

\section{Dorytomus Stephens.}

laticollis LeConte. Rhyn. N. E. Am., 195. (A.B.C.) (H.B.K.) parvicollis Casey. Ibid., 195.

Desmoris LeConte.

constrictus Say. Rhyn. N. E. Am., 206.

Smicronyx Schonherr.

corniculatus Fabricius. Khyn N. E. Am., 2 I I.

Brachybamus Germar.

electus Germar. Rhyn. N. E. Am., 222.

Endalus Laporte.

ovalis LeConte. Rhyn. N. E. Am., 224. (A.B.C.) (H.B.K.)

Onchylis LeConte.

nigrirostris Roheman. Rhyn. ‥ E. Am., 226.

Bagous Germar.

magister LeConte. Rhyn. N. E. Am., 231.

nebulosus LeConte. Ibid., $23 \%$.

pauxillus Blatchley. Ibid., 238 . 
Tychius Schonherr.

picirostris Fabricius. Rhyn. N. E. Am., 246.

Otidocephalus Chevrolat.

chevrolatii Horn. myrmecodes. Rhyn. N. E. Am., 251.

lævicollis Horn. Ibid., 250.

myrmex Herbst. myrmecodes Say. Ibid., 250.

Magdalis Germar.

alutacea LeConte. Rhyn. N. E. Am., 257.

armicollis Say. Ibid., 258 .

austera Fall var. substrigata Fall. Ibid., 256.

barbita Say. Ibid., 258.

olyra Herbst. Hickory snout borer. Ibid, 259.

salicis Horn. Rhyn. N. E. Am., 26o. (A.B.C.) (H.B.K.)

Balaninus Germar.

algonquinus Casey. Lesser chestnut weevil. Rhyn. N. E. Am., 267.

baculi Chittenden. uniformis. Ibid., $27 \mathrm{I}$.

caryæ Horn. Hickory nut weevil. Ibid., 265.

confusor Hamilton. Confused acorn weevil. Ibid., 270.

nasicus Say. Mottled acorn weevil. Ibid., 269.

obtusus Blanchard. Hazelnut weevil. Ibid., 272.

pardalis Chittenden. Ibid., 270.

proboscideus Fabricius. Larger chestnut weevil. Ibid., 264. quercus Horn. Proc. Am. Plill. Soc., I873, 458.

rectus Say. Common acorn weevil. Rhyn. N. E. Am., 266.

Orchestes Illiger.

canus Horn. Rhyn. N. E. Am., 28 I.

niger Horn. Ibid., 280.

pallicornis Say. Ibid., 280.

rufipes LeConte. Ibid., 282.

Tachypterellus Cockerell.

quadrigibbus Say. Anthonomus. Apple curculio. Rhyn. N. E. Am., 285.

Anthonomopsis Dietz.

mixtus LeConte. Rhyn. N. E. Am., 286. 
Anthonomus Germar.

elongatus LeConte. Rhyn. N. E. Anı., 315.

musculus Say. Ibid., 302. (A.B.C.) (H.B.K.)

nubilis LeConte. Ibid., 3I 4 .

pusillus LeConte. Ibid., 289.

scutellaris LeConte. Ibid., 287.

scutellatus Gyllenhal. Ibid., 307.

signatus Say. Strawberry weevil. Ibid., 300.

suturalis LeConte. Ibid., 297.

Prionomerus Schonherr.

calceatus Say. Rhyn. N. E. Am., 322.

Piazorhinus Schonherr.

scutellaris Say. Rhyn. N. E. Am., 323.

Gymnetron Schonherr.

teter Fabricius. Rhyn. N. E. Am., 326.

Miarus Stephens.

hispidulus LeConte. Rhyn. N. E. Am., 326.

puritanus Casey. Ibid., 327.

Stephanocleonus Motschulsky.

plumbeus LeConte. Rhyn. N. E. Am., 328.

Cleonus Schonherr.

calandroides Randall. Rhyn. N. E. Am., 330.

Lixus Fabricius.

concavus Say. Rhubarb weevil or curculio. Rhyn. N. E.

Am., 337.

musculus Say. Ibid., 336. (G.D.)

rubellus Randall. Ibid., 334.

Læmosaccus Schonherr.

plagiatus Say. Rhyn. N. E. Am., 346. (A.B.C.) (H.B.K.)

Baris Germar.

scolopacea Germar. Rhyn. N. E. Am., 357.

umbilicata LeConte. Ibid., 350.

Onychobaris LeConte.

pectorosa LeConte. Rhyn. N. E. Am., 366.

Note:-Diorymellus laevimargo Champion, a small black weevil, infests orchids in greenhouses. 
Madarellus Casey.

undulatus Say. Rhyn. N. E. Am., 367.

Pseudobaris LeConte.

nigrina Say. Rhyn. N. E. Am., 374.

Centrinus Schonherr.

perscitus Herbst. Rhyn. N. E. Am., 383 .

picumnus Herbst. Ibid., 380.

Odontocorynus Schonherr.

salebrosus Casey. Rhyn. N. E. Am., $3^{86 .}$

scutellum-album Say. Ibid., 385.

Nicentrus Casey.

linecollis Boheman. Centrinus. Rhyn. N. E. Am., 390.

\section{Idiostethus Casey.}

subcalvus LeConte. Rhyn. N. E. Am., 404.

tubulatus Say. Stethobaris. Ibid., 404.

Stethobaris LeConte.

ovata LeConte. Rhyn. N. E. Am., 407.

Cylindrocopturus Heller.

binotatus LeConte. Copturodes. Copturus. Rhyn. N. E. Am., 420.

quercus Say. Copturodes. Copturus. Ibid., $42 \mathrm{I}$.

Acoptus LeConte.

suturalis LeConte. Rhyn. N. E. Am., 423. (A.B.C.) (H.B.K.)

Mononychus Germar.

vulpeculus Fabricius. Rhyn. N. E. Am., 426.

Acanthoscelis Dietz.*

acephalus Say. Falciger. Caliodes. Rhyn. N. E. Am., 43 I.

Auleutes Dietz.

asper LeConte. Rhyn. N. E. Am., 432. (C.W.L.)

nebulosus LeConte. Ibid., 435 .

tenuipes LeConte. Ibid., 433.

Acallodes LeConte.

ventricosus LeConte. Rhyn. N. E. Am., 437.

Cœliodes Schonherr.

acephalus Say. Proc. Am. Phil. Soc., xv, 269.

flavicaudis Boheman. Rhyn. N. E. Am., $43^{8 .}$

* See also appendix. 


\section{Ceutorhynchus Germar.**}

rapæ Gyllenhal. affuentus. Rhyn. N. E. Am., 442.

septentrionalis Gyllenlial. Ibid., $45^{2}$.

Cœlogaster Schonherr.

zimmermanni Gyllenhal. Rhyn. N. E. Am., 455.

Pelenomus Thomson.

sulcicollis Fabricius. Rhyn. N. E. An1., 457.

Rhinonchus Schonherr.

pyrrhopus Boheman. Rhyn N. E. Anı., 46 I.

Conotrachelus Schonherr.

albicinctus LeConte. Rhyn. N. E. Am., 459. (A.P.C.) (H.P.K.) anaglypticus Say. Ibid., 480.

aratus Germar. Ibid., 473 .

cratægi IValsh. Quince curculio. Ibid., t7t: Cornell Agr.

Expt. Sta., Bull. I 48 .

elegans Say. Rhyn. N. E. Am., 472.

geminatus LeConte. Ibid., 478.

juglandis LeConte. IV alnut curculio. Ibid., 468: Rept. Conn.

Agr. Expt. Sta., I9I 2, 240.

nenuphar Herbst. Plum curculio. Rhyn. N. E. Am., 469:

Rept. Conn. Agr. Expt. Sta,, I904. 219.

posticatus Boheman. Rhyn. N. E. Am., 477.

seniculus LeConte. Ibid., 472. (A.P.C.) (H.B.K.)

\section{Ryssematus Chevrolat.}

æqualis Horn. Rhyn. $\mathrm{N}$. E. Am., 484.

lineaticollis Say. Ibid., 484 .

Tyloderma Say.

ærea Say. Rlynn. N. E. Ann., 493.

foveolata Say. Ibid., 49 I.

nigra Casey. Ibid., 493.

punctata Casey. Ibid., 494.

Apteromechus Faust.

ferratus Say. Rhyn. N. E. Am., 506.

Cryptorhynchus Illiger.

bisignatus Say. Eubulus. Rhyn. N. E. An., 509.

fuscatus LeConte. Ibid., 509.

lapathi Linnzens. Poplar or willow curculio. Ibid., 510.

parochus Herbst. Ibid., 508.

tristis LeConte. Ibid., 5I4. (A.B.C.)

* See also appendix. 


\section{Dryophthorus Schonherr.}

americanus Bedel. corticalis. Rhyn. N. E. Am., 524 .

Himatinum Cockerell.

conicum LeConte. Himatium. Rhyn. N. E. Am., 527. (C.C.)

Cossonus Clairville.

concinnus Boheman. Rhyn. N. E. Am., 53I. (G.D.)

corticola Say. Ibid., 532 .

impressifrons Boheman. Ibid., 53I. (H.L.J.)

platalea Say. Ibid., 530 .

Stenoscelis Wollaston.

brevis Boheman. Rhyn. N. E. Am., 545.

Rhodobænus LeConte.

tredecimpunctatus Illiger. Rhyn. N. E. Am., 550.

Sphenophorus Schonherr.

æqualis Gyllenhal. Rhyn. N. E. Am., 553.

callosus Olivier. sculptilis. Ibid., 567 .

parvulus Gyllenhal. Ibid., 563.

pertinax Olivier. Ibid., 556 .

setiger Chittenden. Ibid., 557.

venatus Say. placidus. Ibid., 57 I.

zeæ Walsh. Ibid., 566.

Calendra Clairville.

granaria Linnæus. Grain weevil. Rhyn. N. E. Am., 574. oryzæ Linnæus. Rice weevil. Ibid., 575 .

\section{Family SCOLYTID无.}

Scolytus Geoffroy.

\section{Eccoptogaster.}

quadrispinosus Say. Hickory bark beetle. Can. Bark Beetles, ii, 52 .

rugulosus Ratzeburg. Fruit bark beetle. Shot-hole borer. Ibid., 52 .

Chramesus LeConte. icoriæ LeConte. Hickory twig borer. Can. Bark Beetles, ii, $5^{8}$.

Phthorophlœus Rey.

frontalis Zimmerman. Can. Bark Beetles, ii, 59. 
Dendroctonus Erichson.

terebrans Olivier. Turpentine bark beetle. Can. Bark Beetles, ii, 64 .

valens LeConte. Ibid., 63 .

Phlœosinus Chapuis.

dentatus Say. Red cedar bark beetle. Can. Bark Beetles, ii, 68.

Leperisinus Reitter.

aculeatus Say. Hylesinus. Ash timber beetle. Can. Bark Beetles, ii, 72.

Hylastinus Bedel.

obscurus Marsham. Can. Bark Beetles, ii, 73.

Hylurgopinus Swaine.

rufipes Eichhoff. Hylastes. Can. Bark Beetles, ii, 74.

Hylurgops LeConte.

pinifex Fitch. Hylastes. Can. Bark Beetles, ii, 8I.

Micracis LeConte.

suturalis LeConte. Rhyn. N. E. Am., 647.

Ptercyclon Eichhoff.

Monarthrum.

fasciatum Say. Can. Bark Beetles, ii, 86.

mali Fitch. Ibid., 87.

Hypothenemus Westwood.

hispidulus LeConte. Rhyn. N. E. Am., 596.

Stephanoderes Eichhoff.

dissimilis Zimmerman. Rhyn. N. E. Am., 603. sp.

Pityophthorus Eichhoff.

canadensis Swaine. Can. Bark Bectles, ii, 97.

puberulus LeConte. Ibid., 95.

pulicarius Zimmerman. Ibid., 95.

sp.

sp.

Pityogenes Bedel.

hopkinsi Swaine. Can. Bark Beetles, ii, Io6. 
Ips DeGeer.

grandicollis Eichhoff. Can. Bark Beetles, ii, II3. pini Say. Ibid., I I 5 .

sp.

Orthotomicus Ferrari.

cælatus Eichhoff. Tomicus. Xyleborus. Can. Bark Beetles, ii, I 2 I.

Pityokteines Fuchs.

sparsus LeConte. Xyleborus. Tomicus. Pityogenes. Can.

Bark Beetles, ii, I23.

\section{Anisandrus Ferrari.}

pyri Peck. Xylebons dispar. Can. Bark Beetles, ii, I24. sp.

Xyleborus Eichhoff.

celsus Eichhoff. Hickory timber beetle. Can. Bark Beetles, ii, 126.

\section{Order STREPSIPTERA.*}

Family STYLOPIDÆ.

Stylops Kirby.

vicinæ Pierce. U. S. Nat. Mus., Bu1l. No, 66, i Io.

\section{Family XENIDÆ.}

Acroschismus Pierce.

wheeleri Pierce. Xenos peckii Brues. U. S. Nat. Mus., Bull. No. 66, I29.

Schistosiphon Pierce.

peckii Kirby. Xenos. U. S. Nat. Mus., Bull No. 66, I33.

* This order has usually been included in the Coleoptera, and is therefore not given in the table for the separation of the orders, Bulletin 16 , page $3 t$. 


\section{Order HYMENOPTERA.}

Ants, bees, wasps, etc.

Suborder PHYTOPHAGA.

\section{Family XYELIDE.}

Macroxyela Kirby.

infuscata Norton. Conn. Geol. Nat. Hist. Surv., Bull. 22, 32.

\section{Family PAMPIILIID.E.}

\section{Itycorsia Konow.}

albomarginata Cresson. Lyda. Conn. Geol. Nat. IIist. Surv., Bull. 22, 35 .

angulata MacGillivray. Ibid., Bull. 22, 34. (A.B.C.)

luteomaculata Cresson. Ibid., Bull. 22, 35, (R.H.)

ochrocera Norton. Ibid., Bull. 22, 34.

zappei Rohwer. Proc. U. S. Nat. Mfus., 57, 209.

Cænolyda Konow:

semidea Cresson. Lyda. Conn1. Geol. Nat. Hist. Surv., Rull. 22,36 .

\section{Nelirotoma Konow.}

fasciata Norton. Lyda. Conn. Geol. Nat. Hist. Surv., Bu1l. 22, 35. (E.N.)

Pamphilius Latreille.

dentatus MacGillivray. Conn. Geol. Nat. Hist. Surv., Bull. 22,38 .

ocreatus Say. Lydd. Ibid., Bu1l. 22, 37 .

persicus MacGillivray. Peach sawfly. Ibid., Bull. 22, 38 :

Rept. Conn. Agr. Expt. Sta., 1907, 285.

rileyi Cresson. Trans. Am. Ent. Soc., viii, 32.

Anoplolyda Costa.

luteicornis Norton. Lyda. Con11. Geol. Nat. Hist. Surv., Bull. 22, 40. (E.N.)

pallimacula Norton. Lydd. Ibid., Bull. 22, 49. (E.N.) rufofasciatus Norton. Lyda. Ibid., Bull. 22, 4I. (E.N.) scripta Say. Lyda. Ibid., Bull. 22, 40. 


\section{Family TENTHREDINIDÆ.*}

Diprion Schrank.

simile Hartig. Lophyrus. Jour. Econ. Ent., viii, 379: Conn. Geol. Nat. Hist. Surv., Bull. 22, 761.

Neodiprion Rohwer.

abietis Harris. Lophyrus. Conn. Geol. Nat. Hist. Surv., Bull 22,44 .

lecontei Fitch. Lophyrus. Ibid., Bull. 22, 44.

pinetum Norton. abbotti. Lophyrts. Ibid., Bull., 22, 44.

pinus-rigida Norton. Lophyrts. Ibid., Bull. 22, 44.

sp.

sp.

\section{Hemitaxonus Ashmead.}

dubitatus Norton. Taxonus. Conn. Geol. Nat. Hist. Surv., Bull. 22, 46.

Epitaxonus MacGillivray.

albidopictus Norton. Ta.tonus. Conn. Geol. Nat. Hist. Surv., Bull. 22, 46.

\section{Taxonus Hartig.}

nigrisomus Norton. Conn. Geol. Nat. Hist. Surv., Bull. 22, 47.

\section{Monostegia Costa.}

martini MacGillivray. Conn. Geol. Nat. Hist. Surv., Bull. 22, 47.

Empria LePeletier.

Pacilostoma Dahlbon.

calda MacGillivray. Conn. Geol. Nat. Hist. Surv., Bull. 22, 5 I. candidula MacGillivray. Ibid., Bull. 22, 54 .

cariosa MacGillivray. Ibid., Bull. 22, 54.

casca MacGillivray. Ibid., Bull. 22, 53.

casta MacGillivray. Ibid., Bull. 22, 52.

costata MacGillivray. Ibid., Bull, 22, $5 \mathrm{I}$.

evecta MacGillivray. Ibid., Bull. 22, 54.

ignota Norton. Monostcgia. Ibid., Bull. 22, 53.

maculata Norton. Harpiphorus. Ibid., Bull. 22, 53.

sp.

* See also appendix. 
Emphytus Kilug.

apertus Norton. Conn. Geol. Nat. Hist. Surv., Bull. 22, 56.

cinctipes Norton. Ibid., Bull. 22, 56.

gillettii Mac Gillivray. Ibid., Bull. 22, 57.

inornatus Say. Ibid., Bull. 22, 56.

mellipes Norton. Ibid., Bull. 22, 56 .

Emphytina Rohwer.

vanduzeei Rohwer. Proc. U. S. Nat. Mus., 49, 205.

Parataxonus MacGillivray.

erythrogastra Rohwer. Proc. U. S. Nat. Mus., 4I, 408.

multicolor Norton. Taronus. Conn. Geol. Nat. Hist. Surv., Bull. 22, 57.

Eriocampa Hartig.

rotunda Norton. Sciaptery.t. Conn. Geol. Nat. Hist. Surv., Bull. 22, 58.

Monosoma MacGillivray.

inferentia Norton. Pecilostoma. Conn. Geol Nat. Hist. Surv., Bull. 22, 59.

Macremphytus MacGillivray.

semicornis Say. Emphytus. Harpiphoris. Conn. Geol. Nat. Hist. Surv., Bull. 22, 59.

tarsatus Say. Emphytus. Harpiphorus. Ibid., Bull. 22, 6r. varianus Norton. Emplyytus. Harpiphorus. Ibid., Bu1l. 22, 60. Strongylogastroidea Ashmead.

apicalis Norton. Allantus. Strongylogaster. Conn. Geol. Nat. Hist. Surv., Bull. 22, 63.

epicera Say. Allantus. Strongylogaster. Ibid., Bull. 22, 63. mellosa Norton. Allantus. Strongylogaster. Ibid., Bull. 22, 62. rufocincta Norton. Allantus. Strongylogaster. Ibid., Bu1l. 22, 63.

terminalis Say. Allantus. Strongylogaster. Ibid., Bull. 22, 62. unicincta Norton. Ta.ronus. Ibid., Bull. 22, 64 (E.N.)

Dimorphopteryx Ashmead.

pinguis Norton. Strongylogaster. Conn. Geol. Nat. Hist. Surv., Bu11. 22, 64.

Selandria Leach.

flavipes Norton. Conn. Geol. Nat. Hist. Surv., Bull. 22, 66.

Thrinax Konow.

impressatus Provancher. Strongylogaster. Conn. Geol. Nat. Hist. Surv., Bull. 22, 67. 
Strongylogaster Dahlbom.

annulosus Norton. Conn. Geol. Nat. IIist. Surv., Bull. 22, 68. longulus Norton. Ibid., Bull. 22, 67.

luctuosus Provancher. Trans. Am. Ent. Soc., xxii, 3 Iо.

tacitus Say. Allantus. Conn. Geol. Nat. Hist. Surv., Bull. 22, 68.

sp.

Dolerus Jurine.

abdominalis Norton. Con11. Geol. Nat. Hist. Surv., Bull. 22, 74. apricus Norton. Ibid., Bull. 22, 72 .

aprilis Norton. Ibid., Bull. 22, 74 .

bicolor Beauvois. Ibid., Bull. 22, 72.

collaris Say. Ibid., Bull. 22, 73. (A.B.C.)

colosericeus MacGillivray. Ibid., Bull. 22, 70.

conjugatus MacGillivray. Ibid., Bull. 22, 73.

dysporus MacGillivray. Ibid., Bull. 22, 73.

inspiratus MacGillivray. Ibid., Bull. 22, 72 .

luctatus MacGillivray. Can. Ent., xl, 127.

monosericeus MacGillivray. Conn. Geol. Nat. Hist. Surv., Bull. $22,70$.

refugus MacGillivray. Ibid., Bull. 22, 7 7 .

sericeus Say. Ibid., Bull. 22, 69.

similis Norton. Ibid., Bull. 22, 74. (E.N.)

tectus MacGillivray. Ibid., Bull. 22, 70.

unicolor Beauvois. Ibid., Bull. 22, 73 .

versus Norton. Ibid., Bull. 22, 75. (A.E.V.)

sp.

Loderus Konow.

albifrons Norton. Conn. Geol. Nat. Hist. Surv., Bull. 22, 76.

Endelomyia Aslinead.

æthiops Fabricius. Caliroa. Monostegia rosa. Rose sawfly. Conn. Geol. Nat. Hist. Surv., Bull. 22, 77.

Caliroa Costa.

cerasi Linneus. Eriocampoides limacina. Pear and cherry slug.

Conn. Geol. Nat. Hist. Surv., Bull. 22, 77.

fasciata Norton. Ibid., Bull. 22, 79.

obsoleta Norton. Ibid., Bull. 22, 78.

quercus-alba Norton. Ibid., Bull. 22, 78. (E.N.) 
Pachyprotasis Hartig.

rapæ Linnæus. omega. Conn. Geol, Nat. Hist. Surv., Bull. 22, Sr. (E.N.)

Lagium Konow.

atroviolaceum Norton. Tenthredopsis. Comn. Geol. Nat. Hist.

Surv., Bi1l. 22, 81.

atroviolaceum var. tardum Norton. Ibid., Bull. 22, Si.

cinctulum Norton. Ibid., Bull, 22, 8 I.

Tenthredopsis Costa.

semilutea Norton. Conn. Geol. Nat. Hist. Surv., Bull. 22, S2. (E. N.)

Rhogogastera Konow.

evansii Harrington. Conn. Geol. Nat. Hist. Surv., Bull. 22, 83 .

Tenthredo Linnæus.

angulifera Norton. Conn. Geol. Nat. Hist. Surv., Bull. 22, 88. (E.N.)

flavomarginis Norton. Ibid., Bull. 22, 90. (E.N.)

grandis Norton. Ibid., Bull. 22, 84. (E.N.)

formosa Norton. Ibid., Bull. 22, 87.

lobata Norton. Ibid., Pinll. 22, S8.

lobata var. maculosa Smulyan. Can. Ent., xlvii, 324. (W.E.B.)

rufipes Say. Conn. Geol. Nat. Hist. Surv., Bull. 22, 91. (A.B.C.)

rufopecta Norton. Ibid., Bull, 22, 86.

verticalis Say. Ibid., Bull. 22, 86.

sp.

\section{Macrophya Dahlbon.}

alba MacGillivray. Comn. Geol. Nat. Hist. Surv., Bull. 22, 94. (A.B.C.)

albomaculata Norton. Ibid., Bull 22, 97. (E.N.)

bilineata MacGillivray. Ibid., Bull. 22, 96.

cassandra Kirby. List. Hym. Brit. Mus., i, 273, pl. Io, fig. I3.

epinota Say. Ibid., Bull 22, 93.

externa Say. Ibid., Bu1l. 22. 96.

externiformis Rohwer. Proc. U. S. Nat. Mus., 43, 220 .

fascialis Norton. Conn. Geol. Nat. Hist. Surv., Bull. 22, 99.

flavicoxa Norton. Ibid., Bu11. 22, 95.

formosa Klug. Ibid., Bull., 22, 9S. 
fuliginea Norton. Ibid., Bull. 22, 96.

goniphora Say. Ibid., Bull. 22, gS.

incerta Norton. Ibid., Pinll. 22, 96 (E. N.)

intermedia Norton. Ibid., Bu1l. 22, 9S. (E.N.)

lineata Norton. Ibid., Bull. 22, 95.

minuta MacGillivray. Ibid., Bull. 22, 97.

nigra Norton. Ibid., Bull. 22, 97.

pannosa Say. Ibid., Bull. 22, 95.

proximata Norton. Ibid., Bull. 22, 95. (E.N.)

pulchella Klug. Ibid., Bull. 22, 94. (E.N.)

succincta Cresson. Ibid., Bull. 22, 9S.

tibiator Norton. Ibid., Bull. 22, 96.

trisyllaba Say. Ibid., Bull. 22, 98.

trosula Norton. Ibid., Bull. 22, 93.

varia Norton. Ibid., Bull. 22, 99. (E.N.)

zabriskei Rolwwer. Proc. U. S. Nat. Mus., 43, 2 IS.

\section{Allantus Jurine.}

basilaris Say. Conn. Geol. Nat. Hist. Surv., Bull. 22, IOI. dubius Norton. Ibid., Bull. 22, IO2.

\section{Abia Leach.}

inflata Norton. Zarca. Conn. Geol. Nat. Hist. Surv., Bull. 22, IO2.

Trichiosoma Leach.

crassum Kirby. Conn. Geol. Nat. Hist. Surv., Bull. 22, Io3. (A.B.C.)

triangulum Kirby. Ibid., Bull. 22, I03.

\section{Cimbex Olivier.}

americana Leach. Conn. Geol. Nat. Hist. Surv., Bull. 22, IO4. americana var. decimaculata Norton. Ibid., Bu1l. 22, I04. americana var. laportei LePeletier. Ibid., Bull. 22, IO4. americana var. nortoni MacGillivray. Ibid., Bull. 22, IO4.

Hoplocampa Ilartig.

halcyon Norton. Conn. Geol. Nat. Hist. Surv., Bull. 22, 105. (A.B.C.)

Craterocercus Roliwer.

quercivorus Rohwer. Proc. WTash. Ent. Soc., 20, I64.

Mesoneura Hartig.

parva Norton. Dineura. Conn. Geol. Nat. Ilist. Surv., Bull. 22, 107. (E.N.) 
Priophorus Dahllom.

acericaulis MacGillivray. Mitple leaf stem borer. Ent. News, xvii, 313; Conn1. Gerol. Nát. Hist. Surv., linll. 22, log.

æqualis Norton. Cladius. (imn. Cieon. Natt. Ilist. Surv., Bull. 22, IOO. (

simplicicornis Norton. Ibid., liull, 22, Ior).

Cladius Illiger.

pectinicornis fourcroy. isomerus. liristly rose slug. Conn. Geol. Nat. Ilist. Surv., Bull. 22, I Io.

Trichiocampus llartig.

viminalis Fallen. Conn. Gersl. Nát. Ilist, Surv., Bu1l. 22, I Io.

Diphadnus Hartigs.

appendiculatus Ifartis. I'ristiphora srossularia. Conn. Geol.

Nat. llist. Surv., Yu1l. 22, I 2.

californicus Marli1t. Ibid., liull. 22, I13.

proximatus Norton. Nicmatus. Ibid., PBill. 22, I 3.

Pristiphora Litreill:.

betulavora Roluwer. Troc. U. S. Nat. 1list. Mus., 57, 219.

bivittata Norton. Conn. Cerl. Nat. IJist. Surv., l’ull. 22, I I4.

luteola Norton. Ibid., Liull. 22, I I4.

sycophanta Walsh. tibialis. Mid., Liull. 22, I I3.

\section{Lygæonematus Konow.}

erichsoni IIartig. Iarch sawfly. Conn. Ceod. Nat. Hist. Surv, Bull. 22, I I 5 .

Pachynematus Kronow.

affinis Marlatt. Conn. Ci(e)]. Nat. J list. Surv., Pull. 22, I I7. corniger Nrorton. Ibid., H,ull. 22, 1 I 8 .

extensicornis Norton. Ibid., J'ull. 22, I If.

nigritus Norton. Ibid., Pull. 22, I I I. (J....)

rufocinctus Macrillivray. Hid., liull. 22, i i 7. (A.P.C.)

subalbatus Norton. Ibid., Yiull. 22, 1.18.

sp.

sp.

Nematus Jurine.

chloreus Norton. Cronn. Ger,l. Nat. Hist. Surv., Pull. 22, I2I. (E.N.)

longicornis Say. Iongulicornis Norton. (Ptcronus?) Ibid., Pitll. 22, I I . (F...) 
Crœsus Leach.

latitarsus Norton. Conn. Geol. Nat. Hist. Surv., Bull. 22, I 2 I.

\section{Amauronematus Konow.}

concolor Norton. Nematus. Conn. Geol. Nat. Hist. Surv., Bull. 22, I 22. (E.N.)

semirufus Kirby. fulipes Norton. Nematus. Ibid., Bull. 22, I 23. (E.N.)

luteotergum Norton. Nematus. Ibid., Bull. 22, I23. (E.N.) sp.

\section{Pteronidea Rohwer.*}

amelanchieridis Rohwer. Proc. U. S. Nat. Mus., 57, 215.

carpini Marlatt. Conn. Geol. Nat. Hist. Surv., Bu1l. 22, I29. (C.R.E.)

corylus Cresson. Ibid., Bull. 22, I27. (C.R.E.)

integra Say. Nematus integer. Ibid., Bull. 22, I3I.

limbata Cresson. Ibid., Bull. 22, 125.

longicornis Marlatt. Ibid., Bull. 22, I32.

mendica Walsh. Ibid., Bull. 22, I3I.

populi Marlatt. Ibid., Bull. 22, I28. (A.B.C.)

ribesi Scopoli. Nematus acutricosus. Currant worm. Ibid., Bull. 22, I2S.

thoracica Harrington. Ibid., Bull. 22, I29.

trilineata Norton. Ibid., Bull. 22, I30.

ventralis Say. Ibid., Bull. 22, I33.

vertebrata Say. Ibid., Bull. 22, I3I.

sp.

\section{Pontania Costa.}

desmodioides Walsh. Conn. Geol. Nat. Hist. Surv., Bull. 22, I 40 .

hyalina Norton. Ibid., Bull. 22, I37.

pisum Walsh. Ibid., Bull. 22, I39. (A.B.C.)

\section{Euura Newman.}

orbitalis Norton. Conn. Geol. Nat. Hist. Surv., Bull. 22, I.fI. salicicola Smith. Ibid., Bull. 22, I4I.

\section{Hypargyricus MacGillivray.}

fumipennis Norton. Monophadnus. Conn. Geol. Nat. Hist. Surv., Bull. 22, I45.

* See also appendix. 
Isodyctium Ashmeacl.

atratum MacGillivray. Conn. Geol. Nat. Hist. Surv., Bull. 22, 146.

caryicola Dyar. Ibid., Bull. 22, I45.

Periclista Konow.

plesia Rohwer. Proc. U. S. Nat. Mus. 57, 2 I I.

Tomostethus Konow.

bardus Say. Sclandria. Conn. Geol. Nat. IIist. Surv., Bull. 22. $\mathrm{I} 48$.

inhabilis Norton. Sclandria. Ibid., Bull. 22, I 48 .

Monophadnus Hartig.

bipunctatus MacGillivray. Conn. Geol. Nat. Ilist., Surv., Bull. 22, 149 .

tiliæ Norton. Sclandria. Ibid., Bull. 22, I49. (E.N.)

\section{Paracharactus MacGilliviay.}

rudis Norton. Selandria. Conn. Geol. Nat. Hist. Surv., Bull. 22, I5o. (E.N.)

Phymatocera Dahlbom.

rufula Norton. Conn. Geol. Nat. Hist. Surv., Bull. 22, I50.

Monophadnoides Ashmead.

collaris MacGillivray. Conn. Geol. Nat. IIist. Surv., Bull. 22, I 52 .

consobrinus MacGillivray. Ibid., Bull. 22, I52.

coracinus MacGillivray. Ibid., Bull. 22, I52.

rubi Harris. Sclandria. Ibid., Bull. 22, I5I.

\section{Aphanisus MacGillivray.}

odoratus MacGillivray. Conn. Geol. Nat. Hist., Surv., Bull. 22 , I 54 .

\section{Blennocampa Hartig.}

sp.

Erythraspides Ashmead.

caryæ Norton. Sclandria. Conn. Geol. Nat. Hist. Surv., Bull. 22. 156.

pygmæus Say. Sclandria ritis. Ibid., Bull. 22, I56.

Metallus Forbes.

rubi Forbes. Conn. Geol. Nat. Hist. Surv., Bull. 22, I6o. (H.L.V.) 


\section{Atomacera Say.}

debilis Say. Conn. Geol. Nat. Hist. Surv., Bull. 22, r6o.

decepta Rolwwer. Proc. U. S. Nat. Mus., 4I, 382.

ruficollis Norton. Conn. Geol. Nat. Hist. Surv., Bull. 22, I60.

\section{Arge Schrank.}

Hylotoma Latreille.

abdominalis Leach. Conn. Geol. Nat. Hist. Surv., Bull. 22, I6r.

clavicornis Fabricius. Ibid., Bull. 22, І6г.

dulciaria Say. Ibid., Bull. 22, I61.

erythrosoma Leach. Trans. Am. Ent. Soc., i, 72.

humeralis Beauvois. Conn. Geol. Nat. Hist. Surv., Bull. 22, 162.

macleayi Leach. Ibid., Bull. 22, І6г.

rubiginosa Beauvois. Ibid., Bull. 22, I62.

rubra Klug. Ibid., Bull. 22, I63. (J.A.H.)

salicis Rohwer. Hylotoma pectoralis. Proc. U. S. Nat. Mus., 43, 206.

scapularis Klug. Conn. Geol. Nat. Hist. Surv., Bull. 22, 162.

sphinx Kirby. Ibid., Bull. 22, I62.

virescens Klug. Ibid., Bull. 22, I6r.

xanthothorax Leach. List. Hym. Brit. Mus., I, 69, p1. 6, fig. I.

sp.

sp.

\section{Sterictiphora Billberg.}

johnsoni MacGillivray. Can. Ent., xl, 403.

Schizocerus LePeletier.

privatus Norton. Conn. Geol. Nat. Hist. Surv., Bull. 22, I64. (J.A.H.)

zabriskei Ashmead. Ibid., Bull. 22, I64.

\section{Acordulecera Say.}

dorsalis Say. Conn. Geol. Nat. Hist. Surv., Bull. 22, I66. (E.N.)

maura MacGillivray. Ibid., Bull. 22, I66. (H.L.V.)

media MacGillivray. Ibid., Bull. 22, I65.

mixta MacGillivray. Ibid., Bull. 22, I66.

saginata Provancher. Ibid., Bull. 22, 167.

sp.

sp. 


\section{Family XIPHYDRIIDE.}

\section{Xiphydria Latreille.}

attenuata Norton. Conn. Geol. Nat. Hist. Surv., Bull. 22, I68. (IV.H.P.) maculata Say. Ibid., Bull. 22, I68.

Fanily SIRICIDÆ.

Sirex Linnæus.

cyaneus Fabricius. Conn. Geol. Nat. Hist. Surv., Bull. 22, I70. (E.N.)

\section{Urocerus Geoffroy.}

albicornis Fabricius. Conn. Geol. Nat. Hist. Surv., Bull. 22, I7 I.

cressoni Norton. Ibid., Bull. 22, I 7 I.

flavicornis Fabricius. Ibid., Bull. 22, I7r.

Tremex Jurine.

columba Linnæus. Pigeon horn-tail. Conn. Geol. Nat. Hist. Surv., Bull. 22, I72.

\section{Family CEPHID无.}

Janus Stephens.

abbreviatus Say. Conn. Geol. Nat. Hist. Surv., Bull. 22, i73. bimaculatus Norton. Cephus. Phyllocus. Ibid., Bull. 22, I73. integer Norton. flaziventris. Currant stem girdler. Ibid., Bull. $22,173$.

\section{Adirus Konow.}

trimaculatus Say. Phyllacus. Conn. Geol. Nat. Mist. Surv., Bull. 22, I73. (E.N.)

\section{Family ORYSSIDÆ.}

\section{Oryssus Latreille.}

sayi Westwood. Conn. Geol. Nat. Hist. Surv., Bull. 22, I75. sayi var. terminalis Newman. Ibid., Bull. 22, I75. 


\section{Suborder HETEROPHAGA. \\ Family VIPIONID $Æ$.}

Opius Wesmael.

exareolatus Viereck. Conn. Geol. Nat. Hist Surv., Bull. 22. I 3 .

novæangliæ Viereck. Ibid., Bull. 22, 183 .

pequodorum Viereck. Ibid., Bull, 22, 183 .

Cardiochiles Nees.

viator Say. Conn. Geol. Nat. Hist. Surv., Bull. 22, 183. (A.B.C.)

Apanteles Fœrster.

agricola Viereck. (Protapanteles). Conn. Geol. Nat. Hist. Surv., Bull. 22, I86.

algonquinorum Viereck. (Protapanteles). Ibid., Bull. 22, I88. augustus Viereck. (Protapanteles). Ibid., Bull. 22, I87. cacæciæ Riley. Trans. St. Louis Acad. Sci., iv, pt. 2, 306. carpatus Say. (Apanteles). Conn. Geol. Nat. Hist.. Surv., Bull. 22, 191 .

clisiocampæ Ashmead. N. H. Agr. Expt. Sta., Tech. Bull. 6, 229.

conanchetorum Viereck. (Apanteles). Conn. Geol. Nat. Hist. Surv., Bull. 22, I9I.

congregatus Say. (Protapanteles). Ibid., Bull. 22, ISS. flaviconche Riley. (Protapanteles). Ibid., Bull. 22, IS6. forbesi Viereck. (Apanteles). Ibid., Bull. 22, 191. gelechiæ Viereck. (Apanteles). Ibid., Bull. 22, 190. hesperidivorus Viereck. (Protapanteles). Ibid., Bull. 22, I87. housatannuckorum Viereck. (Apanteles). Ibid., Bull. 22, I89. hyphantriæ Riley. Ibid., Pull. 22, 193. lacteicolor Viereck. (Apanteles). Ibid., Bull. 22, I9I. læviceps Ashmead. (Protapanteles). Ibid., Bull. 22, I8S. lanificus Viereck. (Protapanteles). Ibid., Bull. 22, I88. limenitidis Riley. (Protapanteles). Ibid., Bull. 22, I86. limenitidis var. (Protapanteles). Ibid., Bull. 22, I87. lunatus (Packard) Weed. (Protapanteles). Ibid., Bull. 22, I 86.

maquinnai Viereck. (Apanteles). Ibid., Bull. 22, Igo. melanopus Viereck. (Apanteles). Ibid., Bull. 22, 190. miantonomoi Viereck. (Apanteles). Ibid., Bu1l. 22, I90. militaris Walsh. (Protapanteles). Ibid., Bull. 22, I89. nemoriæ Ashmead. (Protapanteles). Ibid., Bull. 22, I86. 
ninigretorum Viereck. (Apanteles). Ibid., Bull. 22, I9I. nipmuckorum Viereck. (Apanteles). Ibid., B11ll. 22, I9I. obscuricornis Viereck. (Protapanteles). Ibid., Bull. 22, I86. oxyacanthoidis Viereck. (Protapanteles). Ibid., Bull. 22, IS7. pequodorum Viereck. (Apanteles). Ibid., Bull. 22, Igo. podunkorum Viereck. (Stenopleura). Ibid., Bı1l. 22, I88. (D.J.C.)

pyraustæ Viereck. (Protapanteles). Ibid., Bull. 22, I88. radiatus Ashmead. (Protapanteles). Ibid., Bull. 22, I89. recurvariæ Ashmead. (Apanteles). Ibid., Bul1. 22, I9r. tischeriæ Viereck. Ibid., Bull. 22, I9I. trachynotus Viereck. (Apantelés). Ibid., Bull. 22, 19r. winkleyi Viereck. (Protapanteles). Ibid., Bull. 22, I86.

Microgaster Latrcille.

brittoni Viereck. Conn. Geol. Nat. Hist. Surv., Bull. 22, 20 I. congregatiformis Viereck. Ibid., Bull. 22, 201.

gelechiæ Riley. Ibid., Bu1l. 22, 20 I.

solidaginis Tiereck. Ibid., Bull. 22, 201.

sp.

Microplitis Fœerster.

melianæ Viereck var. Conn. Geol. Nat. Hist. Surv., Bull. 22, 203.

quintilis Viereck. Ibid., Bull. 22, 203.

varicolor Viereck. Ibid., Bull. 22, 203.

waldeni Viereck. Ibid., Bull. 22, 203.

Microbracon Ashmead.

connecticutorum Viereck. Conn. Geol. Nat. Hist. Surv., Bull. 22, 205.

dorsator Say var. æqualis Provancher. Ibid., Bull. 22, 206.

dorsator var. mellitor Say. Ibid., Bull. 22, 205.

hobomok Viereck. Ibid., Bull. 22, 206.

konkapoti Viereck. Ibid., Bu11. 22, 205.

massasoit Viereck. Ibid., Bull. 22, 205.

matacomet Viereck. Ibid., Bull. 22, 206.

montowesei Viercck. Ibid., Bull. 22, 206.

nanus Provancher. Faun. Ent. Can. Hym., 504.

nawaasorum Viereck. Conn. Geol. Nat. Hist. Surv., Bull. 22, 205.

podunkorum Tiereck. Ibid., Bull. 22, 205. 
quinnipiacorum Viereck. Ibid., Bull. 22, 205.

sanninoideæ Gahan. Proc. U. S. Nat. Nus., liii, Ig6.

scanticorum Viereck. Conn. Geol. Nat. Hist. Surv., Bull. 22, 205.

sebequanash Viereck. Ibid., Bull. 22, 204.

uncas Viereck. Ibid., Bull. 22, 206.

wawequa Viereck. Ibid., Bull. 22, 204.

Habrobracon (Ashmead) W. J. Johnson.

gelechiæ Ashmead. Conn. Geol. Nat. Hist. Surv., Bull. 22, 209.

Habrobraconidea Viereck.

bicoloripes Viereck. Conn. Geol. Nat. Hist. Surv., Bull. 22, 764 .

Iphiaulax Fœrster.

augustus Viereck. Conn. Geol. Nat. Hist. Surv., Bull. 22, 2 ro.

eurygaster Brullé. Ibid., Bull. 22, 2 Io.

Cœloides Wesmæl.

pissodis Ashmead. Conn. Geol. Nat. Hist. Surv., Bull. 22, 2 ro.

Atanycolus Fœrster.

simplex Cresson. Conn. Geol. Nat. Hist. Surv., Bull. 22, 210.

Family ALYSIID无.

Cœlinidea Viereck.

occom Viereck. Conn. Geol. Nat. Hist. Surv., Bull. 22, 212.

Ericœlinius Viereck.

mahackemoi Viereck. Conn. Geol. Nat. Hist. Surv., Bull. 22, $2 \mathrm{I} 2$.

Dacnusa Haliday.

sachemella Viereck. Conn. Geol. Nat. Hist. Surv., Bull. 22, $2 \times 3$.

Pentapleura Fœrster.

foveolata Viereck. Conn. Geol. Nat. Hist. Surv., Bull, 22, 213. Aspilota Fœrster.

ephemera Viereck. Conn. Geol. Nat. Hist. Surv., Bull., 22, 213.

Asobara Fœrster.

lineata Viereck. Conn. Geol. Nat. Hist. Surv., Bu1l. 22, 2r3. 
Aphæreta Fœrster.

muscæ Ashmead. Conn. Geol. Nat. Hist. Surv., Bull. 22, 2 I 4.

Synaldis Fœrster.

incisa Gahan. Conn. Geol. Nat. Hist. Surv., Bull. 22, 214.

pygmæa Viereck. Ibid., Bull. 22, 21 5.

quinnipiacorum Viereck. Ibid., Bull. 22, 2 I 5.

\section{Family BANCHID E.}

Banchus Fabricius.

ferrugineus Provancher. Conn. Geol. Nat. Hist. Surv., Bull. 22,2 I 6.

pallescens Provancher. Ibid., Bull. 22, 2 I6.

\section{Family BRACONIDE.}

\section{Macrocentrus Cu1tis.}

cerasivorana Viereck. Proc. U. S. Nat. Mus., 42, 623.

delicatus Cresson. Conn. Geol. Nat. Hist. Surv., Bull. 22, 220. pyraustæe Tiereck. Ibid., Bull. 22, 220.

\section{Ichneutidea Ashmead.}

secunda Rohwer. Con11. Geol. Nat. Hist. Surv., Bull. 22, 22 I.

\section{Eubadizon Nees.}

lithocolletidis Viereck. Conn. Geol. Nat. Hist. Surv., Bu11. 22, $22 \mathrm{I}$.

\section{Meteorus Haliday.}

communis Cresson. Conn. Geol. Nat. Hist. Surv., Bull. 22, 222.

dimidiatus Cresson. Ibid., Bu1l. 22, 223.

exareolatus Viereck. Ibid., Bull. 22, 223.

hyphantriæ Riley. Ibid., Bull. 22, 223.

indagator Riley. Ibid., Bull. 22, 222.

petiolariferus Viereck. Ibid., Bu1l. 22, 223.

pretiosus Viereck. Ibid., Bull. 22, 223.

triangularis Muesbeck. Can. Ent., li, I I5.

versicolor IVesmael. Ibid., Bull. 22, 223.

Euphorus Nees.

mellipes Cresson. Conn. Geol. Nat. Hist. Surv., Bull. 22, 224. 
Dinocampus Føerster.

pyri Viereck. Conn. Geol. Nat. Hist. Surv., Bull. 22, 225.

Perilitus Nees.

americanus Riley. Conn. Geol. Nat. Hist. Surv., Bull. 22, 218.

Orgilus Haliday.

detectiformis Viereck. Conn. Geol. Nat. Hist. Surv., Bull. 22, 226.

\section{Bassus Fabricius.}

Microdus Authors.

agilis Cresson. Conn. Geol. Nat. Hist. Surv., Bull. 22, 227.

annulipes Cresson. Ibid., Bull. 22, 227.

brittoni Viereck. Ibid., Bull. 22, 227.

buttricki Viereck. Ibid., Bull. 22, 228.

discolor Cresson. Ibid., Bull. 22, 227.

erythrogaster Viereck. Ibid., Bu1l. 22, 227.

pyrifolii Viereck. Ibid., Bull. 22, 226.

rugareolatus Viereck. Ibid., Bull. 22, 228.

sanctus Say. Ibid., Bull. 22, 228.

waldeni Viereck. Ibid., Bu1l. 22, 228.

winkleyi Viereck. Ibid., Bull. 22, 227.

Bracon Panzer.

Agathis Latreille.

branfordensis Viereck. Conn. Geol. Nat. Hist. Surv., Bull. 22, 230.

sassacus Viereck. Ibid., Bull. 22, 230.

semirubra Tiereck. montrealensis Morrison. Ibid., Bull. 22, 230.

solidaginis Viereck. Ibid., Bull, 22, 230.

Spathius Nees.

sp.

\section{Ascogaster V'esmael.}

provancheri Dalla Torre var. pallidicornis Viereck. Conn. Geol. Nat. Hist. Surv., Bull. 22, 23 I.

Chelonus Jurine.

fissus Provancher. Conn. Geol. Nat. Hist. Surv., Bull. 22, 232. konkaputus Viereck. Ibid., Bu11. 22, 232. mysticorum Viereck. Ibid., Bull. 22, 232. sassacus Viereck. Ibid., Bull. 22, 232. 
Sigalphus Latreille.

Spharopy.r Illiger.

bicolor Cresson. Conn. Geol. Nat. Hist. Surv., Bull. 22, 233.

Urosigalphus Ashmead.

mohawkorum Viereck. Conn. Geol. Nat. Hist. Surv., Bull. 22, 234.

wampanoagorum Viereck. Ibid., Bull. 22, 234.

Epirhyssalus Ashmead.

atriceps Ashmead. Con11. Geol. Nat. Hist. Surv., Bull. 22, 235.

Bucculatriplex Viereck.

secundus Viereck. Conn. Geol. Nat. Hist. Surv., Bull, 22, 765. sp. Ibid., Bull. 22, 766.

Aleiodes Wesmael.

burrus Cresson. Conn. Geol. Nat. Hist. Surv., Bull. 22, 236.

ceruræ Ashmead. Proc. U. S. Nat. Mus., I888, 634.

parasiticus Norton. Ibid., Bull. 22, 235.

terminalis Cresson. Ibid., Bull. 22, 235.

waldeni Viereck. Ibid., Bull. 22, 235.

\section{Hormius Nees.}

completus Provancher. Conn. Geol. Nat. Hist. Surv., Bull. 22, 236 .

Heterospilus Halliday.

eurostæ Viereck. Conn. Geol. Nat. Hist. Surv., Bull. 22, 238.

Polystenidea Viereck.

metacomet Viereck. Conn. Geol. Nat. Hist. Surv., Bull. 22, 238.

Family MIERSIIDE.

Thaumatotypidea Viereck.

spinulata Strickland. Conn. Geol. Nat. Hist., Surv., Bull. 22, 238 . (A.B.C.)

Family EVANIID E.

Gasteruption Latreille.

Fonus Fabricius.

micrurus Kieffer. Conn. Geol. Nat. Hist. Surv., Bull. 22, 240. montanus Cresson var. incertus Cresson. Ibid., Bull. 22, 240. tarsatorius Say. Ibid., Bull. 22, 240. 


\section{Hyptia Illiger.}

harpyoidea Bradley. Conn. Geol. Nat. Hist. Surv., Bull. 22, 240.

\section{Family TRIGONALID E.}

\section{Lycogaster Schuckard.}

pullatus Schuckard var. hollensis Melander and Brues. Conn. Geol. Nat. Hist. Surv., Bull. 22, 243.

\section{Family CAPITONIID无.}

Capitonius Brullé.

saperdæ Ashmead. Conn. Geol. Nat. Hist. Surv., Bull. 22, 766.

\section{Family ICHNEUMONID后.}

\section{Praon Haliday.}

alaskensis Ashmead. Conn. Geol. Nat. Hist. Surv., Bull. 22, 259.

pequodorum Viereck. Ibid., Bull. 22, 259.

\section{Aphidius Nees.}

avenaphis Fitch. granariaphis. Conn. Geol. Nat. Hist. Surv., Bull. 22, 26o.

exareolatus Viereck. Ibid., Bull. 22, 260.

nigripes Ashmead. Ibid., Bull. 22, 260.

polygonaphis Fitch. Ibid., Bull. 22, 260.

rapæ Curtis. Ibid., Bull. 22, 260.

rosæ Haliday. Ibid., Bull. 22, 260 .

testaceipes Cresson. Ibid., Bu1l. 22, 259.

Cymodusa Holmgren.

distincta Cresson. Conn. Geol. Nat. Hist., Surv., Bull. 22, 262.

\section{Sagaritis Holmgren.}

aprilis Viereck. Conn. Geol. Nat. Hist., Surv., Bull. 22, 262. conjunctiformis Viereck. Ibid., Bull. 22, 262.

dubitatus Cresson. provancheri. Ibid., Bull. 22, 262.

patsuiketorum Viereck. Ibid., Bull. 22, 262. 
Campoplex Gravenhorst.*

amasecontorum Viereck. (Nepiera). Conn. Geol. Nat. Hist. Surv., Bull. 22, 266.

clisiocampæ Wleed. (Ameloctonus). Ibid., Bull. 22, 266.

diversicolor Viereck. (Hyposoter). Ibid., Bull. 22, 267.

elyi Viereck. (Hypothereutes). Ibid., Bull. 22, 264.

etemankiakorum Viereck. (Bathyplectes). Ibid., Bull. 22, 263.

fugitivus Say. (Ameloctonus). Ibid., Bull. 22, 266.

grossularifloræ Viereck. (Campoplex). Ibid., Bull 22, 263.

kiehtani Viereck. (Angitia). Ibid., Bull. 22, 265.

macer Cresson. (Angitia). Ibid., Bull. 22, 265.

maquinnai Viereck. (Campoplex). Ibid., Bıll. 22, 263.

metacomet Viereck. (Holocremnus). Ibid., Bull. 22, 264.

nolæ Ashmead. (Campoplex). Ibid., Bull. 22, 263.

œdemisiæ Ashmead. (Ameloctonus). Ibid., Bull. 22, 266.

œdemisiformis Viereck. (Angitia). Ibid., Bull. 22, 265.

openangorum Viereck. (Angitia). Ibid., Bull. 22, 265.

parviformis Viereck. (Angitia). Ibid., Bull. 22, 265.

pentagœtorum Viereck. (Campoplex). Ibid., Bull. 22, 263.

pilosulus Provancher. (Ameloctonus). Ibid., Bull. 22, 266.

ruficoxa Provancher. (Angitia). Ibid., Bull. 22, 265.

vernalis Viereck. (Hypothereutes). Ibid., Bull. 22, 264.

woonandi Viereck. (Angitia). Ibid., Bull. 22, 265.

Casinaria Holmgren.

argentea Norton. (Subgenus?). Conn. Geol. Nat. Hist. Surv.,

Pi11. 22, 273.

assita Norton. (Subgenus?) Ibid., Bull. 22, 272.

ceanothi Viereck. (Pseudocasinaria). Ibid., Bull. 22, 270.

diversa Norton. (Subgenus?). Ibid., Bull. 22, 272.

eupitheciæ Viereck. (Casinaria). Ibid., Bull. 22, 270.

genuina Norton. (Neonortonia). Ibid., Bull. 22, 27I.

glauca Norton. (Subgenus?). Ibid., Bull. 22, 272.

porrecta Cresson. (Eripternoides). Ibid., Bull. 22, 269.

radiolata Provancher. (Eripternus). Ibid., Bull. 22, 26 9.

scabriformis Viereck. (Casinaria). Ibid., Bull. 22, 270.

sokanakiakorum Viereck. (Idechtis). Ibid., Bull. 22, 270.

villosa Norton. (Anisitsia). Ibid., Bull. 22, 272.

vitticollis Norton. (Anisitsia). Ibid., Bull. 22, 27 I.

* See also appendix. 
Paracanidia Viereck.

elyi Viereck. Conn. Geol. Nat. Hist. Surv., Bull. 22, 273.

Ceratogastra Ashmead.

fasciata Cresson. ornata Say. Ceratosoma. Conn. Geol. Nat.

Hist. Surv., Bull. 22, 273.

\section{Pristomerus Curtis.}

olamonus Viereck. Conn. Geol. Nat. Hist. Surv., Bull. 22, 274 .

Porizon Fallen.

conotracheli Riley. Thersilochus. Conn. Geol. Nat. Hist. Surv., Bull. 22, 275.

\section{Porizonidea Viereck.}

sp.

Orthopelma Taschenberg.

diastrophi Ashmead. Prodrus. Conn. Geol. Nat. Hist. Surv., Bull. 22, 275 .

luteolator Gravenhorst. Proc. U. S. Nat. Mus., 49, 226.

Plectiscidea Viereck.

contentionis Viereck. Mesoleptus rufipes. Conn. Geol. Nat. Hist. Surv., Bu1l. 22, 276.

Cremaster Gravenhorst.

forbesii Weed. Proc. U. S. Nat. Mus., 53, 5r3.

Mesochorus Gravenhorst.

calais Viereck. Conn. Geol. Nat. Hist. Surv., Bull. 22, 280. scitulus Cresson. Ibid., Bull. 22, 279.

Paniscus Gravenhorst.

geminatus Say. Conn. Geol. Nat. Hist. Surv., Bull. 22, 280. Erigorgus (Fœrster) Brischke.

Anomalon Authors.

anale Say. Conn. Geol. Nat. Hist. Surv., Bull. 22, 284. ferrugineum Norton. Ibid., Bull. 22, 285. hyaline Norton. Ibid., Bull. 22, 284 . luteopectum Norton. Ibid., Bull. 22, 284. prismaticum Norton. Ibid., Bull. 22, 284. rufulum Provancher. Ibid., Bull. 22, 284. Heteropelma Wesmael.

flavicorne Brullé. Conn. Geol. Nat. IIist. Surv., Bu1l. 22, 285. 
Therion Curtis.

Erochilum Wesmael.

morio Fabricius. Conn. Geol. Nat. Hist. Surv., Bull. 22, 286.

sassacus Viereck. Ibid., Bu11. 22, 286.

tenuipes Norton. Ibid., Bu11. 22, 286.

waccagum Viereck. Ibid., Bull. 22, 287.

Brachixiphosoma Viereck.

pyralidis Ashmead. Eiphosoma. Trans. Am. Ent. Soc., xxiii, I9I.

Thyreodon Brullé.

brullei Viereck. morio. Conn. Geol. Nat. Hist. Surv., Bull. 22,287 .

Anomalon Panzer.

Nototrachy's Marshall.

sp. Conn. Geol. Nat. Hist. Surv., Bull. 22, 766.

Ophion Fabricius.

arcuatus Felt. Conn. Geol. Nat. Hist. Surv., Bull. 22, 288.

bilineatus Say. Ibid., Bull. 22, 287.

macrurus Linneus. Ibid., Bull. 22, 287.

purgatus Say. Ibid., Bull. 22, 288.

slossoni Davis. Ibid., Pull. 22, 287.

\section{Rogas Nees.}

honestus Cresson. Comn. Geol. Nat. Ilist. Surv., Bull. 22, 289.

Mesoleptidea Viereck.

Mesoleptus Authors.

albifrons Cresson. Conn. Geol. Nat. Hist. Surv., Bull. 22, 290. decens Cresson. Ibid., Bull. 22, 290.

Catoglyptus Fcerster.

fucatus Cresson. Conn. Geol. Nat. Ilist. Surv., Bull. 22, 29 I.

Spanotecnus Foerster.

concolor Cresson. Conn. Geol. Nat. Hist. Surv., Bull. 22, 292. obscurellus Daris. Ibid., Bu11. 22, 292.

Mesoleius Holmgren.

scapularis Cresson. Conn. Geol. Nat. Hist. Surv., Bull. 22, 292.

Trematopygus IIolngren.

sp. 
Holmgrenia Fœrster.

tarsalis Cresson. Conn. Geol. Nat. Hist. Surv., Bull. 22, 293.

Rhimphalea Fœrster.

erythrogastra Viereck. Comm. Geol. Nat. Hist. Surv., Bull. 22, 296.

Polyblastus Hartig.

pedalis Cresson. Conn. Geol. Nat. Hist. Surv., Bull. 22, 297.

Erromenus Holmgren.

dimidiatus Cresson. Conn. Geol. Nat. IIist. Surv., Bull. 22, 298.

Euceros Gravenhorst.

flavescens Cresson. Conn. Geol. Nat. Hist. Surv., Bu1l. 22, 299.

thoracicus Cresson. Ibid., Bull. 22, 299.

Sympherta Fœrster.

burra Cresson. Conn. Geol. Nat. Hist. Surv., Bull. 22, 299.

Rhorus Fœrster.

bicolor Cresson. Conn. Geol. Nat. Hist. Surv., Bull. 22, 300.

Anecphysis Fœrster.

ruficrus Walsh. Conn. Geol. Nat. Hist. Surv., Bull. 22, 30r.

Exenterus Hartig.

consors Cresson. Conn. Geol. Nat. Hist. Surv., Bull. 22, 30r. Syrphoctonus Fœrster.

agilis Cresson. Bassus. Conn. Geol. Nat. Hist. Surv., Bull. 22,302 .

Diplazon (Nees) Gravenhorst.

Bassus Authors.

antennatus Davis. (Zootrephes). Conn. Geol. Nat. Hist. Surv., Bull. 22, 304.

bicapillaris Walsh. (Homotropus). Ibid., Bull. 22, 304.

concinnus Cresson. Ibid., Bull. 22, 303.

frontalis Cresson. Ibid., Bull. 22, 302.

lætatorius Fabricius. Ibid., Bull. 22, 303.

sycophanta Walsh. Ibid., Bull. 22, 303.

Brephoctonus (Fœrster) Ashmead.

hygrotrecha Viereck. Conn. Geol. Nat. Hist. Surv., Bull. 22, 305. 
Hyperacmus Holmgren.

ovatus Davis. Conn. Geol. Nat. Hist. Surv., Bull. 22, 305.

Exochus Gravenhorst.

pallipes Cresson. Conn. Geol. Nat. Hist. Surv., Bull. 22, 306. propinquus Cresson. Ibid., Bu1l. 22, 306. semirufus Cresson. Ibid., Bull. 22, 306.

\section{Chorineus Holmgren.}

carinatus Cresson. Conn. Geol. Nat. Hist. Surv., Bull. 22, 308. costatus Davis. Ibid., Bull. 22, 308.

Metopius Panzer.

pollinctorius Say. Conn. Geol. Nat. Hist. Surv., Bull. 22, 309.

Grotea Cresson.

anguina Cresson. Conn. Geol. Nat. Hist. Surv., Bu1l. 22, 309.

Euxorides Cresson.

americanus Cresson. (Calliclisis). Conn. Geol. Nat. Hist. Surv., Bu1l. 22, 309.

Odontomerus Gravenhorst.

mellipes Say. Conn. Geol. Nat. Hist. Surv., Bu1l. 22, 3I I.

Phytodietus Gravenhorst.

annulatus Provancher. Proc. U. S. Nat. Muss., 57, 466.

pulcherrimus Cresson. Ibid., 57, 462.

vulgaris Cresson. Conn. Geol. Nat. Hist. Surv., Bull. 22, 312.

Meniscus Schiödte.

elegans Cresson. Conn. Geol. Nat. Hist. Surv., Bu1l. 22, 312. pulcherrimus Cresson. Ibid., Bull. 22, 312. superbus Provancher. Ibid., Bull. 22, 312.

\section{Lissonota Gravenhorst.}

Lampronota Curtis.

americana Cresson. (Alloplasta). Conn. Geol. Nat. Hist. Surv., Bull. 22, 3I4.

appalachia Viereck. (Nadia). Ibid., Bull. 22, 3I4.

exilis Cresson. Ibid., Bull. 22, 3I4.

frigida Cresson. Ibid., Bull. 22, 3I4.

occidentalis Cresson. (Alloplasta). Ibid., Bull. 22, 3I 3 .

philipi Viereck. Ibid., Bull. 22, 3I 4 .

punctulata Cresson. Ibid., Bu1l. 22, 3I4.

rubrica Cresson. Ibid., Bu1l. 22, 3I4.

varia Cresson. (Alloplasta). Ibid., Bull. 22, 3I4. 
Cryptoideus Ashmead.

Yylophruridea Viereck.

agrili Viereck. Proc. U. S. Nat. Mus., 55, 536.

luctuosus Provancher. Ibid., 55, 536.

Glypta Gravenhorst.

rufiscutellaris Cresson. Conn. Geol. Nat. Hist. Surv., Bull. 22, 3I 7 .

Clistopyga Gravenhorst.

annulipes Cresson. Conn. Geol. Nat. Hist. Surv., Bull. 22, 3I7.

Polysphincta Gravenhorst.

pontiaci Viereck. Conn. Geol. Nat. Hist. Surv., Bu1l. 22, 318. rubricapensis Provancher. Ibid., Bull. 22, 317.

texana Cresson. Ibid., Bull. 22, 318.

Scambus Hartig.

Pimpla Authors.

cœlebs Walsh. (Iseropus.) Trans. St. Louis. Acad. Sci., iii, I 4 I.

conquisitor Say. (Itoplectis). Conn. Geol. Nat. Hist. Surv., Bu1l. 22, 319.

grapholithæ Cresson. (Calliephialtes). Ibid., Bull. 22, 320. inquisitoriellus Dalla Torre. (Iseropus). inquisitor. Ibid., Bull. 22, 320.

inquisitoriellus var. investigator Walsh. Ibid., Bu!l. 22, 3 I9. marginatus Viereck. Pimplidea aqualis. Provancher. Ibid., Bull. 22, 319.

notandus Cresson. (Epiurus). Ibid., Bull. 22, 320.

pedalis Cresson. (Pimpiidea). Ibid., Bu1l. 22, 319.

pterelas Say. (Epiurus). Ibid., Bull. 22, 320.

rufopectus Cresson. (Tromatobia). Ibid., Bull. 22, 320.

tecumsehi Viereck. (Epiurus). Ibid., Bull. 22, 320.

tenuicornis Cresson. (Delomerista). Ibid., Buil. 22, 319.

viduiformis Viereck. (Iseropus). Ibid., Bull. 22, 766 .

Theronia Hòlmgren.

melanocephala Brullé. Conn. Geol. Nat. Hist. Surv., Bull. 22, 323 .

Ichneumon Linnzeus.

Ephialtes Gravenhorst.

irritator Fabricius. Conn. Geol. Nat. Hist. Surv., Bull. 22, 324. 
Megarhyssa Ashmead.

atrata Fabricius. Thalessa. Black long-sting. Comn. Geol. Nat.

Hist. Surv., Bull. 22, 325.

greenei Viereck. Ibid., Bu1l. 22. 325. (A.I.B.)

lunator Fabricius. Thalessa. Lunate long-sting. Ibid., Bull. 22,325 .

Rhyssa Gravenhorst.

lineolata Kirby. nitida merrill. albomaculata Cresson. Conn. Nat. Hist. Surv., Bull. 22, 325-6.

Rhyssella Rohwer.

humida Say. Proc. U. S. Nat. Mus., 57, 423.

Arotes Gravenhorst.*

amœnus Cresson. Conn. Geol. Nat. Hist. Surv., Bull. 22, 326.

Coleocentrus Gravenhorst.

rufus Provancher. Conn. Geol. Nat. Ilist. Surv., Bull. 22, 327.

Gelis Thumberg.

Peromachus Gravenhorst.

lymensis Strickland. (Micromeson). Conn. Geol. Nat. Hist. Surv., Bull. 22, 327.

minimus Walsh. Ibid., Bull. 22, 328 .

ottawænsis Harrington. Ibid., Bull. 22, 328.

Mesostenidea Vicreck.

spinaria Brullé. (Polyanus.) Conn. Geol. Nat. Hist. Surv., Butl. 22, 329.

\section{Acroricnus Ratzeburg.}

junceus Cresson. Conn. Geol. Nat. Hist. Surv., Bull, 22, 330. Joppidium WTalsh.

peregrinus Cresson. Mesoleptus. Conn. Geol. Nat. Hist. Surv., Bull. 22, 330.

Agrothereutes Forster.

Cryptus Eabricius.

alacris Cresson. Conn. Geol. Nat. Ilist. Surv., Bu1l. 22, 332. americanus Cresson. Ibid., Bull. 22, 332 . canadensis Provancher. Ibid., Bull. 22, 332.

* See also appendix. 
cressoni Viereck. Ibid., Bull. 22, 33I.

hirtifrons Ashmead. Ibid., Bull. 22, 330.

hyslopi Viereck. Ibid., Bull. 22, 332.

limatus Cresson. Ibid., Bull. 22, 332.

lophyri Norton. Ibid., Bull. 22, 33I.

mundus Provancher. Ibid., Bull. 22, 331.

nuncius Say. Compl. Writ., ii, 693.

rufus Provancher. Ibid., Bull. 22, 330.

Phygadeuon Gravenhorst.

auriculiferus Vicreck. (Bachia). Conn. Geol. Nat. Hist Surv., Bull. 22, 336.

brittoni Vicreck. (Bachia). Ibic., Bull. 22, 335.

melanocerus Viereck. (Plesignathus). Ibid., Bull. 22, 335.

nortoni Viereck. (Plesignathus). Ibid., Bull. 22, 335.

orbitaliformis Viereck. (Scinacopus). Ibid., Bull. 22, 335 .

quintilis Viereck. (Plesignathus). Ibid., Bull. 22, 334 .

ruficornis Provancher. Ibid., Bull. 22, 334.

tæniatus Viereck. (Plesignathus). Ibid., Bull. 22, 335.

tumidiformis Viereck. (Bachia). Ibid., Bull. 22, 335.

vulgaris Cresson. Ibid., Bull. 22, 334.

Enoplex Fœrster.

smithi Packard. Phygadcuon. Proc. Bost. Nat. Hist., ix, 346.

Hemiteles Gravenhorst.

algonquinus Viereck. (Rhadinocera). Conn. Geol. Nat. Hist.

Surv., Bull. 22, 339.

areator tenellus Say. (Orthizema), utilis. Ibid., Bull. 22, 337.

crassiformis Viereck. (Idemum). Ibid., Bull. 22, 339.

eximius Cresson. Mesolcptus. Ibid., Bull. 22, 337.

fulvipes Gravenhorst. (Astomaspis). Ibid., Bull. 22, $33^{8}$.

laticinctus Aslmead. Ibid., Bull. 22, 339.

loniceræ Vicreck. (Ethelurgus). Ibid., Bu1l. 22, 338.

metacomet Viereck. (Eriplanus.) Bull. 22, 338 .

nigricaniformis Viereck. (Zoophthorus). Ibid., Bull. 22, 338. orbiformis Viereck. (Zamicrotoridea). Ibid., Bull. 22, 339. rileyi Ashmead. cressoniformis Viereck. (Otacustes). Ibid., Bu1l, 22, 339. 
Stilpnus Gravenhorst.

americanus Cresson. Conn. Geol. Nat. Hist. Surv., Bull. 22, 34 I.

\section{Phæogenes Wesmael.}

fungor Norton. Conn. Geol. Nat. Hist. Surv., Bull. 22, 34t.

hebe Cresson. Ibid., Bull. 22, 34I.

hebrus Cresson. Ibid., Bull. 22, 34I.

helvolus Cresson. Ibid., Bu1l. 22, $3+2$.

quadriceps Cresson. Ibid., Bull. 22, 34I.

vincibilis Cresson. Ibid., Bull. 22, 34I.

\section{Colpognathus Wesmael.}

helvus Cresson. Conn. Gcol. Nat. Hist. Surv., Bull. 22, 342.

Trogus Gravenhorst.

brullei Cresson. (Automalus). Conn. Geol, Nat. Hist. Surv., Bu1l. 22, 3+3.

vulpinus Gravenhorst. (Psilomastix). excsorius. Ibid., Bull.

22,343 .

Amblyteles Wesmael.

Icluneumon Authors.

anceps Cresson. (Ectopimorpha). Conn. Geol. Nat, Hist.

Surv., Bull, 22, 349.

apertus Cresson. (Pterocormus?). Ibid., Bull. 22, 345 .

brevicinctor Say. (Pterocormus?). Ibid., Bull. 22, 35I.

brittoni Viereck. (Pterocormus). Ibid., Pull. 22, 348. centrator Say. (Stenichneumon?). Ibid., Bull. 22, 346.

cincticornis Cresson. (Stenichneumon?). Ibid., Bull. 22, 344. cinctitarsis Provancher. (Stenichneumon?). Ibid., Bull. 22, 345 .

citrifrons Cresson. (Fterocormus?). Ibid., Bull. 22, 353.

comes Cresson. (Cratichneumon?). Ibid., Bull. 22, 352.

comes var. aleatorius Harris. (Cratichneumon?). Ibid., Bull.

22,352 .

comptus Say. (Pterocormus?). Ibid., Bull, 22, 353.

disparilis Cresson. (Pterocormus?). Ibid., Bull. 22, 348.

duplicatiformis Viereck. (Barichneumon). Ibid., Bull. 22, 354 .

electus Cresson. (Amblyteles). Ibid., Bu1l. 22, 353. 
extrematatis Cresson. (Pterocormus?). Ibid., Bull. 22, 344. flavicornis Cresson. (Stenichneumon?). Ibid., Bull. 22, 35I. flavizonatus Cresson. (Cratichneumon?). Ibid., Bull. 22, 352. footei Viereck. (Pterocormus). Ibid., Bull. 22, 353.

galenus Cresson. (Cratichneumon?). Ibid., Bull. 22, 35 I. hospitus Cresson. (Pterocormus?). Ibid., Bull. 22, 353. infidelis Cresson. (Pterocormus?). Ibid., Bu1l. 22, 35 I. instabilis Cresson. (Pterocormus?). Ibid., Bull. 22, 353. jucundus Brullé. (Pterocormus?). Ibid., Bull. 22, 346. lætus Brullé. canadensis. funestus. (Cratichneumon?) Ibid., Bull. 22, 352.

leviculus Cresson. (Cratichneumon?). Ibid., Bull. 22, 353. lewisii Cresson. (Pterocormus?). Ibid., Bull. 22, 347. malacus Say. (Stenichneumon?). Ibid., Bull. 22, 345. merus Cresson. (Pterocormus?). Ibid., Bull. 22, 35I. mimicus Cresson. (Pterocormus?). Ibid., Bull. 22, 353. nigratoricolor Viereck. (Chasmias?). Ibid., Bull. 22, 344. nortoni Cresson. (Amblyteles). Ibid., Bull. 22, 349. ormenus Cresson. (Stenichneumon?). Ibid., Bu1l. 22, 349. otiosus Say. (Stenichneumon?). Ibid., Bıll. 22, 346. parvus Cresson. (Pterocormus?). Ibid., Bull. 22, 352. pepticus Say. (Cratichneumon?). Ibid., Bull. 22, 35I. pequoitorum Viereck. (Chasmias?). Ibid., Bull. 22, 345. pulcher Brullé. (Cœlichneumon). Ibid., Bull. 22, 350. putus Cresson. (Pterocormus?). Ibid., Bull. 22, 347. quadrizonatus Viereck. (Pterocormus). Ibid,. Bull. 22, $35^{2}$. quintilis Viereck. (Pterocormus). Ibid., Bull. 22, 354. sassacus Viereck. (Cœlichneumon). Ibid., Bı1l. 22, 349. seminiger Cresson. (Pterocormus?). Ibid., Bull. 22, 34 S. signatipes Cresson. (Pterocormus?). Ibid., Bull. 22, 348. soror Cresson. (Barichneumon). Ibid., Bıll. 22, 348. stadaconensis Provancher. (Amblyteles). Ibid., Bu1l. 22, 35 I, 355 .

subcyaneus Cresson. (Cratichneumon?). Ibid., Bull. 22, 35I. succinctus Brullé. (Cratichneumon?). Ibid., Bull. 22, 354. suturalis Say. (Amblyteles). Ibid., Bull. 22, 349. ultus Cresson. (Amblyteles). Ibid., Bull. 22, 35I. ultus var. rogalis Cresson. (Amblyteles). Ibid., Bu1l. 22, $35^{1}$. 
unifasciatorius Say. (Pterocormus?). Ibid., Bu1l. 22, 35 I. versabilis Cresson. (Pterocormus?). Ibid., Bull. 22, 352. vinnulus Cresson. (Pterocormus?). Ibid., Riull. 22, 353. w-album Cresson. (Cratichneumon?). Ibid., Bt1ll. 22, $3+8$, 354 .

winkleyi Viereck. (Pterocormus). Ibid., Bull. 22, $3+8$.

Family FIGITID Te.

Anacharis Dalman.

sp. Conn. Geol. Nat. Hist. Surv., Bull. 22, 365.

Aspicera Dahlbom.

sp. Conn. Geol. Nat. Hist. Surv., Bull. 22, 365.

Family CYNIPIDE.

Periclistus Foerster.

pirata Osten Sacken. Aulax. Rhodites globulus. Conn. Geol. Nat. Hist. Surv., Bull. 22, 373.

Aylax Iartig.

podagræ Bassett. Conn. Geol. Nat. Hist. Surv., Bu11. 22, 374. tumida Bassett. Lettuce tumor gall. Ibid., Bull. 22, 375. (IV.B.)

Ceroptres Hartig.

ficus Fitch. Conn. Geol. Nat. IIist. Surv., Bull. 22, 377. (H.F.B.)

\section{Synergus Hartig.}

lana Fitch. Oak wool gall. Conn. Geol. Nat. Hist. Surv., Bull. 38o. (B.H.IV.)

\section{Philonyx Fitch.}

erinacei IValsh. Oak hedghog gall. N. Y. St. Mus., Bull. 200. 94.

\section{Biorhiza VTestwood.}

forticornis IValsh. (Xanthoteras). Oak fig gall. Conn. Geol. Nat. Hist. Surv., Bull. 22. $33_{4}$.

hirta Bassett. (Xystoteras) Philoni.t. Ifid., Bull. 22, 383.

Neuroterus Hartig.

batatus Fitch. Oak potato gall. Conn. Geol. Nat. Ilist. Surv, Pit1l. 22, 384. 
consimilis Bassett. Ibid., Bull. 22, 387.

distortus Bassett. Ibid., Bull. 22, 388.

dubius Bassett. Ibid., Bull. 22, 388.

exiguissimus Bassett. Ibid., Bu1l. 22, 389 .

exiguus Bassett. Ibid., Bull. 22, 389 .

floccosus Bassett. Ibid., Bull. 22, 390.

majalis Bassett. Ibid., Bull. 22, 385 .

noxiosus Bassett. Noxious Oak Gall. Ibid., Bull. 22, 39I.

pallidus Bassett. Ibid., Bull. 22, 386 .

tectus Bassett. Ibid., Bu11. 22, 389.

umbilicatus Bassett. Oak button gall. Ibid., Bull. 22, 390.

Dryophanta Fœrster.

corrugis Bassett. Conn. Geol. Nat. Hist. Surv., Bull. 22, 397.

ignota Bassett. Ibid., Bull. 22, 398.

longicornis Bassett. Ibid., Bu1l. 22, 397, 398.

pallipes Bassett. Ibid., Bull. 22, 398.

papula Bassett. Ibid., Bu11. 22, 400.

parvula Bassett. Ibid., Bull. 22, 396.

pedunculata Bassett. Ibid., Bull. 22, 400.

Holcaspis Mayr.

duricoria Bassett. Pointed bullet gall. Conn. Geol. Nat. Hist. Surv., Bull. 22, 402.

fasciata Bassett. Ibid., Bull. 22, 402.

globulus Fitch. Oak bullet gall. Ibid., Bull. 22, 4OI.

rugosa Bassett. Ibid., Bull. 22, 4OI.

Cynips Linnæus.

confluens Harris. Oak or May apple. Conn. Geol. Nat. Hist. Surv., Bu1l. 22, 404.

cristata Stebbins. Ibid., Bull. 22, 768. (G.D.)

decidua Bassett. Ins. Galls Springfield, 20.

strobilana Osten Sacken. Pine cone oak gall. Conn. Geol.

Nat. Hist. Surv., Bull. 22, 403. .

\section{Amphibolips Reinhard.}

badia Bassett. Conn. Geol. Nat. Hist. Surv., Bull. 22, 405. cœlebs Osten Sacken. Oak spindle gall. Ibid., Bull. 22, 407. cooki Gillette. Ibid., Bu1l. 22, 768. (W.B.)

illicifoliæ Bassett. Scrub oak gall. Ibid., Bull. 22, 405. (G.D.) inanis Osten Sacken. Empty oak apple. Ibid., Bu1l. 22, 407. 
nubilipennis Harris. Ibid., Bull. 22, 408.

prunus Walsh. Acorn plum gall. Ibid., Bull. 22, 406. (G.D.) sculpta Bassett. Ibid., Bull. 22, 408.

tinctoriæ Ashmead. Ibid., Bull. 22, 768. (G.D.)

verna Bassett. Ibid., Bull. 22, 404. (H.F.B.)

Andricus Hartig.

ashmeadii Bassett. Conn. Geol. Nat. Hist. Surv., Bull. 22, 4 I8. (H.F.B.)

ceropteroides Bassett. (Callirhytis). Ibid., Bull. 22, 434.

cicatricula Bassett. Ibid., Bull. 22, 4I3. (H.F.B.)

corniger Osten Sacken. Horned knot oak gall. Ibid., Bull. $22,4 \mathrm{I} 4$.

exiguus Bassett. Ibid., Bull. 22, 417. (H.F.B.)

formosus Bassett. Ibid., Bull. 22, 409. (H.F.B.)

fruticola Ashmead. Ibid., Bull. 22, 769.

futilis Osten Sacken. (Callirhytis). papillatıs. Oak wart gall. Ibid., Bull. 22, 433 .

glandulus Beutenmüller. Ibid., Bull. 22, 770.

incertus Bassett. Ibid., Bull. 22, 4I4. (H.F.B.)

obtusilobæ Bassett. Ibid., Bull, 22, 4I5. (H.F.B.)

operatola Riley and Bassett. Ibid., Bull. 22, 4I8. (H.F.B.)

operator Osten Sacken. Ibid., Bull. 22, 429.

ostensackenii Bassett. Ibid., Bull. 22, 4I2. (H.F.B.)

palustris Osten Sacken. Succulent oak gall. Ibid., Bull. 22, 4 I9.

patiens Bassett. Ibid., Bull. 22, 4I6. (H.F.B.)

pattoni Bassett. Ibid., Bull. 22, 423. (H.F.B.)

petiolicola Bassett. Oak petiole gall. Ibid., Bull. 22, 4I2.

piger Bassett. Ibid., Bull. 22, 422. (H.F.B.)

piperoides Bassett. Ibid., Bull. 22, 4I5. (H.F.B.)

pruinosus Bassett. Ibid., Bull. 22, 4I I. (H.F.B.)

pulchellus Bassett. Ibid., Bull. 22, 4I6. (H.F.B.)

punctatus Bassett. (Callirhytis). Oak knot gall. Ibid., Bull. 22,431 .

pusulatoides Bassett. (Callirhytis). Ibid., Bull. 22, 433. (H.F.B.)

saccularis Bassett. (Callirhytis). Ibid., Bull. 22, 432. (H.F.B.)

scitulus Bassett. (Callirhytis). Ibid., Bull. 22, 429. 
seminator Harris. (Callirhytis). Oak seed gall. Ibid., Bull. 22, 430 .

similis Bassett. (Callirhytis). Scrub oak club gall. Ibid., Bull. 22, 428 .

singularis Bassett. (Callirhytis). Small oak apple. Ibid., Bull. 22, 431. (H.F.B.)

tuberosus Bassett. (Callirhytis). Ibid., Bull. 22, 430. (I.F.B.)

utriculus Bassett. Ibid., Bull. 22, 423.

ventricosa Bassett. Ibid., Bull. 22, 409. (H.F.B.)

Solenozopheria Ashmead.

vaccinii Ashmead. Huckleberry gall. Conn. Geol. Nat. Hist. Surv., Bull. 22, 434 .

\section{Diastrophus Hartig.}

bassettii Beutenmüller. Bassett's Blackberry gall. Conn. Geol. Nat. Hist. Surv., Bu1l. 22, 438.

cuscutæformis Osten Sacken. Blackberry seed gall. Ibid., Bull. 22, 435 .

fragariæ Beutenmüller. Can. Ent., xlvii, 353.

minimus Bassett. Conn. Geol. Nat. Hist. Surv., Bull. 22, 438. nebulosus Osten Sacken. Blackberry knot gall. Ibid., Bull. 22, 435 .

potentillæ Bassett. Gonaspis. Cinquefoil axil gall. Ibid., Bull. $22,436$.

radicum Bassett. Raspberry root gall. Ibid., Bull. 22. 437. similis Bassett. Ibid., Bull. 22, 437.

\section{Dipolepis Geoffroy.} Rhodites Hartig.

bicolor Harris. Spiny rose gall. Conn. Geol. Nat. Hist. Surv., Bull. 22, 440.

dichlocerus Harris. Long rose gall. Ibid., Bull. 22, 44 I. globuloides Beutenmïller. Globular rose gall. Ibid., Bull. 22, $77 \mathrm{I}$.

gracilis Ashmead. Ibid., Bull. 22, 441, 771 .

ignotus Osten Sacken. Mealy rose gall. Ibid., Bull. 22, 44I. radicum Osten Sacken. Rose root gall. Ibid., Bull. 22, 44 . rosæ Linncus. Mossy rose gall. Ibid., Bull. 22, 44I. 
Family IBALIIDE.

Ibalia Latreille.

maculipennis Haldeman. Conn. Geol. Nat. Hist. Surv., Bull. 22,442 .

Family MYRMARIDA.

Anaphes Haliday.

conotracheli Girault. Inaphoidca. Jour. N. Y. Ent. Soc. xviii, 246. Conn. Geol. Nat. Hist. Surv., Bull. 22, 447. pratensis Foerster. Can. Ent., 44, 88.

Family TETRASTICHIDE.

Tetrastichus Haliday.

malacosomæ Girault. Insec. Insc. Menstr. iv, I Io.

sp. reared from coccids.

sp. reared from Diastrophus cuscutaformis.

Family ENTEDONTID在.

Pleurotropis Fœrster.

lithocolletidis Ashmead. Proc. U. S. Nat. Mus., 43, 178. tarsalis Ashmead. Ibid.. 43, iz8.

Closterocerus VTestwood.

tricinctus Ashmead. Entedon Dalman. Conn. Geol. Nat. Hist. Surv., Bull. 22, 458 . (C.D.J.)

Family ELLOPHIDA.**

Sympiesis Foerster.

massasoit Crawford. Proc. U. S. Nat. Mus., 45, 258.

nigrifemora Ashmead. Systomspiesis. Conn. Geol. Nat. Hist. Surv., Bull. 22, 46r.

\section{Eulophus Geoffroy.}

sp.

Family ELASMID.E.

Elasmus Westwood.

atratus Howard. Conn. Geol. Nat. Hist. Surv., Bull. 22, 464.

* See also appendix. 


\section{Family ELACHERTIDA.}

Cirrospilus Westwood.

flavicinctus Riley. Conn. Geol. Nat. Hist. Surv., Bull. 22, 465. marylandi Girault. Descr. Hymenop. Chalcid., 4.

\section{Family PTEROMALIDE.}

Pteromalus Swederus.

boucheanus Ratzeburg. (Dibrachys). Conn. Geol. Nat. Hist. Surv., Bull. 22, 475.

nidulans (Fœrster) Thompson. egregius Fœrster. Ibid., Bull. 22, 773 .

tabacum Fitch. (Hypopteromalus). Ibid., Bull. 22, 474. verditer Norton. Ibid., Bull. 22, 472.

\section{Merisus Walker.}

isosomatis Riley. Conn. Geol. Nat. Hist. Surv., Bull. 22, 478. Pachyneuron Walker.

nigrocyaneum Norton. Chiropachy's nigrocyaneus. Conn. Geol. Nat Hist. Surv., Bull. 22, 48I.

Sphegigaster Spinola.

sp.

\section{Family APHELINIDE.}

\section{Encarsia Fœrster.}

luteola Howard. Bur. Ent., Tech. ser., i, 29: Conn. Geol. Nat. Hist. Surv., Bull. 22, 489.

\section{Aphelinus Dalman.}

mali Haldeman. Conn. Geol. Nat. Hist. Surv., Bull. 22, 490.

Prospaltella Ashmead.

perniciosi Tower. Parasite of the San José scale. Ann. Ent. Soc. Am., vi, I25.

Family ENCYRTIDA.

Blastothrix Mayr.

sericea Dalman. Conn. Geol. Nat. Hist. Surv., Bull. 22, 502. 
No. 31.] insects of CONnecticut: hymenopterA.

Family EUPELMIDA.

Anastatus Motschulsky.

bifasciatus Fonscolombe. Bur. Ent., Bull. 91, I 70.

$$
\text { Family CALLIMOMID E. }
$$

Ormyrus Westwood.

sp.

\section{Monodontomerus Westwood.}

æreus Walker. Conn. Geol. Nat. Hist. Surv., Bull. 22, 5 I4.

dentipes Boheman. Vet. Akad. Hand1., 4I, 23.

Syntomaspis Fœrster.

lazulella Ashmead. Conn. Geol. Nat. Hist. Surv., Bull. 22, 514.

\section{Callimome Spinola.}

brevicauda Osten Sacken. Torymus. Conn. Geol. Nat. Hist. Surv., Bull. 22, 515 .

flavicoxa Osten Sacken. Torymus. Ibid., Bull. 22. 5I5.

sp. Torymus.

$$
\text { Family MISCOGASTERIDA. }
$$

\section{Megorismus Walker.}

fletcheri Crawford. Conn. Geol. Nat. Hist. Surv., Bull. 22, 773.

\section{Family EURYTOMID王.}

\section{Brucophagus Ashmead.}

funebris Howard. Clover-seed Chalcis. Conn. Geol. Nat. Hist. Surv., Bull. 22, 520.

\section{Eurytoma Illiger.}

diastrophi Walsh. Conn. Geol. Nat. Hist. Surv., Bull. 22, 52 I. pissodis Girault. Bull. Brook. Ent. Soc, xii, 88.

\section{Isosoma Walker.}

sp.

$$
\text { Evoxysoma Ashmead. }
$$

vitis Saunders. Conn. Geol. Nat. Hist. Surv., Bull. 22, 523. 
Family PERILAMPIDA.

Perilampus Latreille.

hyalinus Say: Cyaneus. Conn. Geol. Nat. Hist. Surv., Bull. 22, 524 .

platygaster Say. Ibid., Bull. 22, 524.

Family CHALCIDID E.

Smicra Spinola.

rufofemorata Cresson. Conn. Geol. Nat. Hist. Surv., Bull. 22,526 .

Spilochalcis Thomson.

mariæ Riley. Conn. Geol. Nat. Hist. Surv., Bull. 22, 527. torvina Cresson. Ibid., Bull. 22, 527.

Eusayia Ashmead.

debilis Say. Sayiclla. Chalcis. Compl. Writ., ii, 720.

Chalcis Fabricius.

ovata Say. Conn. Geol. Nat. Hist. Surv., Bull. 22, 528. (A.E.V.)

Family LEUCOSPIDA.

Leucospis Fabricius.

affinis Say. Conn. Geol. Nat. Hist. Surv., Bull. 22, 528.

Family PLATYGASTRIDA.

Anopedius Forster.

error Fitch. Conn. Geol. Nat. Hist. Surv., Bull. 22, 533.

Polygnotus Fœrster.

striaticeps Ashmead. Conn. Geol. Nat. Hist. Surv., Bull. 22, 537.

Isocybus Forster.

pallipes Say. Conn. Geol. Nat. IIist. Surv., Bull. 22, 541.

Family SCELIONIDA.

Telenomus Haliday.

coloradensis Craw ford. Proc. Wrash. Ent. Soc., xi, 206. 


\section{Prosacantha Nees.}

marylandica Ashmead. Conn. Geol. Nat. Hist. Surv., Bull. 22, $55^{2}$.

Teleas Latreille.

coxalis Ashmead. Conn. Geol. Nat. Hist. Surv., Bull. 22, 553.

Caloteleia IVestwood.

marlatti Ashmead. Conn. Geol. Nat. Hist. Surr., Bull. 22, 554 .

Idris Fœrster.

nigricornis Brues. Conn. Geol. Nat. Hist. Surv., Bull. 22, 555. (W.M.IV.)

\section{Scelio Latreille.}

hyalinipennis Ashmead. Conn. Geol. Nat. Hist. Surr., Bull. 22,556 .

ovivorus Riley. Ibid., Bull. 22, 556. (IV.IH.P.)

\section{Family CERAPHRONID.E.}

Conostigmus Dahlbom.

Megaspilus Westwood.

schwarzi Ashmead. Conn. Geol. Nat. Hist. Surv., Bu1l. 22, 559.

Eumegaspilus Ashmead.

erythrothorax Ashmead. Conn. Geol. Nat. Hist. Surv., Bull. 22, 559 . (W.M.W.)

\section{Family DIAPRIIDE.}

Entomacis Forster.

ambigua Brues. Hemile.rodes. Conn. Geol. Nat. Hist. Surv., Bull. 22, 563.

\section{Galesus Curtis.}

vierecki Brues. Conn. Geol. Nat. Hist. Surv., Bull. 22, 564.

Diapria Latreille.

conica Fabricius. Conn. Geol. Nat. Hist. Surv., Bull. 22, 565. (W.H.P.) 
Family BELYTIDE.

Leptorhaptus Fœrster.

conicus Ashmead. Conn. Geol. Nat. Hist. Surv., Bull. 22, 570.

$$
\text { Family SERPHIDA. }
$$

Disogmus Fœrster.

obsoletus Brues. Conn. Geol. Nat. Hist. Surv., Bull. 22, 573.

Serphus Schrank.

californicus Holmgren. Proctotrypes. Conn. Geol. Nat. Hist. Surv., Bull. 22, 575 .

carolinensis Ashmead. Ibid., Bull. 22. 575 .

caudatus Say. Ibid., Bull. 22, 574.

melliventris Ashmead. Ibid., Bull. 22, 575.

quadriceps Ashmead. Ibid., Bull. 22, 576 .

Exallonyx Kieffer.

pallidicornis Brues. Jour. N. Y. Ent. Soc., xxvii, I4.

Family HELORID E.

Helorus Latreille.

paradoxus Provancher. Conn. Geol. Nat. Hist. Surv., Bull. 22,576 .

\section{Family PELECINID无.}

\section{Pelecinus Latreille.}

polyturator Drury. Conn. Geol. Nat. Hist. Surv., Bull. 22, 576.

\section{Family FORMICIDA.}

Stigmatomma Roger.

pallipes Haldeman. Conn. Geol. Nat. Hist. Surv., Bull. 22, $58 \mathrm{I}$.

\section{Ponera Latreille.}

coarctata pennsylvanica (Buckley) Emery. Conn. Geol. Nat. Hist. Surv., Bull. 22, 58I. (IV.M.IV.) 
Myrmacina Curtis.

graminicola americana lim r var. brevispinosa Emery. Conn.

Geol. Nat. Hist. Surv., Bull. 22, 584. (IV.M.IV.)

\section{Monomorium Mayr.}

minutum Mayr var. minimum (Buckley) Emery. Conn. Geol. Nat. Hist. Surv., Bu1l. 22, 584 .

pharaonis Linnæus. Little red house ant. Ibid., Bull, 22, 584.

Solenopsis Westwood.

molesta Say. Conn. Geol. Nat. Hist. Surv., Bull. 22, 584.

Pheidole Westwood.

pilifera Roger. Conn. Geol. Nat. Hist. Surv., Bull. 22, 584.

Crematogaster Lund.

lineolatus Say. Conn. Geol. Nat. Hist. Surv.. Bull. 22, 585.

lineolatus var. cerasi Fitch. Ibid., Bu1l. 22, 585. (W.M.W.)

Stenamma Mayr.

brevicorne Mayr. Conn. Geol. Nat. Hist. Surv., Bull. 22, 585. (W.M.W.)

Aphænogaster Mayr.

fulva Roger. Conn. Geol. Nat. Hist. Surv., Bull. 22, 585. (W.M.W.)

fulva aquia (Buckley) Emery. Ibid., Bu1l. 22, 586.

fulva aquia var. picea Emery. Ibid., Bull. 22, 586. (W.M.W.) mariæ Forel. Ibid., Bull. 22, 585. (W.M.IV.)

tennesseensis Mayr. Ibid., Bull. 22, 585. (IV.M.IV.)

treatæ Forel. Ibid., Bull. 22, 585 .

\section{Myrmica Latreille.}

punctiventris Roger. Conn. Geol. Nat. Hist. Surv., Bull. 22, 587. (W.M.W.)

rubra brevinodis Emery var. canadensis Vheeler. Ibid., Bull. 22,587 . (W.M.IT.)

rubra scabrinodis Nylander var. fracticornis Emery. Ibid., Bull, 22, 587 .

rubra scabrinodis var. sabuleti Meinert. Ibid., Bull. 22, 587. rubra scabrinodis var. schencki Emery. Ibid., Bull, 22, 587. 


\section{Leptothorax Mayr.}

acervorum canadensis Provancher. Conn. Geol. Nat. Hist. Surv., Bull. 22, 588. (W.M.W.)

curvispinosus Mayr. Ibid., Bull. 22, 588.

curvispinosus var. ambiguus Emery. Ibid., Bull. 22, 589.

emersoni Wheeler. Ibid., Bull. 22, 588. (IV.M.W.)

longispinosus Roger. Ibid., Bull. 22, 558. (W.M.IV.)

\section{Dolichoderus Lund.}

mariæ Forel. Conn. Geol. Nat. Hist. Surv., Bull. 22, 589. plagiatus Mayr. Ibid., Bull. 22, 590.

Tapinioma Forster.

sessile Say. Conn. Geol. Nat. Hist. Surv., Bull. 22, 590.

Brachymyrmex Mayr.

heeri depilis Emery. Conn. Geol. Nat. Hist. Surv., Bull. 22, 59. (W.M.W.)

\section{Prenolepis Mayr.}

imparis Say. Nylanderia. Conn. Geol. Nat. Hist. Surv., Bull. 22,591 .

imparis var. minuta Emery. Nylanderia. Ibid., Bull. 22, 59 I. Formicina Schuckard.

Lasins Fabricius.

brevicornis Emery. Conn. Geol. Nat. Hist. Surv., Bull. 22, 593.

claviger Roger. (Acanthomyops). Ibid., Bull. 22, 594. (IV.M.W.)

flavus nearcticus Wheeler. Ibid., Bull. 22, 593. (W.M.W.) interjectus Mayr. (Acanthomyops). Ibid., Bull. 22, 594. (IV.M.M.)

latipes Walsh. (Acanthomyops). Ibid., Bull. 22, 594.

niger Linnæus var. americanus Emery. Garden ant. Ibid., Piull. 22, 592 .

niger var. neoniger Emery. Ibid., Bull. 22, 593.

umbratus mixtus Nylander var, aphidicola Walsh. Ibid. Bull. 22, 593 .

umbratus mixtus var. speculiventris Enery. Ibid., Bull. 22, 593. (W.M.W.) 
Formica Latreille.

difficilis Emery var. consocians Wheeler. Conn. Geol. Nat. Hist. Surv., Bull. 22, 595. (IV.MI.IV.)

exsectoides Forel. Ibid., Bu11. 22, 595 .

fusca Linnæus var. subænescens Emery. Ibid., Bull. 22, 596.

(W.M.W.)

fusca var. subsericea Say. Silky ant. Ibid., Bull. 22, 596.

nepticula Wheeler. Ibid., Bull. 22, 595. (WV.MI.IV.)

pallide-fulva nitidiventris Emery. Ibid., Bull. 22, 595.

pallide-fulva schaufussi Mayr. Ibid., Bull. 22, 595.

pallide-fulva schaufussi var. incerta Emery. Ibid., Pull. 22, 595 .

rufa integra Nylander. Ibid., Bull. 22, 595. (W.M.W.)

rufa obscuriventris Mayr. Ibid., Bull. 22, 595. (IV.M.W.)

sanguinea aserva Forel. Ibid., Bu11. 22, 595. (W.M.IV.)

sanguinea rubicunda Emery. Ibid., Bull. 22, 595. (IV.M.IV.) sanguinea rubicunda var. subintegra Emery. Ibid., Bull. 22. 595 .

subpolita Mayr. Ibid., Bull. 22, 596. (G.MI.)

subpolita var. neogagates Emiery. Ibid., Bull. 22, 596.

truncicola Nylander, subspecies obscuriventris Mayr. Ibid., Bu1l. 22, 595 .

Polyergus Latreille.

lucidus Nayr. Conn. Geol. Nat. Ilist Surv., Bull. 22, 599. (G.M.)

Camponotus Mayr.

castaneus Latreille. Conn. Geol. Nat. Hist. Surr., Bull. 22 , 600.

castaneus, subspecies americanus Mayr. Ibid., Bull. 22, 600.

fallax Nylander var. nearcticus Emery. Ibid., Bull. 22, 600.

herculeanus ligniperdus Latreille var. novæboracensis Fitch. Ibid., Bull. 22, 600.

herculeanus pennsylvanicus DeGeer. Carpenter Ant. Ibid. Bull. 22, 600 .

herculeanus pennsylvanicus var. ferrugineus Fabricius. Ibid.,

Bull. 22, 600 . 


\section{Family CHRYSIDIDE.}

Omalus Jurine.

irridescens Norton. Conn. Geol. Nat. Hist. Surv., Bull. 22, 602. sinuosus Say. Ibid., Bull. 22, 602.

Notozus Fœrster.

marginatus Patton. Conn. Geol. Nat. Hist. Surv., Bull. 22, 603.

Hedychridium Latreille.

dimidiatum Say. Conn. Geol. Nat. Hist. Surv., Bull. 22, 60\%. Hedychrum Latreille.

obsoletum Say. Conn. Geol. Nat. Hist. Surv., Bull. 22, 603, violaceum Brullé. Ibid., Bull. 22, 603.

\section{Chrysis Linnaeus.}

cærulans Fabricius. (Tetrachrysis). Conn. Geol. Nat. Hist. Surv., Bull. 22, 604 .

nitidula Fabricius. (Tetrachrysis). Ibid., Bull. 22, 604. parvula Fabricius. (Trichrysis). Ibid., Bull. 22, 604. verticalis Patton. (Chrysogona). Ibid., Bull. 22, 604.

Family BETHYLIDA.

Pristocera Klug.

armifera Say. Conn. Geol. Nat. Hist. Surv., Bull. 22, 609.

Lælius Ashmead.

tricarinatus Ashmead. Conn. Geol. Nat. Hist. Surv., Bull. 22, 6 Io.

Paralælius Kieffer.

pedatus Say. Bethylus. Conn. Geol. Nat. Hist. Surv., Bull. 22, 610 .

Epyrus Westwood.

rufipes Say. Conn. Geol. Nat. Hist. Surv., Bull. 22, 6r I.

Rhabdepyris Kieffer.

occidentalis Ashmead. Conn. Geol. Nat. Hist. Surv., Bull. 22, 6 I2.

Parasierola Cameron.

cellularis Say. Conn. Geol. Nat. Hist. Surv., Bull. 22, 6r 2. 
Family DRYINIDA.

\section{Gonatopus Ljungh,}

contortulus Patton. Conn. Geol. Nat. Hist. Surv., Bull. 22, 6 I 4. (IV.H.P.)

\section{Aphelopus Dalman.}

americanus Ashmead. Conn. Geol. Nat. Hist. Surv., Bull. 22, 6 I 5 .

\section{Family SCOLIID E.}

Scolia Fabricius.

bicincta Fabricius. Conn. Geol. Nat. Hist. Surv., Bull. 22, 6 I6. Campsomeris LePeletier.

plumipes Drury. Elis. Conn. Geol. Nat. Hist. Surv., Bull. 22, 6 I7.

Elis Fabricius.

interrupta Say. Plesia. Myine hamata. Conn. Geol. Nat. Hist Surv., Bull. 22, 6 г 7.

quinquecincta Fabricius. Plesia. Mysine sercincta. Ibid., Bull. 22, 6 I 7 .

Sierolomorpha Ashmead.

ambigua Ashmead. Conn. Geol. Nat. Hist. Surv., Bull. 22, 6 I8.

Tiphia Fabricius.

brunneicornis Viereck. Conn. Geol. Nat. Hist. Surv., Bull. 22, 6 I9.

egregia Vicreck. Ibid., Bull. 22, 619.

inornata Say. Ibid., Bull. 22, 619.

punctata Robertson. Ibid., Bull. 22, 619.

relativa Viereck. Ibid., Bull. 22, 619.

waldeni Viereck. Ibid., Bull. 22, 6I9.

\section{Family SAPYGID无.}

Sapyga Latreille.

centrata Say. Conn. Geol. Nat. Hist. Surv., Bull. 22, 620. 
Family METHOCID无.

Methoca Latreille.

stygia Say. Conn. Geol. Nat. Hist. Surv., Bull. 22, 620.

Family MYRMOSIDE.

Myrmosa Latreille.

unicolor Say. Conn. Geol. Nat. Hist. Surv., Bull. 22, 62 I.

Family MUTILLIDÆ.

Pseudomethoca Ashmead.

canadensis Blake. Conn. Geol. Nat. Hist. Surv., Bull. $22,622$. Nomiæphagus Ashmead.

simillimus Smith. sanbornii. Conn. Geol. Nat. Hist. Surv., Bull. 22, 623 .

Dasymutilla Ashmead.

cariniceps Fox. scrobinata Rohwer. Ibid., Bull. 22, 623.

castor Blake. Conn. Geol. Nat. Hist. Surv., Bull. 22, 624.

cypris Blake. Ibid., Rull. 22, 623.

ferrugata Fabricius. Ibid., Bull. 22, 623 .

gibbosa Say. Ibid., Bull. 22, 624.

lepeletierii Fox harmoniiformis Rohwer. Ibid., Bull. 22, 624 (A.B.C.).

macra Cresson. Ibid., Bull. 22, 624.

occidentalis Linnæus. Ibid., Bull, 22, 624.

vesta Cresson var. zella Rohwer. champlaini Rohwer. Ibid., Bull. 22, 623.

Ephuta Say.

scrupea Say. Conn. Geol. Nat. Hist. Surv., Bull. 22, 625.

Timulla Ashmead.

hexagona Say. Conn. Geol. Nat. Hist. Surv., Bull. 22, 625.

ornativentris Cresson. Ibid., Bull. 22, 625. 


\section{Family PSAMMOCHARIDA.}

Ceropales Latreille.

bipunctata Say. Conn. Geol. Nat. Hist. Surv., Bull. 22, 626. fraterna Smith. Ibid., Bull. 22, 626.

longipes Smith. Ibid., Bull. 22, 626 .

robinsoni Cresson. Ibid., Bull. 22, 626.

\section{Pseudagenia Kohl.}

architecta Say. Conn. Geol. Nat. Hist. Surv., Bull. 22, 627.

Ageniella Banks.

calcarata Cresson. Conn. Geol. Nat. Hist. Surv., Bull. 22, 628.

iridipennis Cresson. Ibid., Bull. 22, 628.

\section{Priocnemis Schiödte.}

alienata Suith. Conn. Geol. Nat. Hist. Surv., Bull. 22, 628.

conica Say. Ibid., Bull. 22, 628.

germana Cresson. Ibid., Bull. 22, 628.

notha Cresson. Ibid., Bull. 22, 628.

\section{Planiceps.}

niger Cresson. Trans. Am. Ent. Soc., i, I $3^{6 .}$

\section{Episyron Schiödte.}

biguttatus Fabricius. Conn. Geol. Nat. Hist. Surv., Bull. 22, $63 \mathrm{I}$.

quinque-notatus Say. 5-notatus. Ibid., Bull. 22, 63I.

\section{Aporinellus Banks.}

fasciatus Smith. Conn. Geol. Nat. Hist. Surv., Bull. 22, 63I.

Pompiloides Radoszkowshi.

argenteus Cresson. Conn. Geol. Nat. Hist. Surv., Bull. 22, $63 \mathrm{I}$.

cylindricus Cresson. Ibid., Bull. 22, 631.

marginatus Say. Ibid., Bull, 22,631.

subviolaceus Cresson. Ibid., Bill. 22, 631 .

tropicus Linnaens. Ibid., Bull, 22, 63I.

Sericopompilus Aslimead.

humilis Cresson. Conn. Geol. Nat. Hist. Surv., Bull. 22, 632. 
Arachnophroctonus Ashmead.

interruptus Say. Conn. Geol. Nat. Hist. Surv., Bull. 22, 632.

Psammochares Latreille.

æthiops Cresson. (Lophopompilus). Conn. Geol. Nat. Ilist. Surv., Bull. 22, 633 .

atrox Dahlbom. (Lophopompilus). Ibid., Bull. 22, 633 .

luctuosus Cresson. (Psammochares). Ibid., 13ull. 22, 633.

philadelphicus LePeletier. 'Ibid., Bull. 22, 633.

relativus Fox. (Psammochares). lbid., Lull. 22, 633.

(H.W.W.)

scelestus Cresson. (Psammozhrcs). Ibid., Bull. 22, 633. tenebrosus Cresson. (Fsammochares). Ibid., Bull. 22, 633. virginiensis Cresson. (Anoplius). Ibid., Bull. 22, 633.

Family EUMENID蚱.

Zethus Fabricius.

spinipes Say. Conn. Geol. Nat. Hist. Surv., Bull. 22, 634.

Eumenes Fabricius.

fraternus Say. Potter wasp. Conn. Geol. Nat. Hist. Surv., Bull. 22, 634.

Monobia Saussure.

quadridens Linnaus. Conn. Geol. Nat. Hist. Surv., Bull. 22, 635. Nortonia Saussure.

symmorpha Saussure. Conn. Geol. Nat. Hist. Surv., Bull. 22, 635.

Odynerus Latreille.

albomarginatus Saussure. (Symmorphus). Conn. Geol. Nat. Hist Surv., Bull. 22, 636.

anormis Say. Ibid., Bull. 22, 637. (H.W.W.)

birenimaculatus Saussure. (Ancistrocerus). Ibid., Bull. 22, 636.

boscii LePeletier. Ibid., Bull. 22, 637.

campestris Saussure. (Ancistrocerus). Ibid., Bull. 22, 636. (II.W.W.)

capra Saussure. (Ancistrocerus). Ibid., Bull. 22, 636.

catskilli Saussure. (Ancistrocerus). Ibid., Bull. 22, 636. 
collega Saussure. Ibid., Bull. 22, 637.

cristatus Saussure. (Symmorphus). Ibid., Bull. 22, 636.

debilis Saussure. (Symmorphus). Ibid., Bull 22, 636.

dorsalis Fabricius. Ibid., Bull. 22, 637 .

foraminatus Saussure. Ibid., Bull. 22, 637.

leucomelas Saussure. Ibid., Bull. 22, 637.

nortonianus Saussure. Ibid., Bull. 22, 637.

pedestris Saussure. Ibid., Bull. 22, 637.

pennsylvanicus Saussure. Ibid., Bull. 22, 637.

spinolæ Saussure. (Ancistrocerus). Ibid., Bull. 22, 636.

tigris Saussure. (Ancistrocerus). Ibid., Bull. 22, 636.

unifasciatus Saussure. (Ancistrocerus). Ibid., Bull. 22, 636. vagus Saussure. Ibid., Bull. 22, 637.

waldeni Viereck. (Ancistrocerus). Ibid., Bull. 22, 636.

Family VESPID正.

Vespa Linnaus.

crabro Linneus. Giant hornet. Conn. Geol. Nat. Hist. Surv., Bull. 22, 64I.

Vespula Thompson.

consobrina Saussure. (Vespa). Conn. Geol. Nat. Hist. Surv., Bull. 22, 6+3.

diabolica Saussure. (Dolichovespula). Trespa. Common yellow jacket. Ibid., Bull. 22, 642.

germanica Fabricius var. pennsylvanica Saussure. (Vespa). Common yellow jacket. Ibid., Bull. 22, 643 .

maculata Linnæens. (Dolichovespula). I'espa. White-faced hornet. Ibid., Bull. 22, $6+2$.

vulgaris Linnæus var. communis Saussure. I'espa. Ibid., Bull. $22,6+3$.

Polistes Latreille.

annularis Linnæus. Conn. Geol. Nat. Hist. Surv., Bull. 22, $6+4$. pallipes LePeletier. Common wasp. Ibid., Bull. 22, 644.

variatus Cresson. Ibid., Bull. 22, 644.

Family AMPULICIDA.

Rhinopsis Westwood.

melanognathus Rohwer. Conn. Geol. Nat. Hist. Surv., Bull. 22,652 . 


\section{Family SPHECIDÆ.}

Alyson Jurine.

oppositus Say. Conn. Geol. Nat. Hist. Surv., Bull. 22, 654.

Nysson Latreille.

æqualis Say. Conn. Geol. Nat. Hist. Surv., Bull. 22, 655.

lateralis Packard. Ibid., Bull. 22, 655.

tramosericus Viereck. Ibid., Bull. 22, 655.

Paramellinus Rohwer.

bipunctatus Say. Conn. Geol. Nat. Hist. Surv., Bull. 22, 656.

\section{Hoplisus LePeletier.}

costalis Cresson. Gorytes. Conn. Geol. Nat. Hist. Surv., Bull. 22,657 .

fuscus Taschenberg. Gorytes. Ibid., Bull. 22, 656.

gracilis Patton. Gorytes. Ibid., Bull. 22, 656.

nebulosus Packard. Gorytes. Ibid., Bull. 22, 656.

phaleratus Say. Gorytes. Ibid., Bull. 22, 656.

Psen Latreille.

cressoni Packard. (Mimesa). Conn. Geol. Nat. Hist. Surv., Bull. 22, 659.

pauper Packard. (Mimesa). Ibid., Bull. 22, 659.

Oxybelus Latreille.

quadrinotatus Say. Conn. Geol. Nat. Hist. Surv., Bull. 22. 659. Notoglossa Dahlbom.

emarginata Say. Conn. Geol. Nat. Hist. Surv., Bull. 22, 660. Anacrabro Packard.

ocellatus Packard. Conn. Geol. Nat. Hist. Surv., Bull. 22, 66 I. (S.N.D.)

Lindenius LePeletier.

errans Fox. Conn. Geol. Nat. Hist. Surv., Bu11. 22, 664.

Rhopalum Kirby.

pedicellatum Packard. Conn. Geol. Nat. Hist. Surv., Bu1l. 22, 664.

Solenius LePeletier.

bigeminus Packard. (Protothyreopus). Conn. Geol. Nat. Hist. Surv., Bull. 22, 668 . 
chrysargynus LePeletier. (Hypocrabro). Ibid., Bull. 22, 669. decemmaculatus Say. (Hypocrabro). Ibid., Bull. 22, 669. interruptus LePeletier. (Solenius). Ibid., Bu1l. 22, 665. obscurus Smith. (Clytochrysus). Ibid., Bu1l. 22, $66_{5}$. producticollis Packard. (Solenius). Ibid., Bull. 22, 666. rufifemur Packard. (Protothyreopus). Ibid., Bull. 22, 668. sayi Cockerell. (Xestocrabro). sermaculatus. Ibid., Bull. 22, 667. singularis Smith. (Lophocrabro). Ibid., Bu1l. 22, 667. (W.F.) stirpicola Packard. (Hypocrabro). Ibid., Bull. 22, 668.

\section{Crabro Fabricius.}

advenus Smith. (Synothyreopus). Conn. Geol. Nat. Hist. Surv., Bull. 22, 670.

æqualis Fox. (Crabro). Ibid., Bu1l. 22, 670.

argus Packard. (Crabro). Ibid., Bull. 22, 670.

oblongus Packard. Ibid., Bull. 22, 662.

tumidus Packard. (Synothreopus). Ibid., Bull. 22, 670.

unicus Patton. Ibid., Bull. 22, 663.

\section{Aphilanthops Patton.}

frigidus Smith. Conn. Geol. Nat. Hist. Surv., Bull. 22, 672.

Philanthus Fabricius.

bilunatus Cresson. Conn. Geol. Nat. Hist. Surr., Bull. 22, 673. dubius Cresson. Ibid., Bu1l. 22, 673 .

politus Say. Ibid., Bu1l. 22, 673 .

punctatus Say. Ibid., Bull. 22, 673.

sanbornii Cresson. Ibid., Bull. 22, 673 .

solivagus Say. Ibid., Bull. 22, 673.

Trypoxylon Latreille.

clavatum Say. Conn. Geol. Nat. Hist. Surv., Bull. 22, 676.

excavatum Say. Ibid., Bull. 22, 676.

frigidum Smith. Ibid., Bull. 22, 6,6 .

politum Say. albitarse. Ibid., Bu11. 22, 676 .

tridentatum Packard. Ibid., Bull. 22, 676 .

Chlorion Latreille.

auripes Fernald. (Isodontia). Conn. Geol. Nat. Hist. Surv., Bu11. 22, 679 . 
bifoveolatum Taschenberg. (Priononyx). Ibid., Bull. 22, 679. cyaneum Dahlbom. var. ærarium Patton. (Chlorion). Ibid., Bull. 22, 679.

harrisi Fernald. (Isodontia). Ibid., Bull. 22, 679. ichneumoneum Linnæus. (Ammobia). Ibid., Bull. 22, 680. pennsylvanicum Linnæus. (Ammobia). Ibid., Bu1l. 22, 680.

Sphex Linneus.

argentatus Hart. Bu11. 111. State Lab. Nat. Hist., vii, 266.

arvensis Dahlbom. Conn. Geol. Nat. Hist. Surv., Bull. 22, 682.

aureonotata Cameron. Biol. Centr. Amer., Hymenop., ii, 7. nigricans Dahlbom. Psyche, $x, 157$.

procerus Dahlbom. Conn. Geol. Nat. Hist. Surv., Bull. 22, 682.

Sceliphron Klug.

cyaneum Klug. Chalybion corulcum. Conn. Geol. Nat. Hist. Surv., Bull. 22, 682.

Pelopæus Latreille.

cæmentarius Drury. Conn. Geol. Nat. Hist. Surv., Bull. 22, 682.

Lyroda Say.

subita Say. Conn. Geol. Nat. Hist. Surv., Bull. 22, 683 . triloba Say. Ibid., Bull. 22, 683. (H.IV.W.)

\section{Notogonidea Rohwer.}

argentata Beauvois. Conn. Geol. Nat. Hist. Surv., Bull. 22, 684.

\section{Larropsis Patton.}

distincta Smith. Conn. Geol. Nat. Hist. Surv., Bull. 22, 684.

Tachytes Panzer.

breviventris Cresson. Conn. Geol. Nat. Hist. Surv., Bull. 22, 686.

calcaratus Fox. Ibid., Bull. 22, 686.

harpax Patton. Ibid., Bull. 22, 686.

Tachysphex Kohl.

apicalis Fox. Conn. Geol. Nat. Hist. Surv., Bull. 22, 687.

Astata Latreille.

bicolor Say. Conn. Geol. Nat. Hist. Surv., Bull. 22, 688. unicolor Say. Ibid., Bull. 22, 688. 
Stigmus Panzer.

americanus Packard. Conn. Geol. Nat. Hist. Surv., Bull. 22, 689.

Spilomena Shuckard.

pusilla Say. Conn. Geol. Nat. Hist. Surv., Bull. 22, 689.

Passalœcus Shuckard.

annulatus Say. Conn. Geol. Nat. Hist. Surv., Bull. 22, 689.

Pemphredon Latreille.

inornatus Say. Conn. Geol. Nat. Hist. Surv., Bull. 22, 690. tenax Fox. Ibid., Bull. 22, 6go.

Plenoculus Fox.

atlanticus Viereck. Cunn. Geol. Nat. Hist. Surv., Bull. 22, 691.

Family BEMBECIDA.

Sphecius Dahlbom.

speciosus Drury. Conn. Geol. Nat. Hist. Surv., Bull, 22, 692.

Microbembex Patton.

monodonta Say. Conn. Geol. Nat. IIist. Surv., Bull. 22, 693.

Bicyrtes Le Peletier.

Bembidula Burmeister.

quadrifasciata Say. Conn. Geol. Nat. Hist. Surv., Bull. 22, 693.

ventralis Say. Ibid., Bull. 22, 693 .

Bembix Fabricius.

spinolæ LePeletier. Conn. Geol. Nat. Hist. Surv., Bull. 22, 694.

Family CERCERIDA:.

Eucerceris Cresson.

zonatus Say. Proc. Ent. Soc. Phila. v, I05.

Cerceris Latreille.

clypeata Dahlbom. Conn. Geol. Nat. Hist. Surv., Bull. 22, 695.

compar Cresson. Ibid., Bull. 22, 695.

dentifrons Cresson. Ibid., Bull. 22, 696. (J.A.H.)

deserta Say. Ibid., Bull. 22, 695. 
fasciola Cresson. Ibid., Bull. 22, 695.

fulvipediculata Schletterer. Ibid., Bull. 22, 695.

fumipennis Say. Ibid., Bull. 22, 695.

imitatoria Schletterer. Ibid., Bull. 22, 695.

robertsoni Fox. Ibid., Bull. 22, 695. (S.N.D.)

\section{Family HALICTIDA $\mathrm{E}$.}

\section{Halictus Latreille.}

albipennis Robertson. (Chloralictus). Conn. Geol. Nat. Hist. Surv., Bull. 22, 702.

arcuatus Robertson. (Evylæus). Ibid., Bull. 22, 701.

cæruleus Robertson. (Chloralictus). Ibid., Bull. 22, 702.

cephalicus Robertson. (Paralictus). Ibid., Bull. 22, 7 Or.

confusus Robertson. (Oxystoglossa). Augochlora. Ibid.,

Bull. 22, 701 .

coriaceus Smith. (Lasioglossum). Ibid., Bull. 22, 700.

cressoni Robertson. (Chloralictus). Ibid., Bull. 22, 702.

foxi Robertson. (Evylæus). Ibid., Bull. 22, 70 .

lerouxi LePeletier. (Halictus). Ibid., Bull. 22, 7 I.

ligatus Say. (Halictus.) Ibid., Bull. 22, 70I.

marinus Crawford. (Chloralictus). Ent. News. I5, 99.

nymphæarum Robertson. (Chloralictus). palustris. Conn.

Geol. Nat. Hist. Surv., Bull. 22, 702.

obscurus Robertson. (Chloralictus). Ibid., Bull. 22, 702.

pectoralis Smith. (Evylæus). Ibid., Bull. 22, 7OI.

persimilis Vicreck. (Oxystoglossa). Augochlora. similis. Ibid., Bull. 22, 703 .

pilosus Smith. (Chloralictus). Ibid., Bull. 22, 7OI.

provancheri Dalla Torre. (Halictus). fasciatus. Ibid., Bull. $22,7 \mathrm{OI}$.

purus Say. (Augochlora). Orystoglossa. Ibid., Bull. 22, 7or. (H.W.W.)

quadrimaculatus Robertson. (Evylæus). Ibid., Bull. 22, 7 ОI. radiatus Say. (Agapostemon). pulchra. Ibid., Bull. 22, 70 I. sparsus Robertson. (Chloralictus). Ibid., Bull. 22, 702.

splendens LePeletier. (Agapostemon). Ibid., Bull. 22, 70 . tegularis Robertson. (Chloralictus). Ibid., Bull. 22, 702. truncatus Robertson. (Evylæus). Ibid., Bull. 22, 70I. 
versatus Robertson. (Chloralictus). Ibid., Bull. 22, 702.

vierecki Crawiord. (Chloralictus). nymphatis. Ibid., Bull. 22,702 .

virescens Fabricius. (Agapostemon), nigricomis. viridulus Authors. Ibid., Bull. 22, 70I.

viridissimus Viereck. (Augochlora). viridula. Ibid., Bull. 22, 700.

zephyrus Smith. (Chloralictus). Ibid., Bull, 22, 702.

Sphecodes Latreille.

arvensis Patton. (Drepanium). Conn. Geol. Nat. Hist. Surv., Bull, 22, 708 .

confertus Say. (Drepanium). falcifer. Ibid., Bull. 22, 708. mandibularis Cresson. (Sphecodium). Ibid., Bull. 22, 708. minor Robertson. (Sphecodes). Ibid., Bull. 22, 708.

Family ANDRENID.E.

Andrena Fabricius.

arabis Robertson. Con11. Geol. Nat. Hist. Surv., Bull. 22, 7I2. asteris Robertson. Ibid., Bull. 22, 7I3. barbarica Viereck. Trans. Am. Ent. Soc., xliii, 369. bisalicis Viereck. Conı. (jeol. Nat. Hist. Surv., Bull. 22, 7I2. braccata Viereck. Ibid., Bull, 22, 713. bradleyi Viereck. I.bid., Bull. 22, 709. čnadensis Dalla Turre. Ibid., Bull. 22, 7I2. carlini Cockerell. bicolor. Ibid., Bull. 22, 7r3. claytoniæ Robertson. Ibid.. Bull. 22, 776. cratægi Robertson. Ibid., Bull. 22, 7IO. cressoni Robertson. Ibid., Bull. 22, 7I3. erythrogastra Ashmead var. rhodura Cockerell. Ibid., Bull. 22, 7 II.

flavoclypeata Snith. bifunctata. Ibid., Ru1l. 22, 7I2. forbesi Robertson. Ibid., Bull, 22, 713. fragilis Snith. platyparis. Ibid., Bull. 22, 7 I2. geranii maculati Robertson. Ibid., Bu1l, 22, 710. hilaris Smith. Ibid., Hi1ll. 22, 713. hippotes Robertson. Ibid., Bull. 22, 7r3. hirticincta Provancher. americana. fimbriata. Ibid., Bull. 22. 7 Io. 
integra Smith. lineata. Ibid., Bull. 22, 712.

krigiana Robertson. Ibid., Bull. 22, 777. (E.J.S.M.)

mariæ Robertson var. concolor Robertson. Ibid., Bull. 22, 7I3. multiplicatiformis Viereck. Ibid., Bull, 22, 7I4.

nasoni Robertson. iestita. hartfordensis. Ibid., Bull. 22, $7 \mathrm{II}$. nivalis Smith. Ibid., Bull. 22, 7 II.

novæ-angliæ Viereck. Ibid., Bull. 22, 7 I I .

nubecula Smith. Ibid., Bull. 22, 7I2.

obscura Robertson. Ibid., Bull. 22, 7 I4.

perplexa Smith var. viburnella Grænicher. Ibid., Bull. 22, 712. placida Smith. macgillivrayi. Ibid., Bull. 22, 7 I I.

pruni Robertson. dumingi. Ibid., Bull. 22, 712.

robertsoni Dalla Torre. scrotina. Ibid., Bull. 22, 712.

rugosa Robertson. Ibid., Bull. 22, 7 I4.

salictaria Robertson. Ibid., Bull. 22, 7 I I.

solidaginis Robertson. Ibid., Bull. 22, 712.

spireana Robertson. Ibid., Bull. 22, 7I4.

thaspii Granicher. mandibularis. Ibid., Bull. 22, 713.

vicina Smith. Ibid., Bull. 22, 713.

victima Smith. Ibid., Bull. 22, 715.

weedi Viereck. Ibid., Bull. 22, 7I4.

wilkella Kirby. winklcyi Viereck. Ibid., Bull. 22, 712.

ziziæ Robertson. Ibid., Bull. 22, 7I2.

ziziæformis Cockerell. Can. Ent., 49, 234.

Family DUFOUREIDA.

Halictoides Nylander.

novæangliæe Robertson. (Conohalictoides). Conn. Geol. Nat. Hist. Surv., Bull. 22, 720.

Family MACROPIDÆ.

Macropis Panzer.

ciliata Patton. Conn. Geol. Nat. Hist. Surv., Bull. 22, 720. patellata Patton. Ibid., Bull. 22, 720. 
Family PANURGIDA.

Perdita Sinith.

novæangliæ Viereck. Con11. Geol. Nat. Hist. Surv., Bull. 22, 721.

octomaculata Say. Cockercllia. Ibid., Bull. 22, 72 I.

Panurginus Nylander.

asteris Robertson. Conn. Geol. Nat. Hist. Surv., Bull. 22, 721. parvus Robertson. Ibid., Bu1l. 22, 721 .

Calliopsis Sinith.

andreniformis Smith. Conn. Geol. Nat. Hist. Surv., Bull. 22, 722.

Family NOMADIDE.

Nomada Fabricius.

americana Kirby. (Centrias). Conn. Geol. Nat. IFist. Surv., Bu11. 22, 723.

cuneata Robertson. (Gnathias). Ibid., Bull. 22, 726.

denticulata Robertson. Ibid., Bull. 22, 725.

illinoiensis Robertson. Ibid., Bull. 22, 724. (H.W.W.)

imbricata Smith. (Holonomada). Ibid., Bull. 22, 724.

incerta Cresson. (Centrias). Ibid., Bull. 22, 724.

obliterata Cresson. (Heminomada). Ibid., Bull. 22, 725.

pygmæa Cresson. Ibid., Bull. 22, 726.

sayi Robertson. Ibid., Bull. 22, 725.

vicina Cresson. Ibid., Bull. 22, 724.

Epeoloides Girand.

Viercckella Swenk.

pilosula Cresson. Conn. Geol. Nat. Hist. Surv., Bull. 22, 727. (E.L.D.)

Epeolus Latreille.

pusillus Cresson. Conn. Geol. Nat. Hist. Surv., Bull. 22, 728.

Triepeolus Robertson.

donatus Smith. Conn. Geol. Nat. Hist. Surv., Bull. 22, 729. lunatus Say. Ibid., Bull. 22, 730. 
Holcopasites Ashmead.

Neopasites Ashmead.

illinoiensis Robertson. Conn. Geol. Nat. Hist. Surv., Bull. 22, 730.

\section{Family EUCERID无.}

Mellissodes Latreille.

bimaculata LePeleticr. Conn. Geol. Nat. Hist. Surv., Bull. 22, $73 \mathrm{I}$.

dentiventris Smith. Ibid., Bull, 22, 732.

desponsa Smith. Ibid., Bull. 22, 732.

perplexa Cresson. Ibid., Bull. 22, 732.

rustica Say. Ibid., Bull. 22, 73 I.

Tetralonia Spinola.

atriventris Smith. Symhalonia. Conn. Geol. Nat. Hist. Surv., Bull. 22, 733 .

Xenoglossa Snith.

pruinosa Say. (Peponapis). Conn. Geol. Nat. Hist Surv., Bull. 22, 733 .

Family ANTHOPHORIDE.

Anthophora Latreille.

floridana Smith. (Emphoropsis). Conn. Geol. Nat. Hist. Surv., Bull. 22, 735.

terminalis Cresson. (Clisodon). Ibid., Bull. 22, $73^{6}$.

Family HYLÆIDE.

Hylæus Fabricius.

Prosopis Fabricius.

ellipticus Cockerell. Conn. Geol. Nat. Hist. Surv., Bull. 22, 737. modestus Say. affinis. Ibid., Bull. 22, 738 .

pygmæus Cockerell. Ibid., Bull. 22, 737.

saniculæ Robertson. Can. Ent., xxxvi, 273.

ziziæ Robertson. Ibid., Bull. 22, 737.

Family COLLETID瓜.

Colletes Latreille.

æstivalis Patton. Conn. Geol. Nat. Hist. Surv., Bull. 22, 740. compactus Cresson. Ibid., Bull. 22, 739. 
inæqualis Say. Ibid., Bull. 22, 739.

validus Cresson. Ibid., Bull. 22, 740.

sp. Ibid., Bull. 22, 741.

\section{Family STELIDIDE.}

Stelis Panzer.

fœederalis Smith. (Microstelis). mitida. Conn. Geol. Nat. Hist. Surv., Bull. 22, 741.

lateralis Cresson. (Microstelis). Ibid., Bull. 22, 74I.

Family MEGACHILIDE.

Megachile Latreille.

brevis Say. (Megachile). Conn. Geol. Nat. Hist. Surv., Bull. 22,743 .

exclamans Viereck. (Xanthosarus). Ibid., Bull. 22, 743.

infragilis Cresson. (Anthemois). Ibid., Bull. 22, 743.

latimana Say. (Xanthosarus). femoratus. Ibid., Bull, 22, 744. mendica Cresson. (Megachile). Ibid., Bull. 22, 745.

vidua Smith. (Delomegachile). frigida. Ibid., Bull. 22, 743. (H.W.W.)

Cœlioxys Latreille.

dubitata Smith. rufitarsis. Conn. Geol. Nat. Hist. Surv., Bull. $22,7+6$.

dubitata var. melanopoda Viereck. Ibid., Bull. 22, 747. mœsta Cresson. Ibid., Bu1l. 22, 746.

octodentata Say. Ibid., Bull. 22, 747.

sayi Robertson. Ibid., Bull. 22, 747.

Osmia Panzer.

albiventris Cresson. (Leucosmia). Conn. Geol. Nat. Hist. Surv., Bull. 22, 749.

atriventris Cresson. (Osmia). Ibid., Bull. 22, 749.

conjuncta Cresson. (Diceratosmia). Ibid., Bull. 22, 748.

distincta Cresson. (Nothosmia). Ibid., Bull. 22, 748.

lignaria Say. (Ceratosmia). Ibid., Bull. 22, $7+8$.

major Robertson. (Osmia). Ibid., Bull. 22, 749 .

pumila Cresson. (Osmia). Ibid., Bull. 22, 749.

purpurea Cresson. (Osmia). Ibid., Bull. 22, 748.

rustica Cresson. (Osmia). Ibid., Bull. 22, 749. (H.W.W.) 
Andronicus Cresson.

cylindricus Cresson. Conn. Geol. Nat. Hist. Surv., Bull. 22, $75 \mathrm{I}$.

pilosifrons Cresson. (Alcidamea). Ibid., Bull. 22, $75^{\mathrm{I}}$.

productus Cresson. (Alcidamea). Ibid., Bull. 22, $75 \mathrm{I}$.

truncatus Cresson. (Alcidamia). Ibid., Bu1l. 22, $75 \mathrm{I}$.

Heriades Spinola.

carinatus Cresson. (Trypetes). Conn. Geol. Nat. Hist. Surv., Bull. 22, 751 .

Dianthidium Cockerell.

notatum Latreille. Conn. Geol. Nat. Hist. Surv., Bull. 22, $75^{2}$. simile Cresson. Ibid., Bull. 22, 752.

Family CERATINIDA.

Ceratina Latreille.

dupla Say. Conn. Geol. Nat. Hist. Surv., Bull. 22, 753.

metallica H. S. Smith. Ibid., Bull. 22, 753.

Family XYLOCOPIDA.

Xylocopa Latreille.

virginica Drury. Conn. Geol. Nat. Hist. Surv., Bull. 22, 753.

Family APIDAE.

Bremus Panzer.

Bombus Latreille.

affinis Cresson. Conn. Geol. Nat. Hist. Surv., Bull. 22, 755.

bimaculatus Cresson. Ibid., Bull. 22, 757.

fervidus Fabricius. Ibid., Bu1l. 22, 755.

impatiens Harris. virginicus. Ibid., Bull. 22, 756.

pennsylvanicus DeGeer. americanormm DeGeer. Ibid., Bull.

22,755 .

perplexus Cresson. Ibid., Bull. 22, 756.

ternarius Say. Ibid., Bull. 22, $75^{6}$.

terricola Kirby. Ibid., Bull. 22, 755.

vagans Smith. Ibid., Bull. 22, 755.

vagans var. consimilis Cresson. Ibid., Pull. 22, 756. 
Bombias Robertson.

auricomus Robertson. Conn. Geol. Nat. Hist. Surv., Bull. 22, 75S. (H.IV.IV.)

separatus Cresson. Ibid., Bull. 22, 758.

Psithyrus LePeletier.

ashtoni Cresson. Conn. Geol. Nat. Hist. Surv., Bull. 22, 759.

laboriosus Fabricius. citrinus. Ibid., Bull. 22, 759.

laboriosus var. contiguus Cresson. Ibid., Bıll. 22, 760.

variabilis Cresson. Ibid., Bull. 22, 759.

Apis Linnæus.

mellifera Linnzus. mellifica. Honey bee. Conn. Geol. Nat. Hist. Surv., Bull. 22, 760. 



\section{APPENDIX}

After the list was set up in type and arranged in the preceding pages, a number of additional genera and species were recognized or were reported from Connecticut material. Some of these additions have been included in the pages where they belong, but others cannot be inserted without entirely rearranging the matter on many pages. It has also seemed necessary to use certain references which are not explained in the Key on page I3; these references and the additional genera and species are given in the following pages:-

\section{KEY TO ADDITIONAL REFERENCES}

(See Page I3.)

Afid. Ital.- Prospetto dell Afido fauna Italica, by Giacomo Del Guercio. Nuove Relazioni intorno ai larori della R. Stazione di Entomologia Agraria di Firenze. Serie Prima - No. 2. Firenze, 1900.

Ann. Hist. Nat.- Annus Historico Naturalis, by J. A. Scopoli, 5 volumes, Lipsiæe, Hillscher, I $768-1772$.

Biol. Centr. Am. Heterop.-Biologia Centrali-Americana, Heteroptera, volume i, by W. L. Distant, London, I880-I893.

- Hymenop. vol. ii, by P. Cameron, isss.

Bull. Soc. Sci. Buc.-Bulletin de la Société des Sciences de Bucarest-Roumanie, Bucarest.

Cat. Macrolep.-Butterflies and Moths of North America. A complete synonymical catalogue of Macrolepidoptera, with full bibliography, by Herman Strecker, is 8 .

Coleop. Neerland.-Coleoptera Neerlandica, De Schildrlengelige insecten van Nederland enhet aangrenzend gebied, by Ed. Everts, Gravenhage, I898.

Contr. Nat. Hist. Lepidop.-Contributions to the Natural History of the Lepidoptera of North America, by William Barnes and J. H. McDunnough, Decatur, Ill. Begun in 19II. 
Descr. Hymenop. Chalcid.-Descriptiones Hymenopterorum Chalcidoidicortum cum Observationibus, by A. A. Girault, The Entonlologist, pages 36-38, London, I9I7.

Faun. Ent. Can. Hym.- Petite Faune Entomologique du Canada, ii, Hymenopteres. By Abbé Leon Provancher, Quebec, I883.

Faun. Suec.- Fanna Suecica Sistens animalia Sueciae regni, by C. V. Linnaeus, Stockholm, Sweden, I746.

Freg. Eugenies Resa. Hemip.-Kongliga Svenska Fregattens Eugenies resa omkring Jorden, Hemiptera, Stockholm, i859.

Ichneumon. d. Forstins.- Die Ichnewmonen der Forstinsecten in forstlicher and entomologisher Bezichung, by $\mathrm{J}$. T. C. Ratzeburg, 3 volumes, Berlin, I844-I852.

Illus. Besttabl. Kaf. Deutsch.- Illustrierte Bestimmungs tabellen der Käfer Deutschlands, by Paul Kuhnt, Stuttgart, I9r2.

Ins. Lapp.-Insecta Lapponica descripta, by J. W. Zetterstedt, 6 volumes, Lipsiae, Voss, I840.

Kafer.-Natursystem aller bekannten in- und ausländischen insecten; nach dem System des Ritters Carl von Linné bearbeitet (von C. G. Jablonsky), fortgesetzt von J. F. W. Herbst, 2 I volumes, Bd. I-IO, Kafer, Berlin, Pauli, Germany, I 785 - I 06.

Long's Exp.- Narrative of an Expedition to the Source of St. Peter's River, \&c, I824, Appendix. (Usually cited as Long's Expedition.)

Mon. Bupr.- Monograph of the Buprestida, from Histoire Naturelle et Iconographie des Coleopteres, by F. L. de Laporte and H. Gory, Paris, I835-I $8+1$.

Mon. Hister.-Essai monographique sur la fanille des Histerides, by·S. A. de Marsenl. Annals de la Société Entomologique de France, Series 3, I853 - Series 4, 1862, Paris.

Penn. Bur. Pl. Ind.- Pennsylvania Bureau of Plant Industry, Technical Series, Bulletin No. I, by J. G. Sanders and D. M. DeLong. Harrisburg, Pa., I920. 
Spec. Ins.- Species Insectorum, by J. C. Fabricius, 2 volumes, I 78 I.

Syn. Ins.- Synomymia Insectorum, by C. J. Schoenherr, 4 parts and Supplement, Stockholm and Skara, I8o6I8I7.

Tenn. State Bd. Ent.- Tennessee State Board of Entomology, Bulletin 17 , The Leafhoppers or Jassoidea of Tennessee, by Dwight M. DeLong, Knoxville, Tem., 1916.

Zweiflüg. Ins.-Zweiflügeligen Insekten Systematische Beschreibung der bekannten Europäischen, by Johann Wilhelm Meigen, 7 volumes, Achen and Hamm, I8I81838 . 


\section{ADDITIONS TO CHECK LIST}

The following species have been recorded from Connecticut and should be added to the list given on the foregoing pages:-

\section{Order ODONATA.}

$$
\begin{gathered}
\text { Family AGRIONIDE. } \\
\text { (See page 33.) } \\
\text { Argia Rambur. }
\end{gathered}
$$

translata Hagen. Biol. Centr. Am., Neuroptera, 76, I90r.

$$
\begin{gathered}
\text { Family LIBELLULIDA. } \\
\text { (See page 35) } \\
\text { Somatochlora Selys. } \\
\text { (See page 36) }
\end{gathered}
$$

linearis Hagen. Trans. Am. Ent. Soc., xx, 253.

\section{Order HEMIPTERA.}

\section{Family CICADELLIDE.}

(See page 47)

Idiocerus Lewis.

cognatus Fieber. Ent. Am., vi, 226.

duzeei Provancher. perplexus Gillette and Baker. Hemip. Col., 78.

\section{Macropsis Lewis.}

gleditschiæ Osborn and Ball. Pediopsis. Temn. State Bd. Ent., Bull. $17,16$.

Oncopsis Burmeister.

pruni Provancher. Me. Agr. Expt. Sta., Bull. 23 $\$, 87$.

Aucucephalus Germar.

nervosus Schrank. striatus Limneus. Me. Agr. Expt. Sta., Bul1. $238,107$. 
Xestocephalus Van Duzee.

pulicarius Van Duzee. Tenn. State Bd. Ent., Bull. 17, 35.

superbus Provancher. fulvocapitatus V'an Duzee. Ibid., Bull. 17,35 .

Parabolocratus Fieber.

major Osborn. Me. Agr. Expt. Sta., Bull. 238, i io.

Platymetopius Burmeister.

fulvus Osborn. Rept. N. Y. State Ent., 20, 519.

Deltocephalus Burmeister.

areolatus Ball. Can. Ent., xxxi, I88.

Euscelis Brullé. Athysanus.

cuneatus Sanders and DeLong. Penn. Bur. P1. Ind., Tech. Bull. I, I7.

parallelus Van Duzee. Can. Ent., xxiii, I69.

Gypona Germar.

pectoralis Spangberg. albimarginata Woodworth. hullensis Provancher. Proc. U. S. Nat. Mus., 56, 94.

Thamnotettix Zetterstedt.

inornatus Van Duzee. Tenn. State Bd. Ent., Bull. I7, 8r. morsei Osborn. Me. Agr. Expt. Sta., Bull. 238 , I34.

Cicadula Zetterstedt.

lepida Van Duzee. Tenn. State Bd. Ent., Bull. 17, 94. slossoni Van Duzee. Can. Ent., xxv, 28 .

Empoasca Walsh.

aureo-viridis Uhler. Tenn. State Bd. Ent., Bull. I7, Iоo. obtusa Walsh. Ibid., Bull. 17, Ioo.

Erythroneura Fitch.

obliqua Say var. dorsalis Gillette. Tenn. State Bd. Ent., Bull. I7, 105 .

trifasciata Say. Ibid., Bull. I7, Iо6.

vitis Harris var. stricta McAtee. Trans. Am. Ent. Soc., xlvi, 305. 
Family MEMBRACIDE.

(See page 53)

Acutalis Fairmaire.

tartarea Say. Bull. Buff. Soc. Nat. Sci., ix, 5 I.

Achasia Stål.

belfragei Stå1. Bull. Buff. Soc. Nat. Sci., ix, 73.

Family MIRIDÆ.

Phytocoris Fallen.

(See page 7I)

buenoi Knight. Bull. Brook. Ent. Soc., xv, 57.

conspurcatus Knight. Ibid., xv, 6I.

corticevivens Knight. Ibid., xv, 63 .

penipectus Knight. Ioid., xv, $5^{8}$.

salicis Knight. Ibid., $\mathrm{xv}, 56$.

sulcatus Knight. Ibid., xv, 64 .

Order LEPIDOPTERA.

Family GLYPHIPTERYGIDÆ.

(Belongs near Tortricide, see page 96)

Hemerophila Hubner.

pariana Clerck. Simathis. Apple and thorn skeletonizer. Rept.

N. Y. State Ent., 33, 33.

Family TORTRICIDE.

(See page 96.)

Olethreutes Hübner.

(See page 97)

hemidesma Zeller. Rept. Conn. Agr. Expt. Sta., 1917, 364, P1. xxxi, b.

$$
\begin{gathered}
\text { Family PYRALID互 } \\
\text { (See page I03) } \\
\text { Acrobasis Zeller. } \\
\text { (See page IO9) }
\end{gathered}
$$

demotella Grote. WVash. Ent. Soc., x, 4I. 


\section{Order DIPTERA.}

Family CECIDOMYIDE (ITONIDIDE).

(See page i68)

Diarthronomyia Felt.

hypogæa Loew. Chrysanthemum gall midge. Rept. Conn. Agr. Expt. Sta., 19I9, I6I, pl. xxxi.

Cincticornia Felt.

(See page I69)

pillulæ Walsh and Riley. N. Y. State Mus. Bull., 200, 90.

Caryomyia Felt.

(See page 169 )

holotricha Osten Sacken. N. Y. State Mus. Bull., 200, 47.

Family SCATOPSIDA.

(See page I 7 I)

Ectætia Enderlein.

clavipes Loew. Scatopse. Linn. Ent., i, 333. (H.L.J.)

Fanily SYRPIIDÆ.

(See page $18_{7}$ )

Xylota Meigen.

(See page I8S)

tuberans Williston. Syn. N. A. Syrph., 225.

Family TACHINID死.

(See page 190)

Tachina Meigen.

(See page 194)

simulans Meigen. Zweifiüg. Ins., iv, 306.

Family SCIOAIYZIDE.

(See page 20I)

Limnia Desvoidy.

boscii Desvoidy. Ann. Ent. Soc. An1., xiii, 323.

Tetanocera Dumeril

elata Fabricius. Ann. Ent. Soc. Am., xiii, 328.

rotundicornis Loew. Ibid., xiii, 328 .

vicina Macquart. Ibid., xiii, 327. 


\section{Order COLEOPTERA.}

Family DYTISCID无.

Deronectes Sharp.

(See page 220)

griseostriatus DeGeer. Coleop. Ind., 2 I6. (K.F.C.)

Hydroporus Clairville.

(See page 220)

spurius LeConte. Proc. Acad. Nat. Sci., I855, 296. (K.F.C.)

Family HYDROPHILID瓜.

Hydrobius Leach.

(See page 223)

tessellatus Ziegler. Coleop. Ind., 263. (K.F.C.)

Family LAMPYRIDE.

(See page 234)

Photinus Laporte.

marginellus LeConte. Trans. An. Ent. Soc., ix, 35.

Family ELATERIDA.

Melanotus Eschscholtz.

(See page 242)

castanipes Paykull. Coleop. Ind., 746.

depressus Melsheimer. Ibid., 755.

sp. near sagittarius LeConte.

Family EROTYLIDA.

(See page 25I)

Mycotretus Lacordaire.

pulchra Say. Trans. Anı. Ent. Soc., iv, 354.

Family TENEBRIONIDA.

(See page 257)

Scaphidema Redtenbacher.

æneolum LeConte. Coleop. Ind., ז 265. 
Family MELANDRYIDA.

(See page 259)

Serropalpus Hellwig.

barbatus Scholl. Illus. Besttabl. Kaf. Deutsch., 727.

Eustrophinus Seidletz (See page 259)

tomentosus Say. Coleop. Ind., I293. (K. F. C.)

Family SCARABEIDÆ.

Aphodius Illiger.

(See page 262)

hæmorrhoidalis Linnæus. Syst. Nat., I, 348.

Family CERAMBYCIDA.

(See page 266)

Leptostylus LeConte.

(See page 270')

aculiferus Say. Trans. Am. Ent. Soc., viii, I2I.

Oberea Mulsant.

(See page 272)

tripunctata Swederus var. myops Haldeman. Trans. Am. Ent. Soc., vii, 47.

Family CHRYSOMELID

Diabrotica Chevrolat.

(See page 277)

longicornis Say. Coleop. Ind., I 172. (K.F.C.)

Family CURCULIONIDÆ.

Acanthoscelis Dietz.

(See page 286)

curtus Say. Rhyn. N. E. Am., 43I. (K.F.C.)

Ceutorhynchus Germar (See page 287)

semirufus LeConte. Rhyn. N. E. Am., 45I. (K.F.C.) 


\section{Order HYMENOPTERA.}

Family TENTHREDINIDE.

(See page 292)

Hemichroa Stephens.

dyari Rohwer. Proc. Wash. Ent. Soc., xx, I7I

\section{Trichiocampus Hartig (See page 297.)}

gregarius Dyar. Cladius gregarius. Conn. Geol. Nat. Hist. Surv., Bull. 22, I IO.

irregularis Dyar. Priophorns irregularis. Journ. N. Y. Ent. Soc., viii, 28.

Pteronidea Rohwer.

(See page 298)

alnivora Rohwer. Proc. U. S. Nat. Mus., 1vii, 213.

Kaliofenusa MacGillivray.

(Belongs near Erythraspides, page 299)

ulmi Sundewall. Elm leaf-miner. Conn. Geol. Nat. Hist. Surv., Bull. 22, 157 .

Family BRACONIDE.

(See page 305)

Cyanopterus Wesmael.

sp.

Family ICHNEUMONIDÆ.

Campoplex Gravenhorst.

(See page 309)

validus Cresson. Limnerium zalidum. Mesoleptus. Proc. Ent. Soc. Phila., iii, 258.

Arotes Gravenhorst.

(See page 315)

decorus Say. Comn. Geol. Nat. Hist. Surv., Bull. 22, 326.

\section{Family EULOPHIDE. \\ (See page 323) \\ Dimmockia Ashmead.}

incongrua Ashmead. Eulophus. Proc. Wash. Ent. Soc., iv, I 58. 
No. 3I.]

INSECTS OF CONNECTICUT: APPENDIX.

Fanily CHALCIDIDÆ.

(See page 326)

Rhopalicus Fœrster.

suspensus Ratzeburg. Pteromalus. Ichneumon. d. Forstins., i, 189. 



\section{INDEX.}

Abbotana, II5.

Abia, 296.

Acallodes, 286.

Acanalonia, 46.

Acanthocephala, 80.

Acanthocerus, So.

Acanthoderes, 270.

Acanthomyops, 330.

Acanthocelis, 286, 359 .

Acemyia, I93.

Achasia, 356.

Achatodes, I37.

Achlarus, I54.

Acholla, 76.

Achorutes, 26.

Achroia, I08.

Acidia, 203.

Acilius, 222

Acinopterus, 5 I.

Aciura, 204.

Acleris, IOI.

Acmæodera, 243.

Acmeops, 267.

Acontia, I32.

Acoptus, 286.

Acordulecera, 300.

Acrididx, 39.

Acritus, 233.

Acrobasis, I09, 356.

Acrocera, 175.

Acrolepia, 86.

Acrolophus, 85.

Acroneuria, 27.

Acronycta, I 46 .

Acropteroxys, $25 \mathrm{I}$.

Acroricnus, 3I 5.

Acroschismus, 290.

Acrosternum, 83 .

Actenodes, 244 .

Actia, I9I.

Actias, I5I.

Actinotia, I43.

Acucephalus, 49, 354.

Acupalpus, 2I9.

Acutalis, 356 .

Acylomus, 254.

Acylophorus, 228.

Adalia, 256.

Adelocera, 240.

Adelphacoris, $7 \mathrm{I}$.
Adelphagrotis, I42.

Adirus, 301.

Adita, I 43.

Adoneta, II4.

Adoxus, 275.

Adranes, 23I.

Aëdes, I66.

Egomorphus, 270.

Enoplex, 3I6.

Eolothripidæ, 44.

Eolothrips, 44.

Eolus, 24 I.

Eschnidx, 34.

Eshna, 35.

Athaloptera, I 8 .

Agabetes, 22I.

Agabus, 22I.

Agallia, 47.

Agapetidx, 156 .

Agapostemon, 342, 343.

Agaristidæ, I 47 .

Agathidium, 225.

Agathis, 306.

Agelasa, 277.

Ageniella, 335 .

Aglais, I 57.

Aglossa, I06.

Agnomonia, I29.

Agonoderus, 218.

Agnopteryx, 92.

Agrilus, 244.

Agrion, 33.

Agrionidxe, 33, 354 .

Agriotes, 241.

Agromyza, 209.

Agromyzidæ, 208.

Agrothereutes, 3I5.

Agrotis, I 42.

Alabama, I34.

Alaria, I35.

Alaus, 240.

Alcidamea, $3+8$.

Alcis, II8.

Alcothoë, II2.

Alder blight, $6 \mathrm{I}$.

Alebra, 52.

Aleiodes, 307.

Aleochara, 230.

Aleurochiton, 62.

Aleurodes, 62. 
Aleyrodes, 62.

Aleyroodidæ, 62.

Allantus, 293, 294, 296.

Allecula, 256.

Alleculidæ, 256.

Allognosta, I 72 .

Allograpta, I 86.

Alloperla, 27.

Alloplasta, 3I3.

Allorhina, 265.

Allotria, I29.

Alobates, 258.

Alophora, I9o.

Alphitobius, 258.

Alsophila, I22.

Altica, 278.

Alydidie, 8I.

Alydus, 8I.

Alypia, I.47.

Alysiidre, 304.

Alyson, 338 .

Amadrya, 86.

Amalopis, I63.

Amara, 214.

Amauronematus, 298.

Ambesa, i io.

Amblycorypha, 4I.

Amblyscirtes, I 55 .

Amblyteles, 3I7, 3I8.

Ameloctonus, 309.

American Copper, I55. Tortoise-Shell, I 57.

Ammalo, I47.

Ammobia, 340 .

Amnestus, 84.

Amolita, 133 .

Amorbia, 102.

Ampelophaga, I52.

Amphiagrion, 33.

Amphibolips, 320 .

Amphicnephes, 202.

Amphicoma, 263.

Amphion, I53.

Amphisa, 99.

Amphiscepa, 46 .

Amphorophora, 60.

Ampulicida, 337.

Anacampsis, 93.

Anacharis, 310.

Anacomis, 260.

Anacrabro, 338 .

Allagoga, Ir7.

Anaphes, 323.

Anaphoidea, 323.

Anaphothrips, 44.

Anaplodes, I I9.

Anarsia, 94.

Anasa, 80.

Anaspis, 237.
Anastatus, 325.

Anatis, 256.

Anax, 35 .

Anaxipha, 43.

Ancistrocerus, $336,337$.

Ancylis, Ioo.

Ancyloxypha, I55.

Andrena, 343.

Andrenidæ, 343.

Andricus, 321 .

Androchirus, 257.

Andronicus, 348 .

Anecphysis, 3 I2.

Aneurus, So.

Angitia, 309.

Ania, I I6.

Anisandrus, 290.

Anisitsia, 209.

Anisocalvia, 256.

Anisodactylis, 219.

Anisolabis, 37.

Anisomera, I62.

Anisosticta, 255.

Anisota, I50.

Anisotoma, 225.

Anobiidre, 260.

Anccia, 57.

Anomala, 264.

Anomalagrion, 34 .

Anomalon, 3I0, 3II.

Allomcea, 273.

Anomoglossus, 217.

Anopedius, 326.

Anopheles, I67.

Anoplitis, 2\%9.

Anoplius, 336.

Anoplolyda, 291.

Anorthodes, I45.

Anorthosia, 94.

Anosia, I56.

Ant, Carpenter, 33I.

Garden, 330.

Little red house, 329.

Silky, 33I.

Anthaxia, 244.

Anthemois, 347.

Antherophagus, 25 I.

Anthicida, 239.

Anthicus, 239.

Anthobium, 226.

Anthoboscus, 269.

Anthocaris, I59.

Anthocomus, 235.

Anthocorida, 74.

Anthomyia, igs.

Anthomyide, I98.

Anthonomopsis, 284.

Anthonomus, 284, 285 .

Anthopliilax, 267. 
Anthophora, $34^{6}$.

Anthophoridxe, 346 .

Antliothrips, 44.

Anthracophaga, 207.

Anthrax, 175 .

Anthrenus, 247.

Anthribide, $28 \mathrm{I}$.

Anthribulus, 28I.

Antipus, 273.

Artispila, 9o.

Antocha, I6I.

Anturaphis, 59.

Anytus, I 40

Aprecasia, i i 8 .

Apanteles, 302, 303.

Apantesis, 147.

Apatela, I 46.

Apatelodes, 126.

Apateticus, 83 .

Apenes, 217.

Aplialara, 56.

Aphanisus, 299.

Aplianus, 79.

Aphrenogaster, 329.

Aphereta, 305.

Aphelinidæe, 324.

Aphelinus, 324.

Aphelopus, 333.

Aphid, Bean, 59.

Beech, 58.

Black peach, 59.

Cabbage, 59.

Cherry, 60.

Choke-cherry, 59.

Corn root, 59 .

Currant, 60.

Green apple, 59. peach, 60.

Hickory gall, 62.

Melon, 59.

Oleander, 59.

Pine bark, 62.

Rosy apple, 59.

Spruce gall, 62.

Turnip, 59.

Yucca, 59.

$\checkmark$ iolet, 59.

Moolly apple, 6I. beech, 6I. larch, 62.

Aphididre, 57.

Aphidius, 308.

Aphilanthops, 339.

Aphiochreta, I84.

Aphis, 57, 58, 59, 60, 6I.

Aphodius, 262, 359 .

Aphonus, 265.

Aphorista, 253.
Aplirastus, 282.

Aphropliora, 55 .

Apidae, 348 .

Apiomerus, 76 .

Apion, 282.

Apis, 349 .

Aplodes, i in.

Apteromechus, 287 .

Apocremnus, 69.

Aporinellus, 335.

Apple and thorn skeletonizer, 356 . Bucculatrix, 89 .

Apristus, 216.

Aprozerema, 93.

Araba, 194.

Arachnophroctonus, 336 .

Aradidre, So.

Aradus, 80.

Archips, 99.

Archytas, 195.

Arctia, I 47, I.48.

Arctiidae, 147 .

Arctocorixa, 66.

Areus, 227.

Arge, 300.

Argia, 33, 354.

Argyninis, I5S.

Argyra, iso.

Argyresthia, 87 .

Argyria, 107.

Arhopalus, 269.

Aristotelia, 95.

Army worm, I39.

Arotes, 3I5, 360.

Arphia, 40.

Arrhenoplita, 257.

Arsilonclie, I 46 .

Arta, 106.

Arthromacra, 259.

Asaphes, 24I.

Ascalaphida, 30.

Asclera, 237.

Ascogaster, 306.

A semul11, 266 .

A silidae, I77.

Asilus, I79.

Asiphtrm, 6I.

Asobara, 304.

Asparagus beetles, 273.

Asphondylia, I69.

Aspicera, 319.

Aspidiotus, 65.

Aspilota, 304.

Astata, 340.

Astenus, 227.

Asterochiton, 62.

Asterolecanium, 63.

Asteromyia, $\mathbf{z}$ o. 
Astomaspis, 316 .

Astylopsis, 27I.

Asyndetus, I8o.

Atacosa, III.

Atrenius, 262.

Atanycolus, 304.

Atarba, I6I.

A tethmia, I35.

Atherix, I75.

Atheta, 229.

Athous, 240.

Athysanus, 50, 5I, 355.

Atlanticus, 42.

Atomacera, 300.

Atomaria, 252.

Atomosia, I78.

Atropos, 26.

Atrytone, I55.

Attacus, I5I.

Attagenus, 247.

Attalus, 235 .

Attelabus, 28r.

Atymna, 55.

Augochlora, 342, 343 .

Aulax, 3 I9.

Aulacaspis, 65 .

Auletes, 28I.

Auleutes, 286.

Autographa, I34.

Automalus, 317.

Automeris, I50.

Axinopalpus, 216.

Aylax, 319 .

Azelina, I 16.

Babia, 273.

Baccha, 185 .

Bachia, 3I6.

Bactra, 96.

Bactridium, 250.

Badister, 2I5.

Beocera, 23I.

Bxtis, 27, 28.

Bagous, 283.

Baileya, I45.

Bag-worm, II4.

Balaninus, 284.

Balclutha, 52.

Baldratia, I70.

Baliosus, 279.

Balsa, I 45 .

Baltimore, I58.

Banasa, 83 .

Banchicle, 305 .

Banchus, 305.

Bantlera, II I.

Barce, 75.

Barichneumon, 317, 318.
Baris, 285.

Barynotus, 282.

Barypeithes, 282 .

Basiæschna, 35.

Basilarchia, I57.

Basilona, I50.

Bassareus, 273.

Bassus, 306, 312.

Bathyplectes, 309 .

Batrachetra, 9I.

Batrisodes, 230.

Batyle, 270.

Batyleoma, 270.

Bed bug, 74.

Beetle, Ash timber, 289.

Black carpet, 247.

Bumble flower, 265.

Carpet, 247.

Chestnut timber, 237.

Cigarette, 260.

Cloverleaf, 282.

Confused flour, 258.

Cottonwood leaf, 276.

Drug-store, 260.

Fruit bark, 288.

Goldsnith, 265.

Ham, 247.

Hickory bark, 288. timber, 290.

May or June, 264.

Red cedar bark, 289.

Rose, 264.

Soldier, 234.

Spotted grape-vine, 265 .

Stag, 266.

Striped cucumber, 277.

Turpentine bark, 289 .

Belidx, 280.

Belostoma, 66 .

Belostomatidx, 66.

Belvosia, I92.

Belytidre, 328 .

Bembecia, I 12.

Bembecidre, 3fI.

Bembidion, 2I2.

Bembidula, 34I.

Bcmbix, 34I.

Benacus, 66.

Benta, I08.

Beris, I72.

Berosus, 223.

Rerytus, 8o.

Bethylidx, 332.

Bethylus, 332.

Bibio, I7I.

Isibionida, I $7 \mathrm{r}$.

Bicyrtes, 34I.

Bidessus, 220. 
Biorhiza, 319.

Birch, leaf skeletonizer, 89.

Bitoma, 252.

Bittacomorpha, I63.

Bittacus, 30 .

Black-dasl, I 54 .

Blapstinus, 257.

Blastobasidre, 9 I.

Blastothrix, 324 .

Blatta, 38 .

Blattella, 38 .

Blattidx, 38.

Bledius, 226.

Blennocampa, 299.

Blepharida, 277.

Blepharipeza, I94.

Blepharomastrix, I04.

Bleptina, I 27 .

Blissus, 78 .

Body-louse, 45.

Bolboceras, 263.

Bolbocerasoma, 263.

Boletobius, 229.

Boll-worm, Cotton, I35.

Bolithopliagt1s, 257.

Bolitotherus, 257 .

Bombias, 349 .

Bombus, 348 .

Bombyliida, I76.

Bombyliomyia, I 95.

Bombylius, 176 .

Bomoloclia, I26.

Bonnetia, I92.

Book louse, 26.

Borboridæe, 200.

Borborus, 200.

Borer, Alder. 272.

Clover stem, 25I.

Elm, 272.

Flat-headed apple, 244.

Hickory, 260. twig, 288. snout, 284 .

Lilac, II2.

Linden, 272.

Locust, 269.

Maple, 260. leaf stem, 297.

Peach, II 2.

twig, 94.

Poplar, $27 \mathrm{I}$.

Round-headed apple, 27 I.

Shot-hole, 288.

Spotted apple tree, 272.

Squash, II2.

Willow, 27 I.

Borkhausenia, 9I.

Bostrichide, $26 r$.

Bostrichus, $26 \mathrm{I}$.
Botanobia, 208.

Bot flies, igo.

Botys, I0.4.

Boyeria, 35.

Brachixiphosoma, 3 I I.

Brachyacantha, 254.

Brachybamus, 283 .

Brachycoma, I94.

Braclygluta, 230.

Brachyleptura, 268 .

Brachylobus, 217.

Brachyloma, 93.

Brachymyrmex, 330 .

Brachynemurus, 29.

Brachynus, 217.

Brachyopa, I87.

Bracliypalpus, Is8.

Brachypterus, 248 .

Brachys, 245.

Brachytarsus, 28I.

Brachytropis, 74.

Bracon, 306.

Braconidae, 305, 360.

Bradycellus, 219.

Bremus, 3.48 .

Brentlis, I58.

Brentidre, 280.

Brephoctonus, 3 I2.

Breplios, I I5.

Bristle-tail, 25

Brevicoryne, 59.

Brochymena, SI.

Brontes, 250.

Brotolomia, 136 .

Bruchidre, 280.

Bruchomorpha, 46.

Bruclius, 280.

Brucopliagus, 325.

Bryaxis, 230.

Bryoporus, 229.

Bucculatriplex, 307.

Bucculatrix, 89 .

Bucl-worm, Tobacco, I35.

Buffalo bug. 2.47.

Bug, Barn swallow, 74. Chincls, 78 .

Four-lined leaf, 72.

Giant water, 66.

Harlequin cabbage, 82.

Spined soldier, 83 . tobacco, 82 .

Squasli, 80 .

Tarnished plant, 73 .

Buprestidre, 243.

Byrthidre, 247.

Byrthus, 247.

Byturidae, 246.

Byturus, 2.46. 
Cabbage butterfly, Imported, I59. Southern, I59. looper, I34. plusia, 134 .

Caberodes, II 5 .

Cacoplia, 270.

Cæcilius, 26.

Cænia, 233.

Cæniella, 233.

Cænocara, 260.

Cænolyda, 291.

Cænoscelis, 252.

Calaphis, 57.

Calasymbolus, 151 .

Calathus, 2I 5 .

Calendra, 288.

Caliroa, 294.

Calledapteryx, i I5.

Callibetis, 28.

Calliclisis, 313 .

Callidium, 269.

Callidryas, I 59.

Calliephialtes, 3 I 4.

Calligrapha, 276.

Callimiris, 74 .

Callimome, 325 .

Callimonidæe, 325 .

Callimorpha, I 48 .

Calliopsis, 345 .

Calliphora, I97.

Callipterus, 57,58 .

Callipterinella, 57 .

Callirhytis, 321, 322.

Calloides, 269.

Callopistria, 203.

Callosamia, I5I.

Calobata, 205.

Calocalpe, I2r.

Calocampa, I38.

Calocoris, $7 \mathrm{I}$.

Calophya, 56.

Calopteron, 233.

Calopteryx, 33 .

Calosoma, 2I I.

Caloteleia, 327.

Calpe, I34.

Calyminia, I 35 .

Camnula, 40.

Camponotus, 33I.

Campoplex, 309, 360 .

Campsomeris, 333 .

Camptobrochis, 70 .

Camptocladius, 165.

Camptoneura, 202.

Camptoneuromyia, izo.

Camptoprosopella, 202.

Camptylochila, 128 .

Campylenchia, 53 .

Campylomma, 68.

Campylus, 2 fo.

Canarsia, i Io.

Canifa, 260.

Canker worm, Fall, I22.

Spring, I 22.

Cantharide. 238.

Canthon, 262.

Canthophorus, 84 .

Capis, 127.

Capitoniidæ, 308 .

Capitonius, 308.

Capitophorus, 60.

Capnia, 27.

Capnochroa, 257.

Capsidae, 68.

Capsus, 69, 70, 71, 72, 73.

Carabidæ, 2II.

Carabus, 2II.

Caradrina, I 45 .

Caricoa, 199.

Cardiochiles, 302.

Cardiophorus, 242.

Caricea, I99.

Carneades, Iło.

Carpet beetle, 247.

Carpocapsa, Ior.

Carpophilus, 248.

Cartodere, 253.

Carynota, 53.

Caryomyia, I70, 357.

Casinaria, 309.

Casnonia, 216.

Catabena, I 45.

Cat and dog flea, I6o.

Caterpillar hunter, 2I2.

Melon, I04.

Red-humped, 124.

Saddle-back, II4.

Salt marsh, I48.

Yellow-necked, I26.

Catocala, i3o.

Catogenus, 250 .

Catoglyptus, 3II.

Catonia, 46.

Catopsilia, 159.

Catopyrrha, i I8.

Cayuga, III.

Cecidonyia, I68, I69, I70, I7 I.

Cecidomyidre, I68, 357.

Celama, I 49.

Celatoria, I9I.

Celerio, I53.

Celetes, 233.

Celethemis, 37 .

Celina, 220.

Celiptera, I29.

Cenopis, 99. 
Centrias, 345 .

Centrinus, 286.

Centrodera, 267.

Ceophyllus, $23 \mathrm{I}$.

Cephaloidæ, 237.

Cephaloön, 237.

Cephide, 30I.

Cephus, $30 \mathrm{I}$.

Ceracis, 26r.

Cerambycidae, 266, 359 .

Ceraphronida, 327.

Cerastipsocus, 26.

Ceratina, 348 .

Ceratinidx, $3+8$.

Ceratocampidæ, I5o.

Ceratocapsus, 69.

Ceratogastra, 3 Io.

Ceratomegilla, 255 .

Ceratomia, I52.

Ceratomyza, 209.

Ceratopogon, I65.

Ceratosmia, 347 .

Ceratosoma, 3io.

Ceraturgus, I77.

Cerceridæ, 3+I.

Cerceris. 34 I.

Cercopidae, 55.

Cercopis, 55.

Cercus, $2+8$.

Cercyon, 224.

Ceresa, 53.

Ceria, IS9.

Ceriodes, I 89.

Cerodontha, 209.

Ceropales, 335.

Ceroptres, 3 I 9.

Cerotoma, 276 .

Ceruchus, 266.

Cerura, i2.4.

Cerylon, 252.

Ceryonis, 157.

Cetema, 207.

Ceutophilus, +2 .

Centorhynchus, 287, 359.

Chretarthria, 224.

Chretocisema, 278 .

Chretoneurophora, I83.

Chætophleps, I9I.

Chatopsis, 203.

Chaitophorus, 57,58 .

Chalarus, I84.

Chalcididie, 326, 361.

Chalcis, 326.

Chalcophora, $2+3$.

Chalepus, 279.

Chalybion, 3 to.

Chamyris, I33.

Characoma, I32.
Charadra, I 47.

Charidryas, I58.

Chariessa, 82.

Chasmatonotus, I66.

Chasmias, 318.

Chauliodes, 29.

Chauliognatlus, 234 .

Checkered white, I59.

Cheese skipper, 205.

Chelonus, 306.

Chelymorpha, 280.

Chermes, 62.

Chilo, ios.

Chilocorts, 256.

Chilosia, I85.

Chilostigma, 3I.

Chimarrbia, 3I.

Chinch bug, 78 .

Chion, 267.

Chionaspis, 64.

Chirida, 280.

Chironomidx, 165.

Chironomus, I65.

Chiropachys, 324.

Chlenius, 2 I7.

Chlienogramma, I 52 .

Chlamydatus, 68.

Chlamys, 273.

Chlcealtis, 39.

Chloralictus, $3+2,3+3$.

Chloridea, I35.

Chloropidæ, 207.

Chlorion, 339, 340.

Chlorochroa, 82.

Chlorochlamys, I20.

Chloropisca, 207.

Chlorops, 207.

Chlorotettix, 5 I.

Choeridium, 262.

Chœrocampa, I53.

Choleva, 225.

Choreutis, 96.

Chorineus, 3I 3 .

Chortophaga, 40.

Chramesus, 288.

Chromagrion, 33.

Chromatica, 257.

Chrysididee, 332.

Chrysis, 332 .

Chrysobothris, 244.

Chrysochlamys, I88.

Chrysochus, 275 .

Chrysodina, 274 .

Chrysogaster, 185.

Chrysogona, 332 .

Chrysomela, 276 .

Chrysomelidie, 272, 359.

Chrysomphalus, 65. 
Chrysomyia, I96.

Chrysomyza, 203.

Chrysopa, 29.

Chrysoplianus, I55.

Chrysophila, I75.

Chrysopidx, 29.

Chrysops, I73.

Chrysotoxum, I84.

Chrysotus, I80.

Chyliza, 205.

Chytolita, I28.

Chytonix, I45.

Cicadella, 48 .

Cicadellidx, 47, 354 .

Cicadidæ, 56 .

Cicadula, 5I, 355 .

Cicindela, 2I I.

Cicindelidæ, 2 II.

Cicinnus, I 5 .

Cimatlan, 70.

Cimbex, 296.

Cimex, 70, 74 .

Cinicidæ, 74 .

Cinclidia, I58.

Cincticornia, I69, 357 .

Cindaphia, 105.

Cingilia, I 7 .

Cinglis, 120 .

Cinura, 25.

Circotettix, 40.

Cirrospilus, 324 .

Cirrula, 206.

Cis, 26I.

Cisidæ, 26r.

Cissa, I 56.

Cistela, 257.

Cistelidx, 256.

Cisthene, I49.

Cistogaster, Igo.

Citheronia, I5o.

Cixius, 46.

Cladiv's, 297, 360.

Clastoptera, 56 .

Clavigeridæ, 231.

Cleis, 256.

Clemensia, I 49.

Cleonus, 285.

Cleora, i 8 .

Cleridx, 236.

Click beetle, 240 .

Clinocera, I82.

Clinodiplosis, I7o.

Clisiocampa, I22.

Clisodon, 346.

Clistopyga, 3I4.

Clivina, 2I2.

Closterocerus, 323.

Clothilla, 26.
Clover seed chalcis, 325 .

Clusia, 200.

Clytanthus, 269.

Clytochrysus, 339.

Clytus, 269.

Cnemidott1s, 220.

Cnemodus, 79.

Coccidae, 63.

Coccidula, 255 .

Coccinella, 255.

Coccinellidx, 254.

Coccobaphes, 72.

Coccus, 63.

Cochlidiidæ, I 13.

Cochlidion, I 13 .

Cockerellia, 345.

Cockroaches, 38 .

Coelambus, 220.

Coelichneumon, 3I8.

Coelinidea, 304.

Coliodes, 286.

Colioxys, 347 .

Coelogaster, 287.

Coloides, 304.

Colostathma, 99.

Conomyia, I74.

Crenosia, I99.

Contus, 82.

Colaspis, 275 .

Colastus, 2.48 .

Coleocentrus, 3I5.

Coleophora, 9I.

Coleoptera, 2I I, 358.

Coleothrips, 44 .

Colias, 158.

Collaria, 74 .

Collembola, 25 .

Colletes, 346.

Colletidx, 346.

Collops, 235.

Colobopterus, 30.

Colon, 225.

Colopterus, 248 .

Colpognathus, 3 I7.

Colydiidx, 252.

Colydium, 252.

Colymbetes, 222.

Comma, Gray, I57. Green, I57.

Commophila, io2.

Compsilura, 103.

Compton tortoise, I57.

Condylolomia, io6.

Connophron, 225.

Conocephalus, 42.

Conocera, I $8_{4}$.

Conohalictoides, 344 .

Conopida, i 80 . 
Conops, I 89.

Conosoma, 229.

Conostigmus, 327.

Conotelus, 248 .

Conotrachelus, 287.

Conservula, I36.

Contarinia, I7o.

Copelatus, 221 .

Copipanolis, I43.

Copris, 262.

Coptocycla, 280.

Coptotomus, 22I.

Copturodes, 286.

Copturus, 286.

Cordulegaster, 34 .

Cordulia, 36 .

Cordylura, 199.

Coreidæ, 80.

Corethra, I67.

Corimelæna, 84 .

Corinthiscus, 236 .

Coriscium, 88 .

Coriscus, 75 .

Corixa, 66.

Corixide, 66.

Corizidæ, 8I.

Corizus, 8I.

Corphyra, 239.

Corrodentia, 26.

Corticaria, 253.

Corydalis, 29.

Corylophide, 226 .

Cory mbites, 240.

Corynocoris, 80 .

Corythucha, 77.

Coscinoptera, 273.

Cosmia, I36.

Cosmopepla, 82.

Cosmopolite, I57.

Cosmopteryx, 9I.

Cossidie, i 2.

Cossonus, 288.

Cossiss, ilz.

Cosymbia, I20.

Cotalpa, 265.

Cotinis, 265.

Crab-louse, 45.

Crabro, 339.

Crambidia, I49.

Crambus. 107.

Crassiseta, 207.

Craterocercus, 296.

Cratichneumon, 3I7, 3I8, 3I9.

Cratoparis, 28I.

Cregya, 236.

Cremaster, 3io.

Cremastocheilus, 265 .

Crematogaster, 320.
Creniphilus, 223.

Creophilus, 228 .

Crepidodera, 278 .

Crescent, Pearl, I58.

Silver, 158.

Cressonia, I5I.

Crickets, 43.

Cricotopus, I65.

Criocephalus, 266.

Crioceris, 273.

Criorhina, I88.

Crocidophora, I04.

Crocigrapha, I38.

Crœest1s, 298.

Crophius, 78 .

Croton bug, 38 .

Cryphula, 79.

Cryptarcha, 249.

Cryptobium, 227.

Cryptocephalus, 274.

Cryptohypnus, $24 \mathrm{I}$.

Cryptoidens, 3I4.

Cryptolechia, 92.

Cryptomeigenia, 190.

Cryptophagus, 252.

Crytophagide, $25 \mathrm{I}$.

Cryptopleurum, 224.

Cryptorhyuchus, 287.

Cryptorophalum, 247.

Cryptothrips, 44.

Cryptus, 315.

Ctenistes, 23I.

Ctenocephalus, I6o.

Ctenolepisma, 25.

Ctenophora, I63.

Ctenucha, I 50.

Cuchius, 250 .

Cucuijidie, 250.

Cucullia, I38.

Culex, 166.

Cinlicille, 166.

Ciplocera, 195.

Curculio, Apple, 284. Plum, 287.

Poplar or Willow, 287.

Quince, 287.

Rhtwarb, 285.

Tralnut, $28 \%$.

Curculionidx, 28I, 359.

Cuterebra, I9o.

Cutworm, Black, It2. Variegated, IfI.

Cyane, 85.

Cyaneus, 326.

Cyaniris. 155 .

Cyanopterus, 360 .

Cychramus, 249.

Cychrus, 2II. 
Cycloneda, 255 .

Cydia, 98, I0I.

Cydnidre, 84.

Cydosia, I32.

Cylapus, 7o.

Cylindrocopturus, 286.

Cyllene, 269.

Cymatodera, 236.

Cymatophora, I I8.

Cymbiodyta, 224.

Cymindis, 217.

Cymodusa, 308.

Cymu1s, 78.

Cynipidae, 3 I9.

Cynips, 320.

Cynomyia, I96.

Cynorhina, I88.

Cyphon, 246.

Cyphonida, 246.

Cyrtidx, I75.

Cyrtinus, 270.

Cyrtolobus, 54.

Cyrtoneurina, 198.

Cyrtophloba, I92.

Cyrtophorus, 270.

Cyrtophyllus, 42.

Cyrtopogon, I77.

Cystiphora, I69.

Cytilus, 247.

Dacnusa, 304.

Dactylopius, 63.

Dalmannia, 189 .

Danais, 156.

Darapsa, I52.

Dascillida, 246 .

Dasycera, 92.

Dasyllis, I78.

Dasymutilla, 334 .

Dasyneura, 169.

Datana, I 25.

Datomicra, 229.

Daulopogon, I 78 .

Debis, 156 .

Decantha, 92.

Decartliron, 230.

Dectes, $27 \mathrm{I}$.

Deidamia, I 53.

Deilephila, I 53.

Deilinea, I I9.

Delomegachile, 347.

Delomerista, 3I4.

Deloyala, 280.

Delphastus, 254.

Delphax, 47.

Deltocephalus, 50, 355 .

Deltometopus, 243.

Demas, 147.
Dendrocoris, 83 .

Dendroctonus, 289 .

Dendroides, 239.

Dendrophagus, 250.

Dendrophilus, 232.

Depressaria, 92.

Deptalia, I 20.

Deræocoris, 7 I.

Dermestes, 247.

Dermestidx, 247.

Derobrachus, 266.

Derodontidx, 25 I.

Deromyia, I 78 .

Deronectes, 220, 358 .

Derrima, I35.

Desmia, I03.

Desmocerus, 268.

Desmometopa, 209.

Desmopachria, 220.

Desmoris, 283 .

Dexiidæ, I95.

Dexiopsis, $\mathbf{I} 99$.

Diabrotica, 277, 359.

Diachus, 274.

Diacrisia, I 48 .

Dialysis, I75.

Dialyta, I99.

Dianthidimm, 348 .

Diaperis, 257.

Diaphania, Iof.

Diapheromera, 38 .

Diaphorıs, I80.

Diapria, 327.

Diapriidre, 327.

Diapris, 257.

Diarthrononyia, 357 .

Diasemia, Iof.

Diaspis, 64, 65.

Diastata, 208.

Diastictis, I03.

Diatrea, Io8.

Diathrausta, I06.

Diast rophus, 322 .

Dibolia, 279.

Dibrachys, 324.

Dicælus, 2 I 5 .

Diceratosmia, $3+7$.

Dicerca, 243.

Dichreta, 206.

Dichetoneura, I 90.

Dichelonyx, 264.

Dichomeris, 93.

Dichroöscytus, 7 I.

Dicosmcecus, 3I.

Dicraneura, 52 .

Dicranomyia, I6o.

Dicromorpha, 39 .

Dicymolomia, Io8. 
Dicyphus, 70 .

Didea, I 86.

Didymops, 35.

Diedrocephala, 48 .

Dilophus, 17 I.

Dimecænia, 206.

Dimmockia, 360 .

Dimorphopteryx, 293.

Dineura, 296.

Dinentes, 222.

Dinocampus, 306.

Dinoderus, $26 \mathrm{I}$.

Dioctria, 177.

Dicedus, 258.

Diopsidxe, 205.

Dioryctria, I09.

Diorymellus, 285.

Diphadnus, 297.

Diplax, 36.

Diplazon, 3 I2.

Diplochila, 2 I4.

Diplotaxa, 207.

Diplotaxis, 263.

Dipolepis, 322.

Diprion, 292

Diptera, I60, 357.

Dipterygia, I 43 .

Dipthera, I45.

Diraphia, 56.

Dircea, 259.

Dircetis, I,27.

Discobcla, I6o.

Disognus, 328 .

Disonycha, 278 .

Dissosteira, 40.

Distenia, 266.

Ditemnus, 235.

Dixa, I64.

Dixida, 164.

Dobson, 29.

Dog-louse, sucking, 45 .

Dog's head butterfly, I58.

Dohrniphora, 183 .

Dolba, I52.

Dolerus, 294.

Dolichoderus, 330.

Dolichopodidx, I79.

Dolichopus, I8I.

Dolichovespula, 337.

Dolopitus, 241 .

Donacia, 272.

Dorcaschema, 270.

Dorcatoma, 260.

Dorcus, 266.

Dorocordulia, 36 .

Doryodes, I33.

Doryphora, 276.

Doryphorophaga, I93.
Dorytomus, 283.

Draculacephala, 48 .

Dragonflies, 33 .

Drapetis, 182.

Drasteria, I 32.

Drasterius, 24I.

Drepana, I 22.

Drepanaphis, 58 .

Drepanium, 343 .

Drepanosiphum, 58 .

Driotura, 50.

Dromius, 216.

Dromogomphts, 34 .

Drosophila, 208.

Drosophilidx, 208.

Dryinidae, 333.

Drymonia, I 25.

Dryocampa, 150.

Dryomyza, 201.

Dryomyzidæ, 20 I.

Dryophanta, 320.

Dryophthorus, 288.

Dryopidx, 245.

Dryops, 245.

Dufoureidre, $3+4$.

Dynatosoma, I68.

Dyschirius, 212.

Dyscinetus, 265.

Dyspieris, 122.

Dytiscidze, 220, 358 .

Dytiscus, 222.

Eacles, 150.

Earwig, Maritime, 37.

Eccoptogaster, 288.

Ecdytolopha, IoI.

Echinomyia, 195.

Ecpantheria, I 48 .

Ectretia, 357.

Ectobia, 37.

Ectopimorpha, 317.

Ectopria, 246.

Ectropis, I88.

Eight-spotted forester, I.47.

Eiphosona, 3I I.

Elachertide, 324.

Elachiptera, 207.

Elachista, 90.

Elachistidie, 90.

Elaplidion, 267.

Elaphris, 2т2.

Elasmidie, 323.

Elasmoceris, 236 .

Elasmopalpus, i io.

Elasmosthetlins, $8_{3}$.

Elasmucha, 83 .

Elasmuns, 323.

Elater, 242. 
Elateridae, 240, 358 .

Elephantomyia, I6I.

Elfin, Banded, I56. Hoary, 156.

Elidiptera, 45 .

Elis, 333.

Ellema, I5I.

Ellida, 124.

Ellipotoma, 236.

Ellychnia, 234.

Elm aspidiotus, 65 .

Elmidæ, 245.

Elmis, 245.

Elm leaf-miner, 360 .

Elophila, ıо6.

Emblethis, 79.

Emesa, 75.

Emmesa, 259.

Emphoropsis, 346.

Emplytina, 293.

Emphytus, 293.

Empidide, I82.

Empis, I82.

Empoa, 52.

Empoasca, 52, 355.

Empretia, I I4.

Empria, 292.

Enallagma, 33.

Enarmonia, Ioo.

Encarsia, 324.

Enchenopa, 53.

Encoptolophus, 40.

Encyclops, 268.

Encyrtidx, 324.

Encyrus, 27I.

Endalis, 283.

Endecatomus, 26I.

Endelomyia, 294.

Endomychidæ, 253.

Endomychus, 253.

Enicmus, 253.

Ennearthron, 262.

Ennomos, I I 7 .

Enochrus, 223.

Enoclerus, 236.

Enodia, I56.

Entedon, 323.

Entedontida, 323.

Entonnacis, 327.

Entomobrya, 25.

Entomobryidxe, 25.

Entomopthalmus, 243.

Entylia, 55.

Eois, I20.

Eosphoropteryx, I34.

Epagoge, IOI.

Epalpus, I95.

Epargyreus, I 54.
Epeoloides, 345.

Epeolus, 345.

Ephemera, 27.

Ephemerella, 28.

Ephemerida, 27.

Ephemerida, 27.

Ephestia, I I I.

Ephestiodes, I II.

Ephialtes, 3I4.

Ephuta, 334.

Ephydra, 206.

Ephydridre, 206.

Epirschna, 35.

Epiblema, 98.

Epicallima, 92.

Epicauta, 238.

Epicnaptera, I22.

Epicordulia, 35 .

Epidiaspis, 64.

Epiglrea, I35.

Epigrymyia, I92.

Epilachna, 256.

Epimecis, I 8 .

Epinotia, 99.

Epipaschia; Io8.

Epiphragma, I62.

Epiplemidre, II5.

Epipsilia, $1+2$.

Epirhyssalus, 307.

Episcopt1s, 69.

Episinus, 98 .

Episyron, 335.

Epitaxonus, 292.

Epithectis, 94.

Epitrix, 278.

Epiurus, 3 I 4.

Epizenxis, I28.

Epurea, 248.

Epyrus, 332.

Erannis, I I7.

Erastria, 120 .

Erax, I79.

Erchomus, 229.

Erebus, I 20 .

Eremocoris, 79.

Ericcelinius, 304.

Erigorgus, 310.

Erineda, 9I.

Eriocampa, 293.

Eriocampoides, 294.

Eriocephala, 85.

Eriocera, I62.

Eriococcus, 63.

Erioptera, I6I.

Eriosoma, 6r.

Eriplanus, 3I6.

Eripternoides, 309.

Eripternus, 309 . 
Eristalis, I87.

Eritettix, 39.

Ernestia, I92.

Errobius; 260.

Eros, 233

Erotylidæ, 25 I, 358.

Erranis, I I7.

Erroments, 3I2.

Erymuis, I55.

Erythemis, 37 .

Erythraspides, 290.

Erythrodiplax, 36 .

Erythronetra, 52, 355.

Estigmene, I 8 .

Fthelurgus, 3 I6.

Euaresta, 20t.

Eubadizon, 305.

Eubaphe, I40.

Eubutus, 287.

Eucalyptera, I33.

Eucanthus, 263.

Euceraphis, 58 .

Encerceris, 3tI.

Fucerida, $3+6$.

Euceros, 3 I 2.

Euchetias. It7.

Euchlaena, I 6 .

Enchreca, I 2 I.

Eucinetus, 246.

Eucirrodia, I36.

Euclea, I 4 .

Enclentensia, 92.

Euclidea, I32.

Encnemide, $2+3$.

Euconnus, 225.

Eucosma, 97.

Eucrostis, II9.

Eucymatoge, I 2I.

Eurlamiss, I5t.

Eudeilinea, I 22.

Fudemis, 99.

Euderces, 270 .

Euclryas, I3t.

Eudule, I II

Eucretagrotis, I 12.

Eugnamptus, 28I.

Engonia, I I7, I57.

Eugonobapta, i I 7 .

Fuherrichia, I32.

Eulecanium, 63.

Eulia, I02.

Eulimnophila, I62.

Eulophide, 323, 360.

Eulopluns, 323, 360 .

Entmegaspiliss, 327.

limmenes, 336 .

Fimenidie, 336

Finmerus, Iso.
Eumetopia, 203.

Eupanychis, I35.

Euparius, 28I.

Euparthenos, I 29.

Euparyplus, I73.

Eupelmidæe, 325.

Euphoria, 265.

Euphorus, 305.

Euplyydryas, I5S.

Euphyes, I5t.

Eupithecia, I 2 I.

Euplexia, I 43.

Euplexoptera, 37.

Eupogonius, $27 \mathrm{I}$.

Eupristocerus, 241.

Euproctis, I 23.

Eupsalis, 280.

Eupteryx, 52.

Euptoieta, I58.

Eurema, I58.

Europs, 250.

Eurosta, 204.

Eurycnemus, I65.

Eurycyttarus, IIt.

Eurygaster, Sf.

Eurymus, I58.

Enrymyeter, $28 \mathrm{I}$.

Enrypogon, 2.46.

Enrythmia, I I .

Eurytoma, 325.

Enrytomidx, 325.

Eusayia, 326.

Euscelis, 50, 355.

Euschistus, 82.

Eustilbus, $25 t$.

Eustixia, 105 .

Eustrona, I2I.

Eustrophinus, 259, 359.

Eustrophus, 259.

Eustrotia, I33.

Eutettix, 50.

Euthisanotia, I34.

Futhochtha. So.

Euthrips, H.

Etitolype, I-3.3.

Eutrapela, I 6 .

Eutreta, 204.

Entrixa, I9I.

Eutura, 208.

Euvanessa, I57.

Euxesta, 203.

Fuxoa, I40.

Euxorides, 313.

Euzonhera, I

Evaniide, 307 .

Everes, 155.

Fvergestis. I04.

Fvetria, 96. 
Evoxysoma, 325 .

Evylaeus, $3+2$.

Exallonyx, 328 .

Exartema, 96.

Exechia, 167.

Exema, 273.

Exenterus, 3I2.

Exochilum, 3II.

Exochus, 3I3.

Exoprosopa, 176.

Exorista, I93.

Exoristoides, 192.

Eyed brown, I 56 .

Elater, 2 . 0.

Fagitana, I36.

Falagria, 230.

Falcaria, I22.

Falcate, orange-tip, I59.

Falciger, 286.

Fannia, I98.

Feltia, IfI.

Feniseca, I 55.

Fentonia, I 24.

Feralia, I43.

Ferdinandea, I88.

Fidia, 275.

Figitide, 3 I9.

Fish-moth, 25.

Firefly, Common, 234.

Fitchia, 76 .

Flata, 46.

Flea, garden, 25.

Fly, Biting House, 197.

Black horse, I74.

Blow, 197.

Blue bottle, I97.

Cluster, Ig6.

Horn, 197.

Honse, I97.

Lunate onion, I89.

Pretty pomace, 208 .

Screw worm, I96.

Vine-loving poinace, 208.

Finnts, 307.

Fonscolombia, 35 .

Forficulidx, 37 .

Formica, 33 I.

Formicidre, 328.

Fornicina, 330 .

Frit-fly, American, 208.

Fritillaries, I58.

Frontina, I93.

Fucellia, 199.

Filgoridxe, 45.

Fulvius, 70 .

Gaherasa, 127.

Grediopsis, I95.
Galasa, 107.

Galerita, 2I6.

Galerucella, 277.

Galesus, 327.

Galgula, I 33 .

Gall, Blackberry, 32I, 322. Cinquefoil axil, 322 .

Huckleberry, 322.

Lettuce, tumor, 319.

Oak, 319-322.

Ocellate leaf, I70.

Raspberry root, 322 .

Rose, 322.

Willow club, I69.

Pine cone, I68.

Galleria, Ios.

Gallinipper, 167.

Gargants, 7I.

Gargaphia, 77.

Gasterolobium, 227.

Gasteruption, 307 .

Gastroidea, 276 .

Gastropacha, I22.

Gastrophilus, Igo.

Gastrops, 206.

Gaurotes, 267.

Felechia, 93.

Gelechiidæe, 93.

Gelis, 315.

Geocoris, 78 .

Geometer, Chain-dotted, II7.

Geometride, I I5.

Geomyzidre, 208.

Geopinus, 218.

Georyssidae, $2 \nmid 6$.

Georyssus, $2\{6$.

Geotrupes, 263.

Geranomyia, 160.

Gerdana, 92.

Geron, 176.

Gerridie, 68

Gerris, 68 .

Geshna, I06.

Girdler, Cirrant Stem, 301. Strawberry Crown, 282.

Gliea, I35.

Glaphria, 103.

Glipodes, 237.

Glischrochilus, 249.

Glossonotus, 53 .

Gluphisia, 124.

Glutops, I75.

Glycolius, 269.

G] vphidocera, 94.

Glyphipterygidae, 356 .

Cilyphonyx, 24 $\mathrm{T}$.

Gilyphodes, Io4.

Glypta, 314.

Glyptonia, 226. 
Glyptocera, I 10.

Glyptoscelis, 275.

Ginathias, $3+5$.

Ginathodus, 52.

Gnathoncus, 232.

Gnorimoschema, 94.

Gnorimella, 265.

Gnorimus, 265.

Goes, 270.

Gomplieschna, 35 .

Gomphus, 3t.

Gonaspis, 322 .

Gonatopus, 333 .

Gonia, 104.

Goniocotes, 28.

Goniomina, I92.

Gonodontis, I 16.

Gonomyia, I6z.

Gortyna, 137.

Gorytes, 338.

Gossyparia, 63.

Gracillaria, 87.

Grape phyloxera, 62.

Graphiphora, I38.

Graphisurus, 27 I.

Graphocephala, 48 .

Graphoderes, 222.

Graphomyia, I97.

Graphops, 275 .

Grapta, 157.

Grasshoppers, 42.

Grayling, Blue-eyed, I57. Duli-eyed, I 57 .

Greenhouse orthezia, 63 .

Grotea, 3I3.

Grouse locusts, 39.

Gryllidae, 43.

Gryllotalpa, 43.

Gryllus, 43 .

Guenaria, I I9.

Gymnandrosoma, I0o.

Gymmetron, 285.

Gym1lochata, 192.

Gimmoclytia, Igo.

Gymnopternus, I8I.

Gymmosoma, Igo.

Givnandropus, 218.

Gypona, 49, 355.

Gypsochroa, I20.

Gyrinidre, 222.

Gyrinus, 222.

Grroliypnt1s, 227.

Gyroph:ena, 230.

Hahrobracon, 304.

Habrohraconidea, 304.

Habrocerus, 228.

Habrosyne, I23.

Hadena, ItH.
Hatrobregmus, 260.

Hrematobia, I97.

Hzematopinus, 45.

Hemorthagia, 153 .

Hamotopsis, 120.

Hagenius, 34 .

Hag-moth, I If.

Ilaimbaclia, 107.

Hair-streaks, I 55, I56.

Halictida, $3+2$.

Halictoides, 344 .

Halictus, $3+2$.

Haliplidie, 220 .

Haliplus, 220.

Halisidota, I47.

Hallesus, $3 \mathrm{I}$.

Halticus, 69.

Hamamelistes, 6I.

Hapalosalia, 267.

Haploa, I 48 .

H aploglossa, 230 .

Harlequin Cabbage bug, $\mathcal{E} 2$.

Harmonia, 250.

Harmostes, \&r.

Harpalus, 2 I8.

Harpipliorus, 293.

Harpipteryx, 96.

Harpyia, 124 .

Harris butterfly, I 58 .

Harrisina, I 13.

Harvest fly, 56.

Hebrida, 75 .

Hehrus, 75.

Head-louse, 45 .

Hedroneura, 201.

Iledychridium, 332.

Herlyclirum, 332 .

Helicobia, 106.

Helicopsyche, 3I.

Helicoptera, 45.

Jeliomata, I Io.

Heliophila, I39.

Heliothis, 135 .

Heliothrips, $4+$.

Heliotropha, I 43.

Hellgrammite, 29 .

Hellinmorpha, 2 I7.

Helolia, I62.

Helochata. 48.

Itelocordilia. 35.

Helodes, 246.

Ilelodidie, 246.

Helomyza. 200.

Helomyzidie, 200.

Helophilus, Ist.

Helophorus, 223.

Helops, 258.

Helorus, 328 .

Hemantus, 230. 
Henaris, $\mathbf{I} 53$.

Heloridæ, 328.

Heme robiidae, 29.

Hemerobius, 26, 29.

Hemerocampa, 123, I24.

Henerodromia, I82.

Hemerophila, 356 .

Henichionaspis, 64.

Hemichroa, 360.

Hemicrepidius, 24 I.

Hemilenca, I 50.

Henilexodes, 327.

Heminomada, 345 .

Hemiptera, 45, 354.

Hemispilota, 264.

Hemitaxonus, 292.

Hemiteles, $3 \mathbf{I} 6$.

Henyda, I9I.

Heodes, I 55.

Hepialidae, 85.

Heptagenia, 28.

Heræus, 78 .

Herculia, ro6.

Heriades, 348 .

Herse, I52.

Hesperiidx, I53.

Hesperobium, 227.

Hesperotingis, 77.

Hessian fly, I69.

Hetærina, 33.

Hetrerius, 232.

Heteraclithes, 267.

Heterocan1pa, I25.

Heterocera, 85 .

Heteroceridx, 246.

Heterocerus, 246.

Heterogramma, $\mathbf{1 2 7 .}$

Heteroneuridæ, 200.

Heteropelma, 3Io.

Heterophaga, 302.

Heterophleps, I2I.

Heteroptera, 66.

Heterospilus, 307.

Hetoemis, 270.

Hexagenia, 27.

Hexatoma, I62.

Hilara, I83.

Hilarella, I94.

Hillia, I45.

Himatinum, 288.

Himatium, 288.

Hippelates, 207.

Hippiscus, 40.

Hippohosca, 2ro.

Hippohoscidre, 210.

Hippodamia, 255.

Hister, 232.

Histerida, $23 \mathrm{I}$.

Hoary-edge, I 5 t.
Holcaspis, 320.

Holcocepliala, I78.

Holcocera, 9I.

Holcopasites, $3+6$.

Holcostethus, $8 \mathbf{I}$.

Holmgrenia, 3 I2.

Holocremnus, 309.

Hololepta, 23I.

Holonomada, 345.

Holopogon, I78.

Holostropluus, 259.

Homamus, 81.

Homalium, 226.

Homalonyia, I98.

Homceosoma, I I I.

Homoclodes, i IS.

Homœusa, 230.

Homomelina, I 49.

Homoptera. 45, I 29.

Homopyralis, I32.

Homosetia, 86.

Homotropus, 3I2.

Honey bee, $3+9$.

Hopatrinus, 257.

Hoplandria, 229.

Hoplandrus, 258.

Hoplia, 264.

Hoplisus, 338

Hoplocampa, 296.

Hoplocephala, 257.

Hoplosia, 27I.

Hop-merchant, I57.

Horcias, 72.

Horistonotus, 243.

Hormaplis, $6 \mathrm{I}$.

Hormisa, 128.

Hormius, 307.

Hormomyia, I70, I7I.

Hormorts, 282.

Hornet, Giant, 337.

White-faced, 337.

Horn-tail, Pigeon, 30I.

Hulstea, I Io.

Hyaliodes, 70.

Hyalomyodes, I9I.

Hyalopterus, 59.

Hyamia, I32.

Hybernia, I 17.

Hybos, I82.

Hybronia, 85 .

Hydaticus, 222.

Hydnocera, 236.

Hydrena, 223.

Iydrellia, 206.

Hydria, I2I.

Hydriomena, 120.

Hvdrobius, 223, 358 .

Hydrocanthus, 220 .

Mydrocius, 223. 
Hydrecia, I37.

Hydrophilidæ, 223, 358.

Hydropliilus, 223.

Hydrophorus, I8o.

Hydroporus, 220, 358 .

Hydropsyche, 32.

Hydropsychidæ, $3 \mathrm{I}$.

Hydroptilidae, 32.

Hydrotiea, I98.

Hydrous, 223.

Hyetodesia, I98.

Hylaidae, 346

Hylaens, $3+6$.

Hylastes, 289.

Hylastinus, 289.

Hyleccetus, 237.

Hylemyia, Ioś.

Hylesinus, 280.

Hylobius, 283.

Hyloicus, I 52.

Hylotoma, 300.

Hylotrupes, 269.

Hylurgopinus, 289.

Hylurgops, 289.

Hymenarcys, 82.

Hymenoptera, 29I, 360.

Hymenorus, 256.

Hypargyricus, 208.

Hyparpax, I24.

Hypatus, I 56 .

Hypena, I 26.

Hypera, 282.

Hyperacmus, 3 I3.

Hypereschra, 125.

Hyperaspis, 25t.

Hyperchiria, I 50.

Hyperitis, I 6 .

Hypermallus, 267.

Hyperodes, 283.

Hyperplatys, $27 \mathrm{I}$.

Hiphantria, I 48 .

Hypocrabro, 339.

Hypoderma, Igo.

Hypophloens, 258.

Hypoprepia, 149.

Hypopteromalus, 324 .

Hypothereutes, 309 .

Hyposoter, 300.

Hypostena, I0I.

Hypothenemus, 289 .

Hyppa, I+4.

Hypsopygia. 106.

Hyptia, 308.

Hypul11s, 259.

Hysterosia, IO3.

lanassa, I2f.

Ibalia, 323 .

Ivaliidre, 323 .
Ichnea, 236.

Ichneumon, 3I4, 3I7.

Ichneumonida, 308,360 .

Ichneutidea, 305.

Iclitliyura, I 26.

leterica, 204.

Illana, 203.

Irlechtis, 309.

Irlemum, 3 I6.

Idiocerus, 47, 354 .

Idliostethus, 286 .

Idris, 327.

Illice, I 49

Ilybius, $22 \mathrm{I}$.

Ilnacora, zo.

Ilythea, 206.

Immyrla, I Io.

Incisalia, I 56 .

Iphiaulax, 304.

Iphidicles, r 50.

Iphtilinus, 258 .

1 pimorpha, I 35.

Ips, 249, 290.

Ischnaspis, 66.

Ischnodenums, 78 .

Ischnoptera, 37.

Ischinorrlynclins, 78 .

Ischntura, 34.

Iseropus, 314 .

Isia, It 8 .

Isocybus, 326 .

Isodontia, 339, 340.

Isodyctium, 299.

Isomira, 257.

Isoperla, 27.

Isoptera, 26.

Isorhipis, 243.

Isosoma, 325 .

Isotoma, 25.

Isthmocoris, 78 .

Ithycerus, 280 .

Itonida, I7 I.

Itonidide, I68, 357.

I toplectis, 314.

Itycorsia. $29 \mathrm{I}$.

Jalysus, So.

Tanus, 30I

Jassoidea, 47.

Jassis, 50, $5 \mathrm{I}$.

Jodia. I37.

Joppidium, 3 I5.

Judolia, 268.

Junonia, I 57.

Jurinia, I95.

Kaliofenusa, 360 .

Katydids, fI, 12 .

Kelisia, 47. 
Kermes, 63.

Kolla, 48.

Labia, 37.

Labioderma, 276.

Laccobitus, 224.

Laccophilus, 220.

Lachnocrepis, 2I7.

Lachnosterna, 264.

Lachnus, 57.

Lacosoma, I 5 .

Lacosomidx, I 5.

Lelius, 332.

Lxmophleus, 250.

Lzemosaccus, 285 .

Laertias, ${ }_{5} 59$.

Lagium, 295.

Lagoa, II3.

Lagriidre, 259.

Lamenia, 46.

Lampria, I78.

Lampronota, 3I3.

Lampyridæ, 234, 358.

Languria, $25 \mathrm{I}$.

Lanthape, I08.

Lanthus, 34 .

Lapara, $15 \mathrm{I}$.

Laphria, i78.

Laphygma, I 43.

Lappits, 239.

Laricobius, 25 I.

Larropsis, 340 .

I asicampidae, I22.

Lasioderma, 260.

Lasioglossum, 342.

Lasiopogon, I78.

Lasioptera, I7o.

Lasius, 330.

Laspeyresia, I0o.

Lathridius, 253.

Lathridiidx, 253

Lathrobium, 227.

Lathropus, 250.

Lathrotaxis, 227.

Lauxania, 20I.

Leaf bug, Four-lined, 72.

Crumpler, Apple, 109

Leaf-hopper, Apple, iog.

Grape, 52.

Rose, 52.

Leaf-miner, Apple, 87.

Peet, I99.

Columbine, 208.

Chrysanthenum, 209.

Elm, 360 .

Imported Cabbage, 208. Turnip, 208.

Spinach, jog.

White oak blotch, 89.
Leaf-roller, Oblique banded, 100.

Strawberry, I00.

Leaf-tyer, Greenhouse, 104.

Lebia, 216 .

Lecanium, 63, 64.

Lecontea, $23+$.

Leia, 167.

Leiodes, 225.

Leiopus, $27 \mathrm{I}$.

Lema, 273.

Leperisinus, 289 .

Lepidoptera, 85, 356 .

Lepidosaphes, 65 .

Lepipolys, I +3 .

Lepisma, 25.

Lepismidae, 25.

Leptidx, 174 .

Leptinotarsa, 276 .

Leptis, 175.

Leptobrysa, 77.

Leptocera, 200.

Leptoceridx, 3I.

Leptocerus, 3I.

Leptogaster, I77.

Leptomeris, I20.

Leptopeza, I82.

Leptophlebia, 27.

Leptorliaptus, 328.

Leptoschema, 240.

Leptostyla, 77.

Leptostylus, 270, 359.

Leptothorax, 330.

Leptothrips, 4.

Leptoypha, 77 .

Leptura, 267. 268.

Lepturges, 27 I.

Lepyronia, 55.

Leskia, I9I.

Leskioninia, I9I.

Lestes, 33.

Lethocerns, 66.

Lencania, I30.

Leucantliza, 87.

Lencarctia, I+8.

Leucaspis, 65.

Lencophax, 38 .

Leitcopis, 209.

Lencorhinia, 36 .

Leucosmia, $3+7$.

Lencospidre, 326.

Lencospis, 326 .

Lencostola, ISo.

Lencostoma, I9r.

Leuctra, 27.

Libellula. 36.

Libellulide, 35,354 .

Liburnia, 47.

Liburniella, 77 .

Libythea, 156 . 
Libytheidre, 156.

Lichenophanes, 26 I.

Ligyrocoris, 79.

Ligyrodes, 205.

Ligyrus, 265.

Limenitis, I57.

Linmephilidae, 30 .

Limmeplilus, 30,3 I.

Limnerium, 360 .

Limnia, 357.

Limnolia, I60, I62.

Limmophora, IoS.

Limochroes, I5t.

Limnophila, I62.

Limonius, 240.

Limosina, 200.

Lina, 276 .

Lindenius, 338 .

Linnemyia, I92.

Linognathus, 45 .

Lioderma, 82.

Liogma, I63.

Liotheidre, 28.

Liuthrips, H.

Liotropis, 83 .

Liparide, 123.

Lipeurus, 28.

Lipocosma, 103.

Lispa, I99.

Lissonota, 3I3.

Listotroplicis, 228.

Listronotus, 283.

Litanonyia, I 82 .

Litargus, 252.

Lithacodes, I I3.

Litlıcodia, I33.

Lithocliaris, 227.

Lithocolletes, 88.

Lithosiidre, If9.

Little glass-wing, I5t.

ITood-satyr, I 56 .

Livia, 56.

Lixus, 285.

Loberus, $25 \mathrm{I}$.

Lobiopa, 249.

Lobopoda, 256 .

Locustidre, 4 I.

Locusts, 39-4I.

Loderus, 294.

Lomanaltes, 127.

Lomechusa, 230.

Lonclizea, 20L.

Lonchoptera, I83.

Lonchopteridre, I83.

Longistigma, 57 .

Longitarsus, 279

Long-sting, Black, 3 I 5. Lullate, 3 I 5.

Lophocrabro, 339.
Loplieros, 233.

Lophodonta, I 25.

Lophupompilus, 336 .

Lophyrus, 292.

Lopidea, 69.

Lopus, 69.

Louse, chichen liead, 28. Large boly hen, 28. Small body hen, 28 .

Variable chicken, 28.

Loxocera, 205.

Loxostege, I04.

Lucanidxe, 266.

Lucallus, 266.

Lucidota, 234.

Lucilia, I97.

Ludius, $240,2+1$.

Luperaltica, 278 .

Luperodes, 277.

Lycrenidae, I55.

Lycia, I I 7 .

Lycidae, 233.

Lycogaster, 308.

Lyconiorpha, I50.

Lycoperdina, 253.

Licostomus, 233.

Lretidie, $26 \mathrm{I}$.

Lyctus, 26I.

Lyda, 20 I.

Lygxidae, 77.

Lygeus, 77, $78,8 \mathrm{I}$.

Ligreonematus, 297.

Lygidea, 72.

Lygus, 70, 72.

Lynexylidie. 237 .

Lynexylon. 237.

Lymnadidre, I 56.

Lymnecia, 90.

Lyonetia, 87.

Lyroda, 3 to.

Macaria, I I9.

Macliilidie. 25.

Nachilis, 25.

Nachimia, 92.

Macquartia, I9I.

Macratria, 239.

Nacremphytus, 293.

Macrobasis, 238.

Macrocentrus. 305.

$M$ lacrocera, I $6 \dot{8}$.

Macrodactylus. 264.

Macroloplius, 70 .

Macromeigenia. I92.

Macronema. $3 \mathrm{I}$

Macronychus, 245.

Macrophya, 205.

Macropida, 344 .

Macropis, 344 . 
Macropsis, 48, 354 .

Macrosargus, I 72 .

Macrosiagon, 238 .

Macrosiphum, 60.

Macrotylus, 69.

Macroxyela, 29 I.

Madarellus, 286.

Magdalis, 284 .

Naggot, apple, 204. Cabbage, I98.

Cherry, 204.

Greater wheat stem, 207.

Onion, Ig8.

Raspberry cane, I99.

Seed corn, 198.

Magusa, I43.

Malachidae, 235.

Malachius, 235.

Malacosoma, 122.

Mallochiella, 209.

Nallophaga, 28.

Mallota, I87.

Malporus, 239.

Malthodes, 235.

Mamestra, I39.

Manomera, 39.

Mlansonia, 166.

Mantidæe, 39.

Mantis, Chinese praying, 39 .

Mantura, 278.

Marasmalus, I34.

Marmara, 87 .

Martyringa, 95.

Narumba, i5 I.

Masicera, I93.

Mastogenius, 245.

Matus, 22I.

Mayetiola, I69.

Meadorus, 83 .

Meal worm, Dark, 258. Yellow, 258.

Mealy bugs, 63.

Mecoptera, 30 .

Mecostethus, 40.

Megachile. 347.

Megachilidæe, 347.

Megalodacne, $25 \mathrm{I}$.

Megalopygidae, I I3.

Megalotomus, $8 \mathrm{I}$.

Megapenthes, 242.

Megarhyssa, 3I5.

Megaspilus, 327.

Megilla, 255.

Megorismus, 325.

Melalopha, I 26 .

Melanactes, 24 I.

Melandrya, 259.

Melandryidæe, 259, 359.

Melanochieta, $20 \%$.
Melanolestes, 76 .

Melanomma, I32.

Melanophia, I I8.

Melanophila, 244.

Melanophora, 195.

Melanophrys, 192.

Melanophthalma, 253.

Melanoplus, 4I.

Mclanorhopala, 77 .

Melanostoma, I85.

Melanotus, 242, 358 .

Melanoxantherium, 58 .

Melanoxanthus, 58 .

Melasidæe, 243.

Melasis, 243.

Melasoma, 276.

Melieria, 203.

Meligethes, 249.

Melinna, 69.

Meliopotis, I32.

Melitæa, I58.

Melittia, II2.

Melittomma, 237.

Mellissodes, 346 .

Meloe, 238.

Meloidxe, 238.

Melophagus, 210.

Melyrida, 235.

Membracidæ, 53, 356.

Memythrus, I I2.

Menecles, 82 .

Meniscus, 3 I 3 .

Menopon, 28.

Menopsimus, 127.

Meracantha, 259.

Merisus, 324.

Merium, 269.

Merocoris, 80 .

Meromyza, 207.

Meronea, 229.

Meroplius, 205.

Meroptera, I Io.

Merycomyia, 174 .

Mesamia, 49, 50.

Mesochorus, 3 Io.

Mesogramma, i 86 .

Mesograpta, i 86.

Mesoleius, 3II.

Mesoleptidea, 3I I.

Mesoleptus, 3I0, 3II, 3I5, 3I6, 360.

Mesoleuca, I2I.

Mesomiris, 74 .

Mesoneura, 296.

Mesostenidea, 3I5.

Mesothemis, 37 .

Mesovelia, 75.

Mesoveliidx, 75 .

Metabletus, 216 .

Metachæta, I94. 
Netachroma, 275.

Metalius, 299.

Metanema, II6.

Metapodius, So.

Metathorasa, I32.

Meteorus, 305.

Nethoca, 334.

Methocidre, 334.

Metopia, I94.

Metopius, 3 I3.

Metriona, 280.

Metrobates, 68.

Metrocampa, I I 7 .

Metzneria, 95.

Miarus, 285.

Miastor, I68.

Micracis, 289 .

Nicrathyria, 36 .

Microbembex, 34I.

Microbracon, 303.

Microcolia, I46.

Microdon, I84.

Microdus, 306.

Microgaster, 303.

Micromeson, 315.

Micromus, 29.

Micropezidæ, 204.

Microphylelliss, 68.

Microplitis, 303 .

Micropterygide, 85 .

Micropthalma, I95.

Microrhopala, 279.

Microstelis, $3+7$.

Microvelia, 68.

Microweisia, 254 .

Micrutalis, 53 .

Midge, Chrysanthemum gall, 357. Pear, I70.

Violet, I70.

Wheat, I70.

Milesia, I 88 .

Milichia, 209.

Milyas, 76 .

Mimesa, 338.

Mineola, I09.

Minettia, 202.

Mineus, 83 .

Miridx, 68,356 .

Miris, 74.

Miscogasteridæe, 325 .

Misogada, I24.

Mitoura, I56.

Molamba, 226.

Nole cricket, Northern, 43.

Molophilus, I6I.

Molorchus, 268.

Mompha, 90.

Munachus, 27f.
Monalocoris, 70 .

Nonarch, I 56.

Monardia, 168 .

Nonarthrum, 289.

Monecphora, 55 .

Monellia, 58.

Nonobia, 336 .

Monocrepidius, 240.

Monodontomerus, 325 .

Nonohammus, 270 .

Monomorium, 329.

Mononychus, 286.

Monophadnoides, 299.

Monophadnus, 298, 299.

Nonophylla, 236 .

Monopsis, 86.

Nonosonia, 293.

Monostegia, 292, 294.

Monotonide, 250.

Monoxia, 277 .

Moodna, I I I.

Mordella, 237.

Mordellidie, 237.

Mordellistena, 237.

Morellia, I 97.

Mormidea, 82

Mormon, I55.

Norrisonia, I39.

Mosillus, 206.

Mosina, 200.

Mosquito, Banded salt marsh, 167. Brown salt marsh, I66.

House, 166.

Malarial, 167.

Rainbarrel, 166

Moth, American silk worm, I 50.

Angoumois grain, 95 .

Bee, 108

Brown-tail, I23.

Bud. 99.

Cecropia, I5I.

Clothes, 86.

Cotton-worm, I34.

Cynthia, I5I.

Fitr, 86.

Gipsy, I23.

Grape berry, 06. plunne, I03.

Hickory tussock, I 47.

Imperial, I 50 .

Indian meal, i I I.

Io, I 50 .

Isabel tiger, I 48 .

Leopard, II2.

Lime tree, I17.

Llina, 150.

Meal snout, io6.

Mediterranean flour, II I. 
Milk-weed, I 47

Oriental peach, Ioo.

Pine tip, Iog.

Promethia, I5I.

Regal, I50.

Rusty tussock, I23.

Silver spotted ghost, 85 .

Snow white linden, II7.

Tulip tree silk, I5I.

Virgin tiger, $\mathbf{I}+8$.

Walnut bud, Iog.

Wax, Ios.

White-marked tussock, I23.

Mountain silver spot, ${ }_{5} 8$.

Nourning cloak, I 57 .

Murgantia, 82 .

Musca, I97.

Muscida, I06.

Muscina, I97.

Mutillidre, 334.

Myas, 2 I3.

Mycetiea, 253.

Mycetreidæe, 253.

Mycetina, 253.

Mycetobia, I68.

Mycetocliara, 257.

Mycetochares, 257.

Mycetophagidie, 252.

Mycetophagus, 252.

Mycetophila, 168.

Mycetophilidre, I67.

Myconya, 167.

Mycotretus, 25I, 358 .

Mycterus, 260 .

Mydaidx, 177.

Mydas, 177.

Myelois, I09.

Myersiidre, 307.

Myiocera, I95, I 96

Myiolepta, I85.

Myiophasia, Igo.

Myiospila, 197 .

Mylabridae, 280.

Myndus, 46.

Myodites, 238 .

Myodocha, 78 .

Myopa, I 80 .

Myrmaridre, 323.

Myrmecina, 329.

Myrmecomyia, 202.

Myrmecothea, 202.

Myrmeleon, 20.

Myrmeleonidae, 29.

Myrmica, 329.

Myrmosa, $33+$

Myrmosida, 33t.

Mysia, 256.

Mystacides, $3 \mathbf{I}$.
Mytilaspis, 65.

Myzine, 333.

Myzocallus, 58 .

Myzus, 60.

Nrogeus, 75 .

Nabidae, 75.

Nabidea, 74 .

Nabis, 75 .

Nacerdes, 237.

Nacophora, II7.

Nadata, I25.

Nadia, 3I3.

Nremia, 255.

Nannothenis, 36 .

Napomyza, 209.

Nallcorida, 67.

Naucoris, 67.

Neanura, 26.

Neaspilota, 204.

Nebria, 2 I 2.

Necrobia, 236.

Necrophorus, 224.

Nectarophora, 60.

Nehalennia, 33.

Neichnea, 236.

Neides, So.

Neididae, 80.

Nematus, 297, 298.

Nennobius, 43.

Nenropoda, 205.

Nemoria, II9.

Nemosoma, $2+7$.

Nemotelus, I73.

Nemoura, 27.

Neoascia, I86.

Neobisnius, 227.

Neoborus, 72.

Neoclytus, 269.

Neodiprion, 292.

Neœmpheria, 167.

Neofischeria, I94.

Neoglaphyroptera, I67.

Neolasioptera, i69.

Neomysia, 256.

Neonortonia, 300.

Neonymplia, I56.

Neopales, I93.

Neopasites, $3+6$.

Neoprociphilus, 6 I.

Ncothomasia, 58 .

Neottiglossa, 82 .

Nepa, 67.

Nephelodes, r39.

Nephopteryx, 1 Io.

Nephrotoma, i63.

Nepida, 67.

Nepiera, 309. 
Nepticula, 90.

Nepytia, I 8 .

Nerice, I25.

Neurigona, I80.

Neurocolpus, $7 \mathrm{t}$.

Neuroctena, 20 I.

Neuronia, 30.

Neuroptera, 29.

Neuroterus, 3 I9.

Neurotoma, 29I.

Nicentrus, 286.

Nigetia, I49.

Nisaxis, 230.

Nitidula, 249.

Nitidulidæe, 248 .

Nitzschia, 28.

Noctua, I4I.

Noctuidæ, I26.

Nodonota, 275.

Nola, I 49.

Nolophana, 145.

Nomada, 345 .

Nomadidæ, $3+5$.

Nomirephagus, 334 .

Nomophila, 104.

Nomotettix, 39.

Nonagria, I 37.

Northern cloudy wing, 154 .

Nortonia, 336.

Nosodendron, 248.

Nosodendronidæ, 248.

Notoglossa, 338 .

Nothosmia, 347.

Notidobia, 3I.

Notiophilus, 2 I2.

Notipliila, 206.

Notodonta, 125.

Notodontidæ, I24.

Notogonidea, 340.

Notolophus, I 23.

Notonecta, 67.

Notonectidæ, 67 .

Nototrachys, 3II.

Notoxus, 239.

Notozus, 332.

Nudobius, 227.

Nycteola, I32, I33.

Nyctibora, 38 .

Nyctobates, 258.

Nyctobia, I 22.

Nylanderia, 330.

Nymphalidæ, I57.

Nymphula, I05.

Nysius, 78 .

Nysson, 338.

Oak apple, 320 .

Sinall, 322.
Oberea, 272, 359.

Obrium, 267.

Ochthera, 206.

Ochthiphila, 209.

Ocneria, I23.

Octoteminus, 26I.

Ocyptamus, I85.

Ocyptera, I92.

Ocypus, 228.

Odinia, 209.

Odonata, 33, 354.

Odontæus, 263.

Odontocera, 209.

Odontocorynus, 286.

Odontomerus, 313.

Odontomyia, I72.

Odontota, 279.

Odynerus, 336.

Cbalus, 82.

(Ecanthus, 43.

Ecetina, 3I.

Eciacus, 74.

Ecleus, 46.

(Ecophora, 9I, 92.

Ecophoridæ, 9I.

(Ecothea, 200.

CEdancala, 78 .

Edaspis, 204.

Edemeridre, 237.

Cdionychis, 277 .

Cine, 266.

Estodes, 241.

Estride, I90.

Estrophasia, I9I.

Estrus; 190.

Ogdoconta, I34.

Olene, I23.

Olethreutes, 97,356 .

Olfersia, 2 I0.

Oliarus, 46.

Olibrus, 254.

Oligia, I 45 .

Olisthærus, 226.

Olisthopus, 216 .

Olophrum, 226.

Omalus, 3.32.

Omethes, 234.

Ommatius, I78.

Omophron. 2 I9.

Omophronidæ, 2 I9.

Omphalocera, Io6.

Onchylis, 283 .

Oncideres, 27 I.

Oncodes, I75.

Oncognathus, 7I.

Oncometopia, 48 .

Oncomyia, I89.

Oncopeltus, 77. 
Oncopsis, 48, 354.

Oneida, Io8.

Onthophagus, 262.

Unychiurus, 26.

Oödles, 2I 8 .

Onychobaris, 285.

Ophiderma, 55 .

Ophiogomphiss, 34 .

Ophion, 3I I.

Ophistomis, 268.

Ophyra, ig8.

Opius, 302.

Opostega, Sq.

Opsidia, I94.

Orchelimnm, 42.

Orchesia, 259.

Orchestes, 284 .

Orectoderus, 69.

Oreta, I 22.

Orgilus, 306.

Orgyia, I23.

Ormenis, 46.

Ormosia, I6I.

Ormyrus, 325 .

Orilix, 87.

Oropeza, 163.

Orphulella, 39 .

Orsodacna, 273.

Ortalide, 202.

Orthaea, 79 .

Orthaltica, 278 .

Orthellia, I97.

Orthezia, 63.

Orthizema, 3I6.

Orthocis, 26I.

Orthocladius, I 65 .

Orthodes, I 39.

Ortholepis, I Io.

Ortholestes, 228.

Ortholomus, 78 .

Orthonama, I21.

Orthopelma, 3 Io.

Orthops, 73 .

Orthoptera, 38 .

Orthosia, 136 .

Orthosoma, 266.

Orthotomicus, 290 .

Orthotrichia, 32.

Orthotylus, 70.

Oryssidx, 301.

Oryssus, 30I.

Oryzxphilus, 250.

Oscinis, 208.

Osmia, 347.

Osmoderma, 265.

Osmosita, 249.

()stomidie, 2.77 .

Otacustes, 316.
Otidocephalus, 284 .

Otiocerus, 46 .

Otiorhynchus, 282 .

Ox-lonse, Loing nosed, 45.

Short nosed, 45.

Oxybelus, 338 .

Oxcera, I72.

Oxycnemis, 249.

Oxygonus, 24t.

Oxyporus, 228.

Oxyptilus, I03.

Oxystoglossa, $3+2$.

Oxytelus, 226.

Ozophora, 79.

Paclinobia, If2.

Pachybrachys, 274.

Paclyydiplax. 37.

Pachynematus, 297.

Pachyneuron, 324.

Pachyprotasis, 295.

Pachypsylla, 57.

Pachyrhina, 163.

Pacliscelus, 245.

Pachysplininx, I5I.

Packardia, I I3.

Prectes, I34.

Paderis, 227.

Predisca, 97.

Pagasa, 75.

Painted beauty, I57.

Paleacrita, I22.

Palingenia, 27.

Pallodes, 249.

Palorus, 258.

Palponiria, I65.

Palthis, 127.

Paltodora, 95.

Pamera, 79.

Pamerocoris, 70.

Pamphiliidae, 29 I.

Pamplila, I54, I 55.

Pamphilits, 291.

Panagens, 212.

Panapoda, I 29.

Panchlora, 38.

Panchrysia, I 34.

Pandeletejus, 282.

Pandemis, ror.

Pangonia, I73.

Pangrapta, I32.

Paniscus, 310.

Panorpa, 30.

Panorpide, 30.

Panscopns, 282.

Pantographa, I04.

Panurgide, 345.

Panturginus, 345 . 
Panzeria, I02.

Paonias, I5I.

Papaipena, I37.

Papilio, I 59.

Papilionidae, I59.

Parabolocratus, 49, 355.

Paracalocoris, 7 I.

Paracanidia, 3Io.

Paracharactus, 299.

Paracymus, 223.

Paradidyma, I94.

Paragrotis, Ifo.

Paragus, I85.

Paralalius, 3.32.

Paralictus, 312 .

Paralipsa, I08.

Parallulia, I 29.

Parallelina, 268.

Parallelosthetus, $24 \mathrm{I}$.

Paramellinus, $33^{8}$.

Paramestis, 49.

Parandra, 260.

Paraphia, I 8 .

Paraplagia, I02.

Parasierola, 332.

Parasitica, 45.

Paraspinuphora, IS3.

Parataxonus, 293.

Paratenodera, 38.

Paratettix, 39.

Parcoblatta, 38.

Parectecephala, 207.

Paria, 275.

Parlatoria, 66.

Paromalus, 232.

Paroxya, fI.

Parthenothrips, 4.

Parydra, 206.

Passalide. 266.

Passaloecus, 3fI.

Passalus, 266.

Patrohus, 2I3.

Pearly eye, 156.

Pear psylla, 57.

Perlicia, I63.

Pediculidie, 45.

Pediculus, 45 .

Pedilidxe, 239.

Perlilus, 239.

Pediopsis, 48.

Pegomyia, I99,

Pelastoneurus, I8I.

Pelecinicle, 328.

Pelecinus, 328.

Pelenomus, 287

Peleteria, I95.

Pelidisota, 265.

Peliopelta, 78 .
Pelocoris, 67.

Peloninm, 236.

Pelopens, 3 fo.

Peltodytes, 220.

Pempligus, 6r.

Pemplnedon, 3tI.

Pentacora, 67.

Pentagenia, 28.

Pentaplenra, 304.

Pentatoma, 82.

Pentatomidie, SI.

Pentlue, 259.

Penthimia, 49.

Penthina, 97.

Penthoptera, I63.

Pentilia, 25t.

Peoria, II I.

Peponaisis, $3+6$.

Percinolus, 2.47.

Percnoptilota, i2I.

Perclita, 345.

Peribalius, \&I

Periclista, 299.

Periclistus, 3 I0.

Peridroma, If $\mathrm{I}$.

Perigea, I 45.

Perigenes, 79.

Perilampidre, 326 .

Perilampus, 326.

Perilampus, 326.

Perilitus, 306.

Perillus, 83 .

Periodical cicada, 56 .

Periphyllus, 58.

Periplaneta, 38.

Perispasta, I04.

Perithemis, 37.

Perla, 27

Perlesta, 27.

Perlida, 27.

Peronea, ior.

Petrophora, I 20.

Pezomachus, 3 I 5.

Plizerlon, 276.

Phicocyma, I 20.

Phreogenes, 317.

Phalacridie, 254.

Phalacrus, $25 \%$.

Phalienostola, I32.

Phaleria, 257.

Phalonia, I02.

Phanans, 262.

Pharmacis, I02.

Phasmidie, 38 .

Pheilole, 320.

Phelister, 232 .

Plienacoccus, 63.

Phengodes, 235. 
Phengodide, 235.

Phenolia, 249.

Pheosia, I25.

Phigalia, I I7.

Philænus, 55.

Philanthus, 339.

Philaronia, 56 .

Philhydrus, 223.

Philobia, I I9.

Philometra, I28.

Philonyx, 3I9.

Philonome, 87.

Philonthus, 227.

Philopteridx, 28.

Philosamia, I5I.

Philothermus, 252.

Phiprosopus, I33.

Phlegyas, 78.

Phlepsius, 5 I.

Phlegethontius, I52.

Phloopterus, 236.

Phlœosinus, 289.

Phlœothripidæ, 44.

Phlyctænia, I04.

Phoberia, I29.

Phobetron, II4.

Pholisora, I54.

Pholus, I 52, I53.

Phora, I84.

Phorantha, I9o.

Phorbia, I98.

Phorichxta, 194.

Phoridx, I 83.

Phormia, 197.

Phorocera, I93.

Phortica, 208.

Photinus, 234, 358.

Photuris, 234 .

Phragmatobia, I48.

Phryganea, 30.

Phrygancidie, 30.

Phthinolophus, ror.

Phthiritus, 45.

Phthorophlous, 288.

Phycanassa, 154.

Phyciodes, I58.

Phygadewon, 3 I6.

Phygadicus, 78 .

Phylexis, 282.

Phyllaphis, 58.

Phyllobænus, 236.

Phyllobrotica, 277.

Phyllodromia, 37.

Phylloecus, 30I.

Phyllodecta, 276.

Phyllophaga, 264.

Phylloscelis, +5 .

Phyllotreta, 279.
Phylloxera, 62.

Phymata, 76.

Phymatida, 76.

Phymatocera, 299.

Phymatodes, 269.

Physatocheila, 77.

Pliysocephala, Is9.

Physokermes, 64.

Physopoda, 44.

Phytocoris, 7I, 356.

Phytodietus, 3 I 3 .

Phytonyza, 208, 209.

Phytonomus, 283.

Phytophaga, I69, 29I.

Piazorhinus, 285 .

Pieridæ, 158.

Pieris, I59.

Piesula, 76 .

Piesmidx, 76.

Piezostethus, 74.

Pigritia, 9I.

Pilopius, 23I.

Pimpla, 314.

Pimplidea, 3 I 4 .

Pinacodera, 2I6.

Pinipestis, 109.

Pinotus, 262.

Pinphila, 205.

Pipiza, 185.

Pipunculidxe, I84.

Pipunculus, 184 .

Pissodes, 283.

Pissonotus, 47.

Pityogenes, 289, 290.

Pityokteines, 290.

Pityophthorus, 289.

Pitys, 85 .

Plagiodera, 276.

Plagiognathus, 68 .

Plagionotus, 269.

Plagodis, II7.

Planiceps, 335.

Plant bug, Tarnished, 73.

Plateros, 233.

Plathemis, 36.

Platycentropus, $3 I$.

Platycerus, 266.

Platychirus, 185.

Platydema, 257.

Platygastridie, 326 .

Platymetopius, 50, 355 .

Platynota, Ior.

Platynus, 215.

Platypalpus, is2.

Platlypena, I 26.

Platypterygidæ, I22.

Platyptilia, I03.

Platysenta, 145. 
Platysoma, 232.

Platystethus, 226.

Platystomidre, 28r.

Platytes, I07.

Platytylellus, 73 .

Plea, 67.

Plecoptera, 27.

Plectiscidea, 3ro.

Plegaderus, 233.

Plenoculus, 34 I.

Plesia, 333.

Plesignathus, 3 I 6.

Pleuroprucha, I20.

Plenrotropis, 323.

Plodia, III.

Ploiaricola, 75 .

Ploiariodes, 75.

Plusia, I 34.

Plutella, 96.

Poanes, I 55.

Podabrus, 234.

Podisus, 83 .

Podops, 8i.

Podosesia, II2.

Podurida, 26.

Pocilocapsus, 72.

Poecilomyia, 201.

Pociloscyt11s, 7I.

Pœecilostoma, 292, 293.

Pogonocherus, 27I.

Polemitis, 235.

Polidea, I9I.

Polistes, 337.

Polites, I 54 .

Pollenia, ro6.

Polyænus, 3I 5.

Polyblastus, 3 I 2.

Polychretoneura, 190.

Polychrosis, 96.

Polydrusus, 282.

Polyergus, 33I.

Polygnotus, 326 .

Polygonia, I 57.

Polygrammata, I45.

Polyhymno, 94.

Polyphylla, 264.

Polypsocus, 26.

Polyspincta, 314.

Polystenidea, 307.

Pomphopea, 238.

Pompiloides, 335.

Ponera, 328.

Pontania, 298.

Pontia, I 59.

Porizon, 3Io.

Porizonidea, 3 Io.

Porosagrotis, I4I.

Porplyyrops, i8o.
Porthetria, I23.

Potato beetle, Colorado, 276.

Old-fashioned, 273.

Praon, 308.

Prasoctiris, 275.

Prenolepis, 330.

Priocnentis, 335.

Priocycla, i 16.

Prionocheta, 225.

Prionomerus, 285.

Priononyx, $34^{\circ}$.

Prionoxysti1s, II2.

Priont1s, 266.

Priophorus, 297, 360.

Pristiphora, 297.

Pristocera, 332.

Pristomerus, 310.

Prociphilus, $6 \mathrm{I}$.

Proctacanthus, 179.

Proctotrypes, 328.

Prodenia, I 43.

Prodrtis, 3 Io.

Prokelisia, 47.

Prolencoptera, 87.

Prolinacodes, II 4 .

Promachus, I79.

Pronuba, 85.

Prosacantha, 327 .

Prosopis, 346.

Prospaltella, 324 .

Protapanteies, 302, 303.

Protenor, Si.

Proteopteryx, 98.

Prothymia, I33.

Protocallipliora, I97.

Protoparce, I52.

Protothyreopis, $338,339$.

Psacaphora, 90.

Psallus, 68.

Psammochares, 336.

Psammocharida, 335.

Psaphidia, I42.

Pselaplidire. 230.

Pselaplius, 23I.

Pselliopus, 76.

Psen, 338 .

Psenocerns, 270.

Psephenidx, 245.

Psephen11s, 245.

Pseudagenia, 335.

Pseudanaphora, 85.

Pseudebrus, 235.

Pseudibion, 267.

Psendobaris, 286.

Pseudocasinaria, 300.

Pseudocistela. 257.

Pseudocnemodus, 79.

Pseudococcus, 63. 
Pseudogalleria, 97.

Pseudolimnophila, I62.

Pseudometlioca, 334.

Pseudopigritia, 9 I.

Pseudopomala, 4I.

Pseudopyrellia, 197.

Psendotepluritis, 203.

Psendothyatira, I23.

Pseudoxenetus, 69.

Psila, 205.

Psiliclx, 205.

Psilocephala, I77.

Psilocorsis, 92.

Psilomastix, 3I7.

Psilopa, 206.

Psilopus, I79.

Psilopyga, 249.

Psilocelis, 232.

Psilotanypus, I66.

Psinidia, to.

Psithyrus, 349.

Psocidie, 26.

Psocus, 26.

Psorophora, I67.

Psychidæ, IIf.

Psychoda, I65.

Psychodida, I65.

Psychonorpha, I35.

Psylla, 57.

Psyllidæe, 56.

Psylliodes, 279.

Psyllobora, 255.

Psyllopsis, 57.

Psy-sostegania, II9.

Ptecticus, I72.

Pterallastes, IS8.

Pterocolus, 28r.

Ptercyclon, 289.

Pterocormus, 317, 318, 319.

Pterodela, 26.

Pteromalidxe, 324.

Pteromalus, 324. 36I.

Pteronidea, 208, 360.

Pteronus, 297

Pteroploridie, I03.

Pterophorus, I03.

Pterostichus, 213.

Ptilidx, 23I.

Ptilodactyla, 246.

Ptilodexia, In6.

Ptinidie, 260.

Ptinus, 260.

Ptochiomera, 79.

Ptomopliagus, 225.

Ptychoptera, I63.

Ptyocerata, 95.

Publilia, 55.

Pulex, I6o.
Pulicidie, I60.

Pulvinaria, 63.

Purple, Banded, I57.

Hybrid, I 57.

Red-spotted, I57.

Purpuricenus, 270.

Pycnopsyclie, 3I.

Pycnoscelus, 38.

Pygolampis, 75.

Pyractonema, 234.

Pyralidxe, I03, 356 .

Pyralis, Io6.

Pyrameis, 157.

Pyrausta, 105.

Pyrellia, I97.

Pyrgota, 202.

Pyrochroa, 239.

Pyrochroide, 239.

Pyromorpha, II3.

Pyromorplida, I I3.

Pyrophana, I85.

Pyroplila, I +3 .

Pyrrharctia, I 48 .

Pyrrlia, 137 .

Pyropyga, 231

Pylindxe, 238.

Pytho, 238 .

Quedius, 228.

Ranatra, 67.

Raspberry byturus, 246.

Recurvaria, 95.

Red admiral, I57.

Reduviidx, 75.

Reduviolus, 75 .

Reduvius, 75 .

Reichenbaclia, 230.

Reichertella, I7 I.

Remigia, I29.

Renia, I27.

Restlenia. 73.

Retinodiplosis, I7 I.

Reuteroscopus, 60.

Rhaldepyris, 332.

Rhabdophaga, I68.

Rhadinocera, 316.

Rhagio, 175.

Rinagium, 267.

lhhagoletis, 204.

Rhamphidia, I6I

Rhamphomyia, IS3.

Rhantus, 22I.

Rhaplitim, ISI.

Rhegmoclema, $17 \mathrm{I}$.

Rhenmaptera, I2I.

Rhimplialea, 3I2.

Rluingia, I86. 
Rhinocapsus, 68. Rhinonchus, 287. Rhinopsis, 3.37 . Rhinosinus, 238 . Rhipiclia, 160 .

khipiphoridx, 238 . Rhipiphorus, $23 \mathrm{~S}$.

Rhizophagidre, $2+9$.

Rlizophagus, 249.

Rhodites, 3I9, 322 .

Rhodolicenus, 288.

Rhodophora, I35.

Rhogogastera, 295.

Rhopalicus, 36I.

Rhopalocera, I 53.

Rhopilonyia, I69.

Rhopalosiphum, 60.

Rliopalum, 338.

Khorus, 3 I 2.

Rhyacophilider, 3 I.

Rhymosia, I67.

Rhynchagrotis, I 42 .

Rhynchites, 28I.

Rhynchomiliclia, 209.

Rhyparochromus, 79.

Rhypholoplius, I6I.

Rhypobius, 226.

Khytidolomia, \&2.

Rliyssa, 3 I 5.

Rliyssella, 3 I5.

Riblued cocoon-maker, 87 .

Rivellia, 202.

Rivula. I33.

Roselia, I +9 .

Rogas, 3I I.

Romaleum, 267.

Rose chafer, 264.

Ryphidie, I72.

Rypliss, I72.

Kyssematus, 287.

Sableta, 220.

Sabulodes, I I5.

Sacium. 226.

Sackenomyia, I69.

Saddled prominent, I25.

Sagarilis, 308 .

Saissetia, 64.

Salda, 67.

Saldicle, 67.

Saldula, 67.

Salebria, I Io.

Silia, I 27.

Salisneana, 98.

Samia, I5I.

Sanninoilea, II2.

Saperda, $27 \mathrm{I}$.

Sapintus, 239.
Saprin1ss, 233.

Sapromyza, 201, 202.

Sapromyzidie, 201.

Sapyga, 333.

Saprgide, 333 .

Sarcofahrtia, I95.

Sarcophaga, 100.

Sarcophagidae, 196.

Sargus, I72.

Sarrothripus, I33.

Saturniirlie, 150.

Satyrodes, I56.

Sawtly, Peach, 201. Rose, 294.

Saxinis, 273 .

Sayjella, 326 .

Sayomyia, I67.

Scale insects, 63-66.

Scambus, 314.

Scaphidema, 358 .

Scaphidiidre, 2.31 .

Scaphidiun, 23I.

Scaphisoma, 231.

Scaphoideus, to.

Scaptomyza, zos.

Scarabreidie, 262, 359

Scardia, Só.

Scarites, 2 I 2.

Scatella, 206.

Scatopliaga, 200.

Scatopluagidie, I99.

Scatopse. I7I, 357.

Scatopsidee, I-I, 357.

Scelio, 327 .

Scelionidae, 326.

Scelipluron, 3 to.

Scenopinidie, $17 \%$.

Scenopinus, 177.

Scepsis, I 50.

Schinia, 135.

Schistocera, $f$ I.

Scli istosiphon, 200.

Schizocerrs, 300.

Schizogenius, 2I2.

Siclizomenta, 57, 6I.

Schizotus, 239 .

Schizura, 124.

Sclinenolinis, $10 \%$.

Schonomyzil, Ino.

Scictgraphia, I Io.

Sciaptery, 203.

Sciara, I68, I/ I.

Sciaridie, I68.

Scinacopus, 316.

Sciomyza, 20 I.

Sciomyzidxe, 201, 257.

Scioplital, I67.

Scirtes, $2+6$. 
Scirtetica, 40.

Scoleocampa, I34.

Scolia, 333.

Scoliidæe, 333 .

Scoliocentra, 200.

Scoliopteryx, I36.

Scolopostethus, 79 .

Scolops, 45 .

Scolytidae, 288 .

Scolytus, 288.

Scoparia, Io6.

Scopelosoma, I36.

Scotobates, 258.

Scudderia, 4I.

Scutellerida, 84.

Scydmænidæ, 225.

Scydmænus, 225.

Scymnuts, 254.

Scythris, 90.

Scythropis, 282.

Sehirus, 84 .

Seira, 25.

Selandria, 293, 299.

Selenophorus, 218.

Selidosema, i 8 .

Semasia, Ioo.

Semiophora, I42.

Semioscopsis, 92.

Senotainia, I92.

Seoptera, 203.

Sepedon, 20 I.

Sepsidx, 205.

Sepsis, 205.

Serica, 263.

Sericoderus, 226.

Sericomyia, $\mathbf{8 7 .}$.

Sericophanes, 69.

Sericoponpilus, 335 .

Sericosomus, $24 \mathrm{I}$.

Sericostomatidæ, 3I.

Sericus, $2+1$.

Serphidae, 328.

Serpluts, 328.

Serropalpus, 359.

Sesia, I 2.

Sesiidre, I I2.

Setodes, 3I.

Seventen year locust, 56 .

Sheep tick, 2 Io.

Sialidxe, 29.

Sialis, 29.

Sibine, I 4.

Sierolomorpha, 333 .

Sigalphus, 307 .

Silplsa, 225.

Silphide, 224.

Silvants, 250.

Silver-fish, 25.
Simathis, 356.

Simuliidxe, I72.

Simulium, I 72 .

Sinea, 76.

Sinoxylon, 26r.

Siplilonurus, 28 .

Siphocoryne, 60.

Siphona, I92.

Siphonaptera, I60.

Siplionella, 207.

Siphonophora, 58, 60 .

Sirex, 30I.

Siricide, 301.

Sisyrosea, II4.

Sitodrepa, 260.

Sitona, 282.

Sitotroga, 95.

Sixeonotus, 70.

Skeletonizer, apple and th^rn, 356 .

Skippers, I54, I 55.

Slug, Bristly rose, 297. Pear and Cherry, 294.

Sinerinthus, I5I.

Smicra, 326.

Smicronyx, 283.

Smilia, 54, 254 .

Smintluridae, 25.

Sminthurus, 25.

Smodicuin, 266.

Snout butterfly, I56.

Soldier bug. Spined, 83.

Solenius, 338, 339.

Solenobia, I I4.

Solenopsis, 329.

Solenozopheria, 322 .

Solubea, 82.

Solva, I75.

Somatochlora, 36, 354 .

Somula. I 88 .

Sooty-wing, I 54 .

Soronia, 249.

Spallanzania, I94.

Spanioneura, 57 .

Spanotecuns, 3 I I.

Sparganothis, 99.

Spargoloma, I32.

Sparnopolius, 176 .

Spathius, 306.

Spermopliagus, $2 S o$.

Speyeria, I58.

Spliceridinmı, 224.

Spharocera, 200.

Spheropyx, $30 \%$.

Spherophoria, I 86.

Spharagenion, 40.

Sphecidre, 338 .

Sphecius, $3+1$.

Spluecodes, $3 \div 3$. 
Spliecodina, I 53.

Sphecodium, 343 .

Sphecomyia, I88.

Sphegigaster, $32 !$.

Sphegina, I86.

Sphenophorus, 288.

Sphex, 3to.

Sphicla, I37.

Sphindidae, 261 .

Sphindus, 26I.

Sphingidæ, I5I.

Splinx, I52.

Sphragisticus, 79.

Sphyracephala, 205.

Spilochalcis, 326.

Spilogaster, IgS.

Spilographa, 204.

Spilomena, 3+1.

Spilomyia, I88.

Spilosoma, I+8.

Spogostylum, I 76 .

Spring azure, I55.

Squash bug, 80 .

Stagnatophora, 90.

Staphylinidae, 226.

Staphylinus, 228.

Statira, 259.

Stelididae, 347.

Stelidota, 249.

Stelis, 3.7.

Stenamma, 329.

Stenelmis, 245 .

Stenichnenmon, 3I7, 3 I8.

Stenispa, 279.

Stenobotlirus, 39.

Stenocortus, 267.

Stenocranus, 47.

Stenodema, 74 .

Stenolophus, 219.

Stenoma, 93.

Stenomyia, 203.

Stenopa, 203.

Stenopleura, 303.

Stenoscelis, 288.

Stenosphenus, 270.

Stenotarsus, 253.

Stenotus, 71 .

Stenus, 226, 227.

Stephanocleonus, 285.

Steplianoderes, 289.

Stephanucha, 265.

Sterictiphora, 300.

Stethobaris, 286.

Sthenopis, $8_{5}$.

Stichopogon, I78.

Stictocephala, 53.

Stigmatomma, 328 .

Stigmus, 3tI.
Stilbosis, 90.

Stilpnus, 317.

Stiplirosoma, 69.

Stiretrus, 83.

Stobara, 47.

Stomoxys, I97.

Strangalia, 268.

Stratiomyia, I72.

Strationyidx, I72.

Stratussia, 203.

Strenoloma, I29.

Strepsiptera, 290.

Strigota, 229.

Stroggylocephalus, 49.

Stromatium, 267.

Strongalepta, 208.

Strongylitum, 259 .

Strongylocoris, 60.

Strongylogaster, 203, 294

Strongy logastroidea, 293.

Strophiona, 268.

Strymon, I55.

Stmrmia, I93.

Stylogaster, I89.

Stylopidie, 200.

Stylops, 200.

Sulplur, Clouded, 158. Clondless, I 59. Little, I5s.

Sunius, 227.

Swallow-tails, I59.

Swammerdania, 87.

Sword-bearer, 42.

Symmerista, 125 .

Symmorpluss, 336,337 .

Sympetrum, 36.

Simpherobius, 29.

Sympherta, 3 I2.

Symphora, 260.

Symphoromyia, I75.

Sympiesis, 32.3.

Symplecta, 162 .

Sympycilus, I So.

Sinaldis, 305 .

Symanthedon, II2.

Syncalypta, 2.47.

Sinclitat, 252 .

Sinchloë, i50.

Syruchlora, II9.

Synchroa, 259.

Syndyas, 182.

Syneclies, ISz.

Sineda, Iзт.

Sinelis, I 20.

Sincrgus, 3 I0.

Syneta, 273.

Syrulualonia. $3+6$.

Synothyreopus, 339. 
Syntomaspis, 325

Syntomidae, I50.

Syntormon, I\&o.

Syritta, ISS.

Syrphidx, IS4, 357.

Syrphoctonus, 3 I 2.

Syrphus, I86.

Sissaura, II6.

Systena, 279.

Systomspiesis, 323 .

Systropus, 176 .

Tabanidie, I73.

Tabanus, 174.

Tabuda, 176 .

Tachina, I94, 357

Tachinidre, I90, 357.

Tachinoplyyto, I9I.

Tachinus, 220.

Tachycellus, 2 I9.

Tachydromia, IS2.

Tachyporus, 229.

Tachypterellus, 284.

Tachys, $2 \mathrm{I} 3$.

Tachyspliex, $3+0$.

Tachytes, 3.0 .

Tacoma, I 10.

Tieniaptera, 204.

Treniopteryx, 27

Taeniorhynchus, I66.

Tailed blue, I55.

Tanyptera, I63.

Tanypus, I 66.

Taplirocerus, 245.

Tapinoma, 330.

Tapinostola, I36.

Tarache, I32.

Taracticus, I 78 .

Tarpela, 259.

Taxonus, 292, 293.

Telamona, 54 .

Telea, I50.

Teleas, 327.

Telenomus, 326.

Telephanus, 250 .

Telephoridxe, 234.

Telephorus, 234.

Telmatophilus, $25 \mathrm{I}$.

Telplussa, 95.

Temnostonia, I88.

Tenehrio, 258.

Tenelrionidie, 257,358 .

Teneluroides, 248.

Tenodera, 30.

Tenthredinidre, 202, 360 .

Tent caterpillar, I 22.

Tenthredo, 205.

Tenthredopsis, 295 .
Tephritis, 204.

Tephrochlamys, 200.

Tephroclystis, I2I.

Tephronota, 203.

Tephrosia, I 18 .

Terias, I58.

Termes, 26.

Termitidx, 26.

Tetanocera, 20I, 357.

Tetanolita, I27.

Tetanops, 203.

Tetrachrysis, 332.

Tetracis, $\mathbf{i} \mathbf{i}$.

Tetragoneuria, 35 .

Tetragonoderus, 216.

Tetraleurodes, 62.

Tetralonia, $3+6$.

Tetraloplia, 108

Tetraneura, 6I.

Tetraopes, 272.

Tetrastichidx, 323 .

Tetrastichus, 323 .

Tetropium, 266.

Tettigidea, 39.

Tettigonia, 48, 49 .

Tettigoniella, 18 .

Tettix, 39.

Teuchocnenis, I88.

Teucholabis, I6I.

Thalessa, 315.

Thalpochares, 133.

Thannotettix, 5I, 355 .

Thanaos, I 53 .

Thanasinus, 236.

Tharops, $240,243$.

Tliaumatotypidea, 307.

Thecabius, 6 I.

Thecla, I55, I56.

Thecodiplosis, I70.

Theisoa, 90.

Thelaira, I96.

Thelia, 53.

Thenira, 205.

Theresia, 106.

Thereta, I53.

Thereva, I77.

Therevide, 176.

Therina, I 1 .

Therioaphis, 58 .

Therion, 3I r.

Thermobia, 25.

Thermonectes, 222

Theronia, 3I4.

Thersiloclius, 310 .

Tlicanus, 230.

Thiodia, 98 .

Thistle butterfly, I 57

Tholeria, 104. 
Thoracophorus, 226.

Thorybes, 154 .

Thrinax, 293.

Thripidae, 4.

Thrips, 4.

Thioscidie, 243.

Throscus, 243.

Thyalta, 82 .

Thyatira, I 23.

Thyatiride, I23.

Thymalus, 248 .

Thymelicus, I 54 .

Thyreocoris, 84 .

Thyreodon, 3 I I.

Thyreus, I 53 .

Thyridae, I 3 .

Thyridopteryx, II4.

Thyris, I I 3 .

Thysanoptera, 44.

Thysanura, 25.

Tibicen, 56 .

Tibicina, 56 .

Tillomorpha, 270.

Timulla, 334 .

Tinea, 86.

Tineide, 85 .

Tineola, 86.

Tingidx, 77.

Tingis, 76,77 .

Tiphia, 333.

Tipula, I64.

Tipulide, 160.

Tischeria, 87 .

Tinetocera, 99.

Torlolachnus, 57.

Tolype, 122.

Tomarus, 251.

Tomaspis, 55.

Tomicus, 200.

Tomostethus, 299.

Tomoxia, 237.

Tornos, i 8 .

Tortriciclee, 96, 356 .

Tortricidia, I I 3 .

Tortrix, IOI.

Torymus, 325 .

Tosale. Iob.

Tommeyclla, 63.

Toxorhina, I6I.

Toxotus, 267.

Tracheloniris, 74 .

Trachysectus, 227 .

Tranlea, 36 .

Trapezonotus, 79 .

Trechus, 213 .

Tree crickets, 43.

Tree-hopper, Buffalo, 53.

Trematoprgus, 3 I .
Tremlicx, 301 .

Triachus, 27t.

Trialemrodes, 62.

Tribolium, 258.

Trichiocampus, 397, 360 .

Trichionotus, 265 .

Trichiosoma, 296.

Trichocera, 162.

Trichodes, 230.

Trichodesina, 200.

Tricholita, 138 .

Trichopepla, $8 \mathrm{I}$.

Trichopherus, 24 I.

Trichophora, I95.

Trichopoda, 100.

Trichoptera, 30 .

Trichopteryx, 231.

Trichotaphe, 94.

Trichrysis, 332 .

Tricrania, 238.

Tricyphona, 163.

Tridactylus, 43 .

Triepeolus, 345 .

Trifurcula, 89.

Triga, 226 .

Trigonalidae, 308 .

Trigonophora, I 36 .

Trigonotylus, 73 .

Trimerotropis, 40.

Trineura, I8.

Triodonta, I87.

Trioza, 56 .

Triphleps, 74 .

Triptogon, I5I.

Trithabda, 277.

Triloma, 251 .

Tritoxa, 202.

Trixagidie, 243 .

Troctes, 26.

Trogoderma, 247 .

Trogositidae, 247.

Trogus, 317.

Tromatobia. 314.

Tropaca, $15 \mathrm{I}$.

Tropidia, 187.

Tropidosteptes, 72 .

Tropisteriuls, 223 .

Trox, 263.

Trypanisina, 94.

Trypeta, 204.

Trypetes, 348 .

Trypeticle, 203.

Trypherus, 235.

Trypopitys, 260.

Trypoxylon, 339.

Twig-girdler, Hickory, 27 I.

Twig pruner, 267.

Tychius, 284. 
Tyloderma, 287.

Tylonotus, 267.

Tymnes, 275 .

Typhrea, 252.

Typhlocyba, 52.

Typocerus, 268.

Typophorus, 275 .

Typopsilopa, 206.

Tyrus, 23I.

Uloma, 258.

Upis, 258.

Uranotes, I 56.

Urocerus, 301 .

Urographis, 27I.

Uroplata, 279.

Urosigalphus, 307.

Utetheisa, I49.

Valentina, 91.

Valgus, 265.

Vanduzea, 55.

Vanessa, I57.

Varneria, III.

Veliidæ, 68.

Venusia, I21.

Vespa, 337.

Vespidx, 337.

Vespula, 337.

Viceroy, I57.

Viereckella, 345 .

Villa, 176 .

Violet tip, I 57.

Vipionidx, 302.

Vitula, iro.

Volucella, I87.

Walshia, 90.

Wanda, I08.

Wasp, Common, 337. Potter, 336.

Webworm, Juniper, 93.

Weevil, Bean, 280.

Grain, 288.

New York, 280.

Pea, 280.

Rice, 288.

Rhubarb, 285 .

Rose, 28I.

Sawtoothed grain, 250.

Strawberry, 285 .

White pine, 283 .

White ant, 26.

Fly, greenhouse, 62.

Winthemia, I94

Wood-nymph, Beautiful, I34.

Pearl, 134.
Worm, Black-headed cranberry, 99.

Carpenter, II 2 .

Clover hay, Io6.

Cranberry fruit, I09.

Fall web, 148.

Green clover, I 26.

Grape vine root, 275 .

Green-stripped maple, I50.

Lesser apple, Ioo.

Orange-striped oak, I5o.

Pickle, I0+.

Railroad, 204.

Raspberry fruit, 246.

Tobacco or tomato, ${ }^{2}{ }^{2}$.

Wyeomyia, I66.

Xabea, 43.

Xanthogramma, I86.

Xantholinus, 227.

Xantholobus, 54, 55.

Xanthomelana, I9I.

Xanthomelanodes, I9I.

Xanthonia, 275.

Xanthorhoë, I20.

Xanthosarus, $3+7$

Xanthoteras, 3I9.

Xanthotype, II7.

Xenetus, 69.

Xenidæ, 290.

Xenoborus, 72.

Xenodusa, 230.

Xenoglossa, 346 .

Xenos, 290.

Xerophløea, 49.

Xestobium, 260.

Xestocephalus, 355 .

Xestocis, 262.

Xestocoris, 79.

Xestocrabro, 339.

Xestoleptıra, 268.

Xiphidium, 42.

Xiphura, I63.

Xiphydria, 301.

Xiphydriidx, 301.

Xyleborus, 290.

Xyelidæ, 291.

Xylestia, 86.

Xyletinus, 260.

Xylina, I38.

Xylobiops, 26r.

Xylocopa, 348.

Xylocopidx, 348 .

Xylocoris, 74 .

Xylomyia, 175.

Xylophagus, I74.

Xylophanes, 153.

Xylophruridea, $3 \mathbf{I} 4$.

Xylopinus, 258 . 
Xylorictidx, 93.

Xyloryctes, 265.

Xylota, I88, 357.

Xylotrechus, 269.

Xystoteras, 3I9.

Yellow jacket, 337 .

Spot, 154 .

Youngomyia, 17o.

Yponomeuta, 95.

Yponomeutidæ, 95.

Ypsia, I29.

Ypsolophus, 94.

Zabrotes, 280 .

Zale, I29.
Zamicrotoridea, $3 \mathbf{I} 6$.

Zanclognatha, I28.

Zaræa, 296.

Zelus, 76 .

Zenodostis, 236.

Zerene, I58.

Zethus, 336.

Zeugophora, 273.

Zeuzera, I1 2 .

Zodion, I 189.

Zonitis, 238.

Zoöphthorus, 316.

Zoötrephes, 312.

Zophodia, I Io.

Zygogramma, 276. 






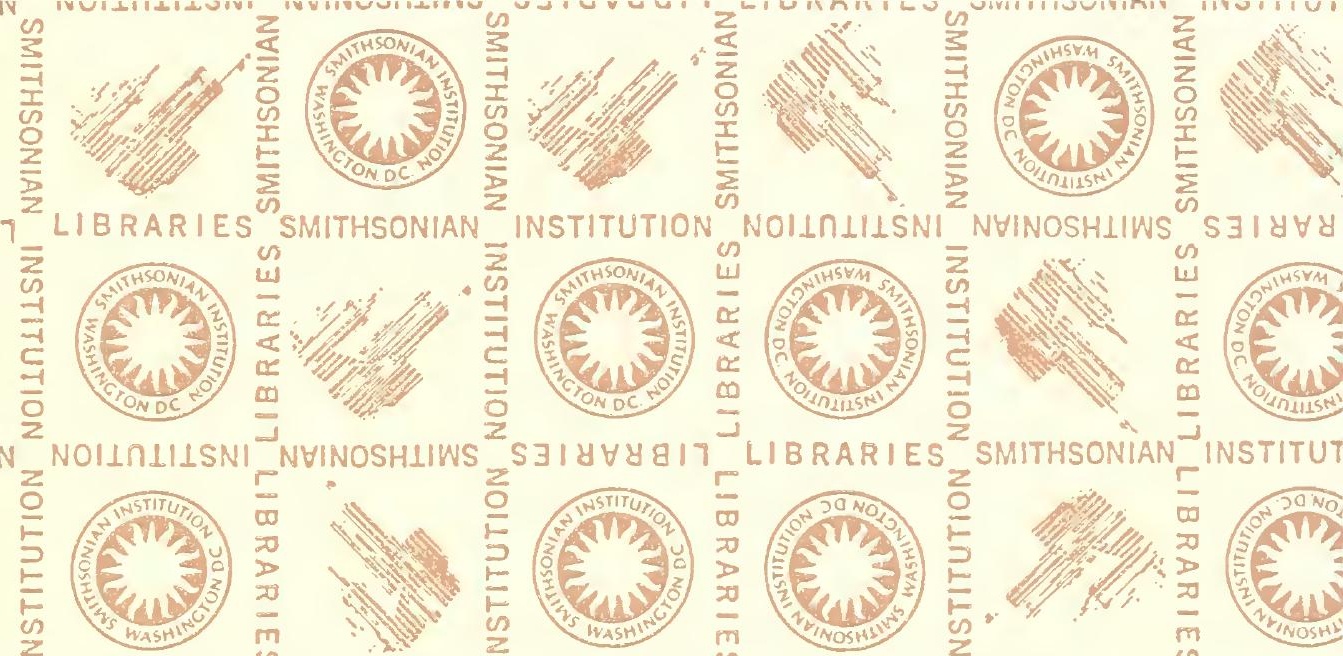

INSTITU
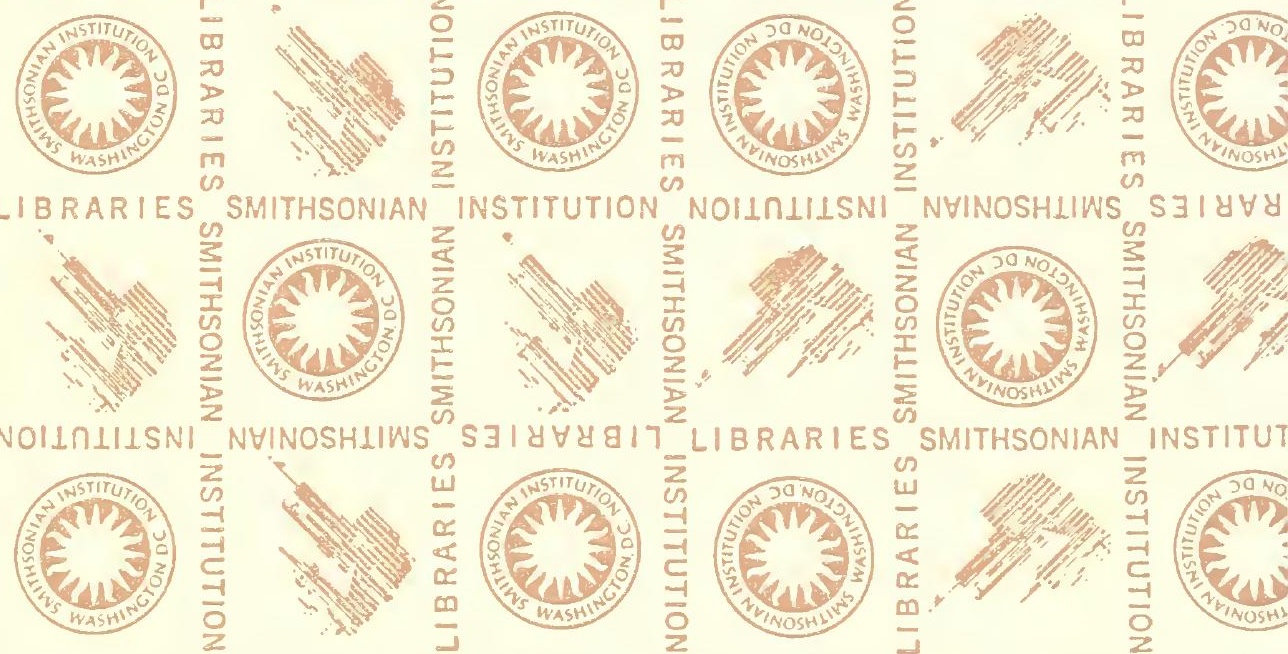

LIBRARIES SMITHSONIAN
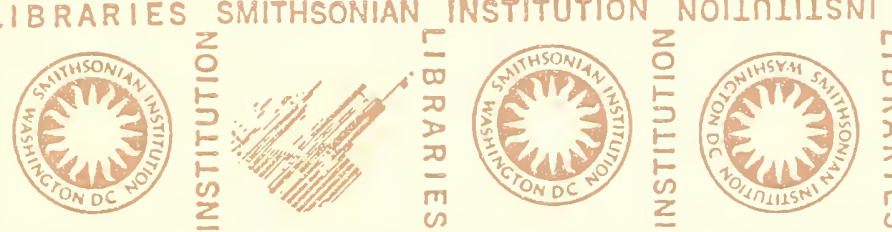

NHINOSHIIWS

INSTITUT
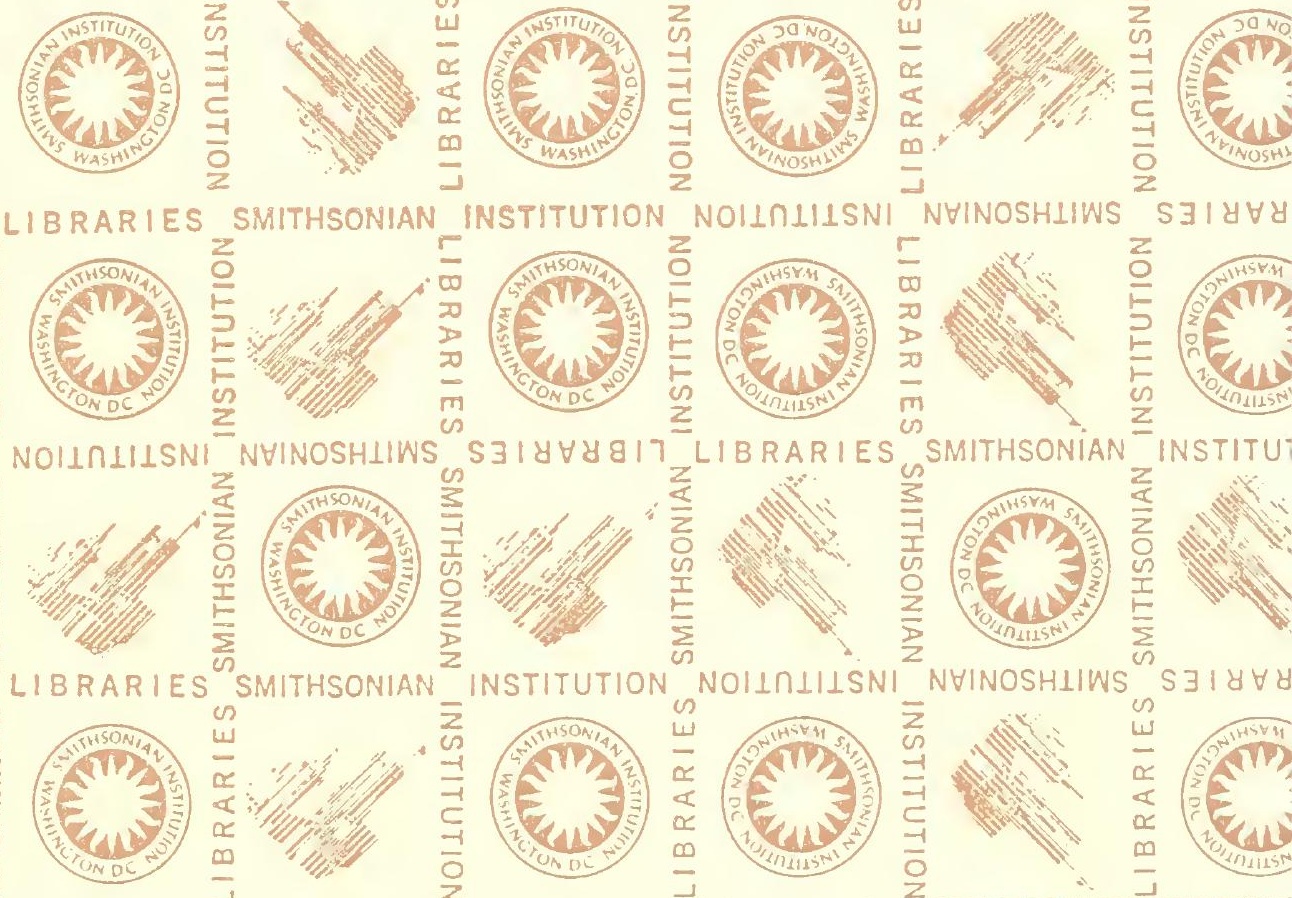

NOIIN1IISNI NYINOSHLIWS
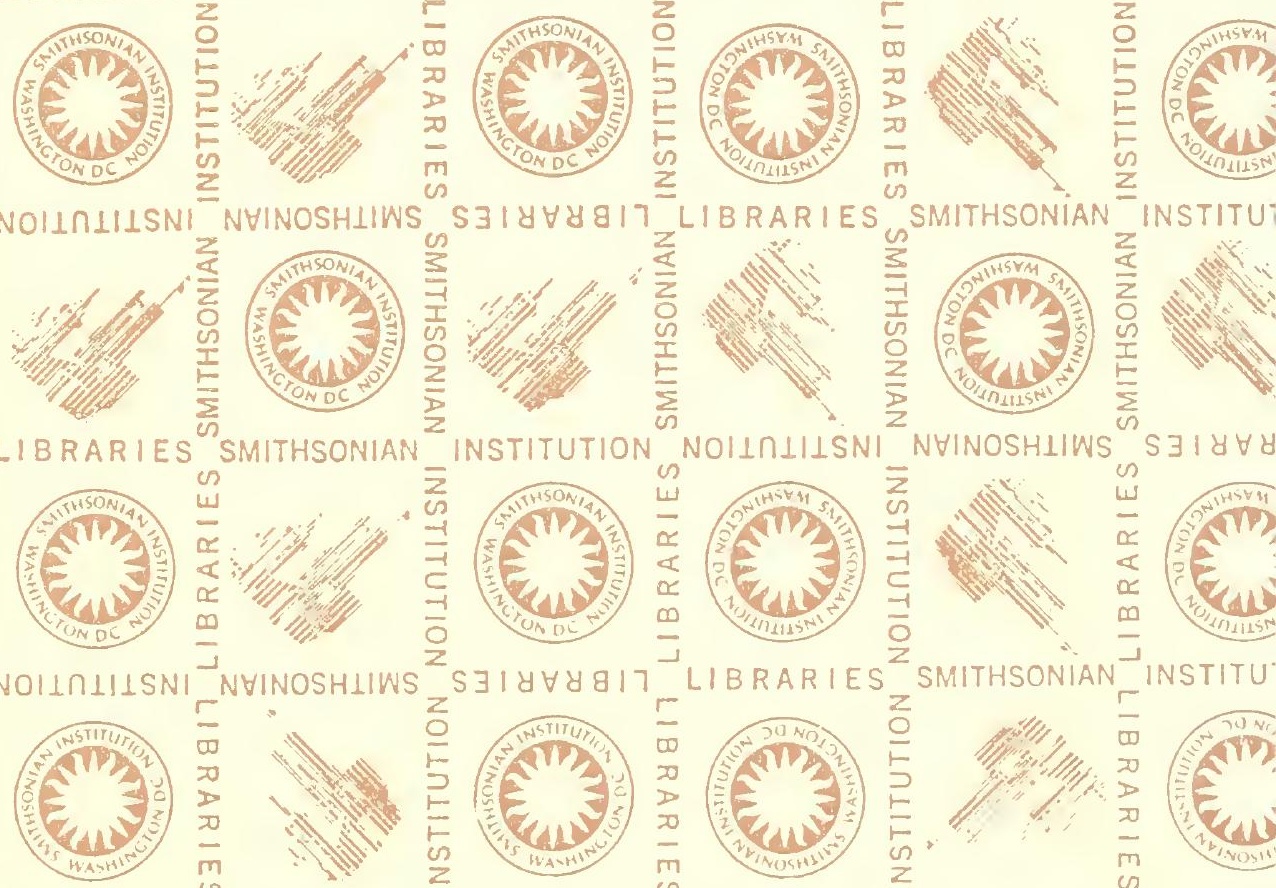

INSTITU
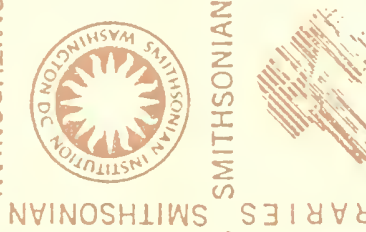

LIBRARIES SMITHSONIAN
INSTITUTION
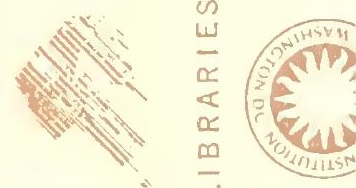
SMITHSONIAN

INSTITU
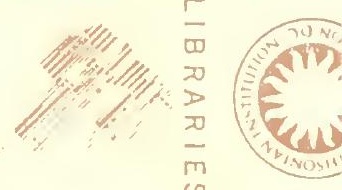


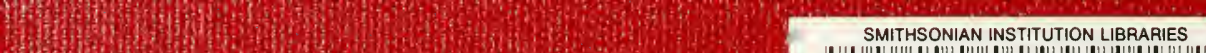

